\author{
This electronic thesis or dissertation has been \\ downloaded from the King's Research Portal at \\ https://kclpure.kcl.ac.uk/portal/
}

\title{
Population Pharmacokinetics of Enoxaparin during the Antenatal Period
}

\section{Patel, Jignesh}

\section{Awarding institution:}

King's College London

The copyright of this thesis rests with the author and no quotation from it or information derived from it may be published without proper acknowledgement.

\section{END USER LICENCE AGREEMENT}

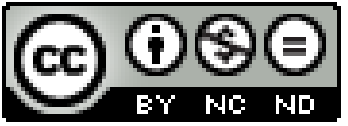

Unless another licence is stated on the immediately following page this work is licensed

under a Creative Commons Attribution-NonCommercial-NoDerivatives 4.0 International

licence. https://creativecommons.org/licenses/by-nc-nd/4.0/

You are free to copy, distribute and transmit the work

Under the following conditions:

- $\quad$ Attribution: You must attribute the work in the manner specified by the author (but not in any way that suggests that they endorse you or your use of the work).

- $\quad$ Non Commercial: You may not use this work for commercial purposes.

- $\quad$ No Derivative Works - You may not alter, transform, or build upon this work.

Any of these conditions can be waived if you receive permission from the author. Your fair dealings and other rights are in no way affected by the above.

Take down policy

If you believe that this document breaches copyright please contact librarypure@kcl.ac.uk providing details, and we will remove access to the work immediately and investigate your claim. 


\section{Population Pharmacokinetics of}

Enoxaparin during the Antenatal Period

Jignesh Prakash Patel

DOCTOR OF PHILOSOPHY

KING'S COLLEGE LONDON

2013 
ગુણાતીતૉડક્ષરં બ્ર્મ

ભગવાનૂ પુરુષ્તમઃ.

જનો જાનાજિદં સત્યં

મુરયતે ભવબન્દનાત્ 


\section{Abstract}

The physiological changes of pregnancy alter the pharmacokinetics of low molecular weight heparins (LMWH). The optimal dosing strategy of LMWH for the treatment of antenatal venous thromboembolism (VTE) is not known and this has led to significant variation in prescribing practice when women are managed for antenatal VTE with LMWH.

The aim of this thesis is to describe the pharmacokinetics of enoxaparin during pregnancy using the method of population pharmacokinetic (PK) modelling. In addition, pregnant women's thrombin generation and D-dimers are measured and described and their views and adherence to enoxaparin are explored.

Pregnant women prescribed enoxaparin at King's College Hospital were eligible for recruitment. Subjects ( $n=123)$ had up to 3 anti-Xa activities drawn per clinic visit (monthly), contributing 795 anti-Xa activities for PK modelling purposes. A one compartment model, with a combined error model, produced a robust enoxaparin antenatal PK model, with weight, baseline lean body weight and gestation found to be significant covariates on enoxaparin antenatal PK. Simulations from the final PK model revealed that a once daily dose of enoxaparin is appropriate in this setting.

D-dimer concentrations were found to increase in line with gestation $(r=0.382)$. Thrombin generation was also increased during the antenatal period, with enoxaparin found to influence the different thrombin generation parameters in a dose-dependent manner. Findings from the adherence aspect of this study revealed that women were highly adherent to enoxaparin antentally (mean $97.92 \%$ ) and demonstrates that women are prepared to inject themselves with a parenteral medication, if they feel it is protecting theirs and their unborn baby's health; this belief does impact on adherence to LMWH during the postnatal period, where in some women adherence drops (mean 92.75\%).

This study describes the pharmacokinetic profile of enoxaparin during pregnancy and provides compelling evidence for enoxaparin once daily dosing for managing antenatal VTE. 


\section{Table of Contents}

Abstract 3

Table of contents 4

Table of figures 10

Table of tables 12

List of acronyms

$\begin{array}{lr}\text { Acknowledgements } & 18\end{array}$

Publications relevant to this thesis $\quad 22$

Preface 23

$\begin{array}{ll}\text { Chapter } 1 & 26\end{array}$

1.1 Blood coagulation 26

1.1.1 An overview 26

1.1.2 Initiation 27

$\begin{array}{lll}1.1 .3 & \text { Amplification } & 27\end{array}$

$\begin{array}{lll}1.1 .4 & \text { Propagation } & 29\end{array}$

1.1.5 Localisation 29

1.2 Thrombin generation (TG) 30

1.2.1 Calibrated automated thrombography (CAT) 31

1.2.2 Altering CAT assay conditions 34

1.2.3 Assay variability 34

1.2.4 Thrombin generation in pregnancy 35

1.2.5 Hexadimethrine bromide (polybrene) and TG 36

1.3 Venous thromboembolism 37

1.3.1 Incidence 38

1.3.2 Survival and recurrence 38

$\begin{array}{lll}1.3 .3 & \text { Risk factors } & 39\end{array}$

1.3.4 Treatment 40

1.4 Heparin 42

1.4.1 The discovery of heparin 42

1.4.2 First in man studies $\quad 44$

1.4.3 Unfractionated heparin 44

1.4.4 Pharmacology 45

1.4.5 Pharmacokinetic profile 46

1.4.6 Adverse effects 46

1.5 Low molecular weight heparins 48

1.5.1 Pharmacokinetics of low molecular weight heparins 50

1.5.2 Adverse effects of low molecular weight heparins 52

1.5.3 Low molecular weight heparin use in the UK 53

1.6 Venous thromboembolism in pregnancy 53

1.6.1 Epidemiology 53

1.6.2 Pathophysiology 
1.6.3 Risk factors for antenatal venous thromboembolism $\quad 55$

1.6.4 Medication use in pregnancy 56

1.6.5 Anatomical and physiological changes of pregnancy 57

1.6.5.1 Anatomical alterations 57

1.6.5.2 Physiological alterations $\quad 57$

1.6.6 Treatment of antenatal venous thromboembolism $\quad 59$

1.7 Pharmacokinetic (PK) studies evaluating LMWH during pregnancy 62

1.7.1 Pharmacokinetic studies of LMWH during pregnancy 62

$\begin{array}{lll}1.8 & \text { Pharmacokinetic analysis } & 73\end{array}$

1.8.1 Non-compartmental pharmacokinetic analysis 73

1.8.2 Compartmental analysis 73

1.8.2.1 Naïve pooled approach $\quad 74$

1.8.2.2 Standard two-stage approach $\quad 74$

1.8.2.3 Nonlinear mixed effects modelling (NLMEM)

1.8.2.4 Population modelling 75

1.8.2.4.1 Structural model $\quad 76$

1.8.2.4.2 Stochastic model 76

1.8.2.5 Estimation methods and software $\quad 77$

$\begin{array}{lll}\text { 1.8.2.6 Covariates } & 78\end{array}$

1.8.2.7 The model development process 79

1.8.3 Advantages of population analysis $\quad 80$

1.8.4 Model evaluation and validation 80

1.8.5 Application of population PK analysis 81

1.8.5.1 Examples from drug development 81

1.8.5.2 Examples from clinical practice 82

1.8.6 Application of population PK analysis in pregnancy 84

1.8.7 Enoxaparin pharmacokinetic PK modelling studies 86

1.8.7.1 PK models of enoxaparin outside of pregnancy 86

1.8.7.2 Antenatal PK model of enoxaparin 92

1.9 Hypothesis 94

1.9.1 Aims of the study 94

$\begin{array}{ll}\text { Chapter } 2 & 95\end{array}$

2.1 Methodology 95

2.1.1 Optimal design 96

2.1.2 Data collection 97

2.1.2.1 Patient recruitment 98

2.1.2.2 Sample collection 99

2.1.2.3 Sample handling and analysis 100

2.1.2.3.1 Clotted sample $\quad 100$

2.1.2.3.2 EDTA sample 100

2.1.2.3.3 Citrate sample I 100

$\begin{array}{ll}\text { 2.1.2.3.4 Citrate sample II } & 101\end{array}$ 
2.1.2.4 Thrombin generation experiments

2.1.2.4.1 Plasma preparation $\quad 101$

2.1.2.4.2 Calibrated automated thrombography 102

2.1.2.5 Birth and postpartum 102

2.1.2.6 Control group 103

2.1.3 Data entry, storage and transfer 103

2.1.4 Population modelling and simulation 104

$\begin{array}{lll}2.1 .5 & \text { Ethical approval } & 104\end{array}$

$\begin{array}{lr}\text { Chapter } 3 & 105\end{array}$

3.1 Clinical information on study patients 105

3.1.1 Eligibility and recruitment 105

3.1.2 Consented patients 105

$\begin{array}{lll}3.1 .3 & \text { Study population } & 106\end{array}$

3.1.4 Patient outcomes 107

3.1.5 Indication for enoxaparin and doses prescribed 108

3.1.6 Women suffering from pathological thrombosis 110

3.1.7 Long-term warfarin patients switched to enoxaparin $\quad 110$

3.1.8 Changes in U\&Es and FBC during pregnancy 111

3.1.9 Birth and delivery information 115

3.1.10 Outcome compared to control group 117

$\begin{array}{ll}\text { Chapter } 4 & 119\end{array}$

4.1 Population pharmacokinetic modelling of enoxaparin 119

4.1.1 Methods 120

4.1.1.1 Data entry and formatting $\quad 120$

4.1.1.2 Development of enoxaparin antenatal base model $\quad 120$

4.1.1.3 Evaluation of enoxaparin antenatal base model 120

4.1.1.4 Parameterisation of base model with covariates 121

4.1.1.5 Visual predictive check 122

4.1.1.6 Simulation 122

4.2 Results: population PK modelling of enoxaparin 123

4.2.1 Breadth of anti-Xa activities drawn 123

4.2.2 Base model development 126

4.2.3 Handling below level of quantification (BLQ) data 127

4.2.4 Inter-occasion variability 128

4.2.5 Goodness of fit evaluation of the base model 131

4.2.6 Covariate analysis 137

4.2.7 Evaluation of the final model 146

4.2.8 Visual predictive check of the final PK model 149

4.2.9 Simulation work 150

4.2.9.1 Developing a weight model 150

4.2.9.2 Simulations 155

4.2.9.3 Percentage of women with a trough $<0.01 \mathrm{IU} / \mathrm{mL} \quad 160$ 
4.3.1 Assumptions made during the PK modelling process 165

4.3.2 Strengths of this study and the final model 165

4.3.3 The clinical case for once daily enoxaparin 165

4.3.3.1 Thrombus regression: once versus twice daily 166

4.3.3.2 Lessons from early cardiology dose-ranging studies 167

4.3.4 Mechanical valve prophylaxis during pregnancy 170

4.3.5 VTE prophylaxis during the antenatal period 172

4.4 Conclusion 174

$\begin{array}{ll}\text { Chapter } 5 & 175\end{array}$

5.1 D-dimer concentrations during pregnancy 175

5.1.1 D-dimer measurements 175

$\begin{array}{lll}5.1 .2 & \text { Background } & 175\end{array}$

5.1.3 D-dimer assays: principle of assay 177

$\begin{array}{lll}\text { 5.1.4 D-dimer concentrations during pregnancy } & 177\end{array}$

5.1.5 Studies of D-dimers during uncomplicated pregnancy 178

5.1.6 Studies of D-dimers during complicated pregnancy 182

5.1.7 The impact of LMWH on D-dimer concentrations 183

5.2 Aim and method 184

5.3 Results 184

5.3.1 Women prescribed prophylactic doses of enoxaparin 187

5.3.2 Women prescribed treatment doses of enoxaparin 188

5.3.3 The influence of ethnicity on d-dimer concentrations 189

5.4 Discussion and implications for practice 190

5.4.1 Strengths, limitations and future work 191

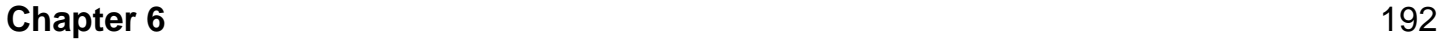

6.1 Thrombin generation during pregnancy 192

6.1.1 Background 192

6.1.2 Limitations of thrombin generation (TG) testing 192

6.1.3 Influence of anticoagulant therapy on the TG test 195

6.1.4 Studies assessing TG in pregnancy 199

6.1.4.1 TG during healthy pregnancy 199

6.1.4.2 TG in women on LMWH in pregnancy 202

6.2 Objectives and methods 204

6.3 Results 205

6.3.1 Internal thrombin generation variability 205

6.3.2 Overall thrombin generation results for the cohort 206

6.3.2.1 TG results for women on prophylaxis and treatment 207

6.3.2.2 Correlation of anti-Xa and thrombin generation 208

6.3.2.3 Lag-time 209

6.3.2.4 Endogenous thrombin potential (ETP) 211

6.3.2.5 Peak height 213 
6.3.2.6 Time to peak 215

6.3.2.7 Start tail 217

6.3.2.8 Thrombin generation velocity 218

6.3.2.9 Ethnicity and the thrombin generation test 220

6.3.2.10 The dynamic state of thrombin generation 222

6.4 Discussion 223

6.5 Can polybrene neutralise enoxaparin in the TG assay? 228

6.5.1 Background 228

6.5.2 Aim 230

6.5.3 Methodology 230

6.5.3.1 Concentration of PB used in the experiments 231

6.5.3.2 In-vitro study of differing PB concentration 231

$\begin{array}{lll}6.5 .4 & \text { Results } & 234\end{array}$

6.5.5 Discussion 238

6.5.5.1 Effect observed: treatment \& prophylactic doses 239

6.5.5.2 Are there alternative enoxaparin neutralising agents? 240

6.5.6 Summary and future work 240

$\begin{array}{ll}\text { Chapter } 7 & 242\end{array}$

7.1 Women's views on adherence to enoxaparin 242

7.1.1 Medication adherence 242

7.1.1.1 Background 242

7.1.1.2 Measures of adherence 244

7.1.1.3 Medication usage during pregnancy 245

7.1.1.4 Medication adherence during pregnancy 245

7.1.1.5 Medication adherence study objectives 247

7.2 Method 248

7.2.1 Assessment of adherence 248

7.2.2 Assessing beliefs about medications 248

7.2.2.1 Adapting the BMQ for the pregnant population 250

7.2.2.2 Administration and completion of the questionnaire 251

7.2.3 Data analysis 251

7.2.3.1 Calculation and classification of patients adherence 252

7.2.3.2 Necessity and concerns differential 252

7.2.3.3 Relationships 253

7.2.3.4 Additional questions 253

7.3 Results 253

7.3.1 Demographic information and response rate 253

7.3.2 Necessity-concerns differential 255

7.3.3 Necessity and concerns about enoxaparin 258

$\begin{array}{lll}\text { 7.3.4 Additional questions } & 259\end{array}$

7.3.5 Voluntary comments made by women 260

7.4 Discussion 263 
7.4.1 Additional questions asked in the questionnaire

7.4.2 Limitations 267

7.4.3 Implications for practice and future work 268

Chapter 8

8.1 Implications for practice 269

8.1.1 Recommendations 269

8.1.1.1 Once versus twice a day for antenatal VTE? 269

8.1.1.2 Prophylactic dosing of low molecular weight heparins 273

8.1.1.3 Do obese patients require special consideration? 274

8.1.1.4 Adherence to LMWH 275

8.1.2.1 Exceptions to once daily LMWH I: Valves 276

8.1.2.2 Exceptions to once daily LMWH II: AT-deficiency 278

8.1.3 Management of 'treatment' doses at delivery 278

8.1.4 Recommended future research priorities 279

8.1.5 Conclusion 280

$\begin{array}{ll}\text { Bibliography } & 281\end{array}$

$\begin{array}{lll}\text { A Appendix I } & \text { Study information document given to eligible subjects } & 320 \\ \text { B Appendix II } & \text { Consent form used during the study } & 326 \\ \text { C Appendix III } & \text { Ethics committee approval } & 327 \\ \text { D Appendix IV } & \text { King's College Hospital R\&D approval } & 328 \\ \text { E Appendix V } & \text { NONMEM control stream for base model } & 330 \\ \text { F Appendix VI } & \text { NONMEM control stream for base model with M3 error method } & 331 \\ \text { G Appendix VII } & \text { NONMEM bootstrap code for base model } & 332 \\ \text { H Appendix VIII } & \text { NONMEM control stream for final enoxaparin pregnancy model } & 333 \\ \text { I Appendix IX } & \text { NONMEM bootstrap code for final enoxaparin model } & 335 \\ \text { J Appendix X } & \text { 'R' code for visual predictive check of final model } & 336 \\ \text { K Appendix XI } & \text { The adapted pregnancy beliefs about medication questionnaire } & 338\end{array}$ 


\section{Table of Figures}

$\begin{array}{llll}\text { Figure } & 1.1 & \text { An overview of blood coagulation } & 28\end{array}$

Figure $1.2 \quad$ The thrombin generation curve 33

$\begin{array}{lll}\text { Figure } & 1.3 & \text { The pathophysiology of venous thromboembolism }\end{array}$

Figure $\quad 1.4 \quad$ The pharmacological action of unfractionated heparin 45

Figure $\quad 1.5 \quad$ The pharmacological action of low molecular weight heparins 49

Figure $\quad 1.6 \quad$ Anti-Xa activity following s/c injection for UFH and LMWH 52

Figure $\quad 1.7 \quad$ Key steps followed in the PK modelling process 79

Figure $2.1 \quad$ An overview of the methodology adopted for this study 95

$\begin{array}{llll}\text { Figure } & 3.1 & \text { Changes in } \mathrm{Cr}, \mathrm{Ur}, \mathrm{Hb} \text {, albumin, MPV and platelets } & 114\end{array}$

$\begin{array}{llll}\text { Figure } & 3.2 & \text { Changes in } \mathrm{CrCl} \text { and weight during pregnancy } & 115\end{array}$

$\begin{array}{llll}\text { Figure } & 4.1 & \text { Steps followed in the PK process to this point in the thesis } & 119\end{array}$

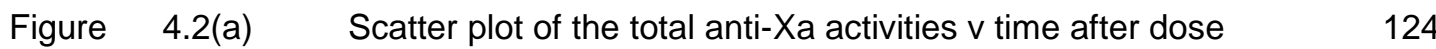

$\begin{array}{llll}\text { Figure } \quad 4.2(\mathrm{~b}) \quad \text { Scatter plot of the total anti-Xa activities } v \text { weeks amenorrhea } & 124\end{array}$

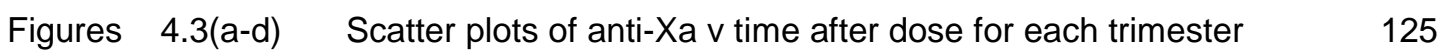

$\begin{array}{lll}\text { Figure } & 4.4(\mathrm{a}) \quad \text { ETA of CL (IOV) versus trimester } & 130\end{array}$

$\begin{array}{lll}\text { Figure } & 4.4(\mathrm{~b}) \quad \text { ETA of } \mathrm{Vd}(\mathrm{IOV}) \text { versus trimester } & 130\end{array}$

$\begin{array}{llll}\text { Figure } & 4.5 & \text { Observations versus population predictions } & 131\end{array}$

$\begin{array}{lll}\text { Figure } & 4.6 & \text { Observations versus individual predictions }\end{array}$

$\begin{array}{llll}\text { Figure } & 4.7 & \text { CWRES versus population predictions } & 132\end{array}$

$\begin{array}{llll}\text { Figure } & 4.8 & \text { CWRES versus time after dose } & 133\end{array}$

Figure $4.9 \quad$ CWRES versus time 133

$\begin{array}{lll}\text { Figure } & 4.10 & \text { CWRES versus gestation }\end{array}$

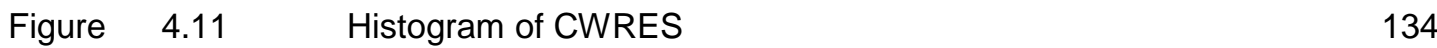

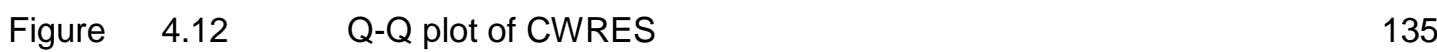

Figure $4.13 \quad$ Histogram of ETA1 (CL) 135

$\begin{array}{llll}\text { Figure } & 4.14 & \text { Histogram of ETA2 }(\mathrm{Vd}) & 136\end{array}$

$\begin{array}{llll}\text { Figure } & 4.15 & \text { ETA1 }(\mathrm{CL}) \text { versus age } & 137\end{array}$

$\begin{array}{llll}\text { Figure } & 4.16 & \mathrm{ETA} 1(\mathrm{CL}) \text { versus creatinine clearance } & 138\end{array}$

$\begin{array}{llll}\text { Figure } & 4.17 & \mathrm{ETA} 1(\mathrm{CL}) \text { versus ethnicity } & 138\end{array}$

$\begin{array}{llll}\text { Figure } & 4.18 & \text { ETA1 }(C L) \text { versus lean body weight } & 139\end{array}$

$\begin{array}{lll}\text { Figure } & 4.19 & \text { ETA1 }(\mathrm{CL}) \text { versus weight }\end{array}$

$\begin{array}{lll}\text { Figure } & 4.20 & \\ & \text { ETA1 }(C L) \text { versus creatinine }\end{array}$

$\begin{array}{llll}\text { Figure } & 4.21 & & 141\end{array}$

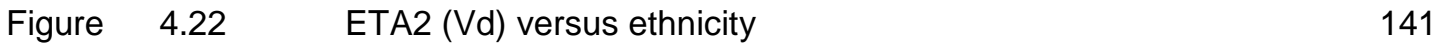

$\begin{array}{lll}\text { Figure } & 4.23 & \text { ETA2 }(\mathrm{Vd}) \text { versus lean body weight }\end{array}$

$\begin{array}{llll}\text { Figure } & 4.24 & \mathrm{ETA} 2(\mathrm{Vd}) \text { versus weight } & 142\end{array}$

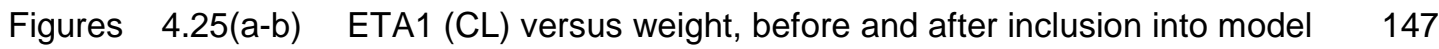

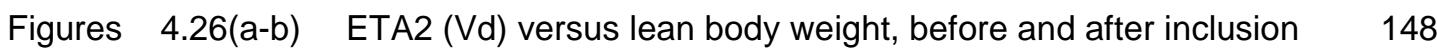

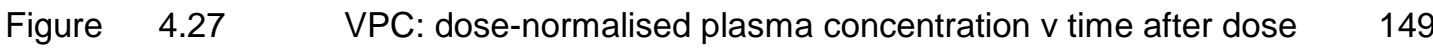

Figure $\quad 4.28 \quad$ VPC breakdown according to each trimester 150 
Figure $\quad 4.29$

Box-plot of first recorded weight for each subject (ethnicity)

Figure $\quad 4.30$

VPC for final weight model developed for simulation purposes

Figures

$4.31(a-b)$

3 hour anti-Xa activity for once (a) and twice daily (b)

Figure 4.32(b) Trough anti-Xa activity for twice daily

Figures $\quad 4.33(a-b) \quad 3$ hour and trough anti-Xa activity for once (a) v twice daily (b) 159

$\begin{array}{llll}\text { Figure } & 4.34 & \text { Simulation of prophylactic enoxaparin using RCOG dosing } & 173\end{array}$

$\begin{array}{lll}\text { Figure } \quad 5.1 \quad \text { Diagrammatic representation of the formation of D-dimers } & 175\end{array}$

Figure $\quad 5.2 \quad$ Scatter plot of log D-dimer concentrations v weeks amenorrhea

185

Figure 5.3

Box-plot of D-dimer concentrations according to each trimester

186

Figure $\quad 5.4$

Box-plot of D-dimer concentrations in prophylactic dosing

187

Figure 5.5

Box-plot of D-dimer concentrations in treatment dosing

Figure $6.1(\mathrm{a})$

Scatter-plot of lag-times $v$ weeks amenorrhea

210

Figure $6.1(\mathrm{~b})$

Box-plot of lag-times according to each trimester

210

Figure $\quad 6.2(\mathrm{a}) \quad$ Box-plot of lag-times according to each trimester (prophylaxis)

Figure $6.2(\mathrm{~b})$

Box-plot of lag-times according to each trimester (treatment)

211

Figure $\quad 6.3(\mathrm{a})$

Scatter-plot of ETP $\vee$ weeks amenorrhea

212

Figure $\quad 6.3(\mathrm{~b})$

Box-plot of ETP according to each trimester

212

Figure $\quad 6.4(\mathrm{a})$

Box-plot of ETP according to each trimester (prophylaxis)

213

Figure $\quad 6.4(b)$

Box-plot of ETP according to each trimester (treatment)

213

Figure $\quad 6.5(\mathrm{a})$

Scatter plot of peak $v$ weeks amenorrhea

214

Figure $\quad 6.5(\mathrm{~b})$

Box-plot of peak according to each trimester

214

Figure $\quad 6.6(\mathrm{a})$

Box-plot of peak according to each trimester (prophylaxis)

215

Figure $\quad 6.6(\mathrm{~b})$

Box-plot of peak according to each trimester (treatment)

215

Figure $\quad 6.7(\mathrm{a})$

Scatter plot of time to peak $v$ weeks amenorrhea

216

Figure $\quad 6.7(\mathrm{~b})$

Box-plot of time to peak according to each trimester

216

Figure $\quad 6.8(\mathrm{a})$

Box-plot of ttp according to each trimester (prophylaxis)

216

Figure $\quad 6.8(\mathrm{~b})$

Box-plot of ttp according to each trimester (treatment)

216

Figure $\quad 6.9(\mathrm{a})$

Scatter plot of start tail $v$ weeks amenorrhea

217

Figure $\quad 6.9(\mathrm{~b})$

Box-plot of start tail according to each trimester

217

Figure

$6.10(a)$

Box-plot of start tail according to each trimester (prophylaxis)

218

Figure $\quad 6.10(b)$

Box-plot of start tail according to each trimester (treatment)

218

Figure

$6.11(a)$

Scatter plot of TG velocity v weeks amenorrhea

219

Figure

6.11 (b)

Box-plot of TG velocity according to each trimester

219

Figure

$6.12(a)$

Box-plot of TG velocity according to each trimester (prophylaxis)

219

Figure

6.12 (b)

Box-plot of TG velocity according to each trimester (treatment)

219

Figure

$6.13(a)$

TG curves of plasma spiked with $0.05 \mathrm{IU} / \mathrm{mL}$ enoxaparin with $\mathrm{PB}$

232

Figure $\quad 6.13(b)$

TG curves of plasma spiked with $0.60 \mathrm{IU} / \mathrm{mL}$ enoxaparin with PB

233

Figure $\quad 7.1$

The practicalities and perceptions model of adherence

Figure $\quad 7.2$

The pregnancy model of adherence 


\section{Table of Tables}

\begin{tabular}{|c|c|c|c|}
\hline Table & 1.1 & Risk factors for venous thromboembolism & 40 \\
\hline Table & 1.2 & Summary of the ACCP recommendations for VTE management & 42 \\
\hline Table & 1.3 & Distribution of heparin in various tissues & 43 \\
\hline Table & 1.4 & The differences between commercially available LMWH & 48 \\
\hline Table & 1.5 & Doses of LMWH recommended in the UK & 53 \\
\hline Table & 1.6 & Rates per 100000 maternities of direct death from thrombosis & 54 \\
\hline Table & 1.7 & Adjusted odds ratio for risk of VTE with different thrombophilias & 56 \\
\hline Table & 1.8 & Doses recommended by RCOG for the management of VTE & 60 \\
\hline Table & 1.9 & Regimen and heparins reported by Voke and Knight's studies & 61 \\
\hline Table & 1.10 & Summary of LMWH PK studies to date in the antenatal setting & 64 \\
\hline Table & 1.11 & Green and Duffull's enoxaparin obesity model & 86 \\
\hline Table & 1.12 & Green's enoxaparin renal impairment model & 87 \\
\hline Table & 1.13 & Hulot's enoxaparin renal impairment model & 88 \\
\hline Table & 1.14 & Bruno's enoxaparin model & 90 \\
\hline Table & 1.15 & Barrass' enoxaparin model & 92 \\
\hline Table & 1.16 & Lebaudy's pregnancy enoxaparin model & 93 \\
\hline Table & 2.1 & Differences between sampling times suggested by the models & 97 \\
\hline Table & 2.2 & Information collected from the women at clinic visits & 98 \\
\hline Table & 3.1 & Demographic information on the recruited women & 107 \\
\hline Table & 3.2 & Indication for enoxaparin and breadth of doses prescribed & 109 \\
\hline Table & 3.3 & Information on the 9 women who suffered VTE & 110 \\
\hline Table & 3.4 & Information on the women switched from warfarin & 111 \\
\hline Table & 3.5 & Blood test results according to each trimester for the women & 112 \\
\hline Table & 3.6 & Outcome information in the women who had live births & 116 \\
\hline Table & 3.7 & Outcomes compared to a control pregnancy group & 118 \\
\hline Table & 4.1 & RCOG antenatal enoxaparin VTE dosing guidelines & 123 \\
\hline Table & 4.2 & King's College Hospital enoxaparin dosing guidelines & 123 \\
\hline Table & 4.3 & Base models explored & 126 \\
\hline Table & 4.4 & Key base models with parameter estimates & 126 \\
\hline Table & 4.5 & PK estimates when M3 error method applied & 128 \\
\hline Table & 4.6 & Nonparametric bootstrap v parametric model estimates & 137 \\
\hline Table & 4.7 & Coding of covariates for inclusion into the base model & 143 \\
\hline Table & 4.8 & Uni-variate addition of covariates into the base model & 143 \\
\hline Table & 4.9 & Table of 4 competing intermediate models & 144 \\
\hline Table & 4.10 & Backward elimination of covariates from model 404 (full model) & 145 \\
\hline Table & 4.11 & Bootstrap results of the final pregnancy model & 146 \\
\hline Table & 4.12 & Results of weight models with 3 error structures & 151 \\
\hline Table & 4.13 & Results with the incorporation of ethnicity into the base model & 153 \\
\hline Table & 4.14 & Assessment of each ethnic group in uni-variate manner & 154 \\
\hline Table & 4.15 & $\%$ of women who would have enoxaparin concentration BLQ & 160 \\
\hline
\end{tabular}




\begin{tabular}{|c|c|c|c|}
\hline Table & 4.16 & Estimates of enoxaparin $\mathrm{CL}$ and $\mathrm{Vd}$ from other studies & 161 \\
\hline Table & 4.17 & Comparison between thesis model $v$ Lebaudy model & 162 \\
\hline Table & 4.18 & Details from Casele's enoxaparin antenatal model & 164 \\
\hline Table & 4.19 & Results from Marder and colleagues Reviparin study & 167 \\
\hline Table & 4.20 & Results from TIMI Ila enoxaparin dose ranging study & 168 \\
\hline Table & 4.21 & Bleeding results from the TIMI Ilb enoxaparin trials & 169 \\
\hline Table & 4.22 & Women switched from warfarin to enoxaparin once daily & 170 \\
\hline Table & 4.23 & Current RCOG suggested doses for VTE prophylaxis & 173 \\
\hline Table & 4.24 & $\%$ of women who would have concentration BLQ (prophylaxis) & 174 \\
\hline Table & 5.1 & D-dimer concentration during pregnancy (Chabloz) & 178 \\
\hline Table & 5.2 & D-dimer concentration during pregnancy (Kline) & 179 \\
\hline Table & 5.3 & D-dimer concentration during pregnancy (Murphy) & 179 \\
\hline Table & 5.4 & D-dimer concentration during pregnancy (Szeci) & 180 \\
\hline Table & 5.5 & D-dimer concentration using the STA-Lia $®$ test & 181 \\
\hline Table & 5.6 & Overall D-dimer results for the cohort of women followed & 185 \\
\hline Table & 5.7 & D-dimer results for subjects prescribed prophylactic doses & 187 \\
\hline Table & 5.8 & D-dimer results for subjects prescribed treatment doses & 188 \\
\hline Table & 5.9 & D-dimer results: Caucasian v African-Caribbean subjects & 190 \\
\hline Table & 6.1 & Inter-and intra-assay variability of TG in 4 healthy controls & 194 \\
\hline Table & 6.2 & TG results from Al-Dieri's and colleagues study & 197 \\
\hline Table & 6.3 & Intra-assay and inter-assay variability in a healthy male control & 206 \\
\hline Table & 6.4 & Overall TG parameter results for the cohort of women & 206 \\
\hline Table & 6.5 & TG results from the cohort of women prescribed prophylaxis & 207 \\
\hline Table & 6.6 & TG results from the cohort of women prescribed treatment & 208 \\
\hline Table & 6.7 & Correlation between TG parameters and anti-Xa activity & 209 \\
\hline Table & 6.8 & TG results: Caucasian versus African-Caribbean & 221 \\
\hline Table & 6.9 & Changes in TG parameters with time around a single dose & 222 \\
\hline Table & 6.10 & TG results from a single subject injecting twice daily enoxaparin & 223 \\
\hline Table & 6.11 & TG changes with the addition of PB to control subjects plasma & 234 \\
\hline Table & 6.12 & $\mathrm{TG}$ results with and without the addition of $\mathrm{PB} 0.0125 \mathrm{mg} / \mathrm{mL}$ & 235 \\
\hline Table & 6.13 & TG results with and without the addition of PB (prophylaxis) & 236 \\
\hline Table & 6.14 & TG results with and without the addition of PB (treatment) & 237 \\
\hline Table & 7.1 & Medications prescribed in the study by Sawicki and colleagues & 246 \\
\hline Table & 7.2 & BMQ general (overuse and harm) questions & 249 \\
\hline Table & 7.3 & BMQ specific (necessity and concerns) questions & 249 \\
\hline Table & 7.4 & Additional questions added to the general section of the $\mathrm{BMQ}$ & 250 \\
\hline Table & 7.5 & Questions repeated in the specific section of the BMQ & 250 \\
\hline Table & 7.6 & Additional exploratory questions added to the adapted BMQ & 251 \\
\hline Table & 7.7 & Demographics on the women who completed the questionnaire & 254 \\
\hline Table & 7.8 & Mean BMQ sub-scale scores & 255 \\
\hline Table & 7.9 & BMQ sub-scale scores: Optimal v Sub-optimal adherence & 256 \\
\hline Table & 7.10 & BMQ sub-scale scores: Caucasian v African-Caribbeans & 257 \\
\hline
\end{tabular}


$\begin{array}{lll}\text { Table } & 7.11 & \text { BMQ sub-scale scores: Once v Twice daily enoxaparin }\end{array}$

Table $7.12 \quad$ BMQ sub-scale scores: Recurrent miscarriage v No history 257

$\begin{array}{llll}\text { Table } & 7.13 & \text { Correlation between necessity and concerns question pairs } & 259\end{array}$

$\begin{array}{llll}\text { Table } & 7.14 & \% \text { agreement with the additional questions added to the BMQ } & 260\end{array}$

$\begin{array}{llll}\text { Table } & 8.1 & \text { Weight banding used for the enoxaparin simulation work } & 270\end{array}$

$\begin{array}{llll}\text { Table } & 8.2 & \text { Current RCOG prophylactic dosing recommendations } & 273\end{array}$ 


\section{List of acronyms}

$\triangle \mathrm{OBJ}$

ACCP

APC

APTT

AT

AUC

BD

$\mathrm{BMI}$

BSV

CAT

$\mathrm{CL}$

$\mathrm{C}_{\max }$

$\mathrm{CrCl}$

CRE

CV

CWRES

$\mathrm{Da}$

D

DVT

ETP

ELISA

FACWTCL

FACLBWV

FACGESTV

FACGESTCL2

FACGESTV2

FACCL Lebaudy

FACV Lebaudy $_{\text {L }}$

FO

FOCE

FOCE-I

GEST

GFR

HIT

I change in objective function value

American College of Chest Physicians

activated protein $\mathrm{C}$

activated partial thromboplastin time

antithrombin

area under the concentration-time curve

bis die (twice a day)

body mass index

between subject variability

calibrated automated thrombinography

clearance

maximum concentration

creatinine clearance

creatinine

coefficient of variation

conditional weighted residuals

Daltons

dose

deep vein thrombosis

endogenous thrombin potential

enzyme linked immunosorbent assay

exponent on the weight covariate on estimates of clearance

exponent on the lean body weight covariate on volume of distribution estimates

exponent on gestation as a covariate on volume of distribution estimates during pregnancy

exponent on gestation as a covariate on clearance estimates post-partum

exponent on gestation as a covariate on volume of distribution estimates post-partum

exponent Lebaudy and colleagues put on clearance estimates during pregnancy in their enoxaparin antenatal model

exponent Lebaudy and colleagues put on volume of distribution estimates during pregnancy in their enoxaparin antenatal model

first order

first order conditional estimation

first order conditional estimation with interaction

gestation in weeks

glomerular filtration rate

heparin induced thrombocytopenia

individual 


\begin{tabular}{|c|c|}
\hline IBW & ideal body weight \\
\hline INR & international normalised ratio \\
\hline IOV & inter occasion variability \\
\hline $\mathrm{IU} / \mathrm{mL}$ & international units per millilitre (unit of measure for $\mathrm{LMWH}$ ) \\
\hline IUGR & intra-uterine growth retardation \\
\hline J & $j^{\text {th }}$ observation \\
\hline $\mathrm{kg}$ & kilogram \\
\hline $\mathrm{Ka}$ & absorption rate constant \\
\hline LBW & lean body weight \\
\hline $\mathrm{L}$ & litres \\
\hline LT & lag-time \\
\hline LMWH & low molecular weight heparins \\
\hline $\mathrm{mg}$ & milligram \\
\hline MGEST & gestation at which baby was born \\
\hline $\mathrm{mL}$ & millilitre \\
\hline NCA & non-compartmental analysis \\
\hline NLME & non-linear mixed effects \\
\hline NONMEM & NONlinear Mixed Effects Modelling software \\
\hline$n$ & number \\
\hline OD & omni die (once a day) \\
\hline OBJ & objective function value \\
\hline PD & Pharmacodynamics \\
\hline PK & Pharmacokinetics \\
\hline PKPD & pharmacokinetic / pharmacodynamic \\
\hline pM & picoMolar \\
\hline PPP & platelet poor plasma \\
\hline PRP & platelet rich plasma \\
\hline Pop & Population \\
\hline popPK & population pharmacokinetics \\
\hline PE & pulmonary embolism \\
\hline Q & inter-compartmental clearance \\
\hline $\mathrm{RCOG}$ & Royal College of Obstetricians and Gynaecologists \\
\hline RUV & residual unexplained variability \\
\hline SD & standard deviation \\
\hline SE & standard error \\
\hline SS & steady state \\
\hline ST & start tail \\
\hline$t$ & time \\
\hline$t_{1 / 2}$ & half-life \\
\hline TF & tissue factor \\
\hline TG & thrombin generation \\
\hline $\mathrm{T}_{\max }$ & time to maximum concentration \\
\hline
\end{tabular}


TTP

$\mathrm{Vd}$

VPC

vS.

VTE

WFN

$\mathrm{Wt}$

$\omega_{\mathrm{X}}$ time to peak

volume of distribution

visual predictive check

versus

venous thromboembolism

wings for NONMEM

total body weight

between subject variability for parameter $\mathrm{x}$ 


\section{Acknowledgements}

I would like to thank the primary members of the research team and my supervisors for their support, mentorship and advice. Graham, right from when I first discussed the research idea with you (in December 2005), you have always been extremely supportive. Over the years, your wisdom, your encouragement to collaborate, your contacts and your direction has made this happen for me, and I will always be grateful. I feel very lucky to have had the opportunity to work with you over the last 11 years and I very much look forward to working with you in the future.

Dr Arya, thank you so much for providing a home for this piece of work and giving me this break. I think you took quite a risk letting me pursue this at King's; I hope the risk has paid off. I appreciate all the effort you put in recruiting patients to the study and maximising the information we collected for modelling purposes. Thank you as well for reviewing the drafts of the work and providing valuable feedback. I feel extremely lucky to have had the opportunity to work with you at the King's Thrombosis Centre and I look forward to more kite-flying in the future.

Dr Patel, thank you for your advice, support and encouragement over the last four years and for doing such a fantastic job in recruiting patients to the study. Thank you also for putting up with my silly questions; I also feel exteremly lucky to have had the opportunity to work with you. I have learnt so much from you and I look forward to learning more, and continued working at King's.

Mr Marsh, thank you for providing an obstetric view point in the study and for ensuring that all angles had been considered; it was good to have your support, encouragement and input when it was needed.

Finally, I would like to thank Bruce. I am extremely grateful for the support you have given me over the course of this research and without your input we would never have been able to tackle this clinical problem. I feel very lucky to have worked with someone as clever as you. I really enjoyed the time I spent in Brisbane in March 2012; thanks for your patience and for introducing me to PK modelling - it's opened up a whole new world for me! I look forward to working on future projects together and learning more. 
I would like to acknowledge Coen Van Hasselt. Coen conducted the D-optimal work, which informed the anti-Xa sampling strategy for this study.

Also a very big thank you to Vivian Auyeung, who helped me adapt the BMQ for the adherence aspect of this study. Thanks Viv for holding my hand through with this aspect of the study and thank you for your patience, encouragement and analytical words. I look forward to continue working with you in the future at IPS.

A very big thank you to Lara; thank you for your patience when showing me how to conduct the thrombin generation experiments and being around, when I first started bleeding the study patients; it was good to know that I could call on your help if I ever needed it. Thank you also, for providing the idea for the polybrene aspect of the thrombin generation experiments. I feel extremley lucky to have been doing my research at the same time as you were in the group.

I would also like to thank Sarah and Glynn at Model Answers, who looked after me and put up with my silly questions when I was in the office in Brisbane in 2012.

I would like to thank Professor Beverley Hunt, who in the early days of the research idea encouraged me to write a review of the literature of low molecular weight heparin use during pregnancy. I am sure, the fact that I had a publication in the research area I wanted to pursue, helped in part, to secure the BRC fellowship.

I would also like to thank the Guy's and St.Thomas' / King's College London comprehensive biomedical research centre. Thank you for establishing the allied healthcare professional fellowships and giving me the opportunity to undertake full-time research; It has been a once in a life-time opportunity for me. I would specifically like to thank the three people who interviewed me for the fellowship and gave me this opportunity; Dr Kate Blake, Dr Wendy Fisher and Professor Charles Wolfe - thank you so much for giving me this break.

Other people who have supported me in this study include: Paradzai (Para) Chitongo, Elizabeth Ford from the King's College Hospital pathology laboratory, who analysed the antiXa activities. Dr Elizabeth Allen and Professor Tim Mant from Quintiles who were very encouraging when I discussed the research idea with them. Dr Catherine Bagot from the haematology department at King's (now in Glasgow) who helped to determine when samples 
for thrombin generation would be drawn for the study. Raliat, Chris and Roz from the pharmacy department at King's, for sorting out contracting issues and letting me join the anticoagulation team at King's. I would also like to thank Dr Alice Oborne who supported my BRC fellowship application.

I would also like to acknowledge the local Sanofi-Aventis reps, who supplied me with the How to inject Clexane ${ }^{\circledR}$ books that the women used to record the times they injected enoxaparin. A big thank you also to the haematology outpatient reception staff: Nici, Margaret, Cerilyn and Esther, for informing me when the women had arrived in clinic and putting up with me altering the subject's clinic appointments! I would also like to thank all the haematology outpatient staff for their support in the study.

I would also like to thank the haematology registrars who rotated into Dr Arya's and Dr Patel's clinic. Thank you for helping in recruiting patients to the study, for reviewing the subjects at their clinic visits and tolerating me ordering d-dimers and coagulation screens on the patients: Dr Shameem Mahmood, Dr Vijay Dhanapaal, Dr J.P.Westwood, Dr Fernando Pinto, Dr Sarah Bennett, Dr Sarah Arnott, Dr Clare Wykes, Dr Vicky Tindall, Dr Wasim Nagi, Dr Sunil Gupta, Dr Preetam Dighe and Dr Shreeyans Gandhi.

I would like to acknowledge the help of Dr Audrey Carlo at Diagnostica Stago. Dr Carlo kindly shared the detail of the polybrene experiments they had completed, so that we could pursue the idea during the course of this study.

A big thank you to the London Pharmacometrics Group, Dr Joe Standing, Dr Julie Bertrand, Dr Jan-Stefan Van der Walt and Joanna Lewis. Your feedback and comments on the work has been extremely useful and whenever I attend our meetings, I always learn something new about PK-PD modelling.

From the polybrene research point of view, I would like to thank Professor Hider, who kindly agreed to embark on trying to work out the binding relationship between polybrene and enoxaparin with me. I would also like to thank Dr Sukhi Bansal, Dr Xiao.I.Kong (Kong) and Dr Vincenzo Abbate, who helped me with the practical side of things in the Hider-Bansal laboratory. 
An extremely big thank you to lan and Judith Coombs, and their children Fiona, Tim and Sam; thank you for looking after me for three weeks in Australia. Your hospitality has no bounds and I will always have fond memories of my time in Woloongabba.

Of course, I will always be indebted to the pregnant women who consented into the study. You are all very altruistic, and I hope your participation in this study will have a positive impact on clinical practice and how we use low molecular weight heparins in other pregnant women. A big thank you particularly in letting me take thrombin generation samples from you all at the time of delivery; it was very kind of you to let me take those samples at such a private time in your lives.

Finally and by no means least, I would like to thank my family. Firstly my mum and dad, who took significant life risks (which I personally would not be able to take), so that Manisha, Tara and I could have a better life; in many ways any of our achievements are yours. You have always encouraged me academically and to do my best - thanks for instilling this in me. Thanks to my big sister Manisha, who proof-read my thesis and provided me with valuable feedback on my English and thanks for your encouragement in the final few weeks of writing up. Thanks also to my younger sister Tara for ongoing support. Finally, a big thank you to my wife Bhavini and my two wonderful children, Hari and Anjali, for tolerating me writing and going to collect samples from the women on weekends and evenings and for not having a proper holiday for two years! Without your understanding and support, we would not have been able to maximise the data that we have collected for this study - thank you.

Hari and Anjali, this thesis is dedicated to you. 


\section{Publications and abstracts relevant to this thesis}

\section{Publications}

Patel JP, Auyeung V, Patel RK, Marsh MS, Green B, Arya R, Davies JGD. Women's views on and adherence to enoxaparin during pregnancy and the puerperium. Journal of Thrombosis and Haemostasis 2012; 10: 2526-2534

Patel JP, Patel RK, Davies JG, Arya R. Prophylaxis with low-dose low molecular weight heparin during pregnancy and the puerperium: is it effective? A rebuttal. Journal of Thrombosis and Haemostasis 2011; 9: 1269-1271 (letter)

\section{Conference abstracts}

Patel JP, Green B, Patel RK, Marsh MS, Davies JG, Arya R. Population pharmacokinetics of enoxaparin during the antenatal period. Oral Presentation.

$54^{\text {th }}$ Annual Meeting and Exposition of the American Society of Haematology, Atlanta, USA, $8^{\text {th }}-11^{\text {th }}$ December 2012

Blood 2012; 120(21): 503

Remedios K, Patel S, Humphries F, Bonner L, Patel JP, Cameron S, Patel RK, Arya R. An audit of venous thromboembolism (VTE) risk assessments in the obstetric department at King's College Hospital. Poster.

British Society for Haematology $52^{\text {nd }}$ Annual Scientific Meeting, Glasgow, $16^{\text {th }}-18^{\text {th }}$ April 2012 British Journal of Haematology 2012; (Suppl. 1), 1-88 (poster number 76)

Patel JP, Patel RK, Marsh MS, Green B, Davies JG, Arya R. The thrombin generation profiles of women on prophylactic enoxaparin during pregnancy and the puerperium. Poster.

XXIII Congress of the International Society on Thrombosis and Haemostasis (ISTH), Kyoto, Japan; 23-28 ${ }^{\text {th }}$ July 2011

Journal of Thrombosis and Haemostasis 2011; 9(Suppl 2): 172: P-MO-367

Patel JP, Auyeung V, Patel RK, Marsh MS, Green B, Arya R, Davies JG. Women's views and adherence to enoxaparin therapy during pregnancy and the puerperium. Poster.

XXIII Congress of the International Society on Thrombosis and Haemostasis (ISTH), Kyoto, Japan; 23-28 ${ }^{\text {th }}$ July 2011

Journal of Thrombosis and Haemostasis 2011; 9(Suppl 2): 172: P-MO-368

Patel JP, Roberts LN, Patel RK, Marsh MS, Green B, Davies JG, Arya R. Polybrene can neutralise enoxaparin in plasma being tested for thrombin generation, however this effect is concentration dependent. Poster.

XXIII Congress of the International Society on Thrombosis and Haemostasis (ISTH), Kyoto, Japan; $23-28^{\text {th }}$ July 2011

Journal of Thrombosis and Haemostasis 2011; 9(Suppl 2): 129: P-MO-228 


\section{Preface}

\section{A brief history of antenatal venous thromboembolism}

Venous thromboembolism (VTE) is almost five times more common in pregnant women compared to women of the same age who are not pregnant [Rodger et al., 2003], and until recently has been the commonest cause of direct maternal mortality in the United Kingdom [Royal College of Obstetricians and Gynaecologists, 2007; Centre for maternal and child enquiries, 2011]. One would therefore expect numerous references to antenatal VTE in the historical literature, as it is difficult to imagine how unilateral swelling of the leg, dyspnoea and sudden death could have escaped the attention of midwifes and the relatives of pregnant women. However, extensive reviews [Anning, 1957; Mannucci, 2002; Dickson, 2004; Bagot and Arya, 2008] have found that reference to VTE in general, let alone that associated with pregnancy and the post-partum period, prior to the eighteenth century was rare. William Smellie's authoritative textbook on the practice of midwifery from the $18^{\text {th }}$ century contains no reference to VTE, although he does describe some pregnant women experiencing difficulty in breathing occasionally accompanied by collections of matter in the chest and thorax [Smellie, 1764]. He perhaps was referring to VTE, but this is speculation. The first reference to VTE is widely thought to be the case of Raoul of Normandy, in the $13^{\text {th }}$ century [Dexter and Folch-Pi, 1974]. This twenty year old man developed oedema in his right ankle, which subsequently extended up his thighs; he is reported to have prayed and his symptoms resolved within a year. It was some four centuries later that the first detailed description of VTE associated with pregnancy and post-partum were reported by Richard Wiseman, the famous SergeantChirurgical to Kings Charles II [Wiseman, 1676]. He describes the case of a wife of an apothecary, who, after a difficult labour developed pain and swelling in the right leg, extending from the knee to the hip (developing post-partum deep vein thrombosis (DVT)).

Prior to $19^{\text {th }}$ century, it was thought that venous thrombosis associated with pregnancy and post-partum was due to retention of evil humors in the legs. The $16^{\text {th }}$ century French surgeon Ambroise Paré, believed that swelling of the legs during pregnancy was due to the retention and concentration of menstrual blood [Mannucci, 2002]. Another widely held view was that post-partum thrombosis was due to the retention of unconsumed milk in the legs, engorgements laiteaux [Findley, 1912]. Towards the end of the $18^{\text {th }}$ century, Charles White, an obstetrician in the then emerging city of Manchester, was one of the first to dispute this 
humoral thinking and suggested that swelling of the leg during pregnancy was due to obstruction, retention and accumulation of lymph in the limb [White, 1784]. He believed this was brought about by rupture of the lymphatics from pressure of the fetus' head and he believed that only pregnant and postpartum women could develop this phenomenon and was the first to describe phelgmatia alba dolens puerperarium [White, 1801]. Also known as phlegmasia dolens, and what would now be recognised as post-partum deep vein thrombosis, case reports of phlegmasia dolens began to be widely reported in the medical literature during the nineteenth century [Bacon, 1832; Hodgson, 1835; Johnson, 1842; Winn, 1852; Crighton, 1871; Barnes, 1880; Elster, 1884; Dixon, 1885; Wilcox, 1897].

Following the first descriptions of pulmonary embolism by Virchow mid way through the nineteenth century [Virchow, 1856], assistant obstetric physician to King's College Hospital, W.S. Playfair began recognising and reporting cases of pulmonary embolism he was observing at the time of labour and during the post-partum period [Playfair, 1867; Playfair 1869]. Very few cases of antenatal pulmonary embolism in the nineteenth century were reported, presumably because many would have been sudden and fatal in nature, with the condition only gaining recognition mid way through that century. However, of note is a case reported in 1872 of an eight month pregnant farmer's wife in her first pregnancy who developed antenatal pulmonary embolism which was successfully managed [Atkinson, 1872].

Dr Atkinson describes how this patient woke at $4 \mathrm{am}$ in the morning, was violently sick, after which she fainted. On examination, she was dyspnoeic (respiratory rate 75) and her pulse was found to be rapid and weak. She had visible oedema of the lower extremities, and given her signs and symptoms, he concludes that "a clot had come loose and was plugging up one of the pulmonary vessels." He prescribes a mixture containing sprit of sulphuric ether with spirit of chloroform, and bottles of hot water to the extremities. For the next few days, she rallied, but four days after her initial collapse, she relapsed which passed before Dr Atkinson reaches her house. Linseed poultices were then applied to the chest and plenty of nourishment ordered. The patient continued in the same state for the next few days, with her continued laboured breathing. Eleven days after her initial symptoms, her respiratory rate fell to 55, pulse of 120 , still coughing, she was perspiring and unable to sleep. Dr Atkinson prescribed 15 grains of chloral hydrate, following which, over the next three days, her respiratory rate steadily fell to 40 . The patient began to recover and by the $2^{\text {nd }}$ May $\mathrm{Dr}$ 
Atkinson discharged her from his care, presumably having delivered her baby successfully. The case demonstrates eloquently how patients might have survived VTE, even without the treatments available today.

Following the accidental discovery of heparin by Jay Mclean in 1916 [Mclean, 1916], the management of VTE and subsequently antenatal VTE was transformed during the second half of the $20^{\text {th }}$ century. Despite this a number of uncertainties remain regarding the optimal use of heparins for the prophylaxis and treatment of VTE during the antenatal period. 


\section{Chapter 1}

\subsection{Blood coagulation}

\subsubsection{An overview}

Coagulation involves a series of complex and inter-linked processes that leads to fibrin formation. Haemostasis is coagulation which occurs in a physiological setting and results in the sealing of a break in the vasculature, which is essential in protecting its integrity. Thrombosis is coagulation in a pathological setting that leads to localised intravascular coagulation and potential occlusion of a blood vessel [Key (ed) et al., 2009].

When the vessel wall is damaged, exposed collagen in the endothelial matrix attracts and binds platelets, causing activation, degranulation and aggregation. In parallel, coagulation is activated by the exposure of tissue factor (TF) in the exposed endothelium, resulting in fibrin formation, leading to stabilisation of the platelet plug [Sere and Hackeng, 2003; Furie and Furie, 2008].

TF is present in a number of tissues throughout the body [Drake et al., 1989], with highest concentrations found in the brain, lung and heart. Low levels are detected in skeletal muscle, joints, spleen and the liver. As well as being found in tissues, TF is also expressed on vascular smooth muscle cells and the pericyte cells that surround blood vessels. This concentration of tissue factor which surrounds the vasculature is referred to as the haemostatic envelope. Under normal conditions, the endothelium acts as a barrier to separate TF from factor VII and factor VIla in the circulating blood. TF is also found expressed on many non-vascular cells (such as monocytes) and microparticles in the circulation. This blood originating TF is thought to participate in coagulation, with coagulation reactions occurring on specific cell surfaces, such as activated platelets and TF-bearing cells [Furie and Furie, 2008; Chou et al., 2004].

The physiological coagulation process can be described under four distinct phases (see overview in figure 1.1):
1) initiation
2) amplification
3) propagation
4) localisation 


\subsubsection{Initiation}

When there is a break in the vasculature, platelets adhere to the site of injury [Varga-Szabo et al., 2008]. The initiation phase of coagulation is localised to cells that express TF. After binding to factor VIla (from the circulating blood), the TF-VIla complex activates small amounts of factor IX and factor X. Factor IXa moves into association with platelets, where it plays a role in the later stages of haemostasis. Factor $\mathrm{Xa}$ activates factor $\mathrm{V}$ and forms a complex with factor Va to convert small amounts of prothrombin (factor II) to thrombin (factor Ila) $(0.1-1 \mathrm{nM})$. The source of factor $V$ is from the alpha granules of collagen adherent platelets [Monkovic et al., 1990]. The thrombin produced promotes local fibrin formation, but is not sufficient to provide full blown haemostasis.

\subsubsection{Amplification}

The thrombin formed during the initiation phase acts as an amplifier by acting on platelets and proteins to facilitate platelet driven thrombin generation. Thrombin interacts tightly with platelet glycoprotein Ib [De Marco et al., 1994]. When bound to glycoprotein Ib, thrombin undergoes a conformational change, which is thought to protect it from inhibition. The conformational change enhances the ability of thrombin to cleave either of two platelet protease-activated receptors (PARs). PARs are members of the seven transmembrane domain G-coupled family of proteins [Coughlin, 2005]. Cleavage of a PAR creates a new amino terminal, which can fold back on itself and bind to a receptor site in the transmembrane domain. This binding initiates a signalling cascade. In platelets, cleavage of PAR1 leads to signalling that results in platelet activation.

Platelet activation leads to:

- platelets undergoing cytoskeletal changes leading to a shape change

- platelets degranulating, releasing the contents of both alpha and dense granules

- dense granules release ADP, which promotes further platelet activation

- alpha granules release partially activated factor $\mathrm{V}$

von Willebrand factor (vWF), in addition to participating in platelet adhesion, acts as a carrier of factor VIII. VWF binds to glycoprotein Ib and brings factor VIII into close proximity to thrombin, which is also bound to glycoprotein Ib. The amplification phase results in activated platelets that have cofactors Va and VIIla bound to their surface. 


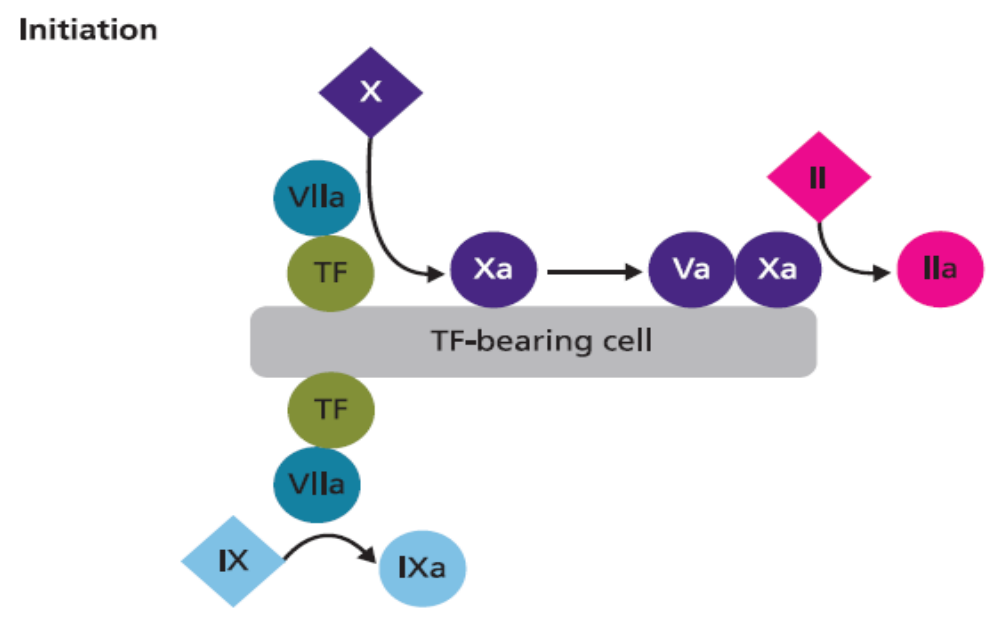

\section{Amplification}
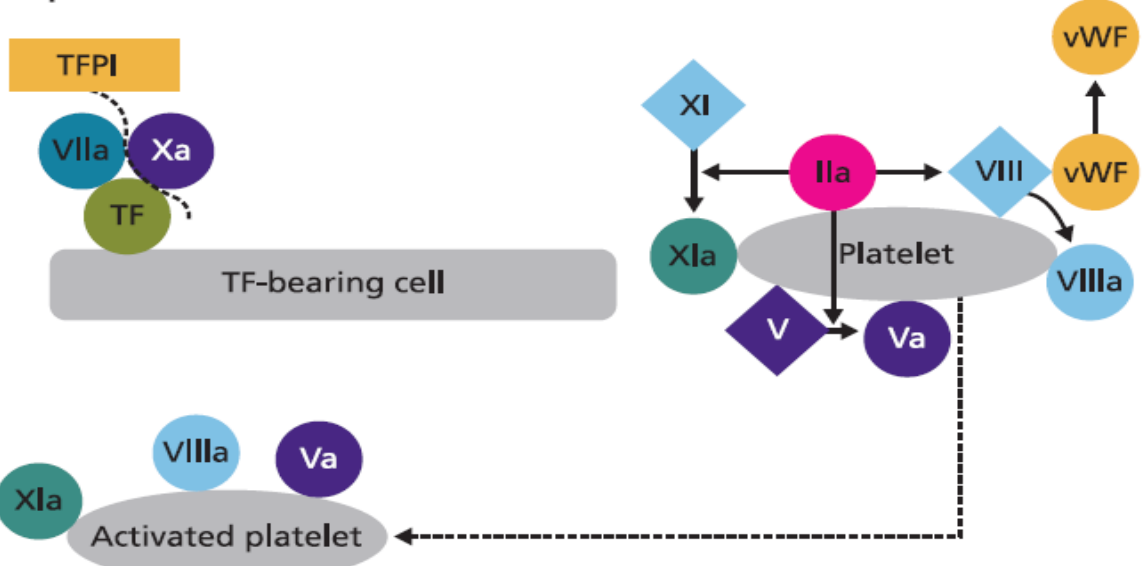

Activated platelet

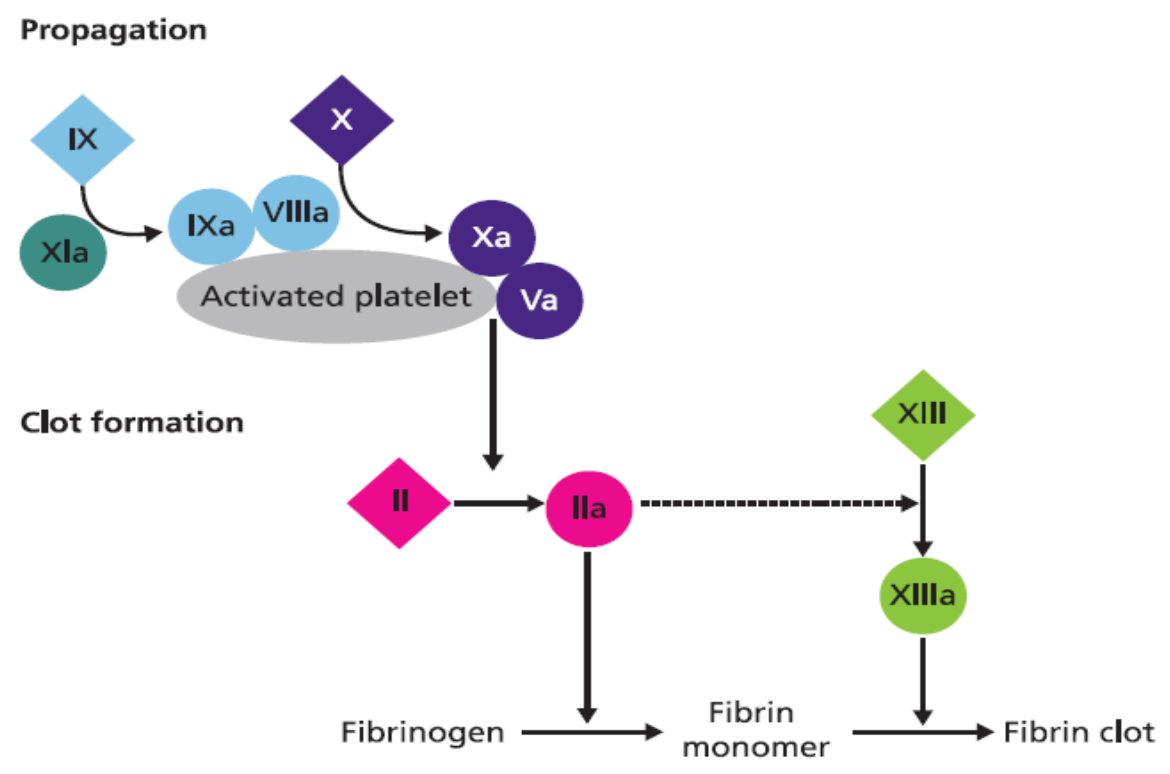

Figure 1.1: An overview of blood coagulation, with permission from John Wiley and Sons, in Practical Hemostasis and Thrombosis', Key NS, Makris M, O'Shaughnessy D, and Lillicrap D, eds. Wiley-Blackwell, Oxford, 2nd edition, 2009 


\subsubsection{Propagation}

The platelets are now ready for full blown thrombin generation. Factor IXa formed during the initiation phase binds to activated platelets, partly through factor VIIla and partly independent of factor VIIIa. The factor IXa/VIIla complex activates factor $X$ on the platelet surface. This platelet surface generated factor Xa moves directly to complex with platelet surface factor Va. In the presence of prothrombin, factor $\mathrm{Xa}$ is protected from inhibition by antithrombin or tissue factor pathway inhibitor (TFPI). Research suggests that factor $\mathrm{Xa} / \mathrm{Va}$ complexes are very stable and in the presence of a new supply of prothrombin, can immediately act to promote thrombin generation [Orfeo et al., 2008].

The burst of thrombin generation during the propogation phase leads to cleavage of fibrinopeptides from fibrinogen, which exposes new binding sites which fit complementary sites on other fibrin molecules [Lord, 2007]. These interactions lead to fibrin molecules assembling in long, branched chains anchored at the platelet receptor glycoprotein Ilb/llla. This stabilises the initial platelet plug into a fibrin plug.

In addition to its role in cleaving fibrinopeptides, thrombin generation participates in a positive feedback loop by activating factor XI on the platelet surface [Oliver et al., 1999]. This factor Xla can activate factor IXa to enhance factor Xa generation. A single thrombin activated platelet exposes more than 12,000 copies of GP IIb/IIla receptors. The thrombin which are bound to fibrin are protected from inhibition by antithrombin. This fibrin bound thrombin provides an important role in maintaining haemostasis.

\subsubsection{Localisation}

Physiologically, a haemostatic plug should seal the break in the vasculature but not continue platelet accumulation and thrombin generation to the point that the entire vessel is occluded. The endothelial cells produce the coagulation inhibitor, tissue factor pathway inhibitor (TFPI) and heparan sulphate. TFPI inhibits the TF-factor VIla/factor Xa complex, inhibiting the initiation of coagulation. Free thrombin and factor Xa are inhibited by antithrombin (AT); the inhibitory activity of AT is markedly enhanced by heparan sulphates [Tanaka et al., 2009]. Furthermore, free thrombin in the circulation binds to the endothelial surface-bound thrombomodulin (TM) and activates the protein $C$ system. This binding of thrombin to TM causes a conformational change in thrombin such that it can no longer cleave fibrinogen or activate platelets, and thrombin within the thrombin-TM complex is also found to be more 
sensitive to inhibition by circulating AT than free thrombin [Turpie and Esmon, 2011]. Protein $\mathrm{C}$ is a vitamin $\mathrm{K}$-dependent protein and is activated after binding to the endothelial protein $\mathrm{C}$ receptor and interacting with the thrombin-TM complex. Activated protein C (APC), along with its co-factor protein $\mathrm{S}$, inactivates factor $\mathrm{Va}$, and factor VIIla, decreasing the formation of factor Xa-factor Va (the prothrombinase complex) and factor IXa-factor VIIla (the intrinsic tenase complex), resulting in coagulation inhibition [Sere and Hackeng, 2003; Sarangi et al., 2010]. APC also enhances fibrinolysis by inactivating plasminogen activator inhibitor-1.

The intact vasculature also has a number of mechanisms which prevents haemostasis and ensures localisation and that coagulation only occurs when necessary. Healthy endothelium expresses ectonucleotidase (CD39) and produces prostacyclin $\left(\mathrm{PGl}_{2}\right)$ and nitric oxide (NO), which block platelet adhesion to and activation by healthy endothelium [Jin et al., 2005].

Under physiological conditions, the afore-mentioned mechanisms ensure that thrombus formation is a controlled and balanced process; deficiency or impairment of these systems may result in a hypercoagulable state.

\subsection{Thrombin generation}

The coagulation system is complex and measuring the individual plasma levels or activities of individual proteins holds little clinical utility [Castoldi and Rosing, 2011]. Research suggests that patients with the same coagulation factor deficiency or thrombophilic defect, often experience different bleeding or thrombotic risks, depending on genetic and environmental factors also present. In current clinical practice, no valid test for the overall haemostatic thrombotic system exists [Hemker et al., 2002]. The clotting times most commonly utilised in clinical practice, the prothrombin time and the activated partial thromboplastin time, are of limited use as they do not indicate the haemostatic potential in an individual and have been developed for a specific use, e.g. the prothrombin time to monitor oral vitamin $\mathrm{K}$ antagonist therapy [Baglin, 2005]. The current lack of a valid test of the overall haemostatic thrombotic system means that it can at times be difficult to judge which patients are at higher risk of thrombosis and require intervention, and whether the intervention initiated is actually effective. Hence there is a need for global assays which evaluate the overall coagulation function that reliably estimates the bleeding or thrombotic risk in an individual patient. 
Thrombin is central to haemostasis and thrombosis; its formation is influenced by numerous coagulation factors and has numerous actions on the blood [Hemker et al., 2002], with no process bypassing thrombin. Thrombin can therefore be considered as a central marker of haemostasis and can be considered to represent the phenotype of the thrombotichaemostatic function of blood [Hemker and Beguin, 2000]. Research into a thrombin generation test dates back to work carried out over fifty years ago [Macfarlane and Biggs, 1953; Pitney and Dacie, 1953]. However the assays developed following this early research were labour intensive and imprecise. In the 1980's Hemker and colleagues re-visited the thrombin generation test and developed a method which was reproducible and accurate [Hemker et al., 2003].

Thrombin generation assays measure the ability of a plasma sample to generate thrombin following in-vitro activation of coagulation with tissue factor (TF) or another trigger. As well as exploring the initiation phase of coagulation, in contrast to classical coagulation tests, thrombin generation assays probe the propagation phase (where the bulk of thrombin is formed) and the termination phase (where thrombin formation is shut down and inhibited) [Castoldi and Rosing, 2011]. The thrombin generation curve which results, reflects both the pro- and anticoagulant reactions that lead to the formation and inhibition of thrombin, and show great potential as a global test of plasma coagulability [Baglin, 2005]. Research has shown, that in platelet poor plasma (PPP), thrombin generation is diminished in all coagulation factor deficiencies and in patients receiving anticoagulant treatment. In platelet rich plasma (PRP), thrombin generation is found to be diminished in both congenital and acquired platelet related disorders and in those patients receiving anti-platelet drugs. Conversely, thrombin generation in PPP is increased in congenital and acquired thrombophilias [Hemker et al., 2003].

\subsubsection{Calibrated automated thrombography (CAT)}

The CAT assay [Hemker et al., 2003; Hemker et al., 2006] utilises a fluorogenic substrate (ZGly-Gly-Arg-AMC) to continuously monitor thrombin activity in plasma. As fluorescence is not affected by the turbid changes associated with clot formation, the test can be conducted in both PPP and PRP. However, appropriate calibration is needed to account for the quenching of the fluorescence signal by substrate molecules that have already been converted and by the plasma colour, which differs among individuals [Castoldi and Rosing, 2011]. The 
fluorescence output readings are automatically converted into thrombin generation curves by dedicated software. The principle of the fluorescence approach has also been developed with chromogenic substrates [Hemker et al., 1993]. However, with the chromogenic approach, the turbidity of the emerging clot can affect optical density measurements, so fibrinogen containing plasma and PRP cannot be used. This is not the case with the fluorescence approach.

Each CAT test is typically carried out in triplicate on a microtitre plate, with two tests carried out on the same plasma sample. In a set of triplicate wells (the measurement wells), TF and synthetic phospholipid vesicles are added to the plasma to initiate coagulation. In the second set of triplet wells (the calibrator wells), a known amount of substrate-converting activity is added to plasma without activating coagulation. The thrombin calibrator consists of thrombin bound to $\alpha_{2}$-macroglobulin, a form of thrombin that cannot be inhibited by plasma protease inhibitors. A mixture of calcium chloride and flurogenic substrate is subsequently dispensed in both wells and the developing fluorescence is recorded by a fluorometer. A fluorescence tracing in the measurement wells is produced and forms the basis of the thrombin generation curve produced. In contrast, the calibration wells containing the flurogenic substrate is converted at a constant rate by the added thrombin calibrator. This leads to an initial linear fluorescence tracing, whose slope is used to convert the raw fluorescence units (RFU) to thrombin concentration units $(\mathrm{nM})$. As more substrate is converted, the fluorescence tracing bends with substrate consumption. The thrombin generation curve is eventually calculated by taking the first derivative of the fully corrected fluorescence tracing [Castoldi and Rosing, 2011]. Figure 1.2 illustrates the parameters of the thrombin generation curve. The thrombin generation curve can be described in terms of lag time, peak height, area under the curve, time to peak and start tail. 


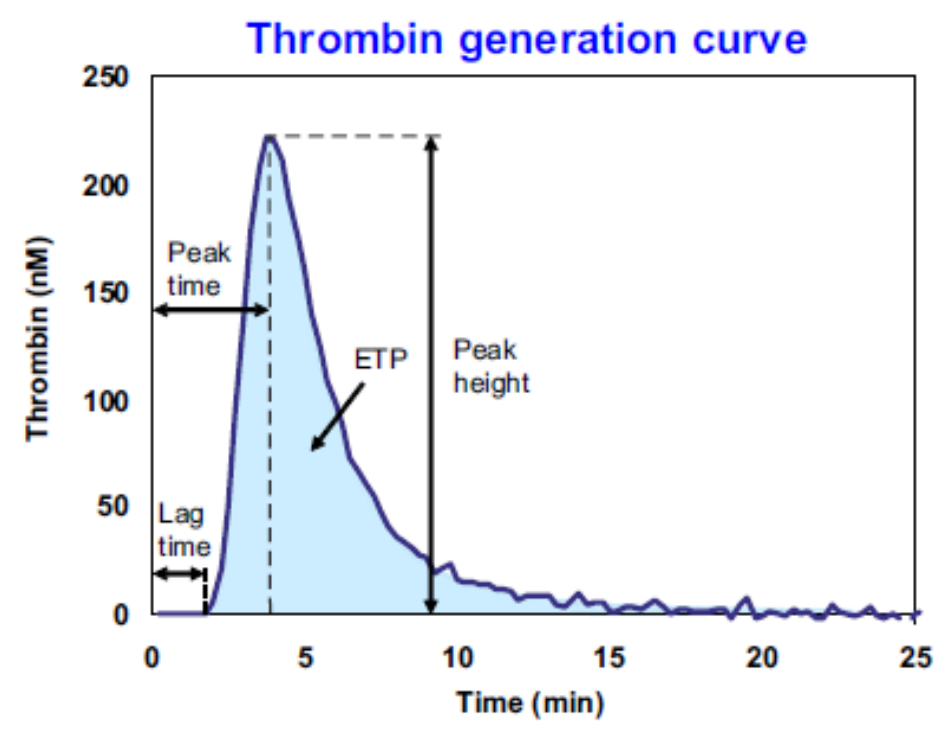

Figure 1.2: The thrombin generation curve, illustrating the lagtime, peak height, time to peak and the endogenous thrombin potential (ETP). Reprinted from Thrombosis Research, Vol 127; (Supplement 1), Castoldi and Rosing, Thrombin generation tests, s21-s25, Copyright 2011, with permission from Elsevier

The lag time (LT) is a period when no observable thrombin generation is seen, followed by a sudden burst in thrombin generation. The lag phase can be considered as representative of clotting times in historical tests and is defined as the time needed for the thrombin concentration to reach $1 / 6$ of the peak concentration. The area under the curve, referred to as the endogenous thrombin potential (ETP), represents the total enzymatic work performed by thrombin and is representative of the bleeding/thrombosis potential within an individual. Although ETP and peak height are strongly correlated, peak height is sometimes a more sensitive indicator of the plasma thrombin generating capacity [Duchemin et al., 2008]. Short lag times and high ETP and peak heights suggest a hypercoagulable state. More recently researchers have become interested in calculating the thrombin generation velocity, derived from the peak, lag-time and time to peak parameters, as shown in equation 1.1:

Velocity of thrombin generation $($ nmol/l per $\mathrm{min})=\frac{\text { peak height }}{(\text { time to peak-lagtime })}$

The CAT assay has demonstrated good reproducibility both in PPP [Spronk et al., 2008] and in PRP [Gerotziafas et al., 2005]. The assay also demonstrates reasonably consistent results (\% coefficient of variance $\sim 10-15 \%$ ) when the same individuals were tested repeatedly over a one-year period [Dargaud et al., 2009]. 


\subsubsection{Altering CAT assay conditions}

One advantage of CAT is that the experimental conditions can be modified for specific purposes. For example, the TF concentration used to trigger coagulation determines the extent to which the intrinsic coagulation pathway contributes to thrombin generation and what role the tissue factor pathway inhibitor (TFPI)/protein S system plays in inhibiting thrombin formation. Alternatively, the addition of thrombomodulin or activated protein $\mathrm{C}$ extends the sensivity of the assay to the protein $C$ anticoagulant pathway. This means that the shape of the thrombin generation curve changes according to the reaction conditions used. Dielis and colleagues measured thrombin generation under four conditions (1pMTF +/-TM and 13.6pMTF +/-APC) as well as assessing the effect of the plasma concentrations of 15 coagulation related proteins in 140 healthy individuals, in order to determine the individual proteins' influence on the thrombin generation parameters [Dielis et al., 2008]. This study reports that the strongest determinants of the ETP were fibrinogen and TFPI at low TF concentrations, but prothrombin and antithrombin at high TF concentrations. These differences in the plasma concentrations of the assay determinants account for the large inter-individual variation reported in thrombin generation parameters in population studies and explain why thrombin generation is affected by gender [Dielis et al., 2008 ], age [Dielis et al., 2008; Haidl et al., 2006], genetic factors [Segers et al., 2010], body mass index (BMI) [Fritsch et al., 2010], pregnancy [Rosenkranz et al., 2008], and other drug use, e.g. oral contraceptive use [Tchaikovski et al., 2007].

Several epidemiological studies have reported a correlation between elevated thrombin generation and the risk of VTE [Dargaud et al., 2006; van Hylckama et al., 2007; Tripodi et al., 2008; Besser et al., 2008], with thrombin generation tests performed at low TF in the presence of TM being the most predictive, as they are shown to be sensitive to both genetic and acquired risk factors for the development of VTE [Castoldi and Rosing, 2011].

\subsubsection{Assay variability}

Although initial studies using thrombin generation assays to guide clinical decisions are encouraging, there are several standardisation problems which need to be overcome before they can be used routinely [van Veen et al., 2008]. Factors affecting assay outcome by promoting contact activation and the release of platelet micro-particles, include; the method of blood collection and plasma preparation and the length for which plasma samples are pre- 
heated in the thrombinoscope [de Smedt and Hemker, 2011]. In addition the variation of assay conditions and protocols and the use of different reagents, makes establishing a reference range for the thrombin generation parameters difficult, as well comparing results between laboratories [Dargaud et al., 2007].

Such variability can be reduced by the addition of corn-trypsin inhibitor (CTI), which is recommended when thrombin generation is initiated at a low TF concentration [Dargaud et al., 2007; Luddington and Baglin, 2004]. Furthermore, inter-laboratory variation can be improved by standardising reagents and by normalising ETP of each sample against a reference plasma measured in parallel [Dargaud et al., 2007].

\subsubsection{Thrombin generation in pregnancy}

Accepting that thrombin generation represents the thrombotic-haemostatic system, this assay has an important role to play in pregnant women, given the prothrombotic state which ensues and as a potential marker for monitoring LMWH during the course of pregnancy.

Thrombin generation has been evaluated in 150 healthy pregnant women by Rosenkranz and colleagues, who found that ETP and peak thrombin generation increased significantly with gestational weeks, while the lag time and time to peak thrombin generation remained unchanged [Rosenkranz et al., 2008]. A result confirmed by others [Hynes et al., 2009; Rosetto et al., 2009]. However in contrast, in a study of 93 pregnant women, Dargaud and colleagues [2010] report that the increase in thrombin potential begins early in the first trimester, further increases in the second trimester and stabilises by the third trimester. They also found that thrombin generation did not correlate with other markers of thrombosis (Ddimers and prothrombin fragment $\mathrm{F} 1+\mathrm{F} 2$ ) and suggest that this may be due to thrombin generation measurements representing the global coagulation capacity of individuals, whilst markers such as D-dimers are markers of ongoing coagulation.

More recently, a group in Plymouth have reported the results of their pilot study measuring thrombin generation in 12 pregnant women prescribed enoxaparin [Ngu et al., 2010]. They found that the thrombin generation parameters were highly correlated with anti-Xa levels (taken three hours post dose) in particular the time to peak and peak concentration. Furthermore, a group in London retrospectively analysed samples for thrombin generation in 39 patients receiving $\mathrm{LMWH}$ during their pregnancy. The thrombin generation parameters 
were analysed by anti-Xa activity, according to two groups; group one, $>0.4 \mathrm{IU} / \mathrm{mL}$ anti-Xa activity, group two, $<0.4 \mathrm{IU} / \mathrm{mL}$. The researchers report that only in patients with therapeutic anti-Xa activities (group I), did the ETP, peak height and time to peak differ significantly from the control group. In those samples with low anti-Xa activity (group II), no difference was found for the ETP or peak height, although the time to peak was significantly different from that in the control group [Adamidou et al., 2011].

Measuring thrombin generation provides a unique insight into the global thrombotichaemostasis system of the patient at a specific time, providing an extremely useful marker for women on anticoagulant therapy during pregnancy, when there is substantial activation of the coagulation system [Hoke et al., 2004].

\subsubsection{Hexadimethrine bromide (polybrene) and thrombin generation}

Hexadimethrine bromide is a synthetic polymerised quaternary ammonium salt. During the late 1950's and early 1960's, it was utilised as an alternative agent to protamine to neutralise unfractionated heparin in patients undergoing open heart surgery and in those patients with an extracorporeal circulation [Weiss et al., 1958; Keats et al., 1959; Blumberg et al., 1960; Lillehei et al., 1960]. Although found to be as effective as protamine, its clinical application was short lived following reports of renal toxicity [Haller et al., 1962; Ransdell et al., 1965]. Over the last few years the use of polybrene within the laboratory setting has re-emerged. As well as those working in the field of gene transfer, reporting successful use of polybrene in viral gene transfer across target cell membranes [Davis et al., 2002], a use within the anticoagulation laboratory has emerged. Messmore and colleagues [2003] evaluated the effectiveness of polybrene to neutralise unfractionated heparin and LMWH (in-vitro) in plasma spiked with unfractionated heparin and enoxaparin at anti-Xa concentrations of 0.5 , 1.0 and $1.5 \mathrm{IU} / \mathrm{mL}$. They found that both unfractionated heparin and enoxaparin were completely neutralised, by the addition of polybrene. More recently, Carlo and colleagues [2009] tested the use of polybrene for heparin neutralisation in the thrombin generation test. They reported that a concentration of $0.025 \mathrm{mg} / \mathrm{mL}$ polybrene completely neutralised the anticoagulant effect of up to 2 anti-Xa $\mathrm{IU} / \mathrm{mL}$ of both unfractionated heparin and LMWH in their thrombin generation experiments. Though these experiments need confirmation from a reproducibility point of view, they suggest that it should be possible to neutralise the effect the $\mathrm{LMWH}$ in plasma being tested for thrombin generation using polybrene, and therefore be able 
to determine what impact the LMWH has on thrombin generation in-vivo, providing a valuable insight.

\subsection{Venous Thromboembolism}

Pulmonary embolism (PE) and deep vein thrombosis (DVT) represent the spectrum of one disease, and exemplifies thrombosis in a pathological setting. VTE is a complex (multifactorial) disease, involving an interaction between environmental and genetic factors. Thrombi commonly form in the deep veins of the calf and then propagate into the proximal veins, from where they are more likely to embolise [Tapson, 2008]. Figure 1.3 illustrates the pathophysiology of VTE. It's thought that three-quarters of patients who present with PE have evidence of DVT in their legs; in the remaining twenty-five percent of patients, usually the whole clot has embolised from the leg.

Chronic sequelae of VTE include the post thrombotic syndrome and chronic thromboembolic pulmonary hypertension [Schulman et al., 2006; Pengo et al., 2004]. Post-thrombotic syndrome causes chronic venous hypertension, which leads to limb pain, swelling, hyperpigmentation, dermatitis and ulceration [Tovey and Wyatt, 2003]. Symptoms of pulmonary hypertension include progressive exertional dyspnoea and exercise intolerance, which can lead to chest pain on exertion or syncope as a result of severe pulmonary hypertension and the inability of a compromised right ventricle to meet the body's demands for cardiac output [Fedullo et al., 2001]. 


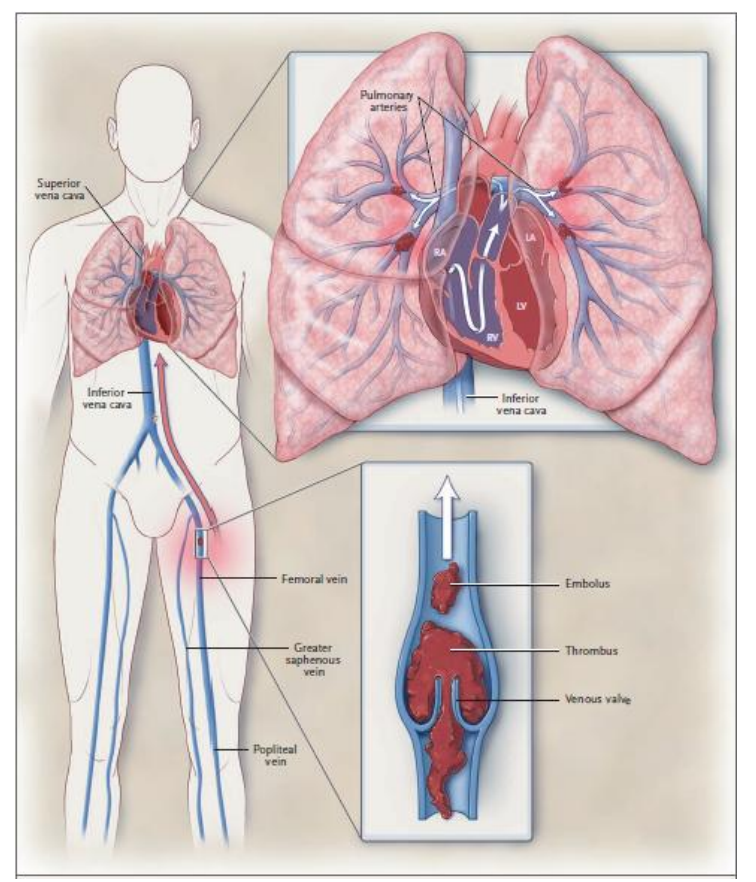

Figure 1.3: The pathophysiology of VTE - With permission, New England Journal of Medicine Copyright (๑) 2010 Massachusetts Medical Society. All rights reserved

\subsubsection{Incidence}

The annual estimated incidence of VTE is reported to be around 1 in 1000 of the population, and although less common in certain regions e.g. Asia, VTE is a worldwide problem [Silverstein et al., 1998].

VTE is predominantly a disease of older age [Silverstein et al., 1998; Cushman et al., 2004], as it is rare prior to adolescence unless thrombophilia or a central venous catheter are present [Heit, 2005]. Incidence rates of VTE increase markedly with age for both men and women. The overall age-adjusted incidence rate is higher for men (130 per 100 000) than women (110 per 100 000) (male:female sex ratio is 1.2:1). However, the incidence is higher in women during the childbearing years, while the incidence rates after 45 years of age are generally higher in men. PE accounts for a signficiant proportion of VTE, increasing with age for both genders [Heit, 2005].

\subsubsection{Survival and recurrence}

Survival after experiencing a PE is worse than for a DVT alone [Heit et al., 1999; Goldhaber et al., 1999]. The risk of early death among patients with symptomatic PE is 18 -fold higher when compared to patients with DVT alone. For almost one-quarter of PE patients, the initial clinical presentation is sudden death. Independent predicators of mortality after a VTE 
include increasing age, male gender, lower body mass index, being in a hospital at VTE onset, congestive heart failure, chronic lung disease, serious neurological disease and active malignancy [Heit, 2005].

VTE frequently recurs, with an estimated $30 \%$ of patients developing a recurrence within 10 years [Heit et al., 2000]. The risk of recurrence changes over time, but is highest within the first 6-12 months. Independent predictors of recurrence include the male gender, increasing age, high body mass index, presence of neurological disease with extremity paresis and active malignancy [Heit, 2005]. For women, VTE during pregnancy and the post partum period, or VTE precipitated through oral contraceptive use or gynaecologic surgery are associated with a reduced risk of recurrence [Heit et al., 2000]. Provoked VTE through surgery, trauma or fracture are reported to have a negligible or reduced risk of recurrence [Prandoni et al., 1996].

\subsubsection{Risk Factors}

There are a number of risk factors which pre-dispose a patient to the development of VTE. Some of these risk factors are hereditary and some acquired. Table 1.1 lists the common risk factors associated with VTE [Tapson, 2008; Geerts et al., 2008]. 
Table 1.1: Risk factors for VTE

\begin{tabular}{|c|c|}
\hline Hereditary factors & Acquired factors \\
\hline Antithrombin deficiency & Immobility, lower-extremity paresis \\
\hline Protein $\mathrm{C}$ deficiency & Advanced age \\
\hline Protein S deficiency & Previous VTE \\
\hline Factor V Leiden & Cancer (active or occult) \\
\hline Activated protein $\mathrm{C}$ resistance without factor $\mathrm{V}$ Leiden & Acute medical illness \\
\hline Prothrombin gene mutation & Surgery \\
\hline Dysfibrinogenemia & Trauma (major trauma or lower-extremity injury) \\
\hline \multirow[t]{16}{*}{ Plasminogen deficiency } & Spinal cord injury \\
\hline & Pregnancy and postpartum period \\
\hline & Polycythemia vera \\
\hline & Antiphospholipid antibody syndrome \\
\hline & $\begin{array}{l}\text { Estrogen-containing oral contraceptives or hormone } \\
\text { replacement therapy }\end{array}$ \\
\hline & Cancer therapy (hormonal, chemotherapy, angiogenesis \\
\hline & inhibitors, radiotherapy) \\
\hline & $\begin{array}{l}\text { Venous compression (tumor, haematoma, arteria } \\
\text { abnormality) }\end{array}$ \\
\hline & Selective estrogen receptor modulators \\
\hline & Erythropoiesis-stimulating agents \\
\hline & Inflammatory bowel disease \\
\hline & Nephrotic syndrome \\
\hline & Myeloproliferative disorders \\
\hline & Paroxysmal nocturnal hemoglobinuria \\
\hline & Obesity \\
\hline & Central venous catherisation \\
\hline
\end{tabular}

\subsubsection{Treatment}

Anticoagulant therapy is currently the mainstay of treatment for VTE. The purpose of anticoagulant therapy is to prevent thrombus extension during the acute phase and recurrence during the chronic phase of treatment [Kearon et al., 2008]. The objective evidence for using anticoagulant therapy for this indication stems back to 1960, when Barritt and Jordan [1960] compared anticoagulant therapy with placebo in patients with symptomatic VTE. In their trial, those patients in the anticoagulant arm received one and a half days of unfractionated heparin therapy along with two weeks of oral anticoagulation with the Vitamin $\mathrm{K}$ antagonist, nicoumalone and this was compared to placebo. They reported that the anticoagulant treatment arm markedly reduced recurrence and mortality relative to the placebo group. Subsequent studies have unequivocally endorsed the findings from Barritt and Jordan's study, that anticoagulant therapy is superior to placebo [Alpert et al., 1976; Kanis, 1974; Kernohan et al., 2007]. 
There is also strong evidence demonstrating a need to use a form of heparin treatment initially, in combination with a vitamin $\mathrm{K}$ antagonist, as opposed to vitamin $\mathrm{K}$ antagonist monotherapy. Brandjes and colleagues [1992] conducted a randomised controlled trial comparing a vitamin $\mathrm{K}$ antagonist alone to a combination of heparin and vitamin $\mathrm{K}$ antagonist therapy and found a three-fold increase in the rate of VTE in those patients who had received vitamin $\mathrm{K}$ antagonist therapy alone.

The American College of Chest Physicians (ACCP) guidelines for the management of VTE state that both heparin and vitamin $\mathrm{K}$ antagonist therapy should be started at the time of diagnosis (or at the time of clinical suspicion - if there is a delay in conducting the diagnostic tests) and to discontinue the heparin after five days provided the INR is $\geq 2.0$ for at least 24 hours [Kearon et al., 2008].

Of the heparins available (unfractionated heparin or Low Molecular Weight Heparins $(\mathrm{LMWH}))$, LMWH offers the advantage of a predictable pharmacokinetic response and longer elimination half-life relative to unfractionated heparin [Ansell et al., 2008]. This allows body weight adjusted doses of LMWH to be administered to patients by subcutaneous injection once or twice a day, without laboratory monitoring in the majority of cases. The Cochrane collaborative carried out a meta-analysis of fixed dose subcutaneous LMWH versus adjusted dose unfractionated heparin for VTE and found that LMWH are associated with fewer thrombotic complications (3.6\% versus $5.4 \%$; OR: $0.68 ; 95 \% \mathrm{Cl}, 0.55$ to 0.840 ) and less major bleeding (1.2\% versus $2.0 \%$; OR: $0.57 ; 95 \% \mathrm{Cl}, 0.39$ to 0.83$)$ [van Dongen et al., 2004].

A meta-analysis of five studies involving LMWH has found no difference in recurrent VTE at 3 months: (OR, $0.85 ; 95 \% \mathrm{Cl}, 0.48$ to 1.49$)$, major bleeding at 10 days: $(\mathrm{OR}, 1.05 ; 95 \% \mathrm{Cl}$, 0.53 to 2.09 ), when comparing once daily LMWH with twice daily LMWH, suggesting that a once daily dose of LMWH is safe and effective [Couturaud et al., 2001].

Options for thrombolysis exist for both DVT and PE. For DVT, catheter -directed thrombolysis is sometimes conducted in selected patients with ileofemoral DVT and who have a low risk of bleeding. The aim of this treatment being to minimise the risk of postthrombotic syndrome. Evidence suggests that catheter-directed thrombolysis is effective in improving quality of life [Comerota et al., 2000], although limited facilities and experience 
means that most patients are not routinely offered such treatment. For PE, thrombolytic therapy is usually indicated in patients who are haemodynamically compromised [Kearon et al., 2008].

Once a patient suffers a VTE event, the duration of warfarin therapy depends on the presence of risk factors, whether the VTE was provoked and whether the event was a recurrence. Table 1.2 summarises the current recommendations from the American College of Chest Physcians [Kearon et al., 2008]:

Table 1.2: Summary of the ACCP recommendations for VTE management

\begin{tabular}{|c|c|}
\hline Clinical scenario & $\begin{array}{l}\text { Recommended duration of } \\
\text { antagonist }\end{array}$ \\
\hline $\begin{array}{l}\text { VTE secondary to a transient (reversible) risk } \\
\text { factor }\end{array}$ & 3 months treatment \\
\hline Unprovoked VTE & $\begin{array}{l}\text { At least } 3 \text { months treatment } \\
\text { After } 3 \text { months, all patients should be evaluated } \\
\text { for the risk-benefit ratio of long term therapy }{ }^{*} \text {. In } \\
\text { those patients with a first unprovoked VTE that is } \\
\text { a proximal DVT and in whom the risk of bleeding } \\
\text { is absent and good anticoagulation control is } \\
\text { feasible, long term treatment* is recommended. }\end{array}$ \\
\hline Second episode of unprovoked VTE & Long term treatment ${ }^{*}$ \\
\hline VTE and cancer & $\begin{array}{l}3 \text { to } 6 \text { months of } \mathrm{LMWH} \text {, following which } \\
\text { anticoagulant therapy with a vitamin } \mathrm{K} \text { antagonist } \\
\text { or LMWH indefinitely* or until the cancer has } \\
\text { resolved }\end{array}$ \\
\hline
\end{tabular}

${ }^{*}$ Patients receiving long term treatment, the risk benefit ratio of continuing anticoagulant treatment should be reassessed at regular intervals.

The advent of the newer oral anticoagulant agents such as the direct thrombin inhibitor, dabigatran [Schulman et al., 2009] and the direct factor Xa inhibitors rivaroxaban [Buller et al., 2010], apixaban and edoxaban are likely to alter this treatment algorithm in the future.

\subsection{Heparin}

\subsubsection{The discovery of heparin}

Heparin was discovered accidentally in 1916 at John Hopkins University, Baltimore in 1916, when, Jay McLean, a second year medical student at the University was trying to isolate thromboplastin from the liver of dogs and instead found a substance, now known as heparin, which inhibited the coagulation of blood [McLean, 1916]. Following McLean's discovery, 
Howell and Holt carried out further work on the newly identified heparin and objectively confirmed the inhibitory action of heparin on coagulation:

"In shed blood it was found that in mixtures containing $0.1 \%$ of heparin, the coagulation is prevented entirely. In more dilute solutions the coagulation was delayed in proportion to the concentration. When injected intravenously (into dogs), kymographic records showed that there was no disturbance of heart rate, blood pressure or respiratory rhythm and no variation in rectal temperature." [Howell and Holt, 1918].

Following this breakthrough, it took some 15 years before practical and cost-effective methods of extracting purified heparin that could be considered for human administration became available, as Howell and Holt's method of extracting heparin was expensive and cumbersome.

Charles and Scott, in Professor Best's laboratory in Toronto, were the first to extract heparin in a highly purified state in 1933 [Charles and Scott, 1933a; Charles and Scott, 1933b; Scott and Charles, 1933].

They developed a practical method to extract heparin, which yielded large quantities from bovine liver. They also found that heparin was distributed in a number of tissues, not exclusively in the liver (table 1.3):

Table 1.3: Distribution of heparin within various tissues

\begin{tabular}{l|c|c|c|c}
\hline \multirow{2}{*}{ Tissue } & \multicolumn{3}{|c|}{ Crude heparin per kilo of tissue } & \multicolumn{2}{c}{ Purified heparin per kilo of tissue } \\
\cline { 2 - 5 } & $\mathrm{mg}$ & Units & $\mathrm{mg}$ & Units \\
\hline Ox liver & 780 & 1800 & 190 & 1900 \\
Ox spleen & 1000 & 4030 & 230 & 700 \\
Ox heart & 200 & 420 & 54 & 380 \\
Ox blood & 260 & 185 & 66 & 60 \\
Ox thymus & 640 & 40 & 310 & 35 \\
Ox blood serum & 24 & 3300 & - & - \\
Ox lung & 840 & 1500 & 230 & 2200 \\
Ox muscle & 2000 & 5000 & 600 & 1900 \\
Hog liver & 1400 & 4500 & 340 & 1700 \\
Dog liver & 900 & & 330 & 4400 \\
& & & & \\
\hline
\end{tabular}




\subsubsection{First in man studies}

The first reported administration of heparin in humans was by Godlowski [1933]; however, he used a low potency, impure form and reported low level toxicity with its use. Hedenius and Wilander [1936], are reported to have initially self-injected heparin (manufactured by Professor Jorpes according to the Charles and Scott method), followed by administration to patients, and found that $100 \mathrm{mg}$ or more of heparin was needed for heparinising man for a few hours.

It was the great Swedish surgeon, Clarence Crafoord, who recognised the potential clinical application of heparin as a prophylactic agent against pulmonary embolism. As early as 1929 he had approached Professor Jorpes of the Chemistry department at the Karolinska Institute in Sweden, asking for Howell's heparin, so he could use it as a prophylactic agent in his patients undergoing surgery. At the time, Professor Jorpes said this was not possible because of expense and impurities present after the extraction process [Jorpes, 1959]. However in 1935, Professor Jorpes approached Clarence Crafoord, after heparin extraction and purification had been made possible by the Scott and Charles method. Crafoord immediately started a series of experiments by heparinising patients post-operatively, with the help of his colleagues, Wetterdal (Gynaecologist) and Leissner (Obstetrician). In total, they treated around 800 patients, and in the case series they treated, one would normally have expected a VTE rate of 3-4\%, perhaps higher. None of the patients suffered from a VTE event [Crafoord, 1937; Crafoord and Jorpes, 1941]. At the same time that Crafoord was conducting his experiments on his post-op patients, Gordon Murray in Toronto was assessing the prophylactic use of heparin post operatively in a case series amounting to 400 cases. His results re-affirmed Crafoord's findings [Murray, 1941].

\subsubsection{Unfractionated Heparin}

Unfractionated heparin belongs to the family of sulphated glycosaminoglycans (mucopolysaccharides). It is present together with histamine in the granules of mast cells, and commercial preparations are extracted from beef lung or porcine intestine. Due to the variation in potency seen from the different sources used, unfractionated heparin is assayed against an internationally agreed standard, so that doses are specific in units of activity [Council of Europe, European Directorate for the Quality of Medicines, 2007]. 


\subsubsection{Pharmacology}

Heparin exerts in anticoagulant effect in vivo and in vitro by activating anti-thrombin. Antithrombin inhibits the serine proteases XIla, Xla, Xa, IXa, and Ila, by binding and inhibiting the active serine sites, through an arginine-reactive site on the anti-thrombin. Heparin modifies this interaction by binding to the lysine site on anti-thrombin, causing a conformational change at the arginine-reactive site that converts anti-thrombin from a slow inhibitor of thrombin and $\mathrm{Xa}$ to a rapid inhibitor. Anti-thrombin binds covalently to the active serine centres of coagulation enzymes; heparin then dissociates and can be re-used [Rosenberg and Bauer, 1994].

About one-third of a heparin dose administered binds to anti-thrombin, with a pentasaccharide fraction (green segment of figure 1.4) [Choay et al., 1981] thought to be responsible for most of its anticoagulant effect [Lam et al., 1976; Andersson et al., 1976], as illustrated in figure 1.4 .
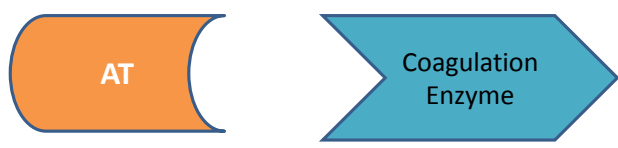

Without heparin

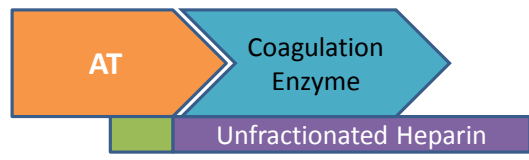

Ternary complex formation

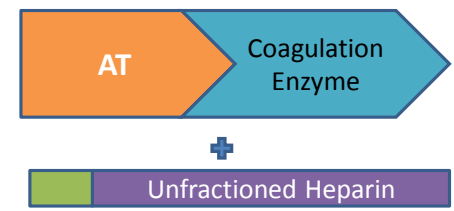

Dissociation of heparin

Figure 1.4: The pharmacological action of unfractionated heparin

In addition to its anticoagulant effect, heparin increases vessel wall permeability, inhibits the proliferation of vascular smooth muscle cells and suppresses osteoblast formation, whilst activating osteoclasts [Hirsh et al., 2008]. 


\subsubsection{Pharmacokinetic profile}

Unfractionated heparin (UFH) has a molecular weight ranging from 3000 to 30,000 daltons (mean 15,000 - 45 monosaccharide chains) [Johnson and Mulloy, 1976]. The high molecular weight moieties are cleared more rapidly than those of a lower-molecular weight [Hirsh et al., 2008].

In vivo, heparin binds to a number of plasma proteins, which reduces its anticoagulant activity, contributing to its variable anticoagulant response [Hirsh et al., 2008]. Heparin is also found to bind to endothelial cells [Mahadoo et al., 1977; Glimelius et al., 1978; Barzu et al., 1985] and macrophages [Friedman et al., 1974], which further complicates its pharmacokinetic profile [de Swart et al., 1982].

Heparin clearance involves a combination of a rapid saturable and a much slower first-order mechanism [Olsson et al., 1963; de Swart et al., 1982; Bjornsson et al., 1982]. The mechanism of the saturable phase of heparin clearance is through binding to receptors on endothelial cells and macrophages, where it is depolymerised. The slower unsaturable mechanism involves renal clearance. At therapeutic doses, heparin is cleared through the rapid saturable, dose-dependent mechanism, making its pharmacokinetic profile non-linear, with both the intensity and duration of effect rising disproportionately with an increase in the dose. As a result, its half life will vary according to the dose (and route) administered, increasing from 30 minutes following an intravenous bolus dose of 25 units $/ \mathrm{kg}$, to 60 minutes with a bolus of 100 units $/ \mathrm{kg}$, and 150 minutes with a bolus of 400 units $/ \mathrm{kg}$ [Olsson et al., 1963; de Swart et al., 1982; Bjornsson et al., 1982].

\subsubsection{Adverse effects}

UFH can cause hyperkalemia, as it is found to inhibit aldosterone secretion [Oster et al., 1995]. Although all patients treated with heparin may develop reduced levels of aldosterone, most are able to compensate through the renin-aldosterone system. However, some patients, e.g. patients with diabetes and those with renal impairment, are thought to have an impaired compensatory mechanism and may therefore develop hyperkalemia [Anon (a), 1999]. The hyperkalemia is usually transient and resolves once heparin is discontinued.

Heparin has also been associated with the development of thrombocytopenia. Although the reported incidence varies greatly, up to $6 \%$ of treated patients are thought to be affected 
[Derlon et al., 1988; Anon (b), 1990]. There are two types of thrombocytopenia attributed to heparin. The first is an acute, but usually mild, fall in platelet count occurring within the first four days of starting treatment that resolves without stopping treatment. A direct effect of heparin on platelet aggregation appears to be responsible [Martindale, 2011]. The second type of thrombocytopenia, the more serious form, has an immunological basis. It usually develops between day 5 to 15 of treatment and is associated with thromboembolism, complicating treatment [Aster, 1995]. The mechanism appears to be as a result of the development of antibodies to a complex formed between heparin and platelet factor-4 (found on platelets and endothelial cells), which then causes platelet activation and thrombin generation. The reaction is independent of dose or route with reports of thrombocytopenia after use of heparin flushes [Keeling et al., 2006] or heparin-coated catheters [Warkentin, 1997].

Osteoporosis is another recognised adverse effect of heparin and is a rare complication of long-term heparin therapy - around 2\% [Nelson-Piercy, 1998; Bates et al., 2008]. Prophylaxis and treatment of VTE during pregnancy is one of the few indications requiring the long term use of heparin, so most studies of heparin-induced osteoporosis have been during pregnancy [Nelson-Piercy, 1998]. In animal studies, heparin causes a dose dependent loss of bone through decreasing rates of bone formation and increased bone resorption [Muir et al., 1996] and it is thought this mechanism also applies to humans. Subclinical reduction in bone density occurs in up to one-third of patients [Bates et al., 2008]. As the state of pregnancy additionally causes bone demineralisation, long term heparin use in this cohort of patients puts them at particular risk.

Skin necrosis is a rare complication of heparin therapy and may present as a localised reaction at the site of subcutaneous injection or more rarely be related to heparin-induced thrombocytopenia [Ulrick et al., 1984; Fowlie et al., 1990]. Very rarely, eczematous plaque reactions develop in some patients several days after starting treatment. A type IV hypersensitivity reaction has been implicated [Bircher et al., 1990]. 


\subsection{Low molecular weight heparins}

In 1976, two research groups both reported the preparation of LMWH fractions from unfractionated heparin, and found progressively less effect on the activated partial thromboplastin time ((APTT), the laboratory measure of unfractionated heparin activity), whilst still inhibiting factor Xa and exerting an anticoagulant effect [Johnson et al., 1976; Andersson et al., 1976]. This finding led to extensive research into fragments of LMWH in the late 1970's and early 1980's and eventually, to the availability of commercial preparations of LMWH in the 1990's.

LMWH are also glycosaminoglycans, repeating fragments of unfractionated heparin produced by controlled enzymatic or chemical depolymerisation processes that yield chains with a mean molecular weight of about $5000 \mathrm{Da}$ (table 1.4) [Weitz, 1997; Baglin et al., 2006].

Table 1.4: The differences between commercially available LMWH

\begin{tabular}{llcc}
\hline Preparation & Method of preparation & $\begin{array}{c}\text { Mean } \\
\text { MW (Da) }\end{array}$ & $\begin{array}{c}\text { Anti-Xa: } \\
\text { Anti-lla ratio }\end{array}$ \\
\hline Ardeparin & Peroxidative depolymerisation & 6000 & 1.9 \\
Dalteparin & Nitrous acid depolymerisation & 6000 & 2.7 \\
Enoxaparin & Benzylation and alkaline depolymerisation & 4200 & 3.8 \\
Nadroparin & Nitrous acid depolymerisation & 4500 & 3.6 \\
Reviparin & Nitrous acid depolymerisation, chromatographic & 4000 & 3.5 \\
Tinzaparin & Eurification & 4500 & 1.9 \\
Certoparin & Nitrous acid depolymerisation & 3100 & 2.4 \\
Parnaparin & Peroxidative depolymerisation & 3700 & 2.3 \\
Bemiparin & Quaternary ammonium fractionation & 2900 & 9.6 \\
Semuloparin & $\begin{array}{l}\text { Partial and controlled chemoselective } \\
\text { depolymerisation }\end{array}$ & 2400 & 80 \\
\hline
\end{tabular}

Similar to unfractionated heparin, LMWH exert their anticoagulant effect through binding to anti-thrombin [Rang et al., 2005]. Unfractionated heparin and LMWH interaction with antithrombin is mediated by a unique pentasaccharide sequence that is randomly distributed along the heparin chains. Approximately one third of the chains of unfractionated heparin, but only 15 to 25 percent of the chains of $\mathrm{LMWH}$, contain the pentasaccharide sequence [Harenberg, 1990]. By binding to anti-thrombin through this unique pentasaccharide sequence, the activity of antithrombin is increased 1000 times [Rosenberg and Bauer, 1994]. 
The key difference between unfractionated and LMWH is their action on factor lla (thrombin). Any pentasaccharide-containing heparin chain can inhibit the action of factor Xa simply by binding to antithrombin and causing a conformational change (figure 1.5).

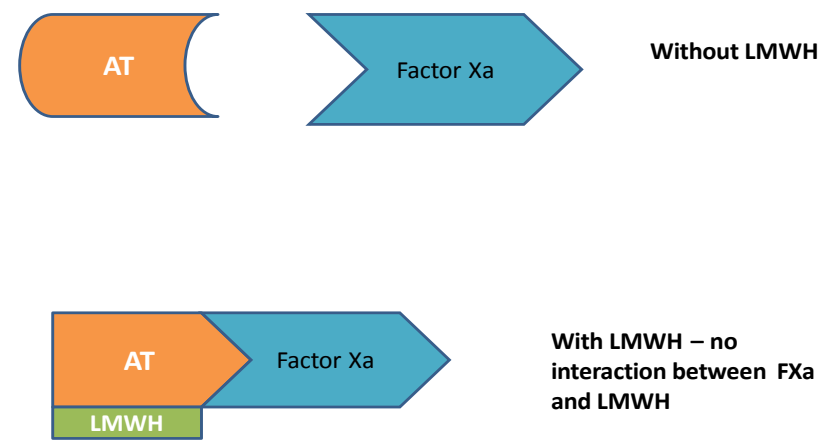

Figure 1.5: The pharmacological action of LMWH

In contrast, to inactivate factor Ila, heparin must bind to both antithrombin and thrombin, leading to the formation of a ternary complex [Danielsson et al., 1986]. This ternary complex can be formed only by pentasaccharide-containing heparin chains composed of at least 18 saccharide units. Whereas most of the chains of unfractionated heparin are at least 18 saccharide units long, less than half of LMWH are of sufficient length to bind to both antithrombin and thrombin [Jordan et al., 1980]. This means that LMWH have a greater activity against factor $\mathrm{Xa}$, whereas unfractionated heparins have equivalent activity against factor Ila and factor Xa.

It has also been reported that the tissue-factor-pathway inhibitor (TFPI) contributes to the inhibitory activity of LMWH and unfractionated heparin against factor Xa [Abildgaard et al., 1991]. The TFPI forms a complex with and inactivates factor Xa, followed by an inactivation of factor VIla [Sandset et al., 1988]. Both unfractionated heparin and LMWH are thought to release TFPI from the endothelium and enhance its inhibitory activity against factor $\mathrm{Xa}$ [Sandset et al., 1988; Huang et al., 1993; Broze, 1995]. 


\subsubsection{Pharmacokinetics of LMWH}

LMWH have a more predictable pharmacokinetic profile compared to unfractionated heparin, which in clinical terms has meant better bioavailability than unfractionated heparin, a doseindependent rate of clearance and a longer half life [Bara et al., 1985; Bara et al., 1988; Weitz, 1997]. The plasma elimination half life of LMWH is longer than that of unfractionated heparin, ranging from 2-4 hours, after intravenous injection (rarely used in clinical practice), to three to six hours after subcutaneous injection [Harenberg, 1990; Matzsch et al., 1987; Bara and Samama 1988]. Over the last twenty years, LMWH have superceeded unfractionated heparin as the heparin of choice in clinical practice. This shift in preference has largely been led by the favourable pharmacokinetic profile of LMWH. This profile is more favourable for the following reasons [Weitz, 1997]:

1. Less binding to plasma proteins and to proteins released from activated platelets and endothelial cells - leading to a more predictable anticoagulant response

2. Less binding to endothelium - leading to a better bioavailability

3. Less binding to macrophages - which means a dose independent clearance and a longer half-life

Unfractionated heparin binds to endogenous plasma proteins, for example histidine-rich glycoprotein, polymeric vitronectin and fibronectin. It also binds to platelet factor 4 , which is released from activated platelets and to high molecular weight multimers of von Willebrand factor, which are released from platelets and endothelial cells [Lane, 1989; Sobel et al., 1991; de Romeuf and Mazurier, 1993]. This binding reduces the anticoagulant activity of unfractionated heparin, as there is less available to interact with antithrombin. Furthermore, the unpredictable response that is observed with unfractionated heparin is attributed to the wide variability in patients' plasma concentrations of heparin binding proteins [Young et al., 1992]. In addition, some of these heparin binding proteins are acute phase reactants, which increase in concentration at times of illness, and some, like platelet factor 4 and von Willebrand factor, are increased during active coagulation [Weitz, 1997].

As LMWH exhibit reduced binding to plasma proteins [Young et al., 1993; Young et al., 1994] and the endothelium [Barzu et al., 1985], their bioavailability is improved. Furthermore, the 
reduced binding of LMWH to macrophages explains why they are not cleared by hepatic mechanisms to the same extent as unfractionated heparin and why renal clearance is more prominent for these agents. In addition, LMWH are thought to be associated with less bleeding than unfractionated heparin. This is because, unlike unfractionated heparin, LMWH do not increase microvascular permeability [Blajchman et al., 1989]. LMWH have a lower affinity for endothelial cells, platelets and von Willebrand factor, which means that LMWH are less likely to interfere with the interaction between platelets and the vessel wall [Salzman et al., 1980; Horne and Chao, 1990; Weitz, 1997].

The better bioavailability, decreased affinity for heparin binding proteins and dose independent clearance makes the anticoagulant response to LMWH more predictable and in clinical practice has meant that routine laboratory monitoring is unnecessary, except in patients at the extremes of body weight and in those with renal impairment [Samama, 1995; Kessler, 1997; Abbate et al., 1998].

The pharmacokinetic properties of LMWH have been studied and described using anti-Xa activity as a biomarker. Following subcutaneous injection, dose-dependent anti-Xa activity is found, peaking at between 3-4 hours post dose with anti-Xa activity found up to 12 hours later [Andrassy and Eschenfelder, 1996]. It is thought, that the persistence of anti-Xa activity in the plasma is central to the pharmacological activity of all LMWH. The area under the plasma concentration versus time curve (AUC) for the different LMWH increases with rising doses, with repeated doses of LMWH also increasing the AUC [Harenberg et al., 1989; Pedersen et al., 1991; Barradell et al., 1992]. Figure 1.6 illustrates the typical anti-Xa activity over time in non-pregnant individuals, for the three most commonly used LMWH in the UK, dalteparin, enoxaparin and tinzaparin, in addition to unfractionated heparin [Andrassy and Eschenfelder, 1996]. Enoxaparin and dalteparin have similar anti-Xa profiles, whereas tinzaparin's profile is more closely related to unfractionated heparin. For all four preparations, anti-Xa activity converges at the 12 hour point.

LMWH are found to be confined to the intra-vascular space, and their apparent volume of distribution corresponds to that of the plasma. The elimination of LMWH is primarily through a non-saturable renal route [Andrassy and Eschenfelder, 1996]. 


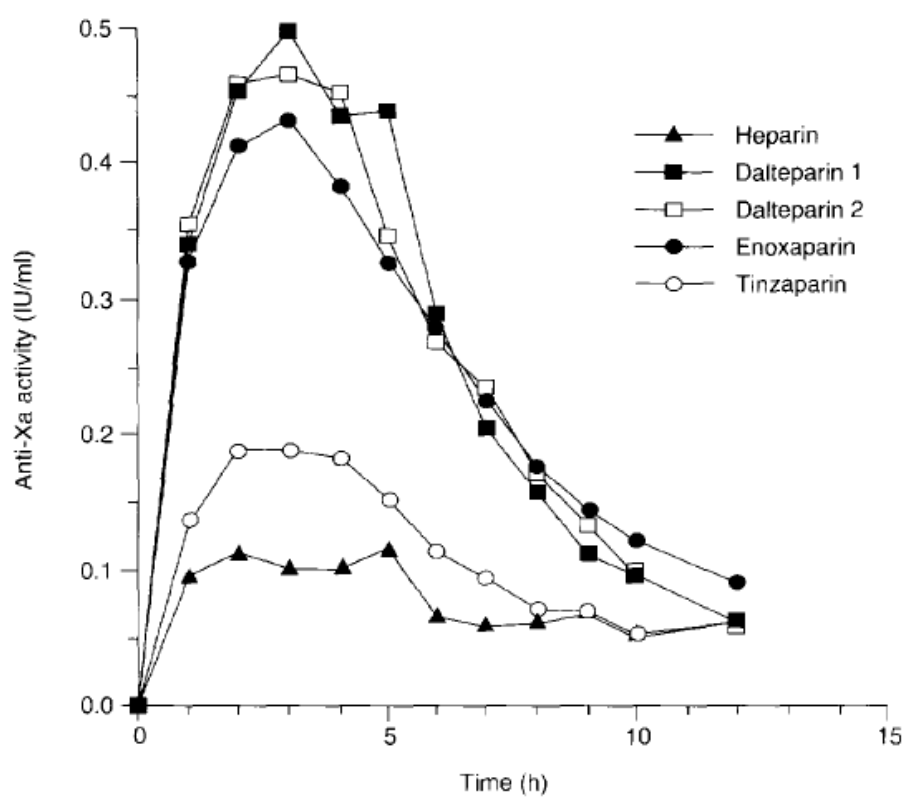

Figure 1.6: Elimination curves of Unfractionated heparin (5000 IU), Dalteparin (5000 IU) - two administrations, Enoxaparin (4000 IU) and Tinzaparin (50 IU/kg) [Andrassy K, Eschenfelder V [1996], with permission from Elsevier]

\subsubsection{Adverse effects of LMWH}

Being derived from unfractionated heparin, the side-effects of LMWH mirror those of unfractionated heparin. The two clinically relevant side-effects are HIT and osteoporosis. The frequency of HIT is approximately three fold lower with LMWH than with unfractionated heparin. This is explained by the fact that the interaction of heparin with platelet factor- 4 is chain length dependent, and so with LMWH, there is less likelihood of that specific interaction occurring. However, although the chance of binding with platelet factor-4 is reduced, LMWHs can form complexes with platelet factor-4 that are capable of binding HIT antibodies, therefore in patients with HIT antibodies, cross-reactivity with LMWH occurs [Warkentin et al., 2008]. The risk of osteoporosis is reported to be lower with LMWH when compared to unfractionated heparin, although research based on rat models is conflicting. Mätzsch and colleagues using a rat model, found that for similar anti-Xa activity, the effect of LMWH and unfractionated heparin on experimental bone loss were similar [1990]. However, Monreal and colleagues [1990], compared the effects of unfractionated heparin and LMWH on bone loss in rats and found both heparin types to cause bone loss, although the effect was greater with unfractionated heparin. Muir and colleagues [Muir et al.,1997] found both unfractionated heparin and LMWH produced a dose-dependent decrease in bone volume in rats, although the effect was greater for unfractionated heparin. The current consensus is that both UFH 
and LMWH have the potential to produce osteopenia, but the risk is greater for unfractionated heparin [Hirsh et al., 2008].

\subsubsection{LMWH use in UK}

In the United Kingdom, there are four LMWH preparations licensed for clinical use; Bemiparin Sodium, Dalteparin Sodium, Enoxaparin Sodium and Tinzaparin Sodium. Of these, Dalteparin, Enoxaparin and Tinzaparin are most commonly prescribed in clinical practice and is where most clinical experience lies [British Medical Association and the Royal Pharmaceutical Society of Great Britain, 2010]. Table 1.5 lists the different licensed indications and the associated doses.

Table 1.5: Doses of LMWH recommended in the UK

\begin{tabular}{llll}
\hline Preparation & Prophylaxis of VTE & Treatment of VTE & $\begin{array}{l}\text { Unstable angina and } \\
\text { STEMI }\end{array}$ \\
\hline Dalteparin & $2,500-5,000 \mathrm{IU}$ od & $200 \mathrm{IU} / \mathrm{kg}$ od & $120 \mathrm{IU} / \mathrm{kg} \mathrm{bd}$ \\
Enoxaparin & $2,000-4,000 \mathrm{IU}$ od & $150 \mathrm{IU} / \mathrm{kg}$ od & $100 \mathrm{IU} / \mathrm{kg} \mathrm{bd}$ \\
Tinzaparin & $3,500-4,500 \mathrm{IU}$ od & $175 \mathrm{IU} / \mathrm{kg}$ od & Not licensed \\
\hline
\end{tabular}

For each $\mathrm{LMWH}$, a wide spectrum of in-vitro and in-vivo coagulation tests can detect measurable pharmacodynamic differences between the LMWH when they are administered using equivalent anti-Xa doses. Whilst debate continues on whether LMWH are all the same [Prandoni and Nenci, 2003; White and Ginsberg, 2003], clinical experience indicates that any differences in effectiveness and safety, if they exist, are small [Baglin et al., 2006], with the small number of studies comparing one against another having found no clinically meaningful differences between them [White and Ginsberg, 2003].

\subsection{Venous Thromboembolism in Pregnancy}

\subsubsection{Epidemiology}

VTE during pregnancy represents a significant cause of maternal morbidity, with an overall reported incidence of between 1-2 episodes per 1,000 deliveries [Lindqvist et al., 1999; Heit et al., 2005, Jacobsen et al., 2008]. These figures represent a five to ten fold increase in risk compared to non-pregnant women of a comparable age [Bates et al., 2008]. Over the last 30 years, thrombosis and thromboembolism has consistently been reported to be the most 
common cause of direct maternal mortality in the western world, including the UK, remaining relatively constant over this time (table 1.6) [Centre for maternal and child enquiries, 2007]. The recently published eighth report of the confidential enquiries into maternal deaths in the United Kingdom [Centre for maternal and child enquiries, 2011], describes a significant fall in deaths as a result of thrombosis and thromboembolism in pregnant and postpartum women, from 41 deaths during the reporting period 2003-2005 to 18 deaths during the period 20062008. This is the first time that thrombosis and thromboembolism is not reported as the leading cause of direct maternal death in the United Kingdom. The first Royal College of Obstetricians and Gynaecologists (RCOG) guideline for VTE prophylaxis during pregnancy and the puerperium was published in 2004 , so that it is likely that this significant fall in mortality, is as a result of more wide-spread ante and post-natal LMWH thromboprophylaxis.

Table 1.6: Rates per 100000 maternities of direct deaths from thrombosis and thromboembolism; United Kingdom: 1985-2008

\begin{tabular}{cccccccccc}
\hline & \multicolumn{3}{c}{ Pulmonary embolism } & \multicolumn{3}{c}{ Cerebral vein Thrombosis } & \multicolumn{2}{c}{$\begin{array}{c}\text { Thrombosis and } \\
\text { thromboembolism }\end{array}$} \\
\cline { 2 - 10 } & $n$ & Rate & $95 \% \mathrm{Cl}$ & $n$ & Rate & $95 \% \mathrm{Cl}$ & $n$ & Rate & $95 \% \mathrm{Cl}$ \\
\hline $1985-87$ & 30 & 1.32 & $0.83-1.89$ & 2 & 0.09 & $0.02-0.32$ & 32 & 1.41 & $1.00-1.99$ \\
$1988-90$ & 24 & 1.02 & $0.68-1.51$ & 9 & 0.38 & $0.20-0.72$ & 33 & 1.40 & $1.00-1.96$ \\
$1991-93$ & 30 & 1.30 & $0.91-1.85$ & 5 & 0.22 & $0.09-0.51$ & 35 & 1.51 & $1.09-2.10$ \\
$1994-96$ & 46 & 2.09 & $1.57-2.79$ & 2 & 0.09 & $0.02-0.33$ & 48 & 2.18 & $1.65-2.90$ \\
$1997-99$ & 31 & 1.46 & $1.03-2.07$ & 4 & 0.19 & $0.07-0.48$ & 35 & 1.65 & $1.19-2.29$ \\
$2000-02$ & 25 & 1.25 & $0.85-1.85$ & 5 & 0.25 & $0.11-0.59$ & 30 & 1.50 & $1.05-2.14$ \\
$2003-05$ & 33 & 1.56 & $1.11-2.19$ & 8 & 0.38 & $0.19-0.75$ & 41 & 1.94 & $1.43-2.63$ \\
$2006-08$ & 16 & 0.70 & $0.43-1.14$ & 2 & 0.09 & $0.02-0.35$ & 18 & 0.79 & $0.49-1.25$ \\
\hline
\end{tabular}

The increased risk of VTE during pregnancy begins in the first trimester and remains high during the second and third trimesters [James, 2009]. A meta-analysis of the risk of pregnancy related DVT showed event rates of $21.9 \%, 33.7 \%$ and $47.6 \%$ over the three trimesters, sequentially [Ray and Chan, 1999]. DVT accounts for $85 \%$ of pregnancy-related symptomatic VTE [James et al., 2005] and affects the left leg in approximately $90 \%$ of cases [Chan et al., 2010], due to the gravid uterus and compression of the left common iliac vein by the right common iliac artery [Arya, 2011]. Rarely, pregnancy associated DVT presents as upper extremity thrombosis which is usually associated with either assisted conception or ovarian hyperstimulation syndrome [Arya et al., 2001]. Cerebral venous thrombosis is a rare 
complication of pregnancy, particularly occurring during the third trimester and after delivery [Cantu and Barinagarrementeria 1993].

\subsubsection{Pathophysiology}

During pregnancy, all three of Virchow's triad factors associated with venous thrombosis are present; hypercoagulability, venous stasis and vascular damage [Virchow, 1846].

Endothelial damage to pelvic vessels can occur during vaginal or abdominal delivery [Goodrich \& Wood, 1964]. Venous blood flow velocity is reduced by around $50 \%$ by the third trimester [Macklon et al., 1997]. Normal pregnancy is accompanied by increased concentrations of factors VII, VIII, IX, von Willebrand factor and fibrinogen. Conversely, free protein $\mathrm{S}$ is reduced during pregnancy [Hellgren and Blomback, 1981; de Moerloose et al., 1998; Bremme, 2003; Szecsi et al., 2010]. These changes are accompanied by impaired fibrinolysis through increases in plasminogen activator inhibitors 1 and 2, the latter being produced by the placenta [Greer, 1999]. Collectively, these haemostatic changes occur in preparation for delivery, to protect against haemorrhage.

\subsubsection{Risk factors for antenatal VTE}

Aside from the haemostatic and venous alterations during pregnancy, the risk of VTE might be further increased by the presence of additional factors. As for non-pregnant individuals, previous thrombosis is a strong risk factor for VTE, increasing risk 3 to 4 fold (Pabinger et al., 2002), with recurrent events accounting for 1 in 5 VTE events in pregnancy. About $50 \%$ of events are associated with thrombophilia; both acquired and inherited. Table 1.7 lists the different inherited thrombophilias and describes the risk conferred by each. 
Table 1.7: Adjusted odds ratio (OR) for risk of VTE in pregnancy and/or postpartum with different thrombophilias (data from Lim et al., 2007 and Robertson et al., 2005).

\begin{tabular}{lccc}
\hline Thrombophilia & \multicolumn{3}{c}{ Risk of VTE } \\
\cline { 2 - 4 } & \multicolumn{1}{c}{ Odds Ratio } & $95 \% \mathrm{Cl}$ \\
\cline { 2 - 4 } & Lim (2007) & Robertson (2005) & $1.3-17$ \\
\hline Antithrombin deficiency & $10-$ unknown & 4.7 & $2.2-10.6$ \\
Protein C deficiency & $2-$ unknown & 4.8 & $1.5-6.9$ \\
Protein S deficiency & - & 3.2 & $2.5-18.8$ \\
$\begin{array}{l}\text { Prothrombin G20210A } \\
\text { (heterozygous) }\end{array}$ & $3-10$ & 6.8 & $9.9-120.1$ \\
$\begin{array}{l}\text { Factor V Leiden } \\
\text { (homozygous) }\end{array}$ & $10-41$ & 34.4 & $1.2-559.2$ \\
$\begin{array}{l}\text { Prothrombin G20210A } \\
\text { (homozygous) }\end{array}$ & - & 26.4 & - \\
$\begin{array}{l}\text { Compound } \\
\text { heterozygote (Factor V } \\
\text { Leiden and prothrombin } \\
\text { G20210A) }\end{array}$ & $9-107$ & & - \\
\hline
\end{tabular}

Additional factors include the presence of heart disease, sickle cell disease, diabetes, smoking, $\geq 35$ years age and obesity [James et al., 2006]. Pregnancy related factors include assisted reproduction, hyperemesis, pre-eclampsia, multiple gestation, prolonged labour and delivery through caesarean section [Royal College of Obstetrics and Gynaecologists, 2009].

\subsubsection{Medication use in pregnancy}

It is estimated that approximately $80 \%$ of pregnant women in the United States of America receive at least one medication during their pregnancy [Andrade et al., 2004] with similar figures reported for Europe where $80 \%$ of pregnant women in Norway and $70 \%$ of pregnant women in Italy [Engeland et al., 2008; Gagne et al., 2008] had used a medicine during this period. Although there is wide-spread use of medications during pregnancy with antibiotics and respiratory drugs most commonly prescribed [Andrade et al., 2004], little research has been conducted to assess how effective the medication remains, given the physiological changes in pregnancy, with safety often being the primary concern during pregnancy following the thalidomide and diethylstilbestrol cases [Ing et al., 1962; Goodman et al., 2011]. This has largely meant that when drugs have been prescribed during pregnancy they have not been objectively evaluated by the relevant pharmaceutical regulatory bodies, meaning the clinician prescribing or recommending the medication, does so, off-label, and as few 
medications are developed and subsequently evaluated in pregnant women, little information on appropriate dosing or efficacy exists [Malek and Mattison, 2010]. Furthermore, little information exists on women's adherence to their medication during their pregnancy. Information from a recently published Australian study [Sawicki et al., 2011] suggests that adherence to medication used for long term conditions during pregnancy may be affected and warrants further investigation.

\subsubsection{Anatomical and physiological changes of pregnancy}

Appreciating the anatomical and physiological changes which occur during pregnancy, should help develop appropriate dosing strategies for drugs used by pregnant women. Some forty years ago researchers began to recognise that the state of pregnancy altered drug disposition, which could then alter the desired outcome [Philipson et al., 1976; Philipson, 1979].

\subsubsection{Anatomical alterations}

Key anatomical changes which occur in pregnant women include, an increase in body weight, an increase in uterine volume and composition, outward growth of the pelvis and displacement of the abdominal and chest organs [Hytten, 1975; Hill and Pickinpaugh, 2008; Malek and Mattison, 2010]. Growth of the uterus alters the centre of gravity of the pregnant woman; the heart enlarges, is elevated and rotated to the left, modifying the electrocardiogram; the diaphragm is elevated, decreasing lung volume; breasts enlarge and the circumference of the rib cage increases. The growth of the uterus increases vascular pressure on tissues below the pelvis, leading to oedema, varicosities and venous stasis.

\subsubsection{Physiological alterations}

Physiological changes begin early in pregnancy and can broadly be broken down into alterations within the cardiovascular, renal, pulmonary, gastrointestinal and haematological systems. The key changes associated with these systems and their implications follow.

Changes in cardiovascular physiology begin in early pregnancy [Abbas et al., 2005] and continue through to the postpartum period. Cardiac output increases from 5 to $7.5 \mathrm{~L} / \mathrm{min}$, stroke volume increases from 65 to $85 \mathrm{ml}$ and heart rate increases from 75 to 87 beats per min [Lindheimer et al., 2009]. During the second half of pregnancy, cardiac output is altered according to maternal position; cardiac output falling when standing or lying supine 
[Lohsiriwat and Imrittha, 2008]. Blood pressure falls by mid pregnancy and then begins to rise [Ganzevoort et al., 2004]. Collectively, this leads to decreased vascular resistance and blood pressure, increased cardiac output, and stimulation of the renin-angiotensin-aldosterone system.

As the uterus grows, the kidneys are moved upward, with progesterone also acting to dilate the ureters. Renal blood flow, glomerular filtration and kidney size increase [Pavek et al., 2009]. During pregnancy, there is an expansion of the intravascular plasma volume by up to $60 \%$ [Anderson, 2005]. In addition, the glomerular filtration rate (GFR) increases by up to $50 \%$ by the end of the first trimester and this increase is maintained throughout pregnancy until the last three weeks, when a decrease in GFR is observed, reaching post-partum levels by the last week [Anderson, 2005]. The renal changes occurring during pregnancy often have important implications for the pharmacokinetic profiles of medications that may be required during pregnancy.

The tidal volume and inspiratory capacity increase during pregnancy. The respiratory rate, vital capacity and inspired reserve volume remains unchanged during pregnancy. The total lung capacity, residual volume, expiratory reserve volume and functional reserve capacity are decreased during pregnancy [Schatz et al., 2006; Jensen et al., 2008]. These changes largely have implications for women who suffer from asthma, which may worsen during pregnancy [Tan and Thomson, 2000]. Increased blood flow and oedema in the upper airway leads to pregnancy rhinitis in approximately one in five women [Demoly et al., 2003].

Gastrointestinal changes include an increased stomach $\mathrm{pH}$ due to decreased acid secretion and delayed stomach emptying. Furthermore, progesterone decreases gut motility and sphincter tone; these changes in part explain the heartburn, nausea and vomiting experienced by women duringearly pregnancy [Bynum, 1977; Frederiksen, 2001; Matok et al., 2009].

Maternal blood volume increases by approximately $50 \%$ during pregnancy, beginning early in the first trimester and plateauing during the third trimester. The increase is approximately $1500 \mathrm{ml}$ for a singleton and $3000 \mathrm{ml}$ for a twin pregnancy [Hytten and Leitch, 1971]. Red cell volume increases approximalety $400 \mathrm{ml}$ in a singleton pregnancy, however plasma volume increases to a greater extent than red cell volume, leading to physiological anaemia [Norwitz 
et al., 2005; Hartman-Craven et al., 2009]. During pregnancy, levels of factor VII, VIII, X and von Willebrand factor increase, while protein $\mathrm{S}$, factor $\mathrm{XI}$ and platelets tend to decrease [Hellgren and Blomback, 1981; de Moerloose et al., 1998; Bremme, 2003]. Due to these haemostatic changes, it is well recognised that a procoagulant state is favoured during pregnancy, particularly as term approaches, with substantial haemostatic activation, even during uncomplicated pregnancy [Eichinger et al., 1999]. Progesterone mediated venous stasis and decreased mobility further increases the risk for the development of pathological clots to form.

\subsubsection{Treatment of antenatal VTE}

The standard treatment algorithm described for the management of VTE in Table 1.2, based on ACCP guidelines [Kearon et al., 2008], does not apply to the treatment of VTE during pregnancy. This is because warfarin (and other Vitamin $\mathrm{K}$ antagonists) is known to cross the placenta causing fetal wasting, fetal bleeding as well as teratogenic effects [Hall et al., 1980; Ginsberg et al., 1989a; Chan et al., 2000]. The most common fetal anomaly reported is coumarin embryopathy, consisting of nasal hypoplasia and/or stippled epiphyses. Coumarin embryopathy typically occurs after in-utero exposure to vitamin $\mathrm{K}$ antagonists during the first trimester of pregnancy (particularly weeks six to twelve) [Hall et al., 1980]. Vitamin K antagonists are also known to cause fetal CNS abnormalities, which can occur following exposure during any trimester. Two patterns of CNS damage have been described; dorsal midline dysplasia and ventral midline dysplasia [Hall et al., 1980; Chan et al., 2000].

The heparins (unfractionated heparin (UFH) and LMWH, due to their molecular size, do not cross the placenta [Flessa et al., 1965; Forestier et al., 1984; Forestier et al., 1987; Ginsberg et al., 1989b] and are therefore considered safe [Lepercq et al., 2001] to use for maternal indications where anticoagulant therapy is indicated, namely [Greer and Hunt, 2005]:

(i) Treatment of VTE

(ii) Thromboprophylaxis (venous and arterial)

(iii) Thromboprophylaxis for women with mechanical heart valves

(iv) Prevention of foetal loss during the first trimester and placental dysfunction in thrombophilic women 
As previously discussed, LMWH are preferred over UFH in clinical practice. However, one fundamental property of LMWH is lost when they are used during the antenatal period, the fixed dose response curve. This is due to the physiological changes which occur during pregnancy altering their pharmacokinetic profile.

In 2001, to account for this, the United Kingdom Royal College of Obstetricians and Gynaecologists (RCOG) published guidelines for the management of antenatal VTE, which were subsequently updated in 2007. The guidelines suggest that a twice daily LMWH regimen should be employed, preferably using enoxaparin or dalteparin, where most antenatal clinical experience exists (table 1.8), [RCOG, 2007]. This change to the antenatal dosing recommendations compares to a fixed dose of $1.5 \mathrm{mg} / \mathrm{kg}$ once daily of enoxaparin and $200 \mathrm{lU} / \mathrm{kg}$ once daily of dalteparin when used in those who are not pregnant [Sanofi-Aventis, 2009; Pharmacia, 2009].

Table 1.8: Doses recommended by RCOG for the management of antenatal VTE

\begin{tabular}{|c|c|c|c|c|}
\hline \multirow[b]{2}{*}{ LMWH } & \multicolumn{4}{|c|}{ Early pregnancy weight $(\mathrm{kg})$} \\
\hline & $<50$ & $50-69$ & $70-89$ & $>90$ \\
\hline Enoxaparin & $40 \mathrm{mg} \mathrm{bd}$ & $60 \mathrm{mg} \mathrm{bd}$ & $80 \mathrm{mg} \mathrm{bd}$ & $100 \mathrm{mg} \mathrm{bd}$ \\
\hline Dalteparin & $5000 \mathrm{IU}$ bd & $6000 \mathrm{IU}$ bd & $8000 \mathrm{IU}$ bd & $10000 \mathrm{IU} \mathrm{bd}$ \\
\hline Tinzaparin & \multicolumn{4}{|c|}{175 IU/kg od (all weights) } \\
\hline
\end{tabular}

The RCOG recommendations also state that routine anti-Xa (accepted biomarker for LMWH therapy) monitoring is not usually required if a weight based approach is used, as most women will reach a satisfactory peak anti-Xa response, three hours post dose, of between 0.5-1.2 IU/mL. The RCOG suggest that anti-Xa monitoring is warranted in those patients at the extremes of body weight $(<50 \mathrm{~kg}$ and $>90 \mathrm{~kg})$, patients with renal disease and those with complicating factors, such as recurrent VTE.

The ACCP pregnancy and postpartum guidelines [Bates et al., 2008] state that the optimal dosing strategy for the antenatal management of VTE using LMWH is not well described and remains controversial. Their guidance is less prescriptive in comparison to those of the RCOG, stating that a once or twice daily approach can be used, with or without anti-Xa monitoring. If anti-Xa monitoring is conducted, aim for a concentration of $0.6-1.0 \mathrm{IU} / \mathrm{mL}$ when a twice daily regimen is used, and slightly higher for a once daily regimen. The authors of the 
guidelines acknowledge that at present definitive advice cannot be provided because of a lack of published evidence.

Surveys of antenatal anticoagulation practice in the treatment of antenatal VTE in the UK and Ireland have found that although LMWH are widely used for this indication the particular LMWH used, the dosing strategy employed and the frequency of anti-Xa monitoring is highly variable [Voke et al.,2007; Knight et al. 2008]. Voke and colleagues surveyed antenatal VTE practice in 25 centres in the UK and Ireland (126 patients) and found that $97 \%$ of patients were treated with a $\mathrm{LMWH}$. Of these, $66 \%$ received a once daily regimen, and anti-Xa monitoring was performed in $90 \%$ of centres with a wide range of target values (table 1.9). More recently Knight and colleagues conducted a case control study evaluating the incidence and management of obstetric pulmonary embolism in the UK. In their study of 143 patients, $134(97 \%)$ were treated with $\mathrm{LMWH}$, with $49 \%$ receiving a once daily regimen.

Table 1.9: Regimen and heparins found to be used in Voke [2007] and Knight [2008]

\begin{tabular}{lcccc}
\hline LMWH & \multicolumn{2}{c}{ Voke (2007) } & \multicolumn{2}{c}{ Knight (2008) } \\
\cline { 2 - 5 } & Once a day LMWH & Twice a day LMWH & Once a day LMWH & Twice a day LMWH \\
\hline Dalteparin & 23 & 9 & 11 & 14 \\
Enoxaparin & 32 & 27 & 32 & 51 \\
Tinzaparin & 28 & 3 & 23 & 3 \\
\hline Total & $83(66 \%)$ & $39(31 \%)$ & $66(49 \%)$ & $68(51 \%)$ \\
\hline
\end{tabular}

These studies suggest that despite the RCOG (and ACCP) guidelines for the management of antenatal VTE, there is currently no consensus on the management of these patients, with many clinicians using a once daily LMWH approach [Patel and Hunt, 2008].

The dosing controversies described for the management of VTE, also exist when LMWH are used for other antenatal indications, raising some important questions regarding the antenatal use of LMWH: (i) given the physiological changes occurring during pregnancy, how frequently should LMWH be administered during the antenatal period?; (ii) What weight should be used to calculate the dose used during pregnancy? 


\subsection{Pharmacokinetic studies evaluating LMWH during pregnancy}

During pregnancy, the expansion of the intravascular plasma volume by up to $60 \%$ and the increase in the glomerular filtration rate (GFR), by up to $50 \%$ by the end of the first trimester, will impact on two key independent pharmacokinetic parameters of $\mathrm{LMWH}$; namely the apparent volume of distribution and clearance. The apparent volume of distribution (Vd) of a drug provides an estimate of the extent to which a drug distributes into extra vascular tissues. The $\mathrm{Vd}$ is largely dependent on the physiochemical attributes of the drug, namely, molecular size, degree of ionisation, lipid solubility and its ability to cross biological membranes [Hanley et al., 2010]. As LMWH are confined to the intravascular space, the increase in the plasma volume observed during pregnancy will increase the $\mathrm{Vd}$ of the $\mathrm{LMWH}$. The clearance $(\mathrm{CL})$ of a drug is inversely related to the steady-state plasma concentration of a drug and determines the drug's maintenance dose. CL is largely influenced by the capacity of the liver and kidneys, the principal organs involved in the removal of drugs and their metabolites. As the GFR increases during the first trimester, and this increase is maintained throughout the remainder of pregnancy, because LMWH are principally cleared through renal clearance, one would predict an increase in LMWH CL during pregnancy.

Having an understanding of how pregnancy affects these two independent parameters could help ensure the safe and effective use of LMWH and answer some of the uncertainties previously raised.

\subsubsection{Pharmacokinetic studies of LMWH during pregnancy}

Following successful use of unfractionated heparin in pregnant women during the 1970's and 1980's [Hirsh et al., 1970; Bonnar, 1976; Spearing et al., 1978; Anon (c), 1979; de Swiet et al., 1980; Bonnar, 1981; Chen et al., 1984; Letsky and de Swiet, 1984], as LMWH therapy gained acceptance in antenatal practice through the 1990's, a number of groups began publishing research or information on the pharmacokinetics and use of different LMWH during pregnancy [Priollet et al., 1986; Melissari et al., 1992; Gillis et al., 1992; Sturridge et al., 1994; Hunt et al., 1997; Nelson-Piercy et al., 1997; Blomback et al., 1998; Casele et al., 1999; Brennard et al., 1999; Bremme et al., 2000; Ellison et al., 2000; Crowther et al., 2000; Leqercq et al., 2001; Rodie et al., 2002; Hunt et al., 2003; Jacobsen et al., 2003; Sephton et al., 2003; Norris et al., 2004; Smith et al., 2004; Ensom et al., 2004; Huxtable et al., 2005; 
Fox et al., 2008; Lebaudy et al., 2008; Ni Ainle et al., 2008; Simpson et al., 2009; Friedrich et al., 2010] (see table 1.10 for a summary of each study).

Table 1.10: Summary of LMWH PK studies published to date in the pregnancy setting 


\begin{tabular}{|c|c|c|c|}
\hline Study & LMWH studied & Kinetic findings & Summary of results reported \\
\hline Priollet et al. (1986) & Enoxaparin & $\begin{array}{l}\text { No } \\
\text { pharmacokinetic } \\
\text { parameters } \\
\text { calculated }\end{array}$ & $\begin{array}{l}\text { Case report of a patient with a massive ileo-femerol DVT managed with once daily adjusted dose } \\
\text { enoxaparin (60-80mg daily) from week } 13 \text { of her pregnancy. } \\
\text { The patient delivers at } 34 \text { weeks gestation with no thrombotic or haemorrhagic complications. }\end{array}$ \\
\hline Melissari et al. (1992) & Dalteparin & $\begin{array}{l}\text { No } \\
\text { pharmacokinetic } \\
\text { parameters } \\
\text { calculated }\end{array}$ & $\begin{array}{l}\text { Case series, reporting on use of dalteparin use in women undergoing termination of pregnancy (as } \\
\text { prophylaxis) and in women at risk of VTE during their pregnancy. } \\
\text { The authors report that dalteparin is safe during pregnancy, but this finding needs further endorsement } \\
\text { from larger clinical studies. }\end{array}$ \\
\hline Gillis et al. (1992) & Enoxaparin & $\begin{array}{l}\text { No } \\
\text { pharmacokinetic } \\
\text { parameters } \\
\text { calculated }\end{array}$ & $\begin{array}{l}\text { Case series of } 6 \text { pregnant women administered enoxaparin for the treatment and prophylaxis of VTE. In } 5 \\
\text { out of } 6 \text { patients, the enoxaparin was successfully used with no complications. The sixth patient relapsed } \\
\text { after } 6 \text { weeks of therapy. } \\
\text { The authors conclude that LMWH could be considered as a alternative agent to unfractionated heparin } \\
\text { during pregnancy. }\end{array}$ \\
\hline Sturridge et al. (1994) & Enoxaparin & $\begin{array}{l}\text { No } \\
\text { pharmacokinetic } \\
\text { parameters } \\
\text { calculated }\end{array}$ & $\begin{array}{l}\text { Observational study following } 16 \text { women in a total of } 18 \text { pregnancies, receiving thromboprophylaxis with } \\
20 \mathrm{mg} \text { or } 40 \mathrm{mg} \text { daily. Mean anti-Xa activity with the } 40 \mathrm{mg} \text { dose was } 0.102 \mathrm{IU} / \mathrm{mL} \text { compared with } 0.036 \\
\mathrm{IU} / \mathrm{mL} \text { with the } 20 \mathrm{mg} \text { dose. The mean concentrations were significantly lower after } 20 \text { weeks gestation. }\end{array}$ \\
\hline Hunt et al. (1997) & Dalteparin & $\begin{array}{l}\text { No } \\
\text { pharmacokinetic } \\
\text { parameters } \\
\text { calculated }\end{array}$ & $\begin{array}{l}\text { Observational study assessing dalteparin in } 34 \text { high risk pregnancies. Dalteparin was given to maintain a } \\
\text { trough anti-Xa level of } 0.15-0.2 \mathrm{IU} / \mathrm{mL} \text { and a peak } 2 \text { hour post injection concentration of } 0.4-0.6 \mathrm{IU} / \mathrm{mL} \text {. } \\
\text { Most women required } 5,000 \text { units dalteparin once daily during the first trimester. } \\
\text { The mean time for a dosage increase was } 20.5 \text { weeks gestation into the pregnancy (SD 8.2). } 26 \text { of the } 34 \\
\text { women required } 5,000 \mathrm{IU} \text { twice daily by the end of the pregnancy. }\end{array}$ \\
\hline
\end{tabular}




\begin{tabular}{|c|c|c|c|}
\hline Study & LMWH studied & Kinetic findings & Summary of results reported \\
\hline Nelson-Piercy et al. (1997) & Enoxaparin & $\begin{array}{l}\text { No } \\
\text { pharmacokinetic } \\
\text { parameters } \\
\text { calculated }\end{array}$ & $\begin{array}{l}\text { Reported on } 61 \text { pregnant women at high risk of thromboembolism who were managed with enoxaparin } \\
\text { (usually } 40 \mathrm{mg} \text { ). No correlation was found between the gestational age and anti-Xa levels in this study. } \\
\text { One of only two studies to date suggesting that there is no link between gestational age and anti-Xa } \\
\text { concentration. }\end{array}$ \\
\hline Blomback et al. (1998) & Dalteparin & $\begin{array}{l}\text { Mean } \\
\text { parameters for the } \\
5000 \mathrm{IU} \text { dose: } \\
\text { Cmax } 0.21 \mathrm{IU} / \mathrm{mL} \\
\text { AUC } 1.97 \mathrm{~h} / \mathrm{mL} \\
\mathrm{tmax} 3.71 \mathrm{~h} \\
\mathrm{t}_{1 / 2} 4.92 \mathrm{~h}\end{array}$ & $\begin{array}{l}\text { Non compartmental pharmacokinetic study assessing dalteparin ( } 2500 \text { or } 5000 \text { IU once daily) in } 17 \\
\text { pregnant women during the third trimester ( } 15 \text { women on } 5000 \text { IU once a day and } 2 \text { women on } 2500 \text { IU } \\
\text { once a day, due to a lower weight). } \\
\text { They report that the peak concentration, time to maximum concentration and area under the curve were } \\
\text { lower in this cohort compared to historical non-pregnant population data they had on file. } \\
\text { The authors also assessed if administering the dose in the morning or evening made a difference and } \\
\text { report no difference. }\end{array}$ \\
\hline
\end{tabular}




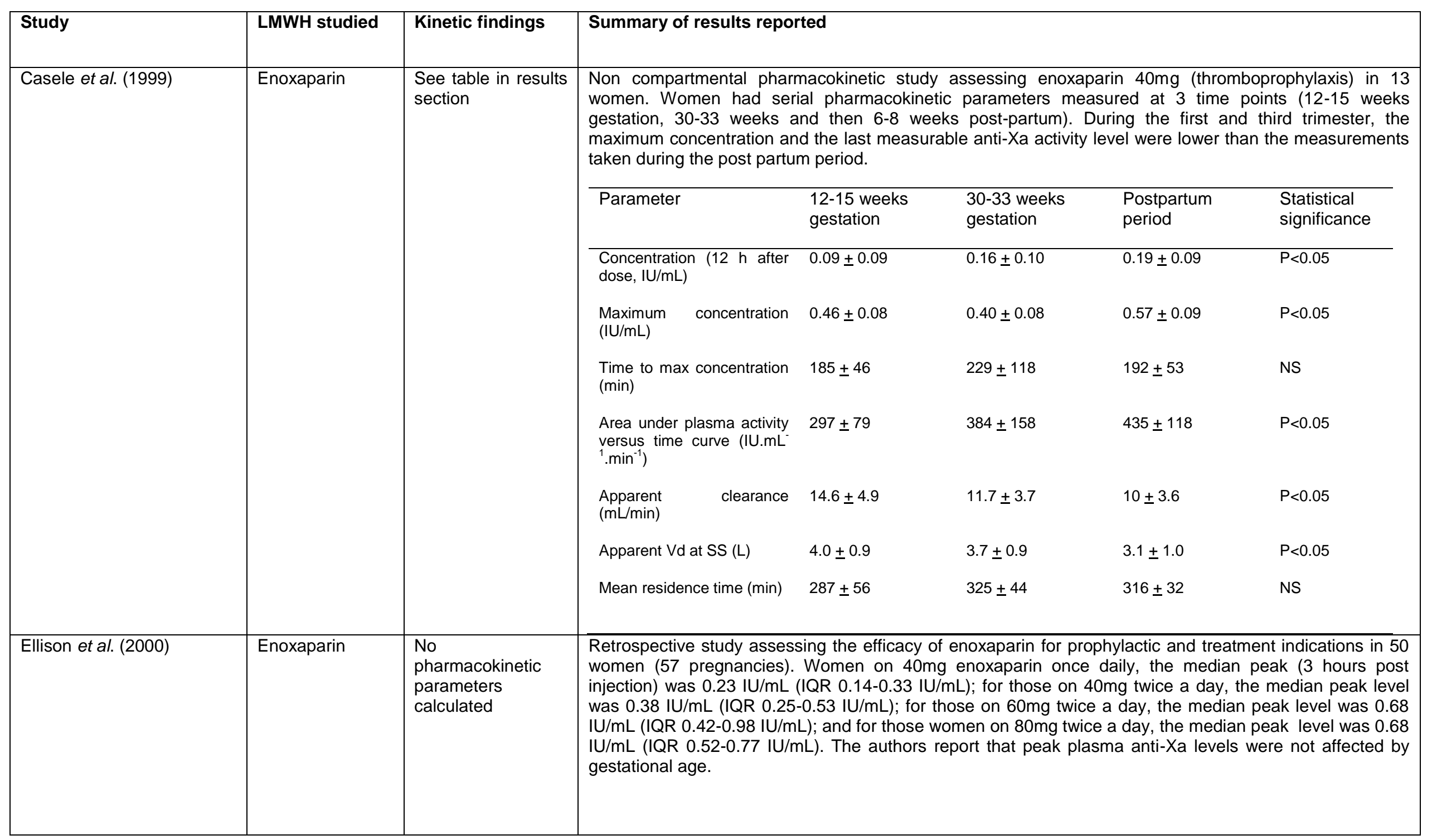




\begin{tabular}{|c|c|c|c|}
\hline Study & LMWH studied & Kinetic findings & Summary of results reported \\
\hline Bremme et al. (2000) & Dalteparin & $\begin{array}{l}\text { No } \\
\text { Pharmacokinetic } \\
\text { parameters } \\
\text { calculated }\end{array}$ & $\begin{array}{l}\text { Report the results from } 4 \text { women who had allergies to conventional unfractionated heparin therapy, who } \\
\text { received dalteparin (varying doses) during their pregnancy. Anti-Xa activity was monitored at various time } \\
\text { points during pregnancy. } \\
\text { The authors report that the last measurable anti-Xa activity in the third trimester at } 37 \text { weeks was } 17 \text { hours } \\
\text { post dose, compared to } 24 \text { hours post dose at } 40 \text { weeks gestation, and suggest that there is accumulation } \\
\text { of dalteparin, as term approaches, with the authors suggesting that this could have implications for how } \\
\text { LMWH are managed around delivery. }\end{array}$ \\
\hline Crowther et al. (2000) & Reviparin & $\begin{array}{l}\text { No } \\
\text { pharmacokinetic } \\
\text { parameters } \\
\text { calculated }\end{array}$ & $\begin{array}{l}\text { This prospective study followed } 42 \text { patients receiving } 4900 \text { IU of reviparin once daily throughout their } \\
\text { pregnancy. Up to four anti-Xa activities per patient were measured scattered throughout these patients } \\
\text { pregnancies. The authors report that reviparin is safe to use in an antenatal population and found that anti- } \\
\text { Xa activity was highly correlated with patient's weight, irrespective of gestational age. } \\
\text { Though in their study, women were receiving fixed prophylactic doses of reviparin, they suggest that } \\
\text { women requiring treatment doses of LMWH might need their dose adjusting in line with weight increase, } \\
\text { given the high correlation between weight and anti-Xa activity found in their study. }\end{array}$ \\
\hline Leqercq et al. (2001) & Enoxaparin & $\begin{array}{l}\text { No } \\
\text { pharmacokinetic } \\
\text { parameters } \\
\text { calculated }\end{array}$ & $\begin{array}{l}\text { Retrospective review of enoxaparin safety in } 604 \text { women in France. } 49 \text { cases were actively treated for } \\
\text { VTE ( } 1 \mathrm{mg} / \mathrm{kg} \text { bd) and } 574 \text { cases were treated prophylactically ( } 357 \text { received } 20 \mathrm{mg} \text { daily and } 217 \text { received } \\
40 \mathrm{mg} \text { daily). } \\
\text { Eight documented VTE events occurred ( } 5 \text { during pregnancy and } 3 \text { post partum). The authors concluded } \\
\text { that the enoxaparin was well tolerated during pregnancy. }\end{array}$ \\
\hline Rodie et al. (2002) & Enoxaparin & $\begin{array}{l}\text { No } \\
\text { pharmacokinetic } \\
\text { parameters } \\
\text { calculated }\end{array}$ & $\begin{array}{l}\text { A case series of } 36 \text { VTE episodes antenatally, managed with enoxaparin } 1 \mathrm{mg} / \mathrm{kg} \text { bd. Target anti-Xa } \\
\text { activity }(0.4-1.0 \mathrm{IU} / \mathrm{mL}) \text {. In } 33 \text { women, the initial dose provided satisfactory peak anti-Xa activity (median } \\
\text { range } 0.44-1.0 \mathrm{IU} / \mathrm{mL}) \text { and was continued. Three women required a dose reduction, due to their peak } \\
\text { being above the therapeutic range }(1.2,1.2,1.1 \mathrm{IU} / \mathrm{mL}) \text {. The authors conclude that routine monitoring with } \\
\text { this regimen is not required. }\end{array}$ \\
\hline Hunt et al. (2003) & Dalteparin & $\begin{array}{l}\text { No } \\
\text { pharmacokinetic } \\
\text { parameters } \\
\text { calculated }\end{array}$ & $\begin{array}{l}\text { A case series of } 25 \text { women ( } 27 \text { pregnancies) assessing the use of unmonitored dalteparin } 5,000 \text { IU daily } \\
\text { increased empirically to } 5,000 \mathrm{IU} \mathrm{bd} \text { at } 16-20 \text { weeks. All women had normal renal function and were } \\
\text { classed as obese. } \\
\text { The authors report that this regimen worked well and does not require monitoring of anti-Xa activity. The } \\
\text { authors also comment that many of women were on therapeutic warfarin prior to pregnancy and the } \\
\text { regime they describe appeared to provide adequate anticoagulation cover. }\end{array}$ \\
\hline
\end{tabular}




\begin{tabular}{|c|c|c|c|}
\hline Study & LMWH studied & Kinetic findings & Summary of results reported \\
\hline Jacobsen et al. (2003) & Dalteparin & $\begin{array}{l}\text { No } \\
\text { pharmacokinetic } \\
\text { parameters } \\
\text { calculated }\end{array}$ & $\begin{array}{l}\text { Observational study following the VTE management of } 20 \text { women. } \\
\text { For } 9 \text { out of } 13 \text { women where treatment was initiated at the time of diagnosis at } 100 \mathrm{IU} / \mathrm{kg} \mathrm{bd} \text {, dose } \\
\text { escalation was required to maintain target anti-Xa activity of } 0.5-1.0 \mathrm{IU} / \mathrm{mL} 2-3 \text { hours post injection. } \\
6 \text { women started on higher doses (mean } 112 \mathrm{IU} / \mathrm{kg} \mathrm{bd} \text { ) and the target was reached in all cases. } 1 \text { woman } \\
\text { required a dose reduction (to } 100 \mathrm{IU} / \mathrm{kg} \mathrm{bd} \text { ) after starting on } 133 \mathrm{IU} / \mathrm{kg} \text { bd. The authors concluded that an } \\
\text { approximately } 10-20 \% \text { higher dose of dalteparin may be needed during pregnancy compared with non- } \\
\text { pregnant women. }\end{array}$ \\
\hline Sephton et al. (2003) & Dalteparin & $\begin{array}{l}\text { Relative to non } \\
\text { pregnancy, the } \\
\text { AUC } 0-24 \text { was lower } \\
\text { during pregnancy } \\
\text { at all gestational } \\
\text { times - with a } \\
\text { decrease observed } \\
\text { as the gestation } \\
\text { progressed }\end{array}$ & $\begin{array}{l}\text { A prospective observational study of } 24 \text { women suffering from recurrent miscarriage and positive for anti- } \\
\text { phospholipid syndrome. The women were administered } 5000 \text { units once daily of dalteparin and serial anti- } \\
\text { Xa levels were measured at } 12,24 \text { and } 36 \text { weeks, as well as } 6 \text { weeks postpartum. } \\
\text { The authors report a lower peak anti-Xa levels (and } A^{-} U_{0-24} \text { ) during the pregnancy phases compared to } \\
\text { the non-pregnant state. }\end{array}$ \\
\hline Norris et al. (2004) & Tinzaparin & $\begin{array}{l}\text { No } \\
\text { pharmacokinetic } \\
\text { parameters } \\
\text { calculated }\end{array}$ & $\begin{array}{l}\text { Observational study of how anti-Xa activity changes for women at moderate risk of VTE during their } \\
\text { pregnancy, managed with tinzaparin. } \\
\text { In } 24 \text { women, one of three doses ( } 50,75 \text { or } 100 \mathrm{IU} / \mathrm{kg} \text { ) were given according to the treating physician's } \\
\text { assessment of their risk profile. Four hour peak anti-Xa levels were measured throughout pregnancy, with } \\
\text { additional } 24 \text { hour profiles measured at } 28 \text { and } 36 \text { weeks gestation. } \\
\text { The authors report that women receiving tinzaparin }(50 \mathrm{IU} / \mathrm{kg}) \text { frequently had peak ( } 4 \text { hour) anti-Xa levels } \\
\text { below } 0.1 \mathrm{IU} / \mathrm{mL} \text { and that } 46 \% \text { of these patients required dose adjustment. Similarly anti-Xa levels were } \\
\text { also found to be low over a } 24 \text { hour period. They suggest that a starting dose of } 75 \mathrm{IU} / \mathrm{kg} \text { once daily, gave } \\
\text { a greater anti-Xa cover over a } 24 \text { hour period and avoided the need for dose adjustment for this } \\
\text { population. }\end{array}$ \\
\hline
\end{tabular}




\begin{tabular}{|c|c|c|c|c|c|c|c|c|c|c|}
\hline Study & LMWH studied & Kinetic findings & \multicolumn{8}{|c|}{ Summary of results reported } \\
\hline Smith et al. (2004) & Tinzaparin & $\begin{array}{l}\text { No } \\
\text { pharmacokinetic } \\
\text { parameters } \\
\text { calculated }\end{array}$ & \multicolumn{8}{|c|}{$\begin{array}{l}54 \text { women (12 for active VTE treatment and } 42 \text { for prophylaxis with tinzaparin). Aim of the study was to } \\
\text { assess whether a dose adjustment would be required through pregnancy to achieve the target anti-Xa } \\
\text { activity, four hours post dose }(0.3-1.0 \mathrm{IU} / \mathrm{mL} \text { for treatment and } 0.1-0.5 \mathrm{IU} / \mathrm{mL} \text { for prophylaxis). } \\
\text { In the treatment and high risk group, the authors report a reduction in peak anti-Xa levels as pregnanc) } \\
\text { progressed, of } 0.10 \mathrm{IU} / \mathrm{mL} \text {. The authors conclude that } 175 \mathrm{IU} / \mathrm{kg} \text { daily used was a sufficient dose for this } \\
\text { group. } \\
\text { In the prophylaxis group, three women (20\%) required a dose increase from } 50 \mathrm{IU} / \mathrm{kg} \text { to } 75 \mathrm{IU} / \mathrm{kg} \text { to reach } \\
\text { target levels. The authors endorsed the use of once daily tinzaparin for treatment indications and sugges } \\
\text { a dose of } 75 \mathrm{IU} / \mathrm{kg} \text { daily for prophylaxis indications for women at moderate risk. }\end{array}$} \\
\hline \multirow[t]{6}{*}{ Ensom et al. (2004) } & \multirow[t]{6}{*}{ Dalteparin } & \multirow[t]{6}{*}{$\begin{array}{l}\text { See table in the } \\
\text { results section for } \\
\text { pharmacokinetic } \\
\text { parameters } \\
\text { calculated by the } \\
\text { authors }\end{array}$} & \multicolumn{8}{|c|}{$\begin{array}{l}\text { Compared the pharmacokinetics (non-compartmental analysis) of dalteparin (9) versus unfractionate } \\
\text { heparin (6) during pregnancy for women with anti-phosphospholipid syndrome. } \\
\text { Women were randomised to a regime of prophylactic dalteparin (2500 IU od pre-pregnancy, } 2500 \mathrm{IU} \text { od } \\
\text { the first trimester, } 5000 \mathrm{IU} \text { od in the second trimester and } 7500 \mathrm{IU} \text { od in the third trimester) } \\
\text { unfractionated heparin ( } 5000 \mathrm{IU} \text { bd pre pregnancy and during the first trimester, } 7500 \mathrm{IU} \text { bd during th } \\
\text { second trimester and } 10000 \mathrm{IU} \text { bd in the third trimester). } \\
\text { The authors report that during pregnancy a higher dose of dalteparin would be required compared to th } \\
\text { non-pregnant state (see table of PK results), whereas for unfractionated heparin, using their dosir } \\
\text { protocol, no further dose change was required. }\end{array}$} \\
\hline & & & Time period & Dose & $\begin{array}{l}\text { Cmax } \\
\text { (IU/mL) }\end{array}$ & $\begin{array}{l}\text { Cmin } \\
\text { (IU/mL) }\end{array}$ & $\operatorname{tmax}(\mathrm{h})$ & $\begin{array}{l}\text { AUC } \\
\text { (IU.h/mL) }\end{array}$ & $\mathrm{Cl}(\mathrm{mL} / \mathrm{h})$ & $\begin{array}{l}\mathrm{Cl} / \mathrm{kg} \\
(\mathrm{mL} / \mathrm{h} / \mathrm{kg})\end{array}$ \\
\hline & & & $\begin{array}{l}\text { Pre- } \\
\text { pregnancy }\end{array}$ & 2500 & $\begin{array}{ll}0.28 & \pm \\
0.13 & \end{array}$ & $\begin{array}{l}0.02 \\
0.02\end{array}$ & $2.5 \pm 1.2$ & $\begin{array}{l}2.70 \quad \pm \\
0.80\end{array}$ & $\begin{array}{l}1005 \quad \pm \\
312\end{array}$ & $15.7 \pm 3.8$ \\
\hline & & & $\begin{array}{l}\text { First } \\
\text { trimester }\end{array}$ & 2500 & $\begin{array}{l}0.20 \\
0.14\end{array}$ & $\begin{array}{ll}0.03 & \pm \\
0.05 & \end{array}$ & $2.9 \pm 3.4$ & $\begin{array}{l}1.64 \quad \pm \\
0.87\end{array}$ & $\begin{array}{ll}2117 & \pm \\
1351 & \end{array}$ & $\begin{array}{l}31.8 \quad \pm \\
17.0\end{array}$ \\
\hline & & & $\begin{array}{l}\text { Second } \\
\text { trimester }\end{array}$ & 5000 & $\begin{array}{l}0.34 \\
0.13\end{array}$ & $\begin{array}{l}0.04 \\
0.03\end{array}$ & $4.1 \pm 1.5$ & $\begin{array}{l}3.04 \\
1.34\end{array}$ & $\begin{array}{l}2031 \\
1040\end{array}$ & $26.4 \pm 9.7$ \\
\hline & & & $\begin{array}{l}\text { Third } \\
\text { trimester }\end{array}$ & 7500 & $\begin{array}{ll}0.43 & \pm \\
0.16 & \end{array}$ & $\begin{array}{l}0.06 \\
0.04\end{array}$ & $4.4 \pm 1.5$ & $\begin{array}{l}4.69 \\
2.00\end{array}$ & $\begin{array}{l}2207 \\
1953\end{array}$ & $\begin{array}{l}26.4 \\
20.4\end{array}$ \\
\hline
\end{tabular}

- 69 - 


\begin{tabular}{|c|c|c|c|}
\hline Study & LMWH studied & Kinetic findings & Summary of results reported \\
\hline Simpson et al. (2009) & Dalteparin & $\begin{array}{l}\text { No } \\
\text { pharmacokinetic } \\
\text { parameters } \\
\text { calculated }\end{array}$ & $\begin{array}{l}\text { A retrospective review of } 25 \text { women requiring treatment dose of dalteparin during their pregnancy. They } \\
\text { found that the dose required to maintain the peak plasma concentration at } 4 \text { hours between } 0.5-0.8 \mathrm{IU} / \mathrm{mL} \text {, } \\
\text { required to be increased, when comparing results from the first trimester to the third trimester. The authors } \\
\text { suggest that the increase in dose requirement could not be explained by weight increase alone, and that } \\
\text { anti-Xa levels be monitored during pregnancy and the dose should be adjusted. }\end{array}$ \\
\hline Friendrich et al. (2010) & Enoxaparin & $\begin{array}{l}\text { No } \\
\text { pharmacokinetic } \\
\text { parameters } \\
\text { calculated }\end{array}$ & $\begin{array}{l}\text { A prospective evaluation of anti-Xa levels in } 15 \text { women requiring therapeutic enoxaparin }(1 \mathrm{mg} / \mathrm{kg}+/-20 \%) \\
\text { twice daily during their pregnancy. Three anti-Xa concentrations were monitored; trough, } 3-4 \text { hour (peak) } \\
\text { and } 8 \text { hours pose dose. } \\
\text { The authors considered an anti-Xa activity of between } 0.5-1.2 \mathrm{IU} / \mathrm{mL} \text { as satisfactory. } \\
\text { Mean anti-Xa activity levels were: trough } 0.45+/-0.18 \text {, peak } 0.9+/-0.25 \text {, and } 8 \text {-h post dose } 0.72+/-0.23 \\
\text { IU } / \mathrm{mL} \text {. The authors report that trough and } 8-\mathrm{h} \text { post dose anti-Xa activity levels were sub-therapeutic in a } \\
\text { substantial number of patients receiving a twice daily regimen of therapeutic enoxaparin. }\end{array}$ \\
\hline Nelson-Piercy et al. (2011) & Tinzaparin & $\begin{array}{l}\text { No } \\
\text { pharmacokinetic } \\
\text { parameters } \\
\text { calculated }\end{array}$ & $\begin{array}{l}\text { A retrospective review of tinzaparin use during the antenatal period from } 1267 \text { pregnancies. In } 254 \\
\text { pregnancies, women recieievd tinzaparin for treatment indications, with } 94.1 \% \text { recieiving tinzaparin as a } \\
\text { once daily indication. In this study, } 5(2 \%) \text { recurrent VTEs were reported. } \\
\text { This study provides valuable information on the use of tinzaparin during the antenatal period. }\end{array}$ \\
\hline
\end{tabular}


Whilst the majority of these studies are observational in nature and they have methodological differences in the way they were conducted and the primary objectives set, the studies confirm that LMWH clearance is altered antenatally. Little attention in these studies has been given to alterations in the Vd of LMWH and what impact (if any), this may have.

The two studies most commonly cited in this area are the studies by Blomback [1998] and Casele [1999]. Blomback and colleagues conducted a non-compartmental pharmacokinetic study of dalteparin (2500 or $5000 \mathrm{IU}$ once daily) in seventeen pregnant women during the third trimester. In this study, although a measurable anti-Xa concentration was detectable at 16 hours in the fourteen women in the study receiving a $5000 \mathrm{IU}$ dose once a day, the maximum concentration $\left(\mathrm{C}_{\max }\right)$, the time to maximum concentration $\left(\mathrm{t}_{\max }\right)$ and the area under the curve were significantly lower compared with when dalteparin was administered to twelve healthy men and women. Casele and colleagues conducted a non-compartmental pharmacokinetic study assessing the pharmacokinetics of enoxaparin $(40 \mathrm{mg})$ in thirteen women who received thromboprophylaxis throughout pregnancy. These women had pharmacokinetic parameters calculated at three time points; $12-15$ weeks gestation, 30-33 weeks gestation and 6-8 weeks postpartum. During early and late pregnancy, the maximum concentration and the last measurable anti-Xa activity were lower antenatally than during post-partum period. Both studies imply a higher dose is required antenally. More recently Lebaudy et al. [2008] carried out a population pharmacokinetic modelling and simulation study assessing enoxaparin during pregnancy and the post-partum period in seventy-five women. They found that enoxaparin clearance was higher in the pregnant women when compared to non-pregnant women: $0.78 \mathrm{~L} / \mathrm{hr} \pm 0.03$ versus $0.52 \mathrm{~L} / \mathrm{hr} \pm 0.03(p=<0.001)$. However, they also report, that the volume of distribution of enoxaparin was influenced by the stage of pregnancy, found to be a two-step increase. An initial rise paralleling the woman's increase in body weight during the first two trimesters, followed by an additional increase of $41 \%$ during the last two months of pregnancy. Lebaudy's study to date provides the most useful information in this area, although the uptake of their recommendations into clinical practice is yet to be seen. 


\subsection{Pharmacokinetic analysis}

\subsubsection{Non-compartmental pharmacokinetic analysis}

Non-compartmental analysis (NCA) is the pharmacokinetic (PK) analysis of individual concentration-time curves, which determine parameters such as maximum concentration $\left(\mathrm{C}_{\max }\right)$, the area under the concentration-time curve $(A \cup C)$, time to maximum concentration $\left(T_{\max }\right)$, in addition to $\mathrm{CL}$ and Vd. NCA summarise the PK data obtained from individuals and generally speaking is fast and readily automated, providing estimates of the desired PK parameters. Historically, PK analysis has been conducted using NCA, with the pharmaceutical industry utilising this method with data obtained from homogenous populations of adults during phase I +/phase II clinical trials informing the dose to be evaluated in phase III studies. The disadvantage associated with NCA is that, it makes no attempt to understand or describe the structural or mechanistic properties of the system into which the drug is being introduced (like compartmental analysis) and is therefore limited in its clinical application, as it is difficult to then predict new situations, for example what might happen if the drug being studied was prescribed for a patient with moderate renal impairment [Gillespie, 1991]. Furthermore, sources of variability between subjects are not explored or identified using this method, further limiting its clinical application.

\subsubsection{Compartmental analysis}

Compartmental PK analysis on the other hand, involves describing drug disposition in terms of the drug moving through a series of connected compartments. In this type of analysis, the drug is either administered into one of the compartments, either directly, e.g. intravascularly, or indirectly, e.g. extravascularly, and from there either distributes into peripheral compartments or is eliminated from the body. First order kinetic processes are used to describe the transfer of drugs between compartments, with the rate of drug transfer dependent on drug concentration and permeability. Drug input (drug administration or absorption) and output (elimination) from the system are modelled using different kinetic processes.

Compartmental analysis does attempt to describe the mechanistic and structural systems into which the drug is being introduced, and therefore models from compartmental analyses can be used to make predictions into different scenarios, e.g. a different patient population. Furthermore, depending on the type of compartmental analysis used, sources of variability can be identified and quantified. Compartmental analysis can be further sub-divided into three different 
approaches; the naïve pooled approach, the standard two-stage approach and nonlinear mixed effects modelling.

\subsubsection{Naïve pooled approach}

This analysis approach involves pooling all concentration data from the population and treating the data, as if it were from one individual [Sheiner and Beal, 1980]. For this approach, data must originate from a well-controlled study, i.e. sampling taken at the same time and performed in a homogenous population [Ette and Williams, 2004]. This analytical approach provides no information on sources of variability in the data and cannot explore relationships between parameters and covariates within subjects, and in many ways is similar to NCA.

\subsubsection{Standard two-stage approach}

This approach involves two stages of data analysis. Firstly, models are fitted to individual data to estimate each subject's pharmacokinetic parameters. Following this, population parameters are then determined, as a mean of the individual values, with individual differences in values described by the variances around these means. With a well designed and executed study, it is possible to obtain good estimates of population parameters using this approach. This method generally requires a homogenous population, in order to obtain reasonable parameter and variability estimates, and is usually performed in a small study population, for example 15 patients with intense sampling per subject. With this approach, estimates of variance tend to be upwardly biased, due to residual unexplained variability (RUV) such as that caused by assay error, not being easily distinguished from true between subject variability (BSV) [Sheiner and Beal, 1981]

\subsubsection{Nonlinear mixed effects modelling (NLMEM)}

NLMEM is the most commonly used population modelling analytical method used. It allows estimation of both fixed (i.e. CL or $\mathrm{Vd}$ ) and random (i.e. between subject or residual unexplained variability) effects simultaneously, hence the term mixed effects, whilst preserving an individual's data within the population. The method was originally developed by Professor Sheiner in the United States, where, the challenges of dosing patients on chronic warfarin therapy, and in those with chronic heart failure prescribed digoxin, provided a stimulus for the clinician Sheiner to get involved with NLMEM [Sheiner, 1969; Peck et al., 1973]. Following the publication of the seminal papers by Sheiner and colleagues [Sheiner et al., 1972; Sheiner et al., 1977], the foundations of NLMEM had been laid for application in pharmacokinetic analyses. In the early days, NLMEM was viewed with scepticism by the wider statistical community, seen as a pragmatic method of 
dealing with sparse data and poorly conducted trials [Pillai et al., 2005]. However, the concept has stood the test of time and is now an invaluable tool in both the pharmaceutical industry and the clinical environment.

This approach is considered the most robust method of population data analysis as it allows the investigation and quantification of variability in model parameters between individuals from data which may be sparse, unbalanced and from a heterogeneous population [Sheiner and Grasela, 1991]. In fact, a heterogeneous population is preferred for this type of modelling, as it provides more information on variability [Sheiner and Ludden, 1992].

The benefit of NLMEM is that data from a range of sources may be analysed, e.g. from highly controlled randomised controlled trials to sparse therapeutic drug monitoring data collected in a clinic environment, therefore having an important clinical application. Furthermore, unlike the naïve or two-stage approach, the approach can allow data from different studies to be combined for analysis, even if precision is different between the two studies [Sheiner and Grasela, 1991].

\subsubsection{Population modelling}

Population modelling can be defined as:

...the application of a mathematical model to describe pharmacokinetic data that arises from more than one individual. The process does not require that each study individual provides sufficient data to characterise completely their own pharmacokinetic profile. Population analysis methods allow borrowing of information between individuals to fill in gaps in the pharmacokinetic profile. In doing so, the method allows sparse sampling study designs. The influence of patient characteristics, e.g. renal function, on the pharmacokinetic profile can be quantified from the data set as well as any remaining unexplained variability between patients. [Duffull et al., 2011].

A population model may be written as a mathematical expression describing an observation, in terms of two components;

(i) Structural model, which determines the shape or form of the model, and usually describes the fixed effects such as $C L$ or $V d$

(ii) Stochastic (or statistical) model, which describes the degree of uncertainty, for example the random effects such as between subject variability, assay error or residual unexplained variability 


\subsection{Structural model}

The fixed effects comprises a function of known independent variables and pharmacokinetic parameters. Independent variables may include those such as time, dose, and patient factors (age, weight, gender) influencing the response and the pharmacokinetic parameters such as CL and $\mathrm{Vd}$. Mathematically, a structural model can be expressed as:

$$
y_{i j}=f\left(x_{i j}, \theta_{i j}\right)
$$

Where $y_{i j}$, the $f^{\text {th }}$ observation of the $i^{\text {th }}$ individual, is a function of the known independent variables $x_{i j}$ and the predicted individual PK parameters, $\theta_{i j}$.

An example of a structural PK model is a one compartment model following IV bolus dosing of a drug. In this model, the concentration of the drug can be described with a mono-exponential decline from the compartment:

$$
\mathrm{C}=\frac{\mathrm{D}}{\mathrm{Vd}} \cdot e^{\left(-\frac{\mathrm{CL}}{\mathrm{Vd}} \mathrm{t}\right)}
$$

where $\mathrm{C}$ is the predicted concentration at time $t$ after dose $\mathrm{D}$. In this example, $\mathrm{D}$ and $t$ are the known independent variables, whereas $\mathrm{CL}$ and $\mathrm{Vd}$ are the pharmacokinetic parameters to be estimated.

\subsection{Stochastic (statistical) model}

Uncertainty in modelling arises from a variety of sources, for example assay error, between subject variability (BSV), inter occasion variability (IOV). As the aim of population modelling is to describe variability of individual pharmacokinetic parameters around the typical population value, this between subject variability can be expressed as:

$$
\theta_{i}=\theta_{\text {pop }}+n_{i}
$$

where $\theta_{i}$ is the parameter value for the $i^{\text {th }}$ individual, $\theta_{p o p}$ is the population parameter value, and $\eta_{i}$ is assumed to be normally distributed with a mean of zero and variance of $\omega^{2}$. When variability in a population is assessed to be log-normally distributed, a log-normal distribution may also be used:

$$
\theta_{i}=\theta_{\text {pop }} \cdot e^{n i}
$$


The variability that remains once BSV and IOV have been added to the model is referred to as residual unexplained variability (RUV) and can be associated with errors in sampling of the assay, or can be attributed to model misspecification due to oversimplification of the system. RUV can be incorporated into a model as an additive error

$$
y_{i j}=y_{\text {pred }_{, i j}}+\epsilon_{i j}
$$

where $y_{\text {pred,ij }}$ is the predicted observation of the model and $\varepsilon_{i j}$ is the random error between the observed value with a mean value of zero and variance of $\sigma^{2}$.

A proportional or exponentional error can also be used, where

$$
y_{i j}=y_{\operatorname{pred}_{, i j}} \cdot e^{\epsilon_{i j}}
$$

which approximates a constant coefficient of variation (CCV).

Both errors terms may also be included together in a combined proportional $\left(\varepsilon_{\mathrm{ij} 1}\right)$ and additive $\left(\varepsilon_{\mathrm{ij} 2}\right)$ error term

$$
y_{i j}=y_{\text {pred }_{, i j}} \cdot e^{\epsilon_{i j 1}}+\epsilon_{i j 2}
$$

\subsubsection{Estimation methods and software}

Fitting mathematical models to data involves a process of parameter estimation to achieve a set of values that best fit the data, i.e. minimising the differences between observations and predictions. In non-linear mixed effects modelling, there is no closed form solution to the likelihood, therefore approximation and iterative approaches have to be used. A number of software packages have been developed for this purpose [Beal and Sheiner, 1989], with NONMEM, an acronym for Nonlinear Mixed Effects Modelling, the most widely used, and in essence is a regression program that specialises in non-linear systems.

In NONMEM, nonlinear equations are expressed linearly as Taylor-series approximations of the likelihood and an iterative search within the parameter space is performed to obtain the estimates of the parameters [Ette and Williams, 2004; Schoemaker and Cohen, 1996]. The simplest linearization is by the first-order (FO) method, in which the same parameter values are assumed for all subjects during the search for the best parameter combination (Taylor series expansion about $\eta=0$ ), whereas the first-order conditional estimation (FOCE) method linearises with respect 
to the random effects $(\eta)$, resulting in a more accurate approximation to the equation [Beal and Sheiner, 1992; Schoemaker and Cohen, 1996].

Final parameter estimates for the model are obtained in NONMEM once the maximum likelihood of model to fit the data is achieved, i.e. when the sum of squares has been minimised. This is approximated by the objective function value (OBJ) of an extended least squares (ELS) analysis which approximates minus twice the natural logarithm of the likelihood. Mathematically, this can be expressed as

$$
O B J=\sum_{i=1}^{N_{s u b}} \frac{\left(y_{i}-f\left(\theta, x_{i}\right)\right)\left(y_{i}-f\left(\theta, x_{i}\right)\right)^{T}}{\left(\left|J \Omega J^{T}+\sum\right|\right)}+\ln \left(\left|J \Omega J^{T}+\sum\right|\right)
$$

where $N_{\text {sub }}$ is the total number of subjects, $y_{i}$ is the vector of observations for the $i^{\text {th }}$ individual; $f$ is a function of $x_{i}$, a vector of independent variables for the $i^{\text {th }}$, individual and $\theta$, the vector of population mean parameter values; $J$ is the Jacobian matrix of the first partial derivatives of the model with respect to the random effects; $\Omega$ is the variance-covariance matrix of between subject effects; $\Sigma$ is a matrix of the residual variance; and I $\cdot$ I denotes the determinant of the matrix [Peck et al., 1984]. A lower OBJ during the parameter search indicates a better model fit to data. Models representing the same data can be compared by determining the difference $(\Delta)$ between model OBJs. As $\triangle \mathrm{OBJ}$ is asymptotically and asymmetrically $x^{2}$-distributed, the significance of change can be determined according to the $x^{2}$-distribution with the appropriate degree of freedom, for example, with one degree of freedom (i.e. one parameter difference between models) a $\triangle \mathrm{OBJ}$ of 3.84 or 6.63 indicates a $P<0.05$ or $<0.01$, respectively. However, it must be remembered, that all mathematical calculations for estimating or predicting parameter values are oversimplifications of reality.

\subsubsection{Covariates}

Covariates are the factors which can explain some of the variability seen during the modelling building process, and may be constant, e.g. subject gender, or change with time, e.g. renal function, age. They can be considered as the factors which help to describe and understand the system into which the drug is being introduced. A catergorical covariate is one that can only be described as a discrete value. Unordered catergorical (nominal) covariates include those such as sex, race, and smoking status for example. Ordered categorical (ordinal) covariates include those such as health or disease status, e.g. progressive cancer stages 0 to IV. Continuous covariates 
are factors which can be measured on a continuous scale, such as weight, height and age, as well as including clinical measures such as creatinine clearance or haemoglobin concentration.

In a population model, covariates can be defined in terms of their use. For example a mechanistic covariate can be regarded as those which explain variability within a population based on physiological differences, such as body size on Vd. Due to their physiological basis, their inclusion makes a population model useful for predictive purposes into different populations. Empirical covariates are descriptive explanations of variability that do not generally have any physiological basis, e.g. eye colour. In population modelling, the inclusion of empirical covariates in a model cannot be used to predict into new situations, as it can only be considered a description of the population from which the data arose.

\subsubsection{The model development process}

Generally speaking, irrespective of the purpose of the model or the number of patients involved, the model building process involves the processes outlined in figure 1.7.

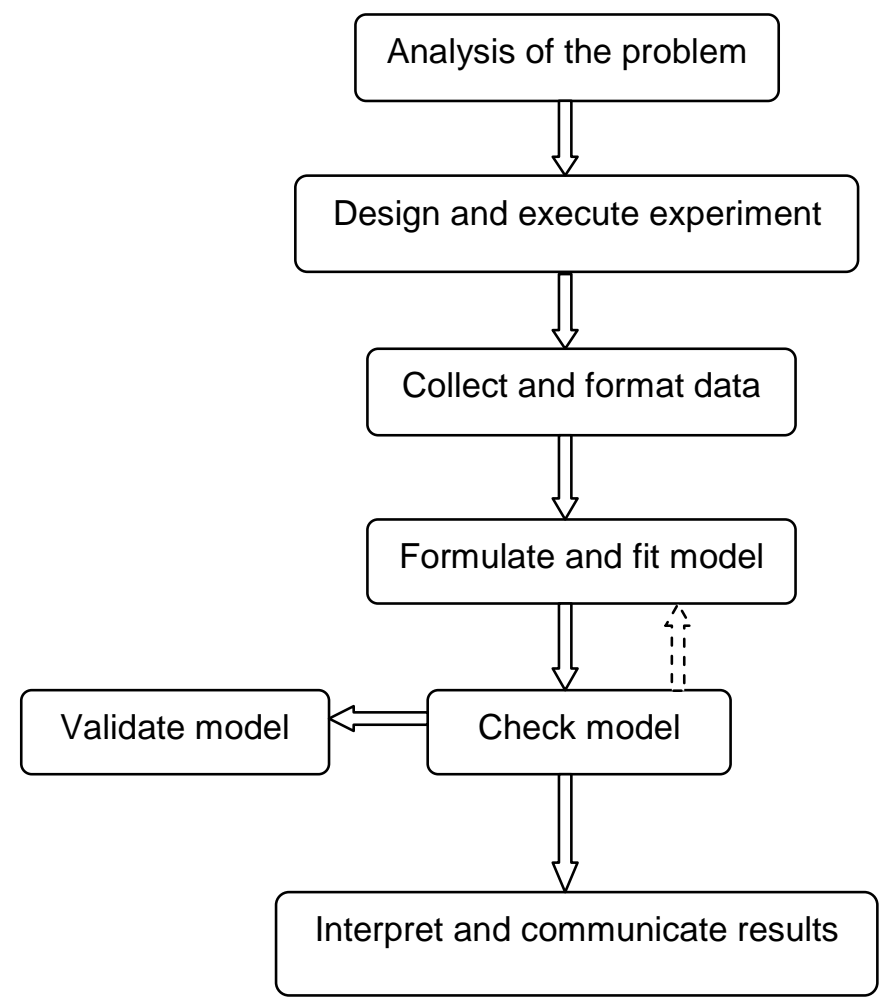

Figure 1.7: Key steps followed in the PK modelling process, adapted from Bonate [2006] 


\subsubsection{Advantages of population analysis}

The population approach offers many advantages over traditional pharmacokinetic analysis, often including data generated from the population of interest. The approach can accommodate unbalanced and sparse data, as well data generated from different sources. Covariate analysis allows explanation of subject variability. Collectively, these advantages help to overcome ethical barriers that may otherwise prohibit any form of study in the population of interest, e.g. pregnant women. Perhaps most importantly, once a model has been developed, if robust enough, can be used to simulate "what-if' clinical scenarios. Weaknesses of the population approach include, being time consuming, being perceived as complicated and the methodology difficult to understand, and that different modellers could develop different models from the same data. However, the advantages outweigh the disadvantages, hence why it is a growing discipline worldwide [Tucker, 2012].

\subsubsection{Model evaluation and validation}

Population pharmacokinetic models can be considered as either descriptive or predictive [Ette et al., 2004]. Descriptive models provide quantitative summaries of the variability in the population which was studied [Ette et al., 2003]. For descriptive models, assessment of goodness of fit, reliability and stability are important factors in model evaluation. Goodness of fit involves checking typical diagnostic plots such as observed versus model individual predicted values, observed versus model population predicted values, conditional weighted residuals [Hooker et al., 2007] versus time, conditional weighted residuals versus covariates. Parameter reliability addresses the issue of confidence in the parameter estimates and hence the model itself. Uncertainty of model parameters must be small; the percentage relative standard error for the fixed and random effect parameters should not exceed $25 \%$ and $50 \%$, respectively [Ette et al., 2004].

Predictive models are those that will be extrapolated beyond the population from which the model was estimated. For these models, validation would be an added requirement. Predictive models require much stronger assumptions about relationships to the underlying population from which the data was collected. The key question being, can the model be extrapolated to another group of patients.

Model validation can be split into two groups; external and internal. External validation is the most robust. With external validation, the final model is estimated from the index data, the model parameters are fixed, and predictions of the drug concentrations are made into test (external) 
data [Ette et al., 2004]. Internal validation, can include data splitting, cross validation, and bootstrapping. Data splitting, involves removing a proportion of data from the original data set, before the model is developed. Once the final model is proposed, it is used to predict concentrations in the data which was kept aside. The disadvantage of this approach is, that all the original data is not used in the modelling process. Cross-validation and bootstrapping have the advantage that they use all the data and are therefore not affected by random splitting. Many consider bootstrapping as the approach of choice for checking for stability, determination of parameter reliability and validation [Miller, 1974; Hinkley, 1977].

Cross validation involves removing a part of the available data and the model is fitted to the remaining data. The model from the remaining data is fixed, and predictions are made into the data that were removed. The process of cross validation provides an estimate of the prediction error. The bootstrap is a re-sampling method, first suggested by Efron and Gong [1983]. In the bootstrap method, psuedosamples that are distributed according to the same distribution of the original samples are repeatedly generated. The process creates a new plausible dataset. Usually, $\geq 200$ bootstrap data sets are created, and is usually done by re-sampling of subjects with replacements - the nonparametric bootstrap.

\subsubsection{Application of population PK analysis}

\subsubsection{Examples from drug development}

Over the last twenty years modelling and simulation has increasingly become part of the drug development process, with both the United States Food and Drug Administration [2004] and the European Medicines Agency [2007] recognising the important role the method can play in successfully developing and bringing a drug to the market. Examples follow which illustrate how the pharmaceutical industry has embraced the discipline of pharmacometrics in recent years. Brief examples include:

- Remifentanil [Egan et al., 2001]. One of the early examples of model-based drug development, where the final dose which was licensed, was based on work conducted through modelling and simulation and had not directly been studied in clinical trials

- Nesiritide [Bhattaram et al., 2005]. Dosing for the final registration trial was derived using exposure-reponse modelling and simulation to balance benefit versus risk

- Gabapentin [Lalonde et al., 2007]. Exposure-response modelling provided confirmatory evidence of effectiveness and alleviated the need for additional trials 
- Mycophenolate [Hale et al., 1998]. One of the first fully simulated clinical trials which was used to design a registration trial based on early trial data

- Pralatrexate [Mould et al., 2009]. During phase I studies, patients experienced a high incidence of grade 3 and 4 mucositis. Preliminary evaluations of the pharmacokinetics of the pralatrexate and its association with mucositis, suggested that its exposure could be controlled by body size (either body surface area or weight) based dosing and that pretreatment with folic acid and vitamin $\mathrm{B}_{12}$ might minimise the incidence and severity of mucositis. The application of modelling was critical in the pharmaceutical development of pralarexate, which lead to important suggestions for dose, dosing schedule and pretreatment modifications.

The aforementioned examples illustrate how the pharmaceutical industry are utilising modelling and simulation method to improve efficiency and effectiveness in the drug development process.

\subsubsection{Examples from clinical practice}

The discipline of pharmacometrics has an important role to play in clinical practice, as the methodology can address what-if questions raised from clinical practice, which may not be able to be addressed through traditional means. Examples follow, which describe the clinical application of pharmacometrics.

Hawwa and colleagues [2008], applied the methodology to the dosing of 6-mercaptopurine (6MP) in paediatric patients with acute lymphoblastic leukaemia (ALL). Conventional dosing methods for oral 6-MP was resulting in highly variable drug and metabolite concentrations in the paediatric patient population and hence variability in treatment outcomes. They prospectively collected data from 19 paediatric patients with ALL treated with 6-MP, titrated to a target dose of $75 \mathrm{mg} \mathrm{m}^{-2} \mathrm{day}^{-1}$. All patients were genotyped for polymorphisms in three enzymes involved in 6 MP metabolism. They found that body surface area and thiopurine methyltransferase genotype were the two significant covariates which influenced between subject variability in how paediatric patients handle 6-MP. Through incorporating these two covariates into the model they developed, they were able to suggest a more rational dosing approach for 6-MP.

In order to develop a better dosing strategy for the paediatric population, Hempel and colleagues [2009], were the first group to develop a population pharmacokinetic model of pegylated (PEG)asparaginase in a paediatric population being treated for acute lymphoblastic leukaemia. They 
analysed 1221 serum activities from 168 children. They report that a one-compartmental model best fitted the data and found clearance to increase with time according to the following formula:

$$
C l=C l_{i} * e^{(0.0793 * t)}
$$

where $\mathrm{Cl}_{i}$ is the initial clearance and $t$ is the time after dose. They also report that a subgroup of patients exhibited a higher clearance and suggest this may be related to the development of inactivating antibodies.

Nath and colleagues [2010] explored the pharmacokinetics of total and unbound plasma melphalan using the population approach, in order to identify factors which affect melphalan disposition and to evaluate the role of melphalan exposure in melphalan-related toxicity and disease response. Modelling was performed with total and unbound concentration-time data from 100 patients who had received a median melphalan dose of $192 \mathrm{mg} \mathrm{m}^{-2}$. They found that a two compartment model best fitted the data and report that creatinine clearance, fat free mass and haematocrit were important determinants of total and unbound $\mathrm{CL}$, reducing inter-individual variability in total $\mathrm{CL}$ from $34 \%$ to $27 \%$ and unbound $\mathrm{CL}$ from $42 \%$ to $30 \%$. They also found that total AUC and unbound AUC were significantly higher in patients who had oral mucositis ( $\geq$ grade 3) and long hospital admissions, providing important information about the relationship between melphalan exposure and outcomes.

Standing and colleagues [2008] developed a population model in paediatric patients receiving diclofenac for acute pain in children, as the optimal dosing strategy was not well described. Their method involved drawing blood from paediatric subjects at the start and end of surgery and on removal of the venous cannula. Data were obtained from 70 children (aged 1 to 12 years), who received a diclofenac preoperative oral dose of $1 \mathrm{mg} \mathrm{kg}^{-1}$. This data was pooled with rich adult data from 30 volunteers. As the optimal adult dose of diclofenac is $50 \mathrm{mg}$ for acute pain, simulation work planned to predict the paediatric dose to achieve a similar AUC to $50 \mathrm{mg}$ in adults. A one compartment model best fitted the data and they found that allometric scaling was able to predict changes in $\mathrm{CL}$ and $\mathrm{Vd}$ with age, and that a dose of $1 \mathrm{mg} \mathrm{kg}^{-1}$ diclofenac produces similar exposure in children aged 1 to 12 , as $50 \mathrm{mg}$ in adults, confirming that higher doses were not necessary in this population. 


\subsubsection{Application of population PK analysis in the fields of pregnancy}

Pregnancy is one of the sub-populations where drug therapy is often not formally evaluated due to ethical constraints and subsequent information on drug use in this population is derived from observational data in the form of case reports.

Pharmacometrics and the use of modelling and simulation therefore provides an opportunity to apply this method in order to optimise drug use in this population, especially when patients are using medicines for a range of chronic conditions, for example asthma, HIV and tuberculosis. Indeed at the $3^{\text {rd }}$ American Conference on Pharmacometrics in April 2011, the clinical application of modelling and simulation in an antenatal population was specifically discussed [van Hasselt et al., 2012]. Some examples published in the literature follow.

Andrew and colleagues [2007] applied modelling and simulation to the problem of developing an appropriate amoxicillin dosing strategy for the prevention of anthrax during pregnancy, in response to possible bioterrorism attacks on the United States. In their study, sixteen women received amoxicillin during 18-22 weeks gestation, 30-34 weeks gestation, as well as 3 months postpartum. Single dose amoxicillin pharmacokinetics were evaluated. Amoxicillin renal clearance and secretion was found to be higher during pregnancy than postpartum, as one might predict. Their simulation work suggested that amoxicillin concentrations required to prevent anthrax may be difficult to achieve during pregnancy and that amoxicillin may not be an appropriate antibiotic for post-antrax exposure prophylaxis in a pregnant population.

Tarning and colleagues [2009] conducted a population pharmacokinetic study of lumefantrine in pregnant women with uncomplicated multidrug resistant Plasmodium falciparum malaria. Their subject population comprised 103 women in the second or third trimester treated with artemetherlumefantrine $(80 / 480 \mathrm{mg})$ twice daily for 3 days. Patients provided 5 capillary samples for drug quantification, with the exact time of sample collection randomly distributed over 14 days. A high proportion of women (40\%) had day 7 capillary concentrations of $<355 \mathrm{ng} / \mathrm{mL}$, a threshold previously associated with an increased risk of therapeutic failure in non-pregnant patients. Following the modelling process, they found that a two compartment model with first order absorption and elimination best fitted the data. In their study, Tarning and colleagues report a high treatment failure rate of $16 \%(95 \% \mathrm{Cl}, 9.9-25.1)$, with their predictive modelling suggesting that a twice-daily regimen given for 5 days would be preferable later on in pregnancy and should overcome the treatment failures observed. 
Allegaert and colleagues [2009] applied the population pharmacokinetic approach to cefazolin disposition. The antibiotic is recommended as an alternative to penicillin based antibiotics on the prevention of perinatal group B streptococcal diseases in pregnant women with a moderate risk of a penicillin allergy. They collected 187 plasma samples and 96 amniotic fluid samples from 82 pregnancies (17-40 weeks gestation). They report cefazolin $\mathrm{CL}$ and $\mathrm{Vd}$ estimates to be $7.44 \mathrm{~L} / \mathrm{hr}$ and $12 \mathrm{~L}$ respectively. On the basis of their results, they suggest that dosing regimens to attain higher amniotic fluid concentrations are required for the prevention of group B streptococcal infection in this population.

A study conducted by Hirt and colleagues [2009] evaluated the pharmacokinetics of tenofovir in HIV-1 infected pregnant women. Tenofovir is commonly used in resource low income countries, as treatment to prevent mother-child transmission of HIV at the time of birth. Thirty-eight women were given tenofovir disoproxil fumerate (TDF 300mg)-emtricitabine (FTC 200mg) tablets; two at labour induction and once daily for 7 days postpartum. Maternal, umbilical and neonatal plasma tenofovir concentrations were assayed by high pressure liquid chromatography. A two compartment model for the mother best fitted the data. The authors found that absorption of tenofovir was greater in women who delivered by caesarean section than those delivering vaginally. $600 \mathrm{mg}$ of tenofovir prior to delivery produces the same concentration as $300 \mathrm{mg}$ in non pregnant adults. Their simulation work suggested that if the time elapsed between maternal administration and delivery is $\geq 12$ hours, two further tablets of tenofovir should be readministered.

Bouillon-Pichault et al., [2009] investigated the need to increase the lopinavir dose during pregnancy in order to achieve pre-determined efficacy concentrations in a HIV pregnant population. They obtained lopinavir concentrations from 145 HIV infected women; 74 of which were pregnant. A one-compartment model with first-order absorption was found to best fit the data. Significant changes in lopinavir PK were observed, with $\mathrm{CL}$ increasing as pregnancy progressed. The study was able to identify that patients previously treated with protease inhibitor were likely to need a dose increase, as their pregnancy progressed.

These studies clearly demonstrate how the population approach can help to address important clinical questions regarding drug use in a pregnant population. 


\subsubsection{Enoxaparin PK modelling studies}

\subsubsection{PK models of enoxaparin outside of pregnancy}

Enoxaparin has been used routinely in clinical practice for a number of years, and given its wide application in the treatment and prevention of VTE, and the management of patients with acute coronary syndromes, it is not surprising that clinical uncertainties have arisen around its optimal dose in certain sub populations with time, for example its use in the obese and those patients with renal impairment. Given these uncertainties, research groups have attempted to address these uncertainties using the population approach and compartmental analysis.

Green and Duffull [2003] applied the population approach to the problem of dosing of enoxaparin in patients who are overweight or obese. Enoxaparin dosing is based on total bodyweight and a commonly asked clinical question is whether the dose should be capped when treating obese patients for the treatment of VTE (1.5 mg/kg once a day) or for acute coronary syndromes (1 $\mathrm{mg} / \mathrm{kg}$ every twelve hours). Patients being managed for deep vein thrombosis, acute coronary syndromes or prescribed enoxaparin as prophylaxis against VTE were recruited to their study. Ninty-six patients were enrolled; thirty-two had a BMI $<25 \mathrm{~kg} / \mathrm{m}^{2}, 31$ a BMI of $25-29.99 \mathrm{~kg} / \mathrm{m}^{2}$ and 33 patients had a BMI of $>30 \mathrm{~kg} / \mathrm{m}^{2}$. On average, 3 anti-Xa samples per patient were collected. Green and Duffull report that a two compartment model with first order absorption, with exponential between subject variability on clearance and volume (central compartment), with an additive error model best fitted the data. A covariate analysis showed clearance was best described by lean body weight and the central volume by total body weight. The final parameter estimates from the covariate model they developed are listed in table 1.11.

Table 1.11: Green and Duffull's enoxaparin obesity model

\begin{tabular}{llll}
\hline Parameter & Units & Value & SE (CV\%) \\
\hline $\mathrm{CL}$ & $\mathrm{L} \mathrm{h}^{-1} 70 \mathrm{~kg}^{-1}(\mathrm{LBW})$ & 1.03 & 6.80 \\
$\mathrm{~V}_{2}$ & $\mathrm{~L} 70 \mathrm{~kg}^{-1}(\mathrm{WT})$ & 3.67 & 24.5 \\
$\mathrm{~K}_{\mathrm{a}}$ & $\mathrm{h}^{-1}$ & 0.195 & 25.6 \\
$\mathrm{~V}_{3}$ & $\mathrm{~L}$ & 13.1 & 34.4 \\
$\mathrm{Q}$ & $\mathrm{Lh}^{-1}$ & 0.363 & 33.3 \\
$\omega \mathrm{CL}$ & $\mathrm{CV} \%$ & 35.6 & 20 \\
$\omega V_{2}$ & $\mathrm{CV} \%$ & 58 & 33.3 \\
$\sigma^{2}$ & $(\mathrm{IU} \mathrm{L})^{-1}$ & 6430 & 30 \\
\hline
\end{tabular}


Their simulation work suggested that patients over 50 years of age whose total body weight is $>90 \mathrm{~kg}$, or patients under 50 years of age whose total body weight is $>120 \mathrm{~kg}$ are likely to have a smoother concentration-time profile and less bruising if a dose of $100 \mathrm{IU} \mathrm{kg}^{-1}$ based on lean body weight is administered every 8 hours; suggesting that obese patients do require a different dosing strategy compared to patients who are normally weighted.

Green and colleagues [2004] conducted a population pharmacokinetic study in order to investigate the influence of glomerular filtration rate on the pharmacokinetics of subcutaneously administered enoxaparin. Thirty-eight patients being managed for acute coronary syndromes were recruited into the study, with each patient contributing 10 anti-Xa activities. In total, 313 antiXa concentrations were collected for modelling purposes. A two-compartment, with exponential between subject variability on Vd and $\mathrm{CL}$ and a combined error model, best fitted the data. In their analysis, Green and colleagues estimate that $71 \%$ of enoxaparin is excreted unchanged through the kidneys, and following their simulation work, they suggest the following recommendations on dosing enoxaparin for the management of acute coronary syndromes (usual dose $1 \mathrm{mg} / \mathrm{kg}^{-1}$ ) in patients with renal impairment (table 1.12).

Table 1.12: Green's enoxaparin renal impairment model

\begin{tabular}{cc}
\hline C-G derived GFR (using IBW) as size descriptor $\mathrm{ml}$ & Enoxaparin dose $\mathrm{mg} \mathrm{kg}^{-1}$ (WT) twice daily \\
\hline $10-19$ & 0.3 \\
$20-29$ & 0.4 \\
$30-39$ & 0.5 \\
$40-49$ & 0.6 \\
$50-59$ & 0.7 \\
$60-69$ & 0.8 \\
$70-79$ & 0.9 \\
$\geq 80$ & 1 \\
\hline
\end{tabular}

Hulot and colleagues [2005] conducted a population pharmacokinetic modelling study of enoxaparin, also addressing the dosing implications for patients with renal impairment, by evaluating 532 patients receiving subcutaneous enoxaparin for non-ST-segment elevation acute coronary syndrome. Patients were typically prescribed enoxaparin $1 \mathrm{mg} / \mathrm{kg} 12^{-1}$, however in patients with renal impairment, clinicians reduced the dose of enoxaparin according to their 
clinical judgement. In their study, $34 \%$ of patients had a normal renal function, $36 \%$ had mild renal failure, $20 \%$ had moderate renal failure and $10 \%$ had severe renal failure. A total 661 anti-Xa activities were obtained and modelled using the population approach. A one compartment model with first order kinetics with proportional between subject variability on clearance and volume of distribution, with an additive error model best fitted the data. Covariate analysis revealed that total body weight was a significant covariate for volume and $\mathrm{CrCl}$ was a significant covariate for $\mathrm{CL}$. Final model parameter estimates are listed in table (1.13).

Table 1.13: Hulot's renal impairment model

\begin{tabular}{|c|c|c|}
\hline Parameter & Model (mean +/- SE of estimate) & $\begin{array}{l}2.5^{\text {th }}-97.5^{\text {th }} \text { percentiles obtained } \\
\text { from bootstrapping }\end{array}$ \\
\hline \multicolumn{3}{|l|}{ Structural model } \\
\hline $\mathrm{V} / \mathrm{F}(\mathrm{L})$ & $5.29+/-0.55$ & $5.00-6.00$ \\
\hline $\mathrm{V}$ (influence of body weight) & $+1.49+/-0.23$ & $1.10-2.00$ \\
\hline $\mathrm{CL} / \mathrm{F}$ & $0.74+/-0.03$ & $0.71-0.79$ \\
\hline $\begin{array}{l}\mathrm{CL} \text { (influence of body } \\
\text { weight/serum creatinine ratio) }\end{array}$ & $+0.24+/-0.04$ & $0.14-0.27$ \\
\hline $\mathrm{CL}$ (factor if female) & $+0.75+/-0.06$ & $0.69-0.80$ \\
\hline$K_{a}\left(h^{-1}\right)$ & $0.34+/-0.05$ & $0.26-0.44$ \\
\hline \multicolumn{3}{|l|}{ Statistical model } \\
\hline Residual variability $(\sigma)(\mathrm{IU} / \mathrm{mL})$ & $0.14+/-0.02$ & $0.13-0.16$ \\
\hline ISV (V) (\%CV) & $50+/-26$ & $42-61$ \\
\hline ISV (CL) (\%CV) & $27+/-12$ & $26-35$ \\
\hline
\end{tabular}

Following their simulation work, aiming for a target peak anti-Xa activity of $0.5-1.2 \mathrm{IU} / \mathrm{mL}$, the authors suggest that for patients with moderate renal impairment, $0.8 \mathrm{mg} \cdot \mathrm{kg}^{-1} .12 \mathrm{~h}^{-1}$ is suggested and in those with severe renal impairment, $0.66 \mathrm{mg} \cdot \mathrm{kg}^{-1} \cdot 12^{-1}$. In all cases, like Green and colleagues, they suggest a loading dose. In this case, Hulot and colleagues suggest that an initial subcutaneous dose of $1 \mathrm{mg} / \mathrm{kg}$ can be administered whatever the renal function.

Feng and colleagues assessed the pharmacokinetics of a continuous intravenous infusion of enoxaparin in a critical care population [2006]. This was in response to the observations from clinical practice, which indicated that unreliable and extensive variability in anti-Xa concentrations are found in patients with critical illness, when the standard recommended dose and route of administration is used. Feng and colleagues suggest that a continuous IV infusion can overcome 
this problem with enoxparin and conducted a study to explore the PK of enoxaparin when administered as a continuous IV infusion. In this study, anti-Xa activity from a previous study was combined with additional data collected. Three-hundred and sixty-three anti-Xa activities were available from patients who received a continuous IV infusion of enoxaparin. A two compartment linear model, with a proportional error model for the critical care patients receiving the continuous IV infusion of enoxaparin, best fitted the data. Both $\mathrm{CrCl}$ and weight were found to significantly affect $\mathrm{CL}$ and $\mathrm{Vd}$ of enoxaparin in this population. Simulation work suggested that the optimal doses for patients in the intensive care unit were, $50 \mathrm{IU} \mathrm{kg}^{-1}$ per $12 \mathrm{~h}$ if $\mathrm{CrCl}<30 \mathrm{ml} \mathrm{min}^{-1} ; 60 \mathrm{IU}$ $\mathrm{kg}^{-1}$ per $12 \mathrm{~h}$ if $\mathrm{CrCl}$ is $30-50 \mathrm{ml} \mathrm{min}^{-1}$; and $100 \mathrm{IU} \mathrm{kg}^{-1}$ per $12 \mathrm{~h}$ if $\mathrm{CrCl}>50 \mathrm{ml} \mathrm{min}^{-1}$.

Bruno and colleagues [2003] conducted a population PK-PD study of data (anti-Xa activity) obtained from the TIMI-11A study [Antman et al., 1997]. TIMI-11A was an open label study assessing the safety and tolerability of two weight-adjusted enoxaparin regimens in patients with unstable angina and non ST elevation MI. Patients received a 30mg intravenous bolus, followed by weight adjusted doses of either $1 \mathrm{mg} / \mathrm{kg}^{-1}$ (309 patients) or $1.25 \mathrm{mg} / \mathrm{kg}^{-1}$ (321 patients) of enoxaparin subcutaneously every 12 hours for a median of 3 days. For the purposes of PK analysis, trough and 3-5 hours post dose (peak) anti-Xa activity was drawn, after the third weightadjusted dose of enoxaparin and again on the last day of enoxaparin dosing. Bruno and colleagues obtained data from 448 patients $(71 \%)$ of the total study population, providing 788 anti-Xa activities. Data from 300 patients were randomly selected and modelled, leaving the remaining 148 patients' data as the validation set for the final model produced. A onecompartment model with between subject variability on drug clearance best fitted the data. Following covariate analysis, weight and $\mathrm{CrCl}$ were significant covariates which were retained for the final model. Their final model estimates are listed in table 1.14 . 
Table 1.14: Bruno's enoxaparin model

\begin{tabular}{lcccc}
\hline & \multicolumn{2}{c}{ Final Model } & \multicolumn{2}{c}{ Bootstrap $(n=500)$} \\
\hline Parameter & Estimates & $95 \% \mathrm{Cl}$ & Median & $95 \% \mathrm{Cl}$ \\
\hline Clearance $\left(\mathrm{L} \mathrm{h}^{-1}\right)$ & 0.733 & $0.698-0.738$ & 0.734 & $0.702-0.766$ \\
Weight & $4.32 \times 10^{-3}$ & $1.13 \times 10^{-3}-7.51 \times 10^{-3}$ & $4.47 \times 10^{-3}$ & $1.39 \times 10^{-3}-7.65 \times 10^{-3}$ \\
$\mathrm{CrCl}$ & $3.38 \times 10^{-3}$ & $1.76 \times 10^{-3}-1.76 \times 10^{-3}$ & $3.38 \times 10^{-3}$ & $1.77 \times 10^{-3}-5.01 \times 10^{-3}$ \\
$\mathrm{~V}(\mathrm{~L})$ & 5.24 & $4.20-6.28$ & 5.19 & $2.55-6.59$ \\
$\mathrm{~K}_{\mathrm{a}}\left(\mathrm{h}^{-1}\right)$ & 0.235 & $0.157-0.313$ & 0.232 & $0.149-0.361$ \\
$\omega_{\mathrm{CL}}(\%)$ & 26.9 & $20.9-31.8$ & 26.6 & $20.3-31.8$ \\
$\Pi_{\mathrm{CL}}(\%)$ & 21.7 & $14.9-26.8$ & 21.7 & $12.3-27.2$ \\
$\omega_{\mathrm{V}}(\%)$ & 56.1 & $36.4-70.6$ & 55.8 & $34.2-77.2$ \\
$\sigma(\%)$ & 21.5 & $16.4-25.6$ & 21.3 & $16.5-25.6$ \\
\hline$\pi_{\mathrm{CL} L \text { coefficient of variability of intra-individual variability }}$ & & &
\end{tabular}

$\Pi_{\mathrm{CL}}$, coefficient of variability of intra-individual variability

According to this model, a typical patient with a median weight and median $\mathrm{CrCl}$ would have an enoxaparin clearance of $0.733 \mathrm{Lh}^{-1}$, a Vd of $5.21 \mathrm{~L}$ and a half-life of $5 \mathrm{~h}$. Variation in weight within the $5^{\text {th }}$ and $95^{\text {th }}$ percentiles of the database $(58-117 \mathrm{~kg})$ resulted in a change in clearance of between $-14 \%$ and $+21 \%$. Patients with a $\mathrm{CrCl}$ of $50 \mathrm{ml} \mathrm{min}^{-1}$ or $30 \mathrm{ml} \mathrm{min}^{-1}$ would observe a enoxaparin clearance decrease of $17 \%$ and $27 \%$ respectively. The authors conclude that body weight and renal function are significantly related to enoxaparin clearance and that a decreased clearance will significantly increase the chances of bleeding complications.

Kane-Gill and colleagues conducted a population PK study of continuous infusion of enoxaparin from patients on either a general medical ward or an ICU setting [Kane-Gill et al., 2005]. In their study, 48 patients contributed data for PK modelling purposes. Clearance and Vd estimates for ICU and ward patients were $0.64+/-0.34 \mathrm{~L} / \mathrm{h}, 10.6+/-1.55 \mathrm{~L}$ and $1.01+/-0.39 \mathrm{~L} / \mathrm{h}, 9.08+/-1.17 \mathrm{~L}$ respectively. The authors from this small retrospective study conclude, that a continuous infusion of enoxaparin is safe and that the PK parameters of enoxaparin of patients on ICU differ from those patients on the ward.

Sanchex-Pena and colleagues conducted a PK modelling study of bolus IV enoxaparin administered to patients undergoing percutaneous coronary intervention (PCI) in France [2005]. In their study, serial blood samples were taken; before iv bolus of enoxaparin, 10 minutes after bolus (start of $\mathrm{PCl}$ ), at the end of $\mathrm{PCl}$ (mean time $=45$ minutes), 3 hours after $\mathrm{PCl}$ and on the morning after PCl. A total of 2016 anti-Xa activities were available for modelling purposes from 
556 patients, who had received a mean IV dose of enoxaparin of $38+/-7 \mathrm{mg}$ (3830 +/-730 IU). The time course of anti-Xa activity was best described by a one compartment model with zeroorder input. Between subject variability was described using an exponential model, and the residual error was best described by an additive error model. During covariate modelling, bodyweight and to a less degree, $\mathrm{CrCl}$ and creatinine influenced $\mathrm{CL}$. In the final model, only body weight significantly affected enoxaparin CL. From their simulation work, the authors conclude that $0.5 \mathrm{mg} \mathrm{kg}^{-1}$ i.v. dose of enoxaparin reached anticoagulation levels adequately in patients undergoing elective PCI. Their simulation work also suggested that an additional second bolus dose could be given in patients with delayed or prolonged procedures.

Berges and colleagues [2007] conducted a population PK study in elderly patients receiving prophylactic enoxaparin $4000 \mathrm{IU}$ sc injection daily as VTE prophylaxis, in order to assess if this sub-population would require additional monitoring, due to their age. One hundred and eighty nine patients were studied, with $22 \%$ weighing $<50 \mathrm{~kg}$ and $50 \%$ having the presence of renal impairment. Four hundred and fifty one anti-Xa activities were available for PK modelling purposes; the authors report a first order input, two compartment model best fitted the data. Clearance was significantly related to body weight and $\mathrm{CrCl}$, and weight significantly affected $\mathrm{Vd}$. According to their model, around $4 \%$ of the population they studied would achieve a peak anti-Xa activity of $>1.0 \mathrm{IU} \mathrm{ml}^{-1}$. The authors conclude that monitoring anti-Xa activity in elderly patients treated with enoxaparin at prophylactic doses does not seem necessary to prevent the occurrence of major bleeding.

Finally, Barrass and colleagues [2009] modelled the severity and occurrence of enoxaparin related bruising in an Australian population. As part of this study, enoxaparin PK estimates were computed for the study population. Patients being managed for PE, DVT, acute coronary syndrome (ACS), or atrial fibrillation (AF) were randomised to a dose individualised arm or a conventional dosing arm of this prospective study. Patients in the conventional arm received the manufacturers recommended doses of enoxaparin. Subjects in the individualised arm were dosed according to either their actual weight or lean body weight (LBW). Subjects $\geq 100 \mathrm{~kg}$ were considered obese and dosed on $1.5 \mathrm{mg} \mathrm{kg}^{-1}$ twice a day, whereas patients $<100 \mathrm{~kg}$ were dosed on their actual weight, at a dose of $1 \mathrm{mg} \mathrm{kg}^{-1}$ twice a day. Patients with a $\mathrm{CrCl}$ of $<50 \mathrm{ml} \mathrm{min}^{-1}$ were dosed at a reduced dose after 48 hours. Four samples were collected, one pre-dose, then one at 15-30, 60-120 and 180-300 min post dose. A total of 349 anti-Xa activities were available for PK 
modelling purposes. A two-compartment model with first order input and linear elimination best described the data. Between subject variability on $\mathrm{CL}$ and $\mathrm{Vd}$ and $\mathrm{Ka}$ was added to the model and an additive error model best described residual unexplained variability. Model parameter estimates are listed in table 1.15.

Table 1.15: Barras' enoxaparin model

\begin{tabular}{lccc}
\hline Parameter & Final Model $(95 \% \mathrm{Cl})$ & $\begin{array}{c}\text { Bootstrap }\left(2.5^{\text {th }}-97.5^{\text {th }}\right. \\
\text { percentile })\end{array}$ & $\begin{array}{c}\text { Relative \% SE of the } \\
\text { parameter estimates }\end{array}$ \\
\hline $\mathrm{CL}\left(\mathrm{L} \mathrm{h}{ }^{-1}\right)$ & 0.3 renal $(0.14-0.45)$ & $0.29(0.13-0.46)$ & 27.4 \\
& 0.42 (non-renal) $(0.24-0.61)$ & $0.42(0.25-0.60)$ & 22.3 \\
$\mathrm{~V}_{\mathrm{c}}(\mathrm{L})$ & $3.43(2.20-4.66)$ & $3.42(2.07-5.17)$ & 18.3 \\
$\mathrm{~K}_{\mathrm{a}}(\mathrm{h})$ & $0.26(0.17-0.35)$ & $0.26(0.16-0.40)$ & 18.3 \\
$\mathrm{~V}_{\mathrm{p}}(\mathrm{L})$ & $5.77(0.75-10.8)$ & $6.42(3.0-17.8)$ & 44.3 \\
$\mathrm{Q}\left(\mathrm{L} \mathrm{h} \mathrm{h}^{-1}\right)$ & $0.31(0.15-0.47)$ & $0.28(0.1-0.44)$ & 27.0 \\
$\omega \mathrm{CL}(\% \mathrm{CV})$ & $37.8(22.3-48.0)$ & $37.4(28.3-50.9)$ & 32.2 \\
$\omega \mathrm{V}_{\mathrm{c}}(\% \mathrm{CV})$ & $35.6(7-51.0)$ & $34.6(5.4-52.9)$ & 52.9 \\
$\omega \mathrm{K}_{\mathrm{a}}(\% \mathrm{CV})$ & $30.3(5.3-42.5)$ & $29.8(1.7-49.8)$ & 49.6 \\
$\sigma\left(\mathrm{IU} \mathrm{mL}{ }^{-1}\right)$ & $0.09(0.086-0.1)$ & $0.09(0.083-0.1)$ & 14.5 \\
\hline
\end{tabular}

In the simulation work that Barrass and colleagues conducted, individualised dosing decreased the probability of a bleeding or major bruising event when compared to conventional dosing, which was most noticeable in those patients who were obese or with renal impairment.

To date, though enoxaparin modelling has been conducted in heterogeneous populations and each were looking to answer specific clinical questions, the common theme emerging from them all is that weight (or lean body weight) appears to be a significant covariate for enoxaparin CL.

\subsubsection{Antenatal PK model of enoxaparin}

The only compartmental PK analysis of enoxaparin during pregnancy that has been conducted to date is that by Lebaudy and colleagues [2008]. They conducted a population pharmacokinetic study of 75 pregnant and 38 non-pregnant women (acting as controls), in order to evaluate the pharmacokinetics of enoxaparin during pregnancy and the postpartum period. They obtained 343 anti-Xa activity samples from the pregnant women, up to 49 hours after the last enoxaparin dose was administered (samples taken at clinicians discretion). The majority of samples were collected during the second and third trimester. They found a one compartment model with first order kinetics, with between subject variability on $\mathrm{CL}$ and $\mathrm{Vd}$, and an additive error model best fitted the 
data. They reported that clearance and volume of distribution of enoxaparin increased by $48 \%$ and $49 \%$ respectively in the pregnant women compared to the nonpregnant controls. Their median parameter estimates using the population approach are presented in table 1.16.

Table 1.16: Lebaudy's pregnancy enoxaparin model

\begin{tabular}{lcc}
\hline Pharmacokinetic parameter & Median $+/-$ SD & $2.5^{\text {th }}-97.5^{\text {th }}$ percentiles \\
\hline Structural model & & \\
Clearance (L/hr) & $0.81+/-0.03$ & $0.763-0.84$ \\
Ratio effect & $0.42+/-0.12$ & $0.18-0.64$ \\
Volume of distribution (L) & $7.81+/-1.23$ & $5.7-10.5$ \\
Gestational age effect & $1.41+/-0.25$ & $1.04-1.86$ \\
Absorption rate constant $\left(\mathrm{hr}^{-1}\right)$ & $0.56+/-0.12$ & $0.31-0.87$ \\
Statistical model & & \\
Between subject variability - CL (\%CV) & 20.3 & $14.7-24.8$ \\
Between subject variability - Vd (\%CV) & 26.0 & $12.7-37.9$ \\
Residual unexplained variability $(\mathrm{IU} / \mathrm{mL})$ & $0.12+/-0.05$ & $0.09-0.14$ \\
\hline
\end{tabular}

In their study, Lebaudy and colleagues found enoxaparin clearance was significantly influenced by body weight and serum creatinine and suggested that the best equation to predict individual clearance was:

$\mathrm{Cl}=0.781 \times[(\text { bodyweight/serum creatinine }) / 1.27]^{0.423}$

They also report that the volume of distribution of enoxaparin increased from $7.25 \mathrm{~L}$ in the first trimester to $7.83 \mathrm{~L}$ by the $31^{\text {st }}$ week of pregnancy, thereafter displaying a sharp increase, reaching $11.4 \mathrm{~L}$ by the time the baby was delivered. The authors suggest that enoxaparin's volume of distribution during pregnancy was best described by the following equation:

$\mathrm{Vd}=7.81 \times$ (bodyweight / 70) $\times 1.41$ at $>31$ weeks of amenorrhea

The study has contributed some important findings; although enoxaparin clearance increases during pregnancy, it does not increase significantly after the first trimester. On the other hand, volume of distribution does continue to increase with pregnancy progression. Therefore, if the same dose is administered throughout pregnancy, a reduction in peak anti-Xa activity will be observed, however, residual anti-Xa activity will also increase with the progression of pregnancy. 
There are limitations to the method Lebaudy and colleagues applied the modelling and simulation method to this problem. In terms of study design and blood sampling, there was no informative sampling strategy; samples were taken at the discretion of the physician, and not optimised using d-optimal methods [Mentré et al., 1997]. In terms of the modelling process, no diagnostic plots of the final model or model evaluation plots were available for inspection. Furthermore, in the model produced, there was a very large unexplained variability (additive error $=120 \mathrm{IU} / \mathrm{L}$ ).

Accepting the limitatations, Lebaudy's study provides an insight into how modelling and simulation could be applied to optimise enoxaparin (and other LMWH) use during the antenatal period and provides a benchmark for future studies.

\subsection{Hypothesis}

The antenatal dosing strategy of enoxaparin for the treatment and prevention of VTE should be the same as in the non-pregnant population.

\subsubsection{Aims of the study}

- To develop a population pharmacokinetic model for enoxaparin during the antenatal period

- To simulate important what-if questions generated from clinical practice from the model developed, specifically:

- Should the dosing strategy of LMWH during pregnancy be once or twice a day for the treatment and prophylaxis of VTE

- Which co-variates significantly affect the PK profile of enoxaparin during pregnancy

- To describe how thrombin generation and d-dimer concentrations are altered during pregnancy and the puerperium for women injecting enoxaparin during pregnancy

- To assess whether polybrene can neutralise the effect of enoxaparin in plasma being assayed for thrombin generation

- To explore women's views on and adherence to enoxaparin during pregnancy and the puerperium 


\section{Chapter 2}

\subsection{Methodology}

The purpose of chapter 2 is to give a broad overview of the methodology adopted for this study.

Further specific details of methodology are detailed in the relevant results chapters when necessary. Figure 2.1 summarises the key methodology stages adopted for this study.

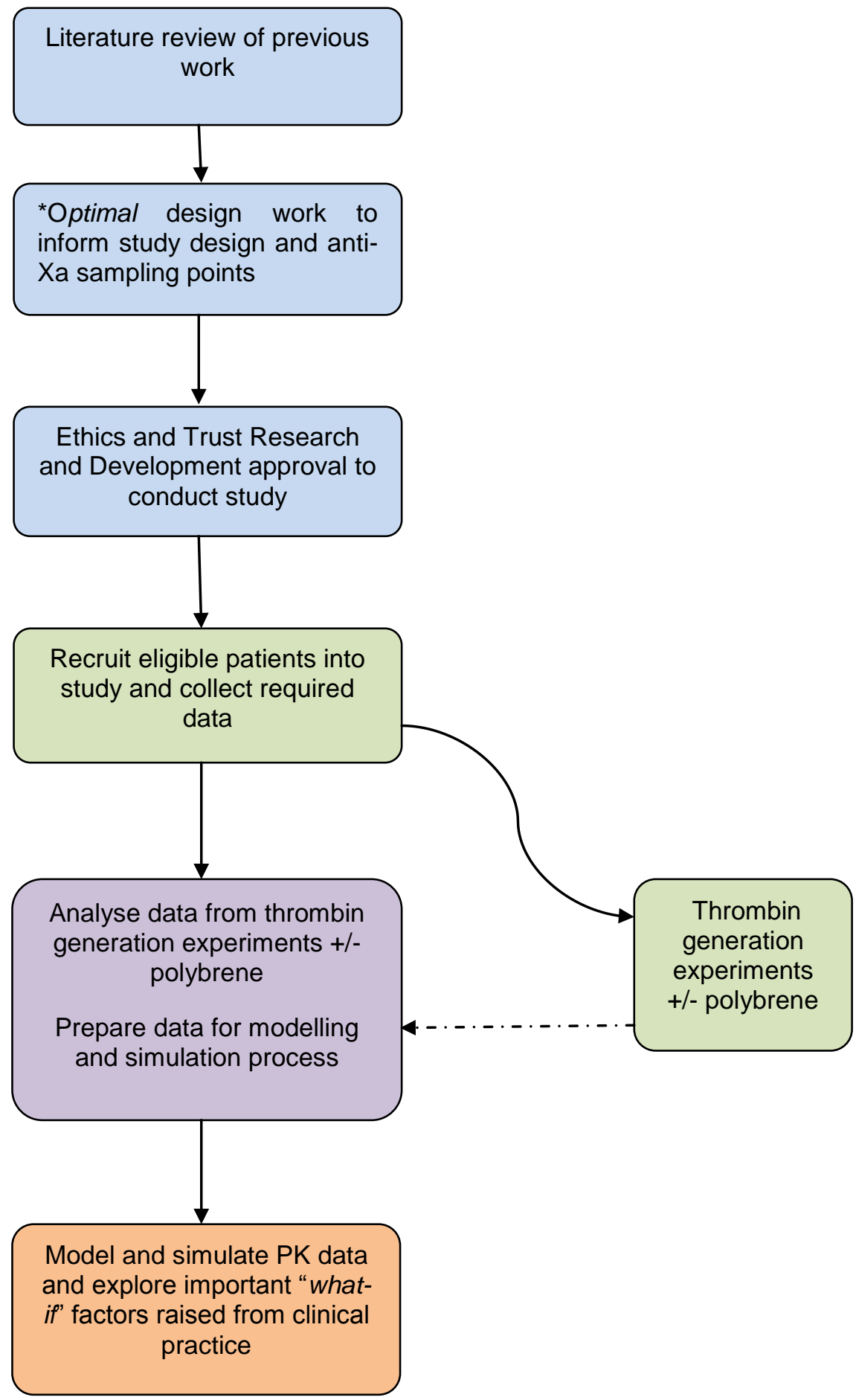

Figure 2.1: an overview of the methodology adopted for the study

*Work carried out by Dr Green's laboratory, University of Queensland, Australia. 


\subsubsection{Optimal design}

The aim of population pharmacokinetic and pharmacodynamic studies is to obtain accurate estimates of the desired parameters in the model being developed. Therefore, although NLMEM can model data from studies where data originates from sparse data points, if the study design is suboptimal in terms of when the samples were drawn, then the final model developed may be inaccurate or imprecise. Therefore, of two approaches of sampling that could be adopted, empirical (where the researchers determine the sampling times on when they think is best or is practical), or optimal (where pre-determined collection times are determined), optimal design methods are the more robust approach in clinical pharmacokinetic modelling [D'Argenio, 1981; Mentré et al., 1997].

The aim of any optimal design work is to analyse and evaluate information for a particular study design that allows selection of a design that allows maximum precision of parameter estimates [Duffull et al., 2005]. In this study, optimal design is of particular value, where one anticipates time varying changes in the key parameter estimates of $\mathrm{CL}$ and $\mathrm{Vd}$ as pregnancy progresses. Therefore to ensure these are accurately quantified, optimal design work was undertaken by $\mathrm{Dr}$ Green's laboratory at the University of Queensland, prior to the commencement of the study [Van Hasselt et al., 2009; Van Hasselt et al., 2012]. This work is described here in brief.

Based on information published in the literature, a semi-physiological model was developed which incorporated all relevant physiological variables likely to affect $\mathrm{CL}$ and $\mathrm{Vd}$ of enoxaparin during pregnancy. The performance of the semi-physiological model was then compared to the enoxaparin model developed by Lebaudy and colleagues [Lebaudy et al., 2009], in terms of estimating volume of distribution and clearance during pregnancy. 1000 individual concentrationtime profiles were then simulated stochastically using both models and the predictions obtained from both models were compared. Sampling widows were calculated for both the semiphysiological and Lebaudy model using optimal design, based on semi-physiological and Lebaudy models predicted changes in clearance and volume of distribution of enoxaparin during pregnancy. The optimal sampling designs for the semi-physiological and Lebaudy models were evaluated by simulating 1000 individual concentration-time profiles under the sampling times of interest, following which clearance and volume of distribution were re-estimated. 
Simulations of the two models were performed in NONMEM (version 7.1.0). D-optimal designs were determined using WinPOPT (version 1.2) [Duffull et al., 2008] and the exchange optimisation algorithm.

Dr Green's laboratory found stochastic simulations of anti-Xa peak and trough concentrations using the semi-physiological and Lebaudy models showed comparable concentration-time profiles. They also found that the D-optimal time-points and sampling windows for the semiphysiological and the Lebaudy models were relatively comparable (table 2.1), except for the second blood sample (there to determine Ka), where the Lebaudy model predictions suggested a later collection of this sample compared to the semi-physiological model. The change in parameter estimates over pregnancy resulted in a negligible change of optimal sampling times for the Lebaudy model ( 0.01 hours) and no change in sampling times was found for the semiphysiological model. Both models showed comparable precision and bias [van Hasselt et al., 2012].

Table 2.1: Differences between the sampling times suggested from the two models evaluated

\begin{tabular}{lcc}
\hline Occasion (month) & \multicolumn{2}{c}{ Sampling times $(\mathrm{h})$} \\
& Semi-physiological model & Lebaudy model \\
\hline 0 & $23.5,0.585,3.00$ & $23.5,1.30,3.00$ \\
2 & $23.5,0.585,3.00$ & $23.5,1.30,3.00$ \\
4 & $23.5,0.585,3.00$ & $23.5,1.30,3.00$ \\
6 & $23.5,0.585,3.00$ & $23.5,1.30,3.00$ \\
8 & $23.5,0.585,3.00$ & $23.5,1.30,3.00$ \\
\hline
\end{tabular}

Based on this work, it was decided that the optimal anti-Xa sampling times for this study were, just prior to the next dose (trough), one hour post injection and three hours post injection.

\subsubsection{Data Collection}

A prospective cohort study was conducted at the King's Thrombosis Centre, King's College Hospital. The thrombosis centre at King's provides expert care for all pregnant women on LMWH on an outpatient basis. The LMWH prescribed at King's is enoxaparin. Clinicians were free to alter the doses of enoxaparin through a woman's pregnancy, if clinically indicated. 


\subsubsection{Patient recruitment}

Nulliparous and multiparous women receiving enoxaparin (all indications and doses) were eligible to participate in the study. Those women who did not wish to participate in the study, who were unlikely to comply with directions or turn up to clinic for follow-up, those who had a documented allergy to $\mathrm{LMWH}$, those who had impaired renal function (Creatinine Clearance $<60 \mathrm{ml} / \mathrm{min}-$ using the Cockcroft Gault method of calculation [Cockcroft and Gault, 1976]) and women $<18$ years of age were excluded. Eligible patients were invited to participate by a member of the haematology medical team, when the patient first attended the haematology outpatient clinic following referral and they were given the study information document (see appendix I). Two days following attendance at the haematology clinic, the principal researcher telephoned the patient to see if she would participate in the study and the patient was then followed up in the haematology clinic, usually the following week. Informed written consent (see appendix II for a copy of the consent form used) was obtained by the principal researcher. Following recruitment, the information outlined in table 2.2 was recorded / collected.

Table 2.2: Information collected from the women at the initial and subsequent clinic visits

\begin{tabular}{lllll}
\hline Demographic & Past Medical History & Obstetric information & Medication & Laboratory \\
\hline Date of birth and age & Past medical history & $\begin{array}{l}\text { Date of last menstrual } \\
\text { period }\end{array}$ & $\begin{array}{l}\text { Indication for starting } \\
\text { enoxaparin }\end{array}$ & Urea and electrolytes \\
Weight $(\mathrm{kg})$ & $\begin{array}{l}\text { Whether there is any } \\
\text { known thrombophilia }\end{array}$ & Expected due date & $\begin{array}{l}\text { Dose of enoxaparin } \\
(\mathrm{mg})\end{array}$ & Full blood count \\
Height $(\mathrm{cm})$ & $\begin{array}{l}\text { Whether there is a } \\
\text { history of thrombosis }\end{array}$ & Parity & Medication history & Liver function tests \\
BMI & $\begin{array}{l}\text { History of thrombosis } \\
\text { in first-degree } \\
\text { relatives }\end{array}$ & & & INR, aPTT, D-dimers \\
Ethnicity & Smoking and alcohol & & & \\
\hline
\end{tabular}

Creatinine clearance was calculated using the Cockcroft-Gault method of calculation [CockcroftGault, 1976], and for the purposes of this study, the weight inserted into this equation was the subject's baseline lean body weight, calculated on a mechanistically dervived formula [Han et al., 2007] 


\subsubsection{Sample collection}

Anti-Xa levels were drawn in clinic. The anti-Xa sampling strategy (informed by previous optimal design work), was based on the following scheme:

\begin{tabular}{ll}
\hline $\begin{array}{l}\text { On arrival at } \\
\text { haematology clinic }\end{array}$ & $\begin{array}{l}24 \text { hours post previous dose (i.e. trough sample). Following collection of this } \\
\text { sample, patient injects their enoxaparin in clinic }\end{array}$ \\
\hline $\mathbf{1}$ hour after injection & Second sample for anti-Xa level drawn \\
\hline
\end{tabular}

3 hours after injection Third sample for anti-Xa level drawn

Not all women recruited to the study consented to having three anti-Xa activities measured at each clinic visit, either because of time or not wishing to have additional venepunctures. In such cases, two (trough and one hour post injection) or a single (typically peak) anti-Xa activities were drawn at each clinic visit.

As well as being taught how to self-inject enoxaparin competently, women were also given an enoxaparin administration book (How to inject Clexane $\Theta$, Sanofi-Aventis Ltd) and asked to record the time each day they injected the enoxaparin at home. They were instructed to bring the completed book with them to their subsequent haematology clinic visits, where the book was replaced.

At subsequent clinic visits (monthly), the patients were reviewed by the haematology doctors and the researcher collected and recorded the following information:

- weight (kg), using electronic scales within the Haematology outpatients, range $2 \mathrm{~kg}-250 \mathrm{~kg}$, Model: MPP-250, Marsden The Weighing Company

- height $(\mathrm{cm})$, using electronic scales within the Haematology outpatients, range $50-230 \mathrm{~cm}$, Model: MPP-250, Marsden The Weighing Company

- $\quad$ urea and electrolytes

- full blood count

- liver function test

- $\quad$ INR, APTT, D-dimers 
- changes in any other medications or doses of mecdications the patient is taking

- anti-Xa level (trough, 1 hour and 3 hour sample)

During the first, second and third trimester, within a week of delivery and eight weeks postpartum, each study patient had additional blood samples taken, for the purposes of assessing thrombin generation. These samples were taken at the same time as the anti-Xa levels.

Blood samples were obtained through antecubital venepuncture using a butterfly Terumo $\AA$ Surflo $\AA$ winged infusion set $(21 \mathrm{G} \times 3 / 4$ ", $0.8 \times 19 \mathrm{~mm}$ UTW tubing, REF: SV-21BL, Terumo Europe N.V., Belgium). Thrombin generation samples were drawn through antecubital venepuncture using free flow or minimal suction into a $10 \mathrm{~mL}$ syringe, avoiding vacuum containers, with the first $5 \mathrm{~mL}$ of blood drawn being discarded. The blood was then dispensed into $0.109 \mathrm{~mol} / \mathrm{L}$ sodium citrate vacuum container and handled as described in section 2.1.2.4.1.

\subsubsection{Sample handling and analysis}

Four samples were collected as part of routine clinical care at each clinic visit; one clotted sample, one EDTA sample, and two citrate samples. These were labelled with the patient's name and analysed as follows:

\subsection{Clotted sample}

For determination of urea and electrolytes and liver function. $5 \mathrm{~mL}$ blood sample collected in $\mathrm{SST}^{\mathrm{TM}}{ }_{\mathrm{Il}}$ Advance Becton-Dickinson Vacutainer ${ }^{\circledR}$. This sample was then centrifuged on a Rotanta 46 CSC centrifuge (Hettich Zentrifugen) at $3500 \mathrm{~g}$ for 10 minutes. The sample was then loaded on Siemens Sample Management System and analysed using the Advia 2400 analyser. The results were reported on the hospital's reporting system, under the patient's name.

\subsection{EDTA sample}

For determination of full blood count. $3 \mathrm{~mL}$ sample collected in K3E 5.4mg Becton-Dickinson Vacutainer®. The sample was loaded onto the Siemens Sample Management System and analysed using the Advia 2120 analyser. The results were reported on the hospital's reporting system, under the patient's name.

\subsection{Citrate sample I}

For determination of INR, APTR and D-dimer. $2.7 \mathrm{~mL}$ blood (9 vol) sample collected in $0.109 \mathrm{M}$ (3.2\% trisodium citrate) Becton-Dickinson Vacutainer®. Following collection, the sample was 
centrifuged at $2500 \mathrm{~g}$ for 7 minutes on a Rotina $38 \mathrm{R}$ centrifuge (Hettich Zentrifugen). The prothrombin time and thus the resultant INR was determined using the STA-Neoplastine ${ }^{\circledR} \mathrm{CI}$ Plus kit (Diagnostica Stago, France). The APTR was determined using the STA-Cephascreen® kit (Diagnostica Stago, France). For determination of d-dimers, the STA-Liatest® D-DI kit (immuneturbidimetric assay of D-dimer (Diagnostica Stago, France)) was used.

Analysis was conducted on the STA-R evolution (Diagnostica Stago) analyser.

\subsection{Citrate sample II}

For determination of anti-Xa activity, $2.7 \mathrm{~mL}$ blood (9 vol) sample was collected in $0.109 \mathrm{M}(3.2 \%$ trisodium citrate) Becton-Dickinson Vacutainer®. Following collection the sample was centrifuged in a Rotina $420 \mathrm{R}$ centrifuge (Hettich Zentrifugen) and double spun for 7 minutes at 2,500g and frozen within 1 hour of sample collection. The samples were stored at $-40^{\circ} \mathrm{C}$ until analysed. Samples were thawed and analysed in weekly batches using the STA-Rotachrom ${ }^{\circledR}$ Heparin colorimetric assay (Diagnostica Stago, France) on the STA-R evolution analyser (Diagnostica Stago). Results were reported as $\mathrm{IU} / \mathrm{mL}$.

The principal of the anti-Xa assay is based on measuring the absorbance of residual factor Xa in the test sample. Initially a standard curve is constructed by the analysing laboratory, by adding known concentrations of LMWH to plasma (which provides the antithrombin), in addition to a fixed amount of factor Xa. This addition of LMWH to the reference plasma sample, results in the formation of an inactive anti-thrombin-Xa complex and residual $\mathrm{Xa}$. The residual $\mathrm{Xa}$ activity is what is measured in the assay, and is inversely proportional to the concentration of LMWH and can be quantified through the calibration curve; the higher the LMWH in the test plasma, the lower the absorbance in the test plasma and vice versa. An example of a typical calibration table which informs the calibration curve follows:

\begin{tabular}{lcccc}
\hline $\begin{array}{l}\text { Reference LMWH } \\
\text { concentration }(\mathrm{IU} / \mathrm{mL})\end{array}$ & 0.00 & 0.45 & 0.82 & 1.54 \\
\hline Absorbance at $405 \mathrm{~nm}$ & 1017 & 564 & 368 & 154 \\
\hline
\end{tabular}

\subsubsection{Thrombin generation experiments}

\subsection{Plasma preparation}

Platelet poor plasma (PPP) was obtained by centrifugation at $4750 \mathrm{~g}$ on a Rotina $420 \mathrm{R}$ centrifuge (Hettich Zentrifugen) for 10 minutes at room temperature. The supernatant was decanted into a 
polypropylene tube and centrifugation repeated. The plasma was decanted into a plastic tube, capped and immediately frozen. This procedure was performed within 60 minutes of venepuncture. The samples were stored at $-35^{\circ} \mathrm{C}$ and analysed using calibrated automated thrombinography (CAT).

\subsection{Calibrated automated thrombinography}

Thrombin generation was measured with the thrombinoscope assay (Thrombinoscope BV, Maastricht, The Netherlands). $20 \mu \mathrm{L}$ of reconstituted PPP reagent (Thrombinoscope BV) was added to each test well of an Immulon 96-well microtitre plate (Thermo Labsystems, Franklin, Massachusetts, USA) followed by $80 \mu \mathrm{L}$ PPP (thawed and warmed to $37^{\circ} \mathrm{C}$ immediately prior to testing). Final concentrations of tissue factor and phospholipid were $5 \mathrm{pmol} / \mathrm{L}$ and $4 \mu \mathrm{mol} / \mathrm{L}$, respectively. Calibration wells were run in parallel utilising Thrombin Calibrator (Thrombinoscope BV) with known thrombin activity instead of PPP reagent. All samples were run in triplicate with the calibrator. $20 \mu \mathrm{L}$ of a premixed fluorochrome and calcium reagent (FluCa; Thrombinoscope BV) was then added automatically to each well to trigger coagulation.

Fluorescence was measured with Fluoroskan Ascent (Thermo Electron Corporation Vantaa, Finalnd) over time. The Thrombinoscope BV (version 3.0.0.29) software was used to convert the fluorescence signal into $\mathrm{nmol} / \mathrm{L}$ thrombin generated over time. Five parameters were recorded: lag time, time to peak, peak thrombin, start-tail and endogenous thrombin potential (ETP). Maximum velocity, as a measure of rate of thrombin formation, was calculated by dividing peak thrombin by the difference between the time to peak and lag time, with results expressed as $\mathrm{nmol} / \mathrm{L}$ per minute.

Details on how the polybrene experiments which were run in parallel, are described in the relevant section within chapter 6 .

In order to determine the intra- and inter- assay variability from the thrombin generation experiments conducted, plasma from the same healthly control (male) was run in each experiment for the duration of the study.

\subsubsection{Birth and post-partum}

Recruited women were asked to phone or text the principal researcher at the time they went into labour, in order for samples to be collected during the post-partum period. When the principal 
researcher reviewed the patient on the post-natal ward, the plan i.e. dose and duration of enoxaparin therapy would be re-confirmed with the patient and obstetric team, following consultation with the haematology consultants.

Patients were then followed up in the haematology outpatient clinic, typically when enoxaparin therapy had been discontinued, at 8 weeks post-partum. At this clinic visit, as well as the patient receiving a final medical review, a blood sample was collected, where apart from anti-Xa activity, all other bloods (UE's / LFTs / FBC / Coagulation screen / D-dimers and thrombin generation) were drawn.

\subsubsection{Control group}

In order to assess whether enoxaparin had a significant effect on blood loss and mode of delivery of delivery for women injecting enoxaparin antenatally, information on women delivering at King's college hospital, not injecting enoxaparin antenatally was collected from 10 women every week (on a Friday) from the post-natal ward from Friday $2^{\text {nd }}$ September 2011 to Friday $2^{\text {nd }}$ March 2012. The key comparisons to be made were, age of women, ethnicity, baby's gender, gestation delivered, weight of baby, method of delivery, estimated blood loss at delivery, and APGAR scores of baby at delivery.

\subsubsection{Data entry, storage and transfer}

All data was recorded by the principal investigator on a pre-designed study form. At the earliest opportunity, the principal investigator transferred the data to an Excel database, with the hard copy of the data collection form being filed and stored in a locked office within the haematology department at King's College Hospital. One week after entry of a patient's data onto the Excel database, the data which had been entered was re-checked for any errors.

Each consented patient was assigned a unique study number comprising of the prefix Preg, followed by the year the patient consented to participate in the study, followed by the sequential number of the patient in the study. For example the first patient recruited to the study had the following study number assigned to them; Preg200901.

All electronic data collected were password protected and conformed to National Health Service confidentiality standards. At the end of data collection, prior to completing the final analysis, $5 \%$ of inputted data was re-checked for any errors. 


\subsubsection{Population Modelling and Simulation}

Analysis was performed using non linear mixed effects modelling. An overview of the principals of this method are described in chapter 1 , with further details described in the enoxaparin modelling and simulation results chapter 4 .

\subsubsection{Ethical approval}

The study received ethical approval from the Isle of White, Portsmouth and South East Hampshire Research Ethics Committee (REC reference: 09/H0501/57) in September 2009 (copy of approval letter in appendix III), and a copy of the King's College Hospital Research and Development office approval letter for conducting this study is in appendix IV. 


\section{Chapter 3}

\subsection{Clinical Information on study patients}

Chapter 3 describes from a clinical perspective, background and pregnancy outcome information on the women who consented and participated in the enoxaparin study.

\subsubsection{Eligibility and recruitment}

King's College Hospital has an extensive women's health service. Specifically, within the obstetric department, approximately 5,400 babies are delivered each year, including 1,500 caesarean sections. Between $7^{\text {th }}$ September 2009 and $31^{\text {st }}$ December 2011, all pregnant women referred to the thrombophilia haematology clinic at King's College Hospital, prescribed enoxaparin or due to commence enoxaparin were eligible to participate in this study. Over this time period, 156 women were referred to this clinic. Twenty-nine of these women were not recruited into the study for the following reasons; (1) one patient had a previous allergy to enoxaparin and was therefore being managed on tinzaparin during the index pregnancy, (2) eight women miscarried prior to informed consent being obtained, (3) six women declined to participate, (4) three women were not approached to take part in the study, (4) seven women prescribed enoxaparin, attended clinic erratically and so were not approached, as any information that would be collected from these women was considered too unreliable, (5) four women were moving out-of-area and so were referred to their new local provider for ongoing obstetric - haematology care.

\subsubsection{Consented patients}

One hundred and twenty-five patients agreed to participate in the study, following informed consent. Six of these women were excluded from the final analysis for the following reasons:

- 1 patient who suffered a left proximal deep vein thrombosis during the second trimester of her pregnancy, was commenced on enoxaparin treatment dose according to the RCOG guidelines. However she developed a troublesome rash at her enoxaparin injection sites and her back, soon after the commencement of enoxaparin. She was switched to dalteparin, which appeared to help for a short time, but her symptoms re-appeared a few weeks later. On switching to tinzaparin therapy for the remainder of her pregnancy, her symptoms resolved. 
- 1 patient miscarried prior to her next clinic appointment, so no samples were drawn from her.

- 1 patient consented into the study, late in the third trimester. The principal researcher found her difficult to bleed and so she was excluded.

- In the remaining three women, clinicians suspected non-adherence to the enoxaparin, and so these women were excluded.

Excluding the patient who miscarried, the remaining 5 women went on to have live births; two had spontaneous vaginal deliveries, two had emergency caesarean-sections and the remaining woman delivered by elective caesarean section.

\subsubsection{Study population}

One hundred and nineteen patients had 123 pregnancies (4 women had two pregnancies during the study, and will be counted as separate women for the remainder of the thesis). Five (4\%) of the 123 pregnancies were twin pregnancies. The mean age for the study population was 33.11 (range 18-46) years. Table 3.1 presents key demographic information for these women in study. 
Table 3.1: Demographic information on the recruited women

\begin{tabular}{|c|c|c|}
\hline Demographic & $\begin{array}{c}\text { Number } \\
n=123\end{array}$ & $(\%)$ \\
\hline \multicolumn{3}{|l|}{ Ethnicity } \\
\hline Caucasian & 66 & $(53.65)$ \\
\hline Black & 37 & $(30.09)$ \\
\hline Asian & 9 & $(7.32)$ \\
\hline Other & 11 & $(8.94)$ \\
\hline \multicolumn{3}{|l|}{ Obstetric history (as a cohort) } \\
\hline Gravida & 448 & \\
\hline Parity & 136 & \\
\hline Miscarriages & 170 & \\
\hline Terminations & 22 & \\
\hline Primi gravid & 26 & $(21.13)$ \\
\hline \multicolumn{3}{|l|}{ Body mass index $\left(\mathrm{kg} / \mathrm{m}^{2}\right)$} \\
\hline Underweight $\quad(<18.5)$ & 2 & $(1.62)$ \\
\hline Normal $\quad(18.5-24.9)$ & 62 & $(50.40)$ \\
\hline Overweight $\quad(25-29.9)$ & 32 & $(26.01)$ \\
\hline Obese Class I $\quad(30-34.9)$ & 7 & $(5.69)$ \\
\hline Obese Class II (35-39.9) & 4 & (3.25) \\
\hline Obese Class III ( $\geq 40)$ & 16 & $(13.01)$ \\
\hline
\end{tabular}

Fifty-seven women (46\%) had a previous history of thrombosis. Thirty-three women (29\%) had a history of miscarriages, with nine of these women having laboratory confirmation of antiphospholipid syndrome. The results in table 3.1 demonstrate that the cohort of women recruited to the study represented a diverse population, particularly with respect to ethnicity and BMI, with a significant number of obese class I-III women in the study (27 women (21.95\%)).

\subsubsection{Patient outcomes}

Eight of the 123 pregnancies miscarried; five women (4.06\%) had first trimester miscarriages and 2 women $(1.62 \%)$ had second trimester miscarriages. The remaining patient had a still birth at 39 weeks gestation. Of the five women who had first trimester miscarriages, four women had a history of recurrent miscarriages in the absence of antiphospholipid antibodies. The two women suffering from a second trimester miscarriages, one had confirmed antiphospholipid syndrome. For the subject who had a still birth at 39 weeks gestation, laboratory, placental histology and 
post-mortem findings from the neonate could not identify a cause for the still birth. In this case, the mother's indication for the enoxaparin prescription was VTE prevention, due to obesity, parity, age and hypertension.

\subsubsection{Indication for enoxaparin and doses prescribed}

Table 3.2 lists information on the women's indication for antenatal enoxaparin therapy, the range of enoxaparin doses prescribed, along with the number of anti-Xa samples each subject agreed to contribute for PK modelling purposes. Ninety women were prescribed enoxaparin for prophylaxis of VTE; of these, forty-three (47.7\%) had no personal history of VTE, but had the presence of significant VTE risk factors warranting ante- and postnatal prophylaxis, according to the current RCOG prophylaxis guidelines. The twelve women who had 'other' indications for antenatal enoxaparin were prescribed enoxaparin for the following indications:

- Seven subjects who had a history of recurrent miscarriages in the absence of antiphospholipid syndrome. The weak evidence for using enoxaparin in this setting was fully discussed with the patient and the decision to proceed was only taken, if the patient wanted to

- $\quad$ subject 22 had a history of retinal artery occlusion during the post-partum period in a previous pregnancy

- $\quad$ subject 27 had a suspected patent patent foramen ovale and her cardiac team requested her to be on treatment dose enoxaparin during her pregnancy, as a pre-cautionary measure

- $\quad$ subject 47 had a stillbirth during a previous pregnancy. Histology findings from the placenta reported perivillous fibrin deposition, placental ageing and dysmaturity. She was prescribed enoxaparin, as a pre-cautionary measure during the index pregnancy

- subject 72 had a history of intrauterine growth retardation (IUGR) and abruption during her last pregnancy. In the index pregnancy, she had a high cardiolipin antibody titre and pregnancy-induced hypertension

- $\quad$ subject 98 had a history of intrauterine growth retardation (IUGR) and pregnancy induced hypertension during her last pregnancy. After discussion with the 
subject, she decided she wanted to inject enoxaparin, as a pre-cautionary measure

Table 3.2: Indication for enoxaparin, dose prescribed and number of anti-Xa samples contributed at each clinic visit

\begin{tabular}{|c|c|c|}
\hline Study patient's prescribing information & $\begin{array}{l}\text { Numbers } \\
n=123\end{array}$ & $(\%)$ \\
\hline \multicolumn{3}{|l|}{ Indication for enoxaparin } \\
\hline Prophylaxis of VTE & 83 & $(67.48)$ \\
\hline Treatment of VTE & 9 & $(7.32)$ \\
\hline Antiphospholipid syndrome & 9 & $(7.32)$ \\
\hline Patients converting from long term warfarin & 10 & $(8.13)$ \\
\hline Other & 12 & $(9.76)$ \\
\hline \multicolumn{3}{|l|}{ Dose of enoxaparin prescribed } \\
\hline 20mg daily & 2 & $(1.62)$ \\
\hline 40mg daily & 81 & $(65.85)$ \\
\hline $60 \mathrm{mg}$ daily & 2 & $(1.62)$ \\
\hline $80 \mathrm{mg}$ daily & 5 & $(4.06)$ \\
\hline 100mg daily & 8 & $(6.50)$ \\
\hline $120 \mathrm{mg}$ daily & 3 & $(2.44)$ \\
\hline 150mg daily & 1 & $(0.81)$ \\
\hline $180 \mathrm{mg}$ daily & 1 & $(0.81)$ \\
\hline 40mg twice daily & 8 & $(6.50)$ \\
\hline 50mg twice daily & 1 & $(0.81)$ \\
\hline $60 \mathrm{mg}$ twice daily & 8 & $(6.50)$ \\
\hline $80 \mathrm{mg}$ twice daily & 2 & $(1.62)$ \\
\hline 100mg twice daily & 1 & $(0.81)$ \\
\hline \multicolumn{3}{|c|}{ Number of anti-Xa samples patients agreed to contribute per clinic visit } \\
\hline One anti-Xa activity drawn per clinic visit & 106 & $(86.17)$ \\
\hline Two anti-Xa activities drawn per clinic visit & 6 & $(4.88)$ \\
\hline Three anti-Xa activities drawn per clinic visit & 11 & $(8.94)$ \\
\hline
\end{tabular}

As a cohort, the recruited subjects contributed 795 anti-Xa activities during the ante- and post natal period for PK modelling purposes. Specifically, 712 were drawn during the antenatal period, with each subject contributing an average of 5.74 anti-Xa antenatal samples (range $1-24)$. 
Twenty women (16.26\%) were prescribed treatment doses of enoxaparin during the course of their pregnancies. Clinical reasons for this follow. The remaining 103 women were prescribed what would be considered as prophylactic doses of enoxaparin.

\subsubsection{Women suffering from pathological thrombosis during the index pregnancy}

Nine women suffered pathological thrombosis during the index pregnancy and were prescribed treatment doses of enoxaparin and referred to the haematology clinic for ongoing management. Table 3.3 lists further details on these nine episodes.

Table 3.3: Details on the 9 women who suffered from pathological thrombosis during the index pregnancy

\begin{tabular}{|c|c|c|c|}
\hline Subject & Indication & $\begin{array}{l}\text { Gestation at time } \\
\text { of presentation }\end{array}$ & Enoxaparin dose prescribed \\
\hline 17 & DVT (left proximal) & 31 weeks & $\begin{array}{l}100 \mathrm{mg} \text { daily antenatally and warfarin } \\
\text { post-partum }\end{array}$ \\
\hline 36 & DVT (left proximal) & 23 weeks & $\begin{array}{l}80 \mathrm{mg} \text { twice a day ante- and } \\
\text { postnatally }\end{array}$ \\
\hline 96 & *DVT (right long saphenous vein) & 7 weeks & $\begin{array}{l}80 \mathrm{mg} \text { daily for six weeks, followed by } \\
40 \mathrm{mg} \text { daily ante- and postnatally }\end{array}$ \\
\hline 117 & DVT (left common iliac vein) & 25 weeks & $80 \mathrm{mg}$ daily ante- and postnatally \\
\hline 18 & $\begin{array}{l}\text { PE (sub-segmental right lower } \\
\text { lobe) }\end{array}$ & 23 weeks & $\begin{array}{l}60 \mathrm{mg} \text { twice a day ante- and } \\
\text { postnatally }\end{array}$ \\
\hline 44 & PE (extensive bilateral) & 8 weeks & $\begin{array}{l}\text { 100mg twice a day ante- and } \\
\text { postnatally }\end{array}$ \\
\hline 49 & PE (VQ probability high) & 23 weeks & $\begin{array}{l}80 \mathrm{mg} \text { twice a day ante- and } \\
\text { postnatally }\end{array}$ \\
\hline 67 & PE (bilateral sub-segmental) & 22 weeks & $150 \mathrm{mg}$ daily ante- and postnatally \\
\hline 79 & $\begin{array}{l}\text { Axillary vein thrombosis plus right } \\
\text { proximal subclavian vein }\end{array}$ & 8 weeks & $\begin{array}{l}50 \mathrm{mg} \text { twice a day antenatally and } \\
80 \mathrm{mg} \text { once daily postnatally }\end{array}$ \\
\hline
\end{tabular}

${ }^{*}$ Not a DVT, but patient was prescribed 6 weeks treatment dose enoxaparin as she had venous malformation. Following a 6 week course of enoxaparin, subject 96 was prescribed prophylactic dose enoxaparin for the remainder of her pregnancy and for 6 weeks post-partum.

\subsubsection{Long term warfarin patients switched to enoxaparin during the index pregnancy}

Table 3.4 lists information on the women who were switched to treatment dose enoxaparin, from being on chronic warfarin therapy prior to their index pregnancy. 
Table 3.4: Details on the women switched from long-term warfarin to treatment dose enoxaparin during the index pregnancy

\begin{tabular}{llcc}
\hline Patient & Indication & $\begin{array}{c}\text { Target INR } \\
\text { range }\end{array}$ & $\begin{array}{c}\text { Enoxaparin dose } \\
\text { prescribed }\end{array}$ \\
\hline 16 & Recurrent VTE & $1.5-2.0$ & $120 \mathrm{mg}$ daily \\
37 & $\begin{array}{l}\text { FVL (homozygous). Symptomatic VTE whilst on } \\
\text { combined oral contraceptive pill }\end{array}$ & $2-3$ & $100 \mathrm{mg}$ daily \\
& Budd-Chiari (Liver transplant in 1998) & $2-3$ & $100 \mathrm{mg}$ daily \\
$45^{*}$ & Paroxysmal nocturnal haemoglobinuria & $2-3$ & $80 \mathrm{mg}$ daily \\
48 & Budd-Chiari (Liver transplant in 2006) & $2-3$ & $100 \mathrm{mg}$ daily \\
53 & Anti-phospholipid syndrome & $2-3$ & $100 \mathrm{mg}$ daily \\
54 & AF/Mitral stenosis/Pulmonary hypertension & $2-3$ & $100 \mathrm{mg}$ daily \\
82 & Recurrent VTE & $2-3$ & $180 \mathrm{mg}$ daily \\
87 & Budd-Chiari (Liver transplant in 1998) & $2-3$ & $100 \mathrm{mg}$ daily \\
$101^{*}$ & Anti-phospholipid syndrome and Budd-Chiari & $3-4$ & $60 \mathrm{mg}$ daily \\
115 & (Liver transplant in 2005) & & \\
\hline
\end{tabular}

One further patient (subject 27), was commenced on treatment dose enoxaparin, following referral from the cardiology team with possible patent foramen ovale. She was managed from mid way through the second trimester through to 6 weeks post partum with enoxaparin $120 \mathrm{mg}$ daily.

\subsubsection{Changes in renal and liver function tests and the full blood count during pregnancy}

At each haematology clinic visit, at birth and at approximately 8 weeks post partum, patients had blood drawn for evaluation of full blood count, urea and electrolytes and liver function tests. All test results for the cohort of women are listed in table 3.5 (mean, (max, min), median values are listed). Alterations in key parameters over the course of pregnancy and the puerperium, i.e. haemoglobin, platelet count, creatinine, albumin, mean platelet volume and urea are additionally illustrated in figure 3.1. 
Table 3.5: Test results according to trimester for the cohort of women followed

\begin{tabular}{|c|c|c|c|c|c|}
\hline Parameter & First Trimester & $\begin{array}{l}\text { Second } \\
\text { Trimester }\end{array}$ & Third Trimester & $\begin{array}{l}\text { Within } 1 \text { week } \\
\text { of delivery }\end{array}$ & $\begin{array}{l}\geq 8 \text { weeks } \\
\text { post-partum }\end{array}$ \\
\hline \multirow{2}{*}{$\begin{array}{l}\text { Serum } \\
\text { creatinine } \\
{[\mu \mathrm{mol} / \mathrm{L}]}\end{array}$} & $53(16-92)$ & $47(20-86)$ & $47(19-80)$ & $54(28-97)$ & $63(27-91)$ \\
\hline & 52 & 46 & 47 & 53 & 63 \\
\hline \multirow{2}{*}{$\begin{array}{l}\text { Creatinine } \\
\text { clearance } \\
\text { [mL/min] }\end{array}$} & $96(37-217)$ & $109(57-212)$ & $109(37-212)$ & $98(35-185)$ & $81(39-160)$ \\
\hline & 89 & 106 & 107 & 92 & 75 \\
\hline \multirow{2}{*}{$\begin{array}{l}\text { Haemoglobin } \\
{[\mathrm{g} / \mathrm{dL}]}\end{array}$} & $12.6(7.8-14.8)$ & $11.8(8.1-14.4)$ & $11.3(8.2-14.7)$ & $10.8(6.2-10.8)$ & $12.6(9.7-17.4)$ \\
\hline & 12.6 & 11.9 & 11.3 & 10.6 & 12.6 \\
\hline \multirow{2}{*}{$\begin{array}{l}\text { Platelets } \\
{\left[\times 10^{9} / \mathrm{L}\right]}\end{array}$} & $277(30-469)$ & $266(34-494)$ & $265(35-936)$ & $263(39-854)$ & $289(47-564)$ \\
\hline & 269 & 264 & 252 & 257 & 287 \\
\hline \multirow[t]{2}{*}{ INR } & $1.0 \quad(0.86-3.09)$ & $0.98(0.83-1.75)$ & $0.97(0.78-1.48)$ & $0.99(0.78-2.18)$ & $1.02(0.82-1.9)$ \\
\hline & 1.0 & 0.98 & 0.98 & 0.98 & 1.0 \\
\hline \multirow[t]{2}{*}{ APTT Ratio } & $1.04(0.72-2.4)$ & $1.03(0.72-2.33)$ & $1.01(0.79-2.14)$ & $1.06(0.82-1.53)$ & $1.02(0.82-1.9)$ \\
\hline & 1.0 & 1.0 & 1.0 & 1.04 & 1.0 \\
\hline \multirow{2}{*}{$\begin{array}{l}\text { Sodium } \\
{[\mathrm{mmol} / \mathrm{L}]}\end{array}$} & $136(133-142)$ & $136(132-141)$ & $136(132-141)$ & $137(127-143)$ & $140(136-145)$ \\
\hline & 136 & 136 & 136 & 138 & 140 \\
\hline \multirow{2}{*}{$\begin{array}{l}\text { Potassium } \\
\text { [mmol/L] }\end{array}$} & $4.2(3.6-5.6)$ & $4.2(3.1-5.0)$ & $4.2(3.2-5.1)$ & $4.1(3.0-5.2)$ & $4.3(3.5-5.1)$ \\
\hline & 4.2 & 4.2 & 4.2 & 4.1 & 4.2 \\
\hline \multirow[t]{2}{*}{ Urea[mmol/L] } & $3.7(1.1-6.8)$ & $3.2(1.1-5.5)$ & $3.0(1.0-6.7)$ & $3.5(1.5-6.9)$ & $4.9(2.1-8.4)$ \\
\hline & 3.5 & 3.2 & 3.0 & 3.3 & 5.0 \\
\hline \multirow{2}{*}{$\begin{array}{l}\text { Calcium } \\
{[\mathrm{mmol} / \mathrm{L}]}\end{array}$} & $2.28(2.04-2.55)$ & $2.25(1.93-2.4)$ & $2.52(1.93-2.28)$ & $2.18(1.89-2.42)$ & $2.29(2.13-2.52)$ \\
\hline & 2.28 & 2.24 & 2.22 & 2.20 & 2.29 \\
\hline \multirow{2}{*}{$\begin{array}{l}\text { Corrected } \\
\text { Calcium } \\
\text { [mmol/L] }\end{array}$} & $2.23(2.07-2.47)$ & $2.256(2.07-2.47)$ & $2.27(2.05-2.56)$ & $2.31(2.07-2.51)$ & $2.26(2.08-2.38)$ \\
\hline & 2.23 & 2.25 & 2.27 & 2.30 & 2.20 \\
\hline \multirow[t]{2}{*}{ Globulin [g/L] } & $28(19-39)$ & $27(19-36)$ & $27(19-36)$ & $25(17-35)$ & $29(18-39)$ \\
\hline & 28 & 27 & 27 & 24 & 29 \\
\hline \multirow{2}{*}{$\begin{array}{l}\text { Phosphate } \\
\text { [mmol/L] }\end{array}$} & $1.22(0.39-2.80)$ & $1.21(0.68-2.80)$ & $1.14(0.58-1.98)$ & $1.23(0.63-1.97)$ & $1.33(0.62-1.82)$ \\
\hline & 1.25 & 1.21 & 1.14 & 1.25 & 1.34 \\
\hline \multirow[t]{2}{*}{ Protein $[\mathrm{g} / \mathrm{L}]$} & $70(43-85)$ & $67(43-84)$ & $64(41-79)$ & 59 (39-78) & $73(50-85)$ \\
\hline & 69 & 66 & 65 & 58 & 73 \\
\hline \multirow[t]{2}{*}{ Albumin [g/L] } & $42(26-50)$ & $39(25-67)$ & 37 (24-67) & $34(21-47)$ & $44(32-50)$ \\
\hline & 42 & 39 & 37 & 34 & 44 \\
\hline \multirow{2}{*}{$\begin{array}{l}\text { Alkaline } \\
\text { Phosphatase } \\
\text { [IU/L] }\end{array}$} & $58(14-586)$ & $65(14-661)$ & $111(12-983)$ & $154(52-983)$ & $97(43-216)$ \\
\hline & 47 & 53 & 94 & 129 & 86 \\
\hline \multirow{2}{*}{$\begin{array}{l}\text { Gamma- } \\
\text { glutanyl } \\
\text { transferase } \\
\text { [IU/L] }\end{array}$} & $30(3-724)$ & $21(2-285)$ & $15(1-222)$ & $20(2-267)$ & $31(1-193)$ \\
\hline & 14 & 11 & 9 & 11 & 13 \\
\hline
\end{tabular}




\begin{tabular}{|c|c|c|c|c|c|}
\hline Parameter & First Trimester & $\begin{array}{l}\text { Second } \\
\text { Trimester }\end{array}$ & Third Trimester & $\begin{array}{l}\text { Within } 1 \text { week } \\
\text { of delivery }\end{array}$ & $\begin{array}{l}\geq 8 \text { weeks } \\
\text { post-partum }\end{array}$ \\
\hline \multirow{2}{*}{$\begin{array}{l}\text { Aspartate } \\
\text { transaminase } \\
\text { [IU/L] }\end{array}$} & $28(8-186)$ & $23(3-258)$ & $20(4-170)$ & $26(4-85)$ & $25(11-114)$ \\
\hline & 20 & 19 & 18 & 25 & 21 \\
\hline \multirow{2}{*}{$\begin{array}{l}\text { Bilirubin } \\
{[\mu \mathrm{mol} / \mathrm{L}]}\end{array}$} & $8(2-27)$ & $7(2-81)$ & $7(3-170)$ & $9(3-267)$ & $9(3-32)$ \\
\hline & 6 & 6 & 6 & 6 & 7 \\
\hline \multirow{2}{*}{$\begin{array}{l}\text { Haematocrit } \\
\text { [L/L] }\end{array}$} & $0.37(0.26-0.46)$ & $0.34(0.25-0.43)$ & $0.33(0.25-0.44)$ & $0.32(0.19-0.43)$ & $0.38(0.32-0.55)$ \\
\hline & 0.37 & 0.35 & 0.33 & 0.32 & 0.38 \\
\hline \multirow{2}{*}{$\begin{array}{l}\text { Mean cell } \\
\text { volume [fL] }\end{array}$} & $87.9(68.9-101.6)$ & $87.7(67.6-114.2)$ & $87.2(67.6-114.1)$ & $85.8(69.9-108.8)$ & $85.5(70.8-101.3)$ \\
\hline & 88.9 & 88.7 & 87.9 & 85.8 & 86.2 \\
\hline \multirow{2}{*}{$\begin{array}{l}\text { Mean cell } \\
\text { height [pg] }\end{array}$} & $29.6(8.3-34.6)$ & $29.5(8.3-35.2)$ & $29.3(21.6-35.9)$ & $28.4(21.6-35.3)$ & $28.1(21.2-33.2)$ \\
\hline & 30.3 & 30.3 & 29.5 & 28.4 & 28.1 \\
\hline \multirow{2}{*}{$\begin{array}{l}\text { Mean cell } \\
\text { haemoglobin } \\
\text { concentration } \\
{[\mathrm{g} / \mathrm{dL}]}\end{array}$} & $33.6(13.1-38.9)$ & $33.8(29.5-38.9)$ & $33.6(30.0-37.2)$ & $33.1(29.4-36.1)$ & $32.8(29.5-35.3)$ \\
\hline & 33.7 & 33.8 & 33.6 & 33.2 & 32.8 \\
\hline \multirow[t]{2}{*}{ RDW [\%] } & $13.6(11.4-22.1)$ & $14.1(12.1-22.5)$ & $14.4(12.2-23.6)$ & $15.1(12.1-20.7)$ & $14.4(11.7-19.8)$ \\
\hline & 13.2 & 13.8 & 14.0 & 14.7 & 14.2 \\
\hline \multirow{2}{*}{$\begin{array}{l}\text { Red cell count } \\
{\left[\times 10^{12} / \mathrm{L}\right]}\end{array}$} & $4.26(2.61-5.43)$ & $4.00(2.76-14.70)$ & $3.91(2.60-13.54)$ & $3.83(2.28-5.27)$ & $4.47(3.43-6.23)$ \\
\hline & 4.23 & 3.97 & 3.84 & 3.73 & 4.48 \\
\hline \multirow{2}{*}{$\begin{array}{l}\text { White cell } \\
\text { count }\left[\times 10^{9} / \mathrm{L}\right]\end{array}$} & $8.18(2.35-8.18)$ & 7.35 (3.04-18.37) & $9.01(2.54-23.07)$ & $12.16(2.78-28.33)$ & $6.47(2.7-12.6)$ \\
\hline & 8.02 & 8.37 & 8.87 & 11.07 & 6.27 \\
\hline \multirow{2}{*}{$\begin{array}{l}\text { Neutrophils } \\
\text { count }\left[\times 10^{9} / \mathrm{L}\right]\end{array}$} & $5.54(1.33-14.48)$ & $6.27(2.17-14.48)$ & $6.56(1.04-20.76)$ & $9.43(0.70-26.12)$ & $3.67(0.85-8.68)$ \\
\hline & 5.36 & 6.03 & 6.41 & 8.46 & 3.4 \\
\hline \multirow{2}{*}{$\begin{array}{l}\text { Lymphocytes } \\
{\left[\times 10^{9} / \mathrm{L}\right]}\end{array}$} & $1.98(0.73-4.00)$ & $1.82(0.49-3.34)$ & $1.76(0.43-3.68)$ & $1.89(0.40-4.43)$ & $2.18(0.66-4.07)$ \\
\hline & 1.85 & 1.74 & 1.68 & 1.79 & 2.11 \\
\hline \multirow{2}{*}{$\begin{array}{l}\text { Monocytes } \\
{\left[\times 10^{9} / \mathrm{L}\right]}\end{array}$} & $0.41(0.15-0.96)$ & $0.41(0.14-0.96)$ & $0.47(0.12-2.65)$ & $0.57(0.13-1.62)$ & $0.33(0.11-0.66)$ \\
\hline & 0.39 & 0.39 & 0.45 & 0.54 & 0.34 \\
\hline \multirow{2}{*}{$\begin{array}{l}\text { Eosinophils } \\
{\left[\times 10^{9} / L\right]}\end{array}$} & $0.16(0.02-0.63)$ & $0.16(0.01-0.69)$ & $0.15(0.00-0.77)$ & $0.13(0.00-0.55)$ & $0.20(0.03-0.96)$ \\
\hline & 0.13 & 0.15 & 0.12 & 0.10 & 0.17 \\
\hline \multirow{2}{*}{$\begin{array}{l}\text { Basophils } \\
{\left[\times 10^{9} / L\right]}\end{array}$} & $0.03(0.00-0.33)$ & $0.03(0.00-0.19)$ & $0.03(0.00-0.26)$ & $0.03(0.00-0.11)$ & $0.03(0.00-0.12)$ \\
\hline & 0.03 & 0.03 & 0.03 & 0.03 & 0.03 \\
\hline \multirow{2}{*}{$\begin{array}{l}\text { Mean platelet } \\
\text { volume [fL] }\end{array}$} & $8.2(5.3-12.6)$ & $8.5(6.3-14.5)$ & $8.8(6.3-19.0)$ & $9.0(6.3-14.1)$ & $8.6(6.7-14.8)$ \\
\hline & 8.1 & 8.4 & 8.7 & 8.7 & 8.4 \\
\hline
\end{tabular}



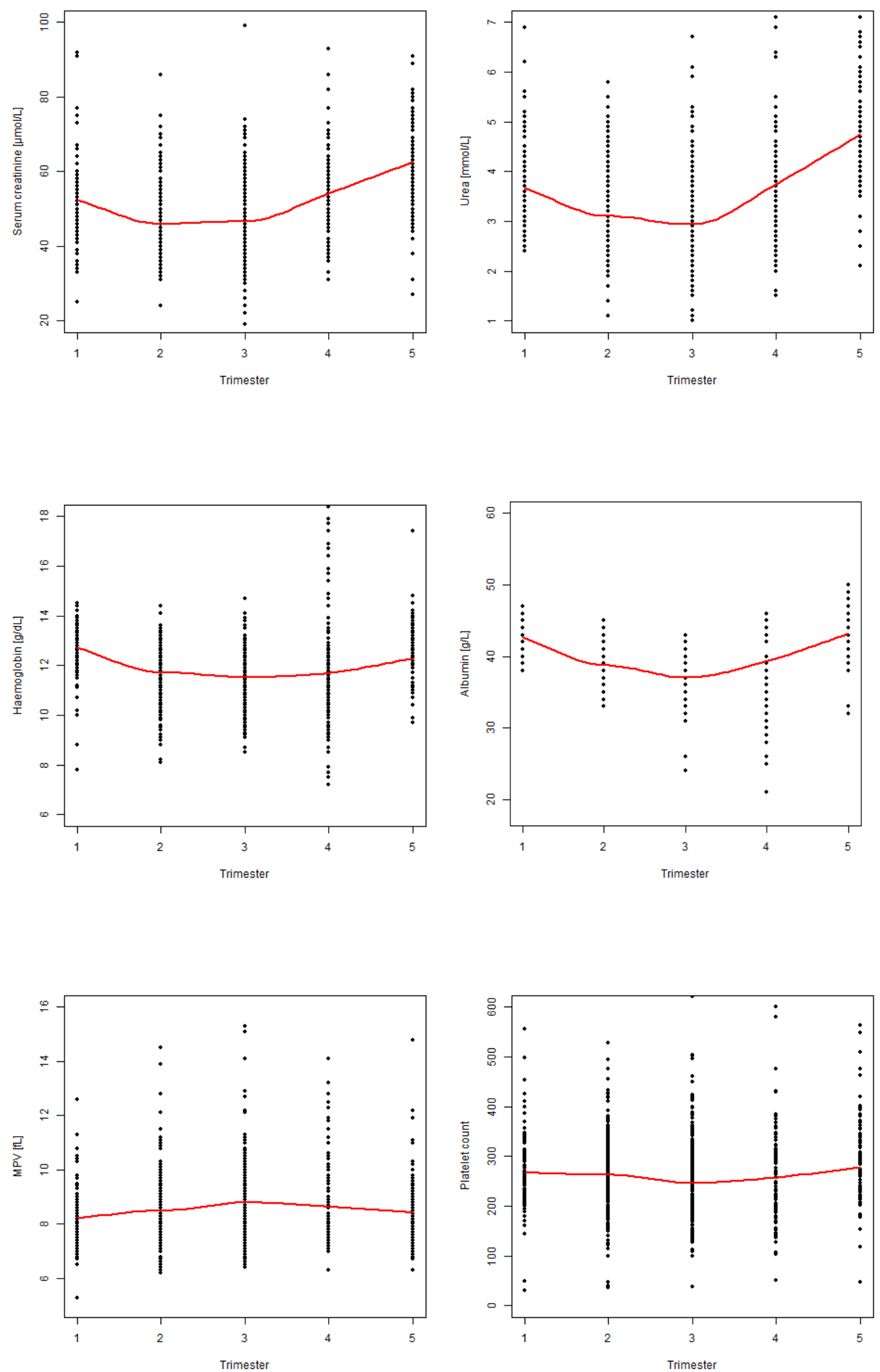

Figure 3.1: Changes in creatinine, urea, haemoglobin, albumin, mean platelet volume (MPV) and platelets over the course of pregnancy. Please note, trimester 4 refers to within a week of delivery and trimester 5 refers to $\geq 8$ weeks post-partum (red lines represents loess smooth) 
Figure 3.2 illustrates the change in calculated $\mathrm{CrCl}$ and actual weight changes in the cohort of women who participated in the study.
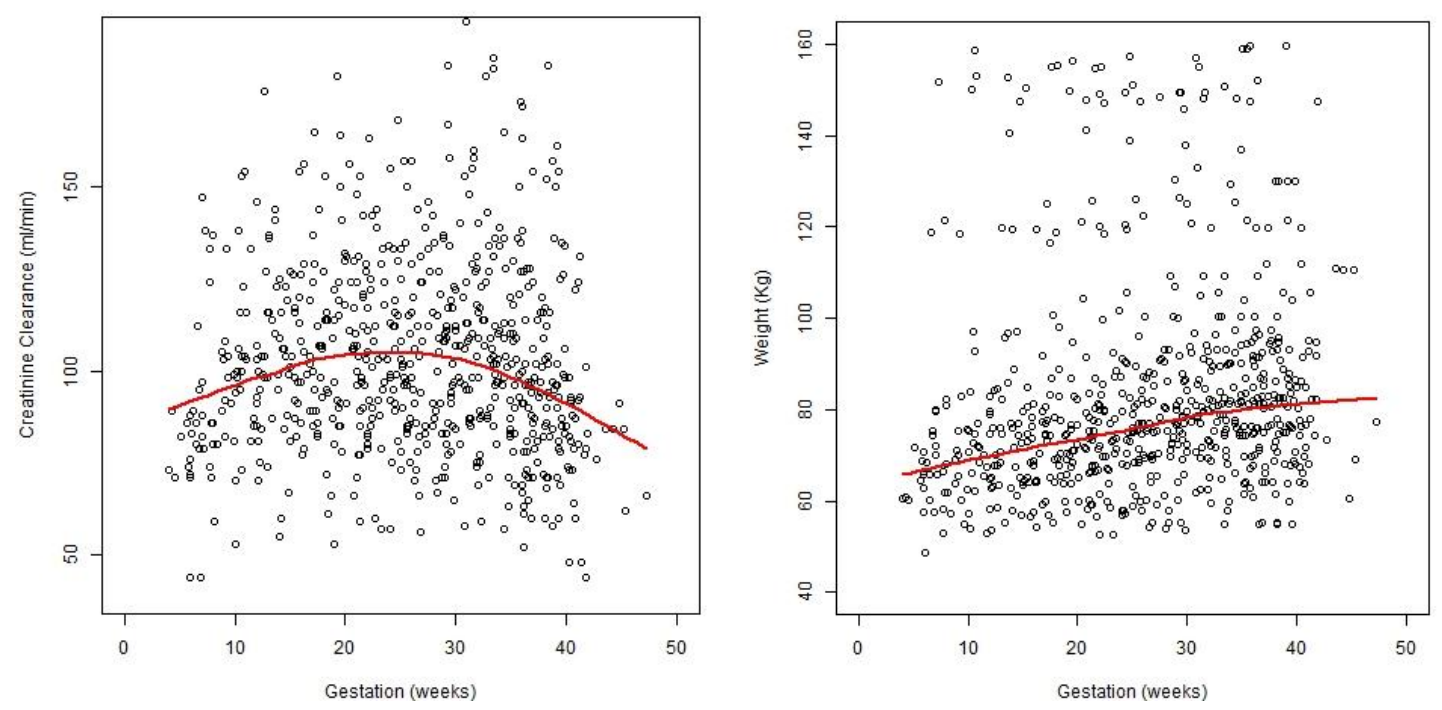

Figure 3.2: Changes in creatinine clearance and weight over the course of pregnancy (red line represents loess smooth)

\subsubsection{Birth and delivery information}

One hundred and fifteen patients had 119 live births. Relevant delivery information is presented in table 3.6. 
Table 3.6: Pregnancy outcome information in the recruited women who had live births

\begin{tabular}{|c|c|c|}
\hline Birth Information & Numbers & $(\%)$ \\
\hline \multicolumn{3}{|l|}{ Gender } \\
\hline Male & 62 & $(52)$ \\
\hline Female & 57 & $(48)$ \\
\hline \multicolumn{3}{|l|}{ Delivery method } \\
\hline Vaginal delivery & 60 & $(52.09$ \\
\hline Elective caesarean section & 23 & $(20.35$ \\
\hline Emergency caesarean section & 20 & $(17.71)$ \\
\hline Ventouse & 6 & $(5.31)$ \\
\hline Forceps & 4 & $(3.54)$ \\
\hline \multicolumn{3}{|l|}{ Blood loss at delivery } \\
\hline $500 \mathrm{~mL}$ or less & 89 & $(78.77$ \\
\hline$>500-1000 \mathrm{~mL}$ & 15 & $(13.27$ \\
\hline$>1000 \mathrm{~mL}$ & 4 & $(3.54)$ \\
\hline Not recorded & 5 & $(4.42)$ \\
\hline \multicolumn{3}{|l|}{ Baby APGAR score (mean(min-max)) } \\
\hline @ 1 minute & $8.4(0-10)$ & - \\
\hline @ 5 minutes & $9.5(0-10)$ & - \\
\hline
\end{tabular}

Four women (3.54\%) suffered postpartum haemorrhage (PPH) greater than $1000 \mathrm{~mL}$. In three of these cases, the reason for blood loss was attributed to problems with delivery of the placenta, as opposed to the antenatal prescription of LMWH. Greer and Nelson-Piercy's meta-analysis of LMWH use during pregnancy reports an incidence of $\mathrm{PPH}(>500 \mathrm{~mL})$ of $0.94 \%(95 \% \mathrm{Cl}, 0.61-1.37)$ [Greer and Nelson-Piercy, 2005]. In a subsequent study assessing whether LMWH is effective during pregnancy and the puerperium in 57 women (82 pregnancies), reported a postpartum haemorrhage rate of greater than $1000 \mathrm{~mL}$ in $9.1 \%(95 \% \mathrm{Cl}, 4.7-16.9)$ [Roeters van Lennep et al., 2011]. More recently another group in the Netherlands published the findings of their study which spanned 10 years (1999-2009) and followed 88 pregnant women on therapeutic anticoagulation [Knol et al., 2012]. In this study, postpartum haemorrhage was defined as $\geq 500 \mathrm{~mL}$ blood loss for vaginal delivery, with severe postpartum haemorrhage defined as $\geq 1000 \mathrm{~mL}$ for vaginal delivery and $\geq 1000 \mathrm{~mL}$ for caesarean section. The authors found the rate of postpartum haemorrhage to be higher in the vaginal delivery group ( $30 \%$ vs $18 \%$, LMWH users vs non-LMWH users), though 
the risk of severe postpartum haemorrhage in the vaginal delivery groups were not found to be different compared to control ( $5.6 \%$ vs $5 \%$, LMWH users vs non-LMWH users). The authors also report that the risk of postpartum haemorrhage after caesarean section was $12 \%$ amongst LMWH users versus $4 \%$ in non-users, with the risk higher if delivery was within 24 hours after the last dose of LMWH. There are likely to be discrepancies in the reported post-partum haemorrhage rate with antenatal LMWH use, as blood loss is estimated at delivery and there will inevitably be differences in what is recorded by different midwives and obstetricians. The results from this thesis suggest that the incidence of severe post-partum haemorrhage is not increased with the use of LMWH. In the remaining post-partum haemorrhage case in this thesis, the patient was prescribed enoxaparin for the prevention of VTE, had stopped her enoxaparin for two days prior to delivering. She eventually delivered by emergency caesarean section and the blood loss recorded was unlikely to be related to enoxaparin.

\subsubsection{Outcomes compared to control group}

Information on 280 pregnant patients who delivered at King's College Hospital, not prescribed antenatal enoxaparin, was collected, in order to assess any differences in the study population compared to a typical pregnant population managed at the hospital. This information is presented in table 3.7 .

The enoxaparin study population was significantly older than the control group. Unsurprisingly, they also had significantly more miscarriages than the control group, given the nature of the intervention being offered in the thrombophilia clinic. Study patients were also more likely to deliver a little earlier than the control group and may be reflective of the "high risk" pregnancy nature of some subjects in the enoxaparin study group. No significant differences were found between blood loss recorded at delivery or between the groups recorded baby weight. 
Table 3.7: Pregnancy outcomes compared to a control group, not prescribed enoxaparin

\begin{tabular}{|c|c|c|c|}
\hline & $\begin{array}{l}\text { Control group } \\
\qquad n=280\end{array}$ & $\begin{array}{l}\text { Study population } \\
\qquad n=123\end{array}$ & Significance \\
\hline $\mathrm{Age} \pm \mathrm{sd}$ & $30.80 \pm 6.02$ & $33.07 \pm 5.48$ & $0.00^{*}$ \\
\hline \multicolumn{4}{|l|}{ Ethnicity $n(\%)$} \\
\hline Caucasian & $134(47.9)$ & $66(53.6)$ & $N S^{\star \star}$ \\
\hline Black & $105(37.5)$ & 37 (30.2) & $N S^{\star *}$ \\
\hline Asian & $19(6.8)$ & $9(7.3)$ & $N S^{\star *}$ \\
\hline Other & $22(7.8)$ & $11(8.9)$ & $N S^{\star *}$ \\
\hline Gravida & 670 & 448 & \\
\hline Parity & 166 & 136 & $N S^{\star \star}$ \\
\hline Miscarriages & 95 & 170 & $0.00^{\star *}$ \\
\hline Terminations & 92 & 22 & $N S^{\star *}$ \\
\hline Gestation delivered (weeks) & 39.5 & 38.8 & $0.0063^{*}$ \\
\hline Blood loss recorded at delivery ( $\mathrm{ml}$ ) & 489.46 & 433.95 & $N S^{*}$ \\
\hline \multicolumn{4}{|l|}{ Delivery Method $n(\%)$} \\
\hline Vaginal & $126(45)$ & $60(53)$ & $0.00^{* *}$ \\
\hline LSCS (elective) & $40 \quad(14)$ & $23(20)$ & $0.046^{* *}$ \\
\hline LSCS (emergency) & $64(23)$ & $20(18)$ & $N S^{* *}$ \\
\hline Ventouse & $37(13)$ & $6(5)$ & $0.00^{\star *}$ \\
\hline Forceps & $13(5)$ & $4 \quad(4)$ & $N S^{* *}$ \\
\hline Twin pregnancies & $7 \quad(3)$ & $5(4)$ & $\mathrm{NS}^{* *}$ \\
\hline \multicolumn{4}{|l|}{ Gender } \\
\hline Boy & $136(47.39)$ & $62(52.10)$ & $N S^{\star *}$ \\
\hline Girl & $151(52.61)$ & $57(47.90)$ & $N S^{\star *}$ \\
\hline Baby weight (g) & 3237.16 & 3162.43 & $N S^{*}$ \\
\hline $\begin{array}{l}\text { APGAR score @ } 1 \text { minute (mean (min- } \\
\text { max), median) }\end{array}$ & $8.49(0-10), 9$ & $8.40(0-10), 9$ & $N S^{\star *}$ \\
\hline $\begin{array}{l}\text { APGAR score @ } 5 \text { minutes (mean } \\
\text { (min-max), median) }\end{array}$ & $9.54(0-10), 10$ & $9.50(0-10), 10$ & $N S^{\star \star}$ \\
\hline
\end{tabular}

*independent $t$-test, ${ }^{\star \star}$ Chi-squared test

Overall, the results from this chapter demonstrate that the population studied in this study represented a diverse pregnant population and that the enoxaparin did not appear to have a negative impact on the women's or the baby's health. 


\section{Chapter 4}

\subsection{Population pharmacokinetic modelling of enoxaparin}

This chapter focuses on the main research question of the thesis; does the antenatal population require a higher total daily dose and more frequent dose of LMWH when being managed for acute VTE. The specific population pharmacokinetic methods followed during this study are described, following which details of how the antenatal enoxaparin model was developed and evaluated are described in detail. Following final model evaluation, simulation work central to the research question, evaluating once versus twice daily regimens of enoxaparin will be presented. Finally the results of the pharmacokinetic modelling work will be discussed in the context of previously published work.

The broad steps of a population pharmacokinetic modelling process were outlined in chapter 1 (figure 1.7). This figure has been updated and presented as figure 4.1. The shaded boxes represent work completed thus far in the thesis, with the remaining boxes, the focus of this chapter.

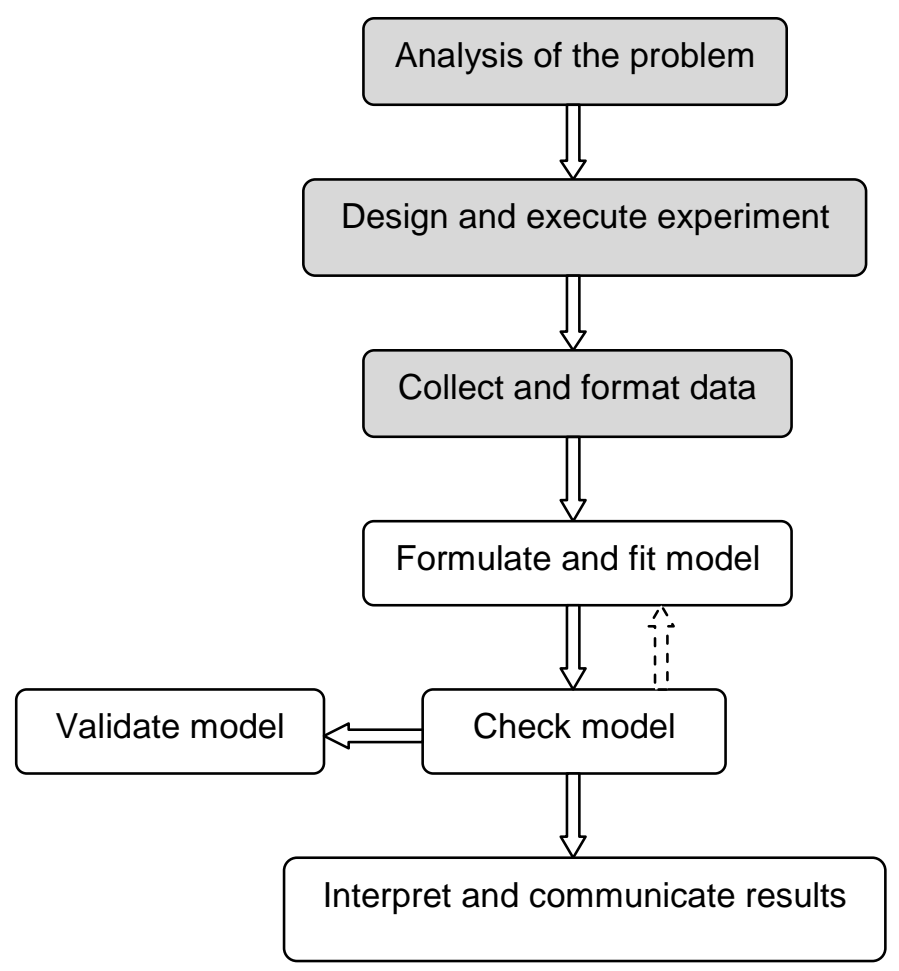

Figure 4.1 Steps in the population PK development process. Shaded boxes represent areas completed thus far in the thesis 


\subsubsection{Methods}

\subsubsection{Data entry and formatting}

Pharmacokinetic analysis was performed using non linear mixed effects modelling, as outlined in chapter 1. Raw data from the study (chapter 2 and chapter 3 ) were initially entered into an excel spreadsheet, usually within two days of subjects attending clinic. One week following initial data entry, the entry records were reviewed for accuracy. Following completion of data collection, the excel spreadsheet was converted to a comma delimited ('.csv') file and formatted ready for use in NONMEM, software version 7.2.2 (ICON development solutions) [Beal, 1998].

\subsubsection{Development of enoxaparin antenatal base model}

The first steps in developing a population model, is to develop a stable base model for the drug being evaluated. A base model provides the foundation for subsequent model development. Several base models were explored, and the chosen model was the one that best fitted the data based upon classical evaluation methods such as assessment of goodness of fit plots, a statistical improvement in the fit of the model to the data using the objective function, assessment of the precision of the parameter estimates and residual variability. The first order conditional method with interaction in NONMEM was used to estimate the base model.

\subsubsection{Evaluation of enoxaparin antenatal base model}

Goodness of fit plots allow a visual examination of how well a model is performing. They include plots of population / individual observed versus population / individual predicted concentrations of the drug being evaluated. A model which is describing the data well, would have population / individual observed versus population / individual predicted concentrations, when plotted, being a mirror imagine of one another, with no systemic trends and the line of unity running through the centre of the points. Goodness of fit plots also include plots of residuals. A residual is the difference between observed and model predicted values [Bonate, 2005]. Positive residuals indicate the model under-predicting the actual observations, whereas negative residuals indicate that the model is over-predicting observations. Residuals are assumed to be normally distributed, with a mean of zero; an unbiased model would have a mean value near zero, with residuals +/within 2, considered good. Once again, graphical examination of systematic trends of residuals provides a valuable insight into how well a particular model is performing. Historically, weighted residuals (WRES) have been the commonly used residual metric in population PK model 
analysis, for evaluating model misspecification. However, the shift in using the first order with conditional estimation (FOCE) in NONMEM has led to a new diagnostic residual tool, the conditional weighted residuals (CWRES) [Hooker et al., 2006]. As part of a residual analysis, histogram analysis of residuals, in this case CWRES, exhibiting a normal distribution, and QQ plot analysis, where there are no departures from normality, provide further evidence that a robust base model has been developed. Finally, as well as evaluating the model estimates for the pharmacokinetic parameters in question and whether the estimates are reasonable (subjective), a non-parametric bootstrap was applied in order to learn about the statistical properties of the distribution of the data and to quantify uncertainty in the parameter estimates [Parke et al., 1999], i.e. median and standard errors.

\subsubsection{Parameterisation of base model with covariates}

Following the establishment of a stable base model, the influence of covariates was then explored. When developing a population pharmacokinetic model, identification of covariates that can explain variability (typically between subject variability) in the pharmacokinetic parameters is one of the primary goals. Appropriately identified covariates can help define optimal dosing schedules and explore if covariate specific dosing regimens are necessary to normalise exposure across a wide population demographic. Parameterisation of the base model with covariates uses both objective and subjective measures to aid in the identification and subsequent retention of covariates in the final model, which include a combination of statistical tests, assessment of biological, mechanistic and clinical relevance of the covariate, along with prior knowledge of the modelled system. A final pharmacokinetic model's predictive performance is not only dependent on the choice of methods used to select covariates, but also incorporation of covariates that underlie biological behaviour. For example, a mechanistic covariate is one which is expected to describe the parameter of interest as a function of known biological phenomena, e.g. renal function on $\mathrm{CL}$ for a drug which is renally cleared. On the other hand, empirical covariates are descriptive explanations of variability that have no clear physiological basis, e.g. eye colour. The utility of descriptive covariates is somewhat limited, compared to mechanistic covariates, particularly when consideration to simulation is given. For the purposes of the development of the antenatal enoxaparin model, mechanistic covariates were considered for parameterisation of the base model. A classical graphical approach was utilised, plotting unexplained variability of CL (ETA1) and $\mathrm{Vd}$ (ETA2) against each of the covariates which were thought to be influencing the 
pharmacokinetics of enoxaparin during pregnancy. Trends identified as part of this graphical analysis, confirmed the relevance of the covariate being tested and the need for their further investigation. Each identified covariate was then tested using a uni-variate stepwise addition into the base-model, with a decrease in objective function of at least $6.64 p<0.01$ required to consider inclusion of the covariate in the intermediate model. Once all relevant covariates had been identified and evaluated, they were all simultaneously added to the base model to form an intermediate model. A stepwise backward elimination of each individual covariate was then conducted, where an increase in the objective function of greater than $10.82 p<0.001$, required to retain the covariate being evaluated in the final enoxaparin model.

The first order conditional method with interaction in NONMEM was used to estimate the intermediate and final models. The final model developed, was once again formally assessed using a non-parametric bootstrap, to assess the statistical properties of the model developed and the final model was also formally evaluated using a visual predictive check.

\subsubsection{Visual predicted check}

The principle of the visual predicted check (VPC) is to assess graphically whether simulations from the final model developed are able to reproduce both the central trend and variability in the observed data, when plotted against an independent variable, typically time, and explores the predictive capability of the final model [Bergstrand et al., 2011]. Percentiles of the simulated data are compared to the corresponding percentiles from the observed data. If the final model is describing the original observed data, then the concentrations should lie on top of one another. A VPC plot is one of the key plots used in pharmacokinetic modelling to evaluate how well the model is performing.

All graphical analysis performed in sections 4.1.1.3, 4.1.1.4 and 4.1.1.5 used ' $R$ 2.14.1' ['R', 2006].

\subsubsection{Simulation}

Following the development of the final enoxaparin antenatal model, the next stage was to simulate the important 'what if' questions of clinical practice. In the case of this study, this was centred on whether pregnant women being managed for VTE needed to receive a total daily dose of LMWH which was more than what they would receive outside of pregnancy and whether the 
dosing interval really needed to be divided, i.e. $1.5 \mathrm{mg} / \mathrm{kg}$ once daily (used outside of pregnancy) versus $1 \mathrm{mg} / \mathrm{kg}$ twice a day (currently recommended during pregnancy). In order to answer this question, the Royal College of Obstetricians and Gynaecologists dosing guidelines (table 4.1 twice daily) and the King's College Hospital once daily dosing guidelines (table 4.2 - used for nonpregnant adult patients) for VTE management with enoxaparin were simulated from the final model. Both regimens incorporate dose-banding, in order to minimise risk and is currently regarded as good clinical practice, in the UK [National Patient Safety Agency, 2010].

Table 4.1: RCOG antenatal enoxaparin VTE dosing guidelines

\begin{tabular}{ll}
\hline Weight $(\mathrm{kg})$ & TWICE daily dose \\
\hline$<50$ & $40 \mathrm{mg}$ \\
$50-69$ & $60 \mathrm{mg}$ \\
$70-89$ & $80 \mathrm{mg}$ \\
$>90$ & $100 \mathrm{mg}$ \\
\hline
\end{tabular}

Table 4.2: King's College Hospital enoxaparin dosing guidelines

\begin{tabular}{ll}
\hline Weight $(\mathrm{kg})$ & ONCE daily dose \\
\hline$<47$ & $60 \mathrm{mg}$ \\
$48-59$ & $80 \mathrm{mg}$ \\
$60-73$ & $100 \mathrm{mg}$ \\
$74-88$ & $120 \mathrm{mg}$ \\
$89-109$ & $150 \mathrm{mg}$ \\
$110-125$ & $180 \mathrm{mg}$ \\
$>125$ & $230 \mathrm{mg}$ \\
\hline
\end{tabular}

The simulation work was based on women commencing enoxaparin from day 1 of their pregnancy and delivering their baby at 40 weeks gestation. The dose of enoxaparin was not altered during the pregnancy. For the specific purposes of simulation, an additional weight progression model for pregnancy was also developed. The development of the weight model followed the same principals of model development, as the main pharmacokinetic model.

\subsection{Results: Population pharmacokinetic modelling}

\subsubsection{Breadth of anti-Xa activities drawn}

During the course of the study, 123 women contributed antenatal anti-Xa activities that were used for the development of the enoxaparin PK model (chapter 3 for details on these women). The 
recruited pregnant subjects provided 795 anti-Xa activities in total for pharmacokinetic modelling purposes, of which 712 were drawn during the antenatal period; the remaining anti-Xa activities were from the post-partum period (typically within two days of delivery). Figures $4.2(a$ and $b$ ) illustrate at what time points these samples were drawn (i.e. the time after the last dose), and the gestational stage at which these anti-Xa activities were collected.

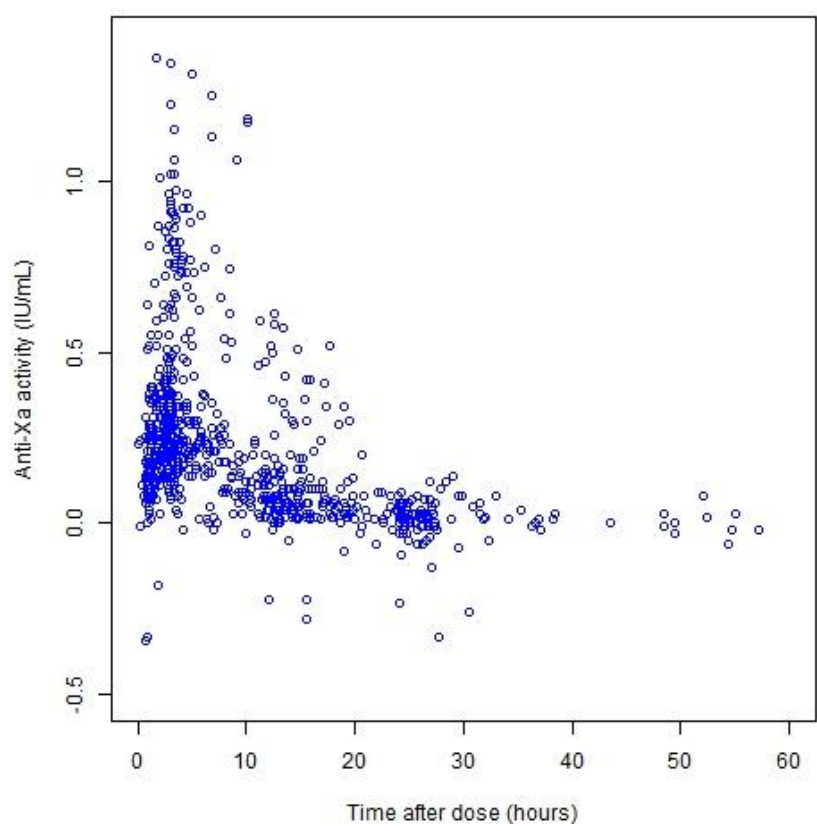

Figure 4.2(a): Scatter-plot of the total anti-Xa activities drawn plotted against time after dose

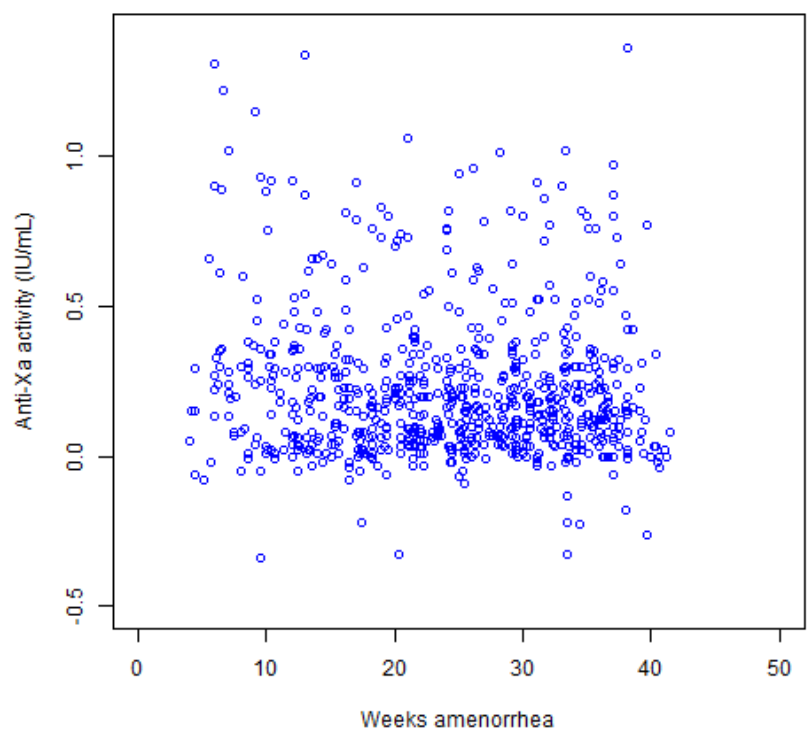

Figure 4.2(b): Scatter-plot of the total anti-Xa activities plotted against weeks amenorrhea

Most antenatal samples were drawn during the second and third trimester, though a reasonable number of anti-Xa activities ( $n=104)$ were also obtained from the first trimester. Figures $4.3(a-d)$, 
illustrate the number of anti-Xa activities according to each trimester and at the post-partum period.
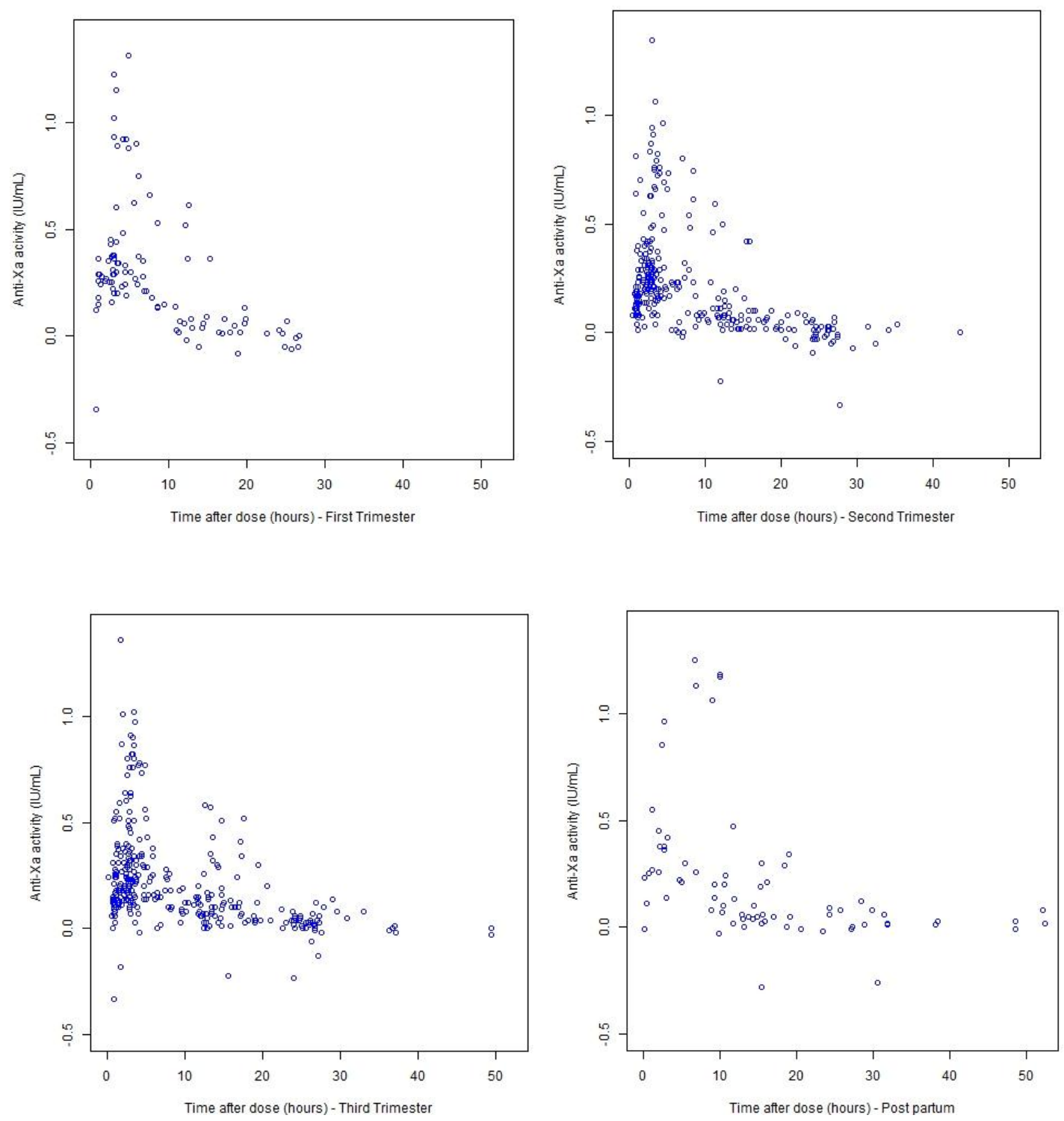

Figure 4.3 (a-d): Scatter plot of anti-Xa activities against time-after-dose, split according to trimester; first trimester (top left), second trimester (top right), third trimester (bottom left) and post-partum (bottom right)

Seventy seven (8.9\%) of the anti-Xa activities drawn were reported below the quantifiable level of the assay, i.e. $<0.01 \mathrm{IU} / \mathrm{mL}$. These individual anti-Xa activities were retained in the data set and their values retained as absolute (i.e. negative anti-Xa activity values recorded from the analyser were retained in the dataset) and their inclusion will be discussed as part of the base model development. 


\subsubsection{Base model development}

Initially, a stable base model, which described the data, was developed. A number of different base models were explored, i.e. one and two compartment, with ETA's (random effects) placed on $\mathrm{CL}, \mathrm{Vd}+/-\mathrm{Ka}$. Table 4.3 lists the different base models initially explored.

Table 4.3: Base models explored

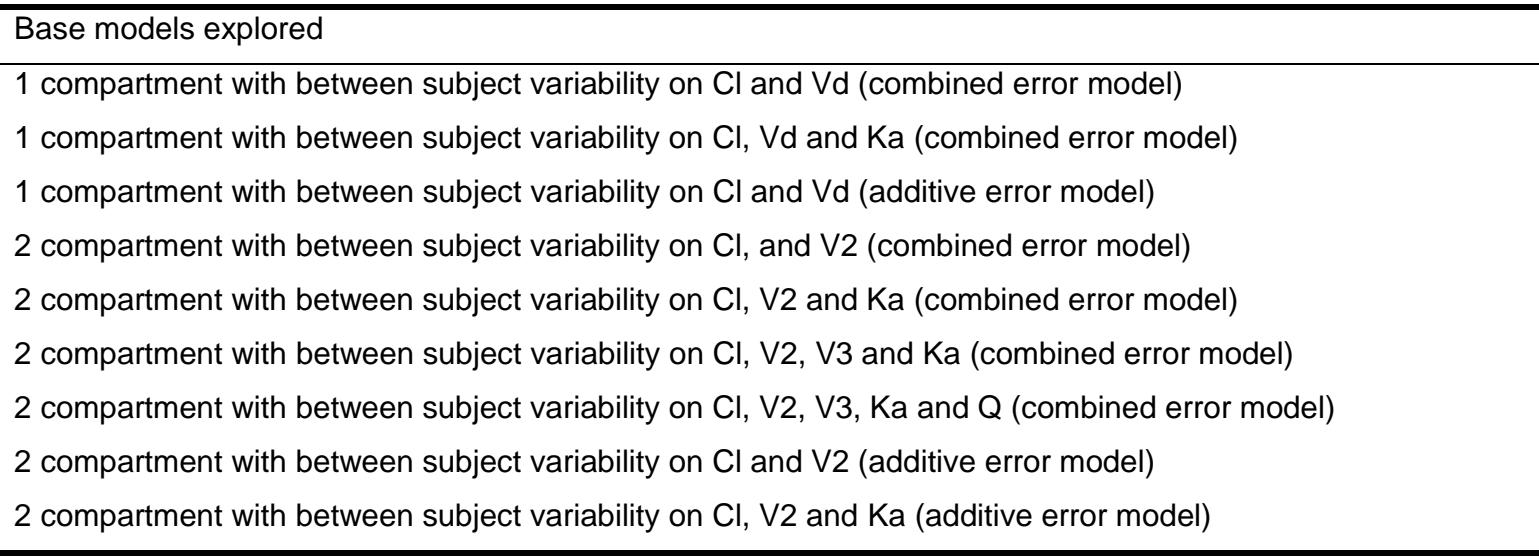

The parameter estimates for the key base models chosen to evaluate further are listed in table 4.4. The NONMEM control stream for the one compartment model with between subject variability on $\mathrm{Cl}$ and $\mathrm{Vd}$ is listed in appendix $\mathrm{V}$.

Table 4.4: Key base models, with associated parameter estimates

\begin{tabular}{|c|c|c|c|c|c|}
\hline \multirow[t]{3}{*}{ Parameter } & \multicolumn{5}{|c|}{ Estimate } \\
\hline & \multirow{2}{*}{\multicolumn{2}{|c|}{$\begin{array}{c}1 \mathrm{CMT}^{*} \\
\text { (Model 1) (\%SE) }\end{array}$}} & \multirow{2}{*}{\multicolumn{2}{|c|}{$\begin{array}{c}2 \text { CMT* }^{*} \\
\text { (Model 2) (\%SE) }\end{array}$}} & \multirow{3}{*}{$\begin{array}{c}1 \mathrm{CMT}^{\star *} \\
\text { (Model 3) } \\
1.63\end{array}$} \\
\hline & & & & & \\
\hline CL (I/hr) & 1.66 & (5.7) & 1.64 & (5.5) & \\
\hline $\operatorname{Vd}(\mathrm{I})$ & 11.0 & (11.5) & 4.66 & (28.0) & 10.6 \\
\hline $\mathrm{Ka}\left(\mathrm{hr}^{-1}\right)$ & 0.505 & (17.1) & 0.21 & (24.0) & 0.502 \\
\hline V3 (I) & - & & 4.30 & (39.5) & - \\
\hline$Q \quad\left(h r^{-1}\right)$ & - & & 0.358 & (47.5) & - \\
\hline$\omega_{C L}(\% C V)$ & 46 & (19.3) & 46 & (19.5) & 47 \\
\hline$\omega_{V d}(\% C V)$ & 35 & $(49.9)$ & 49 & (63.6) & 12 \\
\hline$\omega_{\mathrm{ka}}(\% \mathrm{CV})$ & - & & - & & 65 \\
\hline Proportional error (\%) & 22 & (21) & 21 & (21) & 21 \\
\hline Additive error (IU/mL) & 0.0659 & (18) & 0.0661 & (18) & 0.0658 \\
\hline Objective function & $-29 c$ & 8.573 & -2917.6 & & -2920.156 \\
\hline
\end{tabular}


Although the one compartment model with ETA's on CL, Vd, and Ka (model 3) had a lower objective function than the one compartment model with ETA's on CL and Vd (model 1), model 3's \$COV step did not run, and so was not considered a stable model. It was likely that the standard error around the estimate of between subject variability on Ka could not be well estimated, with the model considered to be over-parameterised given the sparse sampling during the absorption phase. Furthermore, whilst the two compartment model (model 2) saw a decrease in the objective function of $-9.032(p<0.05)$ relative to model 1 , this model was not considered the best base model, as the standard error on V3 was greater than $20 \%$, and the standard error on between subject variability was greater than $50 \%$. Therefore model 1 was considered the optimal base model.

\subsubsection{Handling below limit of quantification (BLQ) data}

Next the BLQ data were considered, which comprised $8.9 \%$ of the total dataset of anti-Xa activities. The specific question explored was, whether these data needed any special consideration. BLQ is not a unique problem to this study and is commonly encountered in the pharmacometric field. A number of methods exist in order to handle BLQ data within population pharmacokinetic modelling analysis. These include:

i) Ignoring the data (i.e. remove them from the dataset)

ii) Setting all the BLQ values to zeros

iii) Setting all the BLQ values to half the BLQ values, i.e. if the BLQ is 0.01 , then all $B L Q$ values are set at 0.005

iv) Use the M3 error method, as developed by Beal [Beal, 2001]. This method assumes that all BLQ are normally distributed. Therefore implementing the $\mathrm{M} 3$ method in NONMEM would involve sampling from this normal distribution of BLQ. This method has gained wide credibility within the pharmacometrics field

v) Obtaining extrapolated values from the analyser of the assay and including these in the dataset as absolute values (including negative values)

As bias could be introduced into the model if the BLQ were ignored, converted to zero or converted to half the BLQ values [Bergstrand and Karlsson, 2009], options four and five were specifically explored. 
The M3 method was explored on the data. Appendix VI lists the NONMEM control stream code for the M3 method. Table 4.5, presents the parameter estimates obtained from this method.

Table 4.5: Pharmacokinetic parameter estimates when using the M3 error method

\begin{tabular}{ll}
\hline Parameter & Estimate \\
\hline $\mathrm{CL}(\mathrm{I} / \mathrm{hr})$ & 1.50 \\
$\mathrm{Vd}(\mathrm{I})$ & 14.3 \\
$\mathrm{Ka}\left(\mathrm{hr}^{-1}\right)$ & 0.649 \\
$\omega_{\mathrm{cl}}(\% \mathrm{cv})$ & 43 \\
$\omega_{\mathrm{Vd}}(\% \mathrm{cv})$ & 36 \\
Proportional error $(\%)$ & 54 \\
Additive error $(\mathrm{IU} / \mathrm{mL})$ & 0.025 \\
Objective function & $-2923.835^{\star}$
\end{tabular}

${ }^{*}$ minimisation terminated due to rounding error, therefore \%SE estimates were not obtained

Minimisation was not successful with the M3 method. This perhaps is not unsurprising, as Bergtrand and Karlsson [2009], have already stipulated that with the M3 method, the number of successful minimisations and how often the \$COV runs, may fall. The M3 error model was rejected, as although the additive error decreased, the proportional error with this method went up significantly ( $54 \%$ compared to $22 \%$ in model 1$)$. The decision to reject the M3 error model was further vindicated by recent work from Keizer and colleagues, which has evaluated different methods of handling BLQ data, including incorporation of BLQ, as a continuous data source [Keizer et al., 2010]. They found that when BLQ is incorporated as a continuous dataset and represents less than $10 \%$ of the total data (as is the case here), all BLQ methods showed similar performance. It was therefore decided to retain the BLQ in the data set as a continuous (extrapolated) data source, where the actual extrapolated values were those extracted from the laboratory analyser (including negative anti-Xa activities obtained).

\subsubsection{Inter occasion variability}

One question which needed to be considered in this study was whether inter occasion variability should be incorporated into the model developed. This is because it is recognised that pharmacokinetic parameters can vary over time in the same individual. In some situations, this may be linked to physiological processes, for example the effect of age, and in such situations, age would be incorporated into a final pharmacokinetic model to account for this. However in 
some situations, individual pharmacokinetic parameters may change randomly between study occasions [Karlsson and Sheiner, 1993]. Within population pharmacokinetics, three levels of random effects are usually identified, firstly, between subject variation, the second is within subject variation and finally residual unexplained variability. Incorporating inter-occasion variation into a final model allows further assessment of within subject variability, by evaluating interoccasion variability for the same subject. Work by Karlsson and Sheiner [1993], has demonstrated that although inter occasion variability can be ignored in the model development process, if it is, it can lead to significant bias on the fixed-effects parameters; which ones and by how much, depending on the design of the study and the model produced. With respect to the random-effect parameters, the effect of ignoring or not incorporating inter occasion variability into the model, can inflate the estimated residual error variance. Indeed only by extracting interoccasion variability, do you truly identify residual error. This is important to consider for this study as each subject in this study would have anti-Xa activity drawn on a number of occasions over the course of their pregnancy. However, the gravid state is a special situation; the primary purpose of the pharmacokinetic model being developed in this sub-group was to understand the pharmacokinetic changes as pregnancy progresses. Therefore, one expects inter occasion variability which can closely be linked to the physiological changes of the gravid state and therefore should be considered as a fixed effect rather than a random effect. Therefore inter occasion variability was investigated as a diagnostic tool to determine appropriate fixed effect changes in clearance and volume of distribution (figures 4.4 (a) and (b)) during pregnancy. These plots took the first ETA values for each subject in each trimester, for both $C L$ and $V d$ and show that $\mathrm{CL}$ did not change significantly over the course of pregnancy, whereas, $\mathrm{Vd}$ did show a change in trend between the second and third trimester and again between the third trimester and within a week of delivery, suggesting that this needed to be accounted for in the final model, with respect to Vd. 


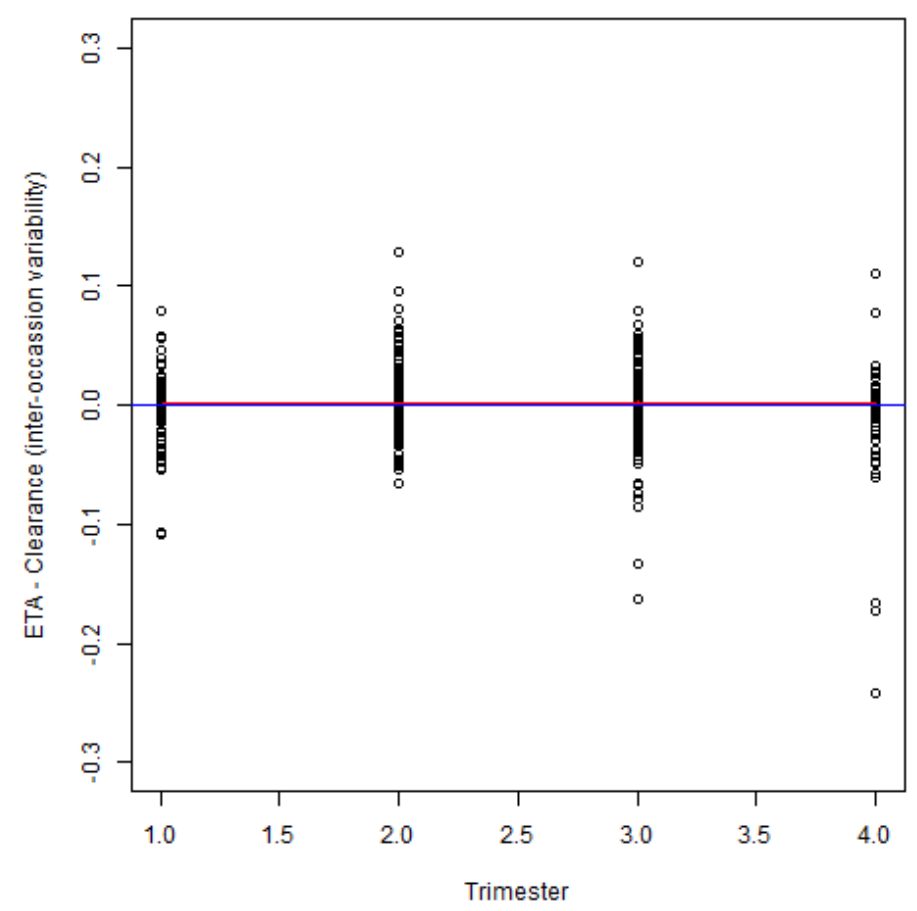

Figures 4.4(a): ETA of CL (IOV) versus trimester (trimester 4 refers to within a week of delivery); redline represents the median values at each time point and the blue line is the reference line

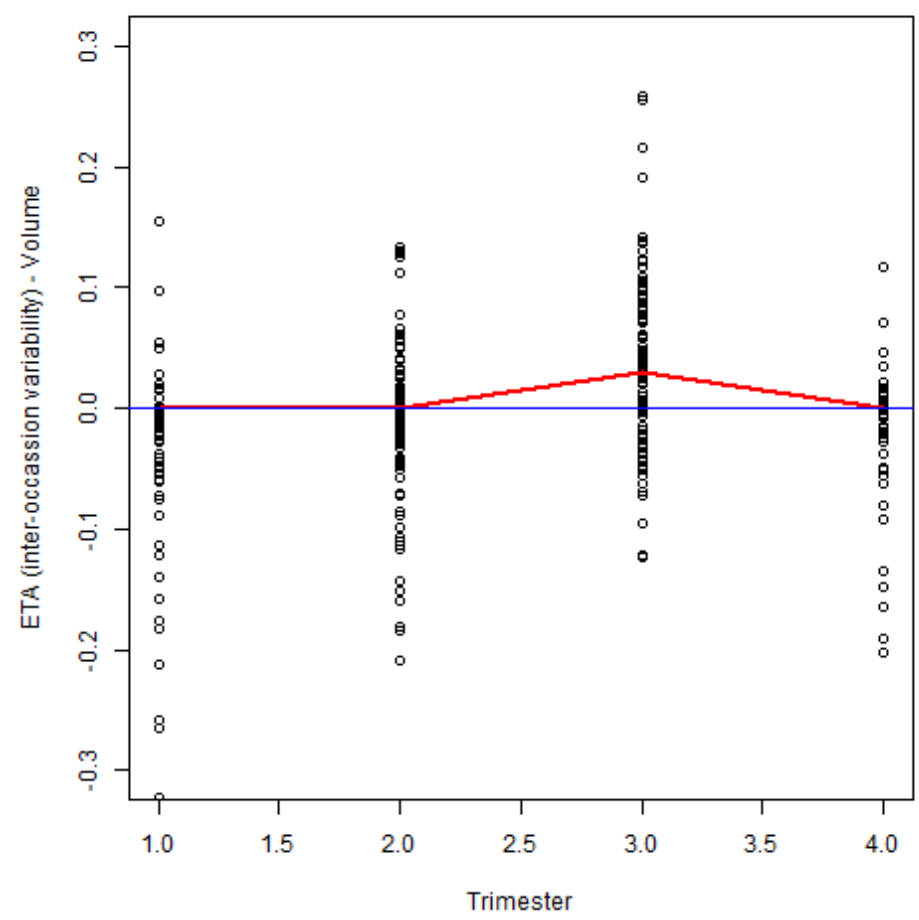

Figures 4.4(b): ETA of Vd (BOV) versus trimester (trimester 4 refers to within a week of delivery); redline represents the median values at each time point and the blue line is the reference line 


\subsubsection{Goodness of fit evaluation of the base model}

A one compartment model, with exponential between subject variability on $C L$ and $V d$ (model 1 ), with a combined additive and proportional error model, was the base model taken forward.

Following this, standard goodness of fit plots of the one-compartment model were produced to graphically evaluate the model (figures 4.5-4.14); blue lines in the plots represent the line of identity and the red lines represent the linear regression line. Concentration of anti-Xa activity is in $\mathrm{IU} / \mathrm{mL}$. For figures 4.5 and figure 4.6 , the model is not able to predict negative anti-Xa activities, as only positive concentrations can be predicted by the population and individual model, which explains the 'tail' observed at the bottom of figures 4.5 and 4.6 .

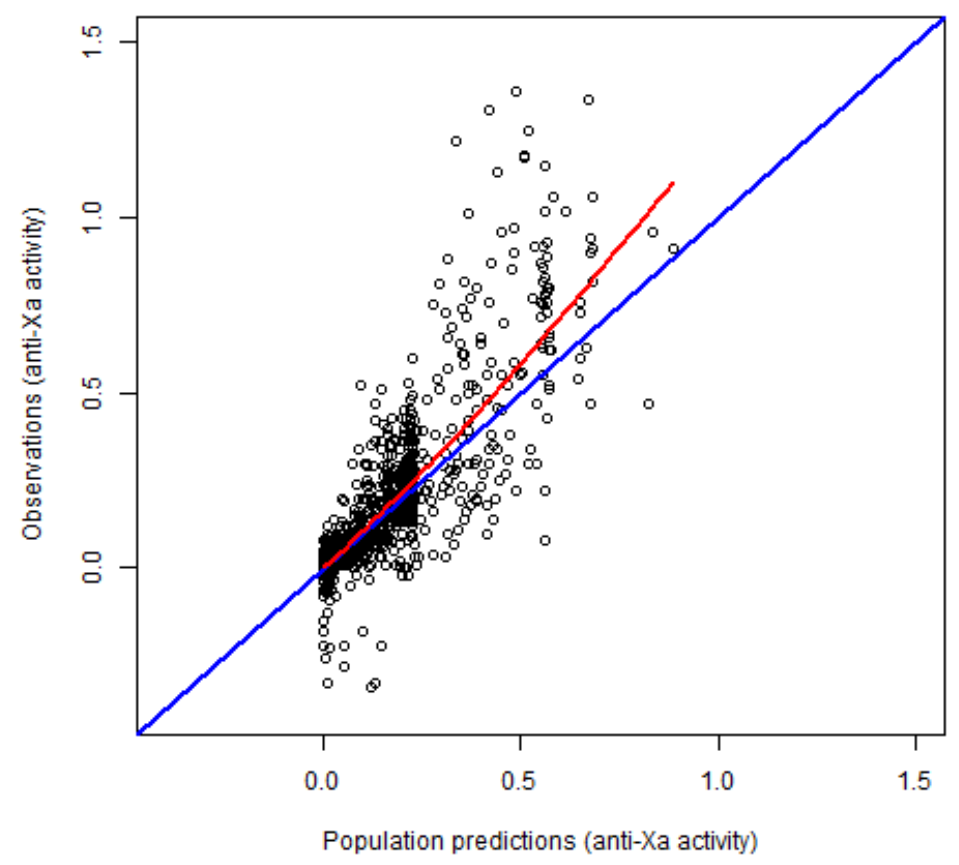

Figure 4.5: Observed versus population predicted values

Within figure 4.5, the population model is under-predicting at higher concentrations. 


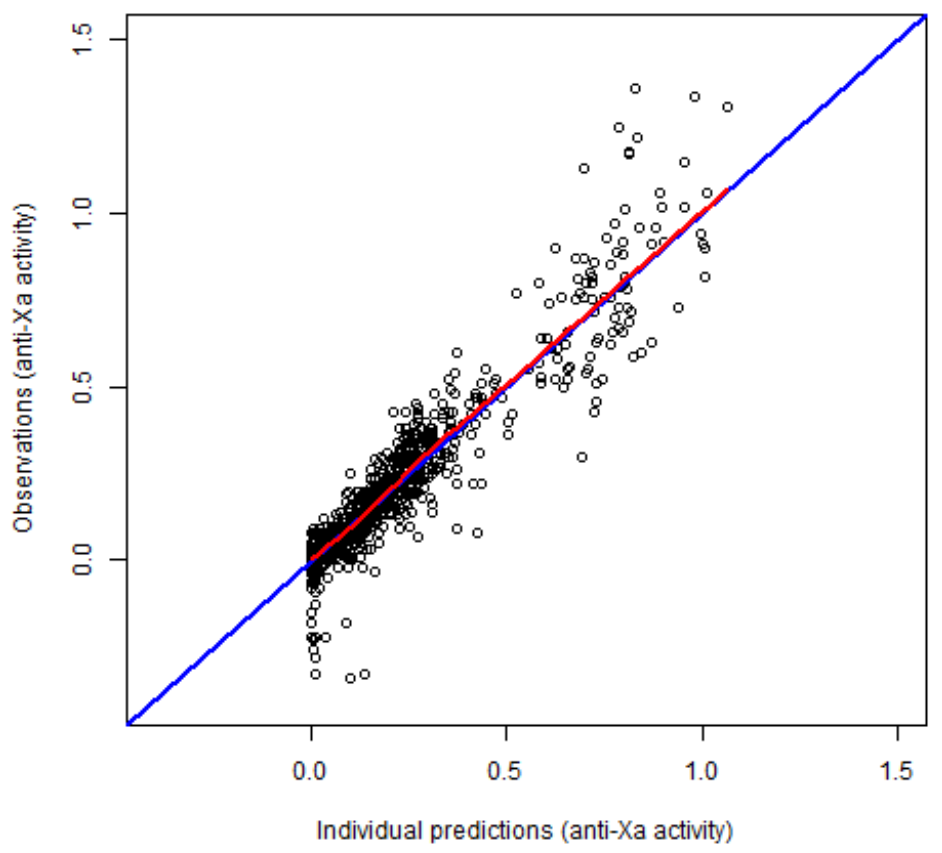

Figure 4.6: Observed versus individual predicted values

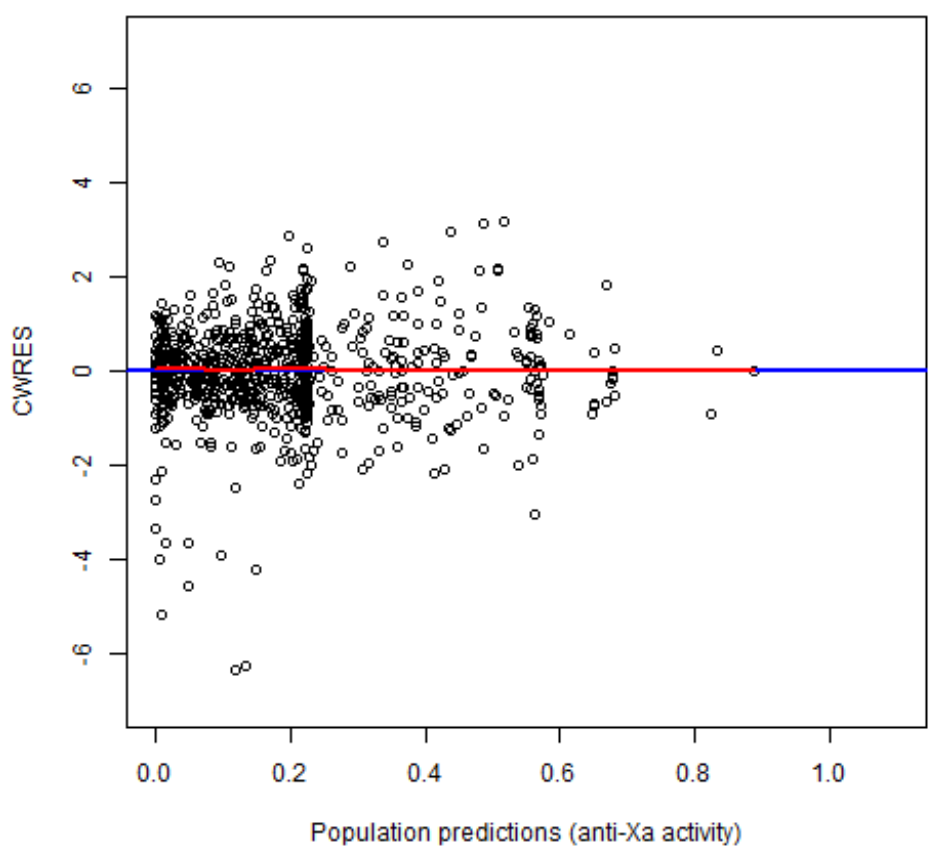

Figure 4.7: Conditional weighted residuals (CWRES) versus population predictions 


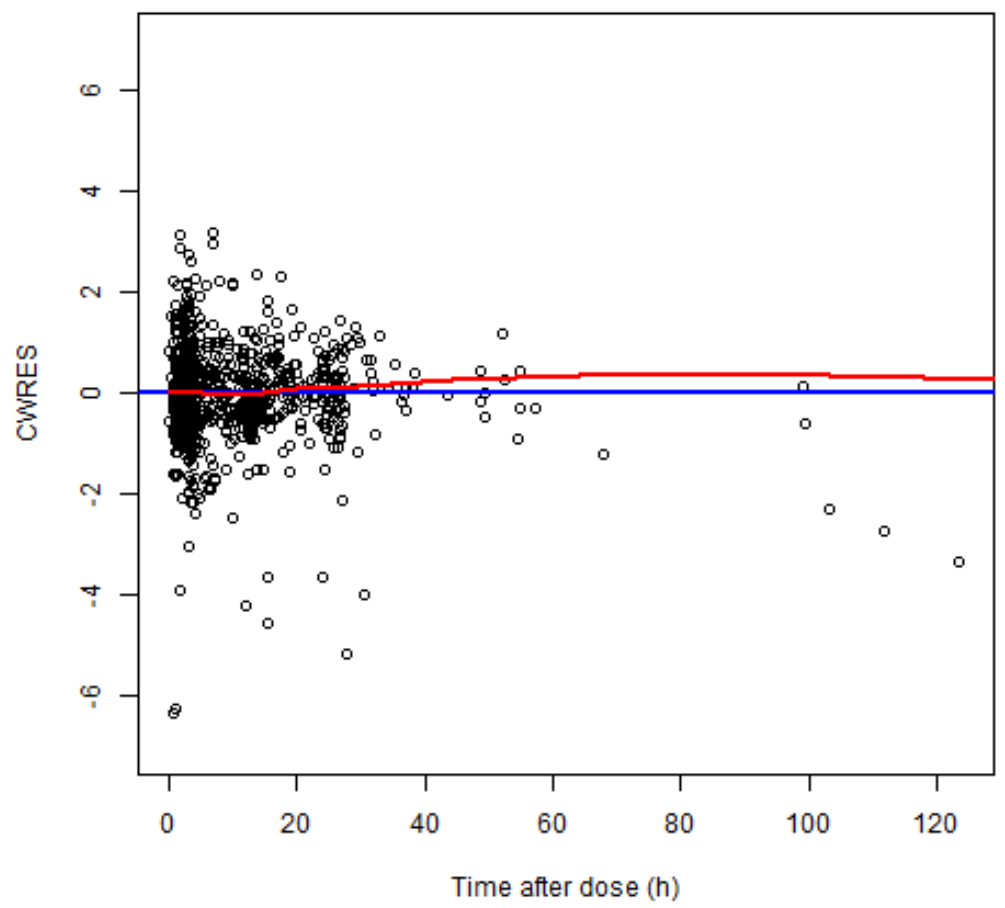

Figure 4.8: Conditional weighted residuals (CWRES) versus time after dose

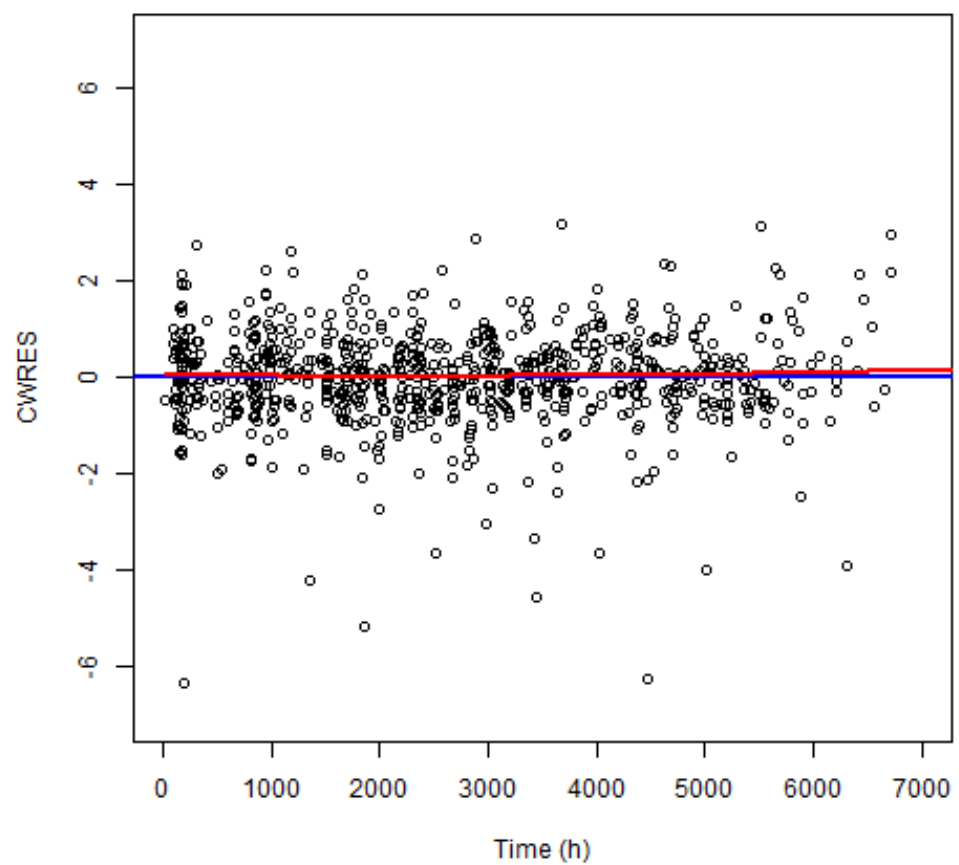

Figure 4.9: Conditional weighted residuals (CWRES) versus time 


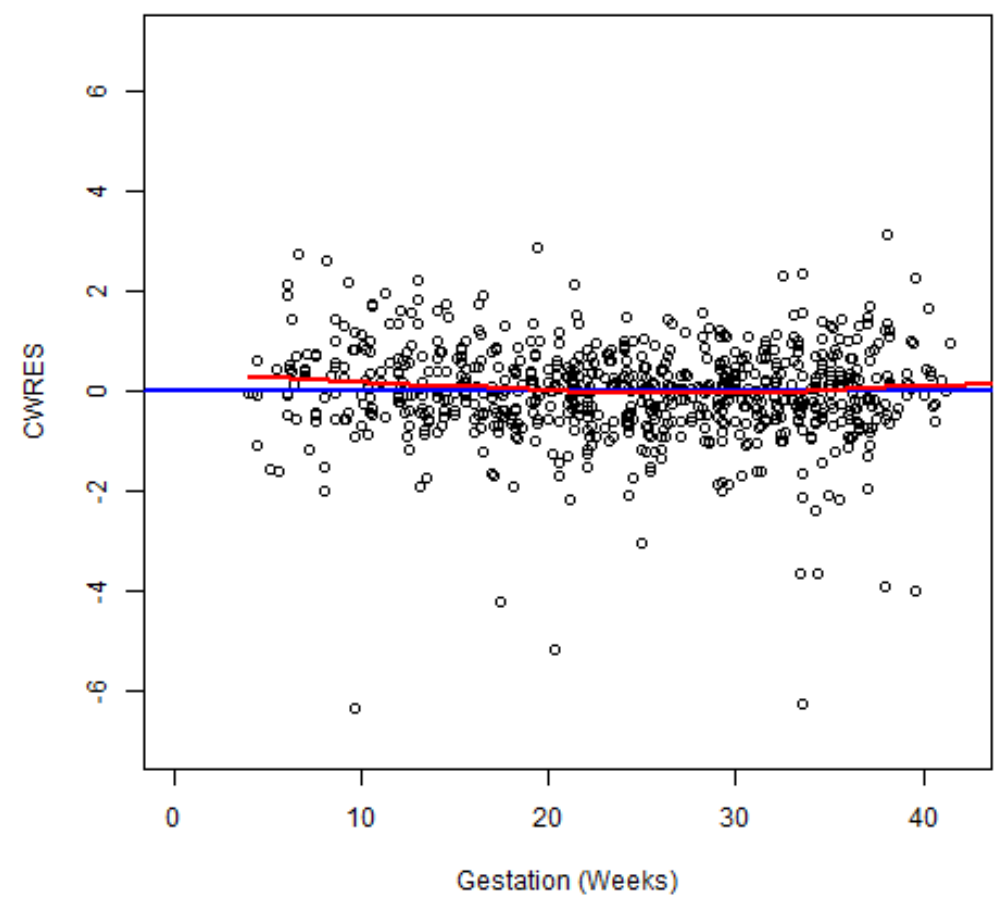

Figure 4.10: Conditional weighted residuals (CWRES) versus gestation

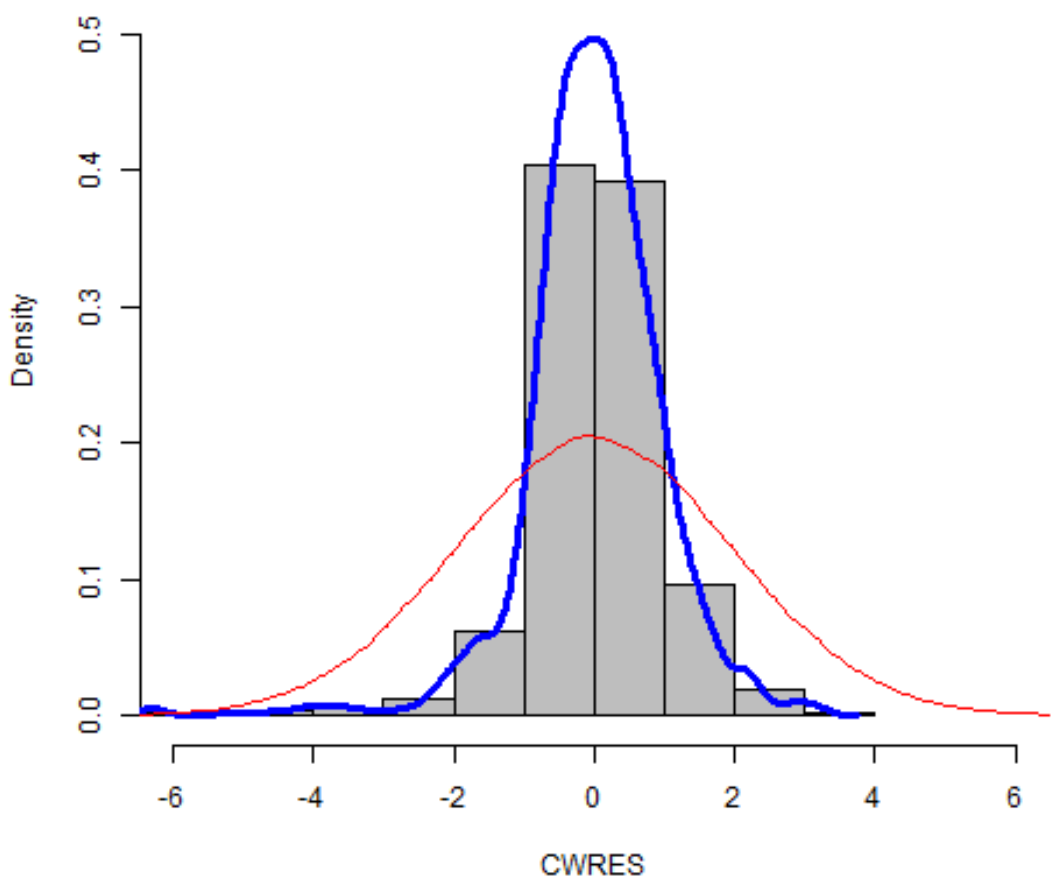

Figure 4.11: Histogram of conditional weighted residuals (CWRES), blue line represents actual standard deviation 


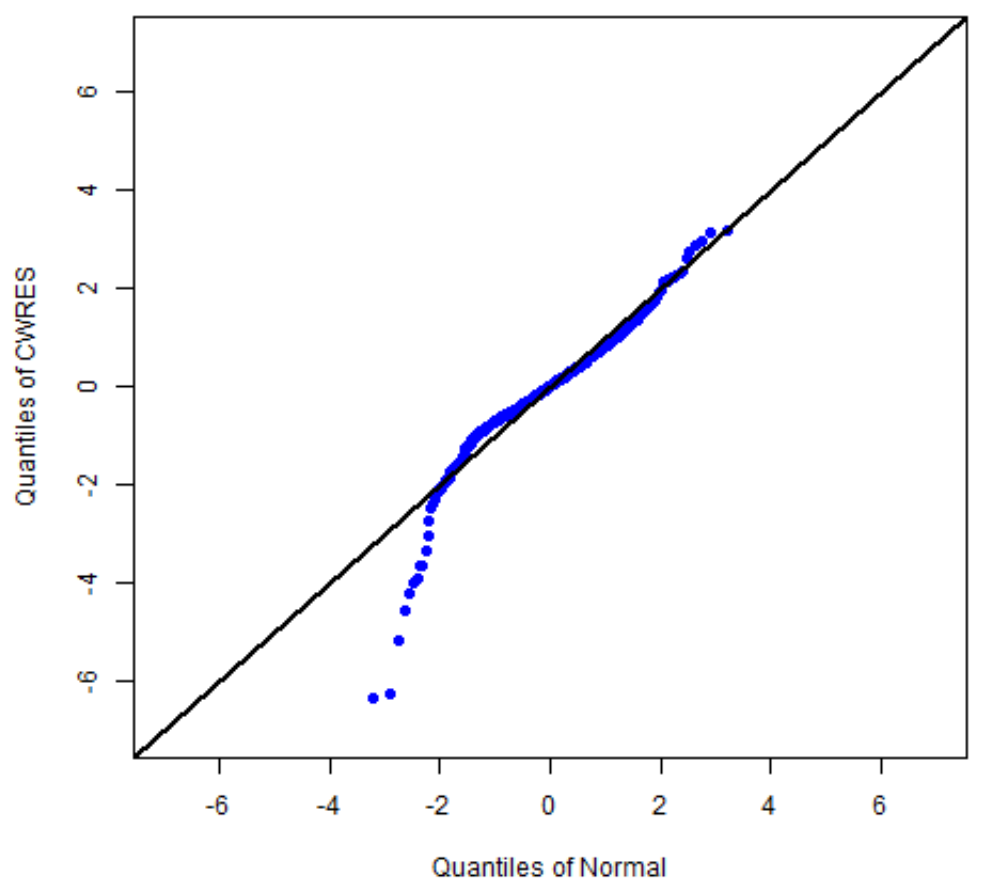

Figure 4.12: Q-Q plot of conditional weighted residuals (CWRES)

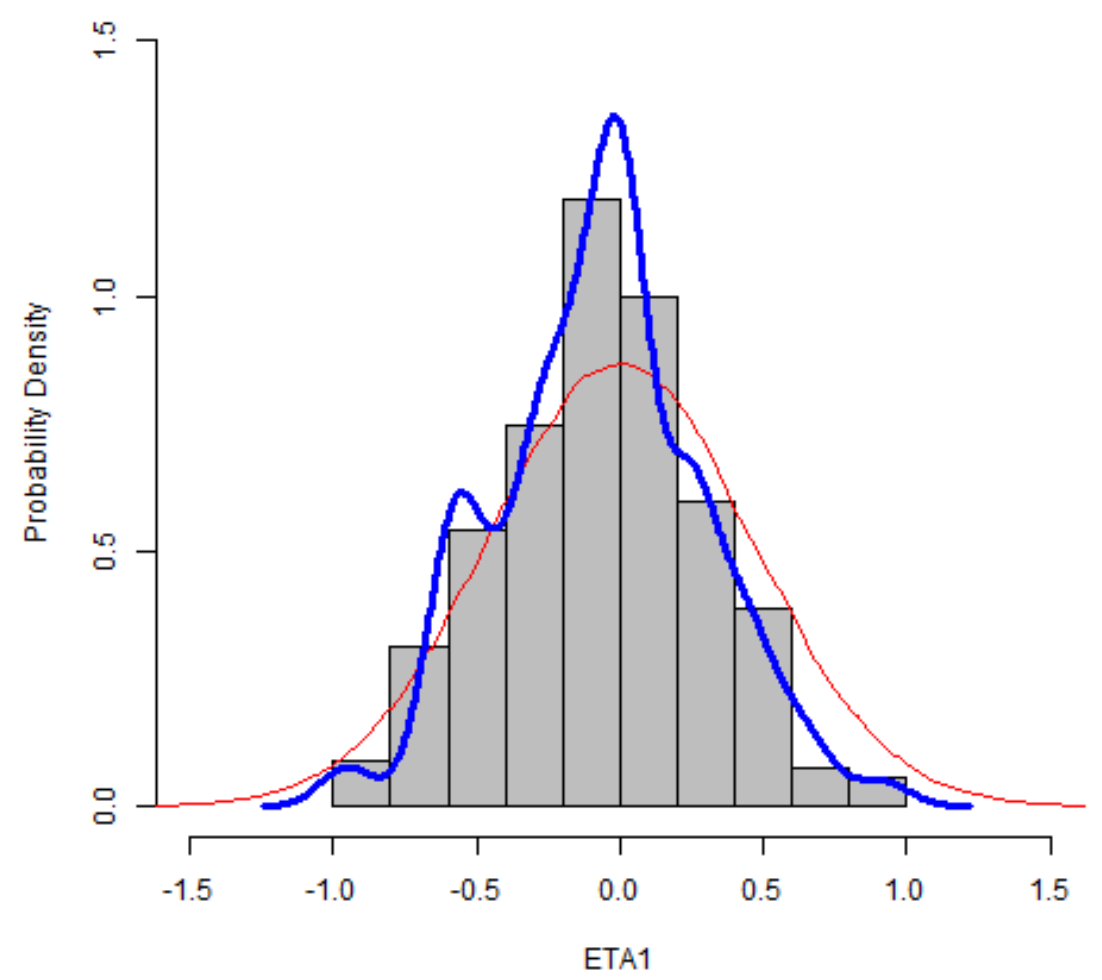

Figure 4.13: Histogram of ETA1 (CL), blue line represents actual standard deviation 


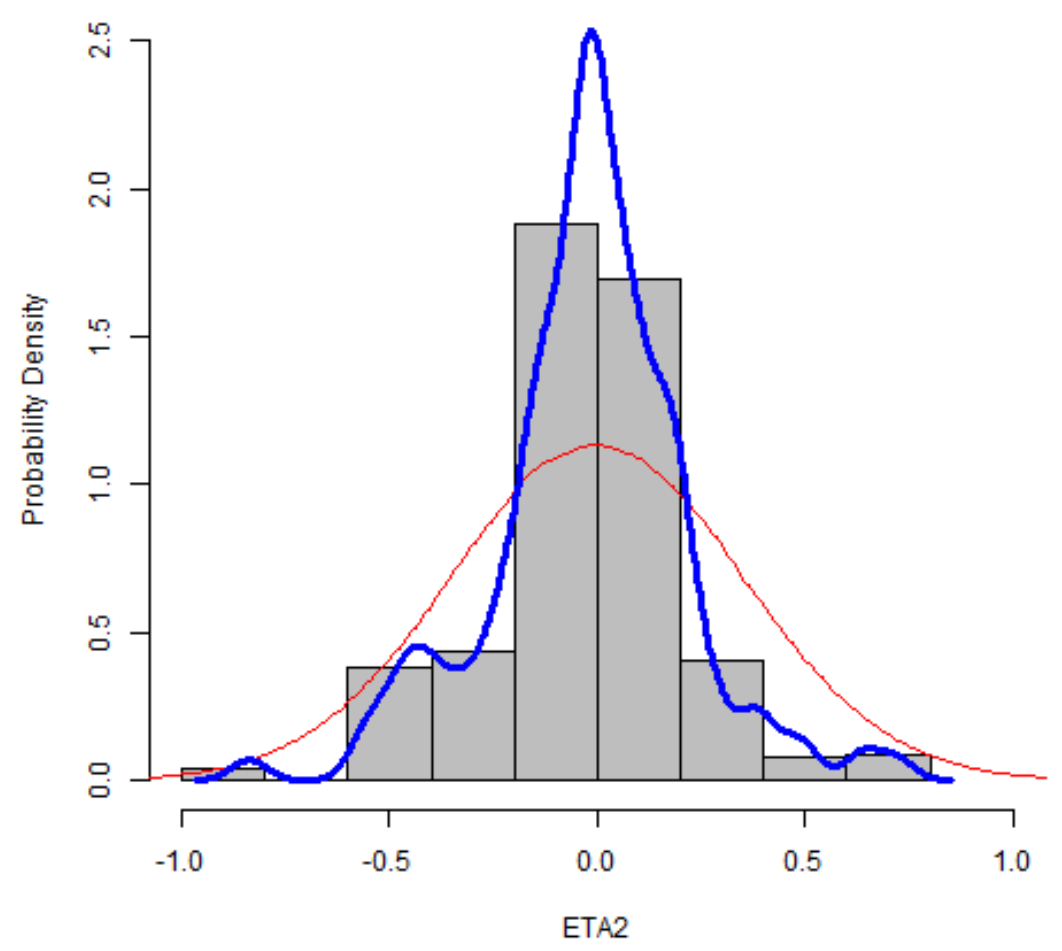

Figure 4.14: Histogram of ETA2 (Vd), blue line represents actual standard deviation

For this base model, shrinkage on ETA1 (CL) was $19.1 \%$ and ETA2 (Vd) was $35.2 \%$. The goodness of fit plots provided confirmation and reassurance that the one compartment model was describing / capturing the observed data well and was a stable base model to take forward.

Next a 1000 non-parametric bootstraps were run on the base model. Appendix VII lists the specific code used for completing this, using Wings for NONMEM for version 7.2.2 (available at http://wfn.sourceforge.net/wfndown.htm). The estimates from this bootstrap are presented in table 4.6. 
Table 4.6: Non-parametric bootstrap results compared with the parametric model estimates

\begin{tabular}{lrlrc}
\hline Parameter & \multicolumn{2}{c}{ 1 CMT model } & \multicolumn{2}{c}{ Bootstrap } \\
& Estimate & $(95 \% \mathrm{Cl})$ & Median & $\begin{array}{c}2.5^{\text {th }}-97.5^{\text {th }} \\
\text { percentile }\end{array}$ \\
\hline $\mathrm{CL}(\mathrm{l} / \mathrm{hr})$ & 1.66 & $(1.48-1.84)$ & 1.67 & $(1.49-1.89)$ \\
$\mathrm{Vd}(\mathrm{I})$ & 11.0 & $(8.53-13.97)$ & 11.20 & $(3.3-18.93)$ \\
$\mathrm{Ka}\left(\mathrm{hr}^{-1}\right)$ & 0.505 & $(0.335-0.675)$ & 0.515 & $(0.15-0.89)$ \\
$\omega_{\mathrm{cl}}(\% \mathrm{cv})$ & 46 & $(18-74)$ & 46 & $(38-57)$ \\
$\omega_{\mathrm{Vd}}(\% \mathrm{cv})$ & 35 & $(2-70)$ & 35 & $(14-65)$ \\
Proportional error $(\%)$ & 22 & $(8-36)$ & 22 & $(17-53)$ \\
Additive error $(\mathrm{IU} / \mathrm{mL})$ & 0.0659 & $(0.0644-0.0674)$ & 0.065 & $(0.04-0.08)$ \\
\hline
\end{tabular}

The results from the bootstrap provided further confirmation of the appropriateness of the one compartment base model.

\subsubsection{Covariate analysis}

A full covariate analysis was then instigated. The following mechanistic covariates were tested; age, ethnicity, weight, lean body weight (baseline), creatinine and creatinine clearance..

Graphical analyses of random effects (ETAs) of CL versus covariates are illustrated in figures 4.15-4.20.

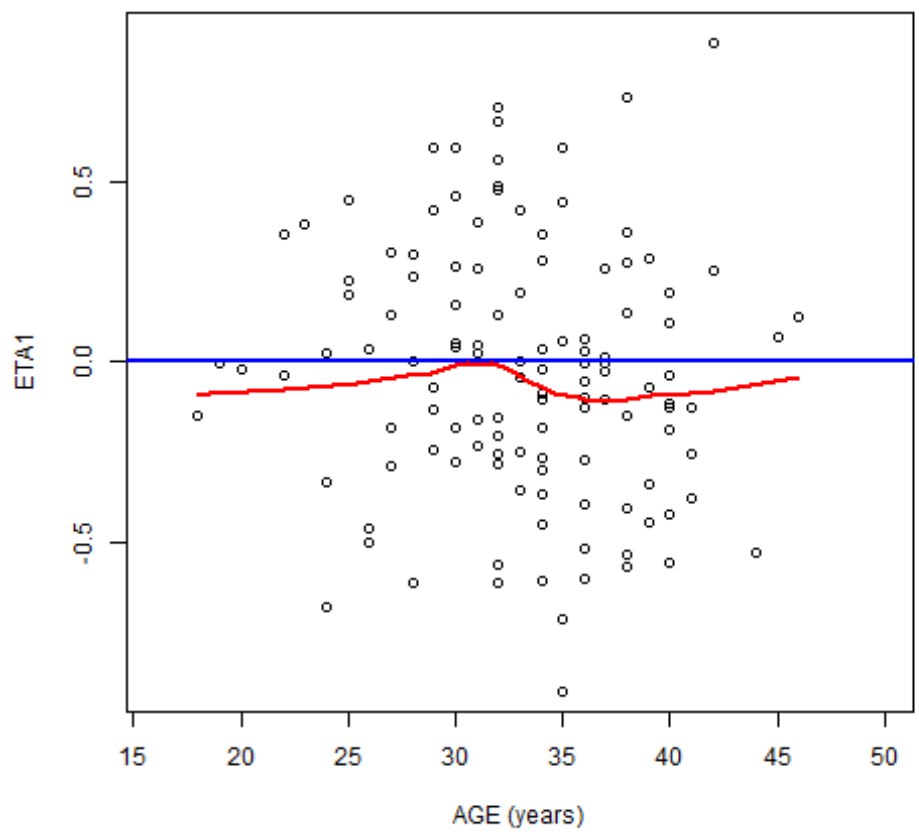

Figure 4.15: ETA1 $(C L)$ versus age 


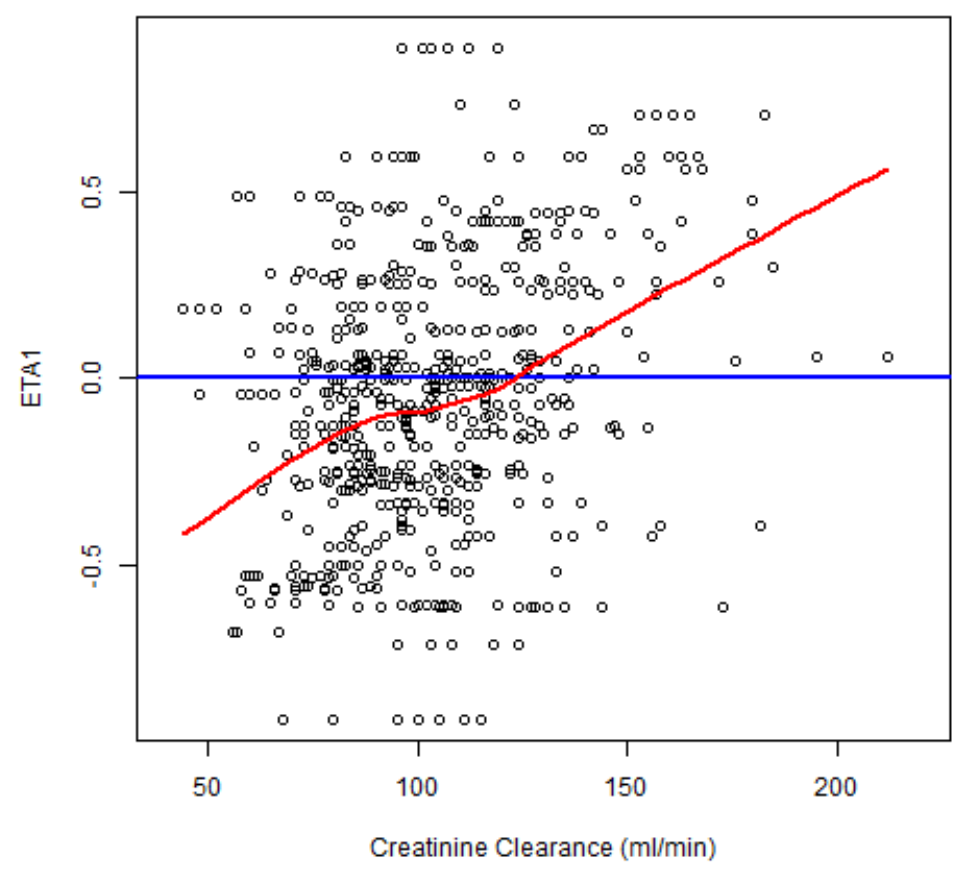

Figure 4.16: ETA1 (CL) versus creatinine clearance

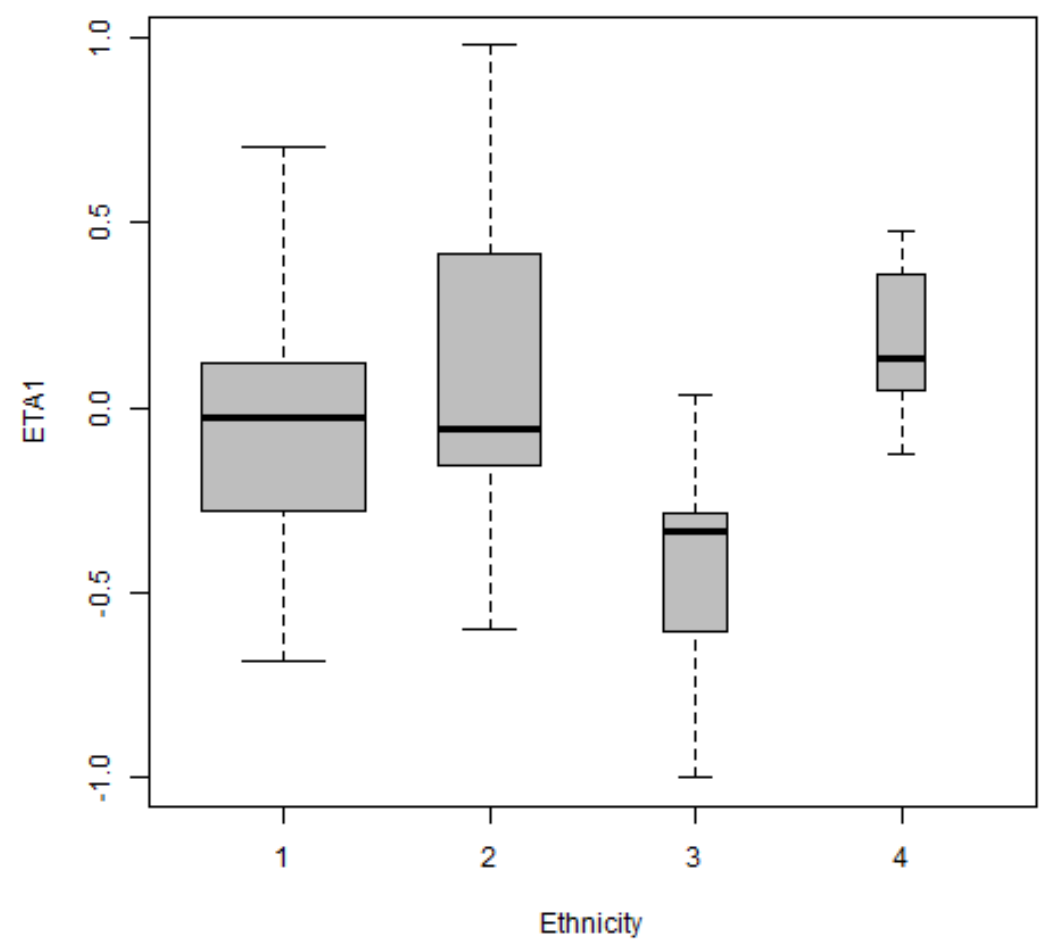

Figure 4.17: ETA1 (CL) versus ethnicity (1 refers to the Caucasian population, 2 refers to the Africian-Caribbean population, 3 refers to the Asian population and 4 refers to the 'other' population), the width of the boxes indicates the relative number of subjects 


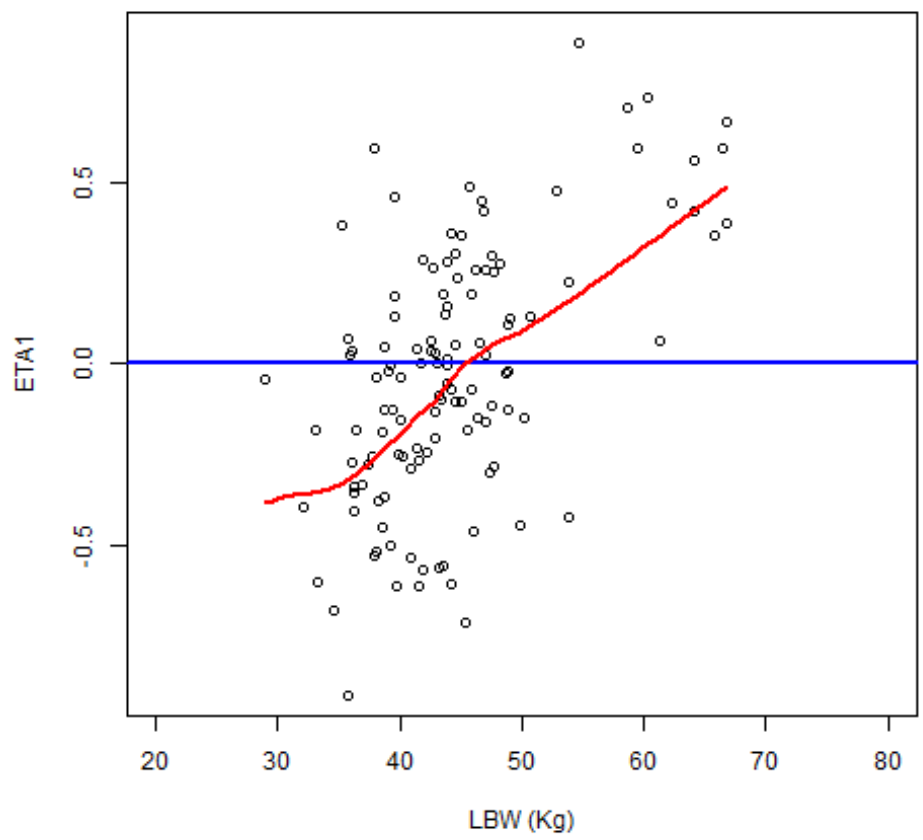

Figure 4.18: ETA1 $(C L)$ versus LBW

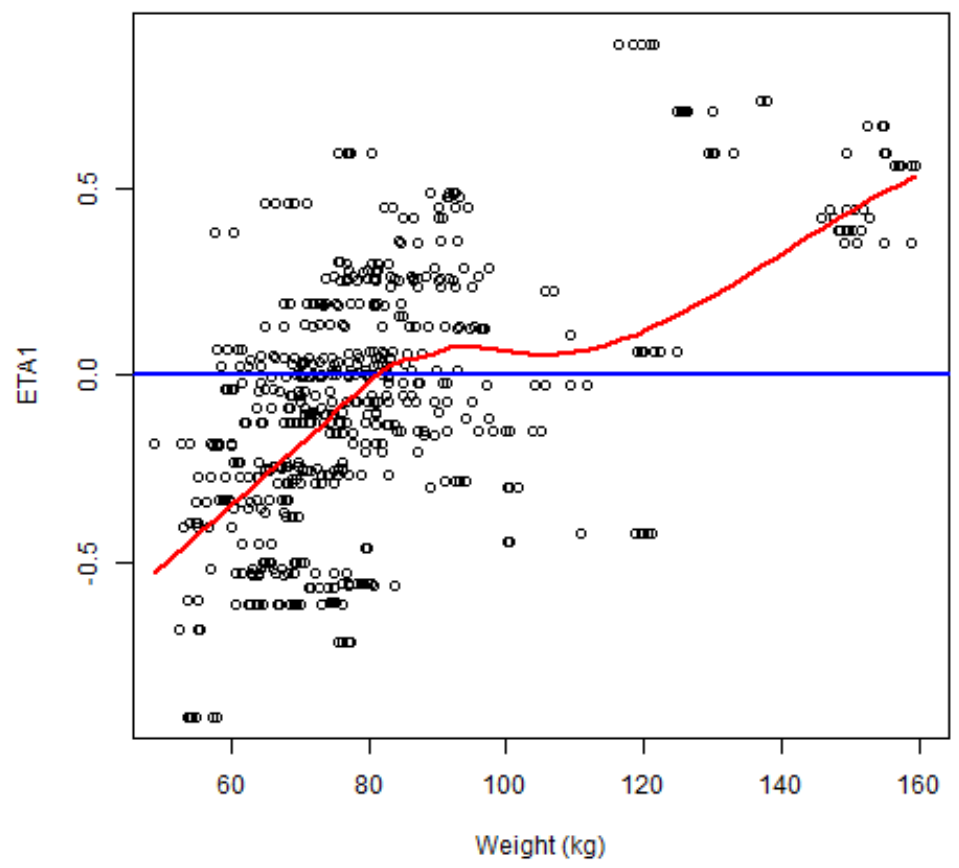

Figure 4.19: ETA1 (CL) versus weight 


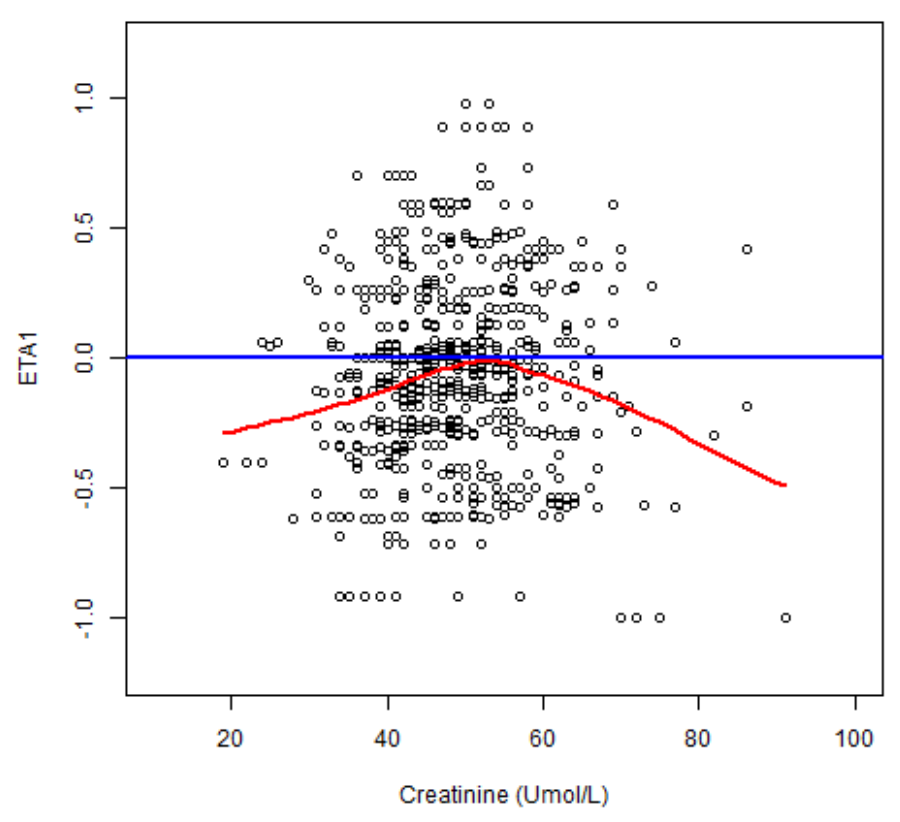

Figure 4.20: ETA1 $(C L)$ versus creatinine

Based on this analysis, trends can be seen with weight, creatinine, baseline lean body weight and creatinine clearance. No trend was observed on age, and so was not considered relevant on CL and not investigated further. The ethnicity box-plots shows a slight trend on with groups 3 and 4 (Asian and 'other' group), however the number of subjects in these two group were too small for further consideration. Gestation was not formally investigated on CL during the antenatal period, as the inter-occasion variability plot (figure 4.4(a)) demonstrated minimal impact during pregnancy.

Graphical analyses of random effects (ETAs) of Vd versus covariates are illustrated in figures 4.21 to 4.24 . 


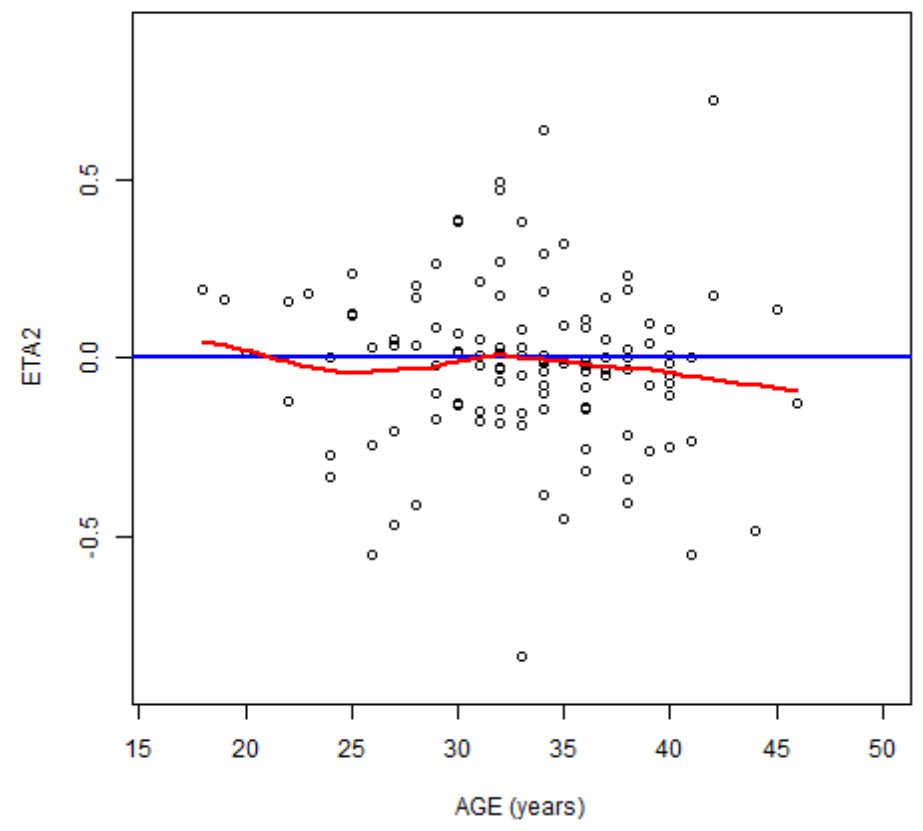

Figure 4.21: ETA2 $(\mathrm{Vd})$ versus age

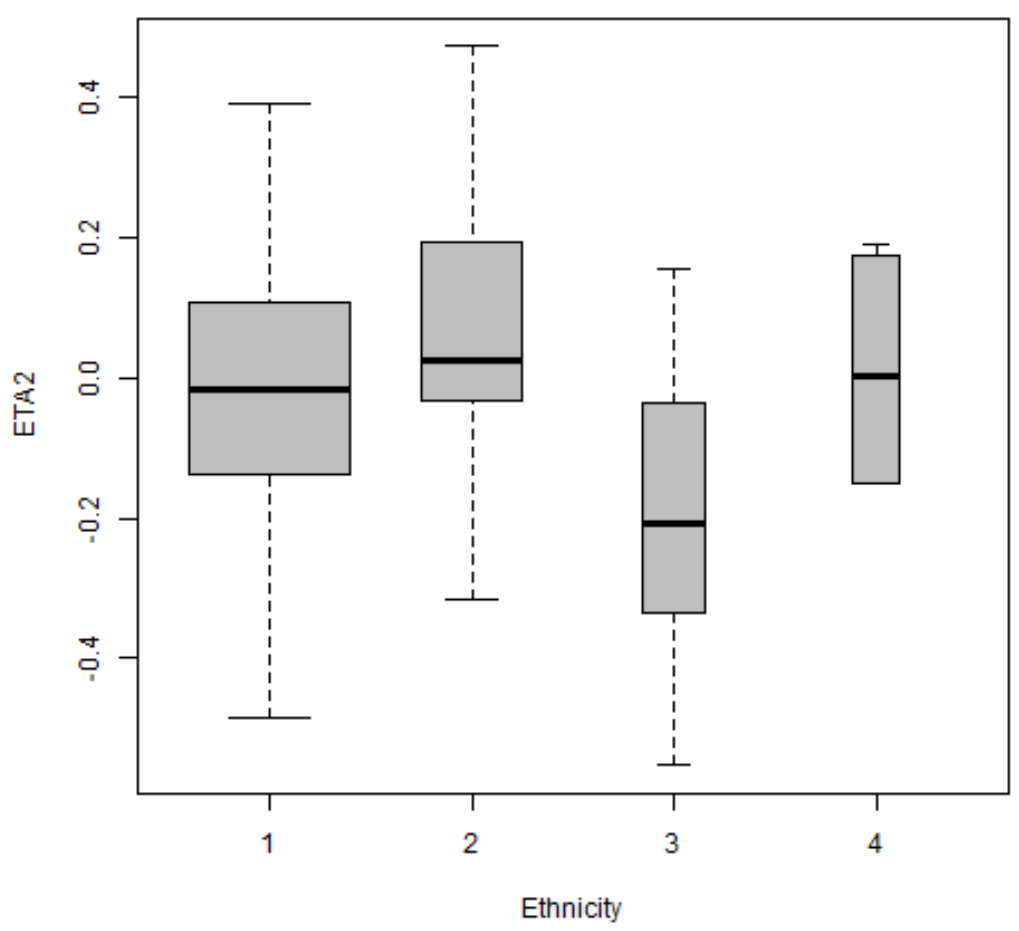

Figure 4.22: ETA2 (Vd) versus ethnicity (1 refers to Caucasian population, 2 refers to AfricanCarribean population, 3 refers to Asian population and 4 refers to 'other' population); The width of the box-indicates the relative number of subjects in each group 


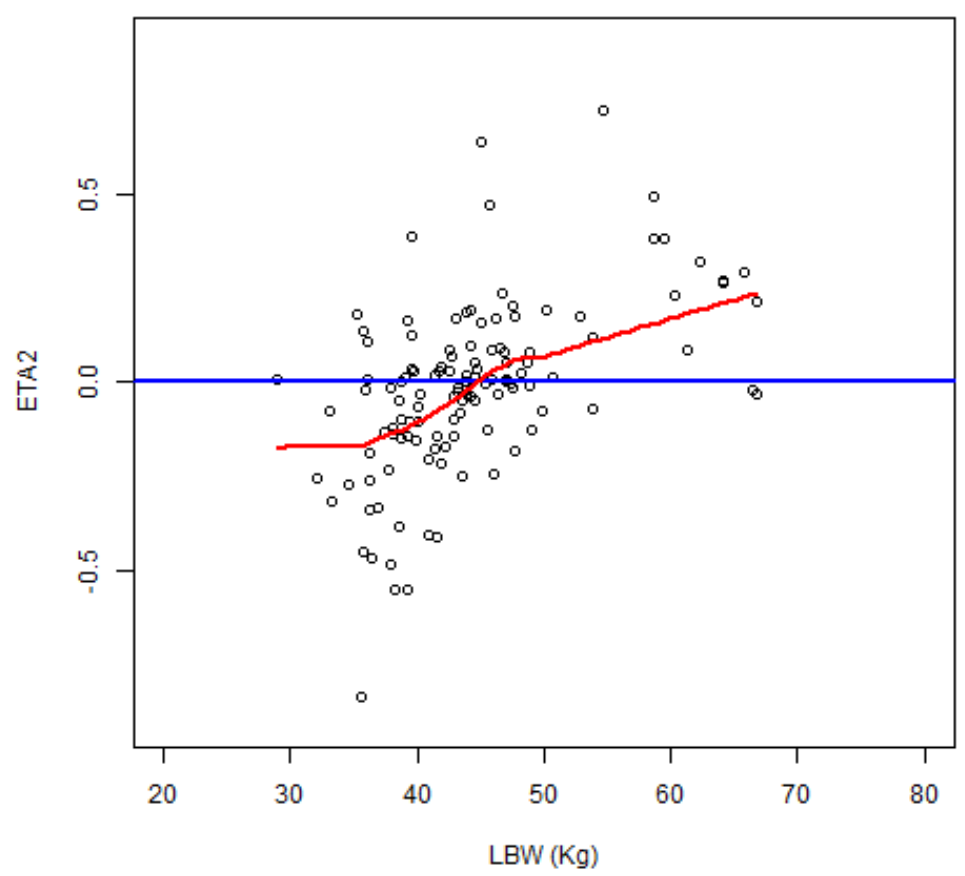

Figure 4.23 ETA2 $(\mathrm{Vd})$ versus lean body weight

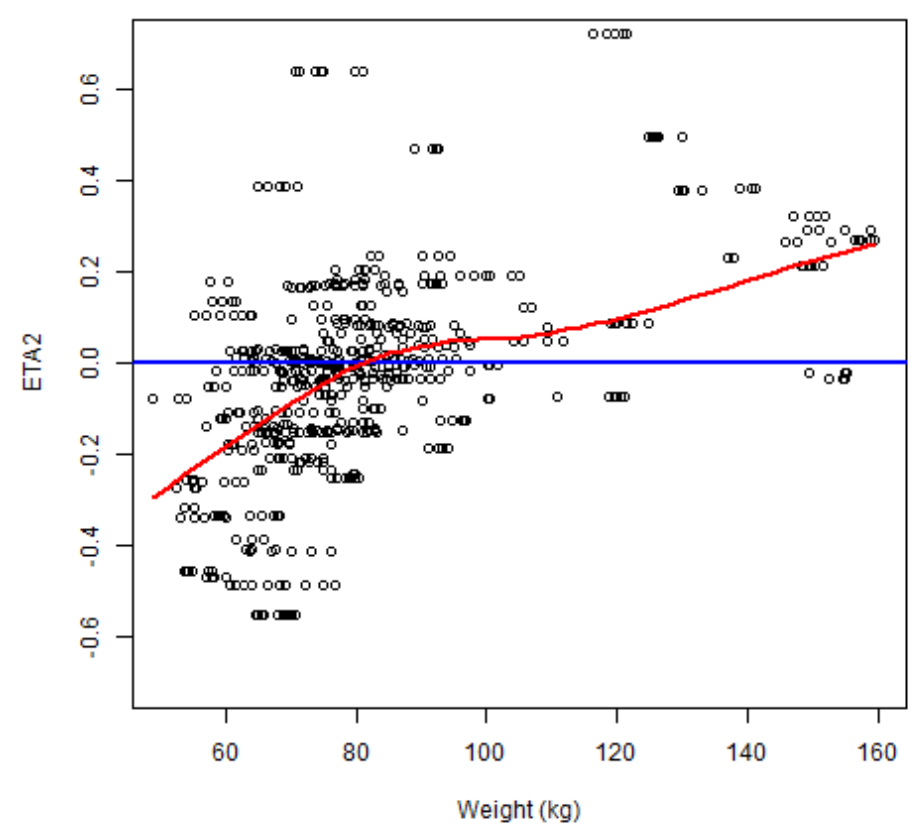

Figure 4.24: ETA2 (Vd) versus weight

Based on this analysis, weight and baseline lean body weight demonstrated trends with the random effects (ETAs) of $\mathrm{Vd}$ and warranted further investigation. Due to a lack of trend, age was not considered relevant and not considered further. Ethnicity once again showed a slight trend 
with groups 3 and 4 (Asian and 'other'), however, like for $\mathrm{CL}$, the numbers were considered too small, to consider this trend formally. Gestation was retained as a covariate for $\mathrm{Vd}$, as the interoccasion variability plot (figure 4.4(b)) clearly showed a change with the progression of pregnancy.

The identified covariates were next added to the base model in a uni-variate fashion. Each covariate was coded, so that it was centred around the median or mean of the covariate being evaluated, as is usual practice in PK modelling. For example creatinine (CRE) was centred around the value 60 . Table 4.7 describes how each covariate was coded and tested.

Table 4.7: How the different covariates were coded for evaluation into the base model

\begin{tabular}{lll}
\hline Covariate & $\mathrm{CL}$ & $\mathrm{Vd}$ \\
\hline $\mathrm{CrCl} / 110$ & $\mathrm{CL} \times(\mathrm{CrCl} / 110)^{\text {thetal }}$ & - \\
$\mathrm{Wt}^{\prime} 80$ & $\mathrm{CL} \times(\mathrm{Wt} / 80)^{\text {theta2 }}$ & $\mathrm{Vd} \times(\mathrm{Wt} / 80)^{\text {theta3 }}$ \\
Gest/MGest & $\mathrm{CL} \times(\mathrm{GEST} / \mathrm{MGEST})^{\text {theta4 }}$ & $\mathrm{Vd} \times(\mathrm{GEST} / \mathrm{MGEST})^{\text {theta5 }}$ \\
$\mathrm{CRE} / 60$ & $\mathrm{CL} \times(\mathrm{Cre} / 60)^{\text {theta6 }}$ & - \\
LBW/42 & $\mathrm{CL} \times(\mathrm{LBW} / 42)^{\text {theta7 }}$ & $\mathrm{Vd} \times(\mathrm{LBW} / 42)^{\text {theta8 }}$ \\
\hline
\end{tabular}

For gestation, centring occurred on MGEST, which represented the specific gestation at delivery for each subject

Table 4.8 presents the change in objective function with the uni-variant addition of each covariate into the base model. A decrease on objective function of $6.64(p<0.01)$ was required for the inclusion of the covariate into the intermediate model.

Table 4.8: Uni-variate addition of covariates to base model

\begin{tabular}{llllc}
\hline Model & Covariate & OBV & AOBV & $\begin{array}{c}\text { Minimisation } \\
\text { successful? }\end{array}$ \\
\hline Base & - & -2908.57 & - \\
\hline 105 & $\mathrm{CL} \times(\mathrm{CrCl} / 110)^{0.133}$ & -2040.14 & -31.57 & $\mathrm{Y}$ \\
80 & $\mathrm{CL} \times(\mathrm{Wt} / 80)^{1.22}$ & -2959.66 & -51.79 & $\mathrm{Y}$ \\
104 & $\mathrm{CL} \times(\mathrm{GEST} / \mathrm{MGEST})^{0.0666}$ & -2923.01 & -15.14 & $\mathrm{Y}$ \\
82 & $\mathrm{CL} \times(\mathrm{LBW} / 42)^{1.78}$ & -2944.63 & -36.76 & $\mathrm{Y}$ \\
92 & $\mathrm{CL} \times(\mathrm{CRE} / 60)^{-0.453}$ & -2919.12 & -11.25 & $\mathrm{Y}$ \\
84 & $\mathrm{Vd} \times(\mathrm{GEST} / \mathrm{MGEST})^{0.261}$ & -2923.49 & -15.62 & $\mathrm{Y}$ \\
83 & $\mathrm{Vd} \times(\mathrm{Wt} / 80)^{1.4 /}$ & -2942.46 & -34.59 & $\mathrm{Y}$ \\
85 & $\mathrm{Vd} \times(\mathrm{LBW} / 42)^{1.43}$ & -2921.06 & -13.19 & \\
\hline
\end{tabular}

$\triangle \mathrm{OBV}$ represents the change in the base model objective function with the addition of the covariate 
$\mathrm{CrCl} / 110$ and $\mathrm{CRE} / 60$ both significantly dropped the objective function on $\mathrm{CL}$, and therefore a case could be made to have either included in an intermediate model. However, it made no physiological sense to have both included; therefore as $\mathrm{CRE}$ is already considered as part of $\mathrm{CrCl}$ (in the Cockcroft-Gault equation) and the objective function drop was greater with $\mathrm{CrCl}$ than with CRE, CRE was dropped as a covariate at this stage of the model building process. The other covariate which required special consideration was how weight and baseline lean body weight would be incorporated into the intermediate and final models. Both were found to be significant covariates for both $\mathrm{CL}$ and $\mathrm{Vd}$.

Based on the uni-variate covariate analysis, four competing models were developed and tested, table 4.9.

Table 4.9: Addition of covariates in different combinations, led to four competing models

\begin{tabular}{lll}
\hline Model & Intermediate Model Description & OBV \\
\hline 401 & LBW/42 + CrCl + Gest/MGest on CL & -3039.899 \\
& LBW/42 + Gest/MGest on Vd & \\
402 & Wt/80 + CrCl + Gest/MGest on CL & -3051.622 \\
& Wt/80 + Gest/MGest on Vd & \\
403 & $\mathrm{LBW} / 42+\mathrm{CrCl}+$ Gest/MGest on CL & -3040.002 \\
& Wt/80 + Gest/MGest on Vd & \\
404 & Wt/80 + CrCl + Gest/MGest on CL & -3051.039 \\
\hline
\end{tabular}

Although intermediate model 402 has the lowest objective function, intermediate model 404 was the model chosen to take forward, as enoxaparin is a hydrophilic drug and it made physiological sense to have LBW on $\mathrm{Vd}$, compared to model 402 which had weight on both $\mathrm{CL}$ and $\mathrm{Vd}$; furthermore the objective function difference between both models was only -0.583 .

A backward elimination of covariates from the intermediate model 404 in a uni-variate fashion was then conducted (table 4.10). An increase in the objective function of greater than $10.82(p<0.001)$ was required for the covariate being evaluated to be retained in the final model. 
Table 4.10: Backward elimination of covariates from model 404 (full model)

\begin{tabular}{lllll}
\hline Model & Covariate removed & OBV & $\Delta$ OBV & Retain? \\
\hline 404 & Full model & -3051.039 & - & - \\
431 & CrCl on CL & -3047.004 & 4.035 & $\mathrm{~N}$ \\
432 & Gest/MGEST on CL & -3028.797 & 22.242 & $\mathrm{Y}$ \\
433 & Wt/80 on CL & -3010.590 & 40.449 & $\mathrm{Y}$ \\
434 & Gest/MGEST on Vd & -3010.948 & 40.091 & $\mathrm{Y}$ \\
435 & LBW/42 on Vd & -3036.259 & 14.780 & $\mathrm{Y}$ \\
436 & Model 431 - GEST/MGEST on CL & -3017.235 & 29.769 & $\mathrm{Y}$ \\
437 & Model 431 - LBW/42 on Vd & -3032.720 & 14.284 & $\mathrm{Y}$ \\
\hline
\end{tabular}

$\triangle \mathrm{OBV}$ represents the change in the final model objective function with the removal of each covariate.

$\mathrm{CrCl}$ was not retained in the final model, as its exclusion from the final model caused an increase in objective function of only 4.035 . Following this, the two covariates with the smallest increases in objective function, following their removal from the final full model (404), were re-evaluated to ensure their continued relevance. Therefore model 431 became the final model and gestation on $\mathrm{CL}$ and lean body weight on Vd were removed singularly, and they both continued to demonstrate their relevance in the final model, with $\triangle \mathrm{OBJ}$ changes of 29.769 and 14.284 respectively. Therefore the final antenatal enoxaparin model was model 431.

The final pharmacokinetic model for enoxaparin during pregnancy can be represented mathematically as:

$\mathrm{CL}=\mathrm{POPCL} \times \mathrm{GESTCL} \times(\mathrm{Wt} / 80)^{\mathrm{FACWTCL}}$

$V d=P O P V d \times$ GESTV $\times(\text { LBW/42) })^{\text {FACLBWV }}$

where during pregnancy:

GESTCL $=1$

GESTV $=(\text { GEST/MGEST })^{\text {FACGESTV }}$

and post delivery:

GESTCL $=(\text { GEST/MGEST })^{\text {FACGESTCL2 }}$

GESTV $=(\text { GEST/MGEST })^{\text {FACGESTV2 }}$ 


\subsubsection{Evaluation of the final model}

In order to evaluate the final model (NONMEM code: appendix VIII), a nonparametric bootstrap was completed; the specific code used for this in listed in appendix IX. This along with the final model parameter estimates are presented in table 4.11.

Table 4.11: Bootstrap results of the final PK model developed

\begin{tabular}{|c|c|c|c|c|}
\hline \multirow[t]{2}{*}{ Parameter } & \multirow{2}{*}{\multicolumn{2}{|c|}{ Model Estimate $(95 \% \mathrm{Cl})$}} & \multicolumn{2}{|c|}{1000 Bootstrap } \\
\hline & & & Median & $2.5^{\text {th }}-97.5^{\text {th }}$ \\
\hline CL (I/hr) & 1.60 & $(1.47-1.73)$ & 1.60 & $1.48-1.74$ \\
\hline V (I) & 13.2 & $(10.93-15.47)$ & 13.4 & $10.3-15.8$ \\
\hline $\mathrm{Ka} \quad\left(\mathrm{hr}^{-1}\right)$ & 0.578 & $(0.428-728)$ & 0.586 & $0.43-0.77$ \\
\hline$\omega C L(\% \mathrm{cv})$ & 31 & $(11-51)$ & 31 & $24-38$ \\
\hline$\omega V \quad(\% c v)$ & 29 & $(1-57)$ & 26 & $5-41$ \\
\hline FACGESTV & 0.357 & $(0.212-0.502)$ & 0.359 & $0.235-0.503$ \\
\hline FACGESTV2 & -5.49 & $(-2.73(-)-8.25)$ & -5.37 & $-28.79-0.78$ \\
\hline FACGESTCL2 & -4 & $(-2.76(-)-5.24)$ & -4.26 & $-9.28-(-) 1.55$ \\
\hline FACWTCL & 1.06 & $(0.78-1.34)$ & 1.06 & $0.76-1.39$ \\
\hline FACLBWV & 1.25 & $(0.47-2.03)$ & 1.27 & $0.42-2.03$ \\
\hline Add error (IU/mL) & 0.0660 & $(0.06449-0.06751)$ & 0.0656 & $0.0536-0.0761$ \\
\hline Prop error (\%) & 18 & $(4-32)$ & 18 & $12-24$ \\
\hline
\end{tabular}

The inclusion of these covariates in the final model led to a decrease in between subject variability in CL from $46 \%$ to $31 \%$ (a drop of $15 \%$ ) and a decrease in between subject variability in Vd from $35 \%$ to $29 \%$ (a drop of $6 \%$ ). Figures 4.25 ( $a$ and $b$ ), and 4.26 ( $a$ and $b$ ) illustrate the ETA trend change, with the covariate being incorporated into the final model; before (top), after (bottom). 

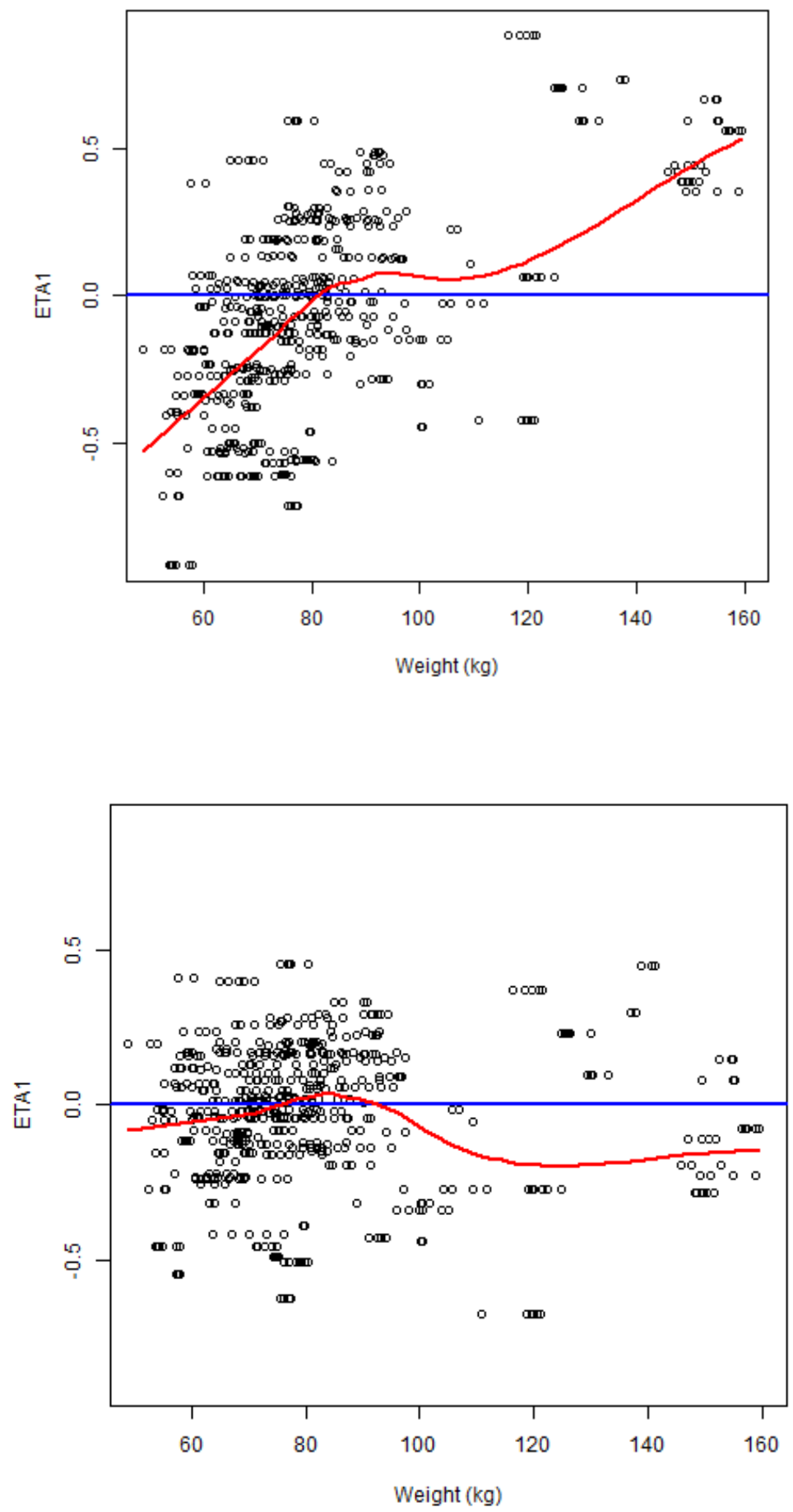

Figure 4.25 (a) and (b): ETA1 (CL) versus weight, before (top) and after (bottom) inclusion into the final model 

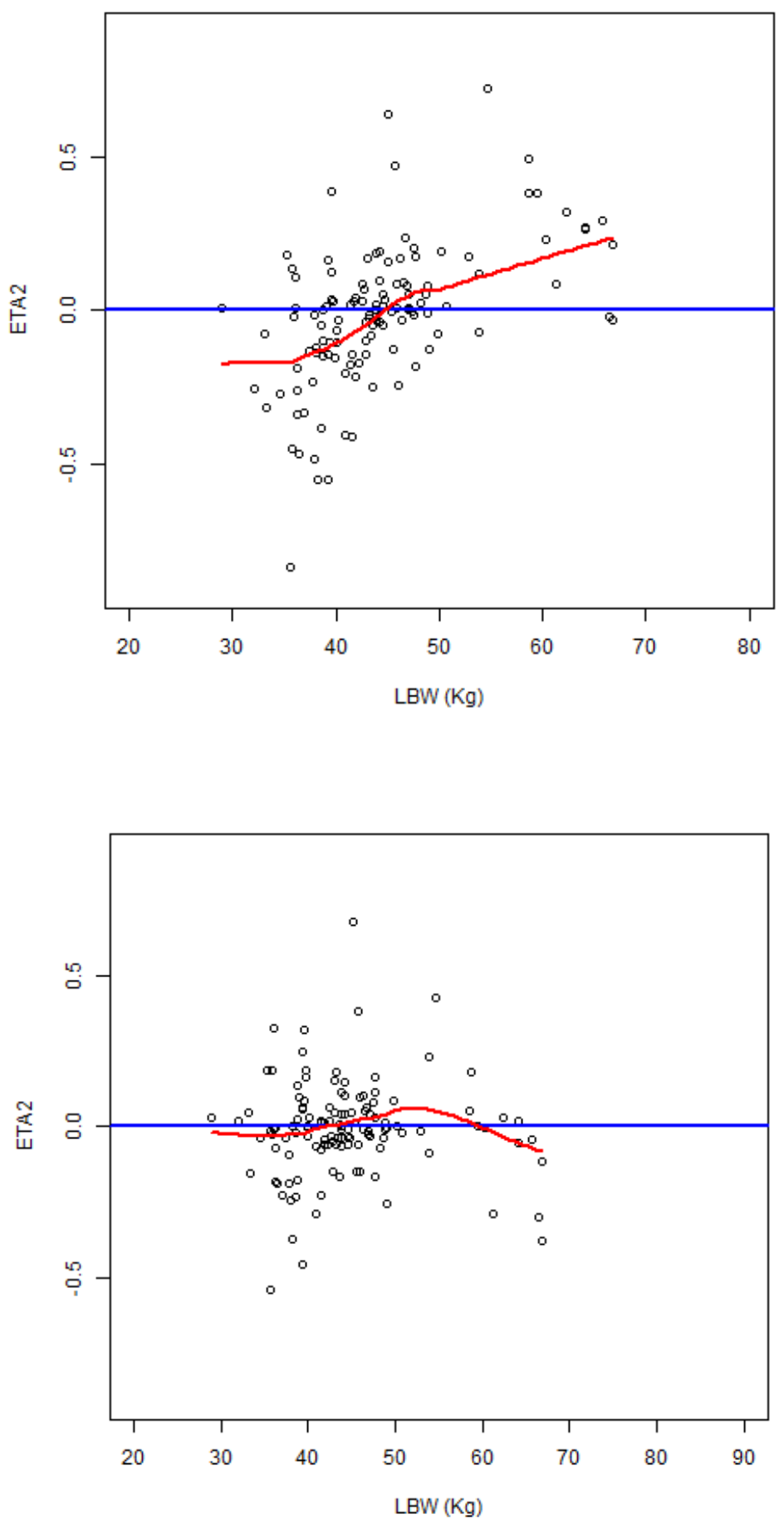

Figure $4.26(\mathrm{a})$ and $(\mathrm{b})$ : ETA2 (Vd) versus LBW, before (top) and after (bottom) inclusion into the final model

The plots demonstrate the relevance of including these covariates into the final model, as with their inclusion, the systematic trends observed in random effects are removed. 


\subsubsection{Visual predictive check of final PK model}

Finally a visual predictive check (VPC) was conducted to ensure the final model developed was capturing the observed data. The 'R' code for the VPC plot is available in appendix X. For all VPCs, 100 datasets that mirrored the demographics of subjects in this clinical study were simulated using the final model parameter estimates. The $5^{\text {th }}, 50^{\text {th }}$ and $95^{\text {th }}$ prediction intervals from the simulated concentrations were plotted against time after dose, with the observed data superimposed. Figure 4.27 illustrates the VPC from the final model, with the red lines representative of the $5^{\text {th }}, 50^{\text {th }}$ and $95^{\text {th }}$ percentile from the observed data and the blue lines representing the $5^{\text {th }}, 50^{\text {th }}$ and $95^{\text {th }}$ percentiles from the simulated data set. As can be seen, the final model is describing the observed data extremely well. Figures 4.28 (a-d) demonstrate the VPC's when broken down by trimester.

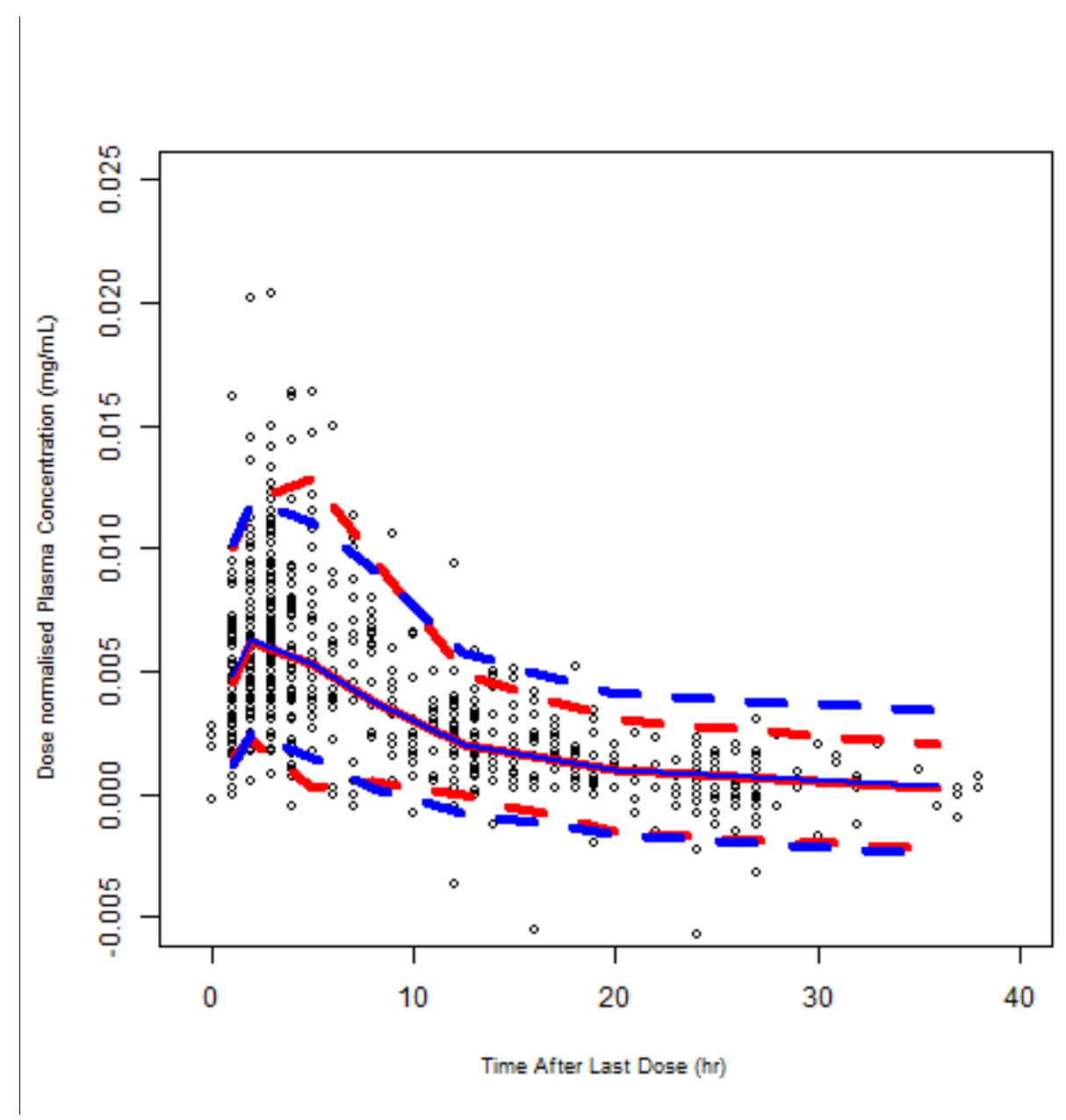

Figure 4.27: VPC of dose normalised plasma concentrations versus time after last dose 

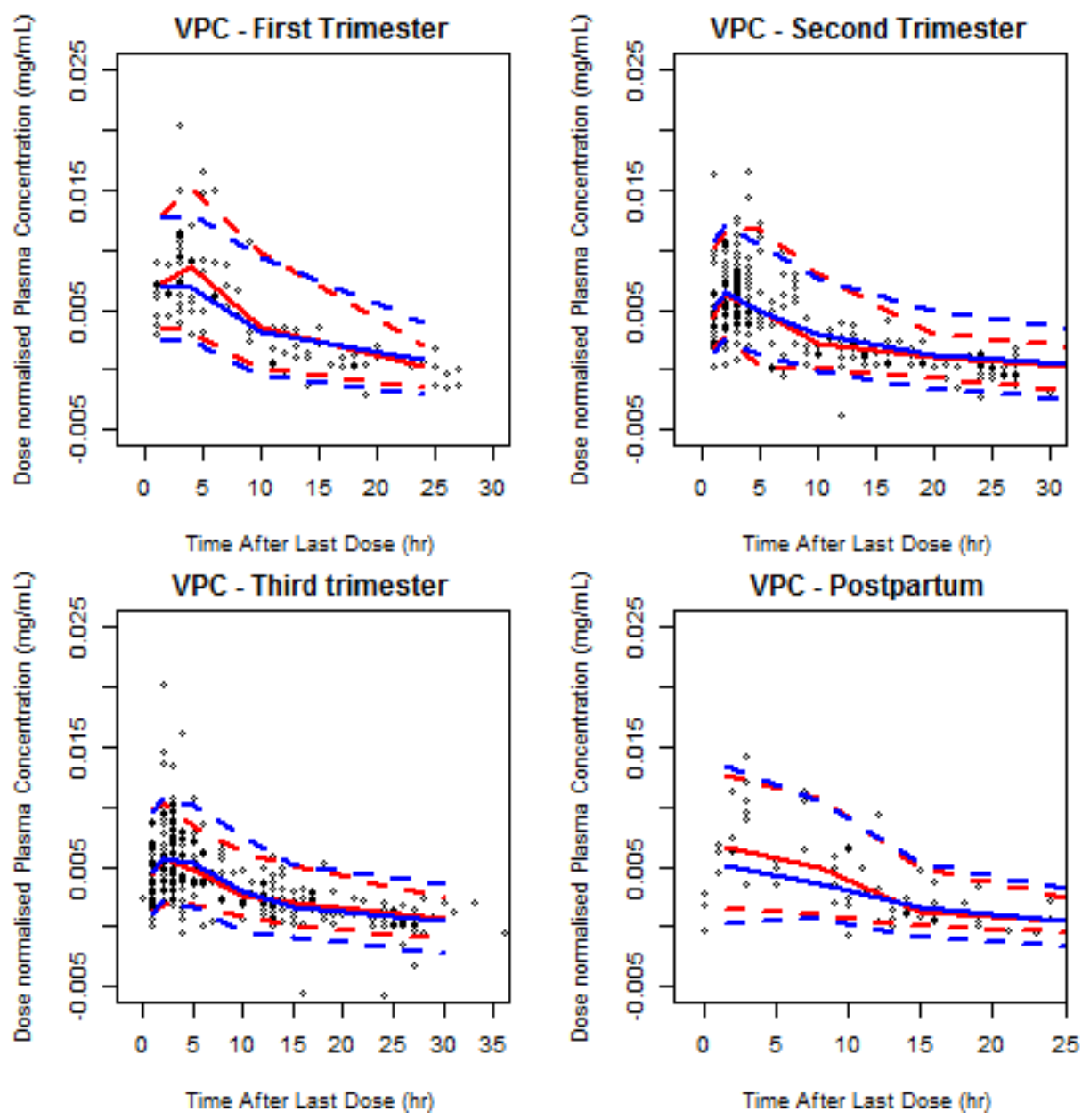

Figure 4.28: VPC breakdown according to each trimester; $1^{\text {st }}$ trimester ((a)top left), $2^{\text {nd }}$ trimester ((b)top right), $3^{\text {rd }}$ trimester ((c)bottom left) and postpartum ((d)bottom right)

The overall VPC and the trimester specific VPC's confirm that a robust final enoxaparin antenatal model had been developed.

\subsubsection{Simulation work}

The aim of the simulation work was to simulate the important 'what if' questions of clinical practice. As part of this, a weight model, describing weight changes over the course of pregnancy needed development. The evolution of this weight model followed the same method principles of development and evaluation as the enoxaparin antenatal model.

\subsubsection{Developing a weight model}

In the first instance, a sub-data file was created from the main file, which listed each subject's weight at each clinic visit, from initial recruitment to when they attended the haematology clinic around eight weeks post-partum for their final clinic visit. Within the data file, each weight entry 
had an associated gestation time listed (weeks amenorrhea), along with the subject's baseline ethnicity and lean body weight.

Initially, three different weight models were explored as suitable base models, differing in the error structure within each model; additive, proportional and combined (additive and proportional)). For all three models, an ETA was added to the exponent describing gestational weight change over pregnancy (FACGESTWT1), with a further ETA added onto baseline weight (BSWT). The OBJ function and parameter estimates from these three models are listed in table 4.12.

Table 4.12: Results of weight models with three error structures

\begin{tabular}{|c|c|c|c|c|c|c|}
\hline Base weight model & \multicolumn{2}{|c|}{$\begin{array}{c}10 \text { Additive error } \\
(\% \mathrm{SE})\end{array}$} & \multicolumn{2}{|c|}{$\begin{array}{c}11 \text { Proportional error } \\
\text { (\%SE) }\end{array}$} & \multicolumn{2}{|c|}{$\begin{array}{c}12 \text { Combined error } \\
\text { (\%SE) }\end{array}$} \\
\hline OBJ & 319 & 211 & 299 & .237 & 297 & 9.109 \\
\hline Baseline weight $\theta_{1}$ & 82.3 & (2.1) & 83.1 & $(2.2)$ & 83.1 & $(2.2)$ \\
\hline FACGESTWT1 $\theta_{2}$ & 0.0569 & (8.3) & 0.0709 & $(7.9)$ & 0.0692 & $(8.8)$ \\
\hline FACGESTWT2 $\theta_{3}$ & -0.313 & (7.3) & -0.350 & $(7.0)$ & -0.345 & $(7.2)$ \\
\hline wBSWT (CV\%) & 25 & $(15.0)$ & 25 & $(14.8)$ & 25 & $(14.8)$ \\
\hline wFACGESTWT1 (CV\%) & 31 & $(23.1)$ & 60 & $(22.2)$ & 63 & $(23.1)$ \\
\hline Additive error (kg) & 10.1 & $(31.3)$ & - & & 3.32 & $(29.3)$ \\
\hline Proportional error (\%) & - & & 3 & (9.7) & 2 & $(44.0)$ \\
\hline
\end{tabular}

As there was little to choose between these three models, all three were considered for covariate analysis.

Next consideration was given to covariates and which ones should to be included in the final weight model developed. Based on observations in clinic, whereby different ethnic groups appeared to have different weight categories, e.g. patients from a south Asian origin were smaller (in terms of weight) than those of African-Caribbean origin; which could be significant. Age was not explored as a covariate, even though it might be in other settings, due to the tight age range of the population followed in this study (mean age 33 (range 18-46)). Figure 4.29 illustrates the weight differences between the four different ethnic groups ( 1 refers to Caucasian, 2 refers to African-Caribbean, 3 refers to Asian and 4 refers to 'other' group). 


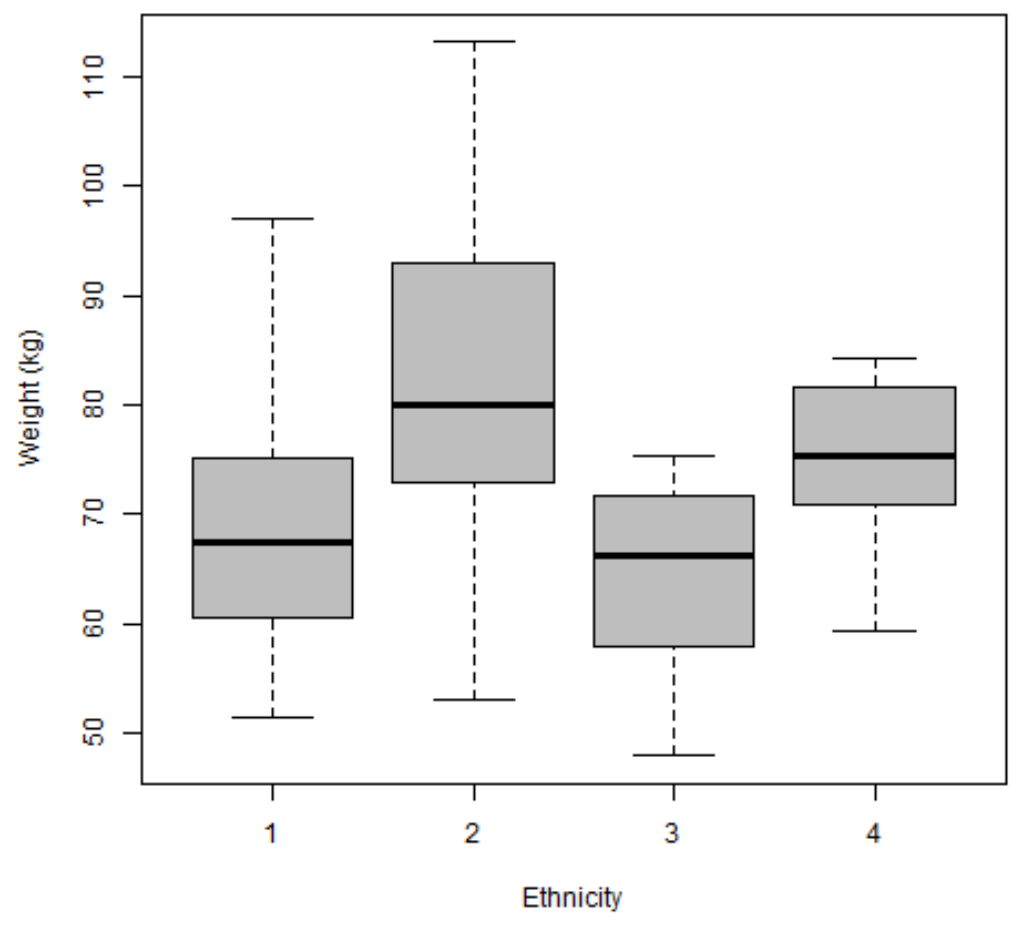

Figure 4.29: box-plot of the first recorded weight for each of subject within each ethic group (1 represents the Caucasian group, 2 represents the African-Caribbean group, 3 represents the Asian group and 4 represents the 'other' group)

As illustrated by figure 4.29, the Asian group weight is much lower (in terms of the spread), relative to the Caucasian and African-Caribbean groups. This suggested that ethnicity should be considered for the weight model and so was added as a covariate to the three base models with different error structures (table 4.13). 
Table 4.13: Results with the incorporation of ethnicity into the base model

\begin{tabular}{|c|c|c|c|c|c|c|}
\hline \multirow{3}{*}{$\begin{array}{l}\text { Model } \\
\text { OBJ } \\
\text { Caucasian } \theta_{1}\end{array}$} & \multicolumn{2}{|c|}{19 - Additive (SE\%) } & \multicolumn{2}{|c|}{19 - Proportional (SE\%) } & \multicolumn{2}{|c|}{19 - Combined (SE\%) } \\
\hline & \multicolumn{2}{|c|}{2959.284} & \multicolumn{2}{|c|}{2979.305} & \multicolumn{2}{|c|}{2950.785} \\
\hline & 79.6 & (3.0) & 79.6 & (3.1) & 79.6 & (3.0) \\
\hline Black $\theta_{2}$ & 91.5 & (3.7) & 91.6 & (3.8) & 91.6 & (3.7) \\
\hline Asian $\theta_{3}$ & 69.6 & $(4.0)$ & 69.6 & $(4.0)$ & 69.6 & $(4.0)$ \\
\hline Other $\theta_{4}$ & 85.5 & (6.3) & 85.5 & (6.3) & 85.6 & (6.3) \\
\hline FACGESTWT1 $\theta_{5}$ & 0.0768 & (8.0) & 0.0787 & (7.0) & 0.0782 & (7.5) \\
\hline FACGESTWT2 $\theta_{6}$ & -0.322 & $(9.2)$ & -0.342 & (7.6) & -0.335 & (8.1) \\
\hline$\omega B S W T(\%)$ & 23 & (15.9) & 23 & (15.9) & 23 & (15.9) \\
\hline wFACGESTWT1 (\%) & 5 & $(17.5)$ & 4 & (19.5) & 4.5 & $(18.0)$ \\
\hline Additive error (kg) & 5.63 & (9.6) & - & & 3.95 & $(65.1)$ \\
\hline Proportional error (\%) & - & & 3.05 & (9.9) & 1.5 & $(26.3)$ \\
\hline
\end{tabular}

Although the combined error model was the model with the lowest objective function, the standard errors around the additive and proportional errors were large, relative to the singular additive and proportional error models. Therefore the additive error model was the model chosen to take forward, as it demonstrated a lower objective function relative to the proportional error model.

Next each ethnic group was tested in this additive error model, with an ETA added on each ethnic group in a uni-variate fashion (models $30-33$ ). Tables 4.14, lists the OBJ functions for these four models. 
Table 4.14: Assessment of each ethnic group in a uni-variate fashion

\begin{tabular}{|c|c|c|c|c|c|c|c|c|}
\hline Model & \multicolumn{2}{|c|}{$\begin{array}{c}30-\text { Asian } \\
(\mathrm{SE} \%)\end{array}$} & \multicolumn{2}{|c|}{$\begin{array}{c}31 \text { - Caucasian } \\
\text { (SE \%) }\end{array}$} & \multicolumn{2}{|c|}{$\begin{array}{c}32 \text { - African- } \\
\text { Caribbean (SE \%) }\end{array}$} & \multicolumn{2}{|c|}{$\begin{array}{c}33 \text { - Other } \\
\text { (SE \%) }\end{array}$} \\
\hline OBJ & \multicolumn{2}{|c|}{2756.768} & \multicolumn{2}{|c|}{2759.749} & \multicolumn{2}{|c|}{2760.830} & \multicolumn{2}{|c|}{2760.649} \\
\hline Caucasian $\theta_{1}$ & 79.5 & (3.0) & 79.4 & (3.0) & 79.5 & (3.0) & 79.5 & (3.0) \\
\hline Black $\theta_{2}$ & 91.4 & (3.7) & 91.4 & (3.7) & 91.4 & (3.7) & 91.4 & (3.7) \\
\hline Asian $\theta_{3}$ & 69.8 & $(4.0)$ & 69.7 & $(4.0)$ & 69.7 & $(4.0)$ & 69.7 & $(4.0)$ \\
\hline Other $\theta_{4}$ & 85.3 & $(6.4)$ & 85.4 & (6.4) & 85.3 & $(6.4)$ & 85.4 & (6.3) \\
\hline FACGESTWT1 $1 \theta_{5}$ & 0.0735 & $(9.2)$ & 0.0734 & (9.3) & 0.0735 & $(9.3)$ & 0.0734 & $(9.3)$ \\
\hline FACGESTWT2 $\theta_{6}$ & -0.319 & $(9.2)$ & -0.318 & (9.2) & -0.318 & $(9.2)$ & -0.318 & $(9.2)$ \\
\hline wBSWTEthn1 (\%) & 24 & $(16.1)$ & 22 & (23.6) & 23 & (20.2) & 24 & $(16.4)$ \\
\hline wBSWTEthn2 (\%) & 11 & $(44.5)$ & 25 & $(21.4)$ & 23 & (25.3) & 21 & $(66.1)$ \\
\hline$\omega F A C G E S T 1(\%)$ & 4 & $(29.8)$ & 4 & $(30.1)$ & 4 & (30.2) & 4 & $(30.2)$ \\
\hline Additive error (kg) & 11.4 & $(49.5)$ & 11.4 & $(49.5)$ & 11.4 & $(49.5)$ & 11.4 & $(49.5)$ \\
\hline
\end{tabular}

Weight model 30 (with a separate ETA on the Asian group) was the best performing model and so was the weight model used for the purposes of simulation. For completeness, a VPC plot analysis of this model was completed (figure 4.30). 


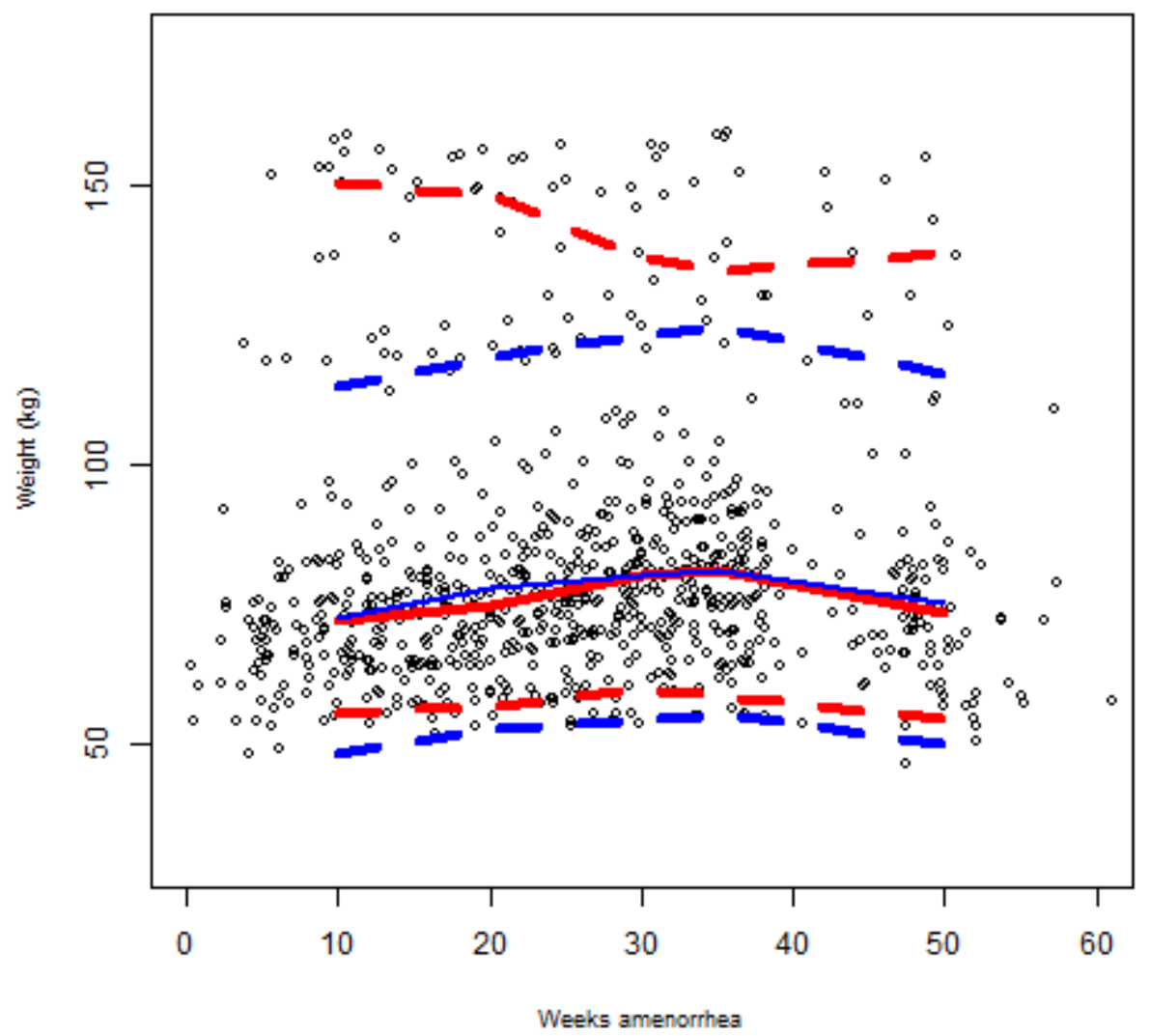

Figure 4:30: VPC for the final weight model developed for simulation purposes. The red lines represent the $5^{\text {th }}, 50^{\text {th }}$ and $95^{\text {th }}$ percentiles from the observed data and the blue lines represent the $5^{\text {th }}, 50^{\text {th }}$ and $95^{\text {th }}$ percentiles from the simulated data set

Overall, the weight model is performing well, demonstrated by the median observed and simulated concentrations. There are discrepancies at the extremes, in the $5^{\text {th }}$ and $95^{\text {th }}$ percentile values, and are likely to be reflective of the large variation in weight gain and loss observed in some women during pregnancy.

\subsubsection{Simulations}

Next simulations based on the the final enoxaparin antenatal model developed were done, and compared once daily enoxaparin (according to the King's College Hospital non-pregnancy guidelines) to twice daily enoxaparin (according to the RCOG antenatal VTE guidelines). The simulations assumed that the dose was not altered through pregnancy and all doses were taken (injected). Figure 4.31 (a) and (b) illustrates the three hour anti-Xa activity of enoxaparin when women are given these two regimens from day one of their pregnancy and they continue to receive this dose and dosing regimens until they deliver at 40 weeks gestation. 


\section{3 hour concentrations - Once daily enoxaparin}

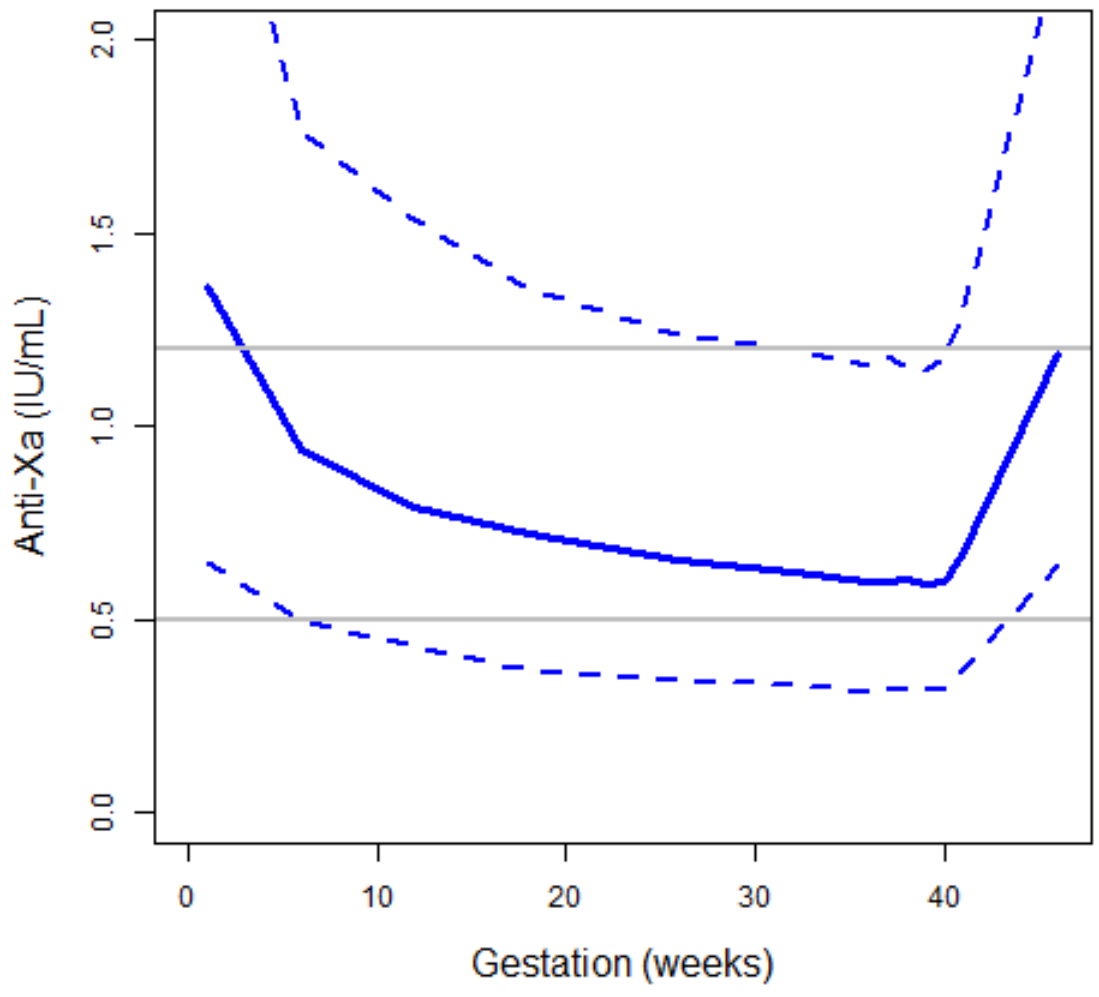

3 hour concentrations - Twice daily enoxaparin

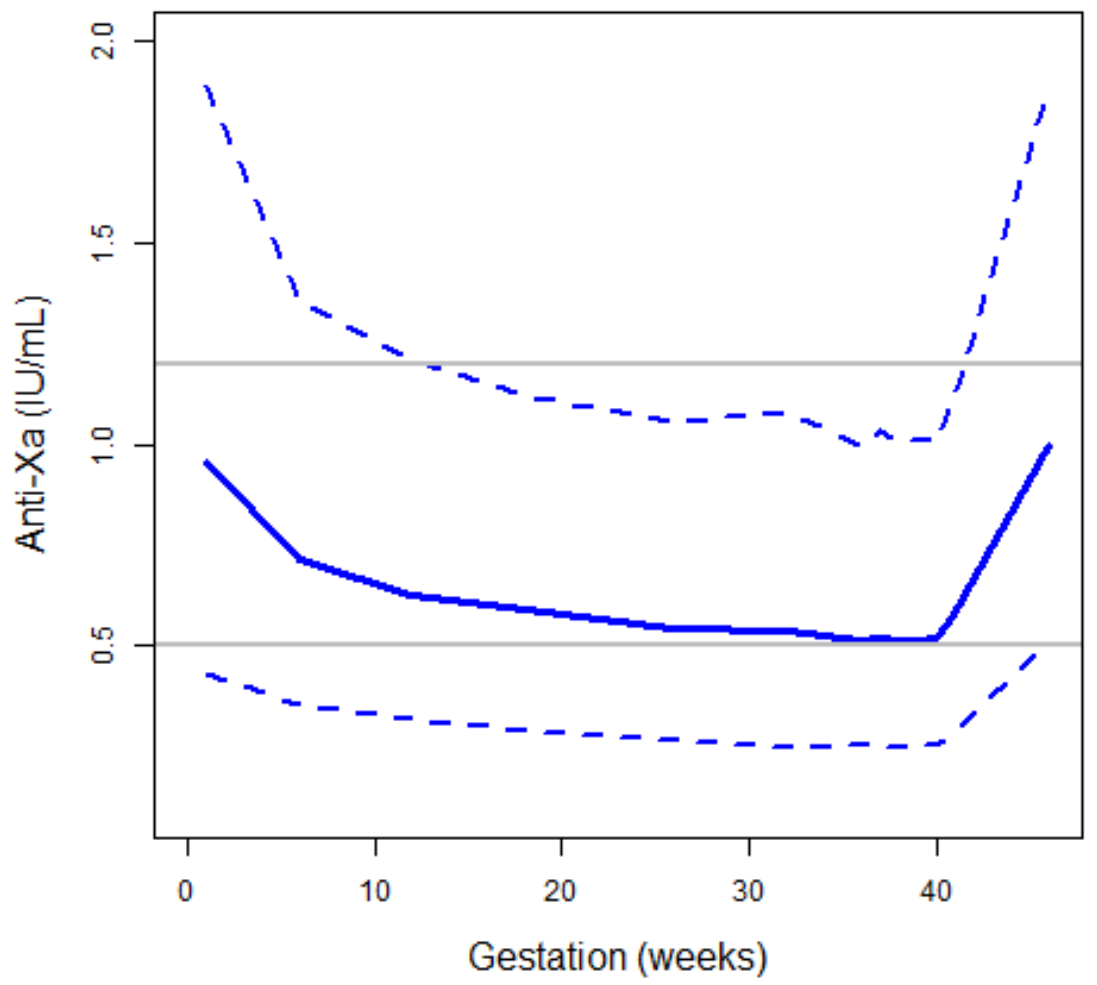

Figure 4.31 (a) and (b): 3 hour anti-Xa activity of enoxaparin during pregnancy, once (top) versus twice a day (bottom). RCOG target anti-Xa activity represented by grey horizontal lines (0.5-1.2 $\mathrm{IU} / \mathrm{mL}$ ). Dashed lines represent the $5^{\text {th }}$ and $95^{\text {th }}$ percentile anti-Xa concentrations 
These plots demonstrate that with both regimens, the three hour anti-Xa activity decreases with gestational age. However, they also demonstrate that for both regimens, the 3 hour peak activity remains within the RCOG desired range of $0.5-1.2 \mathrm{IU} / \mathrm{mL}$ (grey horizontal lines) throughout pregnancy. This suggests that the once daily dosing regimen would be acceptable in this setting, based on current recommendations. However, historically, it's thought that LMWH CL increases during pregnancy, and so pregnant women need to receive a higher and/or more frequent dose. Therefore, the more important concentration to evaluate, is the trough concentration, i.e. before the next dose is due, to assess if LMWH activity exists, before the next dose is injected. When the trough anti-Xa activities are simulated for the two regimens, the results presented in figure 4.32 (a) and (b) are found, suggesting that the majority of women will posses residual anti-Xa activity before the next dose is injected.

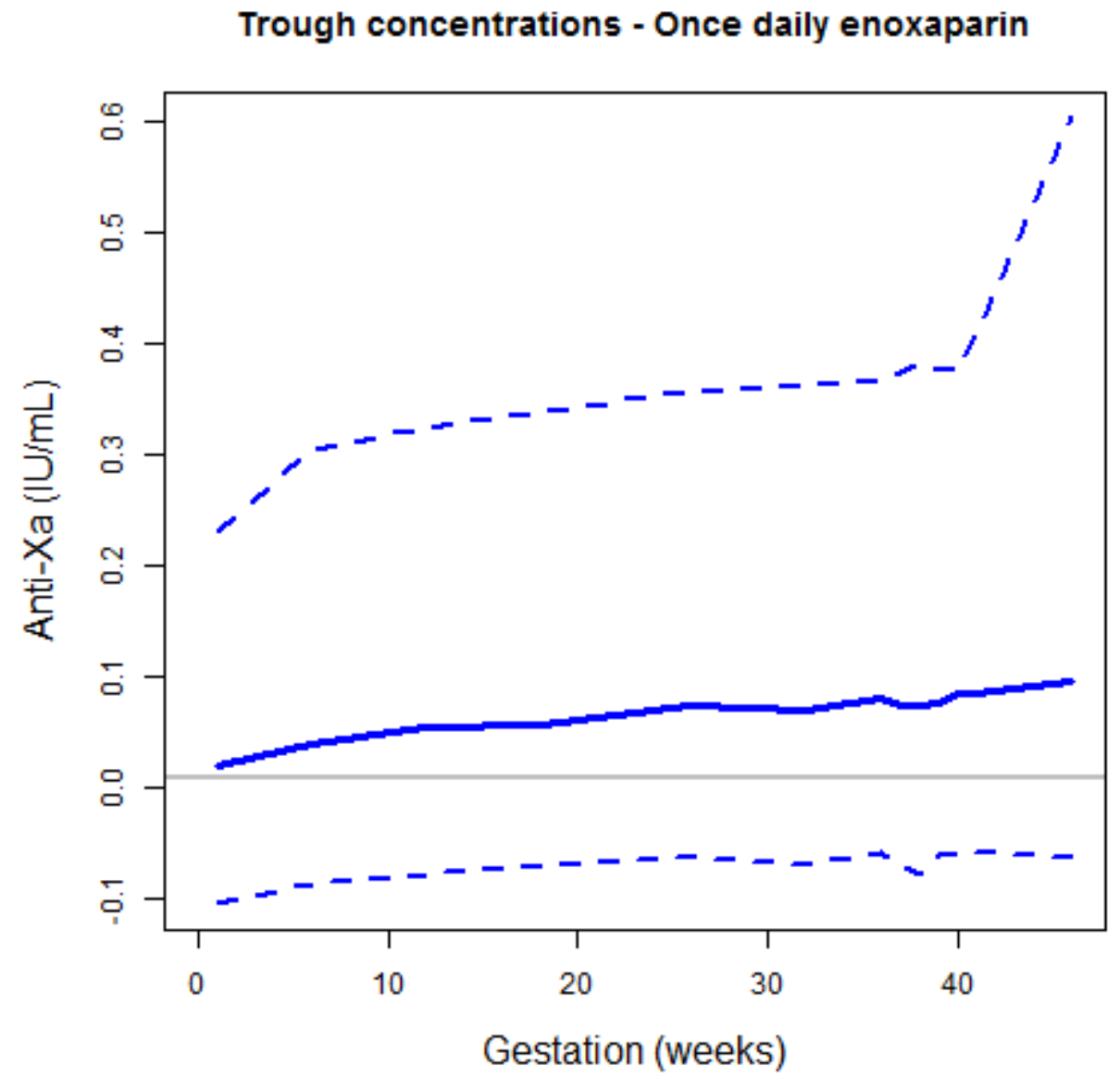

Figure 4.32 (a): Trough anti-Xa activity, once daily enoxaparin, dashed lines represent the $5^{\text {th }}$ and $95^{\text {th }}$ percentile anti-Xa concentrations 
Trough concentrations - Twice daily enoxaparin

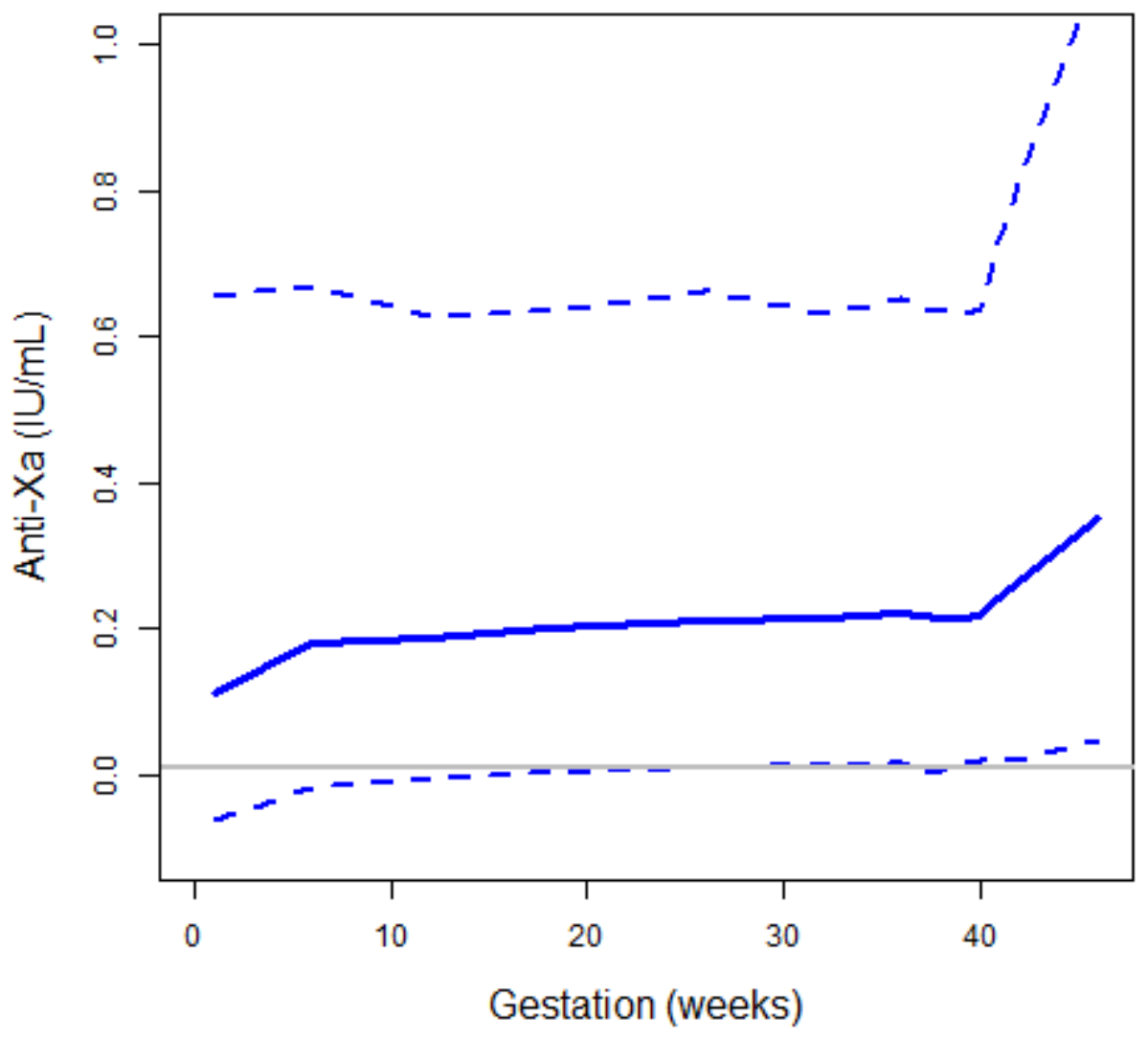

Figure 4.32 (b): Trough anti-Xa activity, twice daily enoxaparin, dashed lines represent the $5^{\text {th }}$ and $95^{\text {th }}$ percentile anti-Xa concentrations

Combining the results and figures 4.31 and 4.32 , the 3 hour and trough plots for once versus twice daily are illustrated in figures 4.33 (a) and (b). 

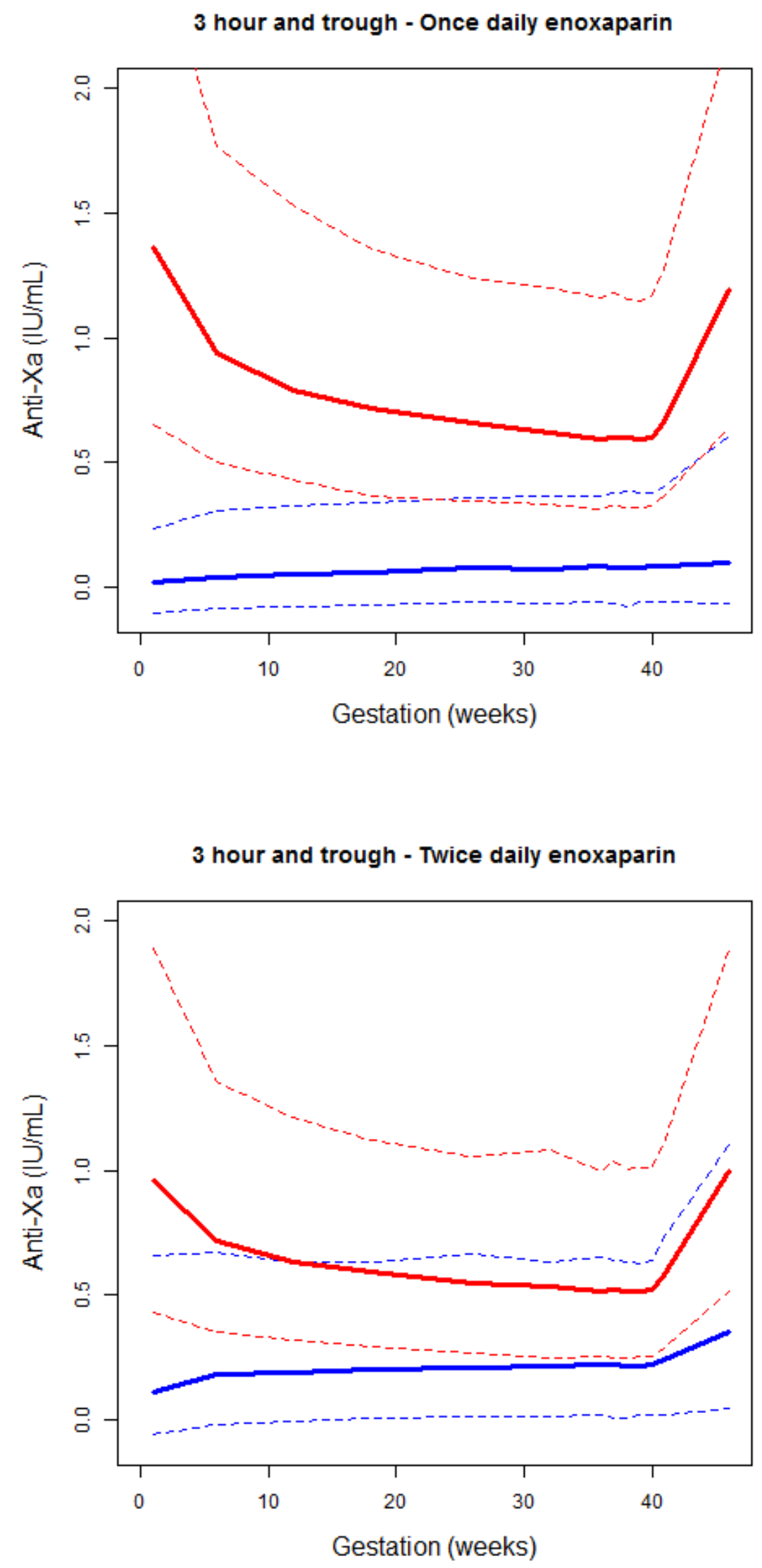

Figure 4.33 (a) and (b): 3 hour and trough anti-Xa activity; once (top) versus twice daily (bottom) enoxapain. The red lines represent the $5^{\text {th }}, 50^{\text {th }}$ and $95^{\text {th }}$ percentile 3 hour anti-Xa activity associated with both regimens. The blue lines represent the $5^{\text {th }}, 50^{\text {th }}$ and $95^{\text {th }}$ percentile concentrations associated with just the trough activity 
The trough activity of both the once and twice daily regimens increases with the progression of pregnancy. Most of the women on the once daily regimen have measurable anti-Xa activity at the trough time point and demonstrates that once daily enoxaparin would be adequate for this purpose in this setting.

\subsubsection{Percentage of women with a trough concentration $<0.01 \mathrm{IU} / \mathrm{mL}$ with these regimes}

The simulation work shows that there will be a percentage of women who would not have a measurable anti-Xa activity at the trough time point. The number of women this would apply to was quantified in ' $R$ ' and presented in table 4.15.

Table 4.15: \% of women who have enoxaparin concentrations BLQ

\begin{tabular}{ccc}
\hline Gestation (weeks) & Once daily regimen $(\%<0.01 \mathrm{IU} / \mathrm{mL})$ & Twice daily regimen $(\%<0.01 \mathrm{IU} / \mathrm{mL})$ \\
\hline 1 & 44.87 & 18.53 \\
6 & 33.98 & 9.18 \\
12 & 29.51 & 6.99 \\
18 & 29.67 & 6.01 \\
26 & 24.39 & 4.87 \\
32 & 24.06 & 4.87 \\
36 & 23.25 & 4.55 \\
37 & 25.28 & 5.53 \\
38 & 25.36 & 5.77 \\
39 & 23.65 & 4.14 \\
40 & 21.62 & 4.30 \\
\hline
\end{tabular}

The clinical implications of not having a measurable anti-Xa activity at the trough time point are not known. For the once daily group, approximately $25 \%$ of women will have a trough activity below $0.01 \mathrm{IU} / \mathrm{mL}$ compared to $5 \%$ of women in the twice daily group. On the other hand, the results also demonstrate that the majority of women will have anti-Xa activity when the next dose of enoxaparin is injected, even with the once daily regimen. 


\subsection{Discussion}

The results from this study demonstrate that both $C L$ and $V d$ of enoxaparin are significantly altered during the antenatal period. The estimates for enoxaparin $\mathrm{CL}$ and $\mathrm{Vd}$ are markedly different to previously published enoxaparin compartmental models published from a nonpregnant population (table 4.16), and demonstrate the impact the physiological changes of pregnancy has on enoxaparin PK. It should be noted that the study by Green and colleagues [2003] had a higher CL and Vd value, relative to the other studies; this is likely because they were specifically assessing enoxaparin PK in an obese population and therefore the higher fixed effects parameter estimates are reflective of this.

Table 4.16: Estimates for enoxaparin Vd and CL from other pop PK studies of enoxaparin

\begin{tabular}{llcc}
\hline Study & $\begin{array}{l}\text { Compartmental or non- } \\
\text { compartmental }\end{array}$ & CL (L/hr) & Vd (L) \\
\hline Casele (1999) & Non-compartmental & 0.6 & 3.10 \\
Green (2003) & Compartmental & 1.03 & 16.77 \\
Hulot (2005) & Compartmental & 0.74 & 5.29 \\
Bruno (2003) & Compartmental & 0.73 & 5.24 \\
Lebaudy (2008)* & Compartmental & 0.52 & 7.03 \\
Barrass (2009) & Compartmental & 0.72 & 9.20 \\
\hline
\end{tabular}

*Only other population PK study of enoxaparin during pregnancy

Some of the compartmental analysis completed to date, e.g. Green and colleagues [2003] and Barrass and colleagues [2009] have described enoxaparin PK in terms of a two compartment model. Although a two compartment base model was initially explored in this thesis, a one compartment model was found to describe the data sufficiently well, and the decision to pursue to a one compartment model was further ratified by the VPC of the final model produced. Indeed the only other compartmental PK analysis of enoxaparin during the antenatal period was also a one compartment model [Lebaudy et al., 2008].

The findings from this thesis report $\mathrm{CL}$ and $\mathrm{Vd}$ to increase to as much as $1.6 \mathrm{~L} / \mathrm{hr}$ and $13.2 \mathrm{~L}$ during pregnancy, and suggest, due to the measurable trough activity (and therefore persistence of anticoagulation), that a once daily dose of enoxaparin is an appropriate dosing regimen for the management of antenatal VTE. 
The concern during pregnancy has always been that $\mathrm{CrCl}$ increases and so therefore does $\mathrm{CL}$, and therefore compensation is required in the dose or dosing strategy of LMWH to counter this. The results from this thesis demonstrate that $\mathrm{Vd}$ also increases significantly during pregnancy and because of this, half life of enoxaparin is actually prolonged. This is the second study to demonstrate this formally. The compartmental PK study by Lebaudy and colleagues [2008] had similar findings to this thesis and they are the only group to date to comment on the significance of the Vd in this setting. In their study they found that the trough anti-Xa activity increased with the progression of pregnancy, attributing this to changes in Vd. Clearance and $\mathrm{Vd}$ are independent PK parameters, and the fact that Vd rises during pregnancy, prolongs the half life of enoxaparin, particularly if renal elimination of enoxaparin has stabilised, which is what is thought to occur, with $\mathrm{CrCl}$ reaching a plateau around 15 weeks gestation. Table 4.17 compares the PK model estimates between Lebaudy's model and those from this thesis.

Table 4.17: Comparison between the model results in this thesis with Lebaudy's model

\begin{tabular}{|c|c|c|c|}
\hline \multirow[t]{2}{*}{ Parameter } & \multicolumn{2}{|c|}{ This thesis } & \multirow{2}{*}{$\begin{array}{l}\text { Lebaudy Model } \\
\text { Estimate (+/-SD) }\end{array}$} \\
\hline & Estimat & (SE\%) & \\
\hline CL (I/hr) & 1.60 & (4) & $0.81(0.03)$ \\
\hline V (I) & 13.2 & (9) & $7.81(1.23)$ \\
\hline $\mathrm{Ka} \quad\left(\mathrm{hr}^{-1}\right)$ & 0.578 & (13) & $0.56(0.12)$ \\
\hline$\omega_{\mathrm{CL}}(\% \mathrm{cv})$ & 31 & (21) & 20.3 \\
\hline$\omega_{v} \quad(\% c v)$ & 29 & (49) & 26 \\
\hline FACGESTV & 0.357 & (21) & - \\
\hline FACGESTV2 & -5.49 & (26) & - \\
\hline FACGESTCL2 & -4 & (16) & - \\
\hline FACWTCL & 1.06 & (14) & - \\
\hline FACLBWV & 1.25 & (31) & - \\
\hline FACCLLebaudy & - & & $0.42(0.12)$ \\
\hline FACV $V_{\text {Lebaudy }}$ & - & & $1.41(0.25)$ \\
\hline Add error (IU/mL) & 0.0660 & (18) & 0.12 \\
\hline Prop error (\%) & 18 & (30) & - \\
\hline
\end{tabular}

FACV $_{\text {Lebaudy }}$ and FACCL Lebaudy are exponents that Lebaudy and colleagues had on CL and Vd

Lebaudy's model provides a good comparator for this thesis' study, and the reproducibility of the Vd finding by two independent groups should provide reassurance to those working in the field that such a finding is not by chance. In their study, Lebaudy's simulation work described that after administration of identical doses of enoxaparin, pregnant women showed a $22 \%$ reduction in peak anti-Xa activity compared to non pregnant women. They were the first to demonstrate that because $C L$ of enoxaparin does not change significantly after the first trimester, the area under 
the curve of anti-Xa activity does not change significantly further during pregnancy for a constant dose and go onto suggest that whereas peak anti-Xa activity will decrease with the progression of pregnancy, the residual anti-Xa activity will increase as pregnancy progresses, a finding which is endorsed and supported by the results from the study in this thesis.

It must be remembered though, that Lebaudy's model does have a number of limitations; the antiXa sampling strategy was left to the clinician's discretion, and was not based on D-optimal design. As described in chapter 2, it is well recognised, that a study based on D-optimisation is more robust, in terms of the information that the study will provide. The study in this thesis was based on D-optimal design and was more likely to capture the gestation related changes in $\mathrm{Vd}$ and $\mathrm{CL}$. Furthermore, Lebaudy's study describes an un-physiological rise in $\mathrm{Vd}$, where a big shift in $\mathrm{Vd}$ is incorporated into their model at 31 weeks gestation, which is not what actually happens in practice, and not what was observed during the course of this study. Finally, relative to this study, the number of anti-Xa activities and number of women recruited in Lebaudy's model was half the number recruited during this study; 343 versus 712 anti-Xa activities and 72 versus 123 women recruited. Accepting these limitations from Lebaudy's model however, the results from the study in this thesis add weight to Lebaudy's group stipulation, that trough anti-Xa activity will increase with the progression of pregnancy and this needs to be considered in the current dosing schedule recommendations in national / international guidelines.

Closer examination of early clinical studies evaluating LMWH PK, have demonstrated the Vd effect, even though the authors were unaware of this, at the time. For example in Sturridge and colleagues [1994] observational study evaluating 18 pregnancies in women receiving thromboprophylaxis with enoxaparin $20 \mathrm{mg}$ or $40 \mathrm{mg}$ once daily, mean peak anti-Xa activity was reported as $0.102 \mathrm{IU} / \mathrm{mL}$ and $0.036 \mathrm{IU} / \mathrm{mL}$ with the $40 \mathrm{mg}$ and $20 \mathrm{mg}$ dose respectively. They go onto to describe how the concentration was significantly lower after 20 weeks gestation, which could be attributed to Vd as well as an increase in CL. Hunt and colleagues [1997] observational study of using dalteparin in 34 pregnancies initially dosed at 5,000 IU daily, aimed to maintain a trough anti-Xa activity of $0.15-0.2 \mathrm{IU} / \mathrm{mL}$ and a 2-hour peak of $0.4-0.6 \mathrm{IU} / \mathrm{mL}$. In this study, the authors report that the majority of women required a dose change to 5,000 IU twice daily from around 20 weeks gestation to reach these target peak levels; this again can be attributed in part to a Vd effect as opposed to just an increase in CL. Perhaps the most compelling evidence comes from Casele and colleagues [1999] seminal study assessing the PK of prophylactic enoxaparin 
during pregnancy in 13 women. The authors of this study reported a lower area-under the concentration-time curve during pregnancy than during the post-partum period. Closer examination of the detailed results from this study reveal some interesting findings, as presented in table 4.18.

Table 4.18: Casele and colleagues findings in their antenatal enoxaparin study

\begin{tabular}{llll}
\hline Parameter & $12-15$ weeks & $30-33$ weeks & $6-8$ weeks PP \\
\hline Anti-Xa activity 12 h after dose $(\mathrm{IU} / \mathrm{mL})$ & $0.09+/-0.09$ & $0.16+/-0.10$ & $0.19+/-0.09$ \\
Maximum concentration $(\mathrm{IU} / \mathrm{mL})$ & $0.46+/-0.08$ & $0.40+/-0.08$ & $0.57+/-0.09$ \\
Mean residence time $(\mathrm{min})$ & $287+/-56$ & $325+/-44$ & $316+/-32$ \\
\hline $\mathrm{PP}=$ post-partum & &
\end{tabular}

The 12 hour activity increases at 30-33 weeks gestation, compared to 12-15 weeks gestation, maximum concentration has decreased by $30-33$ weeks gestation, although the mean resident time has increased at 30-33 weeks gestation, compared to $12-15$ weeks gestation. These changes can be explained by an increase in Vd.

Interestingly, groups worldwide continue to publish their experience of LMWH in the antenatal population and continue to suggest that an increase in the dose of LMWH is required in response to a falling peak anti-Xa activity, with the progression of pregnancy. In a recently published study, De Sancho and colleagues [2012], describe a retrospective review of pregnant women prescribed LMWH from January 2001 to December 2010. Seventy-six women had 89 pregnancies and they found doses of LMWH needed to be adjusted in $75 \%$ of women managed on therapeutic doses of LMWH (aiming for a peak anti-Xa of $0.6-1.1 \mathrm{IU} / \mathrm{mL}$ ) and in $45 \%$ of women managed with prophylactic dose LMWH (aiming for a peak anti-Xa activity of $0.2-0.5 \mathrm{IU} / \mathrm{mL}$ ). In light of the findings from this thesis and coupled with the findings from Lebaudy and colleagues, this strategy has to be wrong when the anti-Xa activity is considered in the context of Vd.

It is thought that persistence of anti-Xa activity is central to LMWH pharmacological action; this being the case, perhaps the focus of monitoring should be centred on trough monitoring, as opposed to peak activity during pregnancy. Such a monitoring strategy would help to identify the women with no anti-Xa activity, and who might then benefit from a dose increase. 


\subsubsection{Assumptions made during the PK modelling process}

As part of the PK modelling process, a number of assumptions have been made. It is important that they are considered as part of the wider results. Firstly, an assumption that the patient population in this study, from which a simulation data-set was created, is representative of a typical obstetric population. The simulation work also assumes all the women begin their enoxaparin from day 1 of pregnancy and deliver at 40 weeks gestation. Next, an assumption is made that the assay is $100 \%$ accurate from which the results were obtained. Finally, the simulation work assumes that women are $100 \%$ adherent to the enoxaparin, and that they inject enoxaparin at the same time each day, during the course of their pregnancy. In reality, this would not be the case and indeed observing women during the course of this study, was not the case, with variation in time administered. Some of these assumptions would account for some of the residual unexplained variability observed in the final model developed.

\subsubsection{Strengths of this study and the final model}

This study is the largest pharmacokinetic study of LMWH in a pregnant population completed to date, and one of only a few which has studied information from the first trimester. Particular strengths include the number of patients recruited, the number of anti-Xa activities drawn and modelled and the negligible complication rate. A further particular strength of the study is in its multi-disciplinary nature, with different disciplines and experts working together (haematologists, obstetricians, PK modeller, and a pharmacist) to achieve a common objective.

\subsubsection{The clinical case for a once daily dosing strategy for antenatal VTE}

An Australasian expert group statement on the management of antenatal VTE [Mclintock et al., 2012], recommends LMWH over UFH as the agents of choice. With respect to LMWH dosing, i.e. once versus twice a day, they suggest that there is currently insufficient evidence to favour one dose regimen over the other. They do however recommend that women with PE or more extensive DVT (i.e. iliofemoral thrombosis), should receive initial treatment with twice daily LMWH for at least 8-12 weeks, after which a reduction to once daily may be considered. They also suggest that women who suffer from a PE should be monitored closely as inpatients for the first couple of days following diagnosis. Their guidelines go on to recommend that there is insufficient evidence to recommend monitoring of anti-Xa levels to guide dosing in women on therapeutic dose $\mathrm{LMWH}$, as there are no data of clinical benefit from dose adjustment. However if a level is 
aimed for, they suggest $0.5-1.0 \mathrm{IU} / \mathrm{mL}$ for a twice daily regimen at four hours post dose, whilst acknowledging that the target is less clear for a once daily regimen $(1.0-2.0 \mathrm{IU} / \mathrm{mL}$ at four hours post dose). The results from this thesis demonstrate that the 3 hour anti-Xa activity will decrease with the progression of pregnancy, due to the increase in the $\mathrm{Vd}$. The results also demonstrate that residual anti-Xa activity increases with the progression of pregnancy, again due to the increase in the $\mathrm{Vd}$. Therefore monitoring peak target anti-Xa activities during pregnancy seems counter-initiative, as if you did adjust the dose to reach a target peak anti-Xa activity, you run the risk of over anticoagulation and ultimately put the patient at risk of bleeding.

\subsubsection{Thrombus regression: once versus twice daily}

Meta-analysises of LMWH in the treatment of VTE outside of pregnancy have concluded that treatment with LMWH increases the frequency of thrombus regression [Leizorovicz et al., 1994; Lensing et al., 1995; van den Belt et al., 2000]; a fundamental aim of LMWH therapy in the management of VTE. A previously published study by Breddin and colleagues [2001], conducted a open-label, multicentre study, with blinded adjudication at end points, whereby patients with acute deep vein thrombosis were randomly assigned to one of three treatment regimens; intravenous unfractionated heparin for a week, twice a day reviparin for one week, or reviparin once a day for four weeks; patients recruited to all three arms received a total of 90 days treatment, with a vitamin $\mathrm{K}$ antagonist started on day 1 of the unfractionated heparin and twice a day reviparin group and from day 20 for the once a day reviparin group. The reviparin dose administered was one injection every 12 hours, or as one injection every 24 hours, based on patients weight; $7000 \mathrm{IU}$ for a weight of $35-45 \mathrm{~kg}$, $8400 \mathrm{IU}$ for a weight between $46-60 \mathrm{~kg}$ and $12,600 \mathrm{IU}$ for a weight of more than $60 \mathrm{~kg}$. The primary end point was a change in the venographically determined thrombus size between baseline and day 21 , using the Marder score [Marder et al., 1977]. This score allocates points to each of the involved deep segments of the lower limb; if all the veins in one leg are occluded, the total score is 40 , with partially occluded veins given a lower score. Patients are considered to have a response when their scores had decreased by at least 30\%. In the study, 40.2\% (129 patients) had thrombus regression (response to treatment) in the unfractionated heparin group, compared to $53.4 \%$ (175 patients) receiving reviparin twice daily, and 53.5\% (167 patients) receiving reviparin once daily. Table 4.19 lists the numbers of patients who were judged to have not responded. 
Table 4.19: Results from Marder and colleagues reviparin study

\begin{tabular}{lccc}
\hline $\begin{array}{l}\text { Patients with no } \\
\text { response }\end{array}$ & $\begin{array}{c}\text { Unfractionated heparin } \\
n=375(\%)\end{array}$ & $\begin{array}{c}\text { Reviparin TWICE daily } \\
n=388(\%)\end{array}$ & $\begin{array}{c}\text { Reviparin ONCE daily } \\
n=374(\%)\end{array}$ \\
\hline Recurrence & $24(6.4)$ & $7(1.8)$ & $13(3.5)$ \\
$\begin{array}{l}\text { No venographic changes } \\
\text { seen }\end{array}$ & $167(52)$ & $140(42.7)$ & $128(41)$ \\
$\begin{array}{l}\text { Deterioration (increase } \\
\text { in Marder score of at } \\
\text { least } 30 \%)\end{array}$ & $25(7.8)$ & $13(4)$ & $17(5.4)$ \\
\hline
\end{tabular}

Clearly the LMWH was more effective in this study compared to unfractionated heparin, with this study demonstrating a correlation between clinical outcome and change in thrombus size, and although the twice daily dose of reviparin has the best outcomes, the once daily dose has extremely good outcomes and is considered as efficacious as the twice daily dose. The results demonstrate objective efficacy of both once and twice daily doses of reviparin, which is further endorsed by a comprehensive Cochrane review [van Dongen et al., 2004].

\subsubsection{Lessons from the early cardiology dose-ranging twice a day trials of enoxaparin}

The dose ranging trial of enoxaparin for unstable angina, originate from the TIMI $11 \mathrm{~A}$ trial [Antman et al., 1997]. This was a phase II trial whose objective it was to compare the safety and tolerability of two weight-adjusted regimens of enoxaparin in patients with unstable angina / nonQ-wave myocardial infarction (NSTEMI), as the optimal dose in patients with arterial disorders had not been established. This study was an open label study, with the original plan in the study for an ascending dose, to evaluate a weight-adjusted dose of enoxaparin of $1.25 \mathrm{mg} / \mathrm{kg}$ every 12 hours (dosing schedule 1 ) in the first cohort of patients studied and then $1.5 \mathrm{mg} / \mathrm{kg}$ every 12 hours (dosing schedule 2) in the second cohort of patients. However, after 321 patients had been enrolled in dosing schedule 1 , the trial had to be reconfigured, as a descending dose-ranging trial, with the second cohort (309 patients) receiving $1.0 \mathrm{mg} / \mathrm{kg}$ every 12 hours (now dosing schedule 2). This was because routine monitoring of the major haemorrhage rate by the trial safety committee found a major haemorrhage rate of $6.5 \%$ (21 patients) at day 14 . Major haemorrhage in the new dosing schedule 2 occurred in six patients (1.9\%) at day 14 . In both dosing tiers the majority of episodes of major bleeding occurred at instrumental sites, with only small numbers attributed to spontaneous bleeds. Further analysis of anti-Xa activities from this trial are presented in table 4.20 . 
Table 4.20: Results from TIMI 11A enoxaparin dose ranging study

\begin{tabular}{|c|c|c|c|c|}
\hline \multirow[t]{2}{*}{$\begin{array}{l}\text { Anti-Xa activity } \\
(\mathrm{IU} / \mathrm{mL})\end{array}$} & \multicolumn{2}{|c|}{$\begin{array}{c}\text { Dose tier } 1 \\
(1.25 \mathrm{mg} / \mathrm{kg} \text { every } 12 \text { hours })\end{array}$} & \multicolumn{2}{|c|}{$\begin{array}{c}\text { Dose tier } 2 \\
\text { (1.0 mg/kg every } 12 \text { hours) }\end{array}$} \\
\hline & $\begin{array}{l}\text { Major } \\
\text { haemorrhage (\%) } \\
(n=21)\end{array}$ & $\begin{array}{l}\text { No major } \\
\text { haemorrhage (\%) } \\
(n=299)\end{array}$ & $\begin{array}{l}\text { Major } \\
\text { haemorrhage (\%) } \\
(n=6)\end{array}$ & $\begin{array}{l}\text { No major } \\
\text { haemorrhage (\%) } \\
(n=303)\end{array}$ \\
\hline \multicolumn{5}{|c|}{$\begin{array}{l}3^{\text {rd }} \text { weight adjusted } \\
\text { dose }\end{array}$} \\
\hline Trough & $0.5(n=14)$ & $0.6(n=202)$ & 0.7 and $1.0(n=2)$ & $0.5(n=162)$ \\
\hline Peak & $1.8(n=14)$ & $1.4(n=213)$ & 1.2 and $1.9(n=2)$ & $1.0(n=161)$ \\
\hline \multicolumn{5}{|l|}{$\begin{array}{l}\text { Last weight } \\
\text { adjusted dose }\end{array}$} \\
\hline Trough & $0.6(n=7)$ & $0.8(n=106)$ & 0.3 and $1.5(n=2)$ & $0.6(n=43)$ \\
\hline Peak & $2.0(n=4)$ & $1.6(n=99)$ & 1.0 and $1.8(n=2)$ & $1.1(n=44)$ \\
\hline
\end{tabular}

The authors from this study concluded that the $1.0 \mathrm{mg} / \mathrm{kg}$ weight adjusted dose was well tolerated and this work then formed the basis of the larger phase III trial the TIMI 11B trial [Antman et al., 1999]. They also suggest that with the twice daily regimen, there was no accumulation of the LMWH. What can be extrapolated for the antenatal population? Antman's study provides valuable information, informing us that increasing the dose of enoxaparin above $1 \mathrm{mg} / \mathrm{kg}$ twice a day can increase the risk of haemorrhage. So in an antenatal setting, if a patient is prescribed a twice daily regimen and the dose is adjusted to maintain a peak level, potentially the risk of bleeding (particularly at instrumental sites) is run, as a drop in peak anti-Xa activity is inevitable with the increase in Vd; once again providing weight to the thinking that trough activity is a better time point to monitor in this setting.

The TIMI IIB trial [Antman et al., 1999], was a phase III study which randomised patients with unstable angina or NON-Q-wave MI to intravenous UFH for $\geq 3$ days followed by subcutaneous placebo injections or uninterrupted therapy with enoxaparin during both the acute and chronic phase, of $30 \mathrm{mg}$ IV bolus, followed by subcutaneous injections of $1.0 \mathrm{mg} / \mathrm{kg}$ every 12 hours and an outpatient phase of $40 \mathrm{mg}$ bd (for patients weighing less than $65 \mathrm{~kg}$ ) and $60 \mathrm{mg}$ bd (for patients weighing $\geq 65 \mathrm{~kg}$ ). The authors of this study conclude that enoxaparin is superior to UFH for reducing a composite end point of death and serious cardiac ischemic events during the acute management of UA/NON-Q-wave MI, without causing a significant increase in the rate of major haemorrhage. However, interestingly, when you analyse the patients who suffered from major 
and minor haemorrhage at 72 hours and at the end of the time period of hospitalisation, more patients significantly suffered from minor bleeds compared to those patients in the unfractionated heparin arm (table 4.21).

Table 4.21: Bleeding results from the TIMI IIB trials

\begin{tabular}{|c|c|c|c|}
\hline \multirow[b]{2}{*}{ Time point } & \multicolumn{2}{|c|}{ Treatment Group } & \multirow[b]{2}{*}{$p$} \\
\hline & UFH $(n=1926)$ & Enoxaparin $(n=1938)$ & \\
\hline \multicolumn{4}{|l|}{$72 h$} \\
\hline Major haemorrhage (\%) & $14(0.7)$ & $16(0.8)$ & 0.714 \\
\hline Minor haemorrhage (\%) & $45(2.3)$ & $99(5.1)$ & $<0.001$ \\
\hline \multicolumn{4}{|l|}{ End of initial hospitalisation } \\
\hline Major haemorrhage (\%) & $19(1.0)$ & $29(1.5)$ & 0.143 \\
\hline Minor haemorrhage (\%) & $48(2.5)$ & $176(9.1)$ & $<0.001$ \\
\hline
\end{tabular}

As with many other unfractionated heparin trials, only around $40-50 \%$ in this study had aPTT values in the target range, so it is difficult to know whether the increase in minor haemorrhage seen with the enoxaparin arm was truly because of the enoxaparin or not, as if all the UFH patients were in range, might a similar number of events have been observed? The minor haemorrhages in most cases were due to ecchymosis at the subcutaneous site or a haematoma at the site of a sheath inserted for cardiac catherisation. Whilst accepting the fact, that the majority of patients in this study are older and not directly comparable to a pregnant population from a physiological point of view, the message that can be discerned from this large and influential study is that giving a twice daily enoxaparin dose, might increase the risk of minor haemorrhage.

Considering all of these factors, more weight is given to the camp supporting a once daily regimen of LMWH in the pregnancy setting for antenatal VTE. Indeed none of the women managed in this cohort who were managed with once daily enoxaparin suffered from any recurrences or complications during the course of this study; including the 10 pregnancies in women on long-term warfarin who were switched to enoxaparin during the index pregnancy.Table 4.22 is a reminder of the specific clinical details of these women. 
Table 4.22: Women switched from warfarin to once daily enoxaparin

\begin{tabular}{llcl}
\hline Patient & Indication & $\begin{array}{c}\text { Target INR } \\
\text { range }\end{array}$ & Enoxaparin dose prescribed \\
\hline 16 & Recurrent VTE & $1.5-2.0$ & $120 \mathrm{mg}$ daily \\
37 & $\begin{array}{l}\text { FVL (homozygous). Symptomatic VTE whilst } \\
\text { on combined oral contraceptive pill }\end{array}$ & $2-3$ & $100 \mathrm{mg}$ daily \\
& Budd-Chiari (Liver transplant in 1998) & $2-3$ & $100 \mathrm{mg}$ daily \\
$45^{\star}$ & Paroxysmal nocturnal haemoglobinuria & $2-3$ & $80 \mathrm{mg}$ daily \\
48 & Budd-Chiari (Liver transplant in 2006) & $2-3$ & $100 \mathrm{mg}$ daily \\
53 & Anti-phospholipid syndrome & $2-3$ & $100 \mathrm{mg}$ daily \\
54 & AF/Mitral stenosis/Pulmonary hypertension & $2-3$ & $100 \mathrm{mg}$ daily \\
82 & Recurrent VTE & $2-3$ & $180 \mathrm{mg}$ daily \\
87 & Budd-Chiari (Liver transplant in 1998) & $2-3$ & $100 \mathrm{mg}$ daily \\
$101^{*}$ & Anti-phospholipid syndrome and Budd-Chiari & $3-4$ & $60 \mathrm{mg}$ daily \\
115 & (Liver transplant in 2005) & & \\
\hline
\end{tabular}

${ }^{\text {*same patient }}$

\subsubsection{Mechanical valve prophylaxis during pregnancy}

Managing pregnant women with a mechanical heart valve in-situ, has to be one of the most high risk and difficult challenges that clinicians can be faced with in obstetric care. The anticoagulation management of these women is highly controversial and each case should be considered on a case-by-case basis on their particular management, depending on co-morbidities, valve type and specific circumstances [Patel and Hunt, 2008]. During pregnancy, the risk of valve thrombosis is least with coumarin anticoagulation throughout pregnancy (4\%) and increased markedly with the use of unfractionated heparin, with the risk of life-threatening thrombosis of $29-33 \%$ and a mortality of between 7-15\% [Chan et al., 2000; Sadler et al., 2000]. Information relating to the use of LMWH has been variable. Current ACCP guidelines [Bates et al., 2012] on this matter suggests one of three approaches should be adopted:

(i) Adjusted twice daily dose of LMWH throughout pregnancy to keep the 4 hour post injection, between $1-1.2 \mathrm{IU} / \mathrm{mL}$

(ii) Aggressive adjusted dose UFH throughout pregnancy, administered by subcutaneous injection every twelve hours to keep the mid-interval aPTT at least twice control or to attain an anti-Xa activity between $0.35-0.70 \mathrm{IU} / \mathrm{mL}$

(iii) UFH or LMWH (as above) until 13 weeks gestation, changing to warfarin, until the middle of the third trimester, and re-converting back to UFH or LMWH 
Regarding LMWH specifically, in 2002, Sanofi Aventis changed the warning label for enoxaparin, stating that it was not recommended in patients with prosthetic heart valves. This was prompted by a South African study which compared the effectiveness of enoxaparin with a combination of warfarin and unfractionated heparin. The safety committee terminated the study after only 12 patients were enrolled, due to two deaths from prosthetic valve thrombosis in the enoxaparin group, prompting concerns about its use during pregnancy for this indication, and implying the same for other LMWH. However, subsequent experience [James et al. 2006; McLintock et al., 2009] suggests that LMWH is by no means inferior to unfractionated heparin, with McLintock reporting worse outcomes in those women non-adherent to the prescribed treatment, an issue which is explored further in chapter 7 of this thesis.

The study in this thesis was not designed to deal with optimising the dosing of LMWH in the obstetric population with mechanical heart valves in-situ. Indeed, none of the 123 patients who were recruited to this study had a mechanical heart valve in-situ, reflecting in part the low incidence of rheumatic fever in the young Western population. However, the results found from this study have implications for the recommendations made in the international ACCP guidelines.

Firstly, the study of this thesis has undoubtedly demonstrated that as pregnancy progresses, $\mathrm{Vd}$ increases, with a reduction in peak anti-Xa activity, if the dose is kept the same throughout pregnancy. Furthermore, the trough anti-Xa activity increases with the progression of pregnancy. This being the case, if the peak anti-Xa activity is titrated against a set target, in this case 1.0-1.2 $\mathrm{IU} / \mathrm{mL}$ four hours post injection, then there is the risk of over-anticoagulation in this setting and increasing the risk of haemorrhage, as has been reported in the literature [McLintock, ISTH 2012]. This is because, during pregnancy, it is inevitable that having such a target, will lead to a dose increase, which perhaps might not be necessary, when the trough is taken into account. The problem in this area is further complicated by the fact, that it is not known, what minimum level of anticoagulation with LMWH is required to prevent valve thrombosis. If this was known, current treatments could be used much more effectively for this population. During the course of this study, the median 3 hour anti-Xa activity in the simulation work, decreased from 1 to $0.6 \mathrm{IU} / \mathrm{mL}$ for women on the once daily dose and 0.9 to $0.5 \mathrm{IU} / \mathrm{mL}$ for women on the twice daily dose of enoxaparin; in both cases, a drop of $0.4 \mathrm{IU} / \mathrm{mL}$. Assuming the same drop for Cmax, then hypothetically speaking, the dose would definitely have been increased, if ACCP guidelines were to be followed to the letter. 
It is becoming more apparent that trough activity might be a better marker for dose adjustments in this setting. In this study, a percentage of patients were found to have no trough anti-Xa activity; this was the case for both once and twice daily regimes. The literature is littered with case reports of patients who have suffered valve thrombosis despite a prescription for $\mathrm{LMWH}$, and perhaps by measuring the trough activity, and identifying women with negligible trough activity in this setting, would identify the women who would benefit from a dose increase, as opposed to increasing the dose for all. Future research should focus on this, with key opinion leaders in this setting are already suggesting their personal practice is likely to focus more on trough activity in the future [McLintock, ISTH 2012].

Finally, the issue of medication adherence needs to be factored into this setting. Only McLintock's study to date, has commented on this, and found that those women who had worse outcomes on LMWH had poor adherence. Perhaps, the lack of efficacy reported in the literature of LMWH could be related to this very issue, as from a scientific point of view, why would vitamin $\mathrm{K}$ antagonists be better than LMWH in this setting. The issue of medication adherence certainly deserves further attention and is explored in detail in this study in chapter 7 of the thesis.

Clearly the management of women with mechanical valves in-situ is complex and requires special attention. Until further work is published to move the management of these women forward, the consensus of managing each woman on a case by case basis seems appropriate. The results from this thesis do however suggest that caveats should be added to the current four hour ACCP recommendations in order for all risks, both thrombotic and haemorrhagic, to be considered.

\subsubsection{VTE prophylaxis during the antenatal period}

VTE prophylaxis has been a real success story in obstetric care over the last decade. Since the introduction of the first RCOG guidelines for the prevention of VTE during pregnancy and puerperium in 2004, the number of women dying or suffering from VTE has been on the decline, suggestive of the good work of those involved in identifying and managing this risk during the antenatal period. How do things look, when women are administered once daily LMWH in this setting? Figure 4.34 is a simulated profile of three hour and trough anti-Xa activity over the course of pregnancy, according to ROCG once daily dosing guidelines based on weight (table 4.23). 
Table 4.23: Current RCOG suggested doses for enoxaparin for VTE prophylaxis

\begin{tabular}{cc}
\hline Weight $(\mathrm{kg})$ & Enoxaparin \\
\hline$<50$ & $20 \mathrm{mg}$ daily \\
$50-90$ & $40 \mathrm{mg}$ daily \\
$91-130$ & $60 \mathrm{mg}$ daily* \\
$131-170$ & $80 \mathrm{mg}$ daily \\
$>170$ & $0.6 \mathrm{mg} / \mathrm{kg} /$ day $^{*}$ \\
High prophylactic (intermediate) dose & $40 \mathrm{mg} \mathrm{12-hourly}$ \\
\hline
\end{tabular}

${ }^{*}$ may be given in two divided doses

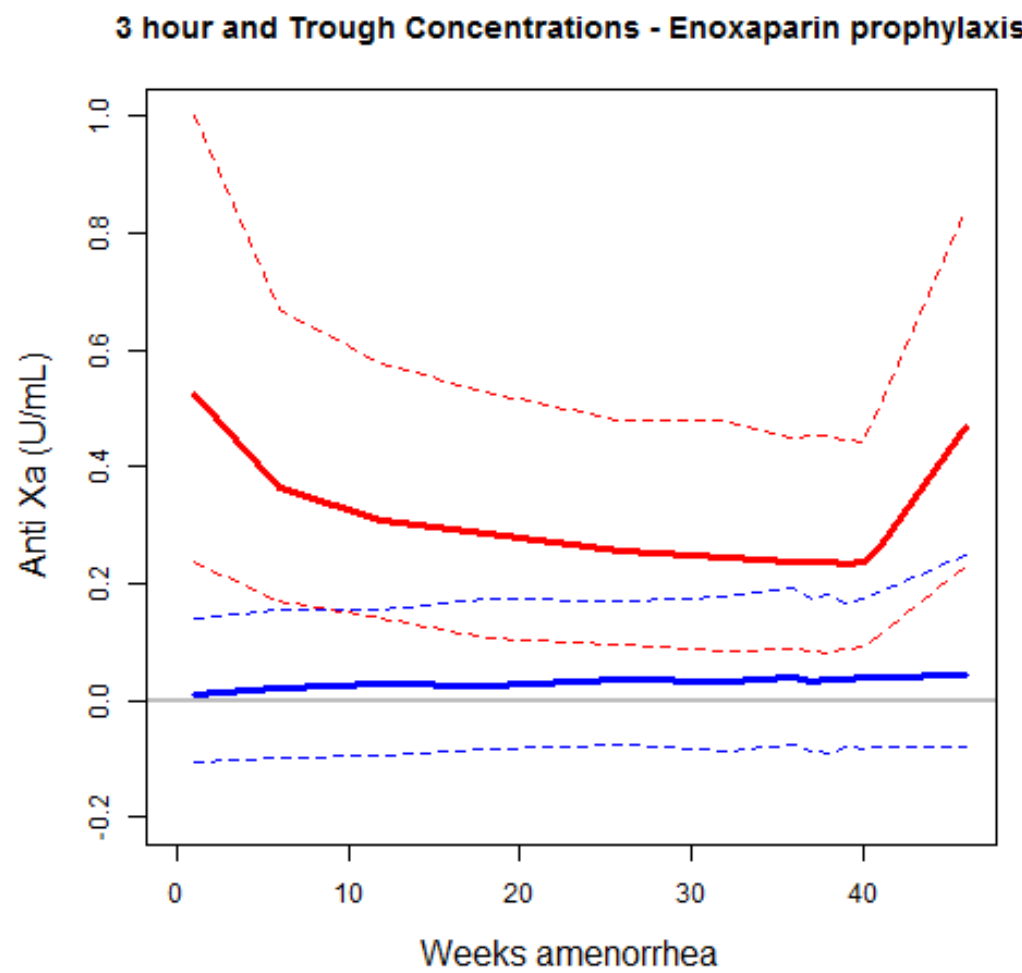

Figure 4.34: Simulation of prophylactic dosing of enoxaparin according to current Royal College of Obstetricians and Gynaecologists recommendations

As can been seen, with prophylactic dosing of $\mathrm{LMWH}$, a large number of women are below a quantifiable level of anti-Xa activity, before the next dose is due. Table 4.24 lists the percentage of patients who this would apply to. 
Table 4.24: \% of women BLQ when dosed according the current RCOG antenatal prophylaxis guidelines

\begin{tabular}{cc}
\hline Gestation (weeks) & Prophylaxis $(\%<0.01 \mathrm{IU} / \mathrm{mL})$ \\
\hline 1 & 49.59 \\
6 & 43.82 \\
12 & 40.89 \\
18 & 41.78 \\
26 & 36.42 \\
32 & 38.94 \\
36 & 35.36 \\
37 & 38.21 \\
38 & 39.11 \\
39 & 37.39 \\
40 & 35.04 \\
\hline
\end{tabular}

The findings from this study provides compelling evidence that a once daily dose, for those weighing $91 \mathrm{~kg}$ or more, is appropriate and there is no need to split the dose. The suggested changes to the guidelines would have the additional benefits of reducing the injection burden for pregnant women, which would be welcome, particularly, as a recent small study [Revell and Smith, 2011] suggested that $41 \%$ of women would meet the threshold for postpartum thromboprophylaxis in the UK if the current RCOG guidelines are followed to the letter.

The clinical case for once daily dosing, coupled with the population PK case for once daily dosing, provides strong evidence for a need for a change in practice.

\subsection{Conclusion}

The aim of this study was to characterise the pharmacokinetic profile of enoxaparin during the antenatal period, using the method of non-linear mixed effects modelling. A robust enoxaparin in pregnancy model was developed. Simulation work, comparing once versus twice a day enoxaparin for the treatment of VTE provides compelling evidence that a once daily dose of enoxaparin is appropriate. The implications of these findings are discussed further in chapter 8. 


\section{Chapter 5}

\subsection{D-dimer concentrations during pregnancy}

Chapter 5 focuses on the measurement and meaning of D-dimer concentrations during pregnancy and the puerperium. After providing background information on the role and meaning of D-dimer concentrations in general, information related to the measurement of D-dimer concentrations within an antenatal context will be discussed. The observational D-dimer concentrations from pregnant women followed during this study are then presented and discussed in the context of previously published work.

\subsubsection{D-dimer measurements}

\subsubsection{Background}

Though not considered a global coagulation assay, D-dimer concentrations provide a valuable insight into individual's active coagulation. When a fibrin clot is degraded by the fibrinolytic enzyme, plasmin, the final product which results are D-dimers and they provide compelling evidence of the activation of the fibrinolytic system, in response to coagulation activation (figure 5.1).

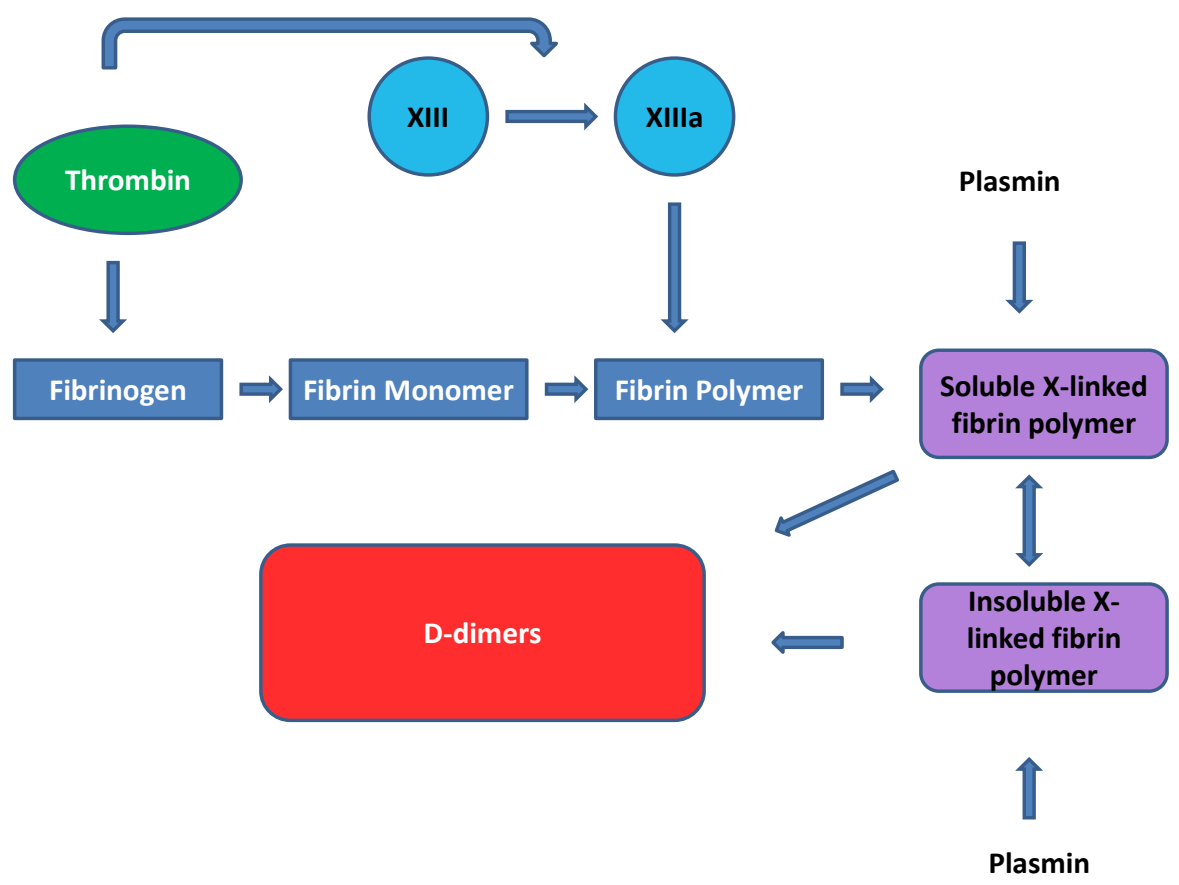

Figure 5.1: diagrammatic representation of the formation of D-dimers

Over the last twenty years, clinical studies have demonstrated that measuring D-dimers concentrations for patients suspected of VTE is an appropriate screening strategy for acute 
thrombosis; D-dimer concentrations demonstrate high specificity and sensitivity, with high negative predictive value, reported in the region of 98\% [Bounameaux et al., 1991; Wells et al., 2003]. Bounameaux and colleagues [1991] prospectively evaluated the clinical utility of D-dimer testing for the diagnosis of acute pulmonary embolism. In their study, they reported a sensitivity of $98 \%$ and a specificity of $39 \%$ for the assay, at a D-dimer cut-off point of $500 \mu \mathrm{g} / \mathrm{L}$ or above, giving positive and negative predictive values of $44 \%$ and $98 \%$ respectively. Following completion of their study, they concluded that plasma measurement of D-dimers had a definite role to play in the diagnostic work-up of patients with suspected acute PE, and suggested that a concentration below $500 \mu \mathrm{g} / \mathrm{L}$ can confidently rule out the diagnosis. Wells and colleagues [2003] evaluated the utility of D-dimer testing in patients presenting with suspected acute DVT. In their study, patients were randomly assigned to two groups; group 1 (control group) comprising patients who underwent ultrasound imaging alone, or group 2, who underwent D-dimer testing followed by ultrasound imaging, unless the D-dimer test was negative. Over one thousand patients were enrolled to the study and the overall prevalence of DVT or PE was $15.7 \%$ in the cohort of patients recruited. Of the patients who had DVT ruled out by the initial diagnostic strategy, there were two confirmed cases of VTE in the D-dimer group and six cases in the control group. Wells and colleagues demonstrated that the utility of D-dimer testing resulted in a significant reduction in the use of ultrasonography, from a mean of 1.34 test per patient in the control group to 0.78 in the Ddimer group ( $p=0.008)$ and concluded that DVT can be ruled out safely in patients who have negative $\mathrm{D}$-dimer results.

In clinical practice today, D-dimer concentrations are considered a routine test in the diagnostic work-up of suspected VTE, with the recently published National Institute for Health and Clinical Excellence guidance on VTE incorporating D-dimer testing, as part of the routine diagnostic workup of individuals suspected of VTE [National Institute for Health and Clinical Excellence, 2012].

In addition to identifying a prothrombotic state and confirmation of an acute VTE episode, D-dimer testing has been investigated as a marker of identifying patients who are at higher risk of VTE recurrence and whom might benefit from long term anticoagulation following an acute VTE episode [Palareti et al., 2003]. In their study, Palareti and colleagues assessed the predictive value of D-dimers for recurrent VTE in subjects with a previous unprovoked VTE event (in subjects with and without inherited thrombophilia). Five hundred and ninty-nine patients were followed for 870.7 patient years. They found that an increased D-dimer concentration at 1 month 
after discontinuation of oral anticoagulation therapy was associated with a higher rate of subsequent recurrence in all subjects; though debate continues on whether this alone is enough to warrant long-term anticoagulation. The other important and increasing utility of D-dimer testing, is when the concentrations are raised, in the absence of VTE (confirmed by objective testing). In such situations a grossly elevated D-dimer concentration could represent the presence of cancer and warrants further investigation [Adam et al., 2009]. Both these examples exemplify the wider utility of D-dimer testing other than in the diagnosis of VTE.

\subsubsection{D-dimer assays: principle of assay}

Commercial D-dimer assays available, use monoclonal antibodies that detect an epitope that is present in the factor XIlla-cross-linked fragment $D$ domain of fibrin, but not in fibrinogen degradation products or non-cross-linked fibrin degradation products. Different commercial assays have their own reported specificity [Adam et al., 2009]. The test principles for D-dimers are based on either a rapid ELISA (enzyme linked immunosorbent assay) or latex agglutination tests. Both these test methods have their own advantages; ELISAs have the greatest sensitivity, whilst the agglutination tests are reported to have the greatest specificity [Kelly and Hunt, 2002].

The STA® - Liatest $\AA^{\circledR}$ D-Di kit (Stago Diagnostica, France) is used at King's College Hospital, utilising the immune-turbidimetric method to quantitatively determine D-dimer concentrations. The test principle involves a beam of monochromic light to traverse a suspension of microlatex particles to which specific antibodies have been attached by covalent bonding. If the wavelength of the light is much greater than the diameter of the latex particles, the light is only slightly absorbed. In the presence of the antigen being tested for, in this case D-dimers, the antibodycoated latex particles agglutinate to form aggregates of diameters greater than the wavelength of the light; more of the latter is absorbed. This increase in light absorption is a function of the antigen level present in the test sample [Stago Diagnostica, 2011].

\subsubsection{D-dimer concentrations use during pregnancy}

Although D-dimer testing is considered a part of routine clinical practice, in those patients suspected of VTE during the gravid state, the utility of D-dimer concentrations as a screening tool for thrombosis is somewhat lost. This is because historically pregnant women have been excluded from clinical studies evaluating D-dimer testing, due to the fact that early studies [Gafney et al., 1987] assessing D-dimers during pregnancy demonstrated D-dimer concentrations to rise, even during uncomplicated pregnancy, due to a pro-thrombotic state being favoured, and 
so the usual reference ranges used in routine clinical practice, were found not to apply in the gravid state.

This has led the Royal College of Obstetricians and Gynaecologists (RCOG) to stipulate in their guidelines for the management of antenatal VTE, that a positive D-dimer is not necessarily consistent with VTE, suggesting that further objective testing is required [Royal College of Obstetricians and Gynaecologists, 2007]. Indeed, recent expert clinician reviews on the management of antenatal VTE, suggest that D-dimer testing, from a reference range view point, cannot be considered as part of the usual diagnostic algorithm for VTE during pregnancy, with both clinical experts offering alternative comprehensive diagnostic algorithms to successfully diagnose antenatal VTE [Arya, 2010; Middeldorp, 2011].

\subsubsection{Studies of D-dimer concentrations during uncomplicated pregnancy}

A small number of studies evaluating D-dimer concentrations in uncomplicated pregnancy have been published. Chabloz and colleagues [2001] measured D-dimer concentrations from 144 pregnant women with uncomplicated pregnancies. A total of 519 samples were drawn from these women. They reported a gradual rise in D-dimer concentrations over the course of pregnancy, peaking at the time of delivery (table 5.1).

Table 5.1: D-dimer concentrations during uncomplicated pregnancy reported by Chabloz and colleagues

\begin{tabular}{lcccc}
\hline Trimester & 1 & 2 & 3 & At delivery \\
\hline $\begin{array}{l}\text { D-dimer concentration }(\mathbf{n g} / \mathbf{m L})- \\
\mathbf{5}^{\text {th }} \text { and } \mathbf{9 5} \mathbf{5}^{\text {th }} \text { percentiles }\end{array}$ & $139-602$ & $291-1231$ & $489-2217$ & $678-5123$ \\
& & & & \\
\hline
\end{tabular}

The authors suggest that given this pattern observed during uncomplicated pregnancy, it might be possible to propose a new reference range for D-dimer concentrations, according to the trimester of pregnancy.

In a further small observational study comparing alterations in D-dimer concentrations, endogenous thrombin potential (ETP) and fragment 1+2 during pregnancy, 50 healthy women had serial D-dimer concentrations measured through their pregnancy [Dargaud et al., 2010]. The authors also found that D-dimer concentrations gradually rose during pregnancy; with the majority of women having results within the normal reference range during the first trimester, followed by a significant rise during the second and third trimesters. 
Another study of 50 women with uncomplicated pregnancy, evaluated D-dimer concentrations serially; preconception and then again at 12,24 , and 36 weeks gestation along with a final measurement at 4 weeks post-partum [Kline et al., 2005]. This is one of the few studies to assess D-dimer concentrations preconception and during the post-partum period. The headline results from this study are presented in table 5.2.

Table 5.2: D-dimer concentrations reported by Kline and colleagues

\begin{tabular}{lccc}
\hline Time point & $\boldsymbol{n}^{\mathbf{a}}$ & $\begin{array}{c}\text { D-dimer mean } \\
\text { concentration } \mathbf{( m g} / \mathbf{L})\end{array}$ & SD \\
\hline Pre-conception & 50 & 0.429 & 0.486 \\
First trimester & 32 & 0.579 & 0.363 \\
Second trimester & 31 & 0.832 & 0.456 \\
Third trimester & 23 & 1.159 & 0.573 \\
Four weeks post-partum & 18 & 0.605 & 0.433 \\
\hline a Number of patients for each time point & & &
\end{tabular}

a Number of patients for each time point

Once again, they demonstrated that during uncomplicated pregnancy, D-dimer concentrations rise to $>0.50 \mathrm{mg} / \mathrm{L}$ in all trimesters and that by the third trimester, D-dimer concentrations will almost certainly be abnormal if a threshold of $0.50 \mathrm{mg} / \mathrm{L}$ is used.

Murphy and colleagues [2011] drew D-dimer concentrations from 694 'low risk' pregnant women in their study. Women were sampled at different time points during their pregnancy and within two days of delivery (table 5.3).

Table 5.3: D-dimer concentrations reported by Murphy and colleagues

\begin{tabular}{llccc}
\hline Period & $\boldsymbol{n}^{\mathbf{a}}$ & Median & $\mathbf{5}^{\text {th }}$ Percentile & $\mathbf{9 5}^{\text {th }}$ Percentile \\
\hline$<12$ weeks & 163 & 101 & 39 & 499 \\
$19-21+6$ & 167 & 282 & 131 & 628 \\
$28-36+6$ & 98 & 475 & 225 & 1033 \\
$38-40+6$ & 102 & 661 & 343 & 1513 \\
Day 2 postpartum & 164 & 480 & 193 & 1746 \\
\hline
\end{tabular}

a Number of patients for each time point

Again, the same pattern as previously reported by other groups, was found.

Finally, the largest study evaluating D-dimer concentrations in pregnancy to date, is that conducted by Szecsi and colleagues [2010]. In their study, 801 women had serial haemostatic 
tests drawn (including D-dimer concentrations). The authors report that D-dimer concentrations increased progressively, peaking at the first day postpartum. Detailed analysis of their results found that, mid way through the second trimester, more than $25 \%$ of women have D-dimer levels at or above $0.5 \mathrm{mg} / \mathrm{L}$, and that by week $36-42$, virtually all the women's D-dimer concentrations were above the traditional cut-off point (table 5.4).

Table 5.4: D-dimer concentrations reported by Szecsi and colleagues

\begin{tabular}{lcccccc}
\hline $\begin{array}{l}\text { Gestation } \\
\text { (weeks) } \\
\boldsymbol{n}^{\mathbf{a}}\end{array}$ & $\mathbf{1 3 - 2 0}$ & $\mathbf{2 1 - 2 8}$ & $\mathbf{2 9 - 3 4}$ & $\mathbf{3 5 - 4 2}$ & Partus & Partus +2 \\
\hline $\begin{array}{l}2.5^{\text {th }}-97.5^{\text {th }} \\
\begin{array}{l}\text { percentiles } \\
(\mathrm{mg} / \mathrm{L})\end{array}\end{array}$ & 537 & 369 & 178 & 362 & 242 & 157 \\
\hline
\end{tabular}

a Number of patients for each time point

Clearly all these published studies to date demonstrate a pregnancy related rise in D-dimer concentrations. The question is can this be harnessed in clinical practice, in order to establish a pregnancy-specific D-dimer concentration range to exclude antenatal VTE.

Morse [2004] was one of the first to suggest that this might be possible and in his study, measured D-dimer concentrations in 48 pregnant women, with concentrations drawn at 16, 26 and 34 weeks gestation in the same women. As per previous studies, a rise in D-dimer concentrations was reported. In the discussion of his results, Morse suggests that it should be possible to work out a pregnancy specific D-dimer concentration level, in order to safely use the test in the diagnosis of pregnancy-associated PE and DVT. This concept has now been taken forward by Chan and colleagues [2011] who recruited pregnant patients who presented to Canadian hospitals with suspected DVT over an eight year period. Plasma samples for D-dimers were collected at the time of presentation and analysed using five different commercially available D-dimer assays [Vidas $\AA$, Asserachrome ${ }^{\circledR}$, IL Test $\AA$, Sta-Lia ${ }^{\circledR}$, Innovance $\AA$ ]. A receiver operating curve (roc) analysis was conducted to determine a pregnancy specific cut-off point for D-dimer tests. A ROC curve is a plot which illustrates the performance of a variable, in this case the Ddimer assay, as its discrimination threshold is varied. Two hundred and twenty eight women contributed data in their study. Of these, fifteen were diagnosed with a DVT, an incidence rate of $6.6 \%(95 \% \mathrm{Cl} 4-10.6)$.

The specific D-dimer concentrations from the Sta-Lia ${ }^{\circledR}$ test (used at King's College Hospital) are presented in table 5.5 . 
Table 5.5: D-dimer concentrations using the Sta-Liaß test

\begin{tabular}{cccccccccc}
\hline & \multicolumn{3}{c}{ T1 (<12 weeks) } & \multicolumn{3}{c}{ T2 (12 to <28 weeks) } & \multicolumn{2}{c}{ T 3 (28 weeks or later) } \\
\cline { 2 - 9 } DVT & $n^{a}$ & $\begin{array}{c}\text { Mean } \\
(\mathrm{SD})\end{array}$ & $\begin{array}{c}\text { Median } \\
(\mathrm{IQR})\end{array}$ & $n^{a}$ & $\begin{array}{c}\text { Mean } \\
(\mathrm{SD})\end{array}$ & $\begin{array}{c}\text { Median } \\
(\mathrm{IQR})\end{array}$ & $n^{a}$ & $\begin{array}{c}\text { Mean } \\
(\mathrm{SD})\end{array}$ & $\begin{array}{c}\text { Median } \\
(\mathrm{IQR})\end{array}$ \\
\hline$+\mathrm{Ve}$ & 4 & 10.27 & 5.40 & 4 & 1.62 & 1.60 & 7 & 3.09 & 0.42 \\
& & $(12.8)$ & $(2.0-18.5)$ & & $(0.73)$ & $(1.1-2.2)$ & & $(1.4)$ & $(0.3-0.7)$ \\
$-\mathrm{Ve}$ & 5 & 0.76 & 0.48 & 79 & 0.97 & 0.52 & 129 & 2.50 & 2.76 \\
& $(0.70)$ & $(0.2-1.1)$ & & $(2.2)$ & $(0.3-0.8)$ & & $(6.5)$ & $(1.7-3.9)$ \\
\hline
\end{tabular}

a Number of women

The receiver operator curve $(\mathrm{ROC})$ analysis found that the area under the ROC curve ranged from 0.82 and 0.87 for all five D-dimer assays, demonstrating that all the assays were able to discriminate between pregnant women with and without DVT. The authors also found that the sensitivities of all five assays were 100\% when the standard D-dimer cut-off point was used, however, the specificity was poor (range, $6-23 \%$ ). Using new optimal cut-off points $[1.89 \mu \mathrm{g} / \mathrm{mL}$ for the Vidas assay, $1.51 \mu \mathrm{g} / \mathrm{mL}$ for the Asserachrome assay, $0.57 \mu \mathrm{g} / \mathrm{mL}$ for the IL assay, $1.38 \mu \mathrm{g} / \mathrm{mL}$ for the Sta-Lia assay, and $1.50 \mu \mathrm{g} / \mathrm{mL}$ for the Innovance assay], the specificity increased substantially to $61-79 \%$, with a small reduction in sensitivity in four of the assays (range $93-100 \%$ ) and a modest reduction in the fifth assay (IL test) of $80 \%$. The authors conclude that setting a higher threshold for D-dimers for the initial screening of VTE during pregnancy is safe and effective, based on a negative predictive value of $98 \%$ and a sensitivity of $60 \%$.

Whilst this study has demonstrated the possible utility of D-dimer testing for the diagnosis of VTE in the antenatal setting, one published case report of a patient with antenatal VTE with a normal D-dimer does provide a note of caution [To et al., 2008]. In this isolated report, the initial VTE event was diagnosed during the first trimester and the patient was prescribed full dose LMWH therapy according to the RCOG guidelines. On re-presentation during the third trimester, where the patient was reported to be non-compliant, the patient was found to have a normal D-dimer concentration. D-dimers do have a long half-life and the patient injecting the LMWH early on during their pregnancy and then on and off thereafter, may well have suppressed to a certain degree, any expected rise during the third trimester. This is speculation however, and the case serves as a reminder that D-dimer concentrations should not be used in isolation, for the initial screening of antenatal VTE. 
Overall, the evidence published to date demonstrates that D-dimer concentrations do indeed increase during pregnancy and although historically they have not been regarded as a useful test to exclude antenatal VTE, they could hold an important clinical utility, if a pregnancy specific reference range can be established.

\subsubsection{D-dimer concentrations in women with antenatal medical complications}

Accepting the rise in D-dimers during pregnancy, are there differences in the rise observed, depending on the underlying antenatal medical complication during pregnancy? Few studies have assessed this. Gafney and colleagues [1987] assessed D-dimer concentrations during the third trimester of their pregnancy, in women with evidence of pre-eclampsia and compared the results to a control pregnant group, at the same stage of their pregnancy. In this small study, compared to the 14 controls, the 24 women with pre-eclampsia had much higher elevations of D-dimers, and the authors suggest that a clear distinction between the pre-eclamptic women and the non-preeclamptic women can be observed.

Nolan and colleagues [1993], evaluated D-dimer concentrations in four groups of women; 17 normal women, 14 with preterm labour, 17 women with pre-eclampsia at term and 14 patients with abruption placentae during the third trimester. As in previous studies, D-dimer concentrations were raised in line with gestational age. Those subjects with preeclampsia, preterm labour and abruption placentae had mean D-dimer concentrations significantly greater than those of controls $(p<0.003)$, suggesting that $D$-dimer concentrations might indeed hold a utility of identifying women at higher risk of pre-eclampsia, pre-term labour and abruption placentae. Though an interesting signal emerges from this study, clearly, studies with higher patient numbers are required before the results from this study can be accepted into wider clinical practice.

Finally, Francalanci and colleagues [1995] collected D-dimer measurements from 37 pregnant women with intra-uterine growth retardation (IUGR) and 22 women with gestational hypertension, in order to test the hypothesis that women with these antenatal complications can be identified through an elevated D-dimer concentration. The IUGR patients were divided into two groups according to gestational age; $\leq 30$ weeks (group A) and $>30$ weeks (group B). Two control groups of 37 and 22 normal matched pregnant women were also followed. In group A IUGR patients, D-dimer plasma concentrations were significantly higher than matched controls $(p=<0.05)$. However, in group B IUGR patients, no significant differences were found between the active and the control groups. In the gestational hypertension cohort of patients, the subjects had 
higher D-dimer concentrations than the controls $(p<0.05)$, but the authors noted that 15 out of the 22 subjects had a D-dimer concentration lower than the mean +2 standard deviations of controls. The authors suggest that using a ELISA D-dimer assay, as utilised in their study, it is not possible to discriminate IUGR and gestational hypertension women when compared to matched controls.

The results published to date, exploring the utility of D-dimer concentrations for discriminating for antenatal complications contradict one another, and due to their observational nature provide little firm direction of the place of D-dimer concentrations in this setting. However, these studies do demonstrate the general rise of D-dimer concentrations during pregnancy. In order to answer whether D-dimer concentrations could be an appropriate biomarker for IUGR or pre-eclampsia further larger controlled studies need to be conducted.

\subsubsection{The impact of LMWH on D-dimer concentrations during pregnancy}

As the gravid state represents a pro-thrombotic state, and D-dimer concentrations rise during pregnancy, is the rise in D-dimer concentrations altered by women who are injecting LMWH? Only one study published to date has assessed this [Hoke et al., 2004]. In this study, 61 women prescribed LMWH thromboprophylaxis throughout pregnancy due to a history of VTE, hereditary thrombophilia and/or previous pregnancy related complications, had their D-dimer concentrations measured at set times during their pregnancy. Dalteparin or enoxaparin were prescribed at a dose range of 4000 to 7500 anti-factor Xa IU daily, commenced as soon as possible after a positive pregnancy test. These results were then compared to a control group comprising 113 healthy pregnant women without a need for thromboprophylaxis. The authors found that D-dimer concentrations in both subjects and controls increased significantly from the first to the second trimester $(p<0.0001)$, as well as from the second to the third trimester $(p<0.0001)$. The authors also describe that the $D$-dimer levels were significantly higher $(p<0.0001)$ among subjects compared with controls. The results are interesting from two viewpoints; firstly despite being prescribed prophylactic LMWH, substantial activation of coagulation is seen during pregnancy, i.e. the prophylactic doses of LMWH do not dampen down the increase in D-dimers one expects to observe. Secondly, those women prescribed LMWH had significantly higher D-dimer concentrations throughout pregnancy compared to women in the control group. This suggests that pregnant women identified at risk of VTE or pregnancy-related complications do indeed have higher activation of coagulation compared to a relatively healthier pregnant control group, not at 
risk. Hoke's work begs the questions of whether treatment doses of LMWH alter the increase in D-dimer concentrations observed.

\subsection{Aim and method}

As very little has been published on how the $\mathrm{D}$-dimer concentrations of pregnant women injecting LMWH (both prophylactic and treatment doses), the aim of this sub-study was to describe Ddimer concentrations in the cohort of women followed during the course of this study.

Chapter 2 provides full details on the methodology for this aspect of the study, and the specific Ddimer assay used. In brief, women recruited to this study had D-dimer concentrations measured at each clinic visit, typically every month during their pregnancy. Additionally, D-dimer concentrations were measured at delivery and $\geq 8$ weeks post-partum, when anticoagulant therapy had ceased.

For the purposes of analysis, the D-dimer concentrations were grouped into 5 time points; first, second and third trimester, within a week of delivery and $\geq 8$ weeks of delivery. The D-dimer concentrations were reported as median and interquartile ranges (IQR), for the cohort as a whole, and then further analysed at these time points, according to the dose of enoxaparin prescribed (prophylaxis or treatment dose). Friedmans mean rank test was used to assess if statistically significant differences existed between the different time points, with the Wilcoxon pair test used to assess significance between specific pairs of trimester timepoints.

Furthermore, in order to assess if ethnic differences existed between the African-Caribbean and Caucasian populations, the median values of those women prescribed prophylactic enoxaparin were compared using the Mann-Whitney $U$ independent rank-sum test, at each pregnancy timepoint.

\subsection{Results}

Over the course of the study, 815 separate D-dimer concentrations were drawn from the women (table 5.6). An increase in D-dimer concentrations was found in line with gestational week, with 
the concentrations peaking at the time of delivering, falling back to 'baseline' levels by 8 weeks postpartum (table 5.6, figures 5.2 and 5.3).

Table 5.6 The overall D-dimer results from this study for the cohort of women followed

\begin{tabular}{llcc}
\hline Pregnancy time point & $n$ & Median & Interquartile range \\
\hline First trimester & 88 & 315 & $245-467$ \\
Second trimester & 240 & 620 & $450-970$ \\
Third trimester & 287 & 990 & $690-1550$ \\
Within a week of delivery & 102 & 1497 & $2225-3910$ \\
$\geq 8$ weeks postpartum & 98 & 335 & $230-482$ \\
\hline
\end{tabular}

$n$ represents the number of D-dimer samples at each trimester time point

Although the standard deviations for the results are large and demonstrates much inter-patient variability, a clear pattern is observed in line with gestational age.

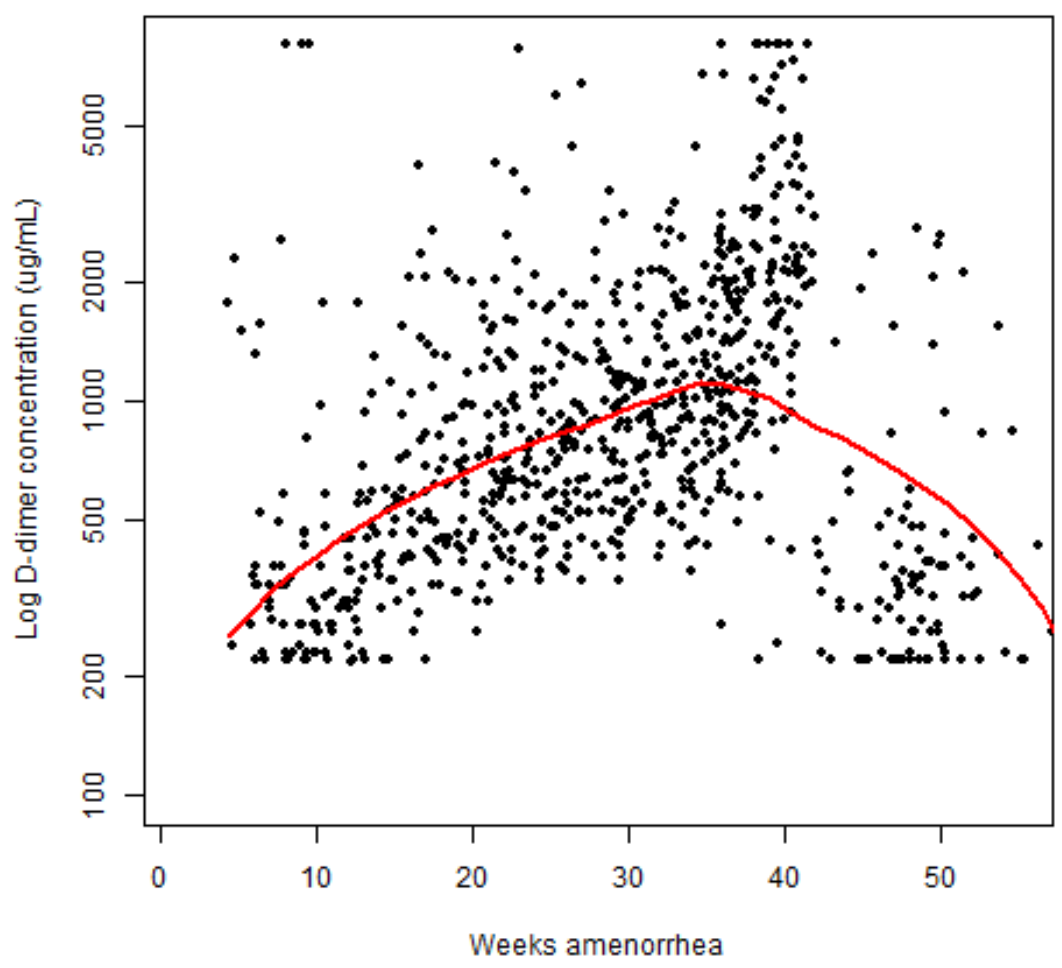

Figure 5.2: Scatter plot of log D-dimer concentration versus weeks amenorrhea (red line represents the loess smooth) 


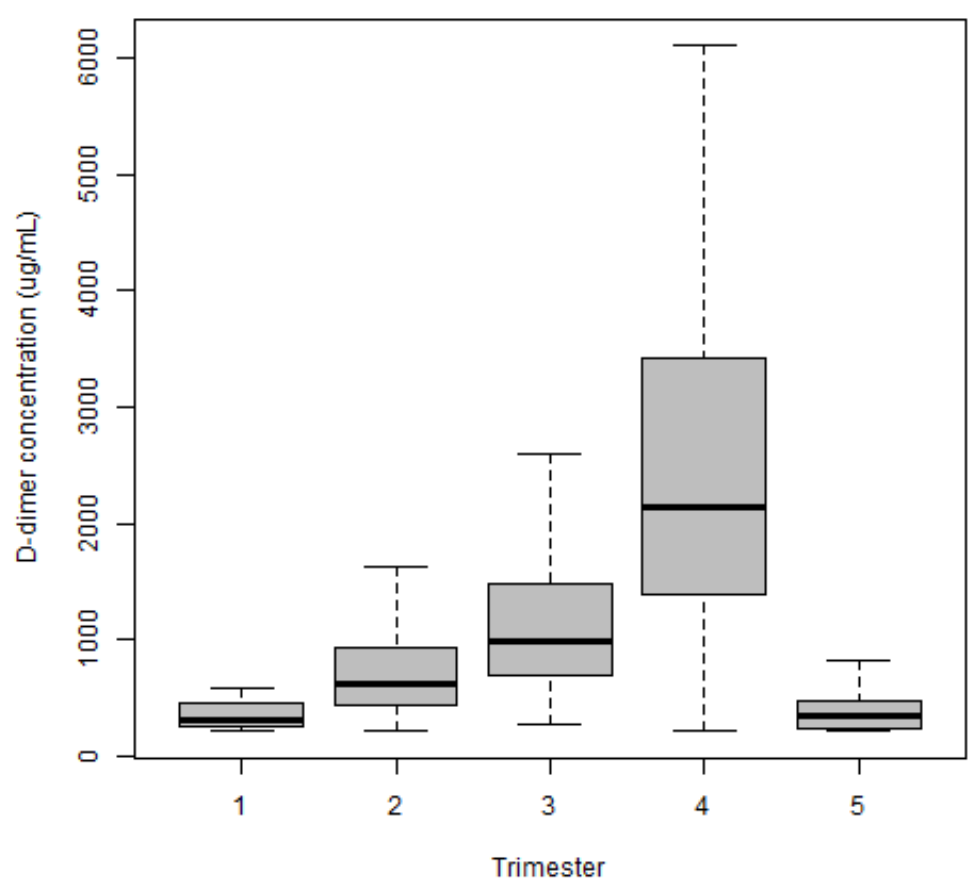

Figure 5.3: Box plot illustration of the D-dimer concentrations, according to each trimester (trimester 4 and 5 represent within a week of delivery and $\geq 8$ weeks post partum respectively)

A significant positive correlation is observed between the D-dimer concentrations and pregnancy gestational age; $r=0.382$ (Pearson's correlation), $p=0.000$. Thirty-six women had consecutive $D$ dimer concentrations measured from the first trimester through to $\geq 8$ weeks of delivery. Friedmans mean rank test demonstrates statistically significant differences between the five different time points assessed $(n=36, d f=4, p=0.000)$. As many women did not book until after the first trimester had passed, when the analysis is re-run excluding the first trimester (which results in more women being considered in the analysis), the same result is found; Friedmans mean rank test $(n=75, d f=3, p=0.000)$ confirming the finding that statistically significant differences do exist between the time points.

A Wilcoxon pair test was conducted comparing results between each trimester. Significant $(<0.05)$ differences were found when each trimester pair, except when the first trimester was compared with the $\geq 8$ weeks post-partum time point (perhaps unsurprisingly).

Sections 5.3.1 and 5.3.2 illustrate how the overall results change when women on prophylactic and treatment doses of enoxaparin are considered as separate groups. 


\subsubsection{Women prescribed prophylactic doses of enoxaparin}

In total $619 \mathrm{D}$-dimer concentrations were drawn from women prescribed prophylactic enoxaparin. The correlation between D-dimer concentrations and gestational age was found to increase to $r=0.441$ (Pearson's correlation), $p=0.000$. Table 5.7 describes the $D$-dimer concentrations for women on prophylactic doses on enoxaparin, and figure 5.4 illustrates the D-dimer concentration rise pattern observed, which largely mirrors the cohort of women overall.

Table 5.7: D-dimer concentrations for the cohort of women prescribed prophylactic doses

\begin{tabular}{lccc}
\hline Pregnancy time point & $N$ & Median & Interquartile range \\
\hline First trimester & 64 & 335 & $270-487$ \\
Second trimester & 193 & 620 & $465-955$ \\
Third trimester & 225 & 1020 & $735-1625$ \\
Within a week of delivery & 79 & 2360 & $1790-4130$ \\
$\geq 8$ weeks postpartum & 78 & 365 & $256-525$ \\
\hline
\end{tabular}

$n$ represents the number of $\mathrm{D}$-dimer samples at each trimester time point

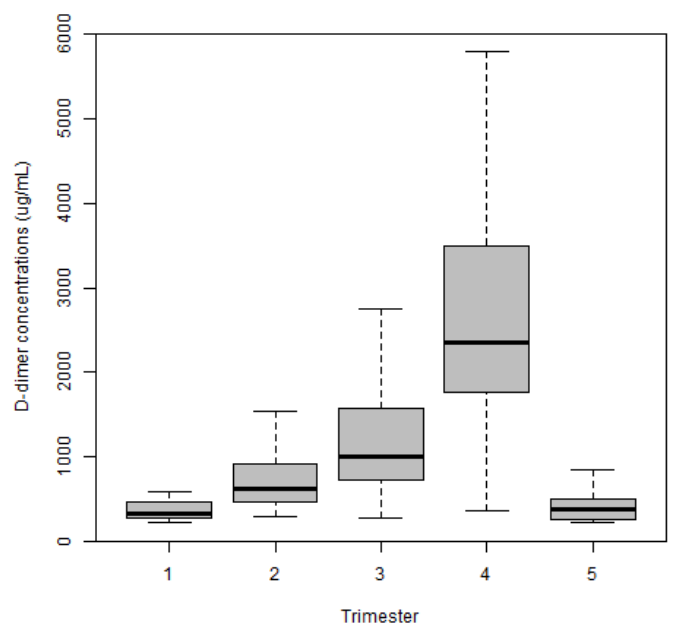

Figure 5.4: The rise in D-dimer concentrations in women prescribed prophylactic doses of enoxaparin; trimester 4 and 5 refers to within a week of delivery and $\geq 8$ weeks post-partum respectively

Twenty-six women had serial D-dimer concentrations measured consecutively from the first trimester through to $\geq 8$ weeks post-partum. Friedmans mean rank test demonstrated statistically significant differences between the five different time points $(n=26, d f=4, p=0.000)$. When this 
analysis is re-run excluding trimester one results, this result is repeated; Friedmans mean rank test $(n=58, d f=3, p=0.000)$ providing further confirmatory evidence that statistically significant do exist between each time point.

A Wilcoxon pair test was conducted comparing results between each trimester. Significant $(<0.05)$ differences were found when each trimester pair, except when the first trimester was compared with the $\geq 8$ weeks post-partum time point, mirroring the results from the overall cohort of women.

\subsubsection{Women prescribed treatment doses of enoxaparin}

Women prescribed treatment doses of enoxaparin did exhibit a different pattern of D-dimer concentrations relative to those women on prophylactic doses. The strength of the relationship between D-dimer concentrations and gestational weeks went down to $r=0.212$ (Pearson's correlation), though still statistically correlated. The rise in D-dimer concentrations for this subgroup of women was also much smaller, relative to women prescribed prophylactic doses of enoxaparin (table 5.8, and figure 5.5), illustrating an enoxaparin effect.

Table 5.8: Specific results from women prescribed treatment doses of enoxaparin

\begin{tabular}{llcc}
\hline Pregnancy time point & $N$ & Median & Interquartile range \\
\hline First trimester & 24 & 270 & $230-422$ \\
Second trimester & 47 & 480 & $350-1180$ \\
Third trimester & 62 & 850 & $545-1270$ \\
Within a week of delivery & 23 & 1610 & $440-2450$ \\
$\geq 8$ weeks postpartum & 20 & 260 & $220-325$ \\
\hline
\end{tabular}

$n$ represents the number of D-dimer samples at each trimester time point 


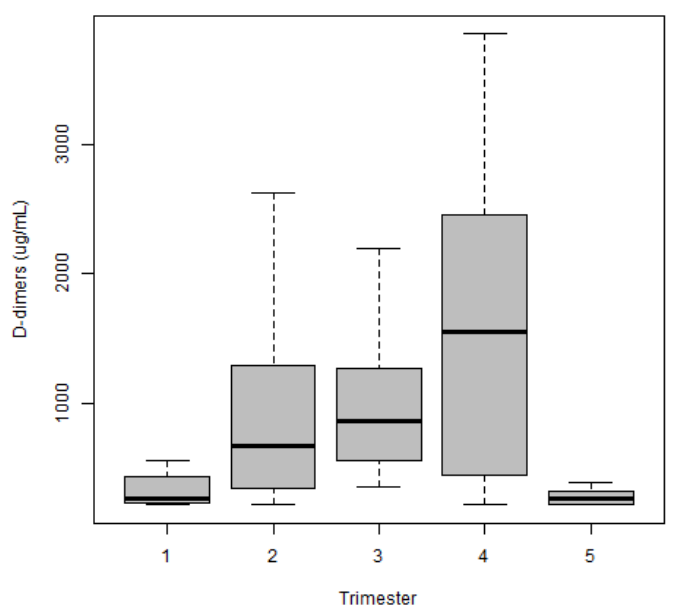

Figure 5.5: The D-dimer concentrations of women prescribed 'treatment' dose enoxaparin during their pregnancy (trimester 4 and 5 refers to within a week of delivery and $\geq 8$ weeks post-partum respectively)

Ten women prescribed treatment dose enoxaparin had serial D-dimer concentrations consecutively measured from the first trimester through to $\geq 8$ weeks of delivery. Friedmans mean rank test demonstrated statistically significant differences between the five different time points $(n=10, d f=4, p=0.000)$. When the analysis was re-run excluding the $1^{\text {st }}$ trimester results, this result is repeated; Friedmans mean rank test $(n=17, d f=3, p=0.000)$ confirming the finding that statistically significant do exist between each time point.

A Wilcoxon pair test was conducted comparing results between each trimester. Significant $(<0.05)$ differences were found between the following time points: second and third trimesters and within a week of delivery and $\geq 8$ weeks postpartum.

\subsubsection{The influence of ethnicity on D-dimer concentrations}

Two ethnic groups, Caucasians and African-Caribbeans, formed the majority of patients recruited to this study, reflecting the location of the hospital in South London. Sub-group analysis between these groups, excluding women on treatment doses, revealed no significant differences (Mann Whitney rank-sum test) between these two groups at all time points (table 5.9). 
Table 5.9: Medium D-dimer concentrations (IQR) for the Caucasian and African-Caribbean ethnic groups of women prescribed prophylactic doses of enoxaparin

\begin{tabular}{lccc}
\hline Time point & \multicolumn{2}{c}{ Ethnicity } & p-value \\
\cline { 2 - 3 } & Caucasian & African - Caribbean & \\
\cline { 2 - 3 } First Trimester & 340 & 335 & $\mathrm{U}=247$, \\
& $(267-512)$ & $(302-430)$ & $\mathrm{p}=0.925$ \\
Second Trimester & 600 & 665 & $\mathrm{U}=2709$, \\
& $(457-865)$ & $(492-992)$ & $\mathrm{p}=0.225$ \\
Third Trimester & 1000 & 1135 & $\mathrm{U}=3541$, \\
& $(715-1540)$ & $(877-1687)$ & $\mathrm{p}=0.071$ \\
Within a week of delivery & 2400 & 2350 & $\mathrm{U}=505$, \\
& $(1690-4250)$ & $(1760-4610)$ & $\mathrm{p}=0.760$ \\
$\geq 8$ weeks post-partum & 365 & 350 & $\mathrm{U}=504$, \\
& $(230-505)$ & $(300-490)$ & $\mathrm{p}=0.750$ \\
\hline
\end{tabular}

Median (IQR) D-dimer values presented; *Mann-Whitney U independent rank-sum test

These results demonstrate that ethnicity does not appear to impact significantly on D-dimer results during pregnancy and, if a pregnancy-specific D-dimer concentration cut-off is established in the future, ethnicity would not need special consideration for this assay. This mirrors current clinical practice in the non-pregnant population.

\subsection{Discussion and implications for practice}

In line with previously published studies, this study has demonstrated substantial activation of the fibrinolytic system during pregnancy, as assessed through D-dimer concentrations. D-dimer concentrations were found to significantly rise over the course of pregnancy, peaking at the time of delivery, before dropping back to baseline concentrations by eight weeks post-partum. Despite the fact that the women were prescribed enoxaparin therapy during the course of their pregnancy, the rise in D-dimer concentrations was not suppressed, a finding endorsing that of Hoke and colleagues [2004], where women were prescribed prophylactic doses of enoxaparin. When the Ddimer concentrations of women prescribed treatment and prophylactic doses are compared, more differences at the different pregnancy time points were observed for women prescribed prophylactic doses of enoxaparin compared to women prescribed treatment doses. This suggests 
that although the increase in D-dimer concentration is not suppressed, the D-dimer concentration is influenced by enoxaparin in a dose dependent manner.

Ethnic differences between the subjects did not appear to influence the D-dimer pattern to any significant degree and if an antenatal specific D-dimer concentration is established, the results here suggest that ethnicity would not need special consideration. It must be remembered though, that this study's data is observational in nature and others have reported differences in D-dimer concentrations between the African-Caribbean and Caucasian population outside of pregnancy [Pieper et al., 2000; Cushman et al., 2003; Khaleghi et al., 2008]. On the other hand, a recent study by Roberts and colleagues comparing D-dimer concentrations between patients who suffered a first deep vein thrombosis and controls from a Caucasian and African-Caribbean population found no significant differences in D-dimer concentrations between the two groups, outside of a pregnancy setting, as well as when the two control groups were compared [Roberts et al., 2012]. The predictable nature of the results from the cohort of women prescribed prophylactic doses provides further compelling evidence that it should be possible to develop a pregnancy specific D-dimer normal concentration reference range, which could then be used as an initial VTE screening test during pregnancy, for women suspected of VTE.

\subsubsection{Strengths, limitations and future work}

The strength of this sub-study are, the number of D-dimers samples measured, the serial measurement nature of D-dimer concentrations in the cohort of women followed, and it is only the second study to assess D-dimer concentrations in pregnant women, prescribed concurrent LMWH.

The study is limited due to its observational nature. It would have been more meaningful if $D$ dimer concentrations were also measured in a concurrent control group. Work is currently underway at King's College Hospital specifically assessing this issue. It must be borne in mind though, that during the course of this study, much inter-individual variability was observed. Future studies might also focus on identifying what factors cause this variability (e.g. the presence of a specific thrombophilia or antenatal complication). Furthermore, if a pregnancy specific reference range for a specific assay is developed, it must be remembered that the initial presentation of antenatal VTE is variable and challenging, and that D-dimer concentrations should not be used in isolation, but in conjunction with other confirmatory testing, as is the case outside of pregnancy [National Institute for Health and Clinical Excellence, 2012]. 


\section{Chapter 6}

\subsection{Thrombin generation during pregnancy}

The focus of chapter 6 is the measurement of thrombin generation as pregnancy progressed in the cohort of women recruited during the course of the enoxaparin in pregnancy study. After discussing some further background to thrombin generation testing, specific questions around assaying thrombin generation within a pregnant population prescribed LMWH are posed. The methodology for this aspect of the study is described, following which, the results are presented and discussed. In addition, work aimed at neutralising enoxaparin in the women's plasma during in-vitro thrombin generation, using polybrene, is also presented and discussed.

\subsubsection{Background}

In chapter one, reference to how thrombin generation is central to coagulation is explained. The capacity to generate thrombin and the enzymatic work that thrombin does, determines an individual's blood coagulability [Baglin, 2005]. In clinical practice, to have a test which determines an individual's coagulation potential is extremely useful, as it can help to determine which patients might benefit from long-term anticoagulation and/or identify patients who are at higher risk of pathological thrombosis. An assay such as thrombin generation has the potential to provide a meaninful pharmacodynamic marker for patient's prescribed anticoagulant therapy. Excellent reviews already exist on the background to thrombin generation testing and the clinical potential that exist [Hemker at al., 2005 Baglin, 2005, van Veen et al., 2008]; although much has been promised, the clinical benefits and utility of the test are yet to be realised in widespread clinical practice. Why might this be the case?

\subsubsection{Limitations of TG testing}

A number of reasons can be attributed as to why the application of thrombin generation has yet to be incorporated into wide-spread clinical practice. Different methods and reagents used in thrombin generation testing can yield different results and influence the total amount of thrombin generated, making comparisons between laboratories and studies difficult to assess. For example the concentration of tissue factor (TF) used as the trigger for the assay can influence the path of thrombin generation that actually gets assessed. The positive feedback activation of thrombin by the intrinsic pathway can only be seen at low TF concentrations [Keularts et al., 2001; van Veen 
et al., 2008]. TF concentrations of $1 \mathrm{pmol} / \mathrm{L}$ in platelet poor plasma (PPP) are required to observe the effects of Factor $\mathrm{XI}$, an observation which is lost at concentrations $>5 \mathrm{pmol} / \mathrm{L}$. Dielis and colleagues [2008] investigated the determinants of thrombin generation in a normal population at two tissue factor concentrations with or without thrombomodulin or activated protein C. Their results showed that thrombin generation is determined by different coagulation factors or inhibitors, depending on the specific experimental conditions.

Furthermore, a number of studies have reported significant contact factor activation with the thrombin generation assay [Luddington and Baglin, 2004; Tappenden et al., 2007; van Veen et al., 2008]. This is problematic as contact factor activation can give falsely elevated levels of thrombin generation, obscuring the true result. Research suggests that contact factor activation is more likely when low tissue factor concentrations are used as the trigger in the assay. To overcome this, some advocate the addition of corn trypsin inhibitor (CTI) to the assay reagents to minimise contact factor activation; the addition of CTI has the added benefit of reducing variability observed in the final results [Baglin, 2005; van Veen et al., 2008].

Additionally, when thrombin generation is assessed using platelet poor plasma (PPP), the phospholipid concentration in the test plasma can influence the final results obtained. During thrombin generation experiments, if the phospholipid concentration present is not adequate, the reaction could become rate limiting. Research has revealed that a phospholipid concentration of between 3-5 $\mu \mathrm{mol} / \mathrm{L}$ is sufficient, and been proven not to be rate-limiting [Chantarangkul et al., 2003; Hemker et al., 2003; Gerotziafas et al., 2005]. The fact that the test can be conducted in PPP and platelet rich plasma (PRP) complicates matters, as samples from the same patient with these two different methods of preparation will yield different thrombin generation results. In PRP, the ETP and peak height are found to be lower when compared to the same subject's sample tested under PPP conditions. In PRP, the lag-time is more prolonged in comparison to the same sample tested under PPP conditions. Furthermore, under PRP conditions, the platelet count in the test plasma can also impact on the thrombin generation result obtained. This is found to be the case when the platelet count is $<100 \times 10^{9} / \mathrm{L}$ [Hemker et al., 2003]. Once above $200 \times 10^{9} / \mathrm{L}$, dependence on platelet numbers is thought to be minimal. Under PRP, under the aforementioned conditions, thrombin generation is also dependent upon concentrations of factor VIII, IX and XI [Hemker et al., 2003]. 
Finally, not all aspects of the coagulation pathway can be evaluated with a single set of reagents in the thrombin generation assay; for example evaluation of the protein $C$ pathway requires a special set of reagents. Standard thrombin generation assays are not sensitive to the actions of the protein $\mathrm{C}$ pathway, as thrombomodulin is not present. However, the protein $\mathrm{C}$ pathway can be assessed in-vitro by the addition of truncated human recombinant thrombomodulin (sTM) [van Hylckama Vlieg et al., 2007]. The addition of thrombomodulin makes the thrombin generation test sensitive to deficiencies in protein $\mathrm{C}$, protein $\mathrm{S}$, factor $\mathrm{V}$ Leiden and conditions associated with acquired protein C resistance [van Veen et al., 2008], providing a different slant to the thrombin generation experiment, but also demonstrating that a single set of experimental conditions cannot evaluate all aspects of thrombin generation.

The other important issue to consider, unrelated to the reagents and experimental conditions of the test, is one of variability within the test, both intra-assay, inter-assay variability, as well as inter-patient variability. The variability results for both PPP and PRP, from 4 individuals who had their thrombin generation assayed over 9 consecutive weeks are listed in table 6.1.

Table 6.1: Inter-assay and intra-assay variability of thrombin generation in 4 healthy controls

\begin{tabular}{lllcc}
\hline $\begin{array}{l}\text { Conditions of } \\
\text { experiment }\end{array}$ & TG parameter & & CV (\%) & \\
\cline { 3 - 5 } & & & & \\
\hline PPP & ETP & 15 & 4.5 & 2.5 \\
& Peak & 13 & 5.5 & 4.5 \\
& Lag Time & 47 & 8.1 & 5 \\
PRP & ETP & 19 & 7.4 & 3 \\
& Peak & 34 & 10.3 & 3.5 \\
& Lag Time & 24 & 9.4 & 7 \\
\hline
\end{tabular}

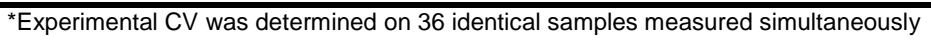

Intra-assay variability was found to be $8 \%$. The inter-individual variability was reported as $17.5 \%$, and suggests that significantly different endogenous thrombin potentials exist between individuals [Hemker et al., 2006]. Others have also reported similar variability results; Dargaud and colleagues [2008] assayed thrombin generation in 12 healthy volunteers every three months for 1 year using CAT, at 3 different tissue factor concentrations $(0.5,1$ and $5 \mathrm{pM})$. They report intraindividual variability of $11 \%$ over the course of the year. Experts in the use and application of the thrombin generation assay have proposed that variability between laboratories could be improved 
by assaying a reference plasma in each thrombin generation experiment and using the same reference plasma across all laboratories, allowing normalisation of the results and a meaningful comparison between the laboratories [Dargaud et al., 2010]. This approach has gained further credence with the publication of a further multi-centre study [Dargaud et al., 2012].

Thrombin generation assays can be performed by numerous methods and reagents and depending on how they are performed, will have different sensitivities for various haemostatic or thrombotic defects. Results from the assay cannot therefore be interpreted without detailed information on how the test was conducted. Currently, multi-centre studies are difficult in the absence of international standards of thrombin and tissue factor. It must be borne in mind that even when a standardised test is available, it is unlikely to be suitable in all situations and depending on the clinical situation under investigation and the specific objectives of the study, the right set of reagents will need to be employed.

\subsubsection{What is the influence of anticoagulant therapy on the thrombin generation test?}

A number of studies have been published which describe the pharmacodynamic effect of anticoagulant therapy on the thrombin generation test, under different experimental conditions. Most of these studies have involved in-vitro spiking of the anticoagulant in question within the experiments, with few actually measuring thrombin generation in patients prescribed anticoagulants.

When evaluating the impact of the oral anticoagulant warfarin on thrombin generation, the endogenous thrombin potential (ETP) exhibits a moderate hyperbolic relationship and negative correlation with the INR in patients prescribed warfarin therapy [Jackson et al., 2003; Altman et al., 2007]. Studies assessing the impact of unfractionated heparin (UFH) [Al Dieri et al., 2004], LMWH [Al Dieri et al., 2006; Gerotziafas et al., 2007] and the direct Xa inhibitor, danaparoid [Stief, 2007] on thrombin generation have been conducted. These studies suggest that UFH, tinzaparin and danaparoid have a more profound effect on thrombin generation relative to $\mathrm{LMWH}$, due to their significantly greater anti-Ila effect, and therefore imply that thrombin generation tests might not detect all the significant activity of LMWH; depending on the molecular weight of $\mathrm{LMWH}$, and the dose prescribed, the anti-thrombin activity and hence the thrombin generation effect will vary.

Studies assessing the newer oral anticoagulants, rivaroxaban and dabigatran, on thrombin generation suggest a concentration dependent effect of these agents on the thrombogram 
parameters [Gerotziafas et al., 2007; Wienen et al., 2007]. Interestingly, in their study, Gerotziafas and colleagues found that the concentration of rivaroxaban required to decrease the lag-time and time to peak to $50 \%$ (IC50), is lower than that required to produce the same effect on the peak height and ETP, suggesting that certain parameters of the thrombogram are more sensitive to the effects of rivaroxaban than others.

Details on the three studies which provide the most information on the effect of LMWH on the thrombogram are described in detail.

Al Dieri and colleagues [2006] tested the hypothesis that the ETP is a sensitive detector of heparin's effect, both LMWH and UFH. In 12 healthy male volunteers, average age 25 years (range:18-30), with a mean body weight of $80 \mathrm{~kg}$ (range:60-95kg), a mono-centric, randomised, double-blind, four-period cross-over trial was conducted. All patients were administered a single subcutaneous injection of 9000 anti-Xa IU of either heparin (UFH, a medium molecular weight heparin (MMWH), certoparin or enoxaparin), followed by a wash-out period of one week, before the next agent was administered. Blood samples assessing thrombin generation were drawn at $t=0,0.5,1.5,2,3,4,5,8,10$ and 24 hours, following the administration of each agent. Anti-Xa activity was performed by amidolytic assays and thrombin generation was measured using a validated chromogenic method. In their experiments using PPP, $30 \mathrm{pM}$ of recombinant TF and a 6 $\mu \mathrm{M}$ concentration of phospholipid was employed. The authors' aim was to assess ETP inhibition relative to time zero, along with the activity of factor Ila and factor Xa.

The authors found that the course of anti-Xa and anti-lla activities in plasma following the four heparin agents showed significant inter-individual variability in the order of $\sim 40-50 \%$ for UFH and $\sim 30-40 \%$ for the other three. Interestingly, the authors also report that the highest heparin levels were found in the volunteer with the lowest weight $(60 \mathrm{~kg})$, and the lowest in volunteer with the highest weight $(98 \mathrm{~kg})$, confirming the strong relationship which exists between weight and heparin concentrations. The authors headline results were, that as the concentration of the heparin in plasma changes with time, so does the ETP; this is interesting, as it confirms that an individual's propensity to generate thrombin is dynamic, not static, and will change with an ever changing heparin concentration in the plasma. Al-Dieri and colleagues report that a hyperbola curve is generated in relation to heparin activity and ETP inhibition. $I_{50}$ values, that is to say, the heparin concentration which reduces the ETP value registered at time zero by $50 \%$, was calculated for each of the four agents in relation to both their Ila and Xa activity. In terms of anti-Xa values, the 
$I_{50}$ was variable and increased with the molecular weight of the heparin, i.e. the higher the molecular weight, the lower the concentration required (table 6.2).

Table 6.2: Anti-Ila and anti-Xa activity of the four agents evaluated by Al-Deiri and colleagues [2006]

\begin{tabular}{lllll}
\hline & UFH & MMWH & Certoparin & Enoxaparin \\
\hline alla $\left(\mathrm{IU} \mathrm{mL} \mathrm{mL}^{-1}\right)$ & $0.10+/-0.01$ & $0.10+/-0.01$ & $0.09+/-0.01$ & $0.06+/-0.01$ \\
aXa $\left(\mathrm{IU} \mathrm{mL} \mathrm{mL}^{-1}\right)$ & $0.10+/-0.01$ & $0.12+/-0.01$ & $0.29+/-0.04$ & $0.36+/-0.04$ \\
\hline
\end{tabular}

The authors of the study stipulate that ETP is not yet validated for the control of anticoagulant therapy, and go onto suggest that a therapeutic window could be possible with this parameter. A traditional INR range of 2-4 corresponds to ETP inhibition of $17-40 \%$ [Odegard et al., 1976]. Studies also suggest that in congenital clotting factor deficiency, bleeding is seen at a ETP inhibition of 20-30\% [Al Dieri et al., 2003; Gouin-Thibault et al., 2003; Ip et al., 2001; Alhenc-Gelas et al., 1994].

In a further study, Gerotziafas and colleagues [2007], assessed the inhibition of thrombin generation induced by the low molecular weights bemiparin, enoxaparin, nadroparin, dalteparin, and tinzaparin in PRP and compared their effects to that from unfractionated heparin and fondaparinux. In their experiments, normal PRP was used, with the platelet count adjusted to 1.5 $x 10^{5} \mathrm{~L}^{-1}$, and a TF trigger concentration of $6 \mathrm{pM}$. The PRP was spiked with increasing concentrations of the agents being assessed to evaluate their effects on thrombin generation. Thrombin generation was assayed according to the CAT method, as described by Hemker and colleagues [2000]. The different LMWH agents influenced the different parameters of the thrombogram in their own unique way. When considering the lag-time and the time to peak, at a concentration $0.2 \mathrm{IU} / \mathrm{mL}$ of anti-FXa, bemiparin, enoxaparin, nadroparin and dalteparin did not appear to significantly affect these parameters, relative to control samples. Tinzaparin, the LMWH most closely related to UFH, did significantly prolong the lag-time and time to peak as compared to control samples. UFH samples, at the equivalent anti-Xa activity, also prolonged the lag-time and time to peak. A doubling of concentration to $0.4 \mathrm{IU} / \mathrm{mL}$, bemiparin, enoxaparin, nadroparin and dalteparin prolonged both the lag-time and time to peak by about $20 \%$. Further increases in the concentration led to further prolongation, suggesting a dose- dependent effect; it is interesting to note however, the influence of both UFH and tinzaparin at the low concentration were 
measurable on the thrombogram relative to the other four LMWH. The key parameters considered important with the thrombogram are the peak height, ETP and the thrombin generation velocity. When considering the rate of TG velocity, a similar picture to the lag time and time to peak emerges, that is to say, at low concentrations UFH and tinzaparin exert a significant effect, and other LMWH reduced the thrombin generation velocity by less than $20 \%$. Further increases in heparin concentrations led to more marked effects, as one would anticipate. When evaluating the ETP and peak height parameters of the thrombogram, the concentration of the LMWH, exhibited a dose dependent effect, with concentrations of $0.2 \mathrm{IU} / \mathrm{mL}$ of bemiparin, enoxaparin, nadroparin and dalteparin not significantly modifying the peak or ETP values as compared to controls. At concentrations $\geq 0.4$ anti-FXa IU/mL, the LMWHs induced a significant concentration dependent reduction of the ETP and peak concentration; as per the lag-time and time to peak results, the bemiparin required an even higher concentration to achieve the same result. Once again, tinzaparin and UFH exhibited a similar concentration-dependent inhibition of peak and ETP at concentrations $\geq 0.2$ anti-FXa IU $/ \mathrm{mL}$.

The authors of this study were able to identify three distinct groups of LMWH, in terms of how they influenced the thrombin generation test. Significantly higher concentrations of bemiparin (group 1) than of enoxaparin, nadroparin, and dalteparin (group 2) were required to inhibit thrombin generation by $50 \%$. Enoxaparin, nadroparin and dalteparin were less active than tinzaparin (group 3). Tinzaparin inhibited thrombin generation by $50 \%$ at similar concentrations to those of UFH. This classification of LMWH was valid for each separate thrombin generation parameter. The important results from this study suggest that anti-Factor Ila activity, which is a major determinant of the ETP and prolongation of TG, and that ETP underestimates the potency of the anti-FXa activity of LMWH. The authors suggest that future research needs to be conducted in patients prescribed LMWH in order to evaluate how much and for how long each phase of thrombin generation has to be inhibited in order to achieve the 'desired' antithrombotic effect.

Green and colleagues [2010] assessed the thromboprophylaxis effect of dalteparin or rivaroxaban in those patients who had undergone elective hip or knee replacements. They did this by measuring thrombin generation pre-operatively, peri-operatively and 24 hour post-operatively, with the thromboprophylaxis agent being assessed, commencing 6-8 hours post-operatively. They report that patients prescribed dalteparin exhibited a variable thrombin generation response after 
surgery, whereas the effects of rivaroxaban were more consistent. Good correlations between rivaroxaban levels and the various parameters of the thrombogram were found, with rivaroxaban found to inhibit thrombin generation more, in relative terms, than dalteparin, at 24 hours post surgery.

Finally a small in-vitro study assessed the inter-individual variability of seven LMWH on CAT [Hacquard et al., 2011]. In this study, samples were drawn from 12 healthy volunteers and the prepared plasma was then spiked with varying concentrations of the LMWH. The authors report a concentration dependent inhibition of thrombin generation, with large inter-individual variability of the effect observed.

The overall messages that emerge from these studies are that there is large inter-individual variability in the response to $\mathrm{LMWH}$, when assessed through the thrombin generation assay, and that different LMWH will have differing effects on the thrombin generation assay, depending on their molecular weight and thus their factor Ila to factor Xa specificity. The studies conducted to date, specifically using enoxaparin, suggest that the full anticoagulant effect of enoxaparin is not seen in the thrombin generation assay.

\subsubsection{Studies assessing thrombin generation during pregnancy}

Very few studies have been conducted which have assessed thrombin generation during pregnancy, with even fewer which have evaluated thrombin generation in women prescribed LMWH during pregnancy. Details of these studies, with specific experimental conditions, are described.

\subsubsection{Thrombin generation during healthy pregnancy}

Eichinger and colleagues [1999] were the first group to assess thrombin generation during pregnancy, and their study was a prospective study evaluating thrombin generation in healthy pregnant women, with and without Factor V Leiden. Thrombin generation (reported as ETP), was measured at fixed time-points $\left(12^{\text {th }}, 22^{\text {nd }}, 34^{\text {th }}\right.$ weeks gestation and 3 months post-partum). The ETP was determined using a chromogenic assay, using 120 pM recombinant TF concentration, as a trigger for the assay. They also followed a control group in their study, which comprised 91 healthy individuals (33 male, 58 female, aged $55+/-11$ years, median 56 years), so their ETP results were expressed as a percentage of normal controls. The pregnant women recruited, comprised 113 women (102 with no FVL, and 11 with FVL). The authors found that ETP levels in 
the pregnant women with and without FVL did not increase over the course of pregnancy $(p=0.2)$, despite evidence of substantial activation of the fibrinolytic system, with increases observed in Fragment 1+2, Thrombin-Antithrombin complex and D-dimer concentrations. This finding is surprising and is likely to be related to the high TF concentration (120 pM) used as a trigger in the study, masking the true thrombin generation pattern of pregnancy. A subsequent smaller study [Ku et al., 2003], also used a high concentration of the trigger and reported no difference in ETP over the course of pregnancy. Both these studies demonstrate the importance of choosing the right TF concentration in evaluating thrombin generation in a pregnant population.

Rosenkranz and colleagues [2007] assessed thrombin generation in 150 healthy pregnant women, aged 18-38 years of age at different gestational ages, contributing a single sample at a routine maternity visit. A sub-group of 20 women were recruited twice, once in the first trimester and again in the third trimester. Thrombin generation was measured by means of calibrated thrombinography in PPP with a $5 \mathrm{pM}$ TF and $4 \mu \mathrm{M}$ phospholipid concentration.

The authors found that the ETP and peak height increased significantly with gestational age, while the lag-time and time to peak remaining unchanged. According to their results, the level of ETP significantly increased from the first to the second trimester, as well as from the first to the third trimester, with no significant difference found between the second and third trimester. A limitation of this study is the fact that the majority of women provided only a single sample during their pregnancy, so it's difficult to conclude whether the pattern of ETP described is true or not, particularly as numbers were small. When analysing the specific results from the sub-group of women contributing samples in the first trimester and repeated again in the third trimester, the authors report significant increases in ETP and peak height levels, with the lag-time and time to peak remaining unchanged, providing relative confirmatory evidence of the wider study results. Accepting the limitations of this study, the results suggest that during normal pregnancy, thrombin generation increases from the first to the second trimester, after which a plateau is reached.

Dargaud and colleagues [2010] followed 93 pregnant women, with a mean age of 29.8 years without a history of VTE or known thrombophilia. Results were obtained from twenty-eight of these women in the first trimester, 33 during the second trimester and 32 during the third trimester. The study authors also recruited a control group in their study, which comprised 50 healthy women, with a mean age of 27.7 years. The thrombin generation assay method employed as part of this study was CAT, using the standard TF trigger concentration of $5 \mathrm{pM}$ and a 
phospholipid concentration of $4 \mu \mathrm{M}$. The study authors report that the ETP was significantly increased amongst the pregnant women, compared to the non-pregnant control group. They found that the ETP was already raised by the first trimester $(p<0.0001)$, with a further rise seen during the second trimester $(p=0.03)$, after which it settled through to delivery $(p=0.67)$. These results provide further evidence of how thrombin generation is altered during normal pregnancy and confirm the findings of Rosenkranz and colleagues [2007] earlier study.

More recently, Wulfkuhle and colleagues [2011] have presented an abstract of their pilot study of thrombin generation during normal pregnancy in young nulliparous women. In this small study, 16 women were recruited and had their thrombin generation measured pre-conception, during early pregnancy, during the $3^{\text {rd }}$ trimester and during the post-partum period (exact times not listed by the authors). For thrombin generation assessment, the study authors used a modified fluorogenic substrate thrombin generation assay, initiated with $5 \mathrm{pM}$ TF and $20 \mu \mathrm{M}$ phospholipid concentration. Unlike previous studies, the authors report the maximum level of thrombin generation (MaxL). They found the maximum level of thrombin generation increased with the progression of pregnancy (pre-conception MaxL 86.3 nM versus early pregnancy MaxL 223.7 versus late pregnancy $322.5 \mathrm{MaxL}$ ), and subsequently decreased during the post-partum period. Though the authors report an increase in thrombin generation from early pregnancy to late pregnancy, as they did not measure thrombin generation during the second trimester, given the findings of other groups, its perhaps inappropriate to conclude that thrombin generation increases progressively during pregnancy, as if it could well be that it reaches a maximal level by the second trimester, something which was not evaluated through data collection in Wulfkuhle's study.

The studies published to date on the thrombin generation pattern during pregnancy are conflicting. Accounting for differences in reagents and methods employed, the conclusion that can be drawn from them are that thrombin generation, as reflected through the ETP increases during pregnancy; there appears to be a significant increase from the first to the second trimester following which the ETP stabilises through to delivery. By approximately six weeks post partum, thrombin generation concentrations will have reached pre-pregnancy values again.

\subsubsection{Thrombin generation of women prescribed LMWH during pregancy}

To date, five studies have been published in the form of 4 abstracts and a short report, which describes thrombin generation results from pregnant women, prescribed concurrent LMWH or who have their plasma spiked, in-vitro, with LMWH. 
Hynes and colleagues [2009] were the first to report their results in this setting. In their study, they assayed thrombin generation, using CAT, presumably using 5pM TF and $4 \mu \mathrm{M}$ phospholipid concentration (not described in their abstract), from healthy pregnant women and women with risk factors for thrombosis. ETP was assayed at 12, 28 and 36 weeks gestation and 6 weeks postpartum. According to the patient's VTE risk, tinzaparin $(50 \mathrm{IU} / \mathrm{kg}$ ) was commenced for women at risk of thrombosis, at the clinician's discretion. The study authors report the ETP to be significantly elevated during pregnancy compared to non-pregnant samples, and report that these values fall by six weeks postpartum to non-pregnancy values. This is interesting, as despite the prescription of tinzaparin in the pregnant women at risk of thrombosis, a rise in ETP over the course of pregnancy occurs.

Ngu and colleagues [2010], assessed thrombin generation, using CAT, in pregnant women prescribed enoxaparin for various obstetric indications, with doses ranging from $40 \mathrm{mg}$ once daily to $1 \mathrm{mg} / \mathrm{kg}$ twice a day. In this abstract report, it appears as if thrombin generation was measured once during the subject's pregnancy, at various gestational ages (7-30 weeks, median 29 weeks). All samples were measured at 3 hours post enoxaparin injection, with the aim of assessing the specific influence of enoxaparin on the thrombogram parameters. The authors report that all parameters of the thrombogram correlated with the measured anti-Xa activity, with the strongest correlation seen with peak height and time to peak parameters. The study authors conclude that all parameters of thrombin generation are affected in a dose-dependent manner, and suggest that time to peak and peak height are the most sensitive thrombin generation parameters, with respective to enoxaparin.

In a retrospective study of 66 high risk pregnancies, contributing 144 samples, Adamidou and colleagues [2011] measured thrombin generation using an 'in-house' fluorogenic method, with ETP, peak height and time to peak expressed as a \% of normal controls. LMWH was administered to 39 pregnant women, with a further 27 women acting as a control group. The study authors report significant differences in all the thrombin generation parameters between the two groups. The group of women who had anti-Xa activity of $>0.4 \mathrm{IU} / \mathrm{mL}$, had significantly different ETP, peak and time to peak parameters compared to the control group. However, in those women who had an anti-Xa activity of $<0.4 \mathrm{IU} / \mathrm{mL}$, no difference in ETP or peak was found compared to the control group, although the time to peak parameter was found to be significantly different in this group, compared to controls. 
The largest study that has been published to date is that by Hron and colleagues [2010]. In this prospective study, 113 healthy pregnant women and 61 pregnant women receiving LMWH heparin prophylaxis were followed. Those prescribed LMWH were receiving either enoxaparin or dalteparin at a dose range of 4000-7500 anti-factor Xa IU subcutaneously, with subsequent dose changes left to the physician's discretion. All subjects had samples drawn in the first, second and third trimester of their pregnancies, and thrombin generation was assayed in PPP using a chromogenic assay (Siemens Healthcare). The concentration of TF and phospholipid used was not disclosed by the assay manufacturers. In women with and without anticoagulation, ETP levels did not change significantly from the first to the second trimester $(p=0.18)$. However, the ETP did decrease significantly during the third trimester. Interestingly, those women prescribed anticoagulation had higher ETP levels than healthy women not prescribed anticoagulation therapy, across all three trimesters. The authors conclude that LMWH thromboprophylaxis did not completely attenuate coagulation activation during pregnancy. It is difficult for the authors to explain their findings, as they contradict what others have reported and their findings are not what you might expect to observe. The fact that the concentrations of TF and phospholipids are not known to the study authors makes it difficult to utilise the results of this study, given what is known about sensitivity of the thrombin generation assay to alterations of assay conditions and reagents.

Finally, Rossetto and colleagues evaluated the effect of LMWH in pregnant women through the in-vitro addition of nadroparin to the women's plasma [Rossetto et al., 2012]. Twelve healthy pregnant women in the first trimester of their pregnancy had blood drawn and thrombin generation was performed on PPP using CAT, using a 5pM TF concentration and a phospholipid concentration of $4 \mu \mathrm{mol} / \mathrm{L}$. The final nadroparin concentration in the plasma was $0.3 \mathrm{IU} / \mathrm{mL}$ and the results were compared to results obtained from 12 healthy non-pregnant women. Before the addition of LMWH, ETP and peak height were higher in the pregnant population than in the controls $(p=<0.003)$. After spiking the women's plasma, a statistically significant reduction of ETP, both in cases and controls was observed. The same pattern was also observed for the peak height. The authors report that the proportional reduction in peak height was the same for both the subjects and the controls, however, the effect observed on ETP was lower in subjects, when compared to controls. This perhaps suggests that during pregnancy, the relationship between anti-Xa activity and peak height is stronger than that between anti-Xa activity and ETP. From their small study, they suggest that there might be a need to give a higher dose of LMWH during 
pregnancy, as despite the addition of LMWH, the ETP in the plasma of pregnant women did not reach the levels observed in plasma of non-pregnant women after the addition of the same amount of LMWH. The authors make an interesting point, however it is important to bear in mind that it is known that thrombin generation is increased during pregnancy, so a lowering effect is what is required, as opposed to reaching a non-pregnant value as suggested by the authors. Clearly further work is required before any significant change in practice would be recommended.

The message which emerges from these five studies is that some (if not all) of the thrombin generation parameters could be influenced by a concurrent LMWH prescription in a pregnant population, depending on the dose prescribed. Thrombin generation assays therefore potentially provide a meaningful pharmacodynamic marker in pregnant women prescribed LMWH.

\subsection{Objectives and Method}

Chapter 2 described the details of how thrombin generation was assayed for the cohort of women recruited during this study. In brief, a TF concentration of $5 \mathrm{pM}$ was used as the trigger for coagulation, coupled with $4 \mu \mathrm{M}$ of phospholipid, in PPP, using CAT. Depending on when women were recruited during the study, they had their thrombin generation assayed in each trimester, within a week of delivery (typically day 0 or day 1 following birth) and $\geq 8$ weeks post-partum. The specific objectives of this sub-study were to:

- Describe the thrombin generation profiles of pregnant women receiving enoxaparin (dose range $20 \mathrm{mg}-180 \mathrm{mg}$ ) during their pregnancy

- Explore the relationship between the enoxaparin concentration and its influence on the different parameters of the thrombogram at different pregnancy time-points and postpartum

- Compare the results from Caucausian women and African-Caribbean women who were prescribed prophylactic doses of enoxaparin

- Compare how the thrombin generation profiles of the pregnant women followed are altered, when the CAT assay conditions are altered by the addition of polybrene, which is thought to neutralise enoxaparin in the plasma 
Units for the different parameters of the thrombogram

The specific units for the different parameters of the thrombogram in the results section which follows are: the lag-time, time to peak and the start tail are recorded in minutes. The peak is in $\mathrm{nM}$. The ETP is measured in nM.min. Finally the thrombin generation velocity is recorded in $\mathrm{nM} / \mathrm{L} / \mathrm{min}$.

\section{Statistical analyses}

Continuous data are reported as means and standard deviations. The means of continuous normally distributed variables were compared between the five pregnancy time points $\left(1^{\text {st }}, 2^{\text {nd }}\right.$ and $3^{\text {rd }}$ trimesters, within a week of delivery and $\geq 8$ weeks postpartum), using the repeated measures ANOVA with a Greenhouse-Geisser correction. Post-hoc analysis was conducted using Bonferroni correction. Differences between the Caucasian and African-Caribbean populations are described at each pregnancy time point, as means and standard deviations. The means between the two groups at each time point were assessed using the independent t-test. Evaluation of correlations between anti-Xa activity and each thrombin generation parameter, a two-way Pearson's correlation was run. Significance was considered when $p<0.05$.

\subsection{Results}

\subsubsection{Internal thrombin generation variability}

A healthy control (male subject, aged 36-37 years) was bled every eight weeks from the $16^{\text {th }}$ April 2010 to the $24^{\text {th }}$ January 2012 . Eight plasma samples were obtained from the control on each occasion and one sample was run in each thrombin generation experiment, from the $20^{\text {th }}$ April 2010 (exp 17) to the $21^{\text {st }}$ February 2012 (exp 125), 79\% of total thrombin generation experiments completed as part of this study. For each batch, the control plasma sample were analysed within 8 weeks of the sample being drawn.

The intra-assay and inter-assay coefficient of variance was calculated for each thrombin generation parameter for this control (table 6.3); the mean (+/-sd) thrombin generation for each parameter are listed. 
Table 6.3: Intra-assay and Inter-assay variability in the male healthy control

\begin{tabular}{lcccc}
\hline Parameter & Mean & SD & Intra-assay \%CV & Inter-assay \%CV \\
\hline Lag time & 3.13 & 0.6 & 4.5 & 7.6 \\
ETP & 1116 & 226 & 1.6 & 11.8 \\
Peak height & 206 & 49 & 3.9 & 13.7 \\
Time to peak & 6.32 & 1.1 & 4.0 & 7.5 \\
Start tail & 20 & 2.3 & 2.1 & 5.1 \\
Velocity* & 69 & 27 & - & - \\
\hline
\end{tabular}

*velocity intra and inter assay \%CV not computed, as it is a secondary parameter derived from primary parameters

These results are similar to previously reported variances for the assay and demonstrate an appropriate laboratory technique to consider the results for further evaluation.

\subsubsection{Overall thrombin generation results for the cohort of women}

The 123 women recruited to the study provided 498 individual thrombin generation profiles; 414 $(83 \%)$ of the profiles were drawn from women prescribed prophylactic doses of enoxaparin with the remaining 84 profiles from women prescribed treatment doses of enoxaparin.

Considering the results overall, the different parameters of the thrombogram were altered during pregnancy and the postpartum period. Table 6.4 , lists the alterations in these parameters, as pregnancy progressed.

Table 6.4: Overall TG parameter results for the cohort of women

\begin{tabular}{|c|c|c|c|c|c|c|}
\hline TG Parameter & $\begin{array}{l}1^{\mathrm{st}} \mathrm{T} \\
n=58\end{array}$ & $\begin{array}{l}2^{\text {nd }} T \\
n=117\end{array}$ & $\begin{array}{l}3^{\text {rd }} \mathrm{T} \\
n=132\end{array}$ & $\begin{array}{c}\text { Within } 1 \text { week } \\
\text { of delivery } \\
n=93\end{array}$ & $\geq 8 \mathrm{u}$ & $\begin{array}{l}\text { veeks pp } \\
n=98\end{array}$ \\
\hline Lag time & $4.27 \quad(3.81)$ & 3.53 (1.26) & 3.88 (2.46) & $3.14 \quad(1.32)$ & 3.13 & (1.34) \\
\hline ETP & 1391 (795) & 1757 (706) & $1692(631)$ & $1857(633)$ & 1293 & $(409)$ \\
\hline Peak height & $(141)$ & (136) & (116) & (127) & 261 & (80) \\
\hline Time to Peak & (5.18) & (3.27) & (3.91) & $5.57 \quad(2.83)$ & 5.68 & (1.58) \\
\hline Start tail & (9.7) & (6.9) & $(6.8)$ & $24.2 \quad(5.8)$ & 20.6 & (2.79) \\
\hline Velocity & (65) & (71) & (59) & (75) & 109 & (45) \\
\hline
\end{tabular}

sd in brackets; T refers to trimester, pp refers to post-partum. $n$ refers to the number of samples analysed in each trimester (some women had two or three profiles drawn around a dose of enoxaparin in each trimester).

The standard deviations on the thrombin generation parameters reflect the hetergenous cohort of women followed and is additionally reflective of the differing doses of enoxaparin prescribed.

The samples drawn at $\geq 8$ weeks post delivery provide an insight into what the cohort of women's baseline thrombin generation was prior to their pregnancy by which point the majority of women 
had discontinued their anticoagulation therapy and all the pregnancy related changes, with respect to coagulation factors, would have subsided. Using the $\geq 8$ week post-partum values as a reference point; a rise in ETP is seen over the course of pregnancy, despite a prescription of enoxaparin. A similar pattern is also observed for the peak height. It must be remembered though, that these samples were drawn from a heterogeneous group of pregnant women and the samples were drawn at different time points (post dose), from pregnant women on different doses of enoxaparin.

The pregnant women in this study can be split into two broad groups; treatment and prophylactic dosing groups. How are the parameters of the thrombogram altered, when the results are subanalysed according to these two groups?

6.3.2.1 Thrombin generation parameters of women on prophylactic and treatment doses

Table 6.5 presents the thrombin generations results from the women (414 profiles) prescribed prophylactic doses of LMWH during the course of their pregnancy. Table 6.6 presents the thrombin generation results from the women prescribed treatment doses of LMWH.

Table 6.5: Thrombin generation results from the cohort of women prescribed prophylactic doses of enoxaparin

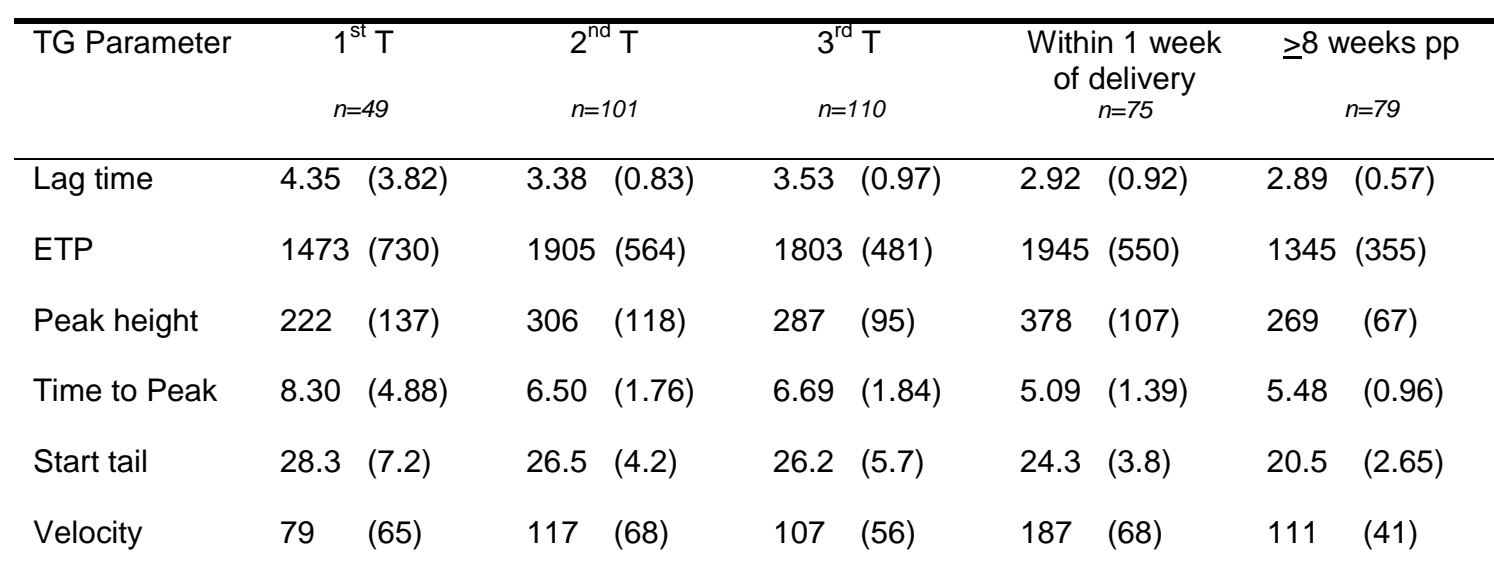

sd in brackets; T refers to trimester, pp refers to post-partum. $n$ refers to the number of samples analysed in each trimester (some women had two or three profiles drawn around a dose of enoxaparin in each trimester). 
Table 6.6: Thrombin generation results from the cohort of women prescribed treatment doses of enoxaparin

\begin{tabular}{|c|c|c|c|c|c|c|c|c|}
\hline \multirow{2}{*}{$\begin{array}{l}\text { TG Parameter } \\
\text { Lag time }\end{array}$} & $\begin{array}{l}1^{\mathrm{st}} \mathrm{T} \\
n=9\end{array}$ & $\begin{array}{l}2^{\text {nd }} T \\
n=16\end{array}$ & \multicolumn{2}{|c|}{$\begin{array}{l}3^{\mathrm{rd}} \mathrm{T} \\
n=22\end{array}$} & \multicolumn{2}{|c|}{$\begin{array}{c}\text { Within } 1 \text { week } \\
\text { of delivery } \\
n=18\end{array}$} & \multicolumn{2}{|c|}{$\begin{array}{c}\geq 8 \text { weeks } \mathrm{pp}^{\star \star} \\
n=19\end{array}$} \\
\hline & 3.83 (3.98) & $4.50(2.57)$ & 3.67 & (5.37) & 4.10 & $(2.14)$ & 4.16 & (2.64) \\
\hline ETP & 941 (1015) & $820 \quad(809)$ & 1141 & (952) & 1497 & $(824)$ & 1078 & $(547)$ \\
\hline Peak height & $129 \quad(146)$ & (103) & 135 & (131) & 266 & (164) & 232 & (117) \\
\hline Time to Peak & $6.96 \quad(6.81)$ & $10.5(6.89)$ & 10.7 & (7.99) & 7.55 & $(5.46)$ & 6.53 & $(2.94)$ \\
\hline Start tail & $21.5 \quad(17.90)$ & $24.9(15.9)$ & 28.8 & (13.1) & 23.9 & $(10.8)$ & 21.1 & (3.36) \\
\hline Velocity & $(61)$ & (35) & 42 & (51) & 122 & (83) & 104 & (61) \\
\hline
\end{tabular}

sd in brackets; T refers to trimester, pp refers to post-partum. $n$ refers to the number of samples analysed in each trimester (some women had two or three profiles drawn around a dose of enoxaparin in each trimester). ${ }^{* *}$ some women were taking warfarin when this sample was drawn

The results demonstrate that differences between the two groups. The ETP rise over pregnancy for women on treatment doses of enoxaparin is more attenuated, relative to those women prescribed prophylactic doses. It is also interesting to observe how the peak height parameter is much lower in the women prescribed treatment doses of enoxaparin, relative to those prescribed prophylactic doses. An influence of enoxaparin on the lag time, time to peak, and start tail is also observed and will be explored further on in this chapter. Examining the results from the women prescribed prophylactic doses, where all anticoagulant therapy had been stopped by eight weeks postpartum, one can see that ETP is already higher by the first trimester, with a further rise seen during the second trimester, after which it settles through to delivery, where it peaks. The other observation which stands out is the wide standard deviations of the results, illustrating the wide inter-patient variability in these parameters, with the variation being more pronounced in those women prescribed treatment doses of enoxaparin.

\subsubsection{Correlation of anti-Xa activity and the thrombin generation parameters}

Previous work assessing the anticoagulant effect in the thrombin generation test has demonstrated thrombin generation is not a static parameter, but is dynamic and can change with an ever changing concentration of anticoagulant in the plasma. In order to better understand this in the antenatal population, anti-Xa activity was correlated for the cohort of women overall, followed by women prescribed prophylactic and treatment doses separated out. These results are presented in table 6.7. Pearson's correlation was used (two-way), and significance was considered at $p<0.05$. 
Table 6.7: Correlation between anti-Xa activity and the different thrombin generation parameters

\begin{tabular}{lccc}
\hline $\begin{array}{l}\text { Correlation between anti- } \\
\text { Xa activity and TG } \\
\text { parameter }\end{array}$ & Cohort overall & Prophylaxis group & Treatment group \\
\hline Lag time & 0.267 & 0.313 & \\
ETP & -0.590 & -0.438 & $0.030^{* *}$ \\
Peak & -0.694 & -0.587 & -0.615 \\
Time to peak & 0.397 & 0.481 & -0.747 \\
Start tail & $-0.004^{* *}$ & 0.324 & $0.137^{* *}$ \\
Velocity & -0.642 & -0.577 & 0.290 \\
\hline
\end{tabular}

${ }^{* *}$ non-statistically significant

The lag time, time to peak and the start tail are all positively correlated with anti-Xa activity, whilst the ETP, peak height and velocity are negatively correlated. The parameters where the strength of the relationships are strongest are between the ETP, peak height and velocity parameters of the thrombogram and anti-Xa activity. For those women prescribed treatment doses of enoxaparin, the strength of these correlations increase, relative to those women receiving prophylactic doses (-0.6 vs. -0.4 for ETP, -0.7 vs. -0.5 for peak, and -0.78 vs. -0.58 for velocity), treatment versus prophylaxis groups respectively. It is interesting to observe that the lag-time and the time to peak in the treatment group of patients were not significantly positively correlated, as might one anticipate. Detailed analysis of each thrombin generation parameter follows.

\subsubsection{Lag time}

The lag time is when no observable thrombin generation is seen and can be considered as being representative of historical clotting times, as it is the time taken until thrombin generation reaches $1 / 6$ of the peak concentration.

When lag time is compared across trimesters and gestations for the study women overall, a decrease in this time is observed as pregnancy progresses. Figures 6.1 (a) and (b) show the overall trend in lag time for the study women overall. 

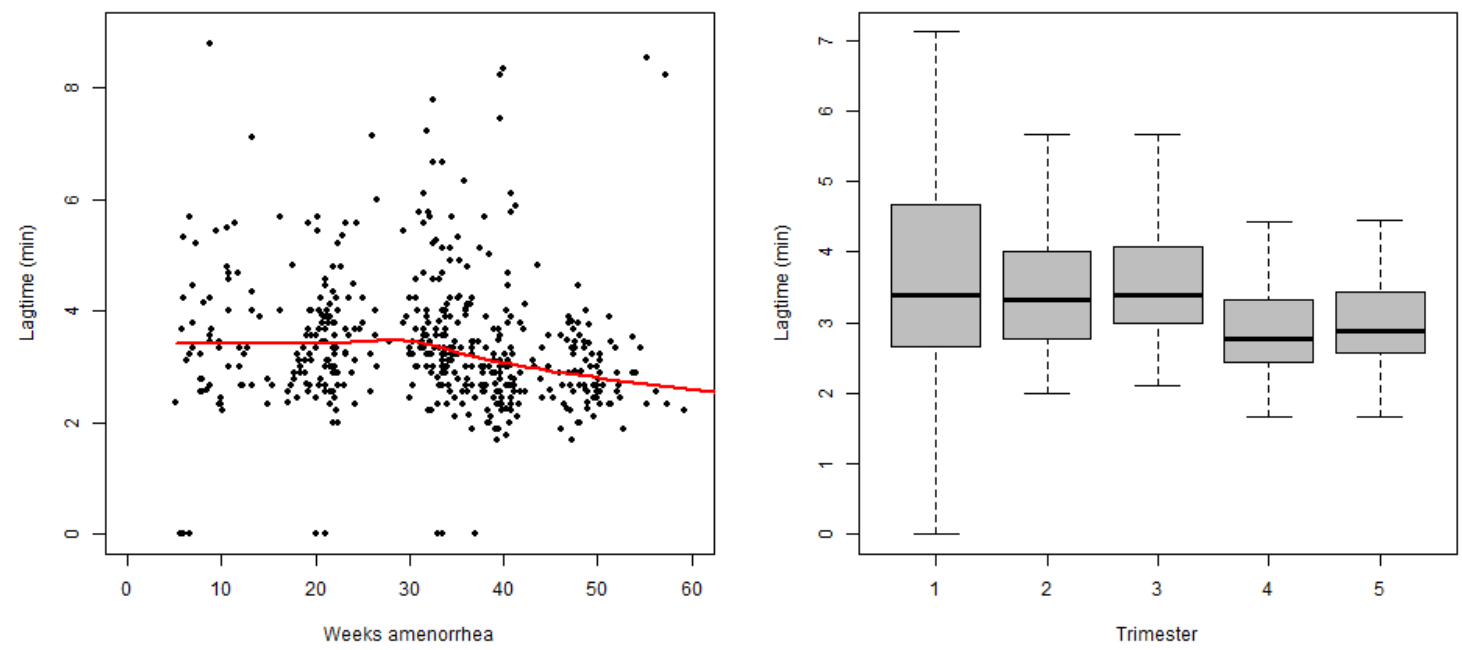

Figure 6.1 (a) left: Scatter-plot of the lag-times versus weeks amenorrhea for the cohort of women recruited. Red line represents the loess smooth

Figure 6.1 (b) right: Box plots of the lag-times, according to when the lag-time was assessed. Trimester 4 and 5 refers to within a week of delivery and $\geq 8$ weeks post partum

Thirty women had results for all five time points and the mean lag-times did not significantly differ between the five antenatal and postnatal time points $(F(1.373,39.807)=0.699, p=0.452)$. When the lag time results are analysed into those from women prescribed prophylactic doses and those women prescribed treatment doses, the results diverge and confirm in part the relationship that exists between this parameter and the enoxaparin dose prescribed. There appears to be more inter-patient variation from those women on treatment doses compared to those on prophylactic doses (figures $6.2(a)$ and (b)). 

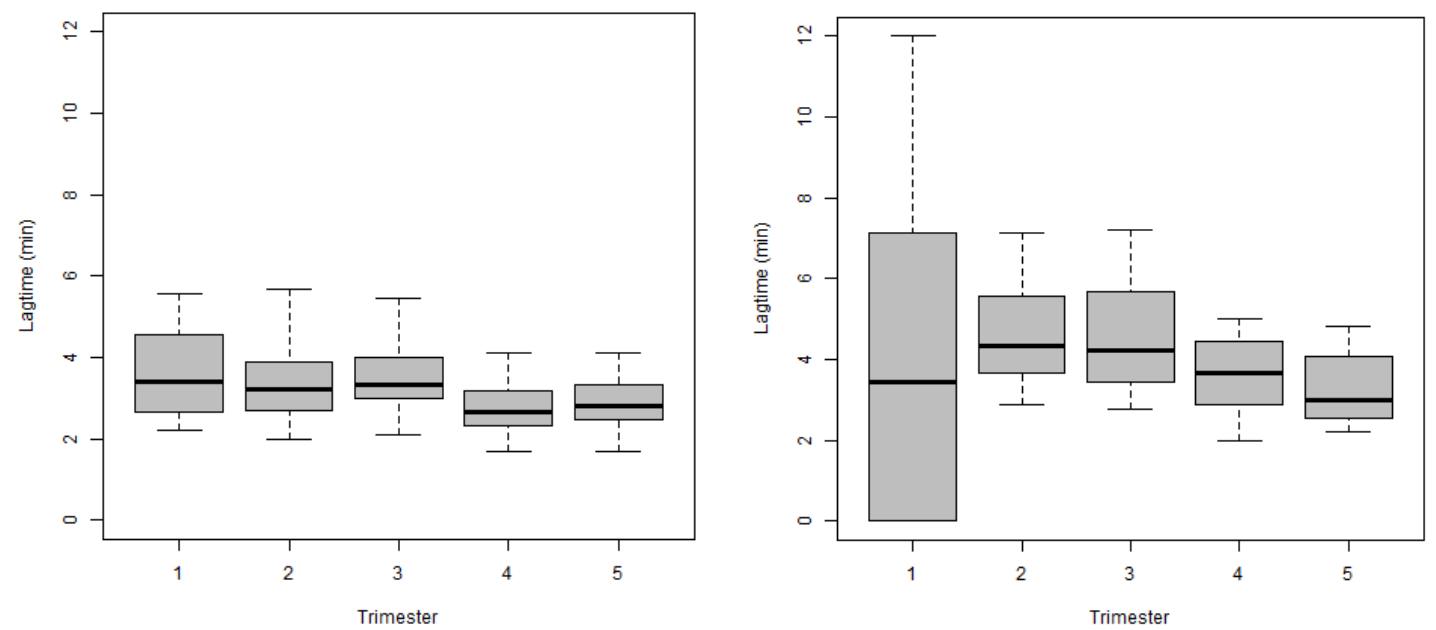

Figure 6.2 (a) left: a box-plot of the lag-times from the women prescribed prophylactic doses of enoxaparin (trimester 4 and 5 refers to within a week of delivery and $\geq 8$ weeks post partum)

Figure 6.2 (b) right: a box plot of the lag-times from the women prescribed treatment doses of enoxaparin (trimester 4 and 5 refers to within a week of delivery and $\geq 8$ weeks post partum)

For women prescribed treatment doses $(n=8)$, no significant differences were found between the five pregnancy and postnatal time points and the lag-time $(F(1.152,8.066)=0.245, p>0.05)$.

Women prescribed prophylactic doses and whom had TG results for all five time points $(n=22)$, significant differences were found between the time points $(F(2.684,56.261)=3.732, p<0.05)$. Post-hoc analysis demonstrated significant differences between the $\geq 8$ weeks postpartum samples and the samples drawn in the first, second and third trimester, where a prolongation of the lag-time was observed.

\subsubsection{Endogenous Thrombin Potential (ETP)}

The ETP represents the area under the thrombin generation curve. A high ETP value is thought to suggest a greater thrombogenic potential in an individual and the greater an individual's propensity to form a clot. A primary aim of anticoagulant therapy would be to reduce the ETP. Figure 6.3 (a) and (b) illustrates the overall ETP pattern for the whole cohort of women followed and confirms a favoured pro-thrombotic state during pregnancy, despite a prescription of enoxaparin. 

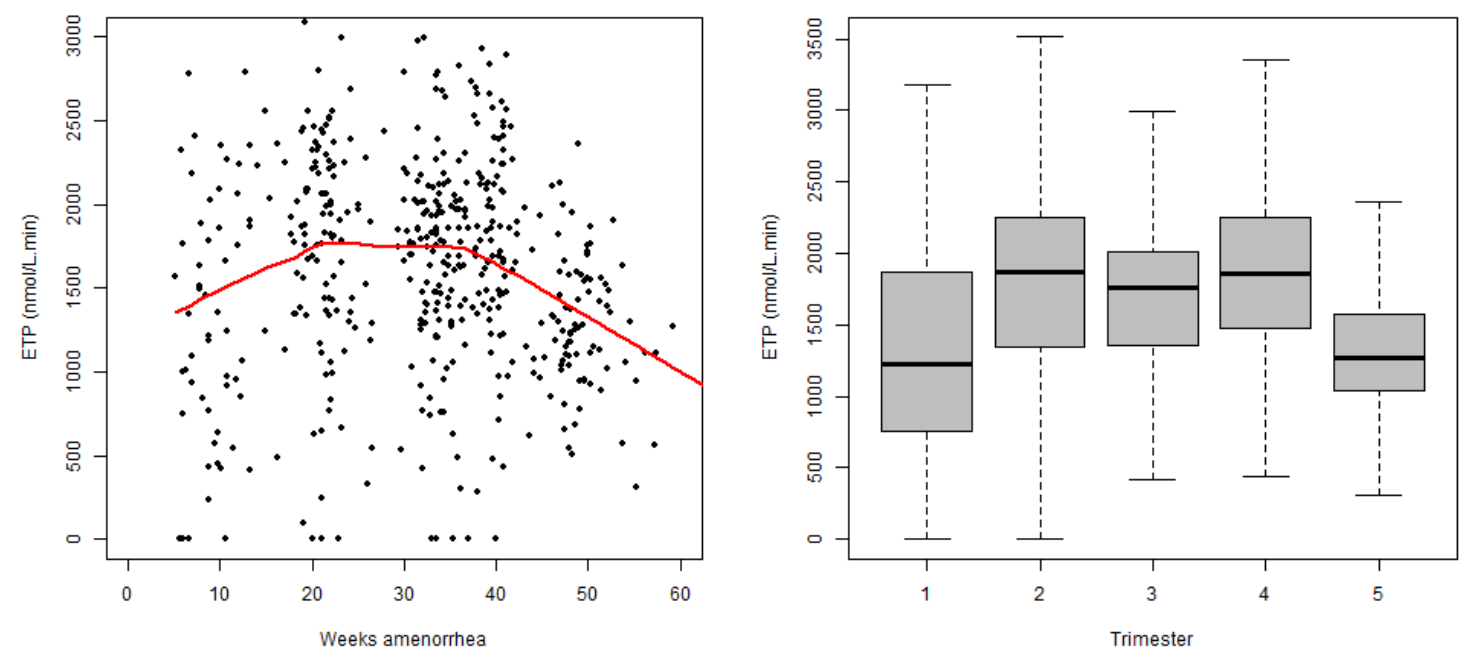

Figure 6.3 (a) left: Scatter-plot of the ETP versus week's amenorrhea for the cohort of women recruited. Red line represents the loess smooth

Figure 6.3 (b) right: Box plots of the ETP, according to when the ETP was assessed. Trimester 4 and 5 refers to within a week of delivery and $\geq 8$ weeks post partum

The mean ETP did significantly differ between the five antenatal and postal time points $(F(2.198$, $63.738)=7.501, p<0.001)$. Post-hoc analysis revealed specific differences between the $\geq 8$ weeks post-partum time point and the second and third trimester and the ETP at the time of delivery. A statistically significant difference was not found between the first trimester and the $\geq 8$ weeks postpartum time point.

When the results were split according to those women receiving prophylactic and those women receiving treatment doses, a clear distinction can be seen (box plot figures 6.4 (a) and (b)). Once again, the treatment (right) dose women have greater variation in response compared to the prophylactic (left) group. 

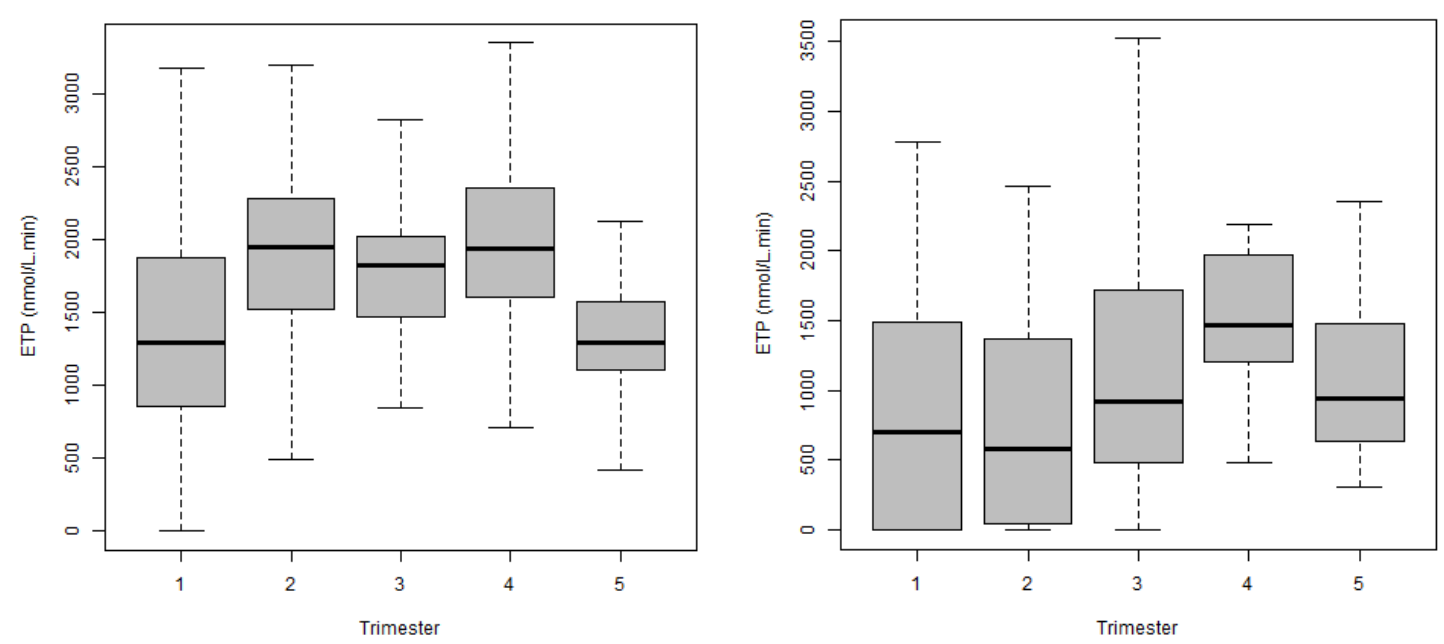

Figure 6.4 (a) left: a box-plot of the ETP from the women prescribed prophylactic doses of enoxaparin (trimester 4 and 5 refers to within a week of delivery and $\geq 8$ weeks post partum)

Figure 6.4 (b) right: a box plot of the ETP from the women prescribed treatment doses of enoxaparin (trimester 4 and 5 refers to within a week of delivery and $\geq 8$ weeks post partum)

The mean ETP concentrations are lower for women on treatment doses, as one might predict. For women prescribed prophylactic doses, a rise in ETP is observed from the first trimester to the second and third trimesters; the ETP remains relatively static during pregnancy for women on treatment doses, with a jump observed at the time point of delivery.

Detailed analyses from the eight women who had thrombin generation samples at all five time points, prescribed treatment doses of enoxaparin, found that mean ETP concentrations in these women did not statistically differ between the time points $(F(1.556,10.891)=1.550, p>0.05)$. For the twenty two women in the prophylaxis group, the mean ETP concentrations did statistically differ between the five time points $(F(2.312,48.561)=9.072, p<0.05)$. Post-hoc analysis revealed that specific differences were between the $\geq 8$ weeks post-partum time point and the second and third trimesters and at the time of delivery, mirroring the result seen for the cohort of women as a whole.

\subsubsection{Peak height}

The peak height was assessed for the cohort of women as a whole. The pattern which emerges is an increase from the first to the second trimester, following which peak height appears to stabilise to the third trimester, with a jump observed at the time of delivery, before a drop at the $\geq 8$ weeks post-partum time point (figures 6.5 (a) and (b)). 

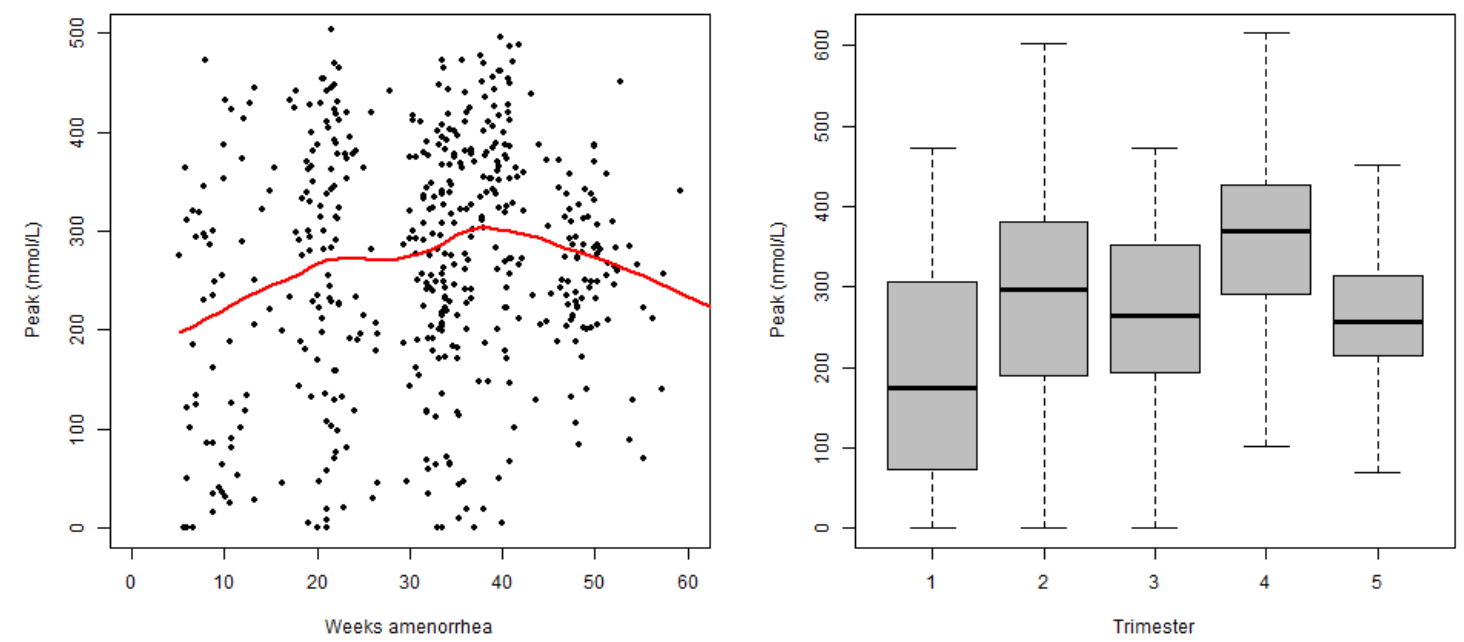

Figure 6.5 (a) left: Scatter-plot of the peak for the cohort of women recruited. Red line represents the loess smooth

Figure 6.5 (b) right: Box plots of the peak, according to when the peak was assessed. Trimester 4 and 5 refers to within a week of delivery and $\geq 8$ weeks post partum

Interestingly, the 'fall back' at the eight week post partum time does not reach the level seen during the first trimester and certainly demonstrates an impressive enoxaparin pharmacodynamic effect during the first trimester. Statistically significant differences did exist between the five time points $(F(2.230,64.684)=7.881, p<0.05)$. Post hoc analysis using a Bonferroni correction revealed that specific differences lie between the results obtained at the time of delivery and the results from the first and second trimester and the $\geq 8$ weeks post partum time points. No statistically significant differences were found between the third trimester and the time of delivery, though the difference was approaching significant $(p=0.063)$.

Once again, when the prophylaxis and treatment groups of women were analysed separately (figures $6.6(a)$ and (b)). 

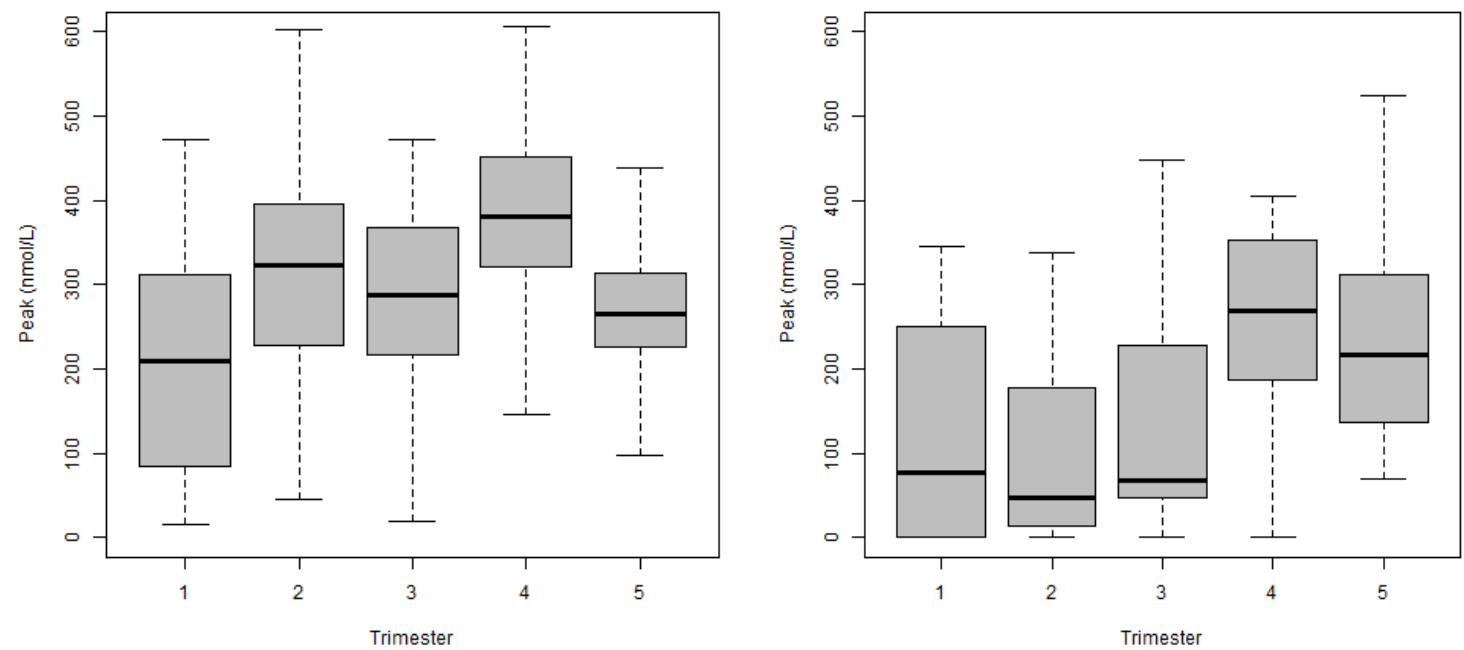

Figure 6.6 (a) left: a box-plot of the peak from the women prescribed prophylactic doses of enoxaparin (trimester 4 and 5 refers to within a week of delivery and $\geq 8$ weeks post partum)

Figure 6.6 (b) right: a box plot of the peak from the women prescribed treatment doses of enoxaparin (trimester 4 and 5 refers to within a week of delivery and $\geq 8$ weeks post partum)

No statistically significant differences were found for the 8 women prescribed treatment doses of enoxaparin, who had repeated measures of peak height throughout their pregnancy and at the $\geq 8$ weeks post-partum time $\operatorname{point}(F(1.689,11.820)=3.290, p=0.079)$. For the 22 women prescribed prophylactic enoxaparin with results at all five time points, statistically significant differences between the five time points $(F(2.375,49.877)=5.209, p=0.006)$ were found. A posthoc analysis however, was unable to identify at which time points the differences lie.

\subsubsection{Time to Peak (tt Peak)}

The time to peak parameter is, as the name suggests, the time it takes for peak thrombin to be reached. Thrombin generation studies do not always report this parameter, however, in this the context of this study, it is worth considering, as one would anticipate an increase in the time to peak thrombin in those patients prescribed anticoagulant therapy. When the results of tt Peak are assessed for the women as a group, a general decrease in this parameter is seen with the progression of pregnancy; once again implying the effect of enoxaparin is greatest during the first trimester (figure 6.7 (a) and (b)). 

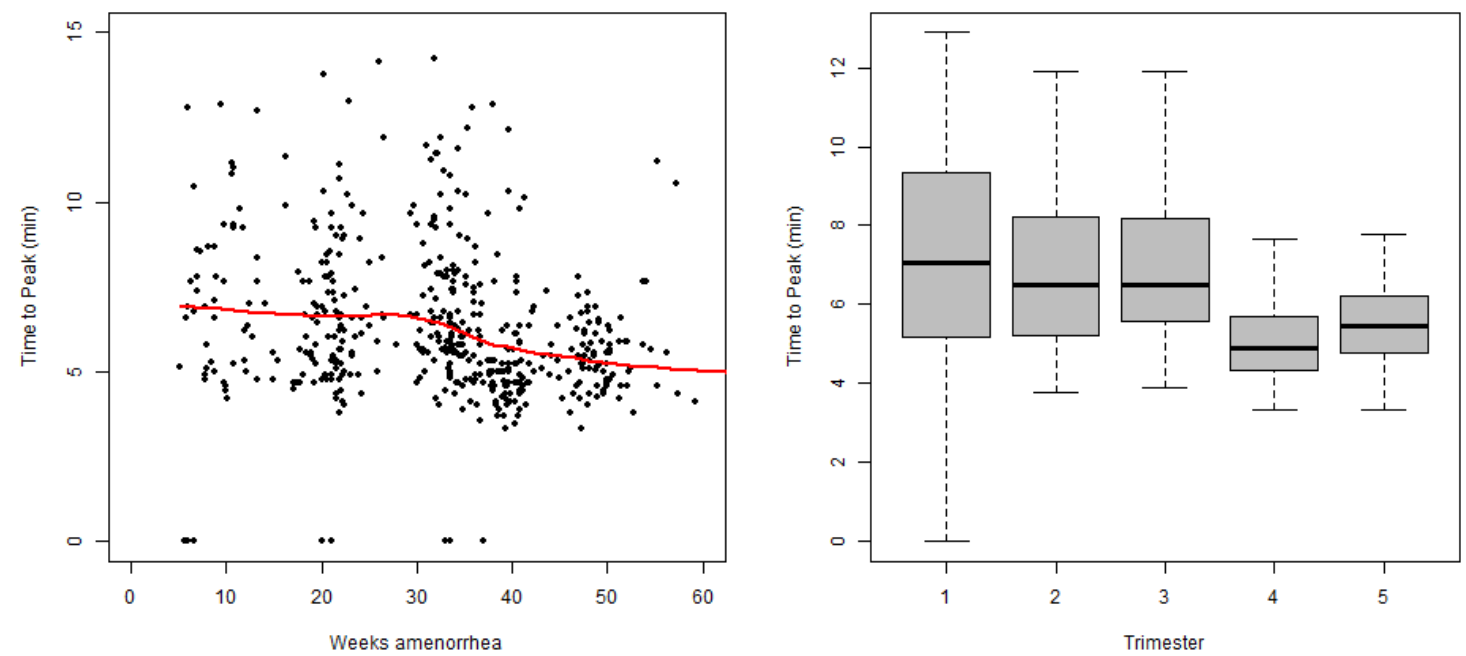

Figure 6.7 (a) left: a scatter-plot of the time to peak for the cohort of women recruited. Red line represents the loess smooth

Figure 6.7 (b) right: a box plots of the time to peak, according to when the time to peak was assessed. Trimester 4 and 5 refers to within a week of delivery and $\geq 8$ weeks post partum

No significant differences existed between the five time points and the time to peak parameter for the 30 women, with results at all the time points $(F(1.692,49.058)=2.445, p=0.105)$.

When women prescribed treatment doses are separated from those subjects prescribed prophylactic doses, differences are observed between the two groups (figures 6.8 (a) and (b)).
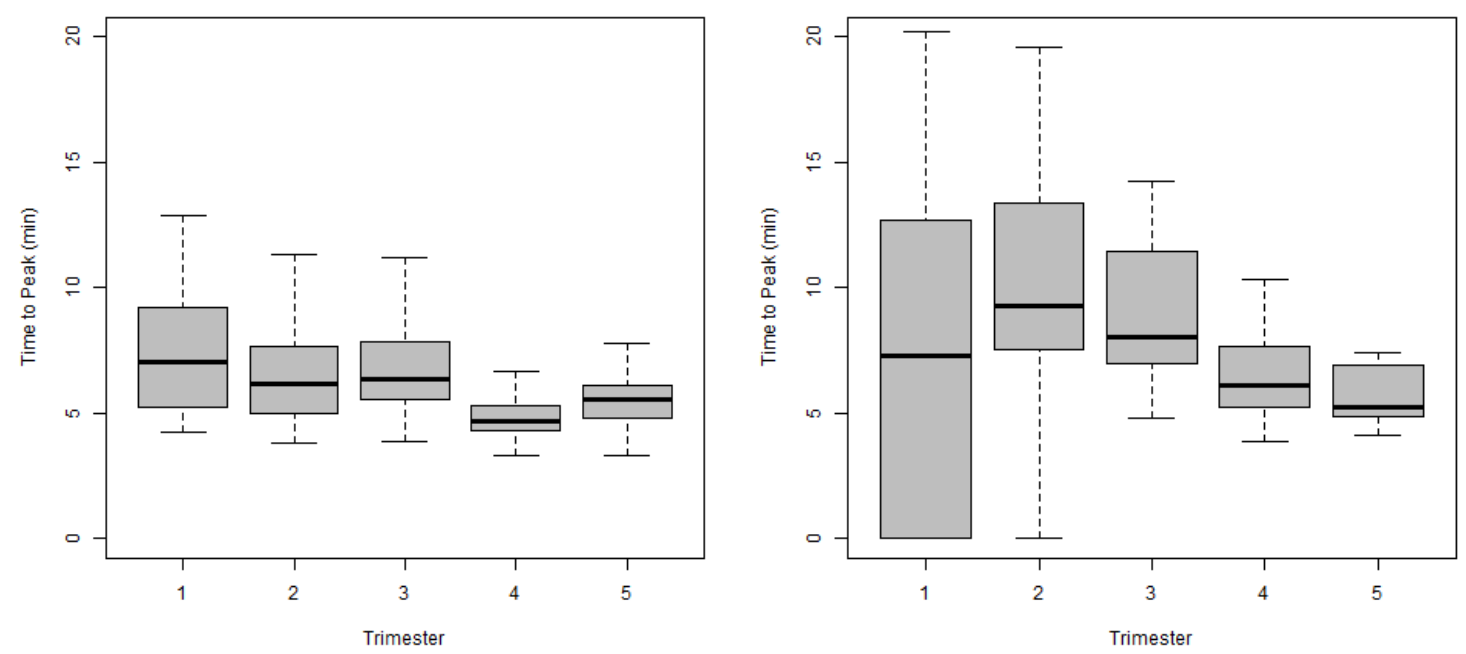

Figure 6.8 (a) left: a box-plot of the time to peak from the women prescribed prophylactic doses of enoxaparin (trimester 4 and 5 refers to within a week of delivery and $\geq 8$ weeks post partum)

Figure 6.8 (b) right: a box plot of the time to peak from the women prescribed treatment doses of enoxaparin (trimester 4 and 5 refers to within a week of delivery and $\geq 8$ weeks post partum) 
The $\mathrm{tt}$ Peak is much longer for the women prescribed treatment doses (right), as one would anticipate.No significant differences between the five pregnancy time points with respect to the time to peak for women prescribed treatment doses. For those women, prescribed prophylactic doses, significant differences were found between the five pregnancy time points; $(F(2.715$, $57.021)=6.181, p=0.001)$. A post-hoc analysis revealed significant differences between the $\geq 8$ weeks post-partum time point and the first, second and third trimesters. No significant differences were found between the samples drawn at delivery and the $\geq 8$ week post-partum time point.

\subsubsection{Start Tail}

The start tail is the time at which thrombin generation has begun to diminish. Overall, for the cohort of women, a downward trend in the start tail is observed with the progression of pregnancy (figures 6.9 (a) and (b)). When patients are prescribed anticoagulant therapy, one would anticipate a prolongation in the start tail time. Significant differences did exist between the five pregnancy time points $(F(2.953,85.632)=3.093, p=0.032)$. Post-hoc analysis revealed significant differences between the $\geq 8$ week post-partum time point and the results from the third trimester and within 1 week of delivery, but not at the trimester 1 and trimester 2 time points.
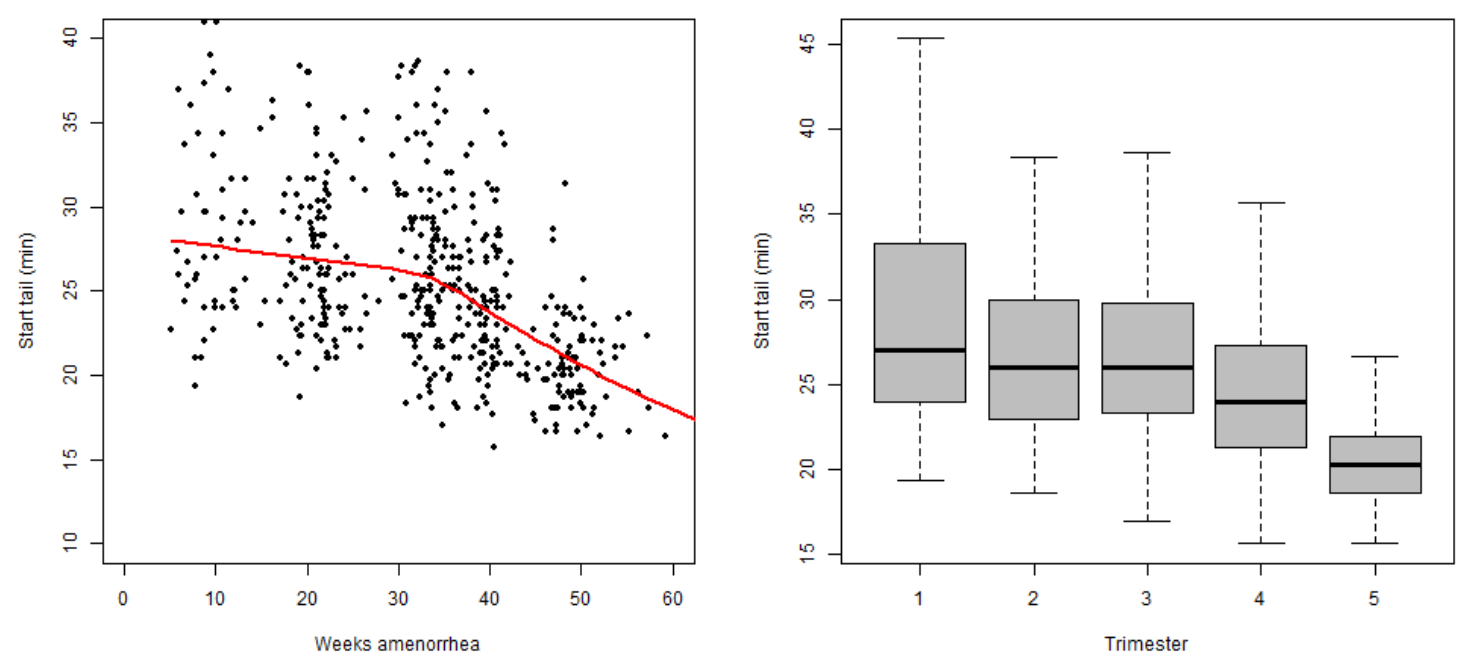

Figure 6.9 (a) left: a scatter-plot of the start tail, for the cohort of women recruited. Red line represents the loess smooth

Figure 6.9 (b) right: a box plot of the start tail, according to when the start tail was assessed. Trimester 4 and 5 refers to within a week of delivery and $\geq 8$ weeks post partum

Once again enoxaparin's effect appeared to be greater during the first trimester than during the second and third trimester. When the data are split into those women prescribed treatment (right) 
and prophylactic doses (left), a difference in response is observed (figures 6.10 (a) and (b)).
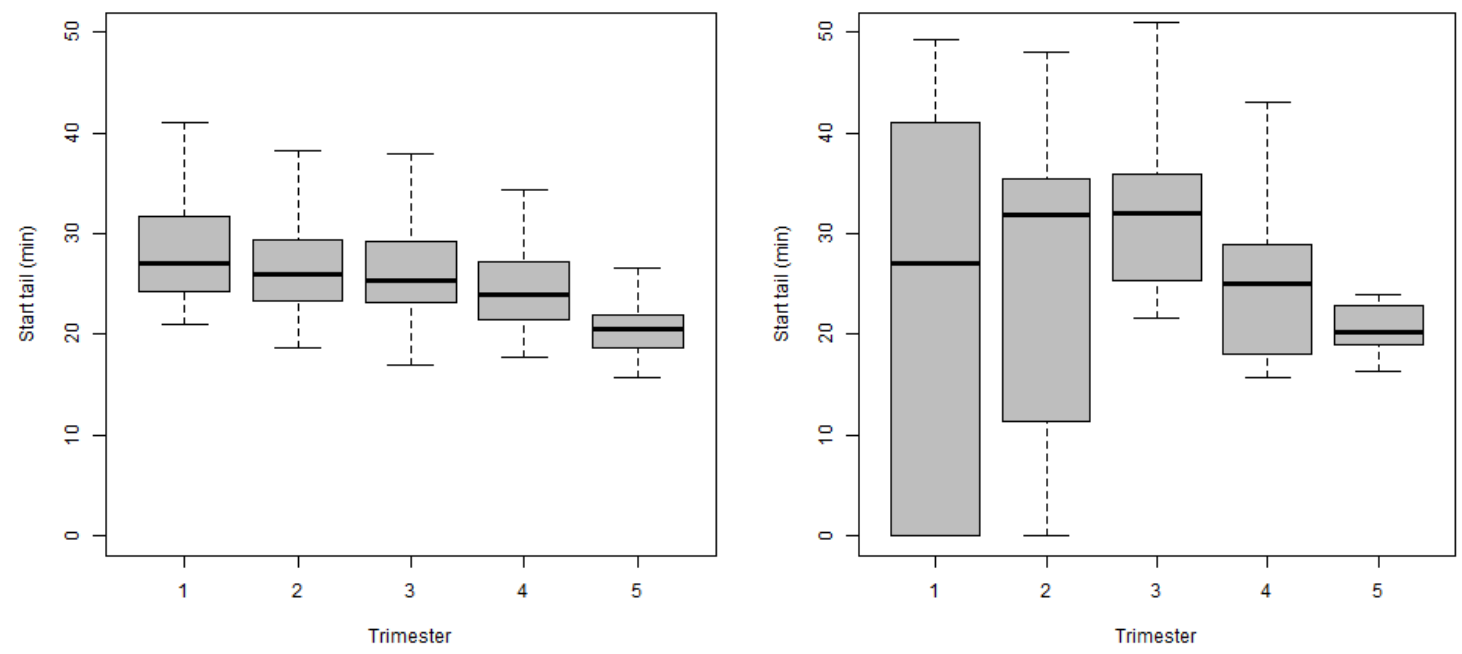

Figure 6.10 (a) left: a box-plot of the start tail from the women prescribed prophylactic doses of enoxaparin (trimester 4 and 5 refers to within a week of delivery and $\geq 8$ weeks post partum)

Figure 6.10 (b) right: a box plot of the start tail from the women prescribed treatment doses of enoxaparin (trimester 4 and 5 refers to within a week of delivery and $\geq 8$ weeks post partum)

No significant differences in the start tail for the 8 women who had results from all five time points, prescribed treatment doses of enoxaparin were found. Interestingly, significant differences between the five pregnancy time points, for those women prescribed prophylactic doses of enoxaparin were found $(F(2.252,47.291)=11.953, p=0.000)$. A post-hoc analysis revealed that the specific differences were between the $\geq 8$ weeks post-partum time point and the other four time points.

\subsubsection{Thrombin generation velocity}

Thrombin generation velocity is the final parameter to consider and is thought by many to be an important parameter; the faster the TG velocity the larger the thrombin generation potential. The overall pattern observed for the women in the study is an increase from the first to the second trimester, after which it stabilises through the third trimester. A 'jump' is observed at the time of delivery, before dropping back by eight weeks postpartum (figure 6.11 (a) and (b)).

Significant differences were found between the five pregnancy time points $(F(2.310$, $66.995)=12.005, p=0.000$ ). A post-hoc analysis revealed that the differences were between the results from the time of delivery, and the other four time points. 

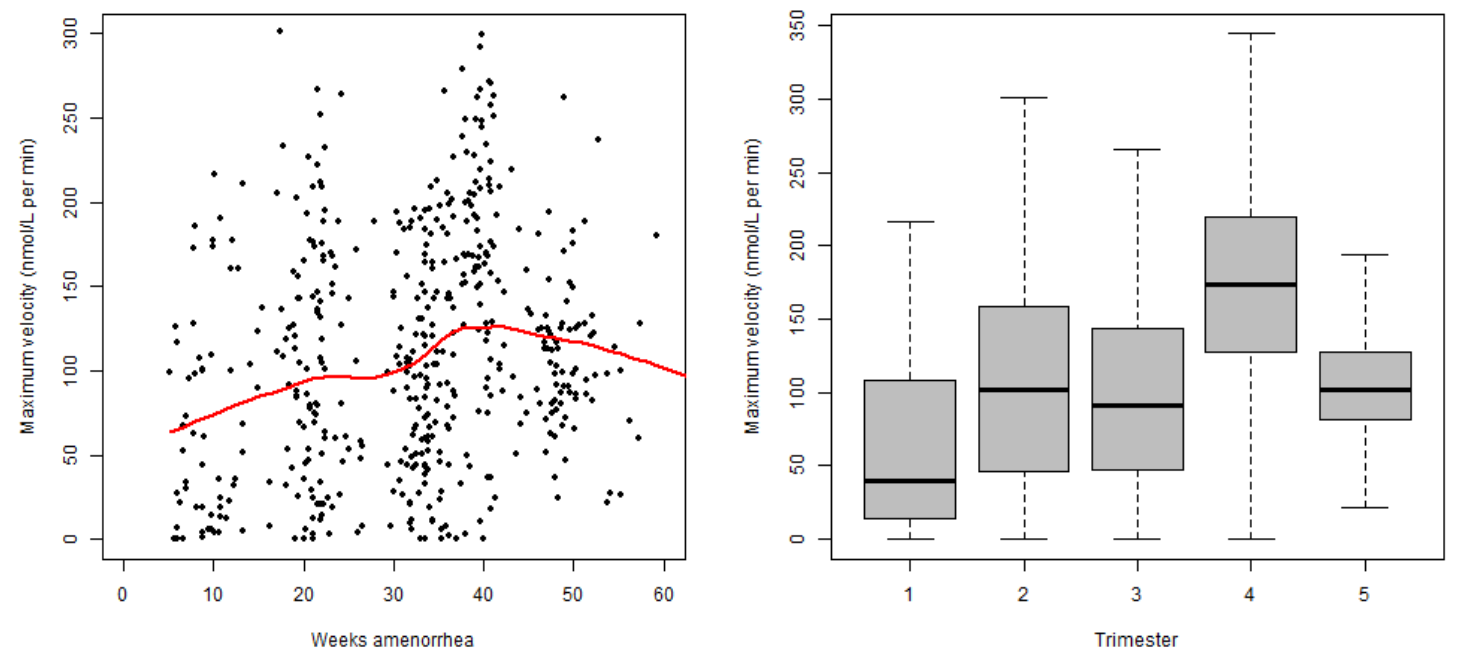

Figure 6.11 (a) left: Scatter-plot of the TG velocity, for the cohort of women recruited. Red line represents the loess smooth

Figure 6.11 (b) right: Box plots of the TG velocity, according to when the TG velocity was assessed. (Trimester 4 and 5 refers to within a week of delivery and $\geq 8$ weeks post partum)

Closer scrutiny of differences between women on prophylactic versus women on treatment doses demonstrated differences. Women prescribed treatment doses (right), show a 'suppression' of velocity during pregnancy, compared to those prescribed prophylactic doses (left). Both groups had a jump in velocity at the time of delivery. Once again, a strong relationship appears to exist between the dose of enoxaparin prescribed and the effect observed (figures 6.12 (a) and (b)).
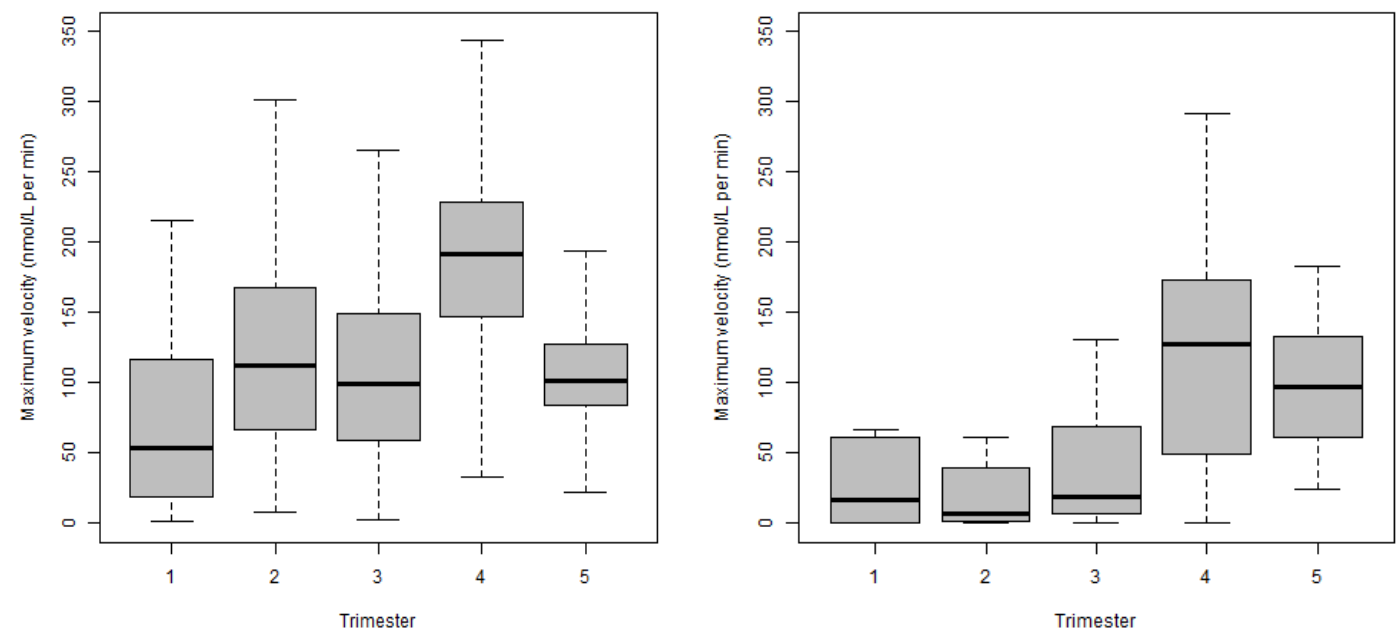

Figure 6.12 (a) left: a box-plot of the TG velocity from the women prescribed prophylactic doses of enoxaparin (trimester 4 and 5 refers to within a week of delivery and $\geq 8$ weeks post partum)

Figure 6.12 (b) right: a box plot of the TG velocity from the women prescribed treatment doses of enoxaparin (trimester 4 and 5 refers to within a week of delivery and $\geq 8$ weeks post partum) 
For women receiving prophylactic doses statistically significant differences between the five time points were found $(F(2.333,48.983)=7.784, p=0.001)$. A post-hoc analysis found significant differences between the first and third trimester and results from the time of delivery. No differences were found between the second trimester and the results from the time of delivery, although the results were approaching significance $(p=0.052)$. For women prescribed treatment doses of enoxaparin, significant differences between the five time points assessed were found $(F(1.762,12.332)=4.752, p=0.033)$. However a post-hoc analysis was unable to identify where the specific differences were.

Overall, the results demonstrate increased thrombin generation during the antenatal period, despite the enoxaparin prescription, and an influence of enoxaparin on the thrombogram parameters, in a dose dependent manner.

\subsubsection{Are there ethnic differences between the women's profiles?}

The Caucasian and African-Caribbean ethnic groups represented the majority of ethnic origin groups in the cohort of women recruited during the course of this study. The thrombin generation results from these two groups of women prescribed prophylactic doses of enoxaparin were also compared and contrasted.

Table 6.8 lists these results according to each trimester (mean +/- SD of each parameter). Significant differences (independent t-test) are highlighted. 
Table 6.8: Thrombin generation results between the Caucasian and African-Caribbean population

\begin{tabular}{|c|c|c|c|c|c|c|}
\hline \multirow[t]{2}{*}{ Parameter } & \multirow[t]{2}{*}{ Ethnicity } & $1^{\text {st }} \mathrm{T}$ & $2^{\text {nd }} T$ & $3^{\text {rd }} T$ & $\begin{array}{l}\text { Within one } \\
\text { week of } \\
\text { delivery }\end{array}$ & $\begin{array}{l}8 \text { weeks } \\
\text { postpartum }\end{array}$ \\
\hline & & $n=34 \vee 8$ & $n=67$ v 24 & $n=75 \vee 27$ & $n=45 \vee 23$ & $n=47$ v 24 \\
\hline \multirow[t]{2}{*}{ Lag time } & Caucasian & $4.11 \quad(1.74)$ & $3.49(0.83)$ & $3.62(0.98)$ & 3.03 (1.09) & $2.98 \quad(0.57)$ \\
\hline & $A-C$ & $3.27 \quad(0.81)$ & $3.18 \quad(0.74)$ & $3.34 \quad(0.77)$ & $2.73 \quad(0.61)$ & $2.72 \quad(0.51)$ \\
\hline \multirow[t]{2}{*}{ ETP } & Caucasian & $1296(651)^{*}$ & $1806(557)^{*}$ & $1784(451)$ & $1944(578)$ & $1306(356)$ \\
\hline & $A-C$ & $2126(754)$ & $2129(556)$ & 1810 & $1911(540)$ & 1402 (336) \\
\hline \multirow[t]{2}{*}{ Peak height } & Caucasian & $193(124)^{*}$ & $284(114)^{*}$ & $280 \quad(92)$ & $379 \quad(119)$ & $256 \quad(60)$ \\
\hline & $A-C$ & 323 (146) & (112) & $301 \quad(96)$ & $(91)$ & (63) \\
\hline \multirow[t]{2}{*}{ tt Peak } & Caucasian & $8.27 \quad(3.6)$ & $6.83(1.8)^{*}$ & $6.95 \quad(1.9)$ & $5.23 \quad(1.6)$ & $5.65(0.9)^{*}$ \\
\hline & $A-C$ & $6.61 \quad(2.2)$ & 5.89 (1.3) & $6.22(1.6)$ & $4.88 \quad(1.0)$ & $5.15 \quad(0.9)$ \\
\hline \multirow[t]{2}{*}{ Start tail } & Caucasian & $27.94(7.4)$ & 26.37 (4.3) & $26.07(4.7)$ & $24.29(3.8)$ & $20.42(2.7)$ \\
\hline & $A-C$ & $28.10(3.1)$ & 26.73 (3.9) & $25.93(4.2)$ & $24.40(4.1)$ & $20.69(2.8)$ \\
\hline \multirow[t]{2}{*}{ Velocity } & Caucasian & (56) & $103(62)^{*}$ & 100 & $(76)$ & $(34)^{*}$ \\
\hline & $A-C$ & $(79)^{*}$ & $148 \quad(71)$ & $(60)$ & (62) & $(40)$ \\
\hline
\end{tabular}

${ }^{*}$ Significance at $<0.05$ level; A-C refers to the African-Caribbean population, C refers to Caucasian population. $n=$ number of samples in each trimester (Caucasian vs African-Caribbean). SD in brackets. ' $T$ ' represents trimester.

When assessing those women receiving prophylactic enoxaparin during the first trimester, there are significant differences between the two ethnic groups for the mean ETP, peak and velocity parameters with the African-Caribbean group having higher values for these relative to their Caucasian counterparts. These differences seem to subside with the progression of pregnancy and re-emerge at the $\geq 8$ weeks postpartum point, when time to peak and velocity were found to be significantly different. It is difficult to draw a firm conclusion from these results, due to a lack of a control group and the heterogeneous nature of women who were followed, however the results do suggest an ethnic difference in thrombin generation over the course of pregnancy, although this difference does not remain static, and appears to change with the progression of pregnancy. It must be remembered though, that in the cohort of women followed in this study, the AfricanCarribean women tended to be heavier relative to their Caucasian counterparts. It is well recognised that obesity in itself can increase thrombin generation, so further objective work is required before a definitive conclusion that women from African-Carribean origin will have an altered thrombin-generation profile relative to their Caucasian counterparts. 


\subsubsection{The dynamic state of thrombin generation}

Eleven women in the study had three anti-Xa samples drawn at each clinic visit. Though the numbers were small, it provided an opportunity to observe how the thrombin generation parameters altered with time, around a single dose of enoxaparin. Overall, the thrombogram parameters appear to change directly in proportion with the anti-Xa activity. Table 6.9 lists the mean parameter results of women injecting prophylactic doses of enoxaparin at three time points, trough, one hour post and 3 hours post dose.

Table 6.9: Changes in the TG parameters over time around a single dose

\begin{tabular}{lccccccc}
\hline $\begin{array}{l}\text { Sampling } \\
\text { time }\end{array}$ & $\begin{array}{c}\text { Anti-Xa } \\
\text { activity }\end{array}$ & Lag time & ETP & Peak & $\begin{array}{c}\text { Time to } \\
\text { Peak }\end{array}$ & Start tail & $\begin{array}{c}\text { TG } \\
\text { Velocity }\end{array}$ \\
\hline Trough & -0.04 & 3.00 & 2042 & 375 & 5.46 & 24.2 & 158 \\
& $(0.29)$ & $(0.64)$ & $(436)$ & $(82)$ & $(0.91)$ & $(2.67)$ & $(47)$ \\
& & & & & & & \\
1 hour post & 0.18 & 4.27 & 1612 & 221 & 8.35 & 28.4 & 66 \\
& $(0.09)$ & $(1.40)$ & $(561)$ & $(103)$ & $(2.73)$ & $(3.62)$ & $(44)$ \\
3 hour post & 0.26 & 4.56 & 1650 & 205 & 9.16 & 29.4 & 53 \\
& $(0.10)$ & $(1.85)$ & $(497)$ & $(87)$ & $(3.50)$ & $(4.93)$ & $(32)$ \\
\hline SD in brackets & & & & & & &
\end{tabular}

The lag time, peak, the time to peak, and the start tail, peak and the thrombin generation velocity follow the anti-Xa activity, i.e. the larger the anti-Xa activity, the longer the times for the lag-time, time to peak and start tail, and the lower the peak and thrombin generation velocity parameters. Interestingly, the ETP does not appear to correlate with the anti-Xa activity. It seems to drop at 1 hour post dose, but then appears to recover by 3 hours post dose. At first glance this is an unexpected result, however, this observation is likely to be caused, by the prolongation of the lag time, time to peak and start tail, which will increase the area under the curve, hence the ETP. Therefore it would seem sensible to be wary of this artefact when comparing the thrombin generation ETP parameter around a dose of an anticoagulant.

One of the study patients was on treatment dose of enoxaparin $(1 \mathrm{mg} / \mathrm{kg} \mathrm{bd})$ and had three antiXa activities drawn during the third trimester. Her results are presented in table 6.10. 
Table 6.10: TG results from a single subject injecting twice daily enoxaparin drawn during the third trimester

\begin{tabular}{|c|c|c|c|c|c|c|c|}
\hline $\begin{array}{l}\text { Sampling } \\
\text { time }\end{array}$ & $\begin{array}{l}\text { Anti-Xa } \\
\text { activity }\end{array}$ & Lag time & ETP & $\begin{array}{l}\text { Peak } \\
\text { Height }\end{array}$ & $\begin{array}{c}\text { Time to } \\
\text { Peak }\end{array}$ & Start tail & $\begin{array}{c}\text { TG } \\
\text { Velocity }\end{array}$ \\
\hline Trough & 0.30 & 4.22 & 1770 & 228 & 8 & 28 & 60 \\
\hline 1 hour post & 0.40 & 4.89 & 756 & 63 & 10 & 35 & 11 \\
\hline 3 hour post & 0.51 & 5.11 & 957 & 65 & 12 & 37 & 10 \\
\hline
\end{tabular}

Once again, a similar pattern is observed, whereby, the lag time, time to peak, start tail all increase with an increase in anti-Xa activity and the peak and thrombin generation velocity decreases with an increase in anti-Xa activity. The ETP values appear to be recovering with an increasing anti-Xa activity, however, this effect is likely to be related to the prolongation of the lag times, time to peak and start tails, causing an increase in ETP seen, as was observed previously.

Though the results from this aspect of the analysis are based on small numbers and confounded by many variables, the message which emerges appears to be that the thrombin generation parameters will alter with an ever changing concentration of enoxaparin in the plasma and these alterations are predictable. If a standard \% inhibition or prolongation is established for each of the thrombin generation parameters at a given time point post dose, such as $\mathrm{IC}_{50}$, then such a parameter could be used to assess objectively the effect the enoxaparin is exerting on the subject's plasma [Gerotziafas et al., 2007].

\subsection{Discussion}

Overall, the results from assaying the thrombin generation of women prescribed enoxaparin during their pregnancy suggest that thrombin generation, as measured by CAT, has already increased by the first trimester, further increases during the second trimester, after which it stabilises through to delivery. By eight weeks post partum it appears to drop back and demonstrates the prothrombotic state which ensues during a gravid state. These results agree with those presented by Dargaud and colleagues [2010], who suggested the same pattern of ETP increase. The results from this thesis do not endorse the thrombin generation pattern described by Eichinger and colleagues [1999], who described no thrombin generation increase during pregnancy in women with and without factor V Leiden and reported that ETP does not increase 
during pregnancy. Ku and colleagues also reported a similar finding from their small study [Ku et al., 2003]. As previously stated, both Eichinger and Ku's studies used an extremely high TF trigger concentration in their thrombin generation experiments. It is likely that this high concentration of TF was masking the thrombin generation rise, demonstrating the importance of experimental conditions in the execution of thrombin generation studies. Wulfkuhle and colleagues [2011], report that the maximum level of thrombin generation increases as pregnancy progresses and then drops back down post-partum. However, this study did not measure thrombin generation during the second trimester, so it is difficult for the authors to conclude without doubt whether thrombin generation would have peaked already by the second trimester, as already discussed. Rosenkranz and colleagues, report a similar pattern of ETP increase as observed by Dargaud and colleagues and during this thesis. They report that ETP is significantly different between the first and second trimester and between the first and third trimester of pregnancy. However, the difference between the second and the third trimester was not significant. This coupled with the results from this thesis, provide more weight that the true pattern of thrombin generation increase during pregnancy is as described by Rosenkranz and Dargaud's studies. Epidemiology studies describe the incidence of antenatal VTE to be equally distributed across all three trimesters. If Wulfkuhle's pattern of thrombin generation were true, then one might anticipate an increase in the incidence of antenatal VTE, in line with gestation, which is not the case, providing weight that Rosenkranz's, Dargaud's and the results presented in this thesis, is the correct thrombin generation pattern rise during pregnancy. More recently, Ismail and colleagues have published the results of evaluating the effect of tinzaparin on thrombin generation post elective caesarean section [Ismail et al., 2012]. In their study, anti-Xa activity, tissue factor pathway inhibitor (TFPI) and thrombin antithrombin complex (TAT) and ETP were measured in 20 healthy women who received 4,500 IU tinzaparin 6 hours post caesarean section, in 20 women who received 4,500 IU tinzaparin 10-12 hours post delivery and in 20 women post spontaneous vaginal delivery. Prior to delivery, TAT levels at 6 hours post delivery were significantly higher in the caesearean section groups than the spontaneous vaginal delivery groups. The authors also found that TAT levels were significantly reduced up to 24 hours following LMWH, despite a falling anti-Xa activity. They also found that peak thrombin and ETP were significantly reduced following LMWH prophylaxis and reverted to pre-delivery levels 10 hours post LMWH. Though this study was conducted in women during the postnatal period, their findings mirror many of those 
presented in this thesis, in terms of the influence of LMWH on the different thrombin generation parameters.

The women in this study were self-injecting enoxaparin, and an argument could be made that the presence of enoxaparin is distorting the true pattern of thrombin generation in the pregnant women. However, as observed in the results, the prophylactic doses of enoxaparin appear to alter the thrombin generation parameters in a consistent manner, allowing the typical thrombin generation pattern to be described, though the confounding factor of women injecting enoxaparin must be born in mind.

Indeed a clear influence of enoxaparin on the thrombogram can be seen in this study, as has been reported by earlier smaller studies. In their study, Ngu and colleagues [2010], reported that all parameters of the thrombogram correlated well with anti-Xa activities with the most sensitive parameters being the peak height and the time to peak parameters. Adamidou and colleagues [2011] report the time to peak to be the most sensitive parameter in pregnant women injecting enoxaparin with anti-Xa activities greater and less than $0.4 \mathrm{IU} / \mathrm{mL}$. Unsurprisingly, the results from this thesis suggest that a treatment dose of enoxaparin has more of a profound effect on thrombin generation compared to prophylactic doses, with strong negative relationships existing between the ETP, peak and velocity parameters of thrombin generation and anti-Xa activity. With respect to the lag time, time to peak and start tail; these relationships were positive. The results agree with the small studies of $\mathrm{Ngu}$ and Adamidou, and with the patient numbers in this study, provide invaluable insight into these relationships. The next steps would be to define by how much thrombin generation is to be inhibited at a set time point with prophylactic and treatment doses of $\mathrm{LMWH}$, for individual $\mathrm{LMWH}$, in order to tailor treatment to individual patients.

This aspect of the enoxaparin study is limited by the fact that it is observational, it has no control group, women were prescribed enoxaparin, and the women that were followed were a heterogeneous group of women, from active pathological thrombosis to asymptomatic VTE prophylaxis. Future thrombin generation studies would be well served to explore sub-groups of women in a controlled manner in order to identify if thrombin generation patterns are different in different groups of women. It would also be good to have confirmation of the pattern in ETP / peak height, with a study which follows a control group. 
The results from this study also found that thrombin generation does indeed alter with time when a dose of enoxaparin is administered. This change is largely reflected in the start tail, time to peak, lag time, thrombin generation velocity and the peak height parameters of the thrombogram. The ETP results, with respect to this, are misleading; as initial inspection of the results suggest that ETP begins to recover by 3 hours post dose. But this is likely to be an artefact of measuring area-under curve, where the lag time and time to peak have increased in a dose dependent manner, thereby increasing the ETP, i.e. not that the ETP is recovering. This should be borne in mind, when reviewing thrombin generation results of patients on anticoagulant therapy where thrombin generation repeatedly measured, over a single dose.

Finally, other groups have reported thrombin generation to be altered by age, obesity, and other medical co-morbidities. These factors were not controlled in this study, and future studies might well look to control these variables, in order to discern the specific impact of covariates on thrombin generation during pregnancy. Obesity is of particular interest, as evidence published in the UK over the last 10 years identifies this sub-group of women as a growing cohort and that as well as an increasing risk of VTE, these women are at increased risk of bleeding [Modder and Fitzsimons, 2010]. More recently, ethnicity has also been identified as an independent factor influencing thrombin generation [Roberts et al., 2012]. Evidence already exists which demonstrates that the African-Caribbean ethnicity is associated with an increased risk of both first and recurrent venous thromboembolism [White et al., 2004; White et al., 2005]. In their study, Roberts and colleagues [2012] evaluated thrombin generation in African-Caribbeans compared to Caucasians in patients with a first DVT, and healthy volunteers. Thrombin generation was measured in a case-control study of 80 patients who had completed anticoagulation therapy for a first DVT (50 Caucasians and 30 African-Caribbean) and 66 controls. In the DVT group, peak thrombin, ETP and maximum velocity were all significantly higher in the African-Caribbeans than the Caucasian group. Within the control group, peak thrombin and maximum velocity were also increased in the African-Caribbean group compared to the Caucasian group. In the pregnancy sub-study, significant differences in some of the thrombin generation parameters were found during the first and second trimester and at the $\geq 8$ weeks post-partum time point. No differences were found during the third trimester and at the time of delivery. The ethnicity results assessed in this thesis were from women prescribed prophylactic doses; and at first glance might suggest that the enoxaparin is 'equalling' out thrombin generation over the course of pregnancy. However, a 
case could be made that enoxaparin has a constant and consistent effect on thrombin generation over the course of pregnancy, and so a true pattern is seen, i.e. African-Caribbeans having a higher TG than their Caucasian counterparts in the first and second trimester, after which the Caucasian group 'catch-up' and they begin to separate out again by eight weeks post-partum. The results certainly provide confirmatory evidence of the findings from Roberts and colleagues and explain some of the findings from the last CMACE report [CMACE, 2011]. In the last CMACE report, 5 women died during the antenatal period, of which 3 were from a African-Caribbean ethnic group, with all 3 dying during the first trimester. The results here demonstrate how much more TG has increased in the first trimester for the African-Caribbean population and may in part explain why this ethnic group dominates the death/incidence rate during the first trimester. Of course the increases incidence in this sub-group could also be dependent on other factors, particularly obesity or social, e.g. language barriers. However, further research should focus on understanding the exact mechanism behind this and further explore what has been found in this thesis.

Strengths of this sub-study include the patient numbers recruited and tested, the fact that the same women were tested on multiple occasions through their pregnancy and during the postpartum period, the fact that thrombin generation was measured 8 weeks post delivery (when most women were off anticoagulant therapy), providing a baseline value for each women. This allowed the evaluation of how much thrombin generation had increased during the antenatal period. Other strengths, include the number of African-Caribbean patients followed in this study, providing valuable information on this particular sub-group. Finally, a major strength of this study is that it involved in-vivo work, i.e. was conducted on plasma from women actually injecting the enoxaparin, not plasma spiked with LMWH. Limitations in this sub-study are, that not all women had thrombin generation assayed at all five pre-determined time points, and that exact impact of obesity on thrombin generation was not controlled, therefore its not known whether the ethics differences observed are due to ethnicity or due to obesity. 


\subsection{Can polybrene neutralise enoxaparin in the thrombin generation assay?}

\subsubsection{Background}

Polybrene (hexadimethrine bromide) is a synthetic polymerised quaternary ammonium cation. As described in chapter one, during the late 1950's and early 1960's, the compound was utilised as an unfractionated heparin neutralising agent for patients undergoing open cardiac surgery and in those patients with an extracorporeal circulation. Its use in clinical practice was short-lived, however, due to significant reports of renal toxicity.

The requirement of reversing or neutralising anticoagulant agents is not new and stems back to work by Chargaff and Olson [1937]. Chargaff and Olson were the first to demonstrate the neutralising effect of protamine on unfractionated heparin. Over the years, with an increase in the use of anticoagulant therapy in patients, a need has arisen to have a safe and effective reversing agent, particularly in an emergency setting. Protamine sulphate achieves this for the heparins by competing with antithrombin for binding to heparin [Okajima et al., 1981]. Protamine has a stronger affinity for binding to heparin than antithrombin, therefore causing antithrombin to dissociate from the heparin-antithrombin complex, and thus reversing the anticoagulant effect. The binding of protamine to heparin is through an electrostatic means, whereby the polycation protamine binds to the polyanion heparin to form a stable complex with no anticoagulant activity [Ando et al., 1973]. Polybrene, also a polycation, is also thought to exert its heparin neutralising effect through electrostatic means. Protamine sulphate fully reverses both the anti-Ila and anti-Xa fractions of unfractionated heparin, and the anti-lla fraction of $\mathrm{LMWH}$, but does not fully reverse the anti-Xa effect of LMWH [van Veen et al., 2011]. The inability of protamine sulphate and thus polybrene to fully reverse the anti-Xa fraction of LMWH is thought to be due to a reduced sulphate charge in ultra low molecular weight fragments present in LMWH but not in unfractionated heparin [Crowther et al., 2000].

The neutralisation of enoxaparin in-vitro holds an important clinical utility for the study in this thesis. The lack of a control group and the fact that the thrombin generation samples were drawn at different time points in the thrombin generation aspect of this study meant that it was difficult to assess what exact impact the enoxaparin has had on the women's thrombin generation. If a suitable LMWH neutralising agent could be established for the thrombin generation assay, then a valuable insight could be obtained. 
This aspect of the sub-study was inspired by an abstract presented at the Interntional Socity of Thrombosis and Haemostasis (ISTH) conference by Carlo and colleagues [2009]. In their study, Carlo and colleagues initially evaluated whether polybrene influenced the thrombin generation assay, in itself. They found that polybrene, at concentrations between $0-5 \mathrm{mg} / \mathrm{mL}$, did influence the thrombin generation assay. This influence was found to be in an inhibitory form; particularly influencing the peak height and ETP. Carlo and colleagues found that at a concentration of $0.1 \mathrm{mg} / \mathrm{mL}$, the peak height and ETP are inhibited by $38 \%$ and $32 \%$ respectively by polybrene. The group then spiked normal pool plasma with UFH and dalteparin (at an anti-Xa concentration range of between $0-2 \mathrm{IU} / \mathrm{mL}$ ), and measured thrombin generation using CAT in order to assess whether the addition of polybrene to the samples would effectively neutralise the heparin effect. They report that a polybrene concentration of $0.025 \mathrm{mg} / \mathrm{mL}$ effectively neutralised the effect of UFH and LMWH up to a concentration of $2 \mathrm{IU} / \mathrm{mL}$ of anti-Xa activity and would be effective in evaluating the pharmacodynamic effect of LMWH on the thrombogram. They go onto to report that at a polybrene concentration of $0.025 \mathrm{mg} / \mathrm{mL}$, the specific influence of polybrene on the thrombin generation parameters was less than $10 \%$.

In a further earlier study, Messmore and colleagues [2003] evaluated the effectiveness of polybrene neutralisation in normal pool plasma. They conducted their experiments, inspired by the fact that some samples arriving in their hospital laboratory were from patients on heparin based treatments and wanted to develop a means of neutralising the heparin effect from the plasma. They spiked plasma with enoxaparin and UFH at the following anti-Xa concentrations; $0.5,1.0,1.5 \mathrm{IU} / \mathrm{mL}$ and then measured anti-Xa activity before and after the addition of polybrene to these samples, at a concentration of $0.1 \mathrm{mg} / \mathrm{mL}$. They found polybrene reversed the anti-Xa activity of both UFH and enoxaparin, demonstrating that the desired effect can be achieved invitro, at therapeutic heparin concentrations.

Using Carlo's study as a template and inspired by the clinical use of polybrene in UFH reversal from the late 1950's and early 1960's, the thrombin generation profiles of recruited pregnant women recruited during this study were additionally assayed with polybrene, in order to establish whether enoxaparin's specific pharmacodynamic effect could be discerned. 


\subsubsection{Aim}

The aim of this sub-study was to:

- Evaluate whether polybrene could neutralise the effect of enoxaparin in the thrombin generation profiles of the women recruited to the study

- If a neutralisation effect was obtained, was it $100 \%$ effective

\subsubsection{Methodology}

Dr Carlo (Diagnostica Stago, France) was personally contacted to obtain details of how her group had conducted their polybrene study. Dr Carlo's kindly sent the following description for conducting their experiments [personal communication, 18/09/2009];

Initially a stock solution of $10 \mathrm{mg} / \mathrm{mL}$ of polybrene, by dissolution of the polybrene powder (Sigma Aldrich) in distilled water is prepared. From the stock solution, prepare a dilution of $1 \mathrm{mg} / \mathrm{mL}$ polybrene solution. Depending on the specific experimental conditions and reagents going to be used in the thrombin generation assay (e.g. either PPP, PPP low, PPP high, micro-particles or PRP), reconstitute the appropriate reagent vial with $850 \mu \mathrm{L}$ of distilled water and make up to $1 \mathrm{~mL}$ with $150 \mu \mathrm{L}$ of the polybrene $1 \mathrm{mg} / \mathrm{mL}$ solution. Then wait for stabilisation of the reconstituted vial for 30 minutes at room temperature. The reagent is then ready to use in the thrombin generation test and executed normally (as previously described), and contains $0.15 \mathrm{mg} / \mathrm{mL}$ of polybrene, which is $0.025 \mathrm{mg} / \mathrm{mL}$ in the final test $(20 \mu \mathrm{L}$ of trigger $+80 \mu \mathrm{L}$ of sample $+20 \mu \mathrm{L}$ of FluCa).

For reasons explained in the next section, the final concentration of polybrene that was utilised in this study was $0.0125 \mathrm{mg} / \mathrm{mL}$, not $0.025 \mathrm{mg} / \mathrm{mL}$. Furthermore, in order to ensure that the PPP reagents were not wasted during each experiment, Dr Carlo's instructions were amended as follows:

i) A stock solution of polybrene $10 \mathrm{mg} / \mathrm{mL}$ was freshly prepared for each experiment (made fresh prior to each thrombin generation test, usually weekly).

ii) A diluted solution of polybrene $1 \mathrm{mg} / \mathrm{mL}$ was prepared from the stock solution.

iii) A vial of PPP was reconstituted with $500 \mu \mathrm{L}$ of di-ionised water and left to stand for 2 minutes. $250 \mu \mathrm{L}$ was then withdrawn from the PPP vial and added to a new tube. A 
further $250 \mu \mathrm{L}$ of de-ionised water was added to the original PPP vial (with no polybrene). To the second tube of $250 \mu \mathrm{L}$ of PPP, $215 \mu \mathrm{L}$ of di-ionised water was added, along with $35 \mu \mathrm{L}$ of the polybrene $1 \mathrm{mg} / \mathrm{mL}$ solution. This was then left to stand for 30 minutes before using in the thrombin generation assay.

\subsubsection{Concentration of polybrene used in the experiments}

Following commencement of the thrombin generation experiments, the research team found that the polybrene concentration suggested by Carlo and colleagues' study might be too high for the in-vivo samples being assayed, as no apparent reversal effect was being observed for women prescribed prophylactic doses of enoxaparin and the ETP and peak parameters were being inhibited significantly; the research team therefore conducted a small in-vitro study to evaluate whether an alternative lower concentration of polybrene $0.025 \mathrm{mg} / \mathrm{mL}$ should be used.

\subsubsection{In-vitro study of differing polybrene concentrations}

The plasma from a healthy control (male, aged 36) was spiked with enoxaparin at the following anti-Xa activities: $0.05,0.10,0.15,0.20,0.30,0.40,0.60,0.80,1.0 \mathrm{IU} / \mathrm{mL}$. Thrombin generation was then evaluated, using CAT, in these samples with and without the addition of polybrene at the following polybrene concentrations; $0.025,0.01875,0.0125,0.00625 \mathrm{mg} / \mathrm{mL}$.

In the absence of polybrene, a strong negative relationship existed between the enoxaparin antiXa activities and the ETP and peak height parameters of the thrombogram ( $r=-0.952, p=0.01$ and $r=-0.872, p=0.01)$. With the addition of polybrene concentrations, all four polybrene concentrations neutralised enoxaparin at higher anti-Xa activities $(\geq 0.30 \mathrm{IU} / \mathrm{mL})$. At lower enoxaparin anti-Xa activities $(<0.30 \mathrm{IU} / \mathrm{mL})$, PB neutralised enoxaparin's effect in a concentration dependent manner, with the two higher PB concentrations $(0.025$ and $0.01875 \mathrm{mg} / \mathrm{mL})$ inhibiting TG. By the lowest anti-Xa activity, all four PB concentrations were inhibiting TG. Figures 6.13 (a) and (b) illustrate the thrombin generation curves of the spiked plasma at two different enoxaparin anti-Xa activities; $0.05 \mathrm{IU} / \mathrm{mL}$ and $0.60 \mathrm{IU} / \mathrm{mL}$. The effect of polybrene does change with anti-Xa activity, being more effective in terms of neutralising enoxaparin at higher enoxaparin concentrations than lower concentrations. This has implications for women for this study, as women in this study were prescribed varying doses of enoxaparin and so the polybrene effect will be variable, dependent on the anti-Xa activity at a given moment in time in the plasma. 


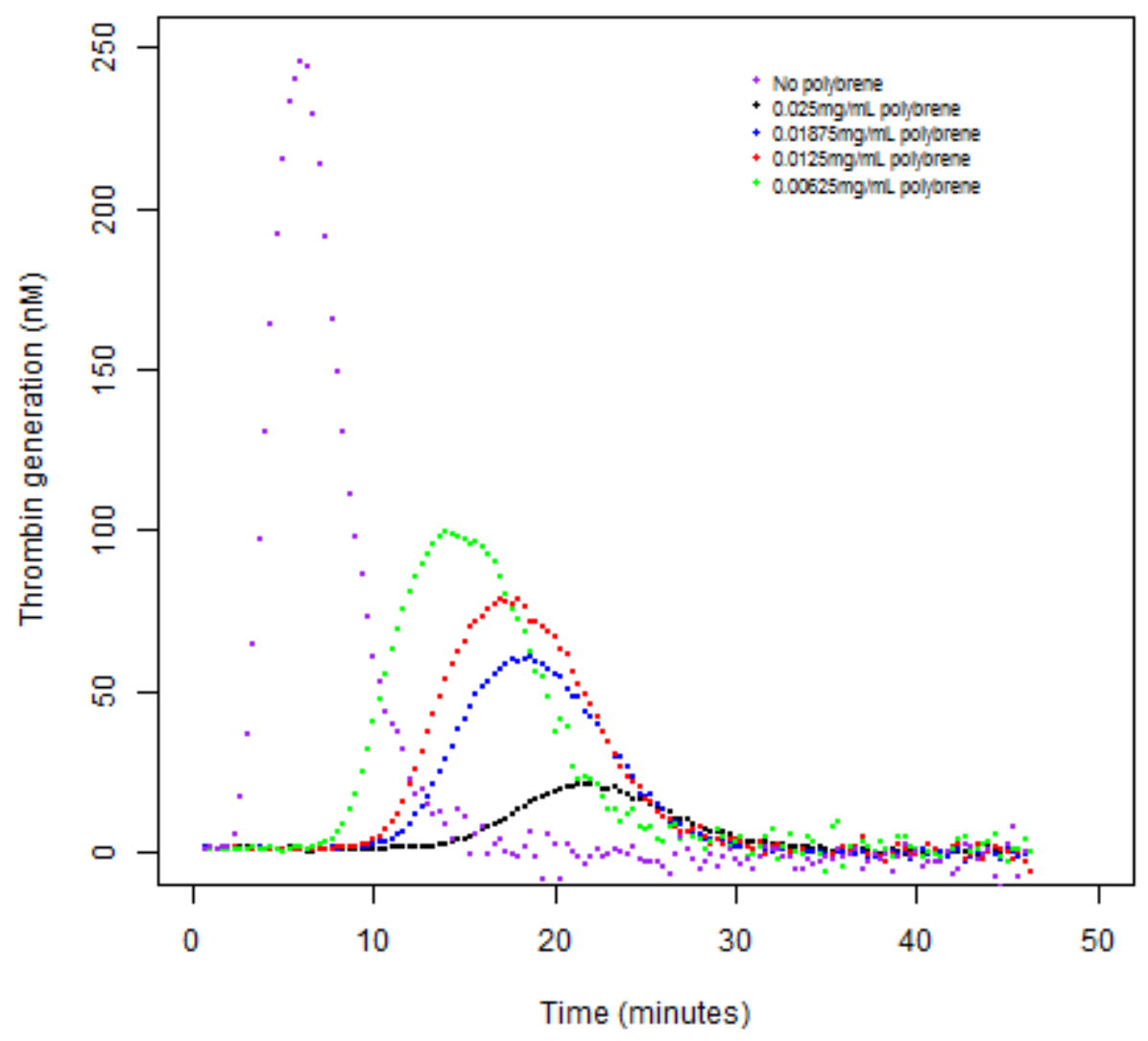

Figure 6.13 (a): Thrombin generation curves of plasma samples spiked with $0.05 \mathrm{IU} / \mathrm{mL}$ of enoxaparin, with the addition of polybrene at differing concentrations 


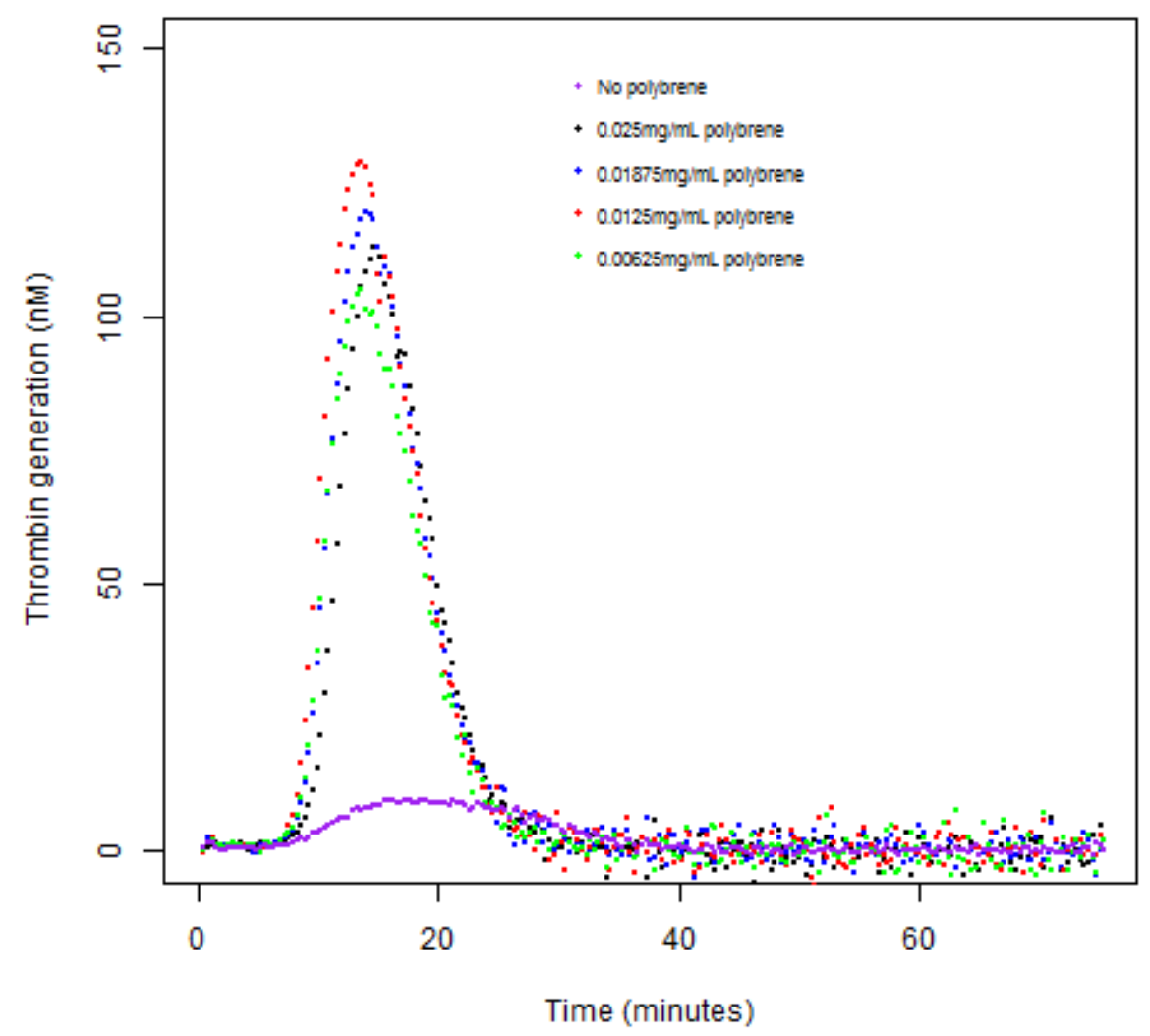

Figure 6.13 (b): Thrombin generation curves of plasma samples spiked with $0.60 \mathrm{IU} / \mathrm{mL}$ of enoxaparin, with the addition of polybrene at differing concentrations

The research team therefore decided to use half the concentration that $\mathrm{Dr}$ Carlo reported in their study, i.e. $0.0125 \mathrm{mg} / \mathrm{mL}$, as this concentration appeared to be able to neutralise therapeutic concentrations of enoxaparin, whilst having a minimal impact itself on thrombin generation.

In order to better determine the impact of $0.0125 \mathrm{mg} / \mathrm{mL}$ polybrene on plasma, as has already been described by Carlo and colleagues, the same healthy control had their thrombin generation assayed in each of the thrombin generation experiments conducted for the enoxaparin in pregnancy study from the period $20^{\text {th }}$ April 2010 to $21^{\text {st }}$ February 2012 (experiments 17 to experiments 125), $79 \%$ of total thrombin generation experiments. Table 6.11 illustrates the thrombin generation results from this healthy control, with and without polybrene, with an indication of what impact the polybrene had on each thrombin generation parameter. 
Table 6.11: Change in thrombin generation parameters with the addition of PB to the control subject's plasma

\begin{tabular}{|c|c|c|c|}
\hline $\begin{array}{l}\text { Thrombin generation } \\
\text { parameter }\end{array}$ & Mean & SD & $\begin{array}{c}\% \text { change with addition } \\
\text { of } P B\end{array}$ \\
\hline Lagtime & 3.13 & 0.60 & \\
\hline Lagtime with PB & 4.44 & 0.81 & +42 \\
\hline ETP & 1115 & 226 & \\
\hline ETP with PB & 996 & 216 & -11 \\
\hline Peak & 206 & 49 & \\
\hline Peak with PB & 145 & 36 & -30 \\
\hline Time to Peak & 6.32 & 1.16 & \\
\hline Time to Peak with PB & 8.57 & 1.43 & +36 \\
\hline Start tail & 20.1 & 2.3 & \\
\hline Start tail with PB & 23.6 & 2.3 & +17 \\
\hline Velocity & 69 & 27 & \\
\hline Velocity with PB & 36 & 12 & +48 \\
\hline
\end{tabular}

These results clearly demonstrate a significant influence of polybrene on the thrombin generation assay in the absence of enoxaparin; with the lag time, peak,time to peak and velocity significantly influenced.

\subsubsection{Results}

Table 6.12 represent the thrombin generation results, when the results with and without polybrene are compared. The table represent the whole cohort of women, i.e. women receiving prophylactic and treatment doses have not been separated out; significant differences are highlighted in this table $\left.{ }^{* \star}\right)$. 
Table 6.12: The thrombin generation results for the cohort of women in this study, with and without polybrene $0.0125 \mathrm{mg} / \mathrm{mL}$

\begin{tabular}{|c|c|c|c|c|c|c|c|c|}
\hline \multirow[t]{2}{*}{ Parameter } & \multicolumn{2}{|l|}{$\mathrm{T} 1$} & \multicolumn{2}{|l|}{$\mathrm{T} 2$} & \multicolumn{2}{|l|}{ T3 } & \multicolumn{2}{|c|}{ At delivery } \\
\hline & $n=58$ & & $n=117$ & & $n=132$ & & $n=93$ & \\
\hline Lag time & 4.27 & (3.81) & 3.53 & $(1.26)^{\star \star}$ & 3.88 & $(2.46)^{\star *}$ & 3.14 & $(1.32)^{\star \star}$ \\
\hline LT with PB & 4.87 & (2.85) & 4.18 & $(1.38)$ & 4.20 & (1.43) & 3.73 & $(1.88)$ \\
\hline ETP & 1391 & $(795)^{* *}$ & 1757 & $(707)^{\star \star}$ & 1692 & $(631)^{\star *}$ & 1857 & $(633)^{\star *}$ \\
\hline ETP with PB & 1666 & $(610)$ & 1922 & $(480)$ & 1889 & $(522)$ & 1926 & $(542)$ \\
\hline Peak & 207 & $(141)^{* *}$ & 277 & $(136)^{* *}$ & 262 & $(117)^{\star \star}$ & 356 & $(127)^{\star \star}$ \\
\hline Peak with PB & 247 & (104) & 301 & (93) & 296 & (82) & 371 & $(100)$ \\
\hline tt Peak & 8.09 & $(5.17)$ & 7.05 & (3.27) & 7.36 & (3.91) & 5.57 & (2.83) \\
\hline tt Peak with PB & 8.45 & (3.47) & 7.41 & (2.07) & 7.34 & (2.01) & 5.99 & (2.39) \\
\hline Start tail & 27 & (9.72) & 26 & (6.96) & 27 & $(6.88)$ & 24 & $(5.82)$ \\
\hline Start tail with PB & 28 & (5.71) & 26 & $(4.41)$ & 26 & $(4.47)$ & 25 & $(4.79)$ \\
\hline Velocity & 77 & (65) & 107 & (71) & 96 & $(57)^{\star \star}$ & 174 & $(75)$ \\
\hline Velocity with PB & 85 & $(50)$ & 107 & (51) & 105 & (44) & 175 & (58) \\
\hline
\end{tabular}

SD in brackets. ${ }^{* *} p<0.05, \mathrm{n}=$ number of samples evaluated. Results from the $\geq 8$ weeks PP not presented, as women were no longer injecting enoxaparin

Overall, the results demonstrate an increase in all thrombogram parameters with the addition of polybrene to the subjects' plasma. The ETP and peak were significantly increased in the polybrene samples, compared to the original samples, across all four time points where women were injecting enoxaparin, suggesting a polybrene neutralising effect, however, the polybrene consistently appeared to prolong the lag time at all time points. If the polybrene was truly neutralising the effect of enoxaparin, one would anticipate a shortening of the lag time. Overall, the tt Peak parameter increased with the polybrene samples; not as expected, as one would predict a shortening of this time with polybrene. This pattern is largely repeated with the start tail. Finally, the thrombin generation velocity parameter increased across all four time points where patients were injecting enoxaparin, in line with the ETP and peak, and suggesting once again, a potential polybrene neutralising effect.

The results clearly demonstrate that the polybrene is exerting a neutralising effect on the women's plasma. However the results are variable depending on which thrombin generation parameter is evaluated, and overall the results appear to suggest that the neutralisation of enoxaparin in-vivo is not complete. 
In order to further evaluate the overall result, they have once again been split, according to women injecting prophylactic or treatment doses of enoxaparin. Results for women on prophylaxis are presented in table 6.13 , whilst the results for women on treatment doses are presented in table 6.14. In both cases, significant differences are highlighted $\left({ }^{* *}\right)$ in these tables.

Table 6.13: Thrombin generation results from women prescribed prophylactic enoxaparin, with and without polybrene

\begin{tabular}{|c|c|c|c|c|c|c|c|c|}
\hline $\begin{array}{l}\text { Thrombin } \\
\text { generation } \\
\text { parameter }\end{array}$ & $\begin{array}{l}\text { T1 } \\
n=49\end{array}$ & & $\begin{array}{l}\text { T2 } \\
n=101\end{array}$ & & $\begin{array}{l}\text { T3 } \\
n=110\end{array}$ & & $\begin{array}{l}\text { At de } \\
n=75\end{array}$ & livery \\
\hline LT & 4.35 & (3.82) & 3.38 & $(0.82)^{\star \star}$ & 3.53 & $(0.97)^{\star \star}$ & 2.92 & $(0.92) * \star$ \\
\hline LT with PB & 4.45 & $(2.25)$ & 3.92 & $(0.85)$ & 4.00 & $(1.00)$ & 3.40 & $(0.92)$ \\
\hline ETP & 1473 & $(730)^{\star \star}$ & 1905 & (564) & 1802 & $(481)^{\star \star}$ & 1945 & $(551)$ \\
\hline ETP with PB & 1701 & (507) & 1955 & (477) & 1889 & $(460)$ & 1957 & $(504)$ \\
\hline Peak & 222 & $(137)^{\star *}$ & 306 & (118) & 288 & $(95)^{\star *}$ & 378 & (107) \\
\hline Peak with PB & 254 & (96) & 313 & (88) & 303 & (74) & 383 & (90) \\
\hline tt Peak & 8.30 & $(4.88)$ & 6.50 & $(1.75)^{\star \star}$ & 6.70 & $(1.84)^{\star *}$ & 5.09 & $(1.39)^{* \star}$ \\
\hline tt Peak with PB & 7.98 & $(2.89)$ & 7.02 & $(1.52)$ & 7.05 & $(1.57)$ & 5.57 & $(1.15)$ \\
\hline Start tail & 28.3 & (7.16) & 26.6 & $(4.2)$ & 26.2 & $(4.7)$ & 24.3 & (3.88) \\
\hline Start tail with PB & 27.6 & $(4.89)$ & 26.4 & (3.9) & 26.1 & $(4.4)$ & 24.1 & (3.26) \\
\hline Velocity & 79 & (65) & 118 & (68) & 107 & (56) & 187 & (68) \\
\hline Velocity with PB & 84 & (51) & 112 & (50) & 108 & (42) & 182 & (52) \\
\hline
\end{tabular}

sd in brackets. ${ }^{* *} p<0.05, n=$ number of samples at the respective time point

As with the 'overall' group, the lag time is prolonged in the polybrene group. The ETP increases by varying amounts at the different time points sampled. The peak height increases by varying amounts at the different time points sampled. Apart from during the first trimester, the time to peak is increased in the polybrene samples. The start tail is consistently found to decrease with the polybrene samples, and apart from the second trimester and within one week of delivery, the velocity increases.

At first glance, it appears as if the results provide no useful information. They do however demonstrate that polybrene can neutralise enoxaparin, even for patients on prophylactic doses, depending on the enoxaparin anti-Xa activity at a given moment in time, but this effect does not appear to be complete. The other useful piece of information to discern is the result demonstrates the previous in-vitro work, that polybrene exerts its own effect on the thrombin generation test. 
Table 6.14: Thrombin generation results of patients prescribed treatment doses of enoxaparin with and without polybrene

\begin{tabular}{|c|c|c|c|c|c|c|c|c|}
\hline \multirow[t]{2}{*}{ Parameter } & \multicolumn{2}{|l|}{ T1 } & \multicolumn{2}{|l|}{ T2 } & \multicolumn{2}{|l|}{ T3 } & \multicolumn{2}{|c|}{ At delivery } \\
\hline & $n=9$ & & $n=16$ & & $n=22$ & & $n=18$ & \\
\hline Lag time & 3.82 & (3.97) & 4.50 & (2.57) & 5.66 & (5.37) & 4.09 & $\begin{array}{l}(2.14) \\
\end{array}$ \\
\hline LT with PB & 7.17 & $(4.53)$ & 5.90 & (2.62) & 5.16 & (2.51) & 5.13 & (3.62) \\
\hline ETP & 942 & $(1015)^{\star \star}$ & 820 & $(809)^{\star *}$ & 1141 & $(952) * \star$ & 1497 & $(824)^{\star \star}$ \\
\hline ETP with PB & 1470 & (1030) & 1711 & $(460)$ & 1889 & (776) & 1800 & (681) \\
\hline Peak & 129 & $(146)^{* *}$ & 95 & $(103)^{\star *}$ & 135 & $(131)^{* *}$ & 266 & $(164)^{\star \star}$ \\
\hline Peak with PB & 210 & $(140)$ & 231 & (97) & 261 & (111) & 321 & $(130)$ \\
\hline tt Peak & 6.96 & $(6.81)$ & 10.5 & $(6.89)$ & 10.7 & (7.99) & 7.55 & $(5.46)$ \\
\hline tt Peak with PB & $10.9 \varepsilon$ & $(5.21)$ & 9.89 & (3.19) & 8.7 & (3.14) & 7.79 & $(4.59)$ \\
\hline Start tail & 21.5 & $(17.9)$ & 24.9 & (15.9) & 28.8 & $(13.0)$ & 23.9 & $(10.8)$ \\
\hline Start tail with PB & 32.6 & $(8.1)$ & 29.9 & (5.96) & 27.9 & $(4.5)$ & 27.6 & $(8.30)$ \\
\hline Velocity & 60 & $(62)^{\star *}$ & 28 & $(35)^{\star \star}$ & 42 & $(52)^{\star \star}$ & 122 & $(83)^{\star \star}$ \\
\hline Velocity with PB & 92 & (38) & 66 & (45) & 85 & (52) & 147 & (74) \\
\hline
\end{tabular}

For all pregnancy time points, apart from the third trimester, the lag time was longer in the PB samples, suggesting that irrespective of enoxaparin plasma concentration, the polybrene effect on thrombin generation will prolong the lag time. The ETP is increased in all the polybrene samples and during the first, second and third trimesters, the enoxaparin effect observed appears to be greater than $50 \%$ at these time points. This really does demonstrate the application of the possibilities of a neutralising agent in this setting. A similar result is also observed for the peak height parameter; the effect is once again more profound during the first, second and third trimesters. During the second and third trimester, the time to peak is shortened, but otherwise is more prolonged, showing a variable response. The start tail is on the whole more prolonged in those samples with polybrene. Finally the velocity of thrombin generation is prolonged significantly during the first, second and third trimesters, and at the time of delivery.

These results demonstrate the concentration dependent effect of polybrene, exerting what appears to be more of a neutralising effect on plasma derived from subjects receiving treatment doses of enoxaparin. The ETP, peak height and velocity are the parameters that appear to be most sensitive to the polybrene effect. At the time of delivery, the effect of polybrene appears to 
be less pronounced. This is likely to be related to the fact, that the concentration of enoxaparin in the plasma was likely to have been less than at other time points, hence the effect observed.

Clearly the PB is exerting an effect on these samples and neutralising part of the enoxaparin present, but this effect is not complete.

Before any firm conclusions can be drawn, further investigation of what the exact binding relationship is between polybrene and enoxaparin is required, along with what influence albumin in the plasma might be having on this relationship, i.e. assessment of the hypothesis, that although polybrene neutralisation might be complete in-vitro, it is not complete in-vivo, due to the presence of albumin (and other plasma proteins).

\subsubsection{Discussion}

The results demonstrate that it is possible to neutralise to a certain degree, enoxaparin's pharmacodynamic effect in the thrombin generation assay with polybrene. However, this effect is dependent on the concentration of LMWH at a given moment in time and the effect is not complete, with polybrene found to exert its own effect on the thrombin generation profiles tested.

The results should not come as a surprise, given what has previously been reported by Carlo and colleagues [2009]. Over the years, many groups have compared and contrasted the effects of polybrene and protamine as heparin neutralising agents. Sie and colleagues [1989] evaluated the effectiveness of protamine sulphate and polybrene in neutralising dermatan sulphate and heparin in plasma and in saline. Protamine sulphate and polybrene were both found to neutralise heparin in-vitro in a predictable fashion, with polybrene found to be as potent as protamine sulphate in plasma and twice as potent as protamine sulphate in saline. When dermatan sulphate in saline was considered, both protamine sulphate and polybrene readily neutralised the dermatan sulphate, but in plasma both agents were approximately five times less active than when compared to saline, with the study authors suggesting that this observation may involve a nonspecific interaction with plasma proteins.

Cumming and colleagues [1986] investigated the effects on the activated partial thromboplastin time (APTT) when heparin in plasma was neutralised with protamine or polybrene. Both polycations exhibited clot promoting activity at low concentrations and acted as anticoagulants in their own right at higher concentrations. At a plasma heparin concentration of $4 \mathrm{IU} / \mathrm{mL}$, protamine 
was the most effective neutraliser, while at a plasma heparin concentration of $10 \mathrm{IU} / \mathrm{mL}$, polybrene was the most effective.

Jacobsen and colleagues [2006] investigated the effect of polybrene on neutralising the effects of heparin in plasma from patients who were being tested for lupus anticoagulant. Lupus anticoagulant positive patients were given $5000 \mathrm{IU}$ intravenous unfractionated heparin and their plasma was drawn just before and five minutes after the injection. They found the lupus ratio to be slightly lower when polybrene was added to plasma without heparin. The patient's lupus ratios returned to the lupus ratios before any additions of heparin and they conclude that the addition of polybrene at a final concentration of $7.9 \mu \mathrm{g} / \mathrm{mL}$ in test plasma can determine the presence of lupus anticoagulant, irrespective of the presence of unfractionated heparin at a concentration of $0.0-1.3 \mathrm{IU} / \mathrm{mL}$.

The messages which emerge from these studies is that polybrene can neutralise the effect of heparin in plasma, but the effect is concentration dependent and it will not completely reverse the LMWH effects in-vivo. Therefore it is unsurprising that in this study, complete neutralisation was not seen.

\subsubsection{Effect observed with treatment and prophylactic doses}

It has been speculated that an INR of between 2-4 corresponds to ETP and peak inhibition by 2040\% [Odegard et al., 1976]. The polybrene experiments gave an opportunity to assess this principle, in those women managed on treatment doses of enoxaparin during pregnancy. This analysis found that ETP and peak were significantly inhibited. This clearly demonstrates the effectiveness of treatment doses of enoxaparin and demonstrates the potential clinical utility of the thrombin generation assay in titrating drug therapy. As one might anticipate, the effect of enoxaparin on thrombin generation is dose related, with much lower inhibition observed for those women prescribed prophylactic doses of enoxaparin. This is important, as currently we do not know the level at which to inhibit the TG parameters, in order to get a prophylactic effect from LMWH. Establishing a prophylactic range would help and overcome a question commonly asked in clinical practice, about what the optimal level of thromboprophylaxis is, and perhaps help overcome prophylaxis failure seen in clinical practice. 
6.5.5.2 Are there alternative neutralising agents that could be used?

Accepting that polybrene does not completely neutralise the effect of LMWH in plasma, is there any other compound that might be $100 \%$ effective at achieving the desired effect in the thrombin generation assay? Protamine could be tried, but given the plethora of literature which suggests that it works in a similar way to polybrene and the fact that protamine exerts its own anticoagulant effect in excess, exploring protamine's use in this setting is unlikely to be fruitful. Reagents that should however be considered are the heparinases, as seen in their application in thromboelastometery $\left(\right.$ ROTEM $\left.^{\circledR}\right)$. In ROTEM ${ }^{\circledR}$, whole blood coagulation is evaluated (www.rotem.de), and can then be compared by the use of HEPTEM®. In the HEPTEM assay (heparinase modified TEM), coagulation is triggered via the instrinsic pathway. During the assay, heparinase rapidly degrades heparin and allows for the assessment of haemostasis in heparinised patients. The results from HEPTEM are compared to INTEM. The heparinise present in the reagent is Heparinase I from falvobacteria (www.rotem.de). This system works well in this setting and could be explored as an option in the thrombin generation test setting, although whether the heparinises could break down enoxaparin further, because of its low molecular weight, and thus neutralise the enoxaparin effect is debatable. If pursued, the key will be establishing the concentration to use in the thrombin generation test, but certainly deserves further consideration.

\subsubsection{Summary and future work}

This chapter set out to explore the thrombin generation profiles of women injecting enoxaparin during their pregnancy. The results confirm that thrombin generation is already raised by the first trimester, with a further rise seen during the second trimester, after which thrombin generation stabilises through to delivery. By eight weeks post partum, baseline (non-pregnancy) thrombin generation levels will be reached. Future work should focus on comparing the thrombin generation profiles of women with pre-eclampsia, IUGR, at high risk of VTE and compare the changes in thrombin generation profiles, to a control group. This might help identify a further important clinical utility of the thrombin generation assay.

As one might predict, the dose of enoxaparin strongly influenced the thrombin generation test, in a dose-dependent manner. Though polybrene did reverse the effects of enoxaparin to a certain 
degree, it was not complete and was more apparent with higher plasma concentrations of enoxaparin, as assessed through anti-Xa activity.

Given the strong correlations between the dose of enoxaparin and the effect observed on thrombin generation parameters, future work should focus on developing an appropriate means of reversing the LMWH anticoagulant effect from subject's plasma. Given the inter-individual differences between the patients, this could help determine a reference range of \% inhibition of ETP or peak that could be used to titrate drug therapy. The results in this sub-study also demonstrate a ethnicity specific pattern of thrombin generation changes over the course of pregnancy; this area certainly warrants further investigation, along with investigating other ethnic group responses. The obese pregnant population continues to present an ever present challenge, as clinicians try to balance the risk of VTE and bleeding concurrently. Specific studies assessing thrombin generation in the obese (with and without LMWH) would additionally be of value to clinicians involved in the care of this group of patients. 


\section{Chapter 7}

\subsection{Women's views on and adherence to enoxaparin}

The focus of chapter 7 is to explore women's views onand adherence to enoxaparin during pregnancy and the puerperium. Following a discussion about medication adherence in general, adherence to medicines during pregnancy will be discussed, before describing how pregnant women's views were explored during the course of this study. The results of this work are then presented, followed by a discussion of the relevance of the results, in the context of previously published work.

\subsubsection{Medication adherence}

\subsubsection{Background}

Research suggests that up to $50 \%$ of patients with chronic conditions who are prescribed medications do not adhere to their medication as prescribed; this not only has a negative impact on the individual in question from a health point of view, but also on society, due to the waste of resources [Cutler and Everett, 2010].

Medication adherence can be defined as:

The extent to which the patient's behaviour matches agreed recommendations from the prescriber [Horne et al., 2005].

The term adherence emphasises the need for agreement and that the patient is free to decide whether or not to adhere to the prescriber's recommendation. Non-adherence can broadly be divided into two categories, intentional and unintentional non-adherence [Horne, 1993; Haynes et al., 2002a; Haynes et al., 2002b]. Unintentional non-adherence, is when the patient has every intention of adhering to their medication, but is unable to. Reasons for this include physical disability, inhibiting them from accessing the medicine, as well as forgetfulness, sensory disability (e.g. poor eyesight) or lack of understanding [Meichenbaum and Turk, 1987]. Intentional nonadherence is when a patient makes a conscious decision not to follow treatment advice. It is intentional non-adherence which is the focus of this chapter.

Medicine taking by patients is a complex behaviour. Research over the last twenty years has demonstrated that intentional non-adherence is influenced by the patient's perception of their illness and treatment, in particular, the patient's specific beliefs about the necessity of the 
medicines prescribed and their perceived concerns about the potential adverse effects, which all influence their medication taking behaviour [Horne, 1997; Horne and Weinman, 1999]. Specifically, patients evaluate whether or not to take a medicine, based on whether the proposed treatment makes sense to them, in the context of their illness beliefs. Patient's personal beliefs about, and experiences of, their illness will influence whether they follow treatment advice. Leventhal and colleagues [1992] conceptualised the common-sense model which suggests that patients form a map when challenged with ill-health before deciding on the most appropriate course of action, including whether to access healthcare and ultimately whether or not to take any prescribed treatment. This map is based on five key components of their condition [Leventhal et al., 1992]:

1. Identity (what is it?)

2. Timeline (how long will it last?)

3. Cause (what caused it?)

4. Consequences (how will it, has it affected me?)

5. Cure/control (can it be cured or controlled?)

Utilising this model, it is unsurprising that adherence rates are lower in patients who suffer with asymptomatic illness. In asymptomatic illness, e.g. hypertension, where no relief of symptoms is required, the intervention offered, e.g. beta-blocker therapy, may lead to negative symptoms and impact on the patient's adherence. Research has shown that adherence in patients with the same illness or condition varies, and this demonstrates patient's individual subjective response to illhealth [Horne et al., 2005]. Furthermore external factors, such as friends, relatives and the media can all impact on how much an individual adheres to their prescribed treatment [Nunes et al., 2009].

Horne and colleagues have further developed Leventhal's model, focusing particularly on medications, by proposing the perceptions and practicalities model of adherence [Horne et al., 2005] (figure 7.1). 


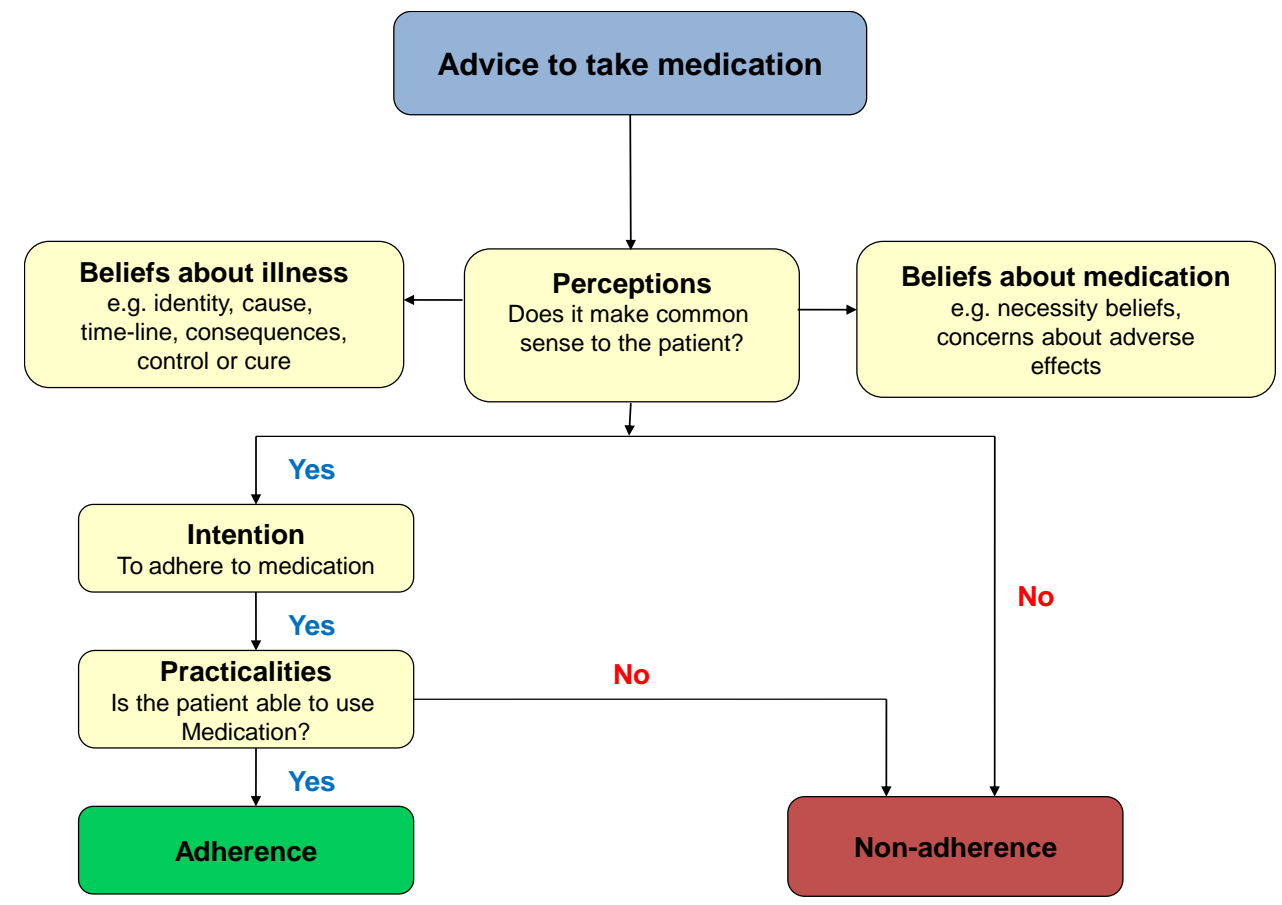

Figure 7.1: The practicalities and perceptions model of adherence [Horne et al., 2005]

The model incorporates both concepts of intentional and non-intentional adherence.

\subsubsection{Measures of adherence}

There are two methods in which adherence to medication can be assessed; directly and indirectly. Direct methods include collecting samples of blood, urine or other body fluids and testing them for the presence of the medicine. Indirect methods on the other hand, use measures such as self reports from patients, pill counts, prescription reordering, pharmacy refill records and a therapeutic effect to form the assessment of adherence [Nunes et al., 2009]. All measures of adherence have their own advantages and disadvantages. Direct measures provide objective evidence of drug in the system, but can be expensive to conduct and depending on the drug being tested, may not reliably be able to indicate continuous adherence. Pill counts and prescription reordering are crude measures of adherence, as the patient may not have actually taken the medicine, even though they have collected or ordered it from the pharmacy. Selfreporting is the most convenient method for reporting adherence in a clinical context. Selfreporting has the advantages of being simple, inexpensive, relatively quick and easily applied to a clinical setting. The advantage of self-reporting is that important information gathered about behavioural factors in medicines use and what is leading to non-adherence in an individual can be deciphered. The disadvantages are, that self-reporting is associated with over-estimation of 
adherence, can be inaccurate due to recall bias and errors in self-observation, and the manner in which information is obtained from patients, particularly around how questions are asked, can affect the answers received [Nunes et al., 2009].

\subsubsection{Medication usage during pregnancy}

Over the last thirty years, medication use amongst pregnant women has increased substantially. A retrospective American study of 152,531 pregnant women, spanning the period 1996-2000 [Andrade et al., 2004], found that $82 \%(124,695)$, had a drug or medical product (vitamin or mineral) dispensed in the 270 days leading up to delivery. In $82,974(54 \%)$ of these cases, it was a vitamin or mineral supplement that was dispensed, whilst in $98,182(64 \%)$ cases, a drug other than a vitamin or mineral supplement was dispensed. A further study from America, which assessed medication use during pregnancy with a particular focus on prescription drugs, spanning the period 1976-2008, reported that the use of prescribed medication amongst the pregnant population had increased by more than $60 \%$ over this period, with $50 \%$ of pregnant women admitting to the use of a prescription only medicines during their pregnancy [Mitchell et al., 2011]. Similar increases in prescription and over the counter medication use has been reported in Europe, Australia and South Africa [Aviv et al., 1993; Refuuerzo et al., 2005; Engeland et al., 2008; Gagne et al., 2008; Broussard et al. 2010; Crespin et al., 2011; Sawicki et al., 2011].

\subsubsection{Medication adherence during pregnancy}

Despite the widespread use of medication during pregnancy, only a small body of research has been published which has explored the issue of medication adherence during pregnancy. Teratogenic risk is a factor considered before women decide whether or not to take a medicine / vitamin / mineral. Research has shown that women often over-estimate this risk [Sanz et al., 2001; Nordeng et al., 2010]. In the study conducted by Sanz and colleagues, the teratogenic risk of fourteen specific medicines were perceived to be higher by pregnant women than the actual risk. This perception could lead to abrupt withdrawal of medication, as has been observed in the field of psychiatry [Einarson et al., 2001; Bonari et al., 2005]; conversely, the gravid state can also lead to improved medication adherence; for example in the field of HIV. Studies report adherence to highly active anti-retroviral therapy during pregnancy to increase, seen as an attempt by mothers to prevent vertical transmission of infection to the fetus [Ickovics et al., 2002; Vaz et al., 2007; Mellins et al., 2008]. Adherence to fertility drugs during in-vitro fertilisation treatment is also found to be complete, presumably because of a strong desire for a successful pregnancy 
outcome [Smith and Williams 2010]. The most comprehensive antenatal adherence study published to date is an Australian study by Sawicki and colleagues [2011]. In this study, all pregnant women attending their 36 week antenatal clinic visit were asked to complete a 61 item questionnaire, which included assessment of adherence using the Morisky scale [Morisky et al., 1986]. Eight hundred and nineteen patients, with a mean age of 30.8 years, mostly born in Australia, comprised the study population. Of these, $322(39.3 \%)$ reported a chronic health condition during pregnancy, most commonly asthma $(104 ; 12.7 \%)$, which required a medication. The commonly used medications by the study population are listed in table 7.1. Non-adherence was reported by 107 (59.1\%) of subjects.

Table 7.1: Medications prescribed in the cohort of women followed in Sawiciki's study

\begin{tabular}{lcl}
\hline Medicine & Number & $(\%)$ \\
\hline Iron therapy & 68 & $(8.3)$ \\
Medicines for chronic airway conditions & 64 & $(7.8)$ \\
Vitamins and minerals & 59 & $(7.2)$ \\
Anti-diabetics & 43 & $(5.2)$ \\
Anti-Depressants & 12 & $(1.5)$ \\
Cardiovascular drugs & 11 & $(1.3)$ \\
Antithrombotics & 10 & $(1.2)$ \\
Thyroid therapy & 10 & $(1.2)$ \\
Corticosteroids for systemic use & 6 & $(0.7)$ \\
Anti-inflammatories and anti-rheumatics & 6 & $(0.7)$ \\
Analgesics & 5 & $(0.6)$ \\
Dermatologicals & 3 & $(0.4)$ \\
Other (e.g. antibiotics, antifungals) & 21 & $(2.6)$ \\
\hline
\end{tabular}

Clearly, the gravid state introduces a new level of complexity to the perceptions and practicalities model that Horne and colleagues have developed, as pregnant mothers have additional considerations on what impact any medication prescribed might have on their developing fetus and depending on the known risk, whether the medicine warrants consumption (figure 7.2). 


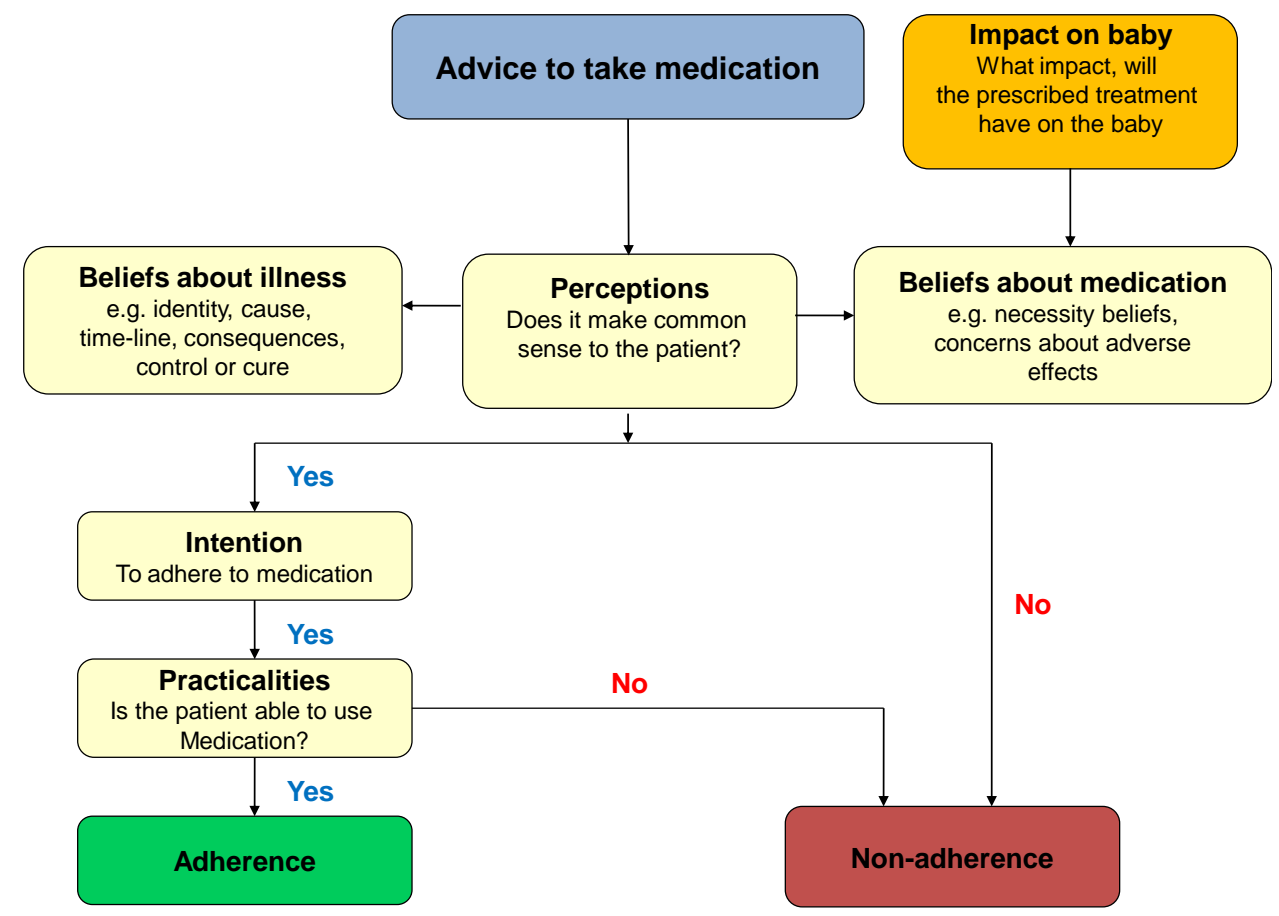

Figure 7.2: Adapted practicalities and perceptions model of adherence for pregnancy

\subsubsection{Medication adherence study objectives}

An assessment of adherence was important during the course of this study, not only because pharmacokinetic interpretation requires a knowledge of whether the medicine was actually taken, but also because prophylaxis of VTE during pregnancy can be viewed as an analogous situation to an asymptomatic health condition, whereby patients are told they are at risk, and then prescribed medication, in this case, a parenteral medication, which significantly impacts on the rest of their pregnancy and for up to six weeks postpartum. It is easy to comprehend why a patient might therefore omit or miss doses, particularly if they have never experienced symptomatic VTE. Furthermore, the dosing of LMWH during pregnancy, as already discussed in this thesis, is controversial. Therefore, gaining an insight into the adherence behaviour of pregnant women prescribed LMWH, would support any recommendations made on the optimal dosing strategy for this population.

Therefore, as part of this study, women's views on and adherence to enoxaparin during pregnancy and the puerperium were explored.

The specific objectives of this sub-study were:

- Monitor and calculate adherence rates to enoxaparin during both the antenatal and postnatal periods 
- Explore and understand women's views of having to inject the enoxaparin during their pregnancy and the postnatal period and what impact this has on their adherence to the enoxaparin prescribed.

\subsection{Method}

\subsubsection{Assessment of adherence}

Adherence in this study was assessed by means of self-report. As outlined in chapter 2 , at each clinic visit, recruited women were given a diary (How to inject Clexane $\AA$, Sanofi-Aventis Ltd) and asked to record the time they injected each day. At the time of consenting the women, the pharmacokinetic nature of the study was explained and recruited women were advised that if, for whatever reason, they missed or omitted a dose or doses, they should record that in their diary. This diary was collected and replaced at each clinic visit. Concurrent laboratory (anti-Xa, and thrombin generation) monitoring was used to provide further objective information about whether adherence might be a problem.

\subsubsection{Assessing beliefs about medications}

Women's beliefs about medication use in general and enoxaparin specifically, were explored using the beliefs about medication (BMQ) instrument [Horne et al., 1999]. This validated tool explores patient's perceived harm and overuse of medication in general and the perceived necessity and concerns associated with a specific medicine prescribed. The other most commonly used instrument in the adherence field is the 4-item Morisky Scale, which was originally developed to assess adherence in relation to anti-hypertension medication [Morisky et al., 1986]. The research team decided to use the BMQ for the purposes of this study, as the instrument allowed more of a detailed exploration of the patient's beliefs about medications relative to the Morisky scale.

The BMQ consists of 18 items, 8 designed to measure overuse and harm of medicines in general (see table 7.2) and 10 items capturing the need and concerns about a medication specifically (see table 7.3). 
Table 7.2: BMQ general (overuse and harm) questions

\begin{tabular}{lc}
\hline BMQ general questions & $\begin{array}{c}\text { Overuse or harm sub- } \\
\text { scale question }\end{array}$ \\
\hline Doctors use too many medicines & Overuse \\
People who take medicines should stop taking their treatment for a while every & Harm \\
now and again & \\
Most medicines are addictive & Harm \\
Natural remedies are safer than medicines & Overuse \\
Medicines do more harm than good & Harm \\
All medicines are poisons & Harm \\
Doctors place too much trust on medicines & Overuse \\
If doctors had more time with patients they would prescribe fewer medicines & Overuse \\
\hline
\end{tabular}

Table 7.3: BMQ specific (necessity and concerns) questions

\begin{tabular}{lc}
\hline BMQ specific questions & Necessity or \\
& concerns sub-scale \\
My health at present, depends on my medicines & question \\
Having to take medicines worries me & Necessity \\
My life would be impossible without my medicines & Concern \\
Without my medicines I would be very ill & Necessity \\
I sometimes worry about the long-term effects of my medicines & Necessity \\
My medicines are a mystery to me & Concern \\
My health in the future will depend on my medicines & Concern \\
My medicines disrupt my life & Necessity \\
I sometimes worry about becoming too dependent on my medicines & Concern \\
My medicines protect me from becoming worse & Concern \\
\hline
\end{tabular}

Responses to the $\mathrm{BMQ}$, are measured on a five point likert scale, ranging from strongly agree (5) to strongly disagree (1). Sub-scale scores for overuse and harm in general, and necessity and concerns specifically, are then computed to gauge subject's beliefs about taking a particular prescribed medicine, as well as the broader context of their general beliefs about medication taking.

When applied to a particular population or setting, the necessity and concerns questions are reworded to fit a particular medicine. For example, the question, Having to take medicines worries me, would be re-worded to, Having to take prednisolone worries me, in the case of an asthmatic patient prescribed prednisolone. 


\subsubsection{Adapting the BMQ for a pregnant population}

The BMQ has not previously been applied to a pregnant population, therefore, as part of this study; it was adapted to meet the study objectives. Specifically, the first part of the questionnaire, listing the general overuse and harm questions was modified to reflect the context of pregnancy (e.g. doctors use too many medicines was changed to doctors use too many medicines during pregnancy). Furthermore, a series of additional questions were added to provide further information about this specific context. Table 7.4 lists the additional questions used in section one of the adapted BMQ.

Table 7.4: Additional questions added to the general section of the adapted BMQ

Question
Over-the-counter medicines from a chemist are safer than prescription medicines during pregnancy
Before considering to take a medicine during pregnancy, I like to know about the safety of that medicine to
me and my unborn baby
Most medicines are safe when taken during pregnancy if prescribed by a doctor
When deciding on whether to take a medicine whilst I am pregnant, I place a higher priority on the impact
the medicine will have on the health of my unborn baby than to any effects the medicine may have on me

The necessity and concerns questions from the BMQ were adapted for the context of enoxaparin generally (for example 'my health at present depends on my medicines' was altered to 'my health at present depends on enoxaparin'), and then specifically in the context of pregnancy, to assess whether women's responses changed when they focussed on the impact of being pregnant (see table 7.5).

Table 7.5: Questions repeated within the specific section of the adapted BMQ in the context of pregnancy

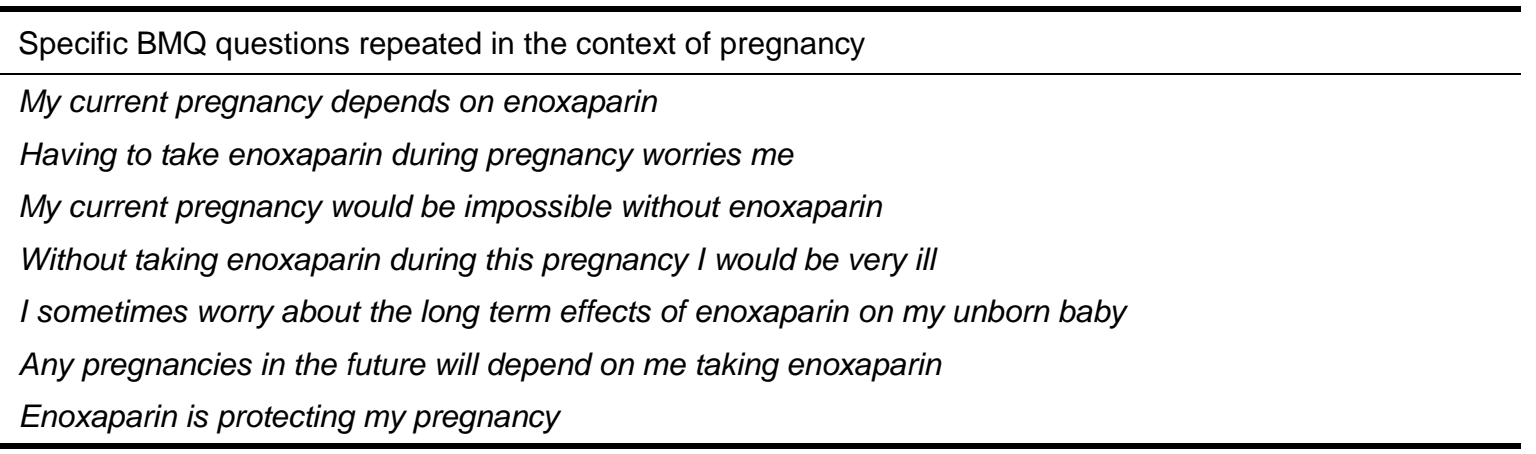

As the BMQ had not been used in a obstetric setting before, it did not capture information relating to women's views and concerns around child delivery, pain relief and breast-feeding issues and what role enoxaparin may have in protecting the unborn baby's health. Therefore, a series of 
additional questions were constructed, which explored these specific issues, which were identified by the research team that might be important for pregnant women, based on previous clinical experience. These are listed in table 7.6.

Table 7.6: Additional exploratory questions added to the adapted BMQ

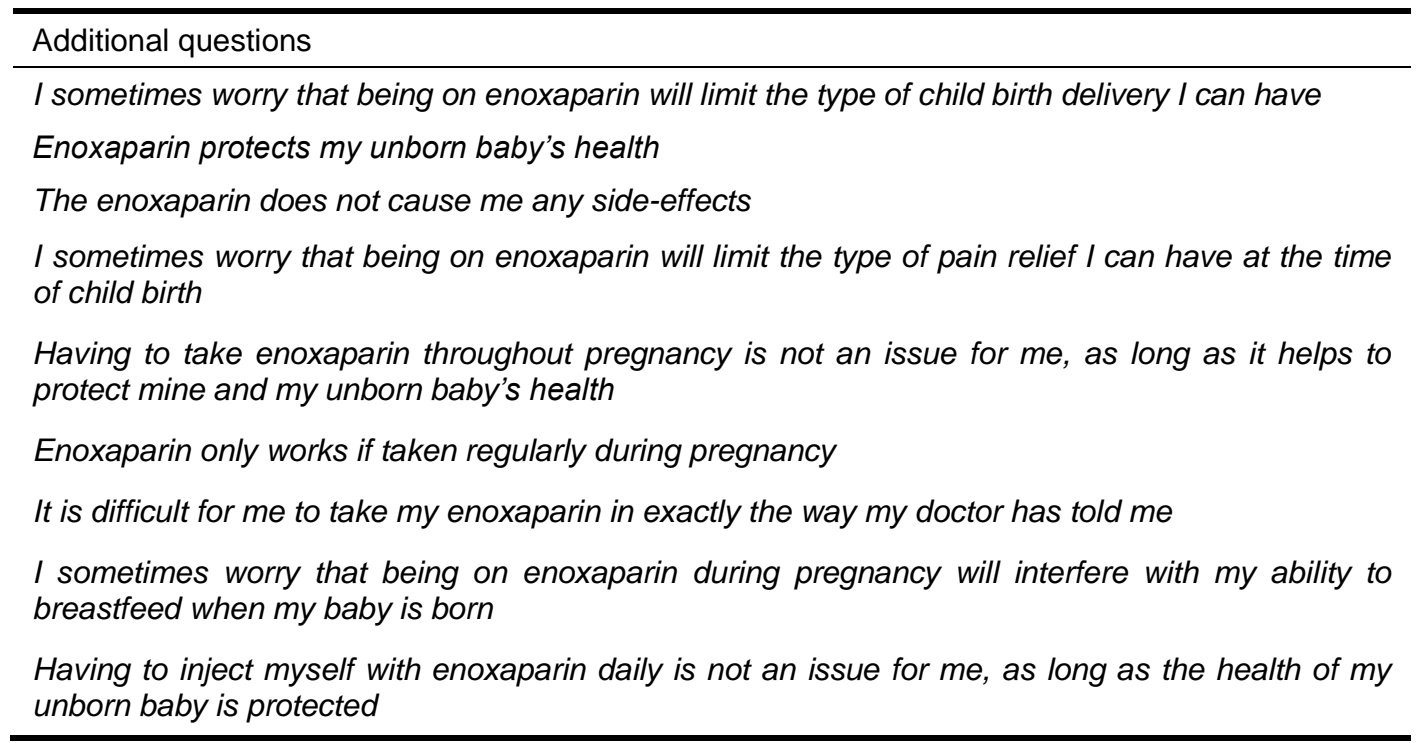

Finally, there was a space at the end of the questionnaire for the women to make any comments, should they wish. See appendix XI for final questionnaire used.

\subsubsection{Administration and completion of the questionnaire}

The questionnaire was given to the pregnant women at the time they consented into the study (during the antenatal period) and they were asked to return the completed questionnaire at one of their subsequent haematology clinic appointments. The timing of administering the questionnaire was very much driven by when women first attended the haematology clinic following referral. For the majority of women, this was following booking with the obstetric service and usually during the second trimester (typically gestational weeks 14-22). Women were advised that their responses would be kept confidential and would not be shared with the clinical team.

\subsubsection{Data Analysis}

Data from the questionnaire was coded, entered and analysed using IBM SPSS (version 18), Chicago, Illinois. Descriptive statistics were used to summarise the demographic (e.g. ethnicity) and clinical characteristics (e.g. indication for enoxaparin) of the cohort of women. 


\subsubsection{Calculation and classification of patients according to adherence}

Percentage adherence rates were calculated separately for both the antenatal and postnatal periods, following recruited women's last clinic visit (eight weeks post-partum). Women were classed as adherent to enoxaparin for the antenatal and/or post-natal period, if they achieved an adherence rate $\geq 80 \%$ [DiMatteo et al., 2004]. This was calculated for each individual woman, by dividing the total number of doses injected over the antenatal or postnatal period by the total number of doses prescribed over that respective time period. The number obtained was then multiplied by 100 to obtain a \% adherence rate. The paired t-test was used to compare antenatal adherence rate with the postnatal adherence rate.

In order to assess women's beliefs about the enoxaparin prescribed, subscale scores for overuse, harm, necessity and concerns were calculated for the cohort of women as a whole. This involved, for example calculating a mean response for all the overuse questions from the tool. In this case, the subscale response from a particular woman could range from a minimum of 4 to a maximum of 20 (as there were four overuse questions). Therefore a score in the region of 4 indicates women's lack of agreement with those questions and that she did not think doctors overuse medicines and a score in the region of 20 indicating that a woman did feel doctor's overused medications. This was repeated for the other three subscales.

Women's adherence to enoxaparin in this study could be classified into two broad groups; women who were equally adherent to enoxaparin through both the antenatal and postnatal periods (optimal adherence group) and women who were adherent to enoxaparin through the antenatal period, but with a $5 \%$ or more decrease in adherence through the postnatal period (suboptimal adherence group). The BMQ subscales scores were additionally computed for these two groups separately and compared using the independent t-test.

\subsubsection{Necessity and concerns differential}

As first suggested by Clifford and colleagues [Clifford et al., 2006], the necessity-concerns differential was also computed for the cohort of women, and then separately calculated for the two groups (optimal and suboptimal groups) and compared. This necessity-concerns differential calculates the difference between the patient's perceived necessity for treatment from their apparent concerns, and is strongly associated with a patient's intention to adhere to treatment. Specifically, this was calculated by subtracting the average necessity subscale score from the average concern subscale score for each woman. A positive number indicates that the necessity 
for treatment outweighs any concerns the patient may have, and vice versa, with a positive differential indicating a patient's or group of patients' intention to adhere to treatment.

\subsubsection{Relationships}

Section 2 of the questionnaire explored the relationship between the general necessity and concern questions of the BMQ and the respective pregnancy specific repeat question. Pearson's correlation was utilised to assess whether each pair of questions was correlated and whether women agreed more with the pregnancy specific questions.

\subsubsection{Additional questions}

The additional questions added to the questionnaire were reported back as $\%$ agreement, with each statement. Voluntary comments made by women in the questionnaire were themed. These themed comments are listed in the results section.

Statistical significance was considered at $\mathrm{p}<0.05$.

\subsection{Results}

\subsubsection{Demographic information and response rate}

In the wider population pharmacokinetic study, 123 patients contributed anti-Xa activities for pharmacokinetic modelling purposes. Of these subjects, four patients had two pregnancies during the study period and were therefore not re-administered the questionnaire during their second pregnancy. A further three patients who consented into the study and had anti-Xa activities measured, miscarried before the questionnaire was administered to them. Two patients did not speak English fluently and so were not asked to complete the questionnaire. Of the remaining 114 patients administered the questionnaire, 8 did not return the questionnaire, response rate $=$ $92.98 \%$. The mean age of the women who returned the questionnaire was 33.14 years (range 1846); Fifty-seven (53.77\%) of the women were actively being treated for VTE or had suffered from thrombosis in the past. Thirty (28.3\%) women had a history of recurrent miscarriage. Table 7.7 summarises key information on the 106 women from the study who returned the questionnaire. 
Table 7.7: Demographic details of the 106 women who returned the questionnaire

\begin{tabular}{|c|c|}
\hline Demographic & $\begin{array}{l}\text { Number }(\%) \\
n=106\end{array}$ \\
\hline \multicolumn{2}{|l|}{ Ethnicity } \\
\hline Caucasian & $58(54.71)$ \\
\hline Black & $32(30.18)$ \\
\hline Asian & $7(6.60)$ \\
\hline Other & $9(8.49)$ \\
\hline \multicolumn{2}{|l|}{ Indication } \\
\hline VTE prophylaxis & $73(68.9)$ \\
\hline VTE treatment & $9(8.5)$ \\
\hline Switched from long term warfarin* & $8(7.5)$ \\
\hline Antiphospholipid syndrome & $7(6.6)$ \\
\hline Other ${ }^{* *}$ & $9(8.5)$ \\
\hline \multicolumn{2}{|l|}{ Frequency of enoxaparin prescribed } \\
\hline Once a day & $88(83.01)$ \\
\hline Twice a day & $16(15.09)$ \\
\hline Once, followed by twice a day & $2(1.88)$ \\
\hline \multicolumn{2}{|c|}{$\begin{array}{l}\text { Prior experience of injecting LMWH during } \\
\text { pregnancy }\end{array}$} \\
\hline No & $72(67.92)$ \\
\hline Yes & $34(32.07)$ \\
\hline \multicolumn{2}{|l|}{ Obstetric history (as a cohort) } \\
\hline Gravida & 369 \\
\hline Parity & 103 \\
\hline Miscarriages & 142 \\
\hline Terminations & 19 \\
\hline \multicolumn{2}{|c|}{$\begin{array}{l}\text { * The specific indications for the women switched from long term warfarin to enoxaparin during the index pregnancy were } \\
\text { recurrent VTE (x2), symptomatic homozygous FVL }(x 1) \text {, Budd-Chiari }(x 3) \text {, paroxysmal nocturnal hamoglobinuria }(x 1) \text {, } \\
\text { valvular atrial fibrillation (x1) }\end{array}$} \\
\hline \multicolumn{2}{|c|}{$\begin{array}{l}\text { ** The specific indications for the women in this category include recurrent miscarriages in the absence of anti- } \\
\text { phospholipid syndrome }(x 5) \text {, stillbirth in previous pregnancy where post-mortem results indicated perivillous fibrin } \\
\text { deposition, placental ageing and dysmaturity }(x 1) \text {, patent foramen ovale }(x 1) \text {, history of IUGR and hypertension in } \\
\text { previous pregnancies ( } x 2)\end{array}$} \\
\hline
\end{tabular}

\section{Adherence to enoxaparin}

A total of 21,914 antenatal doses of enoxaparin were prescribed for the 106 women (mean 211 (range 18-455)), and 4,597 postnatal doses (mean 44 (range 0-114)). The cohort of women were found to be highly adherent $(>80 \%)$ to the enoxaparin therapy; antenatally, mean percentage adherence $97.92 \%$, postnatally mean percentage adherence $92.75 \%$. Adherence to enoxaparin decreased in some women during the postnatal period (paired $t$-test, $\mathrm{t}=6.099, \mathrm{df}=100, \mathrm{p}=0.000$ ). Women could be allocated into two broad categories with respect to their adherence to enoxparin, (i) women who were equally adherent to enoxaparin antenatally and postnatally with no significant change during the postpartum period $(n=66,64.7 \%)$ - optimal adherence group, (ii) women who were adherent to enoxaparin antenatally and postnatally, however, with at least a $5 \%$ drop in adherence during the postnatal phase $(n=36,35.3 \%)$ - suboptimal adherence group. These sub- 
groups will be referred to later on in the analysis. Four women had no further indication for enoxaparin postnatally and were not included in this component of the analysis.

\subsubsection{Necessity-concerns differential}

Cronbach's alpha was calculated for the four established BMQ sub-scales, which revealed good internal consistency (Overuse $=0.792$, Harm $=0.775$, Necessity $=0.800$, Concerns $=0.739$ ). The mean scores from the four established $B M Q$ subscales for the women questioned are shown in table 7.8. There was more agreement from the women regarding the specific necessity and concerns questions, compared to the general overuse or harm questions in the instrument, with an overall necessity-concerns differential of 2.13 , indicating a strong intention to adhere to treatment in the cohort of women followed.

Table 7.8: Mean BMQ sub-scales

\begin{tabular}{lllc}
\hline BMQ sub-scale & Mean & SD & Min-Max \\
\hline Overuse & 9.88 & 2.92 & $4-17$ \\
Harm & 8.06 & 2.62 & $4-16$ \\
Necessity & 14.07 & 3.82 & $5-25$ \\
Concerns & 11.94 & 3.69 & $5-20$ \\
\hline
\end{tabular}

The mean BMQ subscale scores, when the women's responses were subdivided by adherence rates as two groups are described in table 7.9:

(i) Those who were equally adherent ante- and postnatal period (optimal adherence group)

(ii) Those who were adherent antenatally, but had at least a $>5 \%$ drop in adherence during the puerperium (suboptimal adherence group) 
Table 7.9: Differences in BMQ sub-scale scores between the optimal adherence and sub-optimal adherence groups

\begin{tabular}{cccccc}
\hline BMQ sub-scale & Group $^{* *}$ & $n$ & Mean & SD & Significant \\
\hline Overuse & 1 & 66 & 9.58 & 3.17 & NS \\
& 2 & 36 & 10.50 & 2.48 & \\
Harm & 1 & 66 & 7.91 & 2.72 & NS \\
& 2 & 36 & 8.31 & 2.55 & \\
Necessity & 1 & 66 & 15.00 & 3.74 & $<0.05^{*}$ \\
& 2 & 36 & 12.87 & 3.37 & \\
Concerns & 1 & 66 & 11.75 & 3.75 & NS \\
& 2 & 36 & 12.27 & 3.76 & \\
\hline *independent t-test **Group 1- optimal adherence, Group 2- sub-optimal adherence
\end{tabular}

A significant difference was found between the necessity sub-scale mean scores, from those women in the optimal adherence group, compared to those women in the suboptimal adherence group (independent $t$-test, $\mathrm{t}=2.851, \mathrm{df}=100, \mathrm{p}=0.005$ ).

The mean necessity-concerns differential for the optimal and suboptimal adherence groups were 3.26 and 0.60 respectively, (independent $t$-test, $t=2.543, d f=100, p=0.013$ ), suggesting that the perceived need for enoxaparin was less in the latter group.

\section{Differences between different sub-groups?}

Differences in adherence rates were explored between Caucasian and the African-Caribbean population, between those women injecting enoxaparin once a day versus those women injecting twice a day and between those women with a history of recurrent miscarriage versus those who did not. This analysis (independent t-test), revealed no significant different differences in the adherence rates between these sub-groups.

Sub-scale scores (harm, overuse, necessity and concerns) were also compared between these sub-groups, the results of which are presented in tables 7.10, 7.11 and 7.12. 
Table 7.10: Differences in subscale scores between the Caucasian and African-Caribbean population

\begin{tabular}{llccc}
\hline \multicolumn{2}{l}{ Subscale and ethnicity } & Mean & $S D$ & Significant $^{*}$ \\
\hline \multirow{2}{*}{ Overuse } & Caucasian & 8.8305 & 2.47 & 0.000 \\
& African-Caribbean & 11.5625 & 2.61 & 0.000 \\
\multirow{2}{*}{ Necessity } & Caucasian & 6.9096 & 1.89 & \\
& African-Caribbean & 9.6563 & 2.56 & 0.514 \\
& Caucasian & 13.8460 & 3.83 & \\
& African-Caribbean & 14.3984 & 3.87 & 0.000 \\
\hline
\end{tabular}

${ }^{\star}$ Independent $t$-test

Even though significant differences exist between the Caucasian and African-Caribbean women's beliefs about medicines in general (overuse and harm) and specifically about the enoxaparin (concerns), this did not translate into a difference in their adherence.

Table 7:11: Differences in subscale scores between those women injecting once versus those women injecting enoxaparin twice a day

\begin{tabular}{lcccc}
\hline \multicolumn{2}{l}{ Subscale and frequency } & Mean & $S D$ & Significant $^{*}$ \\
\hline Overuse & Once a day & 9.7656 & 2.86 & 0.087 \\
& Twice a day & 11.2308 & 2.80 & \\
Harm & Once a day & 8.0513 & 2.59 & 0.468 \\
& Twice a day & 8.6154 & 2.78 & \\
\multirow{4}{*}{ Cocessity } & Once a day & 13.8645 & 3.86 & 0.109 \\
& Twice a day & 15.6923 & 3.44 & \\
& Once a day & 11.8049 & 3.70 & 0.130 \\
& Twice a day & 13.4615 & 3.35 & \\
\hline
\end{tabular}

${ }^{*}$ Independent $t$-test

No differences in sub-scale scores were found between those women injecting once versus those women injecting twice a day.

Table 7.12: Differences in subscale scores between those women with a history of recurrent miscarriage compared to those women who had no history of recurrent miscarriage

\begin{tabular}{llccc}
\hline \multicolumn{2}{l}{ Subscale and miscarriages } & Mean & $S D$ & Significant $^{*}$ \\
\hline Overuse & History & 8.4881 & 2.52 & 0.003 \\
& No history & 10.3846 & 2.90 & \\
\multirow{4}{*}{ Harm } & History & 7.1786 & 2.56 & 0.037 \\
& No history & 8.3803 & 2.58 & \\
\multirow{4}{*}{ Concersity } & History & 12.7946 & 3.70 & 0.038 \\
& No history & 14.5310 & 3.77 & \\
& History & 11.2321 & 3.24 & 0.241 \\
& No history & 12.1891 & 3.83 & \\
\hline
\end{tabular}


Significant differences in the overuse, harm, and necessity subscale scores could be found between those women with no history, compared to those women with a history of recurrent miscarriage. Interestingly, those women with a history of recurrent miscarriage, one might expect to have a higher necessity subscale scores compared to those that did not, but the results did not reflect this.

\subsubsection{Necessity and concerns about enoxaparin}

In the adapted questionnaire, we asked the original $\mathrm{BMQ}$ questions from the necessity and concerns section of the questionnaire, and repeated seven of these questions in the context of pregnancy specifically to assess if the gravid state altered the women's responses to the question. Table 7.13 lists the mean responses to these questions, along with the correlation relationship between each pair of questions.

The majority of pairs of questions were well correlated. Two pairs, however, were not well correlated; the question exploring future health and future pregnancies and the question exploring the effect of enoxaparin on current health and protecting the current pregnancy. 
Table 7.13: Correlations between the necessity and concerns question pairs

\begin{tabular}{|c|c|c|c|}
\hline Necessity question pairs & Mean & SD & $\mathrm{r}^{*}$ \\
\hline My health at present depends on enoxaparin & 3.22 & 1.11 & $0.487^{\star *}$ \\
\hline My current pregnancy depends on enoxaparin & 3.54 & 1.00 & \\
\hline My life would be impossible without enoxaparin & 2.23 & 0.86 & $0.458^{* *}$ \\
\hline My current pregnancy would be impossible without enoxaparin & 2.81 & 0.97 & \\
\hline Without enoxaparin I would be very ill & 2.59 & 1.05 & $0.754^{* *}$ \\
\hline Without taking enoxaparin during this pregnancy I would be very ill & 2.95 & 0.95 & \\
\hline My health in the future depends on enoxaparin & 2.55 & 0.99 & 0.154 \\
\hline Any pregnancies in the future will depend on me taking enoxaparin & 3.45 & 1.00 & \\
\hline Enoxaparin protects me from becoming worse & 3.41 & 1.07 & 0.114 \\
\hline Enoxaparin is protecting my pregnancy & 3.65 & 0.90 & \\
\hline Concerns question pairs & Mean & SD & $\mathrm{r}^{*}$ \\
\hline Having to take enoxaparin worries me & 2.64 & 1.14 & $0.743^{* *}$ \\
\hline Having to take enoxaparin during pregnancy worries me & 2.71 & 1.07 & \\
\hline I sometimes worry about the long term effects of enoxaparin & 2.82 & 1.17 & $0.745^{\star *}$ \\
\hline I sometimes worry about the long term effects of enoxaparin on my unborn baby & 3.05 & 1.17 & \\
\hline
\end{tabular}

It was interesting to observe that when the questions are asked in the context of pregnancy, the women's responses do change. The women agreed more with all the necessity and concerns questions, when asked specifically in the context of the gravid state. This suggests that the women have stronger necessity and concerns during pregnancy than outside of pregnancy.

\subsubsection{Additional questions}

In addition to the adapted BMQ questions, additional questions were asked in the questionnaire which were felt might be important factors for women prescribed antenatal enoxaparin. Table 7.14 lists the \% agreement of the women to these questions. 
Table 7.14: \% agreement with the additional questions added to the adapted BMQ

\begin{tabular}{|c|c|}
\hline Question & $\begin{array}{l}\text { \% agreement with } \\
\text { statement }\end{array}$ \\
\hline \multicolumn{2}{|l|}{ General questions about medication use during pregnancy } \\
\hline $\begin{array}{l}\text { Before considering to take a medicine during pregnancy, I like to know about the } \\
\text { safety of that medicine to me and my unborn baby }\end{array}$ & 97.2 \\
\hline $\begin{array}{l}\text { When deciding on whether to take a medicine whilst I am pregnant, I place a higher } \\
\text { priority on the impact the medicine will have on the health of my unborn baby than to } \\
\text { any effects the medicine may have on me }\end{array}$ & 84.8 \\
\hline Most medicines are safe when taken during pregnancy if prescribed by a doctor & 66.0 \\
\hline $\begin{array}{l}\text { OTC medicines from a chemist are safer than prescription only medicines during } \\
\text { pregnancy }\end{array}$ & 2.9 \\
\hline \multicolumn{2}{|l|}{ Specific questions about enoxaparin use during pregnancy } \\
\hline $\begin{array}{l}\text { Having to take enoxaparin throughout pregnancy is not an issue for me, as long as it } \\
\text { helps to protect mine and my unborn baby's health }\end{array}$ & 90.4 \\
\hline $\begin{array}{l}\text { Having to inject myself with enoxaparin daily is not an issue for me, as long as the } \\
\text { health of my unborn baby is protected }\end{array}$ & 87.8 \\
\hline Enoxaparin only works if taken regularly & 66.1 \\
\hline The enoxaparin does not cause me any side-effects & 65.7 \\
\hline Enoxaparin protects my unborn baby's health & 38.1 \\
\hline $\begin{array}{l}\text { I sometimes worry that being on enoxaparin will limit the type of child birth delivery I } \\
\text { can have }\end{array}$ & 34.6 \\
\hline $\begin{array}{l}\text { I sometimes worry that being on enoxaparin will limit the type of pain relief I can have } \\
\text { at the time of child birth }\end{array}$ & 31.5 \\
\hline It is difficult for me to take my enoxaparin in exactly the way my doctor has told me & 20.7 \\
\hline $\begin{array}{l}\text { I sometimes worry that being on enoxaparin during pregnancy will interfere with my } \\
\text { ability to breastfeed when my baby is born }\end{array}$ & 14.3 \\
\hline
\end{tabular}

The results from the additional questions demonstrate how protective mothers are of their unborn child. The results also suggest that up to one third of women may have anxiety about what impact the enoxaparin may have on the type of pain relief and method of delivery they could have. Despite the fact that one-fifth of women agreed with the statement that it was difficult for them to take their enoxaparin in exactly the way the doctor had told them, the fact that they persisted, demonstrates the strong necessity beliefs the women had during pregnancy.

\subsubsection{Voluntary comments made by women}

Of the 106 women who completed the questionnaire, 33 women (31.13\%) made a comment at the end of the questionnaire. Some of the comments were unrelated to the context of this study (8 women). The remaining relevant comments were themed into three broad categories: 
(i) Necessity for injecting enoxaparin during pregnancy in order to protect them and/or their unborn baby's health (14 women)

(ii) Concerns or perceived harm associated with injecting enoxaparin (7 women)

(iii) Suggestions for the future (4 women)

Example comments from each theme are listed under each theme below.

(i) Necessity for enoxaparin

The following comments illustrate that although injecting enoxaparin is not a pleasant experience for women, their perceived necessity for it, particularly during pregnancy, overrides any concerns or pain associated with injecting enoxaparin.

"I'm happy to take enoxaparin during pregnancy as a preventative measure. I don't like doing the injections but it's manageable, and with my history it's something I'm prepared to do to ensure a safe arrival of my baby." (- Patient 3 )

"I am very thankful for enoxaparin and grateful it is available. It is not problem for me to take if it means I get my babies with me after so many lost babies." (- Patient 15)

"I'm happy the blood clots were detected in both my lungs. If I never went for that scan during my stay in hospital, i'd be very sick, possibly close to death, because these blood clots can kill if left untreated, they keep growing bigger and bigger until that's it. Mine was found in both my lungs, one of the nurses said I was lucky to be alive. So taking my Clexane don't really worry me as long as it helps to keep the blood clot under control." (- Patient 44)

"As far as I am aware, enoxaparin is to ensure I remain well during pregnancy and do not have a DVT. Therefore it is paramount that my health is taken care of and reviewed. I feel more secure and less worried about my health now I am on this medication. I find the injections uncomfortable but a small price to ensure I have a healthy pregnancy." (- Patient 56)

"I don't have an issue with taking medication during pregnancy, if the unborn baby's health is not compromised. I believe I am on enoxaparin to prevent blood clots from forming (not that they definitely would appear, but to reduce the risk of them appearing)." (- Patient 73)

"Definitely gives me piece of mind during pregnancy; without it I would feel very nervous about developing another DVT." (- Patient 76)

"I took enoxaparin throughout my first baby's pregnancy. My son is now 2.5 years and he does appear to be normal." (- Patient 90)

"Without enoxaparin I could be very ill (as never suffered a clot)." (- Patient 92)

"I really do not like taking enoxaparin every night both because of physical discomfort, bruising and inconvenience. However, if it really is protecting mine and the unborn baby's health then I am willing to keep doing it." (- Patient 93)

"I do not mind taking enoxaparin because I know it will reduce my risk of developing a dvt. The injections sometimes sting and leave a bruise. I use the top of my leg as injection site because I do not like the idea of injecting into my stomach whilst pregnant (this is beacuse I only started injecting at 34 weeks when my stomach is very tight). I am glad that my risk of DVT was identified and treatment started." (- Patient 120) 
"My views may change as my pregnancy continues and I am still injecting everyday. Currently, I am delighted to be taking heparin, since this may protect me from miscarriage." (- Patient 123)

(ii) Concern or perceived harm

The following comments were made by women who had specific concerns about the enoxaparin.

The common theme which runs through these comments, is the unpleasant / painful nature of the enoxaparin.

"It isn't pleasant to inject. During my first pregnancy it was a bit traumatic the first time I injected, especially as it is quiet a painful injection. However, with practice and confidence it was no problem. I am happy to inject during pregnancy, but would not like to do this life-long." (- Patient 16)

"Be glad when its over." (- Patient 30)

"Since the month after taking enoxaparin I'm feeling like I'm having difficulties to breath." (- Patient 38)

"I have no issues injecting if it safeguarding mine and the babies health but I lack some faith in the safety/side-effects/general effects of the medicine. Published information on Clexane seems to be contradictory." (- Patient 42)

"I sometimes worry that in the future they find that enoxaparin has a big side-effect for my baby, for example harmful for the heart or skin." (- Patient 50)

“Taking enoxaparin worries me ...because it's painful." (- Patient 89)

"The problem I have is that I get severe pain for 30 minutes after the jab, and need to lie down. Early on, I decided I would cope better at night. My husband has to administer the injection as I am terrified of needles. I wish there was another way, other than injection of taking this drug! As I am having an elective c-section, I worry that my blood may cause problems during the operation and that if I stop the Clexane before the C-section, this will cause a problem for our baby. I have suffered with severe bruising around every needle mark, this also causes me concern." (- Patient 113)

(iii) Suggestions

Four women made specific suggestions about the enoxaparin. The common theme which runs through these suggestion comments is to make it easier or more palatable to take the enoxaparin, with two women specifically asking for a tablet form of enoxaparin.

"I do not have a problem doing injections and was aware of the possibility of the injections before becoming pregnant. However, I think other women might benefit from more time and support around the use of Clexane in their pregnancy." (- Patient 28)

"If there was a way to get injections for enoxaparin via an epi-pen type device it would be much more tolerable!" (- Patient 64)

"If and when available in tablet/capsule form - it will be much easier to take." (- Patient 108)

"Since the introduction of enoxaparin many women in the UK have been able to have normal, healthy pregnancies which is a huge reassurance for me. The only down fall is that it only comes in injection form - it would be better if they had tablet form as well, as some women although being trained to do injections may not still feel comfortable doing so." (- Patient 111) 


\subsection{Discussion}

This sub-study explored pregnant women's views and adherence to LMWH therapy during pregnancy and the puerperium. We found women to be highly adherent to the enoxaparin therapy during both the antenatal (97.92\%) and postnatal (92.75\%) periods, although in a third of women, adherence decreased significantly postnatally.

The high adherence rate observed in this study is perhaps not surprising. Colwell and colleagues [2005], in the context of extended thromboprophylaxis with LMWH in orthopaedic surgery found that when properly instructed, patients can safely and efficiently self-administer the LMWH at home. This finding was also replicated by Watts and colleagues, who followed a small number of patients who had recently undergone lower limb arthroplasty and were prescribed LMWH for six weeks post surgery. They found that of the patients who could potentially self-inject at home, $85 \%$ were compliant to their prophylactic therapy [2006], and this has been further endorsed by the results of the recently published ETHOS registry [Bergqvist et al., 2012]. The aforementioned studies also primarily relied on self-reported adherence, by means of a patient diary. McLintock and colleagues are one of the few authors to explore the relationship between adherence to prescribed LMWH treatment and outcomes [2009]. In their study of high risk pregnant women with mechanical prosthetic heart valves in-situ, of the women found to suffer from thrombotic complications whilst prescribed enoxaparin treatment, non-compliance and sub-therapeutic antiXa levels were implicated in each case, with the authors concluding that good compliance with treatment was associated with a low risk of valve thrombosis and good fetal outcomes.

Others however have reported problems with self-administration of LMWH. Mengiardi and colleagues [2011] followed 213 non-pregnant patients injecting LMWH in the community and found a self-reported non-compliance rate of $17.1 \%$, with $38.9 \%$ of patients stating that selfadministration of the injections required some effort. A similar finding was also reported by Spahn [2002], who followed 207 patients following knee arthroscopy. Spahn reported problems with selfinjection in $34.8 \%$ initially and $6.3 \%$ the whole time.

In this study, although some women reported some negative experiences about having to inject the enoxaparin, the majority appear to cope with self-administration.

This study had higher adherence rates compared to the aforementioned studies, with apparently fewer problems. This may be in part due to the stronger beliefs about necessity over concerns 
that the women had regarding the enoxaparin, as demonstrated by the necessity-concerns differential of the group of women overall. Another key reason for the high adherence rate may be in part due to the regular monthly follow-up these women had in the haematology clinic. This follow-up provided an opportunity to discuss and check for any problems, and when necessary to reinforce the importance of the LMWH therapy during the antenatal period; an opportunity which was lost to a certain degree during the postnatal period.

It was interesting to observe that although adherence was relatively high during the antenatal period and for two-thirds of women maintained during the postnatal period, there was a group of women, where postnatal adherence dropped by $>5 \%$. On closer examination of the necessityconcerns differential between these two groups, there was a clear difference between the groups, with the antenatal differential appearing to be able to predict postnatal adherence. The theme which emerges from this result is the protective behaviour of women toward their unborn baby. Once women are satisfied that a potential medicine is not doing any harm to their baby, they will inject it, if perceived by them to be necessary. In this study, $96.8 \%$ of women wanted to know to what extent any medicine they took could affect their unborn baby as well as them, with many (78.5\%) placing a higher priority on the impact the medicine has on the unborn baby, compared to any impact the medicine may have on them; $91.4 \%$ of women in the study stated that having to inject enoxaparin was not an issue for them, as long as their own and their unborn baby's health was protected. When this question was repeated, just enquiring about their unborn baby's health, $88.4 \%$ of women stated that having to inject enoxaparin was not an issue for them, as long as their unborn baby's health was protected. This suggests a particular motivation for injecting the enoxaparin during the antenatal period was to protect the health of the unborn baby. It should therefore come as no surprise that for some women, once their baby is born, they were more likely to miss doses of enoxaparin.

In many ways, the women's belief is not completely wrong, because of the close physical relationship between a mother and child during the gravid period, however if this belief continues during the postnatal period, then the women's perceived necessity for enoxaparin may decline and explains in part the reason why postnatally, there was a group of women (suboptimal adherence group), in whom adherence to enoxaparin dropped by $>5 \%$ from their respective antenatal adherence rate. The necessity-concerns differential was significantly different between these two groups. This is further illustrated by the following comment: 
"After delivery it was a lot more difficult to inject myself than during pregnancy, therefore I was more inclined to miss a dose." (- Patient 10)

The drop in adherence in some women during the puerperium have been observed in other clinical specialities, e.g. in the field of HIV [Ickovics et al., 2002; Vaz et al., 2007; Mellins et al., 2008].

When asked informally in clinic by the principal researcher on why they might have missed doses postnatally, two reasons were most commonly cited; firstly, the challenges of motherhood following birth meant that often they simply forgot because their regular routine had been disrupted (unintentional non-adherence). Others reported that following birth, their perceived necessity for the enoxaparin was less (intentional non-adherence); as their baby had been born successfully, they were less worried if they missed a dose. In their study of medication use in general amongst pregnant women, Sawicki and colleagues [2011] reported the two most commonly cited reasons for women non-adhering during pregnancy was forgetting to take and stopping medicine when they were feeling better. Though in Sawicki's study, the reasons cited are by pregnant patients, clearly they resonate for our study population, as both unintentional and intentional non-adherence behaviours are exhibited by the women.

The implication of incomplete adherence is not known. Small deviations in adherence may have little impact on the overall outcome, as has been observed during this study, where none of the women went on to suffer a thrombotic event. No reports in the literature exist which comprehensively define the point below which the desired preventative or desired therapeutic result is unlikely to be achieved, and this makes it difficult to know when non-adherence becomes clinically significant, and therefore which patients might benefit from more intensive adherence support. In the context of this study and the cohort of women followed, one has to aim for high adherence, as although resistance to therapy will not develop, as observed with antibiotic therapy in an infectious diseases setting, in this clinical context, one has to assume that the risk of developing a pathological thrombosis or miscarrying increases in the absence of treatment. Of the 21,914 antenatal and 4,597 postnatal doses prescribed for the 106 women during the sub-study, $456(2.08 \%)$ were missed during the antenatal period and $333(7.24 \%)$ were missed during the postnatal period. As the postnatal period was significantly shorter in terms of time, the number of missed doses per time was significantly more during the postnatal period, and whilst part of this non-adherence is linked to practical reasons of motherhood, it appears a significant reason for this in women whose adherence does fall during the postanatal period, is due to the women's 
perceived necessity for enoxaparin being less at this time point, due to baby being born successfully.

These findings do have important practice implications. In the current Royal College of Obstetricians and Gynaecology guidelines for VTE prevention during pregnancy and the puerperium [Royal College of Obstetricians and Gynaecologists, 2009], the threshold for initiating prophylaxis is lower during the puerperium than antenatally. This means that women not eligible for enoxaparin therapy antenatally become eligible postnatally, e.g. women having had a caesarean section (one week prophylaxis), or women who are asymptomatic heterozygous carriers of Factor V Leiden, will be prescribed LMWH (6 weeks prophylaxis). The observations from this study suggest that a key reason women adhere to enoxaparin is that they feel it protects their unborn baby's health, it might not be too inconceivable that women prescribed prophylaxis cold, i.e. just during the post-natal period might not adhere to their treatment fully. Healthcare professionals, particularly those in contact with women during the early days following birth, requiring $\mathrm{LMWH}$, should ensure patients have an opportunity to discuss prophylactic LMWH fully and are instructed on how to self-inject competently for those newly starting LMWH therapy and reinforce the necessity of adherence to LMWH therapy for those who had already been injecting antenatally, so that women gain maximal benefit postnatally. Future studies assessing adherence, should particularly focus on the post-partum population, as that is the time when adherence could be a particular problem for some women.

\subsubsection{Additional questions asked in the questionnaire}

The additional questions asked in the questionnaire were questions thought to be important factors to women injecting LMWH therapy during pregnancy and the puerperium. Some key issues which came out from this section included, one third of women agreeing with the statement which said that they sometimes worried that being on enoxaparin will limit the type of child birth delivery they could have, with a similar number worried that being on enoxaparin will limit the type of pain relief they could have at the time of child birth.

Most of the women recruited in this study were asked to complete the questionnaire within a month or two of starting LMWH therapy. In the clinic setting of this study, discussions about child birth and options for pain relief are bought to the forefront during the third trimester. The findings from this study suggest that briefly discussing child birth and pain relief issues with women on 
antenatal LMWH early on in their pregnancy might overcome any anxiety that some women may have, particularly as they can be managed.

\subsubsection{Limitations}

This sub-study is limited by the fact that the questionnaire was only administered once during the women's pregnancy. Following the result that postnatal adherence may fall in some women, it would have been interesting to assess how women's views may have changed as pregnancy progresses and following delivery; this should form the basis of future studies. It must be borne in mind, that this study was part of a wider pharmacokinetic modelling study of enoxaparin during the antenatal period. Therefore, the antenatal adherence rates reported might represent an overestimate of the true adherence rate in the real world, due to the fact that women knew enoxaparin (anti-Xa activity) was being monitored. Clinical experience from the study clinic setting suggests that women requiring $\mathrm{LMWH}$ in this setting are often highly motivated, as they wish for a successful obstetric outcome. Due to the pharmacokinetic nature of the wider study, women clearly non-adherent to enoxaparin had to be excluded. During the course of this study, this was found to be the case in 3 patients and so the questionnaire was not administered to them. It would have been interesting to see what their responses would have been to the questionnaire and assess where differences were, compared to the adherent cohort of women. Finally, though it was emphasised to the women participating that their responses would remain confidential and not shared with the clinical team, it is impossible to exclude the fact that some women may have responded with socially acceptable responses.

\subsubsection{Implications for practice and future work}

We found women to be highly adherent to LMWH during the antenatal and postnatal period, though in some women, adherence does drop postnatally. This drop in adherence is related to the women's perceived individual necessity for the enoxaparin and how much they believed it protected their unborn baby's health. Women indicated a strong desire to know about what potential effect a medication may have on them and their unborn child, with the results suggesting that women would take a medicine during the antenatal period, even if it caused them pain and discomfort, if they felt it was protecting the health of their unborn baby. In the cohort of women followed, their perceived necessity for enoxaparin therapy appeared to outweigh any concerns they had antenatally. It is important for healthcare professionals to understand patients beliefs and experiences about LMWH and reinforce the necessity of adherence to LMWH particularly 
during the post-partum period when for some women, the risk of an adverse event is greater. Future work should explore if there are differences in women's responses to the questions at different pregnancy and post delivery time points. It would also be interesting to assess the responses of women who are clearly non-adherent, to see if they respond as a group to certain questions in different ways. Furthermore, it would be interesting to explore how those women prescribed enoxaparin, just during the post-natal period adhere to thrombophylaxis prescribed, not having had to inject during the antenatal period and having successfully delivered their baby. 


\section{Chapter 8}

\subsection{Implications for clinical practice}

The research programme contained within this thesis raises and addresses the key issues of dosing enoxaparin (and other LMWH) for the management of antenatal VTE. This final chapter aims to frame the major findings from this thesis, within a clinical context, in order to optimise the use of enoxaparin (and other LMWH) for the management of antenatal VTE.

\subsubsection{Recommendations}

\subsubsection{Once versus twice a day for the management of antenatal VTE?}

The primary aim of the research conducted in this thesis was to determine how the pharmacokinetics of enoxaparin is altered during the antenatal period, in order to determine the optimal dosing strategy for enoxaparin for the management of antenatal VTE. The key question being addressed was whether enoxaparin needed to be administered twice a day for this population, as currently recommended by national and international guidelines, or whether a once daily dose was more appropriate.

The results from the population pharmacokinetic modelling aspects of the study clearly confirms that although enoxaparin $\mathrm{CL}$ increases during pregnancy, the current recommendation of increasing the dose/dosing frequency, adopted by key guidelines is not appropriate. This is because $\mathrm{Vd}$ also needs to be considered. The Vd increase during pregnancy, impacts on (and prolongs) the half-life of enoxaparin significantly with the progression of pregnancy. The clinical implications of this are that a once a day LMWH regimen for the treatment of VTE during pregnancy is appropriate for the majority of women and a different dosing regimen for enoxaparin is not required during the antenatal period.

This recommendation is made with some confidence; this is because this is the largest pharmacokinetic study that has been undertaken with a LMWH, specifically designed to address this issue in an antenatal population. The studies which were published during the 1990's, which form the basis of current practice recommendations used relatively low patient numbers and did not consider LMWH Vd. Once the Vd of enoxaparin is considered, the situation and subsequent recommendations change. Furthermore, this study is not the first to report the phenomenon of residual anti-Xa activity with the progression of pregnancy (which forms the basis of the once daily recommendation); others have also found this to be the case [Lebaudy et al., 2008]. In their 
study, Lebaudy and colleagues report an increase in residual anti-Xa activity with the progression of pregnancy. Earlier studies assessing the PK of LMWH during the antenatal period, also demonstrated an increase in $\mathrm{Vd}$, but because the respective groups were not formally assessing Vd and were more focussed on the LMWH CL, it appears not to have been considered [Sturridge et al., 1994; Hunt et al., 1997; Blomback et al., 1998; Casele et al., 1999].

What specific changes should be made to current guidelines?

The current RCOG guidelines state that for both dalteparin and enoxaparin, a twice daily dose of these agents should be used for the management of antenatal VTE. Interestingly, the third LMWH commonly used in the UK, tinzaparin, is only prescribed as a once a day LMWH outside of pregnancy and thus is already listed in the RCOG guidelines as a LMWH agent that can be used once daily for the antenatal population for the management of VTE, based on work by Smith and colleagues [Smith et al., 2004].

The current RCOG guidelines should therefore be updated so that both dalteparin and enoxaparin should have once daily doses recommended for the management of VTE. Specifically, for enoxaparin, the simulation work was based on a dose banding strategy as described in table 8.1.

Table 8.1: Weight banding used for the enoxaparin simulation work in this study

\begin{tabular}{ll}
\hline Weight $(\mathbf{k g})$ & ONCE daily dose \\
\hline$<47$ & $60 \mathrm{mg}$ \\
$48-59$ & $80 \mathrm{mg}$ \\
$60-73$ & $100 \mathrm{mg}$ \\
$74-88$ & $120 \mathrm{mg}$ \\
$89-109$ & $150 \mathrm{mg}$ \\
$110-125$ & $180 \mathrm{mg}$ \\
$>125$ & $230 \mathrm{mg}$ \\
\hline
\end{tabular}

The advantage of adopting a dose banding schedule as outlined in table 8.1 for enoxaparin is that it covers a wider weight range compared to the current RCOG guidelines, with respect to the upper end of the weight spectrum; an issue which will need to be considered more with the growing obese pregnant population in the UK [Modder and Fitzsimons, 2010]. Furthermore, like the current RCOG guidelines, dose-banding allows for body-weight increases during the course of 
pregnancy, largely removing the issue of whether the dose of LMWH needs to be increased with the progression of pregnancy. Finally the dose-banding suggestions made in table 8.1 are practical, in that the manufacturers of enoxaparin produce pre-filled syringes in the doses suggested, thus making it possible for women to inject enoxaparin at home safely, without having to worry about drawing up a particular volume of the drug.

If obsterticans / haematologists caring for this population retain concerns about using a once daily LMWH regimen from the start, then a dosing strategy which gives twice a day $\mathrm{LMWH}$ for the first 2 weeks (or until the woman is stable), followed by a once daily regimen would seem reasonable.

With respect to monitoring the $\mathrm{LMWH}$, the results from this thesis suggest that most women would be expected to reach recommended 3 hour target anti-Xa levels on the once daily regimen, so that anti-Xa monitoring would not be necessary for the majority. However, the simulation work from this study revealed a proportion of women who will not have residual anti-Xa activity before the next dose is due. The clinical implication of not having a measurable anti-Xa activity is not known and is unlikely ever to be known, however, given the premise of the recommendations made in this thesis is that having a measurable anti-Xa activity allows once daily dosing of enoxaparin, it is therefore proposed, that the recommended monitoring of anti-Xa activity is changed during pregnancy, from the 3 hour anti-Xa activity measure (as recommended by RCOG) to trough anti-Xa activity for the management of VTE, in patients who clinicians feel need close monitoring, e.g. obese patients. This will ensure that any woman requiring a specific dose adjustment (following confirmation of adherence to LMWH) has a dose increase instigated on the basis of a meaningful anti-Xa activity, in this case trough activity, not peak. Monitoring of peak anti-Xa activity does seem inappropriate during the antenatal period; peak anti-Xa activity will inevitably drop with an increase in the Vd over the course of pregnancy and is not related soley to an increase in $\mathrm{CL}$.

The other key guidelines in the antenatal VTE field, is the ACCP guidelines, which state [Bates et al., 2012]:

"If LMWH is used for the treatment of acute VTE in pregnancy, a weight-adjusted dosing regimen should be used. LMWH requirements may alter as pregnancy progresses because the Vd of LMWH changes and GFR increases in the second trimester. The latter has led some to recommend a bid $L M W H$ dosing schedule. However, many clinicians use a once-daily regimen to 
simplify administration and enhance compliance. Observational studies have not demonstrated any increase in the risk of recurrence with the once-daily regimen over the bid (bis in die - twice daily) regimen."

The current ACCP guidelines already appear to lean towards a once daily dosing strategy.

To summarise, the results from this thesis suggest that it is appropriate to use a once daily dose of enoxaparin for the management of VTE, and the following recommendations / amendments are suggested to the current RCOG / ACCP guidelines:

1. Table 1 in the RCOG VTE treatment guidelines should be revised so that enoxaparin (and dalteparin) once daily doses are recommended as an initial dosing strategy. The ACCP guidelines should just recommend a once daily dose for this indication. A caveat should be added to this recommendation that during the first two weeks of therapy (or until the woman is stabilised), clinicians may consider giving twice a day LMWH.

2. The 3 hour anti-Xa monitoring which is listed as an option in the RCOG guidelines and 4 hour monitoring in the ACCP guidelines, should be revised to suggest that trough anti-Xa activity is recommended in women where clinicians suspect a poor response to LMWH, with subsequent dose increase in those women with no anti-Xa activity registered at this time point. In the majority of women, such monitoring will not be required and clinicians should be mindful of adherence to enoxaparin when anti-Xa activity are drawn and interpreted.

The implementation of the recommendations in this thesis might provide a significant challenge. Historically in medicine, even when land-mark clinical trials are published, there is often a lag time before wide-spread uptake and a change in clinical practice. For example, even though clinical studies from the 1980's had demonstrated the benefits of aspirin and beta-blocker therapy in improving survival in patients with an acute ST-elevation myocardial infarction, the uptake of these interventions was slow [Davis et al., 1995; Pashos et al., 1994]. A significant change in clinical practice, particularly historical practice, can be complex, as it involves behaviour change [Jackevicius et al., 2001]. For a number of years, the twice daily regimen of LMWH has been recommended and used for the management of antenatal VTE, and although studies published in the past 5 years have demonstrated that up to $50 \%$ of patients are managed with a once daily LMWH regimen for antenatal VTE, for the full uptake of the recommendations in this thesis, will 
require key opinions leaders in the UK and further afield to endorse the findings. This will require publishing and presenting the findings in key journals and at conferences and when appropriate, debating the findings, so that a new consensus can be reached and the situation can be moved forward for the benefit of women.

\subsubsection{Prophylactic dosing of LMWH}

As for the management of VTE, the study results in this thesis imply that a once daily regimen should be appropriate for the majority of prophylactic LMWH indications as well. The current RCOG guidelines suggest a once daily dose of enoxaparin (and other LMWH) for prophylactic indications, however they do stipulate that a possible twice daily dose of LMWH could be used in those patients who are obese (>90kg) (table 8.2).

Table 8.2: Current RCOG prophylactic dosing recommendations for enoxaparin

\begin{tabular}{cc}
\hline Weight $\mathbf{( k g )}$ & Enoxaparin \\
\hline$<50$ & $20 \mathrm{mg}$ daily \\
$50-90$ & $40 \mathrm{mg}$ daily \\
$91-130$ & $60 \mathrm{mg}$ daily* \\
$131-170$ & $80 \mathrm{mg} \mathrm{daily}{ }^{*}$ \\
$>170$ & $0.6 \mathrm{mg} / \mathrm{kg} /$ day $^{*}$ \\
High prophylactic (intermediate) dose & $40 \mathrm{mg} \mathrm{12-hourly}$
\end{tabular}

There is no reason to believe that the obese population require a more frequent dose of enoxaparin (and other LMWH). During the course of the study in this thesis, a small number of obese women specifically requested to be managed with a once daily prophylactic dose (e.g. $80 \mathrm{mg}$ once daily, as opposed to $40 \mathrm{mg}$ twice a day) and did not suffer any adverse effects. The findings from the adherence aspect of the study (chapter 7), suggest that some women find it difficult to perform daily injections; therefore a complete once daily strategy is suggested for this sub-group.

The simulations from the prophylactic aspects in this thesis found that a proportion of women would not have a measurable trough anti-Xa activity with a once daily dose. Monitoring anti-Xa activity is currently not recommended in any of the guidelines for prophylactic indications and is unlikely to be beneficial in the prophylactic setting (unlike in the treatment setting). A better marker of efficacy needs to be established for prophylactic doses. This is where thrombin generation could serve a useful purpose. If it can be established what \% inhibition of peak thrombin generation achieves a prophylactic effect at a pre-defined time point, then that could 
potentially be used as a marker of the efficacy of prophylactic regimens and be used to guide future research addressing why LMWH is only $\sim 60-70 \%$ effective in preventing VTE when prescribed for prophylactic indications. More recently, a novel approach has also been suggested by Mandema and colleagues [2011]. They suggest a model based approach to comparing the thromboprophylatic effect of different anticoagulants following orthopaedic surgery. In their study, a therapeutic index was created, which represented the ratio of the dose with an acceptable bleeding risk to the dose with a relevant risk reduction for VTE. Such model based approaches in the future, might also be used to help tackle the problem of what pharmacodynamic response produces an efficacious thromboprophylactic response.

Getting the dosing of thromboprophylaxis correct is important. A recent cross sectional study has been completed by Revell and Smith [2011], evaluating 109 deliveries over a one week period at their district general hospital. Their aim was to quantify the number of women who would reach the threshold for antenatal and postnatal thromboprophylaxis, according to the current RCOG guidelines. They found that $7 \%$ of women met the threshold for antenatal thromboprophylaxis and $41 \%$ met the threshold for postnatal thromboprohylaxis. The large numbers of women, particularly during the postpartum period eligible, illustrates why it is important to get the dosing strategy right.

In summary, the current RCOG prophylactic guidelines should be amended for the obese population (those weighing $>90 \mathrm{~kg}$ ), so that all women prescribed enoxaparin (and dalteparin) for prophylactic indications receive an appropriate once daily regimen. Women are also more likely to adhere to such a regimen and therefore gain maximal benefit from the prophylactic LMWH prescribed.

\subsubsection{Do obese patients require special consideration?}

Dosing of the obese population provides a particular challenge, due to uncertainty outside of pregnancy of whether the dose of LMWH should be increased in proportion to actual weight or whether it should be capped at a maximum dose. The current consensus is that LMWH dose should be increased with increasing body weight, but caution should be exercised when using actual body weight, as LMWH CL is highly correlated with lean body weight, which would be a better descriptor to use for dosing LMWH [Patel et al., 2011].

The obese population does deserve special attention; recent reports suggest that the extremely obese population are more likely to bleed and suffer from complications during the antenatal 
period, even when not prescribed LMWH. Therefore having to inject LMWH for a treatment or prophylactic indication could considerably increase the risk of bleeding in this population [Modder and Fitzsimons, 2010] and particular vigilance would seem appropriate for this population, until the underlying mechanisms are better understood.

\subsubsection{Adherence to LMWH during pregnancy and the puerperium}

The findings from the adherence sub-study of this thesis suggest that women are highly adherent to enoxaparin $(>80 \%)$, during both the antenatal and the puerperium periods. However in a group of women adherence to enoxaparin fell following the birth of their baby. The results suggest that pregnant women's antenatal beliefs around necessity and concerns of enoxaparin could predict their postnatal adherence. No statistically significant differences in adherence rates were found between Caucasian and African-Caribbean women, those women once versus those women injecting twice a day and also between those women who had a history of recurrent miscarriage compared to those women who did not.

The findings from the adherence sub-study are important for two reasons. Firstly, they demonstrate that women will inject themselves with a parenteral medication during pregnancy, if they believe it is protecting their health and that of their unborn baby; demonstrating a protective behaviour towards their unborn baby. Additionally, if such a strong belief is carried through to the postnatal period, then such a belief could lead to non-adherence during the postnatal period. This finding does also imply that adherence could be an issue for women who are commenced on enoxaparin (LMWH) just during the postnatal period. The threshold for commencing LMWH prophylaxis is much lower during the postnatal period than antenatally, according to the current RCOG guidelines:

- two or more risk factors (e.g. age $>35$, obese class III), : at least 7 days postnatal prophylactic LMWH

- If persisting or $>3$ risk factors (e.g. age $>35$, obese class III, parity $>2$ ), consider extending thromboprophylaxis with LMWH

The results in this thesis demonstrate that an important driver of adherence might be the protection of the baby in-utero. Therefore for those women who are simply prescribed thromboprophylaxis during the postnatal period, clinicians may find that adherence to LMWH is not complete and varies, as the perceived necessity may not be that strong. Further studies are 
needed to explore this issue, as it could help optimise for how long thromboprophylaxis is offered in women during the puerperium, particularly as the numbers eligible during the post-partum period increases significantly [Revell and Smith, 2011].

\subsubsection{Exceptions to once daily antenatal LMWH I: mechanical heart valves}

As discussed in chapter four, the antenatal management of women with prosthetic mechanical heart valves in-situ provides a particular challenge. The literature suggests that the risk of valve thrombosis and death is lowest with coumarin anticoagulants and highest with unfractionated heparin during pregnancy. Low molecular weight heparins can be used and the most comprehensive guidelines in the field (the ACCP [Bates et al., 2012]), state that if LMWH is used, that dosing should be twice a day and adjusted to aim for a 4 hour post injection anti-Xa of 1-1.2 $\mathrm{IU} / \mathrm{mL}$. Following the findings from this thesis, the question to consider is whether a once daily dose of LMWH could be used, as suggested for the management of VTE. The PK changes reported in this thesis, will also apply to the mechanical heart valve pregnant population and so one would anticipate an increase in trough anti-Xa activity with the progression of pregnancy and the potential advantage that brings, supporting a once daily dosing strategy. However, the mechanical heart population is very different to the VTE population, in terms of risk. The literature is littered with case reports of women suffering apparent treatment failures whilst on therapeutic dose LMWH. In view of this, to suggest a once daily LMWH just on the basis of this study, particularly as none of the women in this study had a mechanical heart valve in-situ, would seem unwise. Further research specifically in this population is required before such a recommendation can be made.

If the VTE treatment recommendations cannot be directly applied to the mechanical valve population, what can be learnt and applied from this thesis for the antenatal mechanical heart valve population?

First, the Vd issue is important here. The ACCP guidelines [Bates et al., 2012] suggest that a twice daily dose of LMWH should be adjusted to maintain the 4 hour peak anti-Xa activity between 1-1.2 IU/mL. Given observed changes to Vd over the course of pregnancy, this strategy runs the danger of over-anticoagulation, as an inevitable drop in peak anti-Xa activity will occur, with a respective rise in the trough anti-Xa activity. This then potentially places the patient at risk of bleeding, if LMWH doses are aggressively increased in response to the drop in peak anti-Xa activity. Recent published reports suggest this to be the case [Saeed et al., 2011; Basude et al., 
2012], where as well as valve thrombosis being reported, epistaxis, vaginal spotting, and intracerebral haemorrhage were also reported by these studies and may be linked to clinicians trying to maintain the peak anti-Xa activity aggressively within the $1-1.2 \mathrm{IU} / \mathrm{mL}$ range. Haematology- obstetric experts are in the process of re-thinking this strategy; at the recent International Society of Thrombosis and Haemostasis conference, $\operatorname{Dr}$ Claire McLintock, an international expert on managing anticoagulation in women with mechanical heart valves during pregnancy, was invited to give the Shirley Johnsson memorial lecture [McLintock, 2012]. In her lecture, she discussed this issue and suggested that her practice is likely to alter in the future, with more of a focus on monitoring anti-Xa trough activity for women on a twice daily LMWH regimen for this indication. The research findings in this thesis would certainly support such a strategy; those women with low or negligible trough activities can have their dose increased, as opposed to a wholesale increase for all women. The challenge in part will be on knowing what is an acceptable anti-Xa trough activity. At the National Women's Hospital and Auckland City Hospital, Dr McLintock specifically recommends the following monitoring schedule for enoxaparin; $1 \mathrm{mg} / \mathrm{kg}$ bd, peak (4 hours): $07-1.2 \mathrm{IU} / \mathrm{mL}$ and trough (12 hours): $0.4-0.7 \mathrm{IU} / \mathrm{mL}$ [Pavord and Hunt, 2010].

It is surprising that the literature over the last 10-15 years has reported a number of failures with the use of LMWH in this population, suggesting that treatment doses of LMWH are not effective during pregnancy. However, most reports have assumed that medication adherence to be complete in this population. McLintock and colleagues [2009] found that those mechanical valve patients who had poor adherence with LMWH had poorer outcomes compared to those who took their LMWH as prescribed. Medication adherence is complex and very patient specific; McLintock's findings are unsurprising and given the findings outlined in this thesis (chapter 7), perhaps greater emphasis on adherence should be placed, before a conclusion that LMWH has failed is drawn. More emphasis and evidenced based support with adherence is required specifically in this situation, given the large risks involved, with reports continuing to be published in the literature exemplifying the risks involved [Nelson-Piercy and Greer, 2012].

In summary, a once daily recommendation of LMWH cannot be recommended for the mechanical heart valve population until further evidence from this specific population is obtained. A trough monitoring strategy seems a more appropriate anti-Xa activity monitoring strategy in this 
population. Strategies supporting adherence to LMWH should be provided to women suspected of non-adherence, as this is more likely to lead to a successful outcome in these women.

\subsubsection{Exceptions to once daily antenatal LMWH II: antithrombin deficiency}

Women with antithrombin deficiency are at high risk of VTE and may require higher than usual prophylactic doses during pregnancy, particularly those with type-1 deficiency (reductions in both activity and antigen). AT deficiency is discussed here, because the pharmacological activity of LMWH is exerted by potentiating the effects of anti-thrombin, and so a deficiency could render the LMWH less effective. In a pregnancy setting this could be problematic, with few studies specifically exploring this issue. Practice has centred on giving a higher and more frequent dose of LMWH for this group of patients (40mg twice a day, as opposed to $40 \mathrm{mg}$ daily). The question of whether a once daily dose of LMWH could be used in this population is raised again (i.e. $80 \mathrm{mg}$ once daily as opposed to $40 \mathrm{mg}$ twice a day). The principal finding from this study that trough antiXa activity increases in the antenatal population, will not be different for an antenatal antithrombin deficient pregnant woman. Given this, there is no reason to believe that a once daily prophylactic dosing strategy would be ineffective. However, due to the specific high risk nature of this population and because of the nature of LMWH mechanism of action, until further evidence in this area is available, it would be unwise to ignore the current suggested dosing regimen of twice daily dosing, where the majority of experience exists, although at King's College Hospital, AT deficient women are managed with a once daily dose of LMWH. However, like previous discussions, the target peak anti-Xa activity should be re-considered, as one anticipates a drop with the progression of pregnancy, due to the increase in Vd.

\subsubsection{Management of treatment doses of LMWH at the time of delivery}

At some centres, like King's College Hospital, standard practice for women who are on once daily treatment doses of LMWH is to divide the total daily dose of once daily enoxaparin, around 36 weeks gestation; for example if a woman is prescribed enoxaparin $120 \mathrm{mg}$ once daily, then to prescribe $60 \mathrm{mg}$ twice a day. The rational for this, is that given the woman could go into labour at any time, splitting the dose to a twice daily regimen, reduces the chances of high peak concentrations being achieved at the time of delivery.

The current RCOG guidelines for the management of VTE, specify how LMWH should be managed at the time of delivery [Royal College of Obstetricians and Gynaecologists, 2007]. Would a once daily dose of LMWH cause problems for women at the time of delivery? 
For women who have a planned induction or planned caesarean section, once daily enoxaparin (or LMWH) should not present a problem, as they can be advised to omit their enoxaparin treatment dose 24 hours before surgery (i.e. omit the day before) or when induction booked.

Women receiving once a day and twice a day treatment doses of LMWH waiting for spontaneous labour, present more of a challenge for both LMWH regimens, due to the uncertain nature of spontaneous labour making it difficult to know how to manage the LMWH, if an epidural is required. No plan can be formulated, as it will all depend on when the woman goes into labour to what needs to happen and what pain relief can be offered. Either way, a multi-disciplinary approach to support women in this position seems the most logical approach to adopt.

\subsubsection{Recommended future research priorities}

The recent availability of the novel oral anticoagulants in clinical practice, e.g. rivaroxaban and dabigatran, offer many advantages over existing anticoagulant treatments, e.g. oral formulation, and immediate onset of anticoagulant effect. However, their use during the ante- and post-natal periods is not established and is unlikely to be, for the foreseeable future. For these reasons, future antenatal research work should focus on LMWH use, with particular priorities being:

a) Preventing thromboembolic complications in the mechanical heart valve population through better understanding and establishment of a trough target, to help guide clinicians on when a dose increase might be required. Establishing a minimum inhibitory concentration would be the challenge here and the global coagulation assays, e.g. thrombin generation, may have a role to play in establishing this.

b) For women who suffer VTE in the first trimester, an important question to address is whether they really need LMWH for the full nine months at treatment dose $\mathrm{LMWH}$, or whether the dose be reduced after 3 months. As the recurrence rate is so low once on treatment, conducting a clinical trial is likely to be futile, as 1,000 's of women would need to be recruited to demonstrate one intervention is better over another. Perhaps, the best way to address this question would be to observe the outcome of women who are given three months full intensity LMWH, followed by a reduction in dose; such a case series may help clinicians build up experience in this area of uncertainty.

c) Women managed on once daily LMWH for the treatment of VTE, should be followed up across the UK, as a cohort study to confirm (or disprove) the findings from this thesis. 
d) The obese antenatal population in the UK (and worldwide) is on the increase. Obesity is an independent risk factor for VTE, but reports suggest that obese women also suffer more bleeding (particularly at the time of delivery). Further study into why this might be the case would be welcome, as it would help clinicians to appropriately balance the risk of VTE with the risk of bleeding.

\subsubsection{Conclusion}

To conclude, the primary aim of programme of research of this thesis was to establish how the pharmacokinetics of enoxaparin are altered during pregnancy and thus determine what the optimal dosing strategy of enoxaparin is, for the management of VTE. Through applying the method of non-linear mixed effects modelling, mathematical models which estimated $C L$ and $V d$ during pregnancy were developed (equations 8.1-8.4):

$\mathrm{CL}=\mathrm{POPCL} \times \mathrm{GESTCL} \times(\mathrm{Wt} / 80)^{1.06}$

Vd $=$ POPVd $\times$ GESTV $\times(\text { LBW/42 })^{1.25}$

where during pregnancy:

GESTCL $=1$

GESTV $=(\text { GEST/MGEST })^{0.357}$

Simulations of women injecting once versus twice daily doses of enoxaparin demonstrated that for the majority of women, a once daily dose of enoxaparin would be appropriate for the management of antenatal VTE, due to a significant increase in the Vd, prolonging the half-life of enoxaparin. National and international guidelines should reconsider their current recommendations in light of the findings of this thesis. 


\section{Bibliography}

Abbate R, Gori A, Farsi A, Attanasio M, Pepe G. Monitoring of low molecular-weight heparins in cardiovascular disease. American Journal of Cardiology 1998; 82: 33L-36L

Abbas $\mathrm{AE}$, Lester SJ, Connolly $\mathrm{H}$. Pregnancy and the cardiovascular system. International Journal of Cardiology 2005; 98: 179-189

Abildgaard U, Lindahl AK, Sandset PM. Heparin requires both antithrombin and extrinsic pathway inhibitor for its anticoagulant effect in human blood. Haemostasis 1991; 21: 254-257

Adam SS, Key NS, Greenberg CS. D-dimer antigen: current concepts and future prospects. Blood 2009; 113: 2878-2887

Adamidou A, Riddell A, Priest P, Mughaddam L, Aghighi S, Gatt A, Tuddenham EG, Chowdary P. Thrombin generation test (TGT) in pregnant women at risk of thrombosis on LMWH (low molecular weight heparin): correlation with anti-Xa activity. British Journal of Haematology 2011; 153 (suppl. 1): 77 (Abstract 179)

Al Dieri R, Wagenvoord R, van Dedem GW, Beguin S, Hemker HC. The inhibition of blood coagulation by heparins of different molecular weight is caused by a common functional motif - the C-domain. Journal of Thrombosis and Haemostasis 2003; 1: 907-914

Al Dieri R, Alban S, Beguin S, Hemker HC. Thrombin generation for the control of heparin treatment, comparison with the activated partial thromboplastin time. Journal of Thrombosis and Haemostasis 2004; 2: 1395-1401

Al Dieri R, Alban S, Beguin S, Hemker HC. Fixed dosage of low-molecular-weight heparins causes large individual variation in coagulability, only partly correlated to body weight. Journal of Thrombosis and Haemostasis 2006; 4: 83-89

Alhenc-Gelas M, Jestin-Le Guernic C, Vitoux JF, Kher A, Aiach M, Fiessinger JN. Adjusted versus fixed doses of the low molecular weight heparin fragmin in the treatment of deep vein thrombosis. Fragmin-study group. Thrombosis and Haemostasis 1994; 71: 698-702

Allegaert K, van Mieghem T, Verbesselt R, de Hoon J, Rayyan M, Devlieger R, Deprest J, Anderson BJ. Cefazolin pharmacokinetics in maternal plasma and amniotic fluid during pregnancy. American Journal of Obstetrics and Gynaceology 2009; 200: 170e1-170e7

Alpert JS, Smith R, Carlson J, Ockene IS, Dexter L, Dalen JE. Mortality in patients treated for pulmonary embolism. Journal of the American Medical Association 1976; 236: 1477-1480 
Altman R, Scazziota A, Herrera L, Gonzalez C. Relationship between thrombin generation and international normalised ratio in patients receiving oral vitamin $\mathrm{K}$ antagonist therapy. Journal of Thrombosis and Haemostasis 2007; 5: 1552-1569

Andersson LO, Barrowcliffe TW, Holmer E, Johnson EA, Sims GEC. Anticoagulant properties of heparin fractionated by affinity chromatography on matrix-bound antithrombin III and by gel filtration. Thrombosis Research 1976; 9:575-583

Anderson GD. Pregnancy-induced changes in pharmacokinetics: a mechanistic-based approach. Clinical Pharmacokinetics 2005; 44: 989-1008

Ando T, Yamasaki M, Suzuki K. In Kleinzeller A, editor. Protamine: molecular biology biochemistry and biophysics, Vol 12. New York: Spinger-Verlag; 1973. P. 1-109

Andrade SE, Gurwitz JH, Davis, Chan KA, Finkelstein JA, Fortman K, McPhillips H, Raebel MA, Roblin D, Smith DH, Yood MU, Morse AN, Platt R. Prescription drug use in pregnancy. American Journal of Obstetrics and Gynaecology 2004; 191: 398-407

Andrassy K, Eschenfelder V. Are the pharmacokinetic parameters of low molecular weight heparins predictive of their clinical efficacy? Thrombosis Research 1996; 81: s29-s38

Andrew MA, Easterling TR, Carr DB, Shen D, Buchanan ML, Rutherford T, Bennett R, Vicini P, Hebert MF. Amoxicillin pharmacokinetics in pregnant women: modelling and simulations of dosage strategies. Clinical Pharmacology and Therapeutics 2007; 81: 547-556

Anning ST. The historical aspects of venous thrombosis. Medical History 1957; 1: 28-37

Anon (a). Committee of the Safety of Medicines / Medicines Control Agency. Suppression of aldosterone secretion by heparin. Current Problems in Pharmacovigilance 1999; 25: 6

Anon (b). Committee of the Safety of Medicines. Heparin-induced thrombocytopenia. Current Problems in Pharmacovigilance 1990; 28: 1-4

Anon (c). Thromboembolism in pregnancy. British Medical Journal 1979; 6179: 1661

Anon(d). Rapid response report: Reducing treatment dose errors with low molecular weight heparins. National Patient Safety Agency; NPSA/2010/RRR014 July 2010

Antman EM on behalf of the thrombolysis in myocardial infarction (TIMI) $11 \mathrm{~A}$ trial investigators. Dose-ranging trial of enoxaparin for unstable angina: results of TIMI 11A. Journal of the American College of Cardiology 1997; 29: 1474-1482 
Antman EM, McCabe CH, Gurfinkel EP, Turpie AG, Bernink PJ, Salein D, Bayes De Luna A, Fox K, Lablanche JM, Radley D, Premmereur J, Braunwald E. Enoxaparin prevents death and cardiac ischemic events in unstable angina/non-Q-wave myocardial infarction. Results of the thrombolysis in myocardial infarction (TIMI) 11B trial. Circulation 1999; 100: 1593-601

Ansell J, Hirsh J, Hylek E, Jacobson A, Crowther M, Palareti G. Pharmacology and management of the vitamin $\mathrm{K}$ antagonists - American College of Chest Physcians Evidenced Based Clinical Practice Guidelines ( $8^{\text {th }}$ Edition). Chest 2008; 133: 160s-198s

Arya R, Shehata HA, Patel RK, Sahu S, Rajasingam D, Harrington KF, Nelson-Piercy C, Parsons $\mathrm{JH}$. Internal jugular vein thrombosis after assisted conception therapy. British Journal of Haematology 2001; 115: 153-155

Arya R. How I manage venous thromboembolism in pregnancy. British Journal of Haematology 2011; 153: 698-708

Aster RH. Heparin-induced thrombocytopenia and thrombosis. New England Journal of Medicine 1995; 332: 1374-6

Atkinson JP. A case of pulmonary embolism. Lancet 1872: 408

Aviv R, Chubb K, Lindow S. The prevalence of maternal medication ingestion in the antenatal period. South African Medical Journal 1993; 83: 657-660

Bacon NC. Cases of Phlegmasia dolens successfully treated with iodine. Lancet 1832: 102-103

Baglin T. The measurement and application of thrombin generation. British Journal of Haematology 2005; 130: 653-661

Baglin T, Barrowcliffe TW, Cohen A, Greaves M for the British committee for standards in haematology. Guidelines on the use and monitoring of heparin. British Journal of Haematology 2006; 133: 19-34

Bagot CN, Arya R. Virchow and his triad: a question of attribution. British Journal of Haematology 2008; 143: 180-190

Bara L, Billaud E, Gramond G, Kher A, Samama M. Comparative pharmacokinetics of a low molecular weight heparin (PK 10 169) and unfractionated heparin after intravenous and subcutaneous administration. Thrombosis Research 1985; 39: 631-636

Bara L, Samama MM. Pharmacokinetics of low molecular weight heparins. Acta Chirurgica Scandinavica (Suppl) 1988; 543: 65-72 
Barnes F. A clinical note on Phlegmasia dolens. British Medical Journal 1880: 921-922

Barradell LB, Buckley MM. Nadroparin calcium: a review of its pharmacology and clinical application in the prevention and treatment of thromboembolic disorders. Drugs 1992; 44: 858-888

Barrass MA, Duffull SB, Atherton JJ, Green B. Modelling the occurrence and severity of enoxaparin induced bleeding and bruising events. British Journal of Clinical Pharmacology 2009; 68: 700-711

Barritt DW, Jordan SC. Anticoagulant drugs in the treatment of pulmonary embolism: a controlled trial. Lancet 1960; 1: 1309-1312

Barzu T, Molho P, Tobelem G, Petitou M, Caen J. Binding and endocytosis of heparin in human endothelial cells in culture. Biochimica et Biophysica Acta 1985; 845: 196-203

Basude S, Hein C, Curtis SL, Clark A, Trinder J. Low-molecular weight heparin or warfarin for anticoagulation in pregnant women with mechanical heart valves: what are the risks? A retrospective observational study. British Journal of Obstetrics and Gynaecology 2012; DOI: 10.1111/j.1471-0528.2012.03359.x.

Bates SM, Greer IA, Pabinger I, Sofaer S, Hirsh J. Venous Thromboembolism, Thrombophilia, Antithrombotic Therapy, and Pregnancy: American College of Chest Physicians Evidence-Based Clinical Practice Guidelines (8th Edition). Chest 2008; 133: 844-886

Bates SM, Greer IA, Middeldorp S, Veenstra DL, Prabulos AM, Vandvik PO. VTE, Thrombophilia, Antithrombotic therapy and pregnancy. Antithrombotic therapy and prevention of thrombosis, American College of Chest Physicians Guidelines. Chest 2012; 141 Suppl: e691s-e736s

Beal SL, Sheiner LB. NONMEM Users Guide. Icon Development Solutions, 1989-1998

Beal SL, Sheiner LB. NONMEM Users Guide - Part VII. Conditional estimation methods. University of California, San Francisco, 1992

Beal SL. NONMEM Project Group. NONMEM user's guide. University of California at San Francisco, 1998

Beal SL. Ways to fit a PK model with some data below the quantification limit. Journal of Pharmacokinetics and Pharmacodynamics 2001; 28: 481-504 
Berges A, Laporte S, Epinat M, Zufferey P, Alamartine E, Tranchand B, Decousus H, Mismetti P for the PROPHRE 75 study group. Anti-factor Xa activity of enoxaparin administered at prophylactic dosage to patients over 75 years age. British Journal of Clinical Pharmacology 2007; 64: 428-438

Bergqvist D, Arcelus JI, Felicissimo P for the ETHOS investigators. Post-discharge compliance to venous thromboembolism prophylaxis in high-risk orthopaedic surgery - results from the ETHOS registry. Thrombosis and Haemostasis 2012; 107: 280-287

Bergstrand M, Karlsson MO. Handling data below the limit of quantification in mixed effect models. The American Association of Pharmaceutical Scientists Journal 2009; 11: 371380

Bergstrand M, Hooker AC, Wallin JE, Karlsson MO. Prediction-corrected visual predictive checks for diagnosing non-linear mixed effects models. The American Association of Pharmaceutical Scientists Journal 2011; 13(2): 143-151Besser M, Baglin C, Luddington $\mathrm{R}$, van Hylckama-Vlieg A, Baglin T. High rate of unprovoked recurrent venous thrombosis is associated with high thrombin generating potential in a prospective cohort study. Journal of Thrombosis and Haemostasis 2008; 6: 1720-5

Bhattaram VA, Booth BP, Ramchandani RP, Beasley BN, Wang Y, Tandon V, Duan JZ, Baweja RK, Marroum PJ, Uppoor RS, Rahman NR, Sahajwalla CG, Powell JR, Mehta MU, Gobburu JVS. Impact of pharmacometrics on drug approval and labelling decisions: a survey of 42 new drug applications. The American Association of Pharmaceutical Sciences Journal 2005; 7: e503-e512

Bircher AJ, Flückiger R, Buchner SA. Eczematous infiltrated plaques to subcutaneous heparin: a type IV allergic reaction. British Journal of Dermatology 1990; 123: 507-14

Bjornsson TO, Wolfram BS, Kitchell BB. Heparin kinetics determined by three assay methods. Clinical Pharmacology and Therapeutics 1982; 31: 104-113

Blajchman MA, Young E, Ofosu FA. Effects of unfractionated heparin, derman sulphate and low molecular weight heparin on vessel wall permeability in rabbits. Annals of the New York Academy of Sciences 1989; 556: 245-254

Blomback M, Bremme K, Hellgren M, Lindberg H. A Pharmacokinetic study of Dalteparin (Fragmin) during late pregnancy. Blood Coagulation and Fibrinolysis 1998; 9: 343-50

Blumberg JB, Winterscheid LC, Dillard DH, Vetto RR, Merendino KA. The clinical use of polybrene as an antiheparin agent in open heart surgery. Journal of Thoracic and Cardiovascular Surgery 1960; 39: 330-336 
Bonari L, Koren G, Einarson TR, Jasper JD, Taddio A, Einarson A. Use of antidepressants by pregnant women: Evaluation of perception of risk, efficacy of evidence based counselling and determinants of decision making. Archives of Women's Mental Health 2005; 8: 214220

Bonate PL (ed). In Pharmacokinetic-Pharmacodynamic modeling and simulation. Springer 2006

Bonnar J. Long-term self-administered heparin therapy for prevention and treatment of thromboembolic complications in pregnancy. Heparin Chemistry and Clinical Usage (ed. V. V. Kakkar and D. P. Thomas) pp. 247-260. Academic Press, London 1976

Bonnar J. Venous thromboembolism and pregnancy. Clinics in Obstetrics and Gynaecology 1981; 8: $455-473$

Bouillin-Pichault M, Jullien V, Azria E, Pannier E, Firtion G, Krivine A, Compagnucci A, Taulera O, Finkielsztejn L, Chhun S, Pons G, Launay O, Treluyer J-M. Population analysis of the pregnancy-related modifications in lopinavir pharmacokinetics and their possible consequences for dose adjustmenets. Journal of Antimicrobial Chemotherapy 2009; 63: 1223-1232

Bounameaux H, Cirafici P, De Moerloose P, Schneider PA, Slosman D, Reber G, Unger PF. Measurement of $\mathrm{D}$-dimer in plasma as diagnostic aid in suspected pulmonary embolism. Lancet 1991; 337: 196-200

Brandjes DPM, Heiboer H, Buller HR, de Rijk M, Jagt $\mathrm{H}$, ten Cate JW. Acenocoumarol and heparin compared with acenocoumarol alone in the initial treatment of proximal vein thrombosis. New England Journal of Medicine 1992; 327: 1485-1489

Breddin HK, Hach-Wunderle V, Nakov R, Kakkar VV for the CORTES investigators. Effects of a low-molecular weight heparin on thrombus regression and recurrent thromboembolism in patients with deep-vein thrombosis. The New England Journal of Medicine 2001; 344: $626-631$

Bremme K, van Rooijen M, Yu A, Blomback M. Accumulation of low molecular mass heparin during prophylactic treatment in pregnancy. Blood Coagulation and Fibrinolysis 2001; 12: 149-155

Bremme K. Haemostatic changes in pregnancy. Best Practice and Research: Clinical Haematology 2003; 16: 153-168

Brennard JE, Walker ID, Greer IA. Anti-activated factor X profiles in pregnant women requiring antenatal thromboprophylaxis with enoxaparin. Acta Haematologica 1999; 101: $53-55$ 
British Medical Association and the Royal Pharmaceutical Society of Great Britain. Joint formulary committee. British National Formulary, No. 59, Pharmaceutical Press, 2010

Broussard CS, Louik C, Honein MA, Mitchell AA and the National Birth Defects Prevention Study. Herbal use before and during pregnancy. American Journal of Obstetrics and Gynaecology 2010; 202: 443.e1-6

Broze GJ. Tissue factor pathway inhibitor. Thrombosis Haemostasis 1995; 74: 90-93

Bruno R, Baille P, Retout S, Vivier N, Veyrat-Follet C, Sanderink G-J, Becker R, Antman EM. Population pharmacokinetics and pharmacodynamics of enoxaparin in unstable angina and non-ST-segment elevation myocardial infarction. British Journal of Clinical Pharmacology 2003; 56: 407-414

Buller HR on behalf of the Einstein investigators. Oral rivaroxaban for symptomatic venous thromboembolism. The New England Journal of Medicine 2010; 363: 2499-2510

Bynum TE. Hepatic and gastrointestinal disorders in pregnancy. Medical Clinics of North America 1977; 61: 129-138

Cantu C, Barinagarrementeria F. Cerebral venous thrombosis associated with pregnancy and puerperium - Review of 67 cases. Stroke 1993; 24: 1880-1884

Carlo A, Arnaud E, Woodhams BJ. The use of polybrene for heparin neutralisation in the thrombin generation test. Journal of Thrombosis and Haemostasis 2009; 7: Suppl 2: Abstract number PP-WE-164

Casele HL, Laifer SA, Woelkers DA, Venkataramanan R. Changes in the pharmacokinetics of the low molecular weight heparin enoxaparin sodium during pregnancy. American Journal of Obstetrics and Gynaecology 1999; 181: 1113-7

Castoldi E, Rosing J. Thrombin generation tests. Thrombosis Research 2011; 127(suppl 3): s21s25

Chabloz P, Reber G, Boehlen F, Hohlfeld P, De Moerloose P. TAFI antigen and D-dimer levels during normal pregnancy and at delivery. British Journal of Haematology 2001; 115: 150152

Chan WS, Anand S, Ginsberg JS. Anticoagulation of pregnant women with mechanical heart valves: a systematic review of the literature. Archives of Internal Medicine 2000; 160: 191-196 
Chan WS, Lee A, Spencer FA, Chunilal S, Crowther M, Wu W, Johnston M, Rodger M, Ginsberg JS. D-dimer testing in pregnant patients: towards determining the next 'level' in the diagnosis of deep vein thrombosis. Journal of Thrombosis and Haemostasis 2010; 8: 1004-1011

Chan WS, Spencer FA, Ginsberg JS. Anatomic distribution of deep vein thrombosis in pregnancy. Canadian Medical Association Journal 2010; 182: 657-660

Chantarangkul V, Clerici M, Bressi C, Griesen PL, Tripodi A. Thrombin generation assessed as endogenous thrombin potential in patients with hyper- and hypo-coagulability. Haematologica 2003; 88: 547-554

Chargaff E, Olson KB. Studies on the chemistry of blood coagulation VI. Studies on the action of heparin and other anticoagulants. The influence of protamine on the anticoagulant effect in-vivo. Journal of Biological Chemistry 1937; 153-167

Charles AF, Scott DA. Studies on heparin. I. The preparation of heparin. The Journal of Biological Chemistry 1933a; 102: 425-429

Charles AF, Scott DA. Studies on heparin. II. Heparin in various tissues. The Journal of Biological Chemistry 1933b; 102: 431-435

Chen WWC, Chan CS, Lee PK., Wang RYC, Wong VCW. Pregnancy in patients with prosthetic heart valves. An experience with 45 pregnancies. Quarterly Journal of Medicine 1982; 51: 358-365

Chou J, Mackman N, Merrill-Skoloff G, Pedersen B, Furie BC, Furie B. Hematopoietic cell-derived microparticle tissue factor contributes to fibrin formation during thrombus propagation. Blood 2004; 104: 3190-3197

Choay J, Lormeau JC, Petitou M, Sinay P, Fareed J. Structural studies on a biologically active hexasaccharide obtained from heparin. Annals of the New York Academy of Sciences 1981; 370: 644-649

Centre for Maternal And Child Enquiries. Saving Mothers' Lives: Reviewing maternal deaths to make motherhood safer - 2003-2005. The Seventh Report of the Confidential Enquiries into Maternal Deaths in the United Kingdom. December 2007; Available at: http://www.cmace.org.uk/Publications-Press-Releases/Report-Publications/MaternalMortality.aspx

Centre for Maternal And Child Enquiries. Saving Mothers' Lives: Reviewing maternal deaths to make motherhood safer: 2006-2008. British Journal of Obstetrics and Gynaecology: An International Journal of Obstetrics and Gynaecology 2011; 118: 1-203 
Cockroft DW, Gault H. Prediction of creatinine clearance from serum creatinine. Nephron 1976; 16: $31-41$

Colwell CW, Pulido P, Hardwick ME, Morris BA. Patient compliance with outpatient prophylaxis: an observational study. Orthopaedics 2005; 28: 143-147

Comerota AJ, Throm RC, Mathias SD, Haughton S, Mewissen M. Catheter-directed thrombolysis for ileofemoral deep vein thrombosis improves health-related quality of life. Journal of Vascular Surgery 2000; 32: 130-137

Council of Europe (COE) - European Directorate for the Quality of Medicines (EDQM). European Pharmacopoeia $6^{\text {th }}$ Ed, 2007

Couturaud F, Julian JA, Kearon C. Low molecular weight heparin administered once versus twice daily in patients with venous thromboembolism: a meta-analysis. Thrombosis and Haemostasis 2001; 86: 980-984

Coughlin SR. Protease-activated receptors in haemostasis, thrombosis and vascular biology. Journal of Thrombosis and Haemostasis 2005; 3: 2185-2192

Clifford S, Barber N, Elliott R, Hartley E, Horne R. Patient-centred advice is effective in improving adherence to medicines. Pharmacy World and Science 2006; 28: 165-170

Crafoord C. Preliminary report on postoperative treatment with heparin as a prevention of thrombosis. Acta Chirurgica Scandinavica 1937; 79: 407-426

Crafoord C, Jorpes E. Heparin as a prophylactic against thrombosis. Journal of the American Medical Association 1941; 116: 2831-2835

Crespin S, Bourrel R, Hurault-Delarue C, Lapeyre-Mestre M, Montastruc J-L, Damase-Michel C. Drug prescribing before and during pregnancy in south west France - a retrospective study. Drug Safety 2011; 34: 595-604

Crighton RW. Sulphate of iron as a local application in Phelgmasia doelns. British Medical Journal 1871: $466-467$

Crowther MA, Spitzer K, Julian J, Ginsberg J, Johnston M, Crowther R, Laskin C. Pharmacokinetic profile of a Low-Molecular Weight Heparin (Reviparin) in Pregnant Patients: a prospective cohort study. Thrombosis Research 2000; 98: 133-138

Crowther MA, Berry LR, Monagle PT, Chan AKC. Mechanisms responsible for the failure of protamine to inactivate low molecular weight heparin. British Journal of Haematology 2002; 116: 178-186 
Cumming AM, Jones GR, Wensley RT, Cundall RB. In vitro neutralisation of heparin in plasma prior to the activated partial thromboplastin time test: an assessment of four heparin antagonists and two anion exchange resins. Thrombosis Research 1986; 41: 43-56

Cushman M, Tsai AW, White RH, Heckbert SR, Rosamond WD, Enright P, Folsom AR. Deep vein thrombosis and pulmonary embolism in two cohorts: the longitudinal investigation of thromboembolism etiology. American Journal of Medicine 2004; 117: 19-25

Cutler DM, Everett W. Thinking outside the pillbox - medication adherence as a priority for healthcare reform. New England Journal of Medicine 2010; 362: 1553-1555

D'Argenio D. Optimal sampling times for pharmacokinetic experiments. Journal of Pharmacokinetics and Biopharmaceutics 1981; 9: 739-756

Danielsson A, Raub E, Lindahl U, Bjork I. Role of ternary complexes, in which heparin binds both antithrombin and proteinase, in the acceleration of the reactions between antithrombin and thrombin or factor Xa. Journal of Biological Chemistry 1986; 261:15467-15473

Dargaud Y, Trzeciak MC, Bordet JC, Ninet J, Negrier C. Use of calibrated automated thrombinography +/- thrombomodulin to recognise the prothrombotic phenotype. Thrombosis and Haemostasis 2006; 96:562-7

Dargaud Y, Luddington R, Gray E, Negrier C, Lecompte T, Petros S, Hogwood J, Bordet JC, Regnault V, Siegemund A, Baglin T. Effect of standardization and normalization on imprecision of calibrated automated thrombinography: an international multicentre study. British Journal of Haematology 2007; 139: 303-9

Dargaud Y, Francillon S, Negrier C. Intra-individual thrombin generation measurement variability in healthy adults over a one year period. Thrombosis Research 2009; 124:237-8

Dargaud Y, Hierso S, Rugeri L, Chatard B, Battie C, Gaucherand P, Negrier C, Trzeciak MC. Endogenous thrombin potential, prothrombin fragment $1+2$ and D-dimers during pregnancy. Thrombosis and Haemostasis 2010; 103: 469-471

Dargaud $Y$, Wolberg AS, Luddington R, regnault V, Spronk H, Baglin T, Lecompte T, Cate HT, Negrier C. Evaluation of a standardized protocol for thrombin generation measurement using the calibrated automated thrombogram: An international multicentre study. Thombosis Research 2012; 130: 929-934

Davis HE, Morgan JR, Yarmush ML. Polybrene increases retrovirus gene transfer efficiency by enhancing receptor-independent virus adsorption on target cell membranes. Biophysical Chemistry 2002; 97: 159-172 
Davis DA, Thomson MA, Oxman AD, Haynes RB. Changing physician performance: a systematic review of the effect of continuing medical education strategies. Canadian Medical Association Journal 1995; 274: 700-705

Dielis AW, Castoldi E, Spronk HM, van Oerle R, Hamulyak K, Ten Cate H, Rosing J. Coagulation factors and the protein $\mathrm{C}$ system as determinants of thrombin generation in a normal population. Journal of Thrombosis and Haemostasis 2008; 6:125-31

Demoly P, Piette V, Daures JP. Treatment of allergic rhinitis during pregnancy. Drugs 2003; 63: 1813-1820

Derlon A, Le Querrec A, Borg JY, Mosquet B, Thomas M. Thrombopénies induites par l'héparine: symptomatologie, détection, fréquence. Therapie 1988; 43: 199-203 (In French; in Martindale - Pharmaceutical Press)

de Marco L, Mazzucato M, Masotti A, Z M Ruggeri. Localisation and characterisation of alphathrombin-binding site on platelet glycoprotein lb alpha. The Journal of Biological Chemistry 1994; 269: 6478-6484

de Moerloose P, Amiral J, Vissac AM, Reber G. Longitudinal study on activated factors XII and VII levels during normal pregnancy. British Journal of Haematology 1998; 100: 40-4

de Romeuf C, Mazurier C. Heparin binding assay of von Willebrand factor(vWF) in plasma milieu - evidence of the importance of the multimerization degree of VWF. Thrombosis and Haemostasis 1993; 69: 436-440

de Sancho MT, Khalid S, Christos PJ. Outcomes in women receiving low-molecular-weight heparin during pregnancy. Blood Coagulation and Fibrinolysis 2012; 23: 751-755

de Smedt, Hemker HC. Thrombin generation is extremely sensitive to pre-heating conditions. Journal of Thrombosis and Haemostasis 2011; 9: 233-234

de Swart CAM, Nijmeyer B, Roelofs JMM, Sixma JJ. Kinetics of intravenously administered heparin in normal humans. Blood 1982; 60: 1251-1258

de Swiet M, Bulpitt CJ, Lewis PJ. How obstetricians use anticoagulants in the prophylaxis of thromboembolism. Journal of Obstetrics and Gynaecology 1980; 1: 29-32

Dexter L, Folch-Pi W. Venous thrombosis. An account of the first documented case. Journal of the American Medical Association 1974; 228: 195-196

Dickson BC. Venous thrombosis: on the history of Virchow's triad. University of Toronto Medical Journal 2004; 81: 166-171 
DiMatteo MR. Variations in patients' adherence to medical recommendations: a quantitative review of 50 years of research. Medical Care 2004; 42: 200-209

Drake TA, Morrissey JH, Edgington TS. Selective cellular expression of tissue factor in human tissues. Implications for disorders of haemostasis and thrombosis. American Journal of Pathology 1989; 134:1087-97

Duchemin J, Pan-Petesch B, Arnaud B, Blouch MT, Abgrall JF. Influence of coagulation factors and tissue factor concentration on the thrombin generation test in plasma. Thrombosis and Haemostasis 2008; 99:767-73

Duffull SB, Wright DFB, Winter HR. Interpreting population pharmacokinetic-pharmacodynamic analyses - a clinical viewpoint. British Journal of Clinical Pharmacology 2011; 71: 807814

Duffull SB, Waterhouse T, Eccleston J. Some considerations on the design of population pharmacokinetic studies. Journal of Pharmacokinetics and Pharmacodynamics 2005; 32: $441-457$

Dykstra K, Pugh R, Krause A. Visualisation concepts to enhance quantitative decision making in drug development. The Journal of Clinical Pharmacology 2010; 50: 130s-139s

Efron B, Gong G. A leisurely look at the bootstrap, the jack-knife and cross validation. The American Statistician 1983; 37: 36-48

Egan TD, Muir KT, Hermann DJ, Stanski DR, Shafer SL. The electroencephalogram (EEG) and clinical measures of opiod potency: defining the EEG-clinical potency relationship ("fingerprint") with application to remifentanil. International Journal of Pharmaceutical Medicine 2001; 15: 11-19

Eichinger S, Weltermann A, Philipp K, Hafner E, Kaider A, Kittl EM, Brenner B, Mannhalter C, Lechner K, Kyrle PA. Prospective evaluation of haemostatic system activation and thrombin potential in healthy pregnant women with and without Factor $\mathrm{V}$ Leiden. Thrombosis and Haemostasis 1999; 82: 1232-1236

Einarson A, Selby P, Koren G. Abrupt discontinuation of psychotropic drugs during pregnancy: fear of teratogenic risk and impact of counselling. Journal of Psychiatry and Neuroscience $2001 ; 26: 44-48$ 
Ellison J, Walker ID, Greer IA. Antenatal use of enoxaparin for prevention and treatment of thromboembolism in pregnancy. British Journal of Obstetrics and Gynaecology 2000; 107: $1116-1121$

Engeland A, Bramness JG, Daltveit AK, Ronning M, Skurtveit S, Furu K. Prescription drug use among fathers and mothers before and during pregnancy. A population-based cohort study of 106,000 pregnancies in Norway 2004-2006. British Journal of Clinical Pharmacology 2008; 65: 653-660

Ensom MHH, Stephenson MD. Pharmacokinetics of Low Molecular Weight Heparin and Unfractionated Heparin in Pregnancy. Journal of the Society for Gynaecological Investigation 2004; 11: 377-83

Esler R. Two cases of Phlegmasia dolens in the right lower extremity. British Medical Journal 1884: $463-464$

Ette El, Williams PJ, Kim YH, Lane JR, Lui MJ, Capparelli EV. Model appropriateness and PPK modelling. Journal of Clinical Pharmacology 2003; 43: 610-623

Ette El, Williams PJ. Population pharmacokinetics II: Estimation methods. Annals of Pharmacotherapy 2004; 38: 1907-1915

Ette El, Williams PJ, Lane JR. Population pharmacokinetics III: Design, analysis, and application of population pharmacokinetic studies. Annals of Pharmacotherapy 2004; 38: 2136-2144

European Medicines Agency, Evaluation of Medicines for Human Use. Innovative Drug Development Approaches, final report from the EMEA/CHMP think tank group on innovative drug development, 2007, accessed online 27/08/2011 at http://www.ema.europa.eu/docs/en_GB/document_library/Other/2009/10/WC500004913. pdf

Fedullo PF, Auger WR, Kerr KM, Rubin LJ. Chronic thromboembolic pulmonary hypertension. New England Journal of Medicine 2001; 345: 1465-1472

Feng Y, Green B, Duffull SB, Kane-Gill SL, Bobek MB, Bies RR. Development of a dosage strategy in patients receiving enoxaparin by continuous intravenous infusion using modelling and simulation. British Journal of Clinical Pharmacology 2006; 62: 165-176

Findley P. Puerperal thrombophlebitis. American Journal of Obstetrics 1912; 16: 987-995

Flessa HC, Kapstrom AB, Glueck HI, Will JJ. Placental transport of heparin. American Journal of Obstetrics and Gynaecology 1965; 93: 570-57 
Food and Drug Administration (US). Innovation or Stagnation: Challenge and Opportunity on the Critical Path to New Medical Products Report, 2004. Accessed online on 27/08/2011 at http://www.fda.gov/ScienceResearch/SpecialTopics/CriticalPathInitiative/CriticalPathOpp ortunitiesReports/ucm077262.htm

Forestier F, Daffos F, Capella-Pavlovsky M. Low molecular weight heaprin (PK 10169) does not cross the placenta during the second trimester of pregnancy: study by direct fetal blood sampling under ultrasound. Thrombosis Research 1984; 34: 557-560

Forestier F, Daffos F, Rainaut M, Toulemonde F. Low molecular weight heparin (CY 216) does not cross the placenta during the third trimester of pregnancy. Thrombosis and Haemostasis 1987; 57: 234

Fowlie J, Stanton PD, Anderson JR. Heparin-associated skin necrosis. Postgraduate Medical Journal 1990; 66: 573-5

Fox NS, Laughon SK, Bender SD, Saltzman DH, Rebarber A. Anti-factor Xa plasma levels in pregnant women receiving low molecular weight heparin thromboprophylaxis. Obstetrics and Gynecology 2008; 112: 884-889

Francalanci I, Comeglio P, Liotta AA, Cellai AP, Fedi S, Parretti E, Mello G, Prisco D, Abbate R. $\mathrm{D}$-dimer in intra-uterine growth retardation and gestational hypertension. Thrombosis Research 1995; 80: 89-92

Frederiksen MC. Physiologic changes in pregnancy and their effect on drug disposition. Seminars in Perinatology 2001; 25: 120-123

Friedman Y, Arsenis C. Studies on the heparin sulphamidase activity from rat spleen: intracellular distribution and characterisation of the enzyme. Biochemical Journal 1974; 139: 699-708

Friedrich E, Hameed AB. Fluctuations in anti-factor Xa levels with therapeutic enoxaparin anticoagulation in pregnancy. Journal of Perinatology 2010; 30: 253-257

Fritsch P, Kleber M, Rosenkranz A, Fritsch M, Muntean W, Mangge H, Reinehr T. Haemostatic alterations in overweight children: Associations between metabolic syndrome, thrombin generation, and fibrinogen levels. Atherosclerosis 2010; 212: 650-5

Furie B, Furie BC. Mechanisms of thrombus formation. New England Journal of Medicine 2008; 359: $938-949$

Gatt A, van Veen JJ, Cooper P, Kitchen S, Makris M. Protein C deficiency screening using a thrombin generation assay - an upgrade. Thrombosis and Haemostasis 2007; 98: 691692 
Gagne JJ, Maio V, Berghella V, Louis DZ, Gonnella JS. Prescription drug use during pregnancy: a population-based study in Regione Emilia-Romagna, Italy. European Journal of Clinical Pharmacology 2008; 64: 1125-1132

Gafney PJ, Creighton LJ, Callus M, Thorpe R. Monoclonal antibodies to cross-linked fibrin degradation products (XL-FDP). II. Evaluation in a variety of clinical conditions. British Journal of Haematology 1988; 68: 91-96

Ganzevoort W, Rep A, Bonsel GJ, de Vries JL, Wolf H. Plasma volume and blood pressure regulation in hypertensive pregnancy. Journal of Hypertension 2004; 22: 1235-1242

Gerotziafas GT, Depasse F, Busson J, Leflem L, Elalamy I, Samama MM. Towards a standardization of thrombin generation assessment: the influence of tissue factor, platelets and phospholipids concentration on the normal values of ThrombogramThrombinoscope assay. Thrombosis Journal 2005; 3:16

Gerotziafas GT, Petropoulou AD, Verdy E, Samama MM, Elalamy I. Effect of the anti-factor Xa and anti-factor lla activities of low-molecular weight heparins upon the phases of thrombin generation. Journal of Thrombosis and Haemostasis 2007; 5: 955-962

Giavarina D, Mezzena G, Dorizzi RM, Soffiati G. Reference interval of D-dimer in pregnant women. Clinical Biochemistry 2001; 34: 331-333

Gillespie WR. Non-compartmental versus compartmental modelling in clinical pharmacokinetics. Clinical Pharmacokinetics 1999; 20: 253-262

Gillis S, Shushan A, Eldor A. Use of low molecular weight heparin for prophylaxis and treatment and treatment of thromboembolism in pregnancy. International Journal of Gynecology and Obestetrics 1992; 39: 297-301

Ginsberg JS, Hirsh J, Turner CD, Lebine MN, Burrows R. Risks to the foetus of anticoagulant therapy during pregnancy. Thrombosis and Haemostasis 1989a; 61: 197-203

Ginsberg JS, Kowalchuk G, Hirsh J, Brill-Edwards P, Burrows R. Heparin therapy during pregnancy: risks to the fetus and mother. Archives of Internal Medicine 1989b; 149: 22332236

Glimelius B, Busch C, Hook M. Binding of heparin on the surface of cultured human endothelial cells. Thrombosis Research 1978; 12: 773-782

Gobburu JVS. Pharmacometrics 2020. Journal of Clinical Pharmacology 2010; 50: 151s-157s 
Godlowski, Z.: Die Heparin (Antiprothrombin) Verwendung zwecks Funktionsprufung des menschlichen retikulo-endothelialen Systems. Wien. med. Wchnschr. 83: 1034, 1933 In: Best $\mathrm{CH}$. Preparation of heparin and its use in the first clinical cases. Circulation 1959; 19: $79-86$

Goldhaber SZ, Vissani L, De Rosa M, for ICOPER. Acute pulmonary embolism: clinical outcomes in the International Cooperative Pulmonary Embolism Registry (ICOPER). Lancet 1999; 353: $1386-1389$

Goodman A, Schorge J, Greene MF. The long-term effects of in-utero exposures - the DES story. New England Journal of Medicine 2011; 364: 2083-2084

Goodrich SM, Wood JE. Peripheral venous distensibility and velocity of venous blood flow during pregnancy or during oral contraceptive therapy. American Journal of Obstetrics and Gynecology 1964; 90: 740-744

Gouin-Thibault I, Dingler E, Maris FA, Samama MM. In vitro interaction of the pentasaccharide idraparinux (SanOrg 34006) with unfractionated heparin. Journal of Thrombosis and Haemostasis 2003; 1: 2054-2056

Green B, Duffull SB. Developing a dosing strategy for enoxaparin in obese patients. British Journal of Clinical Pharmacology 2003; 56: 96-103

Green B, Greenwood M, Saltissi D, Westhuyzen J, Kluver L, Rowell J, Atherton J. Dosing strategy for enoxaparin in patients with renal impairment presenting with acute coronary syndromes. British Journal of Clinical Pharmacology 2004; 59: 281-290

Greer IA, Nelson-Piercy C. Low-molecular weight heparins for thromboprophylaxis and treatment of venous thromboembolism in pregnancy: a systematic review of safety and efficacy. Blood 2005; 106: 401-407

Green L, Lawrie AS, Patel S, Hossain F, Chitolie A, Mackie IJ, Haddad FS, Machin SJ. The impact of elective knee/hip replacement surgery and thromboprophylaxis with rivaroxaban or dalteparin on thrombin generation. British Journal of Haematology 2010; 151: 469-476

Greets WH, Bergqvist D, Pineo GF, Heit JA, Samama CM, Lassen MR, Colwell CW. Prevention of Venous Thromboembolism - American College of Chest Physicians Evidence Based Clinical Practice Guidelines ( $8^{\text {th }}$ Edition). Chest 2008; 133: 381s-453s

Greer IA. Thrombosis in pregnancy: maternal and fetal issues. Lancet 1999; 353: 1258-1265

Greer IA, Hunt BJ. Low molecular weight heparin in pregnancy: current issues. British Journal of Haematology 2005; 128: 593-601 
Haidl H, Cimenti C, Leschnik B, Zach D, Muntean W. Age-dependency of thrombin generation measured by means of calibrated automated thrombography (CAT). Thrombosis and Haemostasis 2006; 95:772-5

Hale MD, Nicholls AJ, Bullingham RE, et al. The pharmacokinetic-pharmacodynamic relationship for mycophenolate mofetil in renal transplant. Clinical Pharmacology and Therapeutics 1998; 64: 672-683

Hall JAG, Paul RM, Wilson KM. Maternal and fetal sequelae of anticoagulation during pregnancy. American Journal of Medicine 1980; 68: 122-140

Haller JA, Ransdell HT, Stowens D, Rubel WF. Renal toxicity of polybrene in open heart surgery. Journal of Thoracic and Cardiovascular Surgery 1962; 44: 486-491

Han PY, Duffull SB, Kirkpatrick CMJ, Green B. Dosing in obesity: a simple solution to a big problem. Clinical Pharmacology and Therapeutics 2007; 82: 505-508

Hanley MJ, Abernethy DR, Greenblatt DJ. Effect of obesity on the pharmacokinetics of drugs in humans. Clinical Pharmacokinetics 2010; 49: 71-87

Harenberg J. Pharmacology of low molecular weight heparins. Seminars in Thrombosis and Haemostasis 1990;16(Suppl):12-18

Harenberg J, Stehle G, Augustin J, Zimmermann R. Comparative human pharmacology of low molecular weight heparins. Seminars in Thrombosis Haemostasis 1989; 15: 414-422

Hartman-Craven B, Christofides A, O'Connor DL, Zlotkin S. Relative bioavailability of iron and folic acid from new powered supplement compared to a traditional tablet in pregnant women. BMC Pregnancy Childbirth 2009; 9: 33

Hawwa AF, Collier PS, Millership JS, McCarthy A, Dempsey S, Cairns C, McElnay JC. Population pharmacokinetic and pharmacogenetic analysis of 6-mercaptopurine in paediatric patients with acute lymphoblastic leukaemia. British Journal of Clinical Pharmacology 2008; 66: 826-837

Hebert MF, Ma X, Naraharisetti SB, Krudys KM, Umans JG, Hankins GDV, Caritis SN, Miodovnik M, Mattison DR, Unadkat JD, Kelly EJ, Blough D, Cobelli C, Ahmed MS, Snodgrass WR, Carr DB, Easterling TR, Vicini $P$ for the obstetric-fetal pharmacology research unit network. Are we optimising gestational diabetes treatment with glyburide? The Pharmacologic basis for better clinical practice. Clinical Pharmacology and Therapeutics 2009; 85: 607- 614 
Hedenius $\mathrm{P}$, Wilander 0 . The influence of intravenous injections of heparin in man on the time coagulation. Acta Medica Scandinavica 1936; 88: 443-449

Heit JA, Silverstein MD, Mohr DN, Petterson TM, O'Fallon WM, Melton LJ III. Predicators of survival after deep vein thrombosis and pulmonary embolism: A population-based cohort study. Archives of Internal Medicine 1999; 159: 445-453

Heit JA, Mohr DN, Silverstein MD, Petterson TM, O'Fallon WM, Melton LJ III. Predictors of recurrence after deep vein thrombosis and pulmonary embolism: a population-based cohort study. Archives of Internal Medicine 2000; 160: 761-768

Heit JA. Venous thromboembolism: disease burden, outcomes and risk factors. Journal of Thrombosis and Haemostasis 2005; 3: 1611-1617

Hellgren M, Blomback M. Studies on blood coagulation and fibrinolysis in pregnancy, during delivery and in the puerperium. I. Normal condition. Gynaecologic and Obstetric Investigation 1981; 12: 141-54

Hemker HC, Wielders S, Kessels H, Béguin S: Continuous registration of thrombin generation in plasma, its use for the determination of the thrombin potential. Thrombosis and Haemostasis 1993; 70: 617-624

Hemker HC, Beguin S. Phenotyping the clotting system. Thrombosis and Haemostasis 2000; 84 $747-751$

Hemker HC, Giesen P, AlDieri R, Regnault V, de Smed E, Wagenvoord R, Lecompte T, Beguin $S$. The calibrated automated thrombogram (CAT): a universal routine test for hyper- and hypo-coagulability. Pathophysiology of Haemostasis and Thrombosis 2002; 32: 249-253

Hemker HC, Giesen P, AIDieri R, Regnault V, de Smedt E, Wagenvoord R, Lecompte T, Beguin S. Calibrated automated thrombin generation measurement in clotting plasma. Pathophysiology of Haemostasis and Thrombosis 2003; 33: 4-15

Hemker HC, AlDieri R, De Smedt E, Beguin S. Thrombin generation, a function test of the haemostatic-thrombotic system. Thrombosis and Haemostasis 2006; 96: 553-561

Hemker HC, Giesen PL, Ramjee M, Wagenvoord R, Beguin S. The thrombogram: monitoring thrombin generation in platelet rich plasma. Thrombosis and Haemostasis 2000; 83: 589591

Hempel G, Muller H-J, Lanvers-Kaminsky C, Wurthwein G, Hoppe A, Boos J. a population pharmacokinetic model for pegylated-asparaginase in children. British Journal of Haematology 2009; 148: 119-125 
Hinkley DV. Jackknife confidence limits using Student $t$ approximations. Biometrika 1977; 64: 2128

Hill CC, Pickinpaugh J. Physiologic changes in pregnancy. Surgical Clinics of North America 2008; 88: 391-401

Hirsh J, Cade JF, O'Sullivan EF. Clinical experience with anticoagulant therapy during pregnancy. British Medical Journal 1970; 1: 270-273

Hirsh J, Bauer KA, Donati MB, Gould M, Samama MM, Weitz JI. Parenteral Anticoagulants American College of Chest Physicians Evidence-Based Clinical Practice Guidelines (8th Edition). Chest 2008; 133: 141S-159S

Hirt D, Urien S, Ekouevi DK, Rey E, Arrive E, Blanche S, Amani-Bosse C, Nerrienet E, Gray G, Kone M, Leang SK, Mclntyre J, Dabis F, Treluyer J-M. Population pharmacokinetics of tenofovir in HIV-1-infected pregnant women and their neonates (ANRS 12109). Clinical Pharmacology and Therapeutics 2008; 85: 182-189

Hodgson J. Case of acute Phlegmasia dolens. Lancet 1835: 73-74

Hoke M, Kyrle PA, Philipp K, Pabinger I, Kaider A, Schonauer V, Quehenberger P, Eichinger S. Prospective evaluation of coagulation activation in pregnant women receiving low molecular weight heparin. Thrombosis and Haemostasis 2004; 91: 935-940

Hooker AC, Staatz CE, Karlsson MO. Conditional weighted residuals (CWRES): a model diagnostic for the FOCE method. Pharmaceutical Research 2007; 24: 2187-2197

Hooker A, Staatz CE, Karlsson MO. Conditional weighted residuals, an improved model diagnostic for the FO/FOCE methods. Oral presentation: Stuart Beal Methodology Session; Annual Meeting of the Population Group in Europe; Page 15 (2006) Abstract 1001 [ www.page-meeting.org/?abstract=1101]

Horne MK III, Chao ES. The effect of molecular weight on heparin binding to platelets. British Journal of Haematology 1990; 74: 306-312

Horne R, Weinman J, Hankins M. The beliefs about medicines questionnaire: the development and evaluation of a new method for assessing the cognitive representation of medication. Psychology and Health 1999; 14: 1-24

Horne R, Weinman J, Barber N, Elliott R, Morgan M. Concordance, adherence and compliance in medicine taking. Report for the National Co-ordinating Centre for NHS Service Delivery and Organisation R \& D (NCCSDO), December 2005 
Howell WH, Holt E. Two new factors in blood coagulation - heparin and pro-antithrombin. American Journal of Physiology 1918; 328-341

Huang ZF, Wun T-C, Broze GJ. Kinetics of factor Xa inhibition by tissue factor pathway inhibitor. Journal of Biological Chemistry 1993; 268: 26950-26955

Hulot J-S, Montalescot G, Lechat P, Collet J-P, Ankri A, Urien S. Dosing strategy in patients with renal failure receiving enoxaparin for the treatment of non-ST-segment elevation acute coronary syndrome. Clinical Pharmacology and Therapeutics 2005; 77: 542-552

Hunt BJ, Doughty H, Majurndar G, Copplestone A, Kerslake S, Buchanan N, Hughes G, Khamashta M. Thromboprophylaxis with low molecular weight heparin (dalteparin) in high risk pregnancies. Thrombosis and Haemostasis 1997; 77: 39-43

Hunt BJ, Gattens M, Khamashta M, Nelson-Piercy C, Almeida A. Thromboprophylaxis with unmonitored intermediate-dose low molecular weight heparin in pregnancies with a previous arterial or venous thrombotic event. Blood Coagulation and Fibrinolysis. 2003; 14: $735-9$

Huxtable LM, Tafreshi MJ, Ondreyco SM. A protocol for the use of enoxaparin during pregnancy: results from 85 pregnancies including 13 multiple gestation pregnancies. Clinical Applied Thrombosis and Haemostasis. 2005; 11: 171-181

Hynes E, Knaggs K, McCourt S, Bomken C, Loughney AD, Kesteven P, Hanley JP. Endogenous thrombin potential: profiles in normal pregnancy and prothrombotic pregnant women. Journal of Thrombosis and Haemostasis 2009; Suppl 2: PP-WE-372 (abstract)

Hytten FE, Leitch I. The Phsiology of Human Pregnancy. Blackwell Scientific Publications Ltd, Oxfordshire, UK (1971)

Hytten FE. Physiological adjustments in pregnancy. WB Saunders, PA, USA (1975)

Ickovics JR, Wilson TE, Royce RA, Minkoff HL, Fernandez MI, Fox-Tierney R, Koenig LJ for the Perinatal Guidelines Evaluation Group. Pre-natal and postnatal zidovudine adherence among pregnant women with HIV. Journal of Acquired immune Deficiency Syndromes 2002; 30: 311-315

Ing GM, Olman CL, Oyd JR. Drug-Induced (Thalidomide) Malformations. Canadian Medical Association Journal1962; 87: 1259-62

Ip BK, Thomson AR, Moriarty HT. A comparison of the sensitivity of APTT reagents to the effects of enoxaparin, a low molecular weight heparin. Pathology 2001; 33: 347-352 
Ismail SK, Norris L, Muttukrishna S, Higgins JR. Thrombin generation post elective caesarean section: effects of low molecular weight heparin. Thrombosis Research 2012, doi:10.1016/j.thromres.2012.01.008

Jackevicius CA, Anderson GM, Leiter L, Tu JV. Use of Statins in patients after acute myocardial infarction. Archives of Internal Medicine 2001; 161: 183-188

Jackson CM, Esnouf MP, Lindahl TL. A critical evaluation of the prothrombin time for monitoring oral anticoagulant therapy. Pathophysiology of Haemostasis and Thrombosis 2003; 33: $43-51$

Jacobsen AF, Qvigstad E, Sandset PM. Low molecular weight heparin (dalteparin) for the treatment of venous thromboembolism in pregnancy. British Journal of Obstetrics and Gynaecology 2003; 110:139-44

Jacobsen AF, Skjeldestad FE, Sandset PM. Incidence and risk patterns of venous thromboembolism in pregnancy and puerperium - a register-based case-control study. American Journal of Obstetrics and Gynecology 2008; 198: 233.e231-233.e237

Jacobsen EM, Trettenes EJ, Wisloff F, Abildgaard U. Detection and quantification of lupus anticoagulants in plasma from heparin treated patients, using addition of polybrene. Thrombosis Journal 2006; doi:10.1186/1477-9560-4-3

James AH, Tapson VF, Goldhaber SZ. Thrombosis during pregnancy and the postpartum period. American Journal of Obstetrics and Gynecology 2005; 193: 216-219

James AH, Jamison MG, Brancazio LR, Myers ER. Venous thromboembolism during pregnancy and the postpartum period: incidence, risk factors, and mortality. American Journal of Obstetrics and Gynecology 2006; 194: 1311-1315

James AH. Pregnancy-associated thrombosis. Hematology: American Society of Hematology Educational Programme 2009; 1: 277-285

James AH, Brancazio LR, Gehrig TR, Wang A, Ortel TL. Low molecular weight heparin for thromboprophlaxis in pregnant women with mechanical heart valves. Journal of Maternal Fetal Neonatal Medicine 2006; 19: 543-549

Jensen D, Duffin J, Lam YM, Webb KA, Simpson JA, Davies GAL, Wolfe LA, O'Donnell DE. Physiological mechanisms of hyperventilation during human pregnancy. Respiratory Physiology and Neurobiology 2008; 161: 76-86

Jin RC, Voetsch B, Loscalzo J. Endogenous mechanisms of inhibition of platelet function. Microcirculation 2005; 12: 247-258

Johnson HJ. Westminster Medical Society. British Medical Journal 1842: 317-318 
Johnson EA, Mulloy B. The molecular weight of commercial heparin preparations. Carbohydrate Research 1976; 51: 127-199

Johnson EA, Kirekwood TBL, Stirling Y, Perez-Requejo JL, Ingram GI, Bangham DR, Brozović M. Four heparin preparations: anti-Xa potentiating effect of heparin after subcutaneous injection. Thrombosis Haemostasis 1976; 35: 586-591

Jordan RE, Oosta GM, Gardner WT, Rosenberg RD. The kinetics of hemostatic enzymeantithrombin interactions in the presence of low molecular weight heparin. Journal of Biological Chemistry 1980; 255: 10081-10090

Kane-Gill SL, Feng Y, Bobek MB, Bies RR, Pruchnicki MC, Dasta JF. Administration of enoxaparin by continuous infusion in a naturalistic setting: analysis of renal function and safety. Journal of Clinical Pharmacy and Therapeutics 2005; 30: 207-213

Kanis JA. Heparin in the treatment of pulmonary embolism. Thrombosis et diathesis haemorrhagica $1974 ; 32: 519$

Karlsson MO, Sheiner LB. The importance of modelling inter-occasion variability in population pharmacokinetic analyses. Journal of Pharmacokinetics and Biopharmaceutics 1993; 21: 735-750

Kearon C, Kahn SR, Agnelli G, Goldhaber S, Raskob. Antithrombotic Therapy for Venous Thromboembolic Disease - American College of Chest Physicians Evidenced Based Clinical Practice Guidelines ( $8^{\text {th }}$ Edition). Chest 2008; 133: 454s-545s

Keats AS, Cooley DA, Telford J. Relative anti-heparin potency of polybrene and protamine in patients undergoing extracorporeal circulation. Journal of Thoracic and Cardiovascular Surgery 1959; 38: 362-368

Keeling D, Davidson S, Watson H, on behalf of the Haemostasis and Thrombosis Task Force of the British Committee for Standards in Haematology. The management of heparin induced thrombocytopenia. British Journal of Haematology 2006; 133: 259-69

Keizer RJ, Jansen RS, Rosing H, Beijnen JH, Schellens JHM, Huitema ADR. Incorporation of extrapolated concentration data below the limit of quantification in population PK analyses. Presented as a poster at the Population Approach Group in Europe Annual Meeting. Page 19 (2010) Abstr 1722 [www.page-meeting.org/?abstract=1722]

Kelly J, Hunt BJ. Role of D-dimers in diagnosis of venous thromboembolism. Lancet 2002; 359: 456-458

Kernohan RJ, Todd C. Heparin therapy in thromboembolic disease. Lancet 2007; 1: 621-623 
Kessler C. Low molecular weight heparins: practical considerations. Seminars in Haematology $1997 ; 34: 35-42$

Keularts IM, Zivelin A, Seligsohn U, Hemker HC, Beguin S. The role of factor XI in thrombin generation induced by low concentrations of tissue factor. Thrombosis and Haemostasis 2001; 85: 1060-1065

Key N, Makris M, O'Shaughnessy D, Lillicrap D (editors). Practical Haemostasis and Thrombosis $2^{\text {nd }}$ ed, Wiley-Blackwell 2009

Kline JA, Williams GW, Hernandez-Nino J. D-dimer concentrations in normal pregnancy: new diagnostic thresholds are needed. Clinical Chemistry 2005; 51: 825-829

Knight M; UKOSS. Antenatal pulmonary embolism: risk factors, management and outcomes. British Journal of Obstetrics and Gynaecology 2008; 115: 453-61

Knol HM, Schultinge L, Veeger NJGM, Kluin-Nelemans, Erwich JJHM, Meijer K. The risk of postpartum hemorrhage in women using high dose of low molecular weight heparins during pregnancy. Thrombosis Research 2012; 130: 334-338

Lalonde RL, Kowalski KG, Hutmacher MM, Ewy W, Nichols DJ, Milligan PA, Corrigan BW, Lockwood PA, Marshall SA, Benincosa LJ, Tensfeldt TG, Parivar K, Amaantea M, Glue $\mathrm{P}$, Koide $\mathrm{H}$, Miller R. Model-based drug development. Clinical Pharmacology and Therapeutics 2007; 82: 21-32

Lam LH, Silbert JE, Rosenberg RD. The separation of active and inactive forms of heparin. Biochemical and Biophysical Research Communications 1976; 69: 570-577

Lane DA. Heparin binding and neutralising proteins. In: Lane DA, Lindahl U, eds. Heparin: chemical and biological properties, clinical applications. Boca Raton, Fla.: CRC Press, 1989: 363-391

Lebaudy C, Hulot JS, Amoura Z, Costedoat-Chalumeau N, Serreau R, Ankri A, Conrad J, Cornet A, Dommergues M, Piette JC, Lechat P. Changes in enoxaparin pharmacokinetics during pregnancy and implications for antithrombotic therapeutic strategy. Clinical Pharmacology and Therapeutics 2008; 84: 370-377

Leizorovicz A, Simonneau G, Decousus H, Boissel JP. Comparison of efficacy and safety of low molecular weight heparins and unfractionated heparin in initial treatment of deep venous thrombosis: a meta analysis. British Medical Journal 1994; 309: 299-304

Lensing AWA, Prins MH, Davidson BL, Hirsh J. Treatment of deep venous thrombosis with low molecular weight heparins: a meta-analysis. Archives of Internal Medicine 1995; 155: 601-607 
Lepercq J, Conard J, Borel-Derlon A, Darmon JY, Boudignat O, Francoual C, Priollet P, Cohen

C, Yvelin N, Schved J-F, Tournaire M, Borg JY. Venous thromboembolism during pregnancy: a retrospective study of enoxaparin safety in 624 pregnancies. British Journal of Obstetrics and Gynaecology 2001; 108: 1134-1140

Letsky EA, de Swiet M. Thromboembolism during pregnancy and its management. British Journal of Haematology 1984; 57: 543-552

Li F, Nandy P, Chien S, Noel GJ, Tornoe CW. Pharmacometrics-based dose selection of levofloxacin as a treatment for post-exposure inhalational anthrax in children. Antimicrobial Agents Chemotherapy 2010; 54: 375-379

Lindheimer MD, Taler SJ, Cunningham FG; American Society of Hypertension. ASH position paper: hypertension in pregnancy. Journal of Clinical Hypertension 2009; 11: 214-225

Lim W, Eikelboom JW, Ginsberg JS. Inherited thrombophilia and pregnancy associated venous thromboembolism. BMJ 2007; 334: 1318-21

Lindqvist $\mathrm{P}$, Dahlback B, Marsal K. Thrombotic risk during pregnancy: a population study. Obstetrics and Gynecology 1999; 94: 595-599

Lillehei CW, Sterns LP, Long DM, Lepley D. Comparative study of polybrene and protamine for heparin neutralisation in open heart surgery. Annals of Surgery 1960; 151: 11-16

Lohsiriwat S, Imrittha N. Effect of posture on creatinine clearance in late pregnancy and after pregnancy. Journal of Obestetrics and Gynaecological Research 2008; 34: 337-342

Lord ST. Fibrinogen and fibrin: scaffold proteins in hemostasis. Current Opinion Haematology 2007; 14: 236-241

Luddington R, Baglin T. Clinical measurement of thrombin generation by calibrated automated thrombography requires contact factor inhibition. Journal of Thrombosis and Haemostasis 2004; 2:1954-9

Macfarlane RG, Biggs R. A thrombin generation test. Journal of Clinical Pathology 1953; 6: 3-7

Macklon NS, Greer IA, Bowman AW. An ultrasound study of gestational and postural changes in the deep venous system of the leg in pregnancy. British Journal of Obstetrics and Gynaecology 1997; 104: 191-197

Mahadoo J, Hiebert L, Jaques LB. Vascular sequestration of heparin. Thrombosis Research 1977; 12: 79-90 
Malek A, Mattison DR. Drug development for use during pregnancy: impact of the placenta. Expert reviews in Obstetrics and Gynaecology 2010; 5: 437-454

Mandema JW, Boyd RA, DiCarlo LA. Therapeutic index of anticoagulants for prevention of venous thromboembolism following orthopaedic surgery: a dose-response meta-analysis. Clinical Pharmacology and Therapeutics 2011; 90: 820-827

Mannucci PM. Venous thrombosis: the history of knowledge. Pathophysiology of Haemostasis and Thrombosis 2002; 32: 209-212

Marder VJ, Soulen RL, Atichartakarn V. Quantitative venographic assessment of deep vein thrombosis in the evaluation of streptokinase and heparin therapy. Journal of the Laboratory of Clinical Medicine 1977; 89: 1018-1029

Martindale: the complete drug reference. Ed: Sean Sweetman, Pharmaceutical Press, $37^{\text {th }}$ Edition, April 2011

Matok I, Gorodischer R, Koren G, Sheiner E, Wiznitzer A, Levy A. The safety of metoclopramide use in the first trimester of pregnancy. New England Journal of Medicine 2009; 360: 2528-2535

Matzsch T, Bergqvist D, Hedner U, Nilsson B, Ostergaard P. Effects of low molecular weight heparin and unfragmented heparin on induction of osteoporosis in rats. Thrombosis and Haemostasis 1990; 28:505-509

McLean J. The thromboplastic action of cephalin. American Journal of Physiology 1916; 250-257

McLintock C, McCowan L, North R. Maternal complications and pregnancy outcome in women with mechanical prosthetic heart valves treated with enoxaparin. British Journal of Obstetrics and Gynaecology 2009; 116: 1585-1592

McLintock C. Prosthetic heart valves, In Parvod and Hunt (Eds), The Obstetric Hematology Manual. Cambridge University Press 2010

McLintock C. Shirley Johnson Memorial Lecture: How I manage women with mechanical heart valves during pregnancy. Setting standards of care on thrombosis and haemostasis issues in women's health; $58^{\text {th }}$ Meeting of the Scientific \& Standardisation Committee of the International Society of Thrombosis and Haemostasis, $27^{\text {th }}$ June 2012

Mellins CA, Chu C, Malee K, Allison S, Smith R, Harris L, Higgins A, Zorrilla C, Landesman S, Serchuck L, LaRussa P. Adherence to antiretroviral treatment among pregnant and postpartum HIV-infected women. AIDS Care 2008; 20: 958-968 
Mengiardi S, Tsakiris DA, Lampert ML, Hersberger KE. Drug use problems with self-injected lowmolecular-weight heparins in primary care. European Journal of Clinical Pharmacology $2011 ; 67: 109-120$

Mentre F, Mallet A, Baccar D. Optimal design in random-effects regression models. Biometrika 1997; $65: 141-151$

Messmore HL, Fabbrini NJ, Coyne E, Balbale S, Crosby T, Love B, Iqbal O. Effectiveness of ploybrene neutralisation of heparin and low molecular weight heparin in plasma. Journal of Thrombosis and Haemostasis 2003; 1: Suppl 1: Abstract number: CD019

Middeldorp S. How I treat pregnancy related venous thromboembolism. Blood 2011; 118: 53945400

Melissari E, Parker CJ, Wilson NV, Monte G, Kanthou C, Pemberton KD, Nicolaides KH, Barrett JJ, Kakkar VV. Use of low molecular weight heparin in pregnancy. Thrombosis and Haemostasis 1992; 68:652-656

Miller RG. The jackknife: a review. Biometrika 1976; 63: 581-582

Mitchell AA, Gilboa SM, Werler MM, Kelley KE, Louik C, Hernandez-Diaz S, and the National Birth Defects Prevention Study. Medication use during pregnancy, with particular focus on prescription drugs: 1976-2008. American Journal of Obstetrics and Gynaecology 2011; 205: e1-8

Modder $\mathrm{J}$ and Fitzsimons KJ. CMACE/RCOG joint guideline: Management of women with obesity in pregnancy. Centre for Maternal and Child Enquiries and the Royal College of Obstetricians and Gynaecologists, March 2010

Monkovic DD, Tracy PB. Functional characterisation of human platelet-released factor $\mathrm{V}$ and its activation by factor Xa and thrombin. The Journal of Biological Chemistry 1990; 265: $17132-17140$

Monreal M, Vinas L, Monreal L, Laviv S, Lafoz E, Angles AM. Heparin-related osteoporosis in rats: a comparative study between unfractionated heparin and a low-molecular-weight heparin. Haemostasis 1990; 20:204-207

Morisky D, Green L, Levine D. Concurrent and predictive validity of a self-reported measure of medication adherence. Medical Care 1986; 24: 67-74

Morse M. Establishing a normal range for D-dimer levels through pregnancy to aid in the diagnosis of pulmonary embolism and deep vein thrombosis. Journal of Thrombosis and Haemostasis $2004 ; 2: 1202-1204$ 
Mould DR, Sweeney K, Duffull SB, Neylon E, Hamlin P, Horwitz S, Sirotnak F, Fleisher M,Saunders ME, O'Connor OA. A Population Pharmacokinetic and Pharmacodynamic Evaluation of Pralatrexate in Patients With Relapsed or Refractory Non-Hodgkin's or Hodgkin's Lymphoma. Clinical Pharmacology and Therapeutics 2009; 86: 190-196

Muir J, Andrew M, Hirsh J, Weitz JI, Young E, Deschamps P, Shaughnessy SG. Histomorphometric analysis of the effects of standard heparin on trabecular bone in vivo. Blood 1996; 88:1314-1320

Muir J, Hirsh J, Weitz J, Andrew M, Young E, Shaughnessy SG. A histomorphometric comparison of the effects of heparin and low-molecular weight heparin on cancellous bone in rats. Blood 1997; 89:3236-3242

Murphy N, Broadhurst D, Ezyani WN, Jabarudin W, Khashan A, Wallace B, Kenny L, O'Donoghue K. Cross-sectional study to identify normal ranges for D-dimers in pregnancy. American Journal of Obstetrics and Gynaecology 2011; Supplement: s320s321 (poster abstract 822)

Murray DWG. Heparin and thrombosis and major blood vessel surgery. Surgery, gynaecology and obstetrics $1941 ; 721: 341-344$

Nath CE, Shaw PJ,Trotman J, Zeng L, Duffull SB, Hegarty G, McLachlan AJ, Gurney H, Kerridge I, Kwan YL, Presgrave P,Tiley C, Joshua D, Earl J. Population pharmacokinetics of melphalan in patients with multiple myeloma undergoing high dose therapy. British Journal of Clinical Pharmacology 2010; 69: 484-497

National Institute for Health and Clinical Excellence. Venous Thromboembolic Diseases: the management of venous thromboembolic diseases and the role and of thrombophilia testing. NICE clinical guideline 144, June 2012 (accessed via www.nice.org.uk/cg144)

Nelson-Piercy C, Letsky EA, de Swiet M. Low molecular weight heparin for obstetric thromboprophylaxis: experience of sixty-nine pregnancies in sixty-one women at high risk. American Journal of Obstetrics and Gynaecology 1997; 176: 1062-1068

Nelson-Piercy C. Heparin-induced osteoporosis. Scandinavian Journal of Rheumatology 1998; 27 (suppl 107): 68-71

Nelson-Piercy C, Powrie R, Borg J-Y, Rodger M, Talbot DJ, Stinson J, Greer IA. Tinzaparin use in pregnancy: an international, retrospective study of the safety and efficacy profile. European Journal of Obstetrics and Gynaecology and Reproductive Biology 2011; 159: 293-299 
Nelson-Piercy C, Greer I. Anticoagulation with tinzaparin for women with mechanical valves in pregnancy: a retrospective case series. Thrombosis Research 2012: dx.doi.org/10.1016/j.thromres.2012.11.022

Ni'Ainle F, Wong A, Appleby N, Byrne B, Regan C, Hassan T, Milner M, O'Sullivan A, White B, O'Donnell J. Efficacy and safety of once daily low molecular weight heparin (tinzaparin sodium) in high risk pregnancy. Blood Coagulation and Fibrinolysis 2008; 19 : 689-692

Nolan TE, Smith RP, Devoe LD. Maternal plasma D-dimer levels in normal and complicated pregnancies. Obstetrics and Gynaecology 1993; 81: 235-238

Nordeng H, Ystrom E, Einarson A. Perception of risk regarding the use of medications and other exposures during pregnancy. European Journal of Clinical Pharmacology 2010; 66: 207214

Norris LA, Bonnar J, Smith MP, Steer PJ, Savidge G. Low molecular weight heparin (tinzaparin) therapy for moderate risk thromboprophylaxis during pregnancy - a pharmacokinetic study. Blood Coagulation, Fibrinolysis and Cellular Haemostasis 2004; 92: 791-796

Norwitz ER, Edusa V, Park JS. Maternal physiology and complications of multiple pregnancy. Seminars in Perinatology 2005; 29: 338-348

Ngu LSP, Etuk I, Caveen C, Nokes TJ, Copplestone JA,Thomas DW. Use of the thrombin generation assay (calibrated automated thrombogram) in enoxaparin (low molecular weight heparin) treated pregnant patients. British Journal of Haematology 2010; 149 (suppl 1): 38 (abstract)

Nunes V, Neilson J, O'Flynn N, Calvert N, Kuntze S, Smithson H, Benson J, Blair J, Bowser A, Clyne W, Crome P, Haddad P, Hemingway S, Horne R, Johnson S, Kelly S, Packham B, Patel M, Steel J. Clinical Guidelines and Evidence Review for Medicines Adherence: involving patients in decisions about prescribed medicines and supporting adherence. London: National Collaborating Centre for Primary Care and Royal College of General Practitioners 2009

Odegard OR, Lie M, Abilgaard U. Anti-factor Xa activity measured with amidolytic methods. Haemostasis 1976; 5: 265-275

Oliver JA, Monroe DM, Roberts HR, Hoffmann M. Thrombin activates factor XI on activated platelets in the absence of factor XII. Arteriosclerosis, Thrombosis, and Vascular Biology 1999; 19: 170-177

Olsson $\mathrm{P}$, Lagergren $\mathrm{H}$, Ek S. The elimination from plasma of intravenous heparin: an experimental study on dogs and humans. Acta Medica Scandinavica1963; 173: 619-630 
Okajima Y, Kanayama S, Maeda Y, Urano S, Kitani T, Watada M, Nakagawa N, ljichi H. Studies on the neutralising mechanism of antithrombin activity of heparin by protamine. Thrombosis Research 1981; 24: 21-29

Orfeo T, Brummel-Ziedins KE, Gissel M, Butenas S, Mann KG. The nature of the stable blood clot procoagulant activities. The Journal of Biological Chemistry 2008; 283: 9776-9786

Oster JR, Singer I, Fishman LM. Heparin-induced aldosterone suppression and hyperkalemia. American Journal of Medicine 1995; 98: 575-86

Pabinger I, Grafenhofer H, Kyrle PA, Quehenberger P, Mannhalter C, Lechner K, Kaider A. Temporary increase in the risk for recurrence during pregnancy in women with a history of venous thromboembolism. Blood 2002; 100: 1060-1062

Paniccia R, Prsico D, Bandinelli B, Fedi S, Giusti B, Pepe G, Abbate R. Plasma and serum levels of $\mathrm{D}$-dimer and their correlations with other haemostatic parameters in pregnancy. Thrombosis Research 2002; 257-262

Palareti G, Legnani C, Cosmi B, Valdré L, Lunghi B, Bernardi F, Coccheri S. Predictive value of D-dimer test for recurrent venous thromboembolism after anticoagulation withdrawal in subjects with a previous idiopathic event and in carriers of congenital thrombophilia. Circulation 2003; 108: 313-318

Parke J, Holford NH, Charles BG. A procedure for generating bootstrap samples for the validation of nonlinear mixed-effects populations models. Computer Methods and Programs in Biomedicine 1999; 59: 19-29

Patel JP, Hunt BJ. Where do we go now with LMWH use in obstetric care? Journal of Thrombosis and Haemostasis 2008; 6: 1461-1467

Patel JP, Roberts LN, Arya R. Anticoagulating obese patients in the modern era. British Journal of Haematology 2011; 155: 137-149

Pashos CL, Normand ST, Garfinkle JB, Newhouse JP, Epstein AM, McNeil BJ. Trends in the use of drug therapies in patients with acute myocardial infarction: 1988-1992. Journal of the American College of Cardiology 1994; 23: 1023-1030

Pavek P, Ceckova M, Straud F. Variation of drug kinetics in pregnancy. Current Drug Metabolism 2009; 10: 520-529

Peck CC, Sheiner LB, Comb DT, Marton CM, Melmon KL. Computer assisted digoxin therapy. New England Journal of Medicine 1973; 289: 441-446 
Peck CC, Beal SL, Sheiner LB, Nichols Al. Extended least squares nonlinear regression: a possible solution to the "choice of weights" problem in analysis of individual pharmacokinetic data. Journal of Pharmacokinetics and Biopharmaceutics 1984; 12: 545558

Pedersen PC, Ostergaard PB, Hedner U, Bergqvist D, Matzsch T. Pharmacokinetics of a low molecular weight heparin, Logiparin after intravenous and subcutaneous administration to healthy volunteers. Thrombosis Research 1991; 61: 477-497

Pengo V, Lensing AWA, Prins MH, Marchiori A, Davidson BL, Tiozzo F, Albanese P, Biasiolo A, Pegoraro $C$, lliceto $S$, Prandoni $P$. Incidence of chronic thromboembolic pulmonary hypertension after pulmonary embolism. New England Journal of Medicine 2004; 350: 2257-2264

Pharmacia. Dalteparin sodium - a summary of product characteristics 2009. Accessed on www.medicines.org.uk on the 21/04/2010

Philipson A, Sabath LD, Charles D. Erythromycin and clindamycin absorption and elimination in pregnant women. Clinical Pharmacology and Therapeutics 1976; 19: 68-77

Philipson A. Pharmacokinetics of antibiotics in pregnancy and labour. Clinical Pharmacokinetics 1979; 4: 297-309

Pillai G, Mentre F, Steimer J-L. Non-linear mixed effects modelling - from methodology and software development to driving implementation in drug development science. Journal of Pharmacokinetics and Pharmacodynamics 2005; 32: 161-183

Pitney WR, Dacie J. A simple method of studying the generation of thrombin in recalcified plasma. Journal of Clinical Pathology 1953; 6: 9-13

Playfair WS. Observations on thrombosis and embolism of the pulmonary artery as a cause of death during the puerperal state. Lancet 1867: 66, 93-95, 153-155

Playfair WS. Observations on a case of sudden death after delivery, from embolism of the pulmonary artery. British Medical Journal 1869: 282-283

Prandoni P, Lensing AW, Cogo A, Cuppini S, Villalta S, Carta M, Cattelan AM, Polistena P, Bernardi E, Prins MH. The long-term clinical course of acute deep vein thrombosis. Annals of Internal Medicine 1996; 125: 1-7

Prandoni P, Nenci GG. Low molecular weight heparins: are they interchangeable? Journal of Thrombosis and Haemostasis 2003; 1: 10-13 
Priollet P, Roncato M, Aiach M, Housset E, Poissonnier MH, Chavinie J. Low molecular weight heparin in venous thrombosis during pregnancy. British Journal of Haematology 1986; 63: 605-606

R Development Core Team. R: a language and environment for statistical computing. A Foundation for Statistical Computing 2006, Vienna, Austria (http://www.R-project.org)

Rajgopal R, Bear M, Butcher MK, Shaughnessy SG. The effects of heparin and low molecular weight heparins on bone. Thrombosis Research 2008; 122: 293-8

Rang HP, Dale MM, Ritter JM, Moore PK (Eds). Pharmacology. $5^{\text {th }}$ edition, London. Churchill Livingstone: 2005

Ransdell HT, Haller JA, Stowens D, Barton PB. Renal toxicity of polybrene (hexadimethrine bromide). Journal of Surgical Research 1965; 5: 195-199

Ray JG, Chan WS. Deep vein thrombosis during pregnancy and the puerperium: a meta-analysis of the period of risk and the leg of presentation. Obstetrical and Gynaecological Survey 1999; 54: 265-271

Refuuerzo J, Blackwell S, Sokol R, Lajeunesse L, Firchau K, Kruger M, Sorokin Y. Use of over the counter medications and herbal remedies in pregnancy. American Journal of Perinatology 2005; 22: 321-324

Retout S, Duffull S, Mentre F. Development and implementation of the population Fisher information matrix for the evaluation of population pharmacokinetic designs. Computer Methods and Programs Biomedicine 2001; 65: 141-151

Roberts LN, Patel RK, Chitongo P, Bonner L, Arya R. African-Caribbean ethnicity is associated with a hypercoagulable state as measured by thrombin generation. Blood Coagulation and Fibrinolysis 2012; 23: DOI:10.1097/MBC.0b013e32835a07fa

Robertson L, Wu O, Langhorne P, Twaddle S, Clark P, Lowe GD, Walker ID, Graves M, Brenkel I, Regan L, Greer IA. . Thrombosis: risk and economic assessment of thrombophilia screening (TREATS) study: Thrombophilia in pregnancy: a systematic review. British Journal of Haematology 2006; 132: 171-96

Rodger MA, Walker M, Wells PS. Diagnosis and treatment of venous thromboembolism in pregnancy. Best Practice and Research Clinical Haematology 2003; 16: 279-296

Rodie VA, Thomson AJ, Stewart FM, Quinn AJ, Walker ID, Greer IA. Low molecular weight heparin for the treatment of venous thromboembolism in pregnancy a case series. 2002 British Journal of Obstetrics and Gynaecology 2002; 109:1020-1024 
Roeters van Lennep JE, Meijer E, Klumper FJCM, Middeldorp JM, Bloemenkamp KWM, Middeldorp S. Prophylaxis with low-dose low-molecular weight heparin during pregnancy and postpartum: is it effective? Journal of Thrombosis and Haemostasis 2011; 9: 473-480

Rosenberg RD, Bauer KA. The heparin-antithrombin system: a natural anticoagulant mechanism. In: Colman RW, Hirsch J, Marder VJ, Salzman EW, eds. Haemostasis and thrombosis: basic principles and clinical practice. 3rd ed. Philadelphia: J.B. Lippincott, 1994: 837-60

Rosenkranz A, Hiden M, Leschnik B, Weiss EC, Schlembach D, Lang U, Gallistl S, Muntean W. Calibrated automated thrombin generation in normal uncomplicated pregnancy. Thrombosis and Haemostasis 2008; 99: 331-337

Rossetto V, Spiezia L, Dabrilli P, Gavasso S, Simiono P. Effect on thrombin generation of the "invitro" addition of low-dose LMWH to plasma of healthy pregnant and nonpregnant women. Clinical and Applied Thrombosis and Haemostasis 2012; 18: 331-333

Rossetto V, Dabrilli P, Spiezia L, Castelli M, Campello E, Perlati M, Gavasso S, Simioni P. Increased thrombin generation during pregnancy. Journal of Thrombosis and Haemostasis 2009; Suppl 2: PP-TH-382 (abstract)

Royal College of Obstetricians and Gynaecologists. Green-top guidelines, number 28: Thromboembolic disease in pregnancy and the puerperium: acute management. November 2007

Royal College of Obstetricians and Gynaecologists. Thrombosis and Embolism during Pregnancy and the Puerperium, Reducing the Risk (Green-top 37) 2009; Available at: http://www.rcog.org.uk/womens-health/clinical-guidance/reducing-risk-of-thrombosisgreentop37

Sadler L, McCowan L, White H, Stewart A, Bracken M, North R. Pregnancy outcomes and cardiac complications in women with mechanical, bioprosthetic and homograft valves. British Journal of Obstetrics and Gynaecology 2000; 107: 245-53

Saeed CR, Frank JB, Pravin M, Aziz RH, Serasheini M, Dominique TG. A prospective trial showing the safety of adjusted dose enoxaparin for thromboprophylaxis of pregnant women with mechanical prosthetic heart valves. Clinical and Applied Thrombosis and Haemostasis $2011 ; 17: 313-319$

Salzman EW, Rosenberg RD, Smith MH, Lindon JN, Favreau L. Effect of heparin and heparin fractions on platelet aggregation. Journal of Clinical Investigation 1980; 65: 64-73

Samama M. Contemporary laboratory monitoring of low molecular weight heparins. Clinics in Laboratory Medicine 1995; 15:119-123 
Sanchez-Pena P, Hulot J-S, Urien S, Ankri A, Collet J-P, Choussat R, Lechat P, Montalescot G. Anti-factor Xa kinetics after intravenous enoxaparin in patients undergoing percutaneous coronary intervention: a population model analysis. British Journal of Clinical Pharmacology 2005; 60: 364-373

Sandset PM, Abildgaard U, Larsen ML. Heparin induces release of extrinsic coagulation pathway inhibitor (EPI). Thrombosis Research 1988; 50: 803-813

Sanz E, Gomez-Lopez T, Martinez-Quintas MJ. Perception of teratogenic risk of common medicines. European Journal of Obstetrics and Gynaecology and Reproductive Biology 2001; 95: 127-131

Sanofi-Aventis. Enoxaparin sodium - a summary of product characteristics 2009. Accessed on www.medicines.org.uk on the 21/04/2010

Sawicki E, Stewart K, Wong S, Leung L, Paul E, George J. Medication use for chronic health conditions by pregnant women attending an Australian maternity hospital. Australian and New Zealand Journal of Obstetrics and Gynaecology 2011; 51: 333-338

Sarangi PP, Lee HW, Kim M. Activated protein C action in inflammation. British Journal of Haematology 2010; 148: 817-833

Schatz M, Dombrowski MP, Wise R, Momirova V, Landon M, Mabie W, Newman RB, Rouse DJ, Lindheimer M, Miodovnik M, Caritis SN, Leveno KJ, Meis P, Wapner RJ, Paul RH, O'Sullivan MJ, Varner MW, Thurnau GR, Conway DL. Spirometry is related to perinatal outcomes in pregnant women with asthma. American Journal of Obstetrics and Gynaecology 2006; 194: 120-126

Schoemaker RC, Cohen AF. Estimating impossible curves using NONMEM. British Journal of Clinical Pharmacology 1996; 42: 283-290

Schulman S, Lindmarker P, Holmström M, Larfars G, Carlsson A, Nicol P, Svensson E, Ljungberg B, Viering S, Norlander S, Leijd B, Jahed K, Hjorth M, Linder O, Beckman M. Postthrombotic syndrome, recurrence, and death 10 years after the first episode of venous thromboembolism treated with warfarin for 6 weeks or 6 months. Journal of Thrombosis and Haemostasis 2006; 4: 734-742

Schulman S, Kearon C, Kakkar AK, Mismetti P, Schellong S, Eriksson H, Baanstra D, Schnee J, Goldhaber SZ for the RE-COVER study group. Dabigatran versus Warfarin in the treatment of acute venous thromboembolism. The New England Journal of Medicine 2009; 361: 2342-2352

Scott DA, Charles AF. Studies on heparin. III. The purification of heparin. The Journal of Biological Chemistry 1933; 102; 437-448 
Segers $\mathrm{O}$, van Oerle R, ten Cate H, Rosing J, Castoldi E. Thrombin generation as an intermediate phenotype for venous thrombosis. Thrombosis and Haemostasis 2010; 103:114-22

Sephton V, Farquharson RG, Topping J, Quenby SM, Cowan C, Back DJ Toh CH. A longitudinal study of maternal dose response to low molecular weight heparin in pregnancy. Obstetrics and Gynaecology 2003;101: 1307-11

Sere KM, Hackeng TM. Basic mechanisms of hemostasis. Seminars in Vascular Medicine 2003; 3: 3-12

Sheiner LB. Computer-aided long-term anticoagulation therapy. Computers and Biomedical Research 1969; 2: 507-518

Sheiner LB, Rosenberg B, Melmon KL. Modeling of individual pharmacokinetics for computeraided drug dosage. Computers and Biomedical Research 1972; 5: 441-459

Sheiner LB, Rosenberg B, Marathe VV. Estimation of population characteristics of population parameters from routine clinical data. Journal of Pharmacokinetics and Biopharmaceutics 1977; 5: 445-479

Sheiner LB, Beal SL. Evaluation of methods for estimating population pharmacokinetics parameters I. Michaelis-Menten model: Routine clinical pharmacokinetic data. Journal of Pharmacokinetics and Biopharmaceutics 1980; 8: 553-571

Sheiner LB, Beal SL. Evaluation of methods for estimating population pharmacokinetic parameters II. Biexponential model and experimental pharmacokinetic data. Journal of Pharmacokinetics and Biopharmaceutics 1981; 9: 635-651

Sheiner LB, Grasela TH. An introduction to mixed effect modeling: Concepts, definitions, and justification. Journal of Pharmacokinetics and Biopharmaceutics 1991; 19: 11s-24s

Sheiner LB, Ludden TM. Population pharmacokinetics/dynamics. Annual Review of Pharmacology and Toxicology 1992; 32: 185-209

Sie P, Cremers PSB, Dupouy D, Caranobe C, Dol F, Boneu B. Neutralisation of dermatan sulphate in vitro and in vivo by protamine sulphate and polybrene. Thrombosis Research 1989; 54: 63-74

Silverstein MD, Heit JA, Mohr DN, Petterson TM, O'Fallon WM, Melton LJ III. Trends in the incidence of deep vein thrombosis and pulmonary embolism: a 25-year population-based study. Archives of Internal Medicine 1998; 158: 585-593

Simpson VMA, Pescott M, Peebles D, Cohen H. Anti-Xa monitoring is required in pregnant women on low molecular weight heparin (LMWH). Journal of Thrombosis and Haemostasis 2009; Suppl 2: PP-TH-384 (abstract) 
Smellie, W. A collection of cases and observations in Midwifery. 1764, Vol II, $3^{\text {rd }}$ ed. London, Wilson

Smith MP, Norris LA, Steer PJ, Savidge GF, Bonnar J. Tinzaparin sodium for thrombosis treatment and prevention during pregnancy. American Journal of Obstetrics and Gynaecology 2004; 190: 495-501

Sobel M, McNeill PM, Carlson PL, Kermode JC, Adelman B, Conroy B, Marques D. Heparin inhibition of von Willebrand factor-dependent platelet function in vitro and in vivo. Journal of Clinical Investigation 1991; 87: 1787-1793

Spahn G. Compliance with self-administration of heparin injections in outpatients. European Journal of Trauma 2002; 28: 104-109

Spearing G, Fraser I, Turner G, Dixon G. Long-term self-administered subcutaneous heparin in pregnancy. British Medical Journal; 1978; i: 1457

Spronk HM, Dielis AW, De Smedt E, van Oerle R, Fens D, Prins MH, Hamulyak K, ten Cate H. Assessment of thrombin generation II: Validation of the Calibrated Automated Thrombogram in platelet-poor plasma in a clinical laboratory. Thrombosis and Haemostasis 2008; 100: 362-4

Smith A, Williams S. Side effect perception and adherence to fertility drugs during IVF treatment. (abstract) International Journal of Pharmacy Practice 2010; 18(s1): 46-49

Smith MP, Norris LA, Steer PJ, Savidge GF, Bonnar J. Tinzaparin sodium for thrombosis treatment and prevention during pregnancy. American Journal of Obstetrics and Gynecology 2004; 190: 495-501

Stago - summary of product characteristics - D-dimer kit. Diagnostica Satgo. STA-Liatest@ D-DI kit (immuneturbidimetric assay). France, 2011

Standing JF, Howard RF, Johnson Atholl, Savage I, Wong CK. Population pharmacokientics of oral diclofenac for acute pain in children. British Journal of Clinical Pharmacology 2008; 66: 846-853

Stief TW. Inhibition of thrombin generation in re-calcified plasma. Blood Coagulation and Fibrinolysis 2007; 18: 751-760

Sturridge F, DeSwiet M, Letsky E. The use of low molecular weight heparin for thromboprophylaxis in pregnancy. British Journal of Obstetrics and Gynaecology 1994; 101: 69-71 
Szecsi PB, Jorgensen M, Klajnbard A, Andersen MR, Colov NP, Stender S. Haemostatic reference intervals in pregnancy. Thrombosis Haemostasis 2010; 103: 718-727

Tan KS, Thomson NC. Asthma in pregnancy. American Journal of Medicine 2000; 109: 727-733

Tanaka KA, Key NS, Levy JH. Blood coagulation: hemostasis and thrombin regulation. Anesthesia and Analgesia 2009; 108: 1433-1446

Tarning J, McGready R, Lindegardh N, Ashley EA, Pimanpanarak M, Kamanikom B, Annerberg A, Day NPJ, Stepniewska K, Pratap Singhasivanon P, White NJ, Nosten F. Population Pharmacokinetics of Lumefantrine in Pregnant Women Treated with ArtemetherLumefantrine for Uncomplicated Plasmodium falciparum Malaria. Antimicrobial Agents and Chemotherapy 2009; 53: 5837-5846

Tappenden KA, Gallimore MJ, Evans G, Mackie IJ, Jones DW. Thrombin generation: a comparison of assays using platelet-poor and -rich plasma and whole blood samples from healthy controls and patients with a history of venous thromboembolism. British Journal of Haematology 2007; 139: 106-112

Tapson VF. Acute Pulmonary Embolism. New England Journal of Medicine 2008; 358: 1037-52

Tchaikovski SN, van Vliet HA, Thomassen MC, Bertina RM, Rosendaal FR, Sandset PM, Helmerhorst FM, Tans G, Rosing J. Effect of oral contraceptives on thrombin generation measured via calibrated automated thrombography. Thrombosis and Haemostasis 2007; 98:1350-6

To MS, Hunt BJ, Nelson-Piercy C. A negative D-dimer does not exclude venous thromboembolism (VTE) in pregnancy. Journal of Obstetrics and Gynaecology 2008; 28: 222-240

Tovey C, Wyatt S. Diagnosis, investigation and management of deep vein thrombosis. British Medical Journal 2003; 326: 1180-1184

Tripodi A, Legnani C, Chantarangkul V, Cosmi B, Palareti G, Mannucci PM. High thrombin generation measured in the presence of thrombomodulin is associated with an increased risk of recurrent venous thromboembolism. Journal of Thrombosis and Haemostasis 2008; 6:1327-33

Tucker GT. An agenda for UK clinical pharmacology: Research priorities in pharmacokinetics. British Journal of Clinical Pharmacology 2012; 73: 924-926

Turpie AGG, Esmon C. Venous and arterial thrombosis - pathogenesis and the rationale for anticoagulation. Thrombosis and Haemostasis 2011; 105: 586-596 
Ulrick PJ, Manoharan A. Heparin-induced skin reaction. Medical Journal of Australia 1984; 140: 287-9

van den Belt AGM, Prins MH, Lensing AWA, Castro AA, Clark OAC, Atallah AN, Burihan E. Fixed dose subcutaneous low molecular weight heparins versus adjusted dose unfractionated heparin for venous thromboembolism (Cochrane review). In: Cochrane database system review, No. 1. London: Update Software, 2000

van Dongen CJJJ, van den Belt AGM, Prins MH, Lensing AWA. Fixed dose subcutaneous low molecular weight heparin versus adjusted dose unfractionated heparin for venous thromboembolism (review). Cochrane Database and Systematic Reviews 2004; 4: Art No. CD001100

van Hasselt JGC, Morrish G, Green B. D-Optimal design for time varying enoxaparin pharmacokinetics during pregnancy. MSc thesis (Coen van Hasselt), University of Queensland, 2009

van Hasselt JGC, Morrish G, Green B. Leveraging physiological data from literature into a pharmacokinetic model to support informative clinical study design in pregnant women. Pharmaceutical Research 2012; 29: 1609-16

van Hasselt JGC, Andrew MA, Hebert MF, Tarning J, Vicini P, Mattison DR. The status of pharmacometrics in pregnancy: highlights from the $3^{\text {rd }}$ American conference on pharmacometrics. British Journal of Clinical Pharmacology 2012; 74: 932-939

van Hylckama Vlieg A, Christiansen SC, Luddington R, Cannegieter SC, Rosendaal FR, Baglin TP. Elevated endogenous thrombin potential is associated with an increased risk of a first deep venous thrombosis but not with the risk of recurrence. British Journal of Haematology 2007; 138: 769-74

van Veen JJ, Gatt A, Makris M. Thrombin generation testing in routine clinical practice: are we there yet? British Journal of Haematology 2008; 142: 889-903

van Veen JJ, Gatt A, Cooper PC, Kitchen S, Bowyer AE, Makris M. Corn trypsin inhibitor in fluorogenic thrombin generation measurements is only necessary at low tissue factor concentrations and influences the relationship between FVIII:C and thrombogram parameters. Blood Coagulation and Fibrinolysis 2008; 19: 183-189

van Veen JJ, Maclean RM, Hampton KK, Laidlaw S, Kitchen S, Toth P, Makris M. Protamine reversal of low molecular weight heparin: clinically effective? Blood Coagulation and Fibrinolysis 2011 ; 22: 565-570

Varga-Szabo D, Pleines I, Nieswandt B. Cell adhesion mechanisms in platelets. Arteriosclerosis, Thrombosis, and Vascular Biology 2008; 28: 403-412 
Vaz MJR, Barros SMO, Palacios R, Senise JF, Lunardi L, Amed AM Castelo A. HIV-infected pregnant women have greater adherence with antiretroviral drugs than non-pregnant women. International Journal of STD \& AIDS 2007; 18: 28-32

Virchow R. I. Ueber die Verstopfung der Lungenarterie. Froriep's N. Notizen, 1846, No. 794, pp. 221-226 and II. Weitere Untersuchungen ueber die Verstopfung der Lungenarterie und ihre Folgen. Treube's Beitrage zur expermentellen Pathologie und Physiologie, Berlin, 1846, Hft II. Pp. 227-380, collected in Gesammelte Abhandlungen zur Wissenchaftlichen Medicin, Meidinger \& Sohn, Frankfurt

Voke JM, Keidan J, Parvod S, Spencer NH, Hunt BJ. The management of antenatal venous thromboembolism in the UK and Ireland: a prospective multi-centre survey. British Journal of Haematology 2007; 139: 545-558

Warkentin TE. Heparin-induced thrombocytopenia: pathogenesis, frequency, avoidance and management. Drug Safety 1997; 17: 325-41

Warkentin TE, Greinacher A, Koster A, Lincoff AM. Treatment and prevention of heparin-induced thrombocytopenia: American College of Chest Physicians evidence-based clinical practice guidelines (8th edition). 2008; 133(suppl):340s-380s

Watts AC, Howie CR, Simpson AHRW. Assessment of a self-administration protocol for extended subcutaneous thromboprophylaxis in lower limb arthroplasty. The Journal of Bone and Joint Surgery 2006; 88-B: 107-110

Weinen W, Stassen JM, Priepke H, Ries UJ, Hauel N. In-vitro profile and ex-vivo anticoagulant activity of the direct thrombin inhibitor dabigatran and its orally active pro-drug, dabigatran etexilate. Thrombosis and Haemostasis 2007; 98: 155-162

Weiss WA, Gilman JS, Catenacci AJ, Osterberg AE. Heparin neutralisation with Polybrene administered intravenously. Journal of the American Medical Association 1958; 166: 603607

Weitz JI. Low Molecular Weight Heparins. New England Journal of Medicine 1997; 337: 688-698

Wells PS, Anderson DR, Rodger M, Forgie M, Kearon C, Dreyer J, Lovacs G, Mitchell M, Lewandowski B, Kovacs MJ. Evaluation of D-dimer in the diagnosis of suspected deep vein thrombosis. New England Journal of Medicine 2003; 349: 1227-1235

White C. An inquiry into the nature and cause of the swelling in one or both of the lower extremities, which sometimes happens to lying-in-women. Eyres Press, Warrington, 1784 [In: Anning ST. The historical aspects of venous thrombosis. Medical History 1957; 1: 2837] 
White C. An inquiry into the nature and cause of swelling in one or both of the lower extremities, which sometimes happens to lying-in-women. Part II. [s.n.], Manchester, 1801, [In; Webster Bride, J. Some Manchester pioneers in obstetrics and gynaecology. 1954; 61: 69-80]

White RH, Ginsberg JS. Low molecular weight heparins: are they all the same. British Journal of Haematology 2003; 121: 12-20

White $\mathrm{RH}$, Zhou $\mathrm{H}$, Gage BF. Effect of age on the incidence of venous thromboembolism following major surgery. Journal of Thrombosis and Haemostasis 2004; 2: 1327-1333

White RH, Zhou H, Muir S, Harvey D. Effect of ethnicity and gender on the incidence of venous thromboembolism in a diverse population in California. Thrombosis and Haemostasis 2005; 93: 298-305

Winn JM. On a case of Phlegmasia dolens of the upper extremity, occurring after parturition. Lancet 1852: 192

Willcox FM. A case of tedious labour followed by double phlegmasia alba dolens and gangrene. Lancet 1897: 1188

Wiseman, R. Severall Chirurgicall Treatises $1676,2^{\text {nd }}$ edn. Royston and Took, London, pp. 32, 64

Wulfkuhle KC, Butenas S, Bernstein I, Brummel-Ziedins K. Tissue factor dependent and independent thrombin generation across pregnancy. Journal of Thrombosis and Haemostasis 2011; 9 (Suppl(2)): 431, abstract PO-TU-389

Young E, Prins M, Levine MN, Hirsh J. Heparin binding to plasma proteins, an important mechanism for heparin resistance. Thrombosis and Haemostasis 1992; 67: 639-643

Young E, Cosmi B, Weitz J, Hirsh J. Comparison of the non-specific binding of unfractionated heparin and low molecular weight heparin (enoxaparin) to plasma proteins. Thrombosis and Haemostasis 1993; 70: 625-630

Young E, Wells P, Holloway S, Weitz J, Hirsh J. Ex-vivo and in-vitro evidence that low molecular weight heparin exhibit less binding to plasma proteins than unfractionated heparin. Thrombosis and Haemostasis 1994; 71: 300-304 
$17^{\text {th }}$ March 2010

\section{King's College Hospital W/HS \\ NHS Foundation Trust}

King's College Hospital NHS Foundation Trust King's College Hospita Denmark Hill London SE5 9RS

Jignesh Patel

Mobile tel: 07984949011

Direct fax: 02032994689

Email: jig.patel@kcl.ac.uk

\section{Patient Information Sheet}

We would like to invite you to take part in a research study. Before you decide if you want to take part you need to understand why the research is being done and what it would involve for you. Please read the following information carefully. You may ask us if there is anything that is not clear or if you would like more information.

Take time to decide whether you would like to take part or not.

Title: Optimising the use of enoxaparin during the antenatal period

Please read Part 1. If the information given there interests you and you are considering taking part in the study, please read the additional information in Part 2 before making any decision.

At your next appointment, a member of the research team will go through the information sheet with you and answer any questions you may have.

\section{Part 1}

\section{Purpose of the study}

During pregnancy changes which occur in a woman's body increases the chance of developing blood clots in the veins of the body. So pregnant women who are at high risk of getting these clots or already have these clots are given the medicine, enoxaparin.

Enoxaparin works by reducing the stickiness of the blood, stopping these clots from forming.

Currently it is not known how the amount of enoxaparin in the body changes as pregnancy progresses. It is important that we know how the amount of enoxaparin in the body changes during pregnancy, so that pregnant women receive the best dose of this medicine.

The purpose of this study is to find out how being pregnant affects the amount of enoxaparin in the body so that we can make sure that pregnant women get the best dose of enoxaparin to reduce the chance of these blood clots developing. 


\section{Why have I been invited?}

You have been invited to take part in this study because you are having or about to have enoxaparin.

\section{Do I have to take part?}

No. It is up to you to decide. If you do decide to take part you are free to withdraw at any time without giving a reason. Whatever you decide it will not affect the care that you receive.

\section{What will happen to me if I take part?}

If you wish to take part in the study you will be asked to sign a consent form.

\section{During pregnancy}

During pregnancy you will be asked to inject the enoxaparin at a particular time of day. You will normally attend the haematology clinic every 4 weeks, where the doctor will normally measure enoxaparin activity in the blood through a blood test.

If you decide to take part in this study, we will ask you to have two additional blood tests each time you visit the haematology clinic. These additional blood tests also measure the amount of enoxaparin in your blood, but they will be taken at different times during your clinic visit. This will help us to determine how the amount of enoxaparin is changing with time.

All blood tests will be taken from a vein in your arm by a trained member of the research team. This blood test will be similar to other blood tests you may have had at your GP or other clinics. We will ask you if you prefer to have the blood taken from a particular arm.

A small quantity of blood (one and a half tablespoons) will be taken at each clinic visit, as follows:

- one table spoon of blood will be taken on arrival at clinic,

- half a tea spoon of blood will be taken 1 hour later and,

- a further half a tea spoon will be taken 3 hours after you arrived in clinic.

In addition, at each clinic visit you will be weighed and will be asked about any changes in any medications you may be on.

If you decide to participate in the study, it is expected that your appointment in the haematology clinic will last $\mathbf{3}$ hours in total each time - due to the additional tests being done.

The following diagram shows what a typical visit to the haematology clinic will involve, if you decide to take part in the study: 
You will see the researcher in clinic and the following information will be updated / collected:

- weight

- height

- $\quad$ asked about changes in medications

- blood tests taken

You will then be asked to inject your usual dose of enoxaparin in clinic

1 hour later

A blood test will be taken one hour later by the researcher.

You will also see the haematology doctor - as part of routine clinical care

\begin{tabular}{ll}
\hline hours after arriving & $\begin{array}{l}\text { Another blood test will be taken and you will be given the date and time } \\
\text { of your next appointment }\end{array}$
\end{tabular}

During month 2, 5 and 8 of your pregnancy, we will also do additional tests, looking at how your blood is clotting. Three samples will be taken - at the same time as the other blood tests.

At these clinic visits, a total of 2 tablespoons of blood will be taken - due to these extra tests being done.

After your baby is born

Once your baby is born, we will measure how your blood is clotting twice:

- once whilst you are still in hospital following the birth of your baby, and

- again 8 weeks after your baby is born.

In addition during your pregnancy, you will also be asked to complete a short questionnaire which explores your views about having to take enoxparin during your pregnancy.

This is being done, as currently very little information exists on what women's views are, around having to inject enoxaparin during their pregnancy.

\section{Expenses and payment}

Travel costs will be provided for all visits to meet the research team that are not on the same day as your usual outpatient appointments at the haematology or antenatal clinic.

Whilst you are waiting for all three blood tests to be taken at each clinic visit, we will give you a voucher to get refreshments from the hospital shop / coffee shop. 
What are the possible disadvantages and risks of taking part in the study?

As with any blood test, there could be discomfort and/or bruising at the site from where the blood is taken.

Your clinic appointments will take longer than if you were not in the study.

\section{What are the possible benefits of taking part?}

The usual blood tests which you will have as part of your normal care will be taken in the haematology clinic - so you will not have to go to the blood test area of the hospital for these.

The results from this study will give valuable information on the use of enoxaparin during the antenatal period.

Overall, we cannot promise that the study will be of direct benefit to you.

\section{What happens when the research stops?}

You will continue to see the doctor at the haematology clinic.

\section{What if there is a problem?}

Any complaint about the way you are being dealt with during the study or any possible harm you might suffer will be dealt with. The detailed information on this is given in Part 2.

\section{Will my taking part in the study be kept confidential?}

Yes. We will follow ethical and legal practice and all information about you will be kept strictly confidential. The details are included in Part 2.

\section{Part 2}

\section{What if relevant new information becomes available?}

If any new information on this medicine becomes available, then your doctor will let you know. If this happens a member of the research team will discuss with you whether you should continue in the study. If you decide to continue in the study, you may need to sign a new consent form. If you decide to withdraw from the study, you will continue to see your usual doctor and the care you receive will not be affected.

\section{What will happen if I don't want to carry on with the study?}

If you decide to withdraw from the study, please contact the research team. You are free to withdraw at any time, without giving a reason. Withdrawing from the study will not affect the care that you receive. If you wish, any stored blood that can be identified as yours will be destroyed. All data collected up to the time of your withdrawal from the study will be used.

After entering the study, should you lose the ability to give consent you will be withdrawn from the study at this time. We will use any blood or information collected up to the time of your withdrawal from the study. 


\section{What if there is a problem?}

If you are worried about any part of the study, you should speak to a member of the research team (contact names and telephone numbers are at end of this sheet).

If you remain unhappy and wish to complain formally, you can do this through the NHS Complaints procedure. Details can be obtained from the hospital.

In the event that something goes wrong and you are harmed due to someone's negligence, you may have grounds for legal action but you may have to pay for your legal costs.

The normal National Health Service complaints mechanisms will be available to you.

\section{What will happen to any blood samples I give?}

All blood samples collected will be securely stored in a freezer at the hospital. Only members of the research team will have access to these samples. Each research sample will be identified with a unique number. It will only be possible for members of the research team to link the samples back to you.

Samples will not be moved out of the UK; but they may be transferred to a different laboratory in the UK for testing if equipment at the hospital is unavailable e.g. breakdown of equipment.

At the end of the study all blood samples will be destroyed. Should our team or another team of researchers wish to use the samples for any other work, ethical approval will be required and you must give further consent.

\section{What will happen to the questionnaire I complete?}

The information from the questionnaire will be entered onto a computer, so it can be analysed. The information from your completed questionnaire will be anonymised and will not be shared with your clinical team.

\section{Will genetic tests be done?}

No.

\section{What will happen to my data?}

All information collected during the study will be kept strictly confidential. Your data will be anonymised using a unique number.

The data collected will only be used for this study. The data will be stored securely on a password protected computer at the hospital. Only members of the research team will be able to access the data.

The data will be retained for 5 years and then destroyed. Use of the data in other studies will not be possible without further ethical approval and consent from you.

\section{Involvement of the General Practitioner (GP)}

We would like to inform your GP that you are taking part in this study. We will only inform your GP with your consent. 


\section{Informing your hospital doctor (referring doctor)}

We would like to inform your hospital doctor that you are taking part in this study. We will only inform your hospital doctor with your consent.

\section{What will happen to the results of the research study?}

If you decide to participate in this study, we will ask you if you wish to be informed of any results arising from the research. When results are obtained we will send you a letter providing an outline of the broad scientific results of the study, with references for any work that has been published in medical journals.

It is hoped that results will be presented at scientific meetings and published in medical journals. At no time will it be possible to identify that you have taken part in this study.

This study will be described in the researcher's (Jignesh Patel) $\mathrm{PhD}$ thesis.

\section{Who is organising and funding the research?}

King's College Hospital Foundation NHS Trust is sponsoring the research.

The GSTFT/KCL National Institute of Health Research Biomedical Research Centre is funding the research.

\section{Who has reviewed the study?}

All research in the NHS is looked at by an independent group of people, called a Research Ethics Committee to protect your safety, rights, wellbeing and dignity. This study has been reviewed and given favorable opinion by the Isle of Wight, Portsmouth and South East Hampshire Research Ethics Committee.

\section{Further information and contact details}

1) General information about research

National Electronic Library for Health www.library.nhs.uk/trials

The National Research Register (UK database of research projects) www.nrr.nhs.uk

INVOLVE (Promoting public involvement in the NHS) www.invo.org.uk

2) Sponsor and Funder's website

King's College Hospital www.kch.nhs.uk and www.kingshealthpartners.org NIHR GSTFT/KCL Biomedical Research Centre www.biomedicalresearchcentre.org

3) Specific information about this research study

Jignesh Patel 07984949011

Dr. Roopen Arya 02032993570

Dr. Raj Patel 02032993418

Mr. Michael S Marsh 02032993629

Professor Graham Davies 02078484049 


\section{King's College Hospital W/HS \\ NHS Foundation Trust}

King's College Hospital NHS Foundation Trust

King's College Hospital Denmark Hill London SE5 9RS

Mobile tel: 07984949011 Direct fax: 02032994689 Email: jig.patel@kcl.ac.uk

CONSENT FORM (version 2)

Title of Study: Optimising the use of enoxaparin during the antenatal period

Name of Researcher: Jignesh Patel

Please initial each box

Initials

1. I confirm that I have read and understand the information sheet dated 17th March 2010 Version 3, for the above study. I have had the opportunity to consider the information and ask questions.

2. I understand that my participation is voluntary and that I am free to withdraw at any time without giving any reason, without my medical care or legal rights being affected.

3. I understand that relevant sections of my medical notes and data collected during the study may be looked at by individuals from the research team and also by regulatory research and ethical authorities in the NHS Trust, where it is relevant to my taking part in this research. I give permission for these individuals to have access to my records.

4. I agree to my GP being informed of my participation in the study.

5. I agree to my hospital doctor (referring doctor) being informed of my participation in the study

6. I am happy to complete the questionnaire as part of the study

7. I agree to take part in the above study.
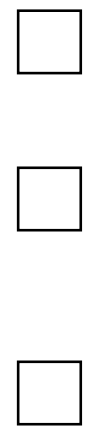

Name of Patient

Signature

Date

I confirm that I have explained the study to the participant and have answered their questions honestly and fully.

Name of Person

Signature

Date

taking consent

Patient would like information on the broad results of the study:

Yes / No

When completed, 1 for patient; 1 for researcher site file; 1 (original) to be kept in medical notes 
National Research Ethics Service

DC/MFK

20 August 2009

Mr Jignesh. P Patel

Department of Pharmacy

King's College London

150 Stamford Street

SE1 $9 \mathrm{NH}$

Dear Mr Patel

\section{ISLE OF WIGHT, PORTSMOUTH \& SOUTH EAST HAMPSHIRE}

RESEARCH ETHICS COMMITTEE

$1^{\text {ST }}$ Floor, Regents Park Surgery

Park Street, Shirley

Southampton

Hampshire

SO16 4RJ

Tel: $\quad 02380362863$

Fax: 02380364110

Email: scsha.SEHREC@nhs.net
Applying the population pharmacokinetic modelling technique to determine the optimal dose and dosing frequency of enoxaparin during the antenatal period

REC reference number: Protocol number: 09/H0501/57

3

Thank you for your letter of 13 August 2009, responding to the Committee's request for further information on the above research and submitting revised documentation.

The further information has been considered on behalf of the Committee by the Chair.

Confirmation of ethical opinion

On behalf of the Committee, I am pleased to confirm a favourable ethical opinion for the above research on the basis described in the application form, protocol and supporting documentation as revised, subject to the conditions specified below.

Ethical review of research sites

The favourable opinion applies to all NHS sites taking part in the study, subject to management permission being obtained from the NHS/HSC R\&D office prior to the start of the study (see "Conditions of the favourable opinion" below).

\section{Conditions of the favourable opinion}

The favourable opinion is subject to the following conditions being met prior to the start of the study.

Management permission or approval must be obtained from each host organisation prior to the start of the study at the site concerned.

For NHS research sites only, management permission for research ("R\&D approval") should be obtained from the relevant care organisation(s) in accordance with NHS research governance arrangements. Guidance on applying for NHS permission for research is available in the Integrated Research Application System or at http://www.rdforum.nhs.uk. Where the only involvement of the NHS organisation is as a Participant Identification Centre, management permission for research is not required but the $R \& D$ office should be notified of the study. Guidance should be sought from the R\&D office where necessary.

Sponsors are not required to notify the Committee of approvals from host organisations.

This Research Ethics Committee is an advisory committee to South Central Strategic Health Authority

The National Research Ethics Service (NRES) represents the NRES Directorate within the National Patient Safety Agency and Research Ethics Committees in England 
$03^{\text {rd }}$ September 2009

\section{King's College Hospital W/ES \\ NHS Foundation Trust}

Mr Jignesh. P Patel

Clinical Pharmacist / Clinical Lecturer

King's College Hospital Denmark Hil

Kings College Hospital /

Kings College London

Tel: 02032999000
Fax: 02032993445

Department of Pharmacy

Franklin-Wilkins Building

Minicom: 02032999009

www.kch.nhs.uk

Stamford Street

London

Direct tel: 02032993841

SE1 9NH

\section{Ref: NH/KCH1350}

\section{Full Research \& Development Approval}

\section{R\&D: KCH1350}

Title: Applying the population pharmacokinetic modelling technique to determine the optimal dose and dosing frequency of enoxaparin during the antenatal period.

REC 09/H0501/57

Site/s: KCH

Dear Jignesh,

Thank you for submitting your research project to the R\&D Department. The project has now been approved by the Trust. Please quote the R\&D registration number noted above in any communications with the R\&D Department regarding your project.

\section{Conditions of Approval:}

- The Principal Investigator must notify R\&D of the actual start and end date of the project.

- The Principal Investigator is responsible for ensuring that Data Protection Principles are observed throughout the course of the project and adhere to relevant Trust policies. Please note that patient databases with any identifiers, such as hospital number, date of birth, address or name must not be held on hard drives even if password protected and in a locked office - these identifiers must only be held on the server.

- The agreed protocol must be followed. R\&D must be notified of any changes to the protocol prior to implementation.

- The Principal Investigator and research team must have appropriate substantive or honorary contracts with the Trust. The Principal Investigator is responsible for ensuring that the team is covered, including new staff recruited to the study.

- If your study is a medicinal clinical trial all members of the research team must have completed GCP, Pharmacovigilance and Trial Master File training - please contact scott.vezina@kcl.ac.uk if training or annual updates are required. 
- Please submit a copy of the progress report on the anniversary of the Ethics favourable opinion (sent via the $\mathrm{CI}$ )

Trust approval for the research is subject to the research being undertaken in line with the Department of Health's Research Governance Framework, and Trust policies relating to Research Governance.

The Research Governance Framework and details of you and your researchers responsibilities within this framework can be found on the Department of Health's website at:

http://www.dh.gov.uk/en/Publicationsandstatistics/Publications/PublicationsPolicyAndGuidance/DH $-4108962$

If appropriate it is recommended that you register with the Current Controlled Trials website; http://isrctn.org/

In line with the Research Governance Framework, your project may be randomly selected for monitoring for compliance against the standards set out in the Framework. For information, the Trust's process for the monitoring of projects and the associated guidance is available from the Trust's intranet or on request from the R\&D Department. You will be notified by the R\&D

Department if and when your project has been selected as part of the monitoring process. No action is needed until that time.

Many thanks for registering your research project

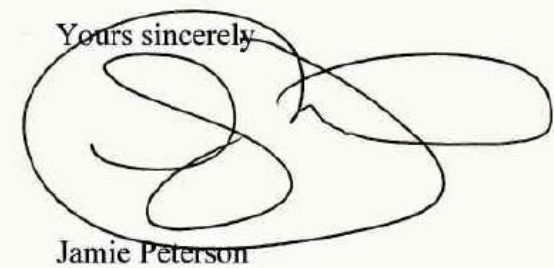

Research and Development Delivery Manager (non-commercial)

Research and Development Department

First Floor Jennie Lee House, 34 Love Walk

Kings College Hospital NHS Trust

London SE5 8AD 
Appendix V: NONMEM control stream for the base model (model 1)

\$SIZES LIM6 $=2000000$

\$PROB BASE PREGNANCY MODEL

\$INPUT ID AGE WT DAT1=DROP TIME AMT DOSE DVID DV MDV GEST TRI CRE CRCL LBW ETHN

\$DATA ..Ifinal22.csv IGNORE=@

ACCEPT $=(D V I D . L E .1)$

\$SUBR ADVAN2 TRANS2

\$PK

;COVARIATE MODEL

TVCL $=$ THETA(1)

TVV $=$ THETA $(2)$

TVKA $=$ THETA(3)

;TIME AFTER DOSE

IF(AMT.GT.0)THEN

TDOS $=$ TIME

$\mathrm{TAD}=0.0$

ENDIF

IF(AMT.EQ.0)TAD=TIME-TDOS

;MODEL FOR RANDOM BETWEEN SUBJECT VARIABILITY

$\mathrm{CL}=\mathrm{TVCL}{ }^{*} \operatorname{EXP}(\mathrm{ETA}(1))$

$\mathrm{V}=\mathrm{TVV}{ }^{*} \operatorname{EXP}(\mathrm{ETA}(2))$

$K A=T V K A$

;SCALE CONCENTRATIONS X 1000 TO COVERT ANTI-XA FROM IU/ML TO IU/L

$\mathrm{SC}=\mathrm{V}^{*} 1000$

\$ERROR

IPRE $=F$

$Y=I P R E * E X P(E P S(1))+E P S(2)$

IWRE=DV-IPRE

\$THETA

$(0.1,10)$

$(1,15)$

$(0.01,3)$

\$OMEGA BLOCK(2)

$0.1 \quad ; P P V C L$

$0.050 .1 \quad ; P P V V$

\$SIGMA

$0.1 \quad$;PROPRUV

$0.2 \quad$;ADDRUV

\$EST MAX=9990 SIG=3 PRINT=1 METHOD $=1$ INT

$\$ C O V$ PRINT=E

\$TABLE ID TIME AMT DOSE DVID DV MDV WT AGE CRE CRCL TRI CL V KA ETA1 ETA2 IPRE TAD CWRES IWRE LBW ETHN GEST

ONEHEADER NOPRINT FILE=final.fit 
Appendix VI: NONMEM control stream for the base model with M3 error method

\$SIZES LIM6 $=2000000$

\$PROB BASE PREGNANCY MODEL

\$INPUT ID AGE WT DAT1=DROP TIME AMT DOSE DVID DV MDV GEST=DROP TRI CRE CRCL

\$DATA final22M3.csv IGNORE=@

ACCEPT $=($ DVID.LE.1)

\$SUBR ADVAN2 TRANS2

\$PK

;COVARIATE MODEL

TVCL $=$ THETA $(1)$

TVV $=$ THETA $(2)$

TVKA $=$ THETA(3)

;MODEL FOR RANDOM BETWEEN SUBJECT VARIABILITY

$\mathrm{CL}=\mathrm{TVCL}{ }^{*} \mathrm{EXP}(\mathrm{ETA}(1))$

$V=T V V^{*} \operatorname{EXP}(E T A(2))$

$K A=T V K A$

;SCALE CONCENTRATIONS $\times 1000$ to covert anti-Xa from IU/mL to IU/L

$\mathrm{SC}=\mathrm{V}^{*} 1000$

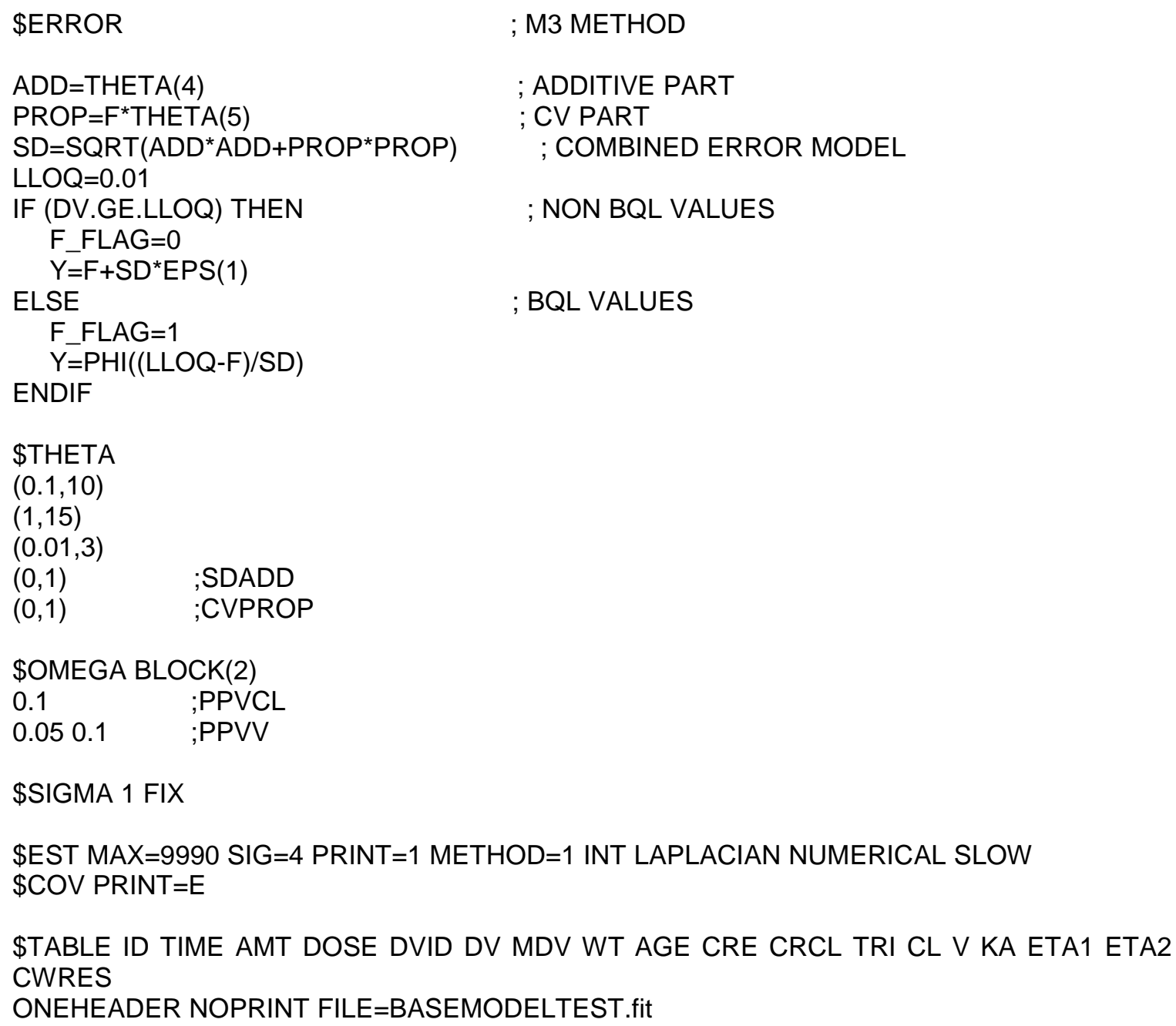

\$TABLE ID TIME AMT DOSE DVID DV MDV WT AGE CRE CRCL TRI CL V KA ETA1 ETA2 CWRES

ONEHEADER NOPRINT FILE=BASEMODELTEST.fit 
Appendix VII: NONMEM bootstrap code for the base model

\$SIZES LIM6=2000000

\$PROB BASE PREGNANCY MODEL

\$INPUT ID AGE WT DAT1=DROP TIME AMT DOSE DVID DV MDV GEST TRI CRE CRCL LBW ETHN

\$DATA ..Ifinal22.csv IGNORE=@

ACCEPT $=(D V I D . L E .1)$

\$SUBR ADVAN2 TRANS2

\$PK

;COVARIATE MODEL

TVCL $=$ THETA(1)

TVV $=$ THETA $(2)$

TVKA $=$ THETA(3)

;TIME AFTER DOSE

IF(AMT.GT.0)THEN

TDOS $=$ TIME

$\mathrm{TAD}=0.0$

ENDIF

IF(AMT.EQ.0)TAD=TIME-TDOS

;MODEL FOR RANDOM BETWEEN SUBJECT VARIABILITY

$\mathrm{CL}=\mathrm{TVCL}{ }^{*} \operatorname{EXP}(\mathrm{ETA}(1))$

$V=T V V^{*} E X P(E T A(2))$

$K A=T V K A$

;SCALE CONCENTRATIONS X 1000 TO COVERT ANTI-XA FROM IU/ML TO IU/L

$\mathrm{SC}=\mathrm{V} * 1000$

\$ERROR

IPRE $=F$

$\mathrm{Y}=\operatorname{IPRE}{ }^{\star} \operatorname{EXP}(\mathrm{EPS}(1))+\mathrm{EPS}(2)$

IWRE=DV-IPRE

\$THETA

$(0.1,10)$

$(1,15)$

$(0.01,3)$

\$OMEGA BLOCK(2)

0.1

;PPVCL

$0.050 .1 \quad$;PPVV

\$SIGMA

0.1 ;PROPRUV

0.2 ;ADDRUV

$\$ E S T$ NOABORT METHOD $=1$ INT PRINT =0

$\$ W A R N$ WARN=NONE 
Appendix VIII: NONMEM control stream for the final pregnancy model

\$SIZES LIM6=2000000

\$PROB BASE PREGNANCY MODEL

\$INPUT ID AGE WT DAT1=DROP TIME AMT DOSE DVID DV MDV TRI CRE CRCL LBW BABY GEST ETHN MGEST

\$DATA ..Ifinal23.csv IGNORE=@

ACCEPT $=(D V I D . L E .1)$

\$SUBR ADVAN2 TRANS2

\$PK

;COVARIATE MODEL

IF(GEST.LE.MGEST)THEN

GESTCL $=1$

GESTV $=(\text { GEST/MGEST })^{\star *}$ FACGESTV

ELSE

GESTCL $=(\text { GEST/MGEST })^{\star *}$ FACGESTCL2

GESTV $=(\text { GEST/MGEST })^{\star *}$ FACGESTV2

ENDIF

TVCL=POPCL *GESTCL* $(W T / 80)^{* *} F A C W T C L$

$T V V=P O P V^{*} G E S T V^{*}(\text { LBW } / 42)^{\star *}$ FACLBWV

TVKA=POPKA

;TIME AFTER DOSE

IF(AMT.GT.0)THEN

TDOS $=$ TIME

$T A D=0.0$

ENDIF

IF(AMT.EQ.0)TAD=TIME-TDOS

;MODEL FOR RANDOM BETWEEN SUBJECT VARIABILITY

$\mathrm{CL}=\mathrm{TVCL}{ }^{*} \mathrm{EXP}(\mathrm{PPVCL})$

$V=T V V^{*} E X P(P P V V)$

$K A=T V K A$

;SCALE CONCENTRATIONS X 1000 TO COVERT ANTI-XA FROM IU/ML TO IU/L $\mathrm{SC}=\mathrm{V}^{*} 1000$

\$ERROR

IPRE $=F$

$Y=I P R E \star E X P(E P S(1))+E P S(2)$

IWRE=DV-IPRE

\$THETA

$(0.1,1)$

$(1,15)$

$(0.01,3)$

$(0.445)$

$(-0.1)$

$(-0.5)$

(1.47)

$(0.165)$

;POPCL

;POPV

;POPKA

;FACGESTV

;FACGESTCL2

;FACGESTV2

;FACLBWV

;FACWTCL

\$OMEGA BLOCK(2)

$0.1 \quad ; \mathrm{PPVCL}$

$0.050 .1 \quad$;PPVV

\$SIGMA

0.1 ;PROPRUV 
0.03

;ADDRUV

\$EST MAX=9990 SIG=3 PRINT=1 METHOD=1 INT

$\$ C O V$ PRINT=E

\$TABLE ID TIME AMT DOSE DVID DV MDV WT AGE CRE CRCL TRI CL V KA ETA1 ETA2 IPRE TAD CWRES GEST ETHN MGEST

ONEHEADER NOPRINT FILE=finalmodel.fit 
Appendix IX: NONMEM bootstrap code for the final model

\$SIZES LIM6=2000000

\$PROB BASE PREGNANCY MODEL

\$INPUT ID AGE WT DAT1=DROP TIME AMT DOSE DVID DV MDV TRI CRE CRCL LBW BABY GEST ETHN MGEST

\$DATA ..Ifinal23.csv IGNORE=@

ACCEPT $=(D V I D . L E .1)$

\$SUBR ADVAN2 TRANS2

\$PK

;COVARIATE MODEL

IF(GEST.LE.MGEST)THEN

GESTCL $=1$

GESTV $=(\text { GEST/MGEST })^{\star *}$ FACGESTV

ELSE

GESTCL $=(\text { GEST/MGEST })^{\star \star}$ FACGESTCL2

GESTV $=(\text { GEST/MGEST })^{\star \star}$ FACGESTV2

ENDIF

TVCL $=P O P C L *{ }^{*} E S T C L *(W T / 80)^{* *} F A C W T C L$

$T V V=P O P V^{*} G E S T V^{*}(\text { LBW } / 42)^{\star *}$ FACLBWV

TVKA=POPKA

;MODEL FOR RANDOM BETWEEN SUBJECT VARIABILITY

$C L=T V C{ }^{*} E X P(P P V C L)$

$V=T V V^{*} \operatorname{EXP}(P P V V)$

$K A=T V K A$

;SCALE CONCENTRATIONS X 1000 TO COVERT ANTI-XA FROM IU/ML TO IU/L $\mathrm{SC}=\mathrm{V}^{*} 1000$

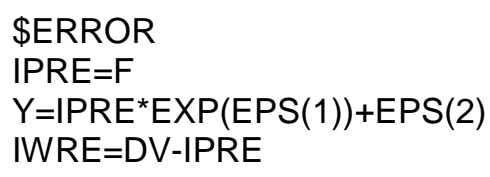


Appendix X: 'R' code for VPC of final model

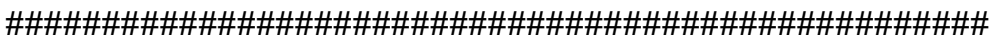

\#\#\#\#\#\#\#\# Enoxaparin in Pregnancy Study VPC \#\#\#\#\#\#\#\#\#\#

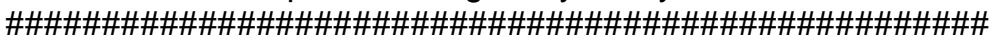

\# $\quad$ Clear History

rm(list=ls(all=TRUE)

simdataname<-"final450.nm7/"

\# READ RAW DATA

obdata <- read.csv(file=paste("c:/nm72/nm7/run/","final23.csv", sep=""),header=TRUE)

\# READ SIM DATA

simdata <- read.table(file=paste("c:/nm72/nm7/run/",simdataname,"final450.fit", sep="'), header $=$ FALSE, skip $=0$ )

tablerow<-c("ID", "TIME", "AMT", "DOSE", "DVID" , "DV", "MDV", "WT", "AGE","CRCL", "TAD", "GEST","ETHN", "REPI", "BABY", "TRI", "MGEST")

dimnames(simdata)<-list(1:nrow(simdata),tablerow)

simdata $<$-simdata[simdata $\$ A M T==0$,]

simdata $\$$ IDS $<-$ simdata $\$ R E P I^{*} 1000+$ simdata $\$ I D$

simdata\$DV<-simdata\$DV/(simdata\$DOSE/100)

obdata<-obdata[obdata $\$$ dvid $==1$,]

obdata $\$$ dose $<$-as.numeric(as.character(obdata $\$$ dose))

obdata $\$ D V<-o b d a t a \$ D V /($ obdata $\$$ dose $/ 100)$

obdata $\$ T A D<-$ simdata $\$ \mathrm{TAD}[$ simdata $\$ \mathrm{REPI}==1]$

obdata $\$$ TIME $<-$ simdata\$TIME[simdata $\$ R E P I==1]$

setwd("C:/nm72/nm7/run/GOF plots")

png $($ filename $=$ "Rplot $\% 03 \mathrm{~d}$. png", width $=480$, height $=480$, units $=$ "px", pointsize $=12$,

$\mathrm{bg}=$ "white", res = NA, family = "'", restoreConsole = TRUE,

type = c("windows", "cairo", "cairo-png"))

\#\#\#\#\#\#\#\#\#\#\#\#\#\#\#\#\#\#\#\#\#\#\#\#\#\#\#\#\#\#\#

\#\#\# GENERATE PLOT For VPC \#\#\#\#\#\#

\#\#\#\#\#\#\#\#\#\#\#\#\#\#\#\#\#\#\#\#\#\#\#\#\#\#

perc5<-function $(x)\{$ quantile $(x$, probs $=0.05)\}$

perc $10<-$ function $(x)\{$ quantile $(x$, probs $=0.1)\}$

perc50<-function $(x)\{$ quantile $(x$, probs $=0.5)\}$

perc $90<-$ function $(x)\{$ quantile $(x$, probs $=0.9)\}$

perc95<-function $(x)\{$ quantile $(x$, probs $=0.95)\}$

\#\#\#\#\#\#\#\#\#\#\#\#\#\#\#\#\#\#\#\#\#\#\#

\# Generate Percentiles \#

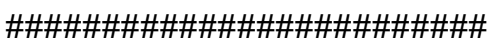

obdata $\$$ TIM $<$-round(obdata\$TIME)

obdata $\$$ TAD $<$-round(obdata\$TAD)

simdata $\$$ TIM<-round(simdata $\$$ TIME)

simdata $\$ T A D<-$ round(simdata $\$ T A D)$

simdata\$TIMBIN<-

ifelse(simdata $\$ \mathrm{TAD}<=2,1$, ifelse(simdata $\$ \mathrm{TAD}>2 \&$ simdata $\$ \mathrm{TAD}<=4,2$, ifelse(simdata $\$ \mathrm{TAD}>4 \&$ sim 
data $\$ \mathrm{TAD}<=6,5$, ifelse (simdata $\$ \mathrm{TAD}>6$ \& simdata $\$ \mathrm{TAD}<=10,8$, ifelse (simdata $\$ \mathrm{TAD}>10$ \& simdata $\$ \mathrm{~T}$ $A D<=15,12.5$, ifelse $($ simdata $\$ T A D>15 \&$ simdata $\$ T A D<=24,20,36)))))$ )

obdata\$TIMBIN<-

ifelse (obdata $\$ \mathrm{TAD}<=2,1$, ifelse(obdata $\$ \mathrm{TAD}>2$ \&obdata $\$ \mathrm{TAD}<=4,2$, ifelse (obdata $\$ \mathrm{~T} A \mathrm{D}>4$ \&obdata $\$ T A D<=6,5$, ifelse (obdata $\$ T A D>6$ \&obdata $\$ T A D<=10,8$, ifelse (obdata $\$ T A D>10$ \&obdata $\$ T A D<=15$, 12.5 , ifelse (obdata $\$ T A D>15 \&$ obdata $\$ T A D<=24,20,36))))$ ))

obpercentile5 <-(tapply(obdata\$DV,list(obdata\$TIMBIN),perc5))

obpercentile50<-(tapply(obdata\$DV, list(obdata\$TIMBIN),perc50))

obpercentile95<-(tapply(obdata\$DV, list(obdata\$TIMBIN), perc95))

simpercentile5 <-(tapply(simdata\$DV,list(simdata\$TIMBIN),perc5))

simpercentile50<-(tapply(simdata\$DV,list(simdata\$TIMBIN),perc50))

simpercentile95<-(tapply(simdata\$DV,list(simdata\$TIMBIN), perc95))

time<-sort(unique(obdata $\$$ TIMBIN))

timesim<-sort(unique(simdata\$TIMBIN))

plot (timesim,simpercentile5, ylim=c(-0.005,0.025),type="n", axes=F,ylab="',xlab="',xlim=c(-1,40))

points (obdata $\$ T A D, o b d a t a \$ D V, p c h=1, \operatorname{cex}=0.75$ )

lines (time,obpercentile95, Ity="dashed",Iwd=4, col="red")

lines (time,obpercentile50, Ity="solid",Iwd=4, col="red")

lines (time,obpercentile5, Ity="dashed",Iwd=4, col="red")

lines (timesim,simpercentile95, Ity="dashed",Iwd=4,col="blue")

lines (timesim, simpercentile50, Ity="solid",Iwd=2,col="blue")

lines (timesim,simpercentile5, Ity=2,Iwd=4,col="blue")

box ()

mtext(side $=1$,"Time After Last Dose (hr)",cex=0.75,line=3)

mtext(side $=2$,"Dose normalised Plasma Concentration $(\mathrm{mg} / \mathrm{mL})$ ",cex $=0.75$,line $=3$ )

$\operatorname{axis}(1, \operatorname{cex}=0.4)$

$\operatorname{axis}(2, \operatorname{cex}=0.4)$

dev.off() 


\section{Understanding your views on having to take enoxaparin whilst you are pregnant}

You have been asked to complete this questionnaire, as you are currently having enoxaparin therapy during your pregnancy.

Currently, very little information exists on pregnant women's views about taking medicines, in particularly enoxaparin during pregnancy.

As part of the enoxaparin in pregnancy study, we want to explore your views about medicines use, in particularly enoxaparin, during pregnancy and would like you to complete this questionnaire.

The questionnaire comprises of two main sections. The first section will investigate your views about medicine use in general during pregnancy. The second section will investigate your views about enoxaparin therapy specifically.

\section{Instructions}

In both sections, a series of statements have been made.

> A 5-point rating scale is used, going from strongly agree to strongly disagree. Please consider each statement in turn and tick the box that best describes your views to that statement.

At the end of the questionnaire, there is an opportunity for you to write any other comments you may have

Please answer every question. It will take approximately 10 minutes to complete.

Your answers will be completely anonymous and will be kept confidential.

There are no "right" answers to the questions - we are simply interested in your views.

Thank you for taking the time to complete this questionnaire 


\section{Section 1: General questions relating to medicine use in general during pregnancy}

This section explores your views and concerns (if any) about taking medicines in general whilst you are pregnant.

Please answer every question by ticking the box that best describes your views to each statement.

Please remember, there are no "right" answers to the questions, we are simply interested in your views.

\begin{tabular}{|c|c|c|c|c|c|c|}
\hline & & $\begin{array}{l}\text { Strongly } \\
\text { agree }\end{array}$ & Agree & Uncertain & Disagree & $\begin{array}{l}\text { Strongly } \\
\text { disagree }\end{array}$ \\
\hline G1 & Doctors use too many medicines during pregnancy & & & & & \\
\hline G2 & $\begin{array}{l}\text { Pregnant women who take medicines should stop their } \\
\text { treatment for a while every now and again }\end{array}$ & & & & & \\
\hline G3 & Most medicines are addictive & & & & & \\
\hline G4 & $\begin{array}{l}\text { Natural remedies are safer than medicines during } \\
\text { pregnancy }\end{array}$ & & & & & \\
\hline G5 & $\begin{array}{l}\text { Medicines used during pregnancy do more harm than } \\
\text { good }\end{array}$ & & & & & \\
\hline G6 & $\begin{array}{l}\text { All medicines are poisons when taken during } \\
\text { pregnancy }\end{array}$ & & & & & \\
\hline G7 & $\begin{array}{l}\text { Doctors place too much trust on medicines during } \\
\text { pregnancy }\end{array}$ & & & & & \\
\hline G8 & $\begin{array}{l}\text { If doctors had more time with patients they would } \\
\text { prescribe fewer medicines during pregnancy }\end{array}$ & & & & & \\
\hline A1 & $\begin{array}{l}\text { Over-the-counter medicines from a chemist are safer } \\
\text { than prescription medicines during pregnancy }\end{array}$ & & & & & \\
\hline & $\begin{array}{l}\text { Before considering to take a medicine during } \\
\text { pregnancy, I like to know about the safety of that } \\
\text { medicine to me and my unborn baby }\end{array}$ & & & & & \\
\hline A3 & $\begin{array}{l}\text { Most medicines are safe when taken during pregnancy } \\
\text { if prescribed by a doctor }\end{array}$ & & & & & \\
\hline A4 & $\begin{array}{l}\text { When deciding on whether to take a medicine whilst I } \\
\text { am pregnant, I place a higher priority on the impact the } \\
\text { medicine will have on the health of my unborn baby } \\
\text { than to any effects the medicine may have on me }\end{array}$ & & & & & \\
\hline
\end{tabular}




\section{Section 2: Specific questions relating to enoxaparin during pregnancy}

This section explores your views and concerns (if any) specifically around enoxaparin during pregnancy.

Please answer every question by ticking the box that best describes your views to each statement.

Please remember, there are no "right" answers to the questions, we are simply interested in your views.

\begin{tabular}{|c|c|c|c|c|c|c|}
\hline & & $\begin{array}{l}\text { Strongly } \\
\text { agree }\end{array}$ & Agree & Uncertain & Disagree & $\begin{array}{l}\text { Strongly } \\
\text { disagree }\end{array}$ \\
\hline s1 & My health at present depends on enoxaparin & & & & & \\
\hline$\$ 2$ & Having to take enoxaparin worries me & & & & & \\
\hline s3 & My life would be impossible without enoxaparin & & & & & \\
\hline$\$$ & Without enoxaparin I would be very ill & & & & & \\
\hline s5 & $\begin{array}{l}\text { I sometimes worry about the long term effects of } \\
\text { enoxaparin }\end{array}$ & & & & & \\
\hline 56 & The enoxaparin is a mystery to me & & & & & \\
\hline S7 & My health in the future depends on enoxaparin & & & & & \\
\hline s8 & The enoxaparin disrupts my life & & & & & \\
\hline s9 & $\begin{array}{l}\text { I sometimes worry about becoming too dependent on } \\
\text { enoxaparin }\end{array}$ & & & & & \\
\hline s10 & Enoxaparin protects me from becoming worse & & & & & \\
\hline R1 & My current pregnancy depends on enoxaparin & & & & & \\
\hline R2 & $\begin{array}{l}\text { Having to take enoxaparin during pregnancy worries } \\
\text { me }\end{array}$ & & & & & \\
\hline R3 & $\begin{array}{l}\text { My current pregnancy would be impossible without } \\
\text { enoxaparin }\end{array}$ & & & & & \\
\hline R4 & $\begin{array}{l}\text { Without taking enoxaparin during this pregnancy I } \\
\text { would be very ill }\end{array}$ & & & & & \\
\hline R5 & $\begin{array}{l}\text { I sometimes worry about the long term effects of } \\
\text { enoxaparin } \\
\text { on my unborn baby }\end{array}$ & & & & & \\
\hline R6 & $\begin{array}{l}\text { Any pregnancies in the future will depend on me } \\
\text { taking enoxaparin }\end{array}$ & & & & & \\
\hline$R^{R}$ & Enoxaparin is protecting my pregnancy & & & & & \\
\hline
\end{tabular}


Section 2 continued:

\begin{tabular}{|c|c|c|c|c|c|c|}
\hline & & $\begin{array}{l}\text { Strongly } \\
\text { agree }\end{array}$ & Agree & Uncertain & Disagree & $\begin{array}{l}\text { Strongly } \\
\text { disagree }\end{array}$ \\
\hline SA1 & $\begin{array}{l}\text { I sometimes worry about that being on enoxaparin will } \\
\text { limit the type of child birth delivery I can have }\end{array}$ & & & & & \\
\hline $\mathrm{SA}_{2}$ & Enoxaparin protects my unborn baby's health & & & & & \\
\hline SA3 & The enoxaparin does not cause me any side-effects & & & & & \\
\hline SA4 & $\begin{array}{l}\text { I sometimes worry that being on enoxaparin will limit } \\
\text { the type of pain relief I can have at the time of child } \\
\text { birth }\end{array}$ & & & & & \\
\hline SA5 & $\begin{array}{l}\text { Having to take enoxaparin throughout pregnancy is } \\
\text { not an issue for me, as long as it helps to protect } \\
\text { mine and my unborn baby's health }\end{array}$ & & & & & \\
\hline SA6 & $\begin{array}{l}\text { Enoxaparin only works if taken regularly during } \\
\text { pregnancy }\end{array}$ & & & & & \\
\hline SA7 & $\begin{array}{l}\text { It is difficult for me to take my enoxaparin in exactly } \\
\text { the way my doctor has told me }\end{array}$ & & & & & \\
\hline SAB & $\begin{array}{l}\text { I sometimes worry that being on enoxaparin during } \\
\text { pregnancy will interfere with my ability to breastfeed } \\
\text { when my baby is born }\end{array}$ & & & & & \\
\hline SA9 & $\begin{array}{l}\text { Having to inject myself with enoxaparin daily is not an } \\
\text { issue for me, as long as the health of my unborn baby } \\
\text { is protected }\end{array}$ & & & & & \\
\hline
\end{tabular}

If you have any other comments you would like to make, please make them below: 